June 2001 • NREL/SR-500-29439

\title{
WindPACT Turbine Design Scaling Studies Technical Area 2: Turbine, Rotor, and Blade Logistics
}

\section{March 27, 2000 to December 31, 2000}

Kevin Smith

Global Energy Concepts LLC

Kirkland, Washington

\section{NPEI}

National Renewable Energy Laboratory

1617 Cole Boulevard

Golden, Colorado 80401-3393

NREL is a U.S. Department of Energy Laboratory

Operated by Midwest Research Institute $\bullet$ Battelle $\bullet$ Bechtel

Contract No. DE-AC36-99-G010337 
June 2001 • NREL/SR-500-29439

\section{WindPACT Turbine Design Scaling Studies Technical Area 2: Turbine, Rotor, and Blade Logistics}

March 27, 2000 to December 31, 2000

Kevin Smith

Global Energy Concepts LLC

Kirkland, Washington

NREL Technical Monitor: Alan Laxson

Prepared under Subcontract No. YAM-0-30203-01

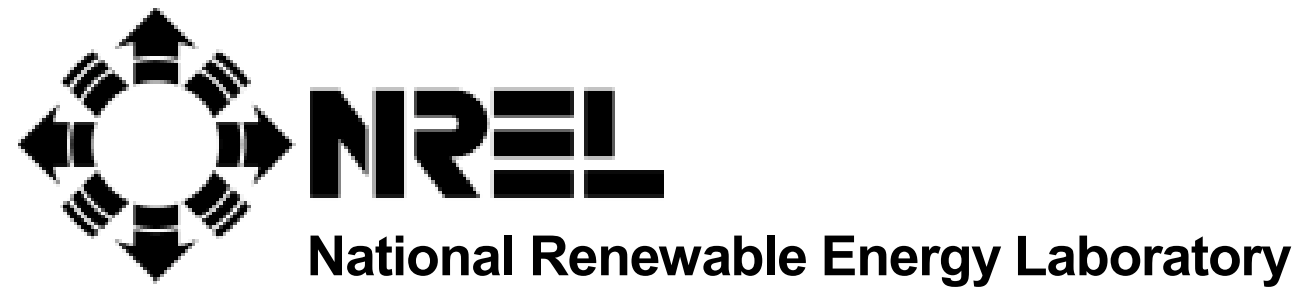

1617 Cole Boulevard

Golden, Colorado 80401-3393

NREL is a U.S. Department of Energy Laboratory

Operated by Midwest Research Institute • Battelle • Bechtel

Contract No. DE-AC36-99-G010337 


\section{NOTICE}

This report was prepared as an account of work sponsored by an agency of the United States government. Neither the United States government nor any agency thereof, nor any of their employees, makes any warranty, express or implied, or assumes any legal liability or responsibility for the accuracy, completeness, or usefulness of any information, apparatus, product, or process disclosed, or represents that its use would not infringe privately owned rights. Reference herein to any specific commercial product, process, or service by trade name, trademark, manufacturer, or otherwise does not necessarily constitute or imply its endorsement, recommendation, or favoring by the United States government or any agency thereof. The views and opinions of authors expressed herein do not necessarily state or reflect those of the United States government or any agency thereof.

Available electronically at http://www.doe.gov/bridge

Available for a processing fee to U.S. Department of Energy

and its contractors, in paper, from:

U.S. Department of Energy

Office of Scientific and Technical Information

P.O. Box 62

Oak Ridge, TN 37831-0062

phone: 865.576.8401

fax: 865.576.5728

email: reports@adonis.osti.gov

Available for sale to the public, in paper, from:

U.S. Department of Commerce

National Technical Information Service

5285 Port Royal Road

Springfield, VA 22161

phone: 800.553.6847

fax: 703.605.6900

email: orders@ntis.fedworld.gov

online ordering: http://www.ntis.gov/ordering.htm

Printed on paper containing at least $50 \%$ wastepaper, including $20 \%$ postconsumer waste 


\section{Executive Summary}

Through the National Renewable Energy Laboratory (NREL), the United States Department of Energy (DOE) implemented the Wind Partnership for Advanced Component Technologies (WindPACT) program. This program will explore advanced technologies that may reduce the cost of energy (COE) from wind turbines. The initial step in the WindPACT program is a series of preliminary scaling studies intended to determine the optimum sizes for future turbines, help define sizing limits for certain critical technologies, and explore the potential for advanced technologies to contribute to reduced COE as turbine scales increase. This report documents the results of Technical Area 2-Turbine Rotor and Blade Logistics.

For this report, we investigated the transportation, assembly, and crane logistics and costs associated with installation of a range of multi-megawatt-scale wind turbines. We focused on using currently available equipment, assembly techniques, and transportation system capabilities and limitations to hypothetically transport and install 50-wind turbines at a facility in southcentral South Dakota.

We found that, as turbine scales increase, logistics costs also increase. The application of transportation and assembly techniques (not currently used by the wind industry) to minimize costs served only to change the rate of increase and extend the point at which dramatic cost increases are incurred. The breakpoint we determined, falls between the 2500-kilowatt and 3500$\mathrm{kW}$ turbines. A decrease in logistics costs to values less than those of the $750-\mathrm{kW}$ turbines may not, however, be realized as turbine scale increases. The logistics costs associated with a smaller number of multi-megawatt turbines would likely be higher than those for the 50 turbines assumed in this study, and would result in breakpoints at lower megawatt turbine sizes because the fixed costs would be distributed over fewer turbines.

Tower transportation factors have the greatest influence on logistics costs. Alternative tower configurations may offer the best opportunity to reduce the overall logistical costs. Significant breakpoints occur at the $2500-\mathrm{kW}$ turbine size and 80 -meter hub height. Turbine designers should be aware of these points and factor in the impacts of exceeding them into the machine design. 


\section{Table of Contents}

1. Introduction ......................................................................................................................... 1-1

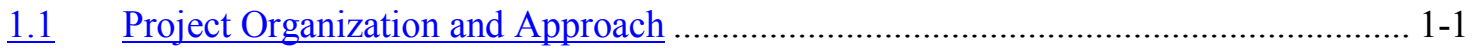

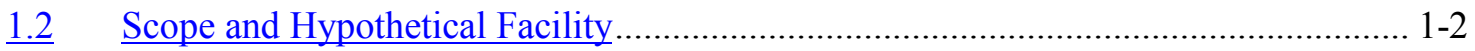

2. Multi-megawatt Turbine Scaling ................................................................................... 2-1

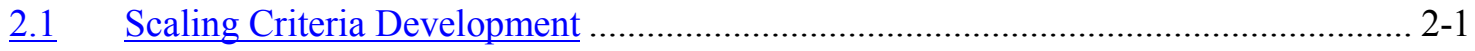

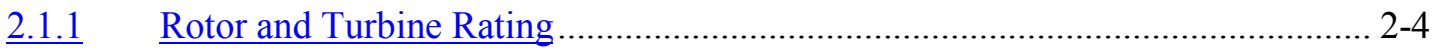

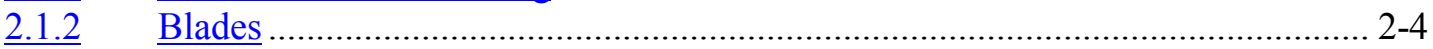

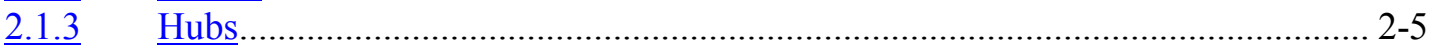

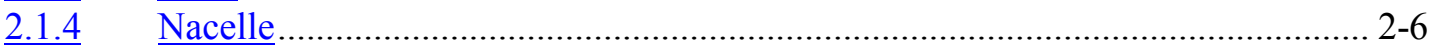

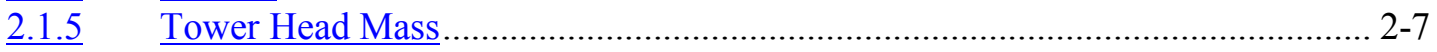

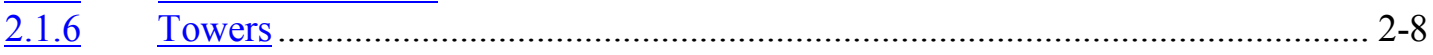

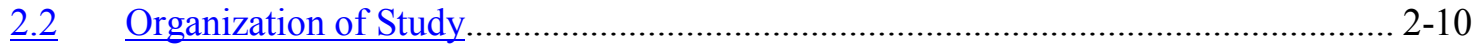

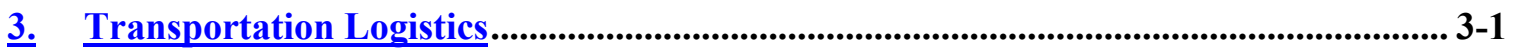

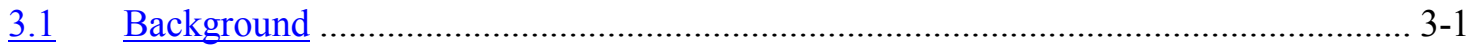

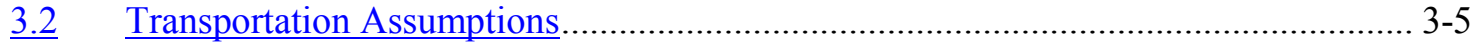

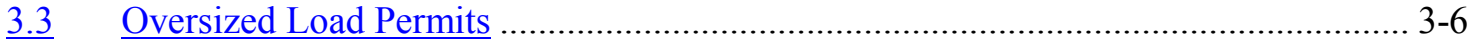

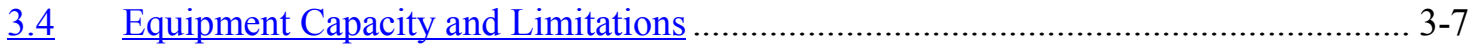

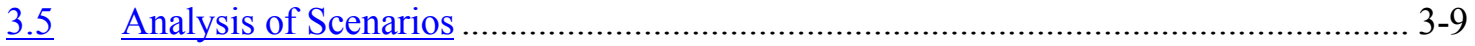

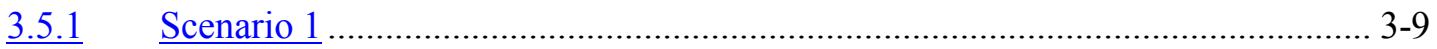

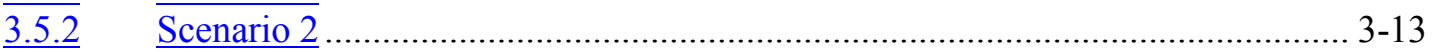

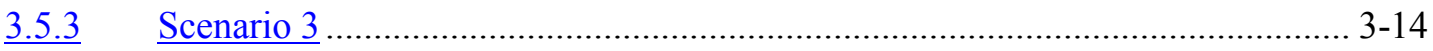

3.6 Emerging Transportation Technology-Airships ….............................................. 3-15

4. $\quad$ Assembly and Crane Logistics ........................................................................................ 4

4.1 Assembly Assumptions ....................................................................................... 4-1

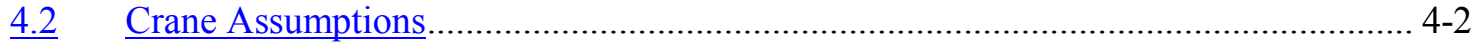

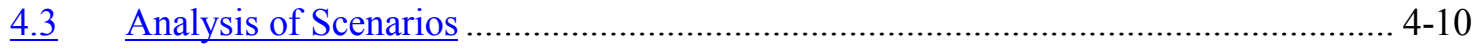

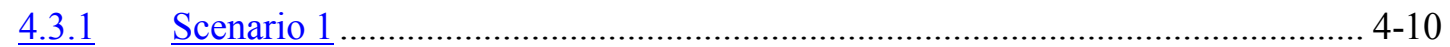

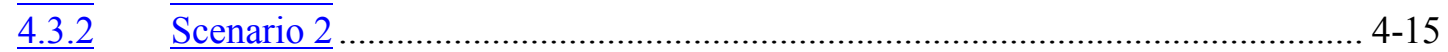

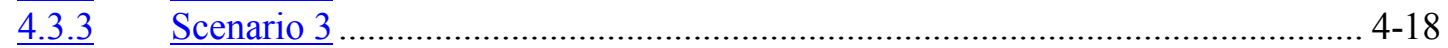

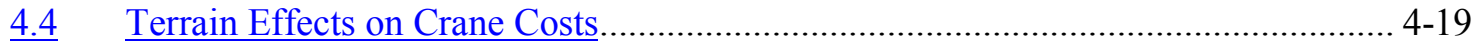

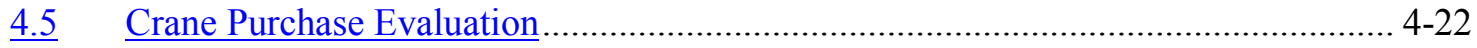

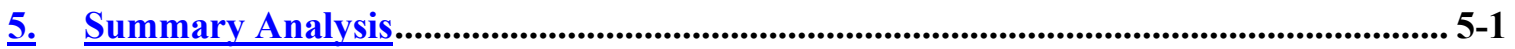

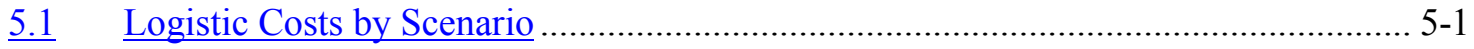

5.2 Logistic Costs for South Dakota Site …............................................................ 5-2

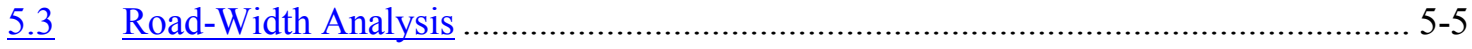




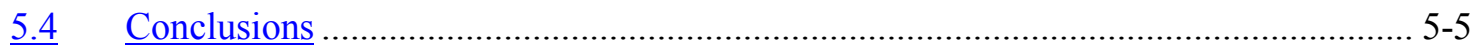

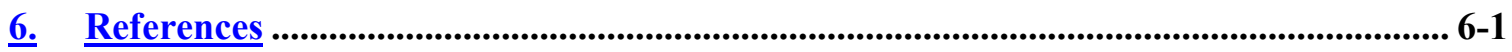

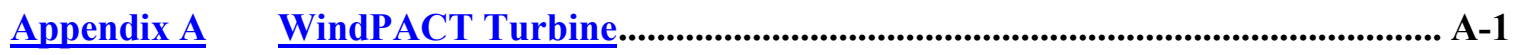

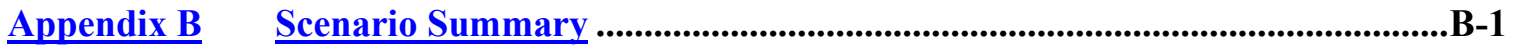

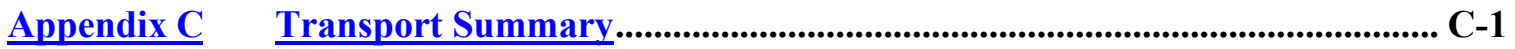

Appendix D $\quad$ Truck Transport...................................................................................................... D-1

Appendix E Dolly Transport

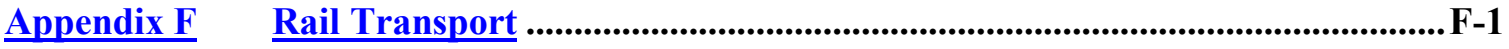

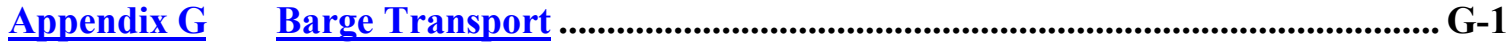

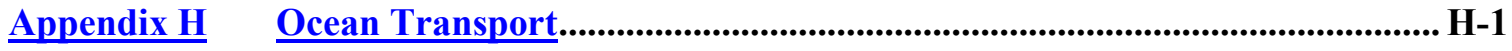

Appendix I $\quad$ Scenario 1..............................................................................................................I-1

Appendix J $\quad$ Scenario 2-Bolting............................................................................................. J-1

Appendix K $\quad$ Scenario 2-Welding.................................................................................................. K-1

Appendix L Scenario 2-Automated Welding..........................................................................L-1

Appendix M Scenario 3 .........................................................................................................

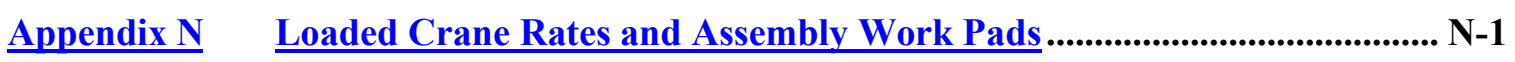

$\underline{\text { Appendix } O} \quad$ Terrain Effects on Cranes....................................................................................... O-1

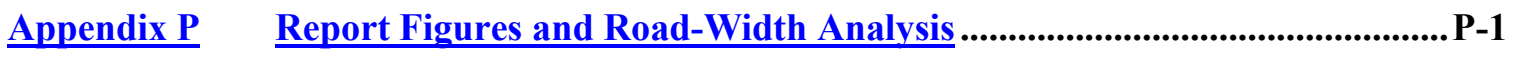

Appendix Q $\quad$ Mortenson Estimates............................................................................................. Q-1

Appendix R Lampson Estimates

$\underline{\text { Appendix } S} \quad$ Crane Purchase Evaluation ...................................................................................... S-1 


\section{List of Figures}

Figure 2-1.Comparison of blade mass equations ............................................................. 2-5

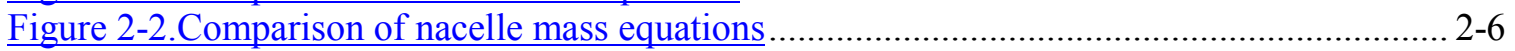

Figure 2-3.Comparison of tower head mass equations..................................................... 2-8

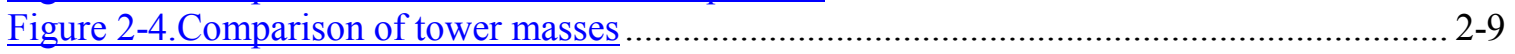

Figure 3-1.Union Pacific Railroad network …...................................................................... 3-2

Figure 3-2.Burlington Northern-Santa Fe Railroad network.................................................... 3-3

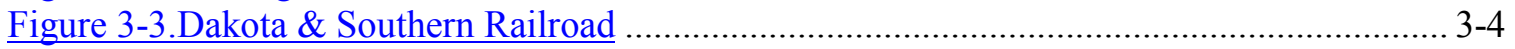

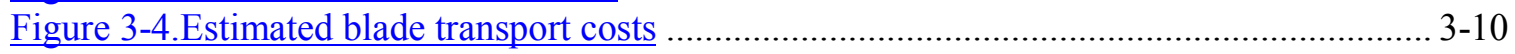

Figure 3-5.Hub transportation costs from Chicago, Illinois .................................................... 3-11

Figure 3-6.Comparison of nacelle transport modes from Chicago, Illinois (short haul)........... 3-12

Figure 3-7.Scenario 1 and Scenario 2 tower transportation cost comparisons.......................... 3-14

Figure 3-8. Comparison of nacelle transport approaches .................................................... 3-15

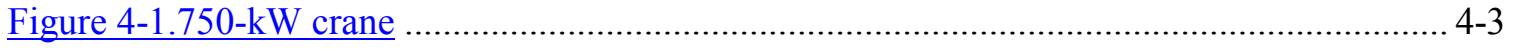

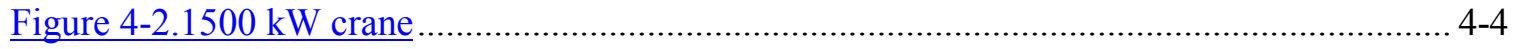

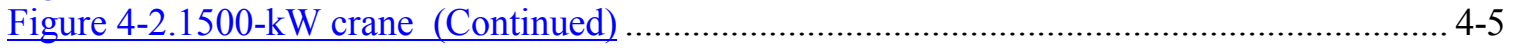

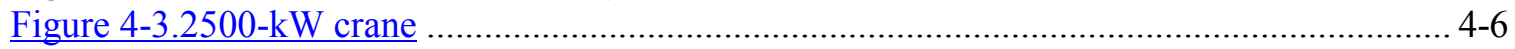

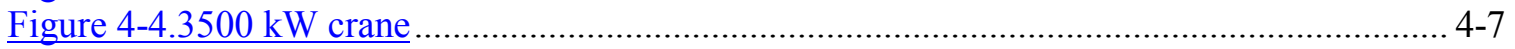

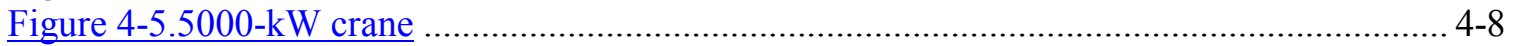

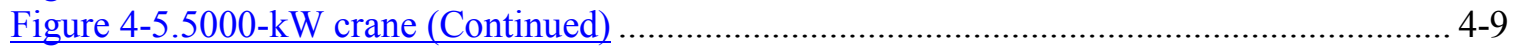

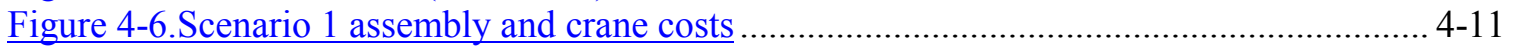

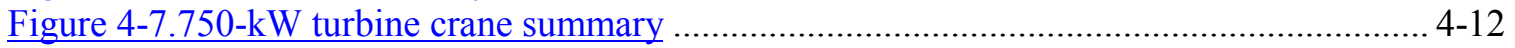

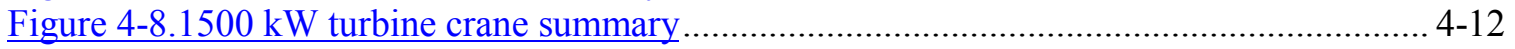

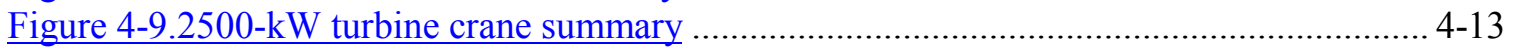

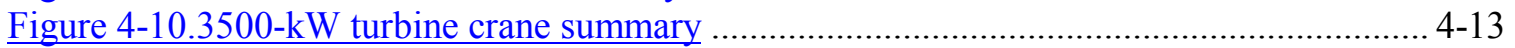

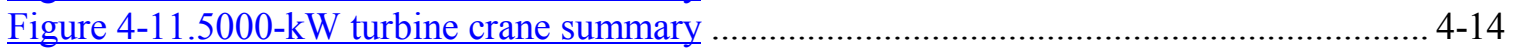

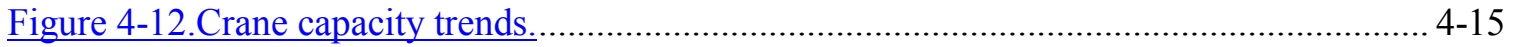

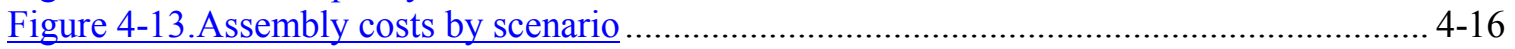

Figure 4-14.Combined assembly and crane costs by scenario ............................................. 4-17

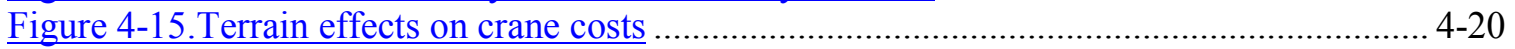

Figure 4-16.Influence of terrain impact to turbine assembly rates......................................... 4-21

Figure 4-17.Influence of terrain impacts on project duration.................................................... 4-21

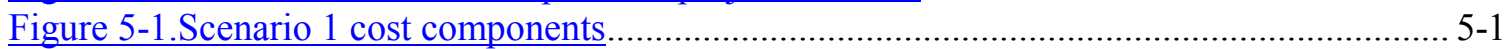

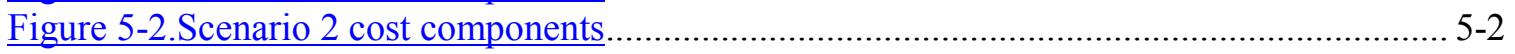

Figure 5-4.Logistic costs for multi-megawatt turbines in South Dakota...................................... 5-3

Figure 5-5.Scaling relationships by rotor diameter ................................................................ 5-4

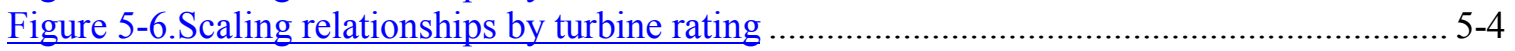




\section{List of Tables}

Table 2-1.WindPACT Turbine, Rotor, and Nacelle Specifications .......................................... 2-2

Table 2-2.WindPACT Tubular Steel Tower Specifications ................................................... 2-3

Table 2-3.Comparison of Manufacturer Tower Data with GEC Calculations ........................... 2-10

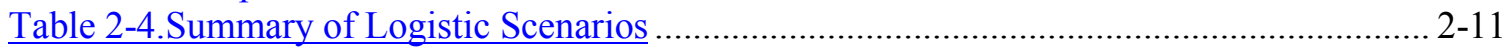

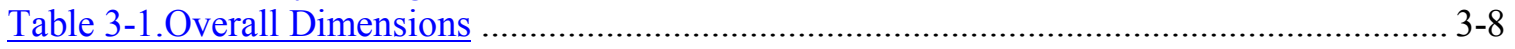

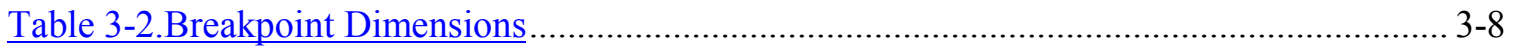

Table 3-3.Example Rail Costs from Houston, Texas ............................................................... 3-15

Table 4-1.Impacts of Sectional Towers on Project Costs (Units: $\$ / \mathrm{kW}$ ) .................................. 4-17

Table 4-2.Nacelles and Components Lifting Parameters ............................................................ 4-18

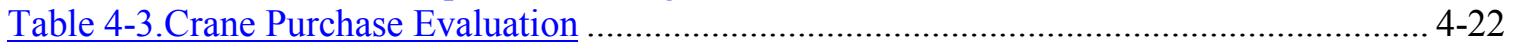

Table 5-1.On-site Road Widths Based on Logistics Equipment ................................................. 5-5 


\section{Introduction}

Through the National Renewable Energy Laboratory (NREL), the United States Department of Energy (DOE) has implemented the Wind Partnership for Advanced Component Technologies (WindPACT) program. This program will explore advanced technologies for reducing the cost of energy $(\mathrm{COE})$ from wind turbines. The initial step in the WindPACT program is a series of preliminary scaling studies intended to determine the optimum sizes for future turbines, help define sizing limits for certain critical technologies and explore the potential for advanced technologies to contribute to reduced COE as turbine scales increase. We identified four technical areas for examination in this initial phase; Technical Area 1, Blade Scaling; Technical Area 2, Turbine Rotor and Blade Logistics; and Technical Area 3, Self-Erecting Towers; and Technical Area 4, Balance of Station.

Global Energy Concepts, LLC (GEC), was awarded contract number YAM-0-30203-01 to examine Technical Areas 1, 2, and 3. This report documents the results of GEC's Technical Area 2 study, which includes an investigation of the transportation, assembly, and crane logistics and costs associated with installing multi-megawatt scale wind turbines. We focused on using currently available equipment, assembly techniques, and transportation system capabilities and limitations to hypothetically transport and install 50 wind turbines at a facility in south-central South Dakota.

\subsection{Project Organization and Approach}

To complete this work, GEC assembled a project team including experts in the areas of transportation, turbine construction and erection, and crane work. These consultants include Lampson International Ltd. (Lampson) of Kennewick, Washington; American Transport Systems (ATS) of Vineland, New Jersey; and M.A. Mortenson (Mortenson) of Minneapolis, Minnesota. Lampson has provided cranes and other equipment for several wind energy installations, including the 1.65-MW Vestas wind turbines in Texas. ATS is a nationally recognized company that handles all aspects of shipping and hauling; they have been involved the transporting wind turbine components to and from various points in the United States for several different vendors. Mortenson is a large construction company that has served as general contractor for multiple U.S. wind projects in the last few years.

GEC used the following general approach to the work:

1. Researched turbine scaling relationships, assembled information for existing megawattscale turbines; and used this information to compute component sizes and weights for use in evaluating transportation, crane, and assembly requirements.

2. Researched and compiled pertinent information such as (a) size, availability, and costs of large cranes and other required equipment; (b) weight and size limitations of existing transportation options including rail links, road trucking, and water transport; (c) costs and requirements for customized transport and special circumstances allowable through permitting; and (d) routing restrictions, permit requirements, and costs.

3. Considered historical experience to identify potential problem areas and build on the lessons already learned in the industry. This included reviewing historic reports related to the Boeing MOD 2 and MOD 5 turbines. 
4. Solicited information from project partners on logistic approaches used in other industries and applications for objects that were comparable in scale to the multi-megawatt WindPACT turbines.

5. Contacted transportation authorities and government agencies to further qualify and quantify options and identify limitations.

6. Established a set of realistic and viable options for transportation and erection logistics and developed cost assumptions for each of these options.

7. Conducted parametric cost analysis to demonstrate the impact of turbine scale on the costs of transportation and turbine erection.

8. Evaluated the viability of nontraditional options such as field assembly of nacelle subcomponents or towers.

9. Established the purchase cost for an appropriate crane, crew requirements, mobilization, and maintenance costs, and evaluated the economics of such a purchase by amortizing these costs over the facility and others in the region.

\subsection{Scope and Hypothetical Facility}

We investigated the transportation, assembly, and crane logistics associated with megawatt-scale wind turbines. Construction of turbine foundations, substation, site roads, the on-site electrical grid, and other miscellaneous items were not considered in this study but were evaluated by others in the WindPACT Technical Area 4 (Balance of Station Costs) study.

We assumed that the hypothetical facility was located near Mission, South Dakota, in an area of rolling hills characterized by a wind power class of 5 to 6 ( 7.5 to 8.5 meters per second at $50 \mathrm{~m})$. We also assumed that the 50 turbines were installed on a grid with $2 \mathrm{D}$ by $10 \mathrm{D}$ spacing ${ }^{1}(\mathrm{D}$ corresponding to rotor diameters). The five turbine sizes evaluated in this study were 750,1500 , 2500,3500 , and 5000 kilowatts. The corresponding facility capacities were $37.5,75,125,175$, and $250 \mathrm{MW}$, respectively. In the following sections, we identify other assumptions as they relate to specific investigations.

\footnotetext{
${ }^{1}$ The WindPACT Technical Area 4-Balance of Station Costs study calculated a grid spacing of 2.3 D by 12 D based on wind resource and topography data. From a logistics perspective there is no significant difference between these grid dimension and those assumed in this logistics report.
} 


\section{Multi-megawatt Turbine Scaling}

\subsection{Scaling Criteria Development}

In order to conduct a study into the logistics associated with multi-megawatt turbines, it was essential that reasonable estimates of turbine component dimensions and masses were derived. The basic configuration of the multi-megawatt WindPACT turbines draws heavily on the existing generation of megawatt-scale turbines. We assumed that all WindPACT turbines were three bladed, upwind, pitch-controlled turbines installed onto tubular steel towers.

We derived the component dimensions and masses for five turbine sizes from the various scaling assumptions and equations discussed below. We also performed literature searches to identify applicable documents that contain scaling equations. As part of developing the scaling relationships, a database of commercial megawatt-scale turbines was prepared to evaluate actual component masses, rotor and hub height relationships, and rotor and power relationships. We present a summary of the specifications used for the WindPACT turbines in Table 2-1 and a summary of the WindPACT tower specifications in Table 2-2. Appendix A contains the turbine database in conjunction with component analysis. 
Table 2-1.WindPACT Turbine, Rotor, and Nacelle Specifications

\begin{tabular}{|c|c|c|c|c|c|c|c|}
\hline & \multirow{2}{*}{$\begin{array}{c}\text { Units } \\
\mathbf{k W} \\
\end{array}$} & \multicolumn{5}{|c|}{ Turbine Ratings } & \multirow{2}{*}{ Notes, References, Assumptions } \\
\hline & & 750 & 1500 & 2500 & 3500 & 5000 & \\
\hline Calculated Rating & kW & 864 & 1505 & 2497 & 3456 & 49976 & Back calculated from rotor diameter using $0.44 \mathrm{~kW} / \mathrm{m}^{2}$ \\
\hline No. of Turbines & each & 50 & 50 & 50 & 50 & 50 & \\
\hline Facility Capacity & MW & 37.5 & 75 & 125 & 175 & 250 & Local 115kV line can handle up to 150MW per WAPA survey \\
\hline \multicolumn{8}{|l|}{ Rotor } \\
\hline Diameter (D) & $\mathrm{m}$ & 50 & 66 & 85 & 100 & 120 & Selected rotor diameter, back calculated turbine power using $0.44 \mathrm{~kW} / \mathrm{m}^{2}$ \\
\hline Swept Area & $\mathrm{m}^{2}$ & 1963 & 3421 & 5675 & 7854 & 11310 & \\
\hline No. of Blades & each & 3 & 3 & 3 & 3 & 3 & Assumes 3-bladed, upwind rotor configuration. \\
\hline Hub Height & $\mathrm{m}$ & 65 & 86 & 111 & 130 & 156 & Used ratio of tower height/rotor diameter of 1.3 per SOW. \\
\hline Rotor Mass & $\mathrm{kg}$ & 12635 & 30819 & 58061 & 88727 & 142783 & No. of blades $\mathrm{x}$ blade mass + hub mass \\
\hline Solidity & - & 0.05 & 0.05 & 0.05 & 0.05 & 0.05 & Assumed typical for 3-bladed rotors. \\
\hline Projected Area & $\mathrm{m}^{2}$ & 98 & 171 & 284 & 393 & 565 & Calculated based on assumed solidity. \\
\hline \multicolumn{8}{|l|}{ Hub } \\
\hline Hx Dia. & $\mathrm{m}$ & $2.25 \times 2.25$ & $3.2 \times 3.8$ & $3.8 \times 4$ & $3.8 \times 4$ & $4.2 \times 4.5$ & \\
\hline Mass & $\mathrm{kg}$ & 3816 & 12516 & 22457 & 34136 & 54604 & Hub mass for $2.5 \mathrm{MW}+$ turbines based on Hub Mass Graph. $m=0.24 \mathrm{D}^{2.5765}$ \\
\hline \multicolumn{8}{|l|}{ Blade (each) } \\
\hline Length & $\mathrm{m}$ & 24.5 & 32.3 & \begin{tabular}{l|l}
41.7 \\
\end{tabular} & 49.0 & 58.8 & Assumes $2.0 \%$ of blade length is comprised of the hub. \\
\hline Maximum Chord & $\mathrm{m}$ & 2.5 & 3.3 & 4.3 & 5.0 & 6.0 & Value based on $5 \%$ of rotor daimeter. \\
\hline Maximum Diameter & $\mathrm{m}$ & 1.35 & 1.78 & 2.29 & 2.70 & 3.23 & $5.5 \%$ of blade length \\
\hline Mass & $\mathrm{kg}$ & 2940 & 6101 & 11868 & 18197 & 29393 & EWEA document. Figure $4.5 .2 \mathrm{~m}=0.1 \mathrm{D}^{2.63}$ \\
\hline \multicolumn{8}{|l|}{ Nacelle } \\
\hline Overall $\mathrm{L} \times \mathrm{W} \times \mathrm{H}$ & $\mathrm{m}$ & $6 \times 3 \times 3$ & $9 \times 3.5 \times 3.5$ & $10 \times 4 \times 4$ & $12 \times 4 \times 4$ & $15 \times 4.5 \times 4.5$ & \\
\hline Total Nacelle Mass & $\mathrm{kg}$ & 31081 & 60517 & 111065 & 164049 & 254102 & EWEA document. Figure $4.6 .3 \mathrm{~m}=2.60 \mathrm{D}^{2.4}$ \\
\hline Rated Nacelle Mass & $\mathrm{kg} / \mathrm{kW}$ & 41 & 40 & 44 & \begin{tabular}{l|l}
47 & -10 \\
\end{tabular} & 51 & \\
\hline Empty Nacelle Mass & & 23311 & $\begin{array}{ll}46173 \\
\end{array}$ & 85839 & 127575 & 199170 & \\
\hline Gearbox Mass & $\mathrm{kg}$ & 4662 & 9078 & 16660 & 24607 & 38115 & Estimated as $15 \%$ of Nacelle mass \\
\hline Generator Mass & $\mathrm{kg}$ & 3108 & 5267 & 8567 & 11867 & 16817 & Estimated at $10 \%$ of Nacelle mass \\
\hline \multicolumn{8}{|l|}{ Tower Head Mass } \\
\hline Mass & $\mathrm{kg}$ & 45428 & 91747 & 174091 & 262708 & 416815 & NREL and TVP Turbines Head Mass Graph, $m=2.2692\left(D^{2.5318}\right)$ \\
\hline Rated Mass & $\mathrm{kg} / \mathrm{kW}$ & 61 & 61 & 70 & 75 & 83 & \\
\hline Specific Mass & $\mathrm{kg} / \mathrm{m}^{2}$ & 23 & 27 & 31 & 33 & 37 & \\
\hline
\end{tabular}




\section{Table 2-2.WindPACT Tubular Steel Tower Specifications}

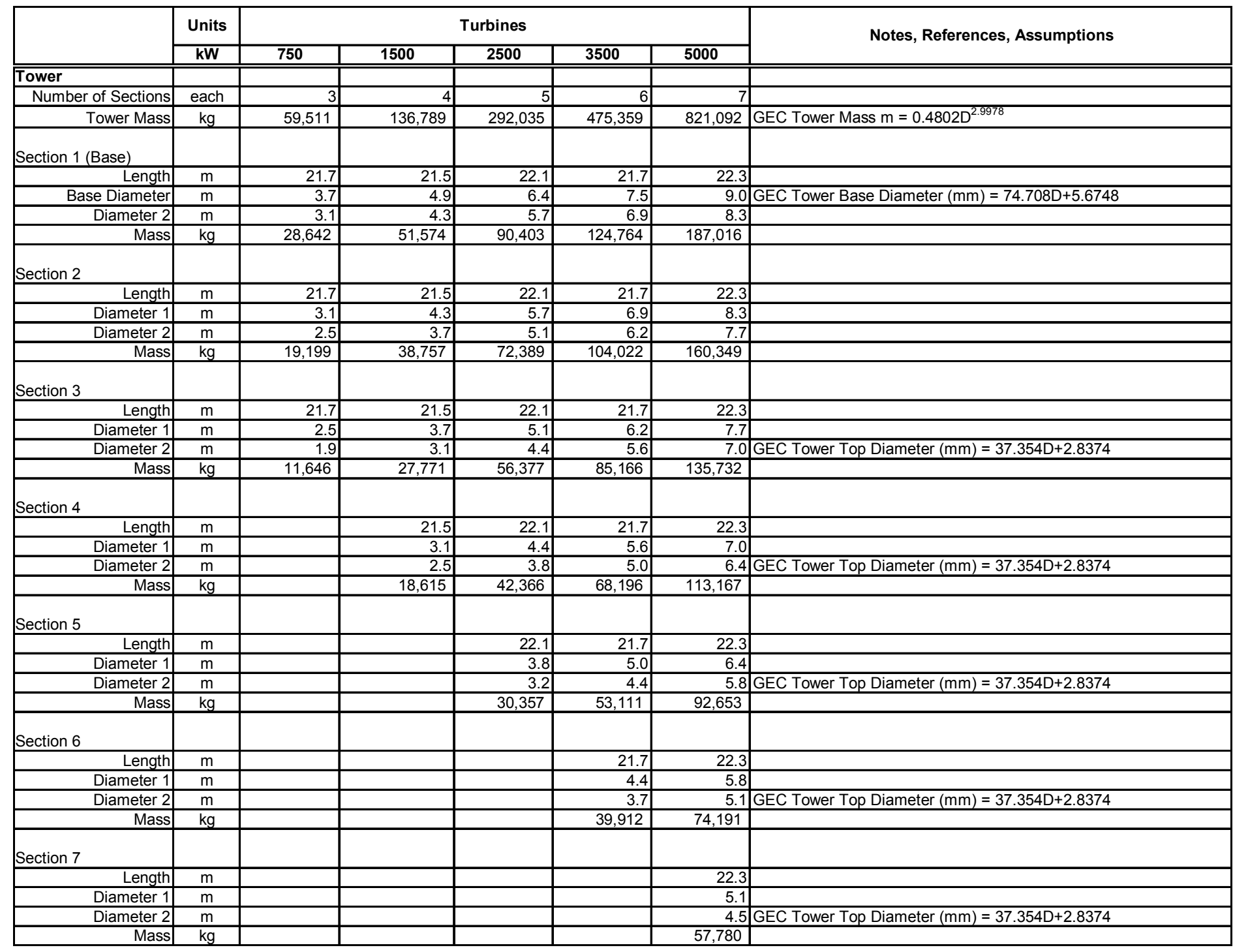


When evaluating the results of this study, a good understanding of the turbine specifications, dimensions, and masses - and how these may differ slightly from existing machines - is required in order to conduct proper comparisons.

\subsubsection{Rotor and Turbine Rating}

Because the rotor diameter has the largest single influence on the design and scale of a turbine and most component scaling equations are a function of the rotor diameter, GEC (working with NREL) elected to specify the rotor dimensions and calculate all other dimensions based on published equations or those derived from the turbine database. The rotor diameters selected were $50 \mathrm{~m}(164 \mathrm{ft}), 66 \mathrm{~m}(217 \mathrm{ft}), 85 \mathrm{~m}(279 \mathrm{ft}), 100 \mathrm{~m}(328 \mathrm{ft})$, and $120 \mathrm{~m}(394 \mathrm{ft})$. An assumed ratio between swept area and rated power of 0.44 was used to calculate the rated power for each turbine. This ratio was derived from the average of the ratio values contained in the turbine database. Applying this ratio resulted in power ratings for the WindPACT turbines of 864,1505 , 2497,3456 , and $4976 \mathrm{~kW}$, respectively. For discussion purposes, we classified these turbines as $750,1500,2500,3500$, and $5000 \mathrm{~kW}$.

Because the power to swept area ratio of 0.44 was derived from a megawatt-scale turbine database, it produced an overrated turbine at the $50-\mathrm{m}$ rotor diameter scale $(864 \mathrm{~kW})$. In general, machines less than a megawatt have had power to swept area ratios between 0.36 and 0.4 . Using the 0.44 relationship, a $47-\mathrm{m}$. diameter rotor would result in a turbine rating of $763 \mathrm{~kW}$, which is closer to the "750-kW" turbine; however, the difference in component dimensions and masses between a $47-\mathrm{m}$ and $50-\mathrm{m}$. diameter rotor as calculated with the various scaling equations, was negligible from a logistics perspective. Where costs per $\mathrm{kW}$ are presented in this report, the calculated turbine power $(864,1505,2497,3456$, and $4976 \mathrm{~kW})$ is utilized.

Hub height was fixed across each turbine by the hub height to rotor diameter ratio of 1.3. Current design practices use ratios between 1 and 1.3. A relatively high ratio was assumed due to the intended land-based installation, the developing industry sense that wind shear may be higher than previously believed, and the desire to examine the upper limits of component scaling. The 1.3 ratio resulted in the $1500-\mathrm{kW}$ turbine (66-m rotor diameter) having a hub height of $86 \mathrm{~m}$ $(282.2 \mathrm{ft})$. Currently, 65-m (213.3 ft) and 80-m (262.5 ft) hub heights are typical for this class of turbine. Although the $1500-\mathrm{kW}$ WindPACT turbine breaks from common practice in this regard, it pushes the scaling limits, and use of the 1.3 ratio resulted in identification of breakpoints associated with the transportation and crane logistics. The latter two issues are discussed further in Sections 3 and 4, respectively.

A rotor solidity value of $5 \%$ of the swept area for the three-bladed rotor was selected to facilitate calculation of rotor thrust as part of determining the tower dimensions and masses. This percentage was selected as an average value based on manufacturer information compiled in Technical Area 1 (Blade Scaling) and Figure 5.2 (from Cost Modeling of Horizontal Axis Wind Turbines [1]).

\subsubsection{Blades}

Assuming that the blades are composed of glass-reinforced fiber, the blade mass was estimated based on the following mass equation [2]:

$$
\mathrm{m}=0.1 \mathrm{D}^{2.63}
$$

Equation 2.1

In Figure 2-1, we presented a comparison of this equation to the blade-scaling results of GEC's WindPACT Technical Area 1 study. Recent commercial blade-mass data indicate that actual 
masses are about 20\% less than shown in Equation 2.1; however, this trend does not appear to vary as the blade length increases. Because we determined blade mass to be insignificant in comparison to the physical dimensions, using the above blade mass equation (that overestimates blade mass) does not raise any logistical issues

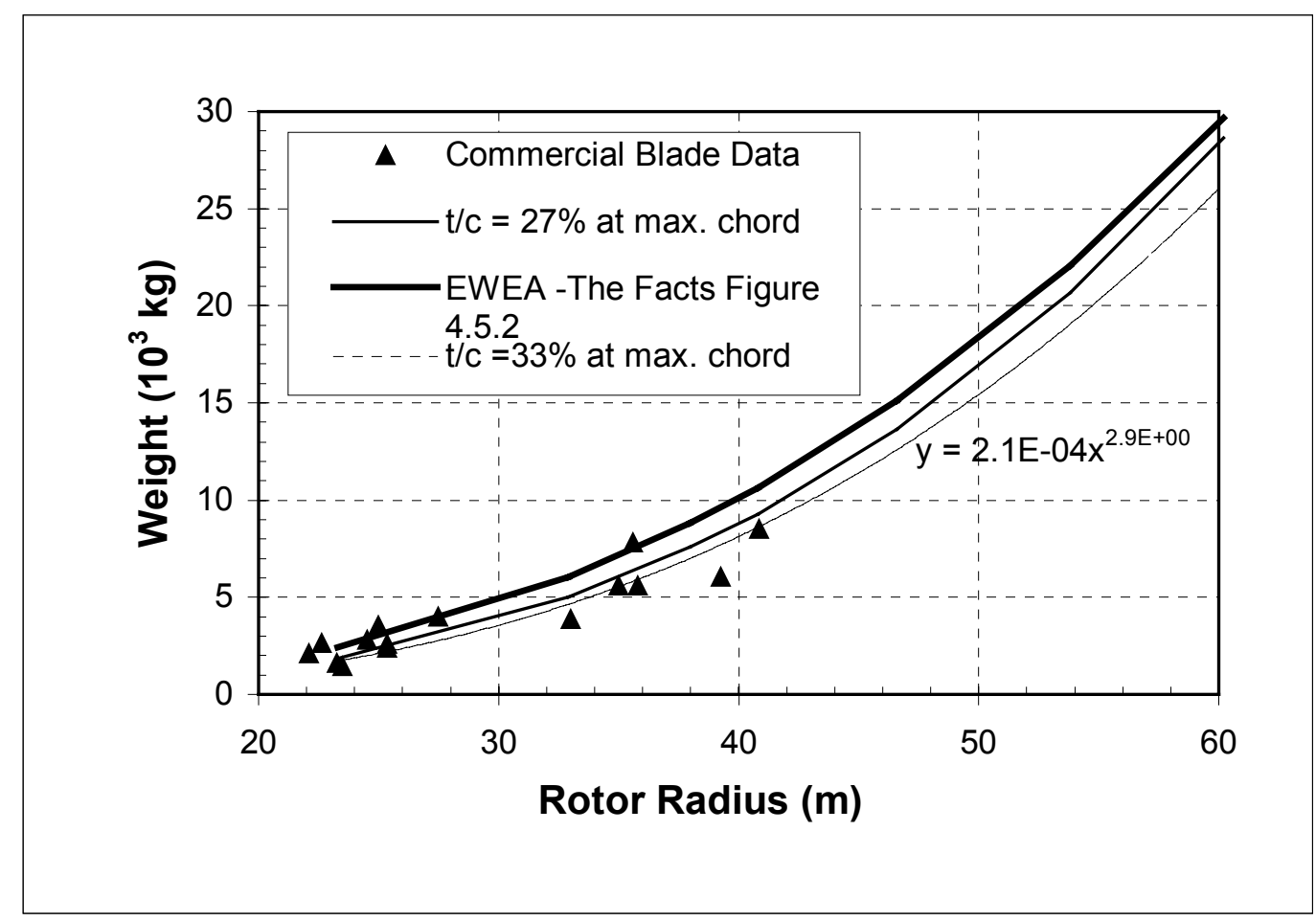

Figure 2-1.Comparison of blade mass equations

(see Appendix A, page 10)

We determined blade dimensions (length, chord, and maximum diameter) were as a function of either rotor diameter or rotor radius (depending on the parameter). The percentage ratios used were based on typical values determined during the blade analysis conducted by GEC as part of Technical Area 1 - Blade Scaling Study.

\subsubsection{Hubs}

We estimated hub masses using relationships developed from a group of existing turbines for which relatively accurate mass values were known. Blade masses and rotor masses were analyzed to calculate the remaining hub mass. We assumed that the hub mass values used included pitch bearings and the pitch mechanism.

The hub generally is not a component that is prominently discussed in manufacturer literature and its scaling with rotor size does not command much discussion in research literature. The massestimating approach used by GEC (see Appendix A page 8) resulted in a hub-mass scaling relationship of:

$$
\mathrm{m}=0.24 \mathrm{D}^{2.58}
$$

Equation 2.2

We estimated hub dimensions based on their current proportions to blade and nacelle dimensions. 


\subsubsection{Nacelle}

The nacelle masses we presented in Table 2-1 were estimated based on the following scaling formula [2]. The resulting mass does not include the rotor mass.

$$
\mathrm{m}=2.6 \mathrm{D}^{2.4}
$$

Equation 2.3

The corresponding nacelle mass associated with the $2500-\mathrm{kW}$ turbine (85-m rotor diameter) exceeded the ability to transport the nacelle over the road via high-capacity tractor trailers, which conflicted with past transport experience with the 2-megawatt Boeing MOD 2 (and more recently, the Nordex N80/2500 turbines). More detailed analysis of nacelle mass was performed by compiling manufacturer mass data and plotting it with respect to rotor diameter. A power fit trend line was applied to the corresponding data point, along with the line added that was associated with the EWEA equation, resulting in Figure 2-2.

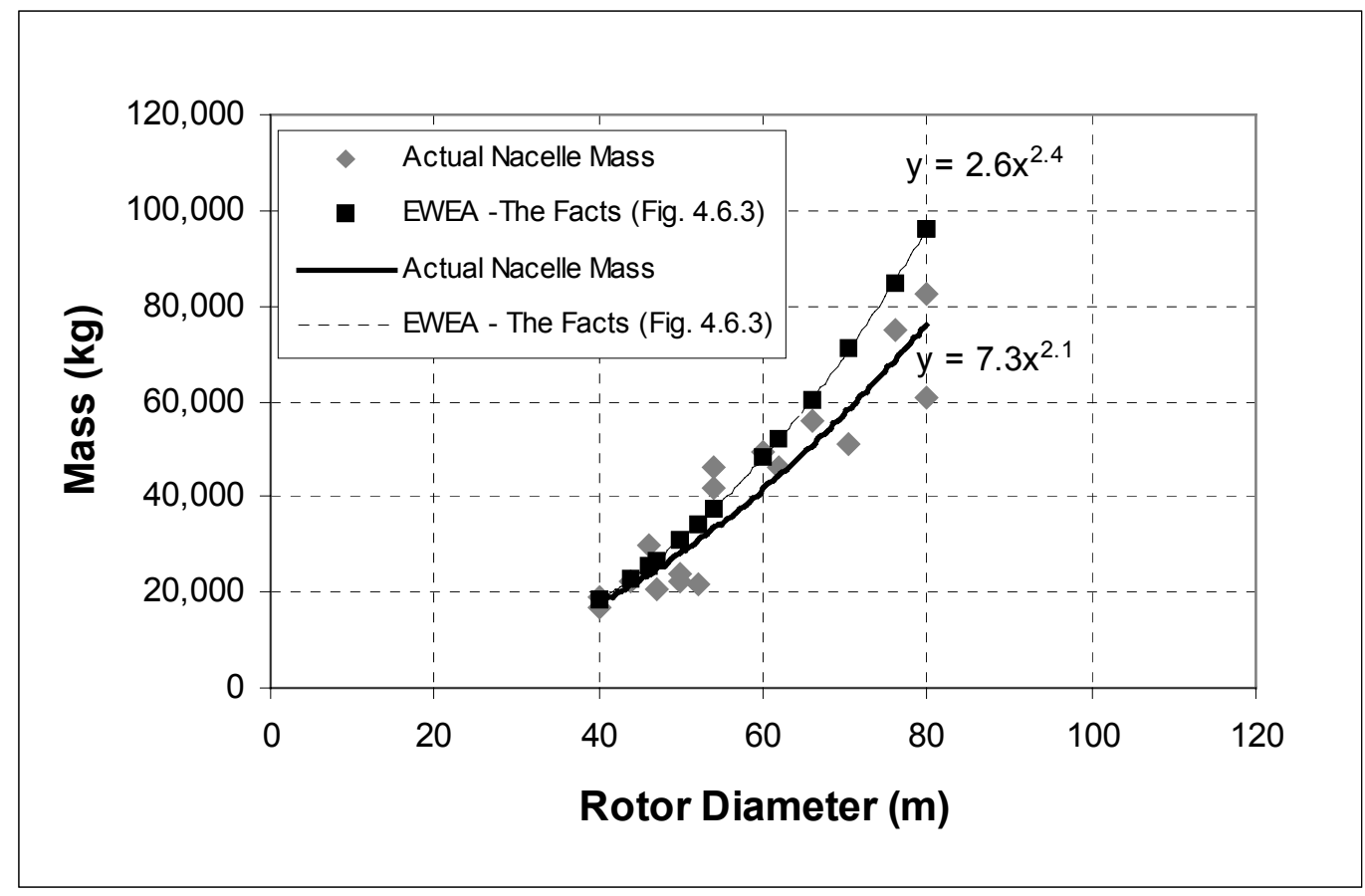

Figure 2-2.Comparison of nacelle mass equations

(see Appendix A, Page 7)

The recent manufacturer data indicate that nacelle masses are not following the trend Equation 2.3. It appears that the manufacturers have been integrating mass-reducing techniques into their nacelle designs, resulting in lighter nacelles. The manufacturer data indicate that the nacelle masses are following an equation closer to:

$$
\mathrm{m}=7.3 \mathrm{D}^{2.11}
$$

Equation 2.4

Application of the nacelle mass equation based on recent manufacturer data is important for the $2500-\mathrm{kW}$ (85-m rotor diameter) WindPACT turbine because it results in a nacelle mass of $86,000 \mathrm{~kg}(190,000 \mathrm{lbs})$, which is virtually the upper limit of high-capacity tractor-trailer transport capability. Because nacelles at this power rating have been and are being transported by trailers, we decided to use this value when evaluating logistics. Application of Equation 2.3 
results in a nacelle mass of $111,000 \mathrm{~kg}(245,000 \mathrm{lbs})$ for the $2500-\mathrm{kW}$ turbine (85-m rotor diameter), which is significantly greater than the tractor-trailer capacity limit and would require the use of steerable dollies (which is not current practice). The use of either nacelle mass equation for the $3500-\mathrm{kW}$ and $5000-\mathrm{kW}$ turbines does not affect this logistics study because both yield nacelle mass values that exceed tractor-trailer capacity.

The nacelle contents are assumed to be comparable to those of current generation of turbines. Nacelle dimensions were estimated using existing turbine designs as the basis. Previously, past $2500-\mathrm{kW}$ and $5000-\mathrm{kW}$ research turbines were evaluated to obtain scaling reference points.

For purposes of evaluating alternative assembly, crane, and transportation scenarios, we performed estimates of the gearbox and generator masses. GEC used published data on gearbox and generator scaling relationships [1] to estimate component masses as percentages of the total nacelle mass. The component masses are shown in Table 2-1.

\subsubsection{Tower Head Mass}

Tower head mass was estimated based on a compilation of specific tower head masses $\left(\mathrm{kg} / \mathrm{m}^{2}\right)$ and rotor diameters [7]. Additional turbine data from the Turbine Verification Program and new data from the current generation of megawatt-sized turbines were added to the original data. We then converted the data into total tower head mass as a function of rotor diameter, and applied a power fit trend line to obtain the following formula:

$$
\mathrm{m}=2.3 \mathrm{D}^{2.5}
$$

Equation 2.5

We compared the results from this equation with those from published equations to evaluate its validity. First, we calculated the combined results from Equations 2.1 through 2.3. Another perspective on tower head mass was derived from a combination of recent manufacturer blade, hub, and nacelle mass data. The results are presented in Figure 2-3. The recent manufacturer information yields a lower tower head mass, primarily because the nacelle mass (as calculated by Equation 2.4) is lower.

Component mass is more critical to this logistics study than combined tower head mass; however, evaluation of the equations used to estimate head mass is important to confirm the validity of the values being presented. Tower head mass has been calculated for comparison purposes but does not enter into the analysis of logistics. Based on this analysis, the tower head mass in Table 2-1 appears to be greater than recent manufacturer data may be indicating. The difference in nacelle discussed in Section 2.1.4 is the largest contributor to the differences in tower head mass. 


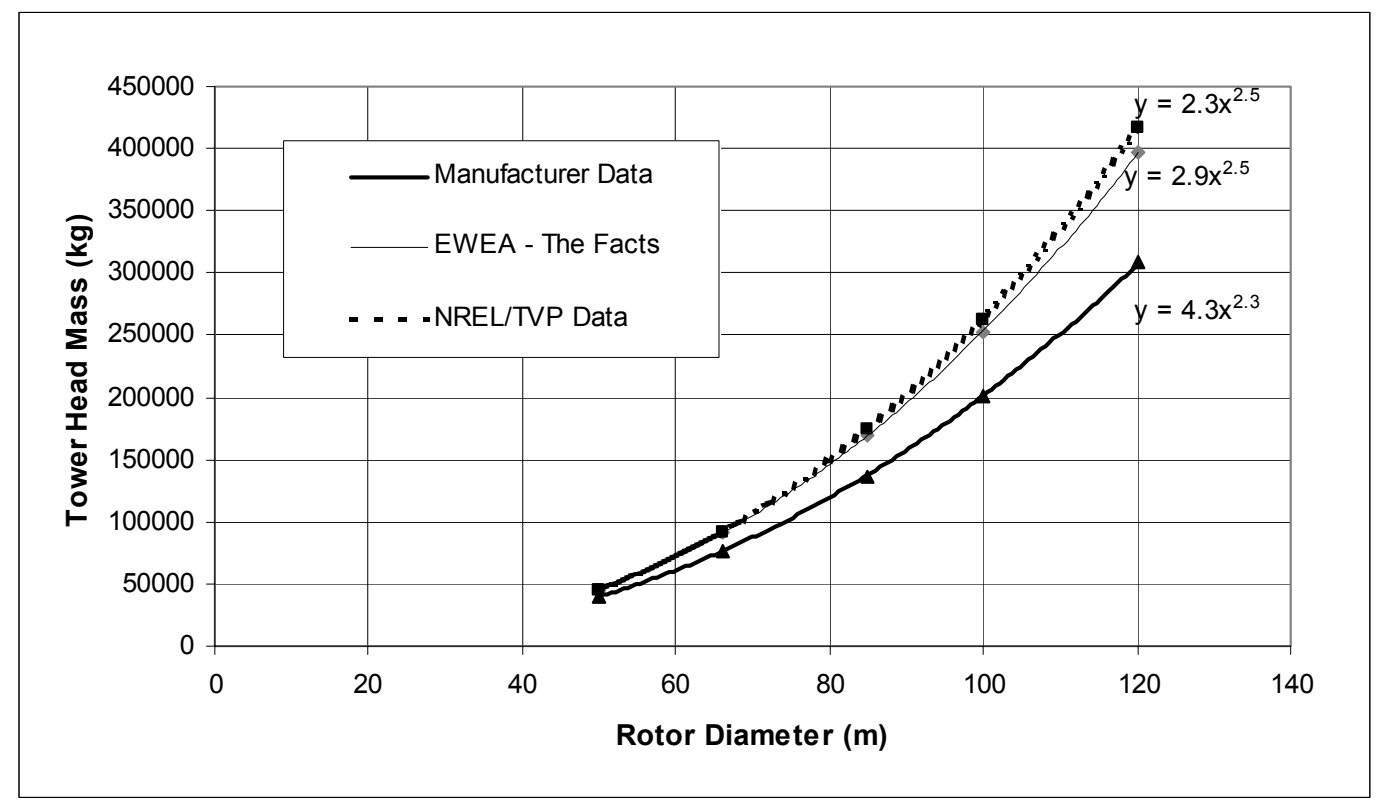

Figure 2-3.Comparison of tower head mass equations

(See Appendix A, Page 4)

\subsubsection{Towers}

It was assumed that the current design of tapered tubular steel towers would be used for all WindPACT turbines, resulting in the use of the following assumptions and design criteria.

An International Electrotechnical Commission (IEC) class 2 wind regime was assumed for the tower design based on the previously stated wind resource information associated with the area of interest in South Dakota. The analysis generally followed the IEC design approach to calculate rotor thrust, tower drag, and total overturning moments. Application of material assumptions, hub height to rotor diameter assumptions, tower wall thickness to diameter assumptions, and use of load and material factors resulted in calculation of tower diameters and total mass. We assumed a linear tower taper for simplicity. Finally, we derived total characteristic base moments exclusive of the load factor to obtain values applicable to the design of the tower foundation in Technical Area 4 (Balance of Station Costs).

We used a peak-load scenario to design the towers; however, we made certain deviations from IEC protocol to account for fatigue and dynamics. We also assumed the peak-loading scenario would occur during a pitch control system failure with the blades in an operating position. IEC allows the use of the Ve1 wind speed [44.5 m/s $(99.5 \mathrm{mph})$ for class 2 along with possible modification of the load factor] under this fault scenario; however, GEC elected to use the Ve50 wind speed value $59.5 \mathrm{~m} / \mathrm{s}(133.1 \mathrm{mph})$ in addition to the load and material factors. This returns a conservative design for the peak-load condition. However, if an actual design was performed, it is likely that fatigue and/or dynamics would determine the tower shape and size. Based on a preliminary evaluation, we determined that fatigue would likely dictate tower sizes similar to those resulting from the modified peak load scenario being used.

Logistically, the critical results of this design process were the total tower mass, base diameter, and tower top diameter. Incorporating this information with hub height and analysis of typical 
tower section lengths being used resulted in an estimate of the number of tower sections per turbine class and the corresponding diameters. We calculated tower section masses by determining the steel volume for each section based on the dimensions then multiplying by the steel density. Non-structural steel mass was excluded from the total mass calculated by GEC.

Figure 2-4 presents a comparison of the GEC-calculated tower mass to recently manufactured towers for which relatively accurate mass information was known. The manufacturer data contain a rather high degree of scatter, which indicates different design approaches. In general, the GEC calculations appear to be underestimating tower mass by about $20 \%$. Because a modified peak-load approach to calculating the loads on the tower is being used, it is possible that actual tower designs are being driven by fatigue and dynamics, resulting in higher masses. A comparison of manufacturer's tower diameter data to those calculated by GEC's model is presented in Table 2-3. This table indicates that the model results are within an acceptable range of variation that could be expected. The calculation spreadsheet is in Appendix A.

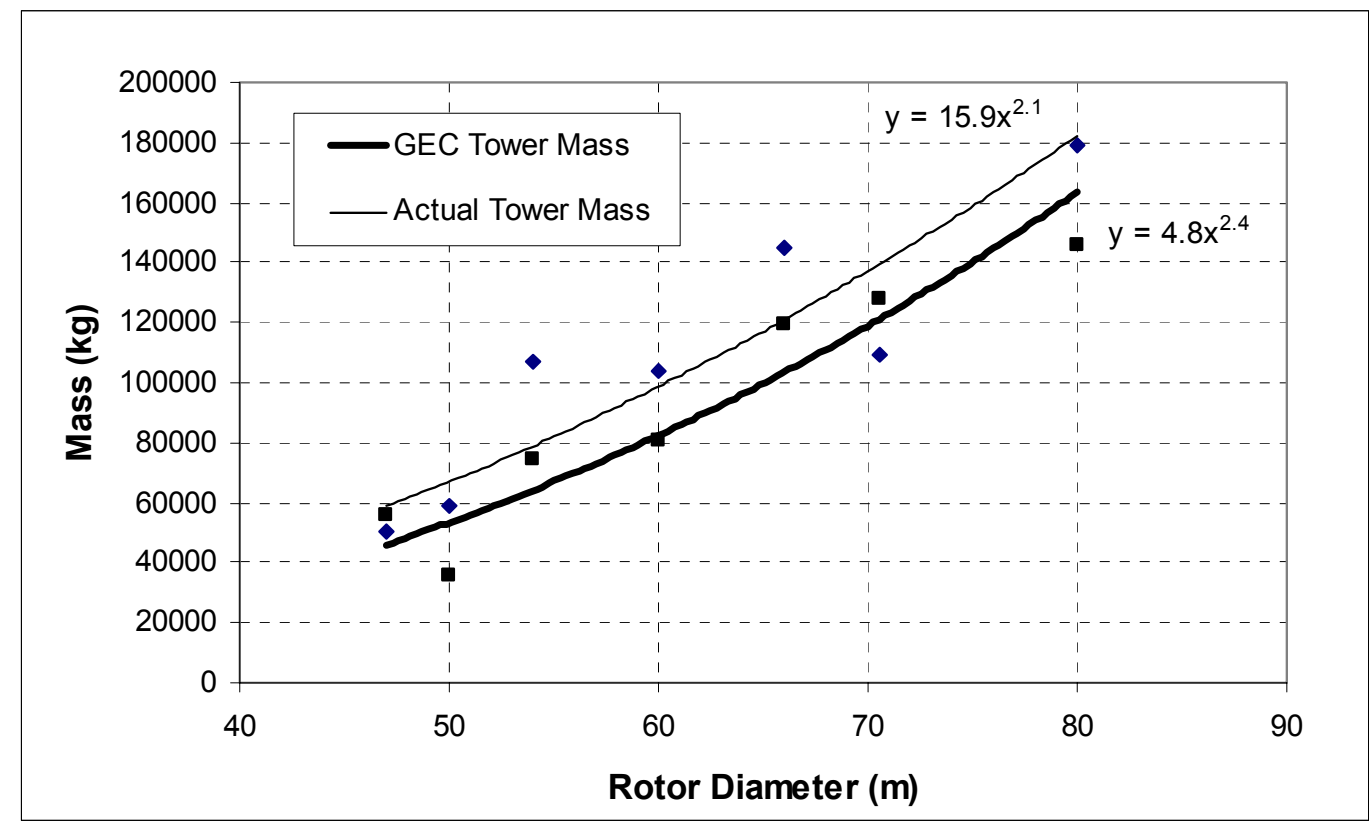

Figure 2-4.Comparison of tower masses

(see Appendix A, Page 6) 
Table 2-3.Comparison of Manufacturer Tower Data with GEC Calculations

\begin{tabular}{|c|c|c|c|c|c|c|c|}
\hline \multirow{2}{*}{\multicolumn{2}{|c|}{ Turbine }} & \multicolumn{3}{|c|}{ Manufacturer Data } & \multicolumn{2}{c|}{ GEC Tower Calculations } \\
\cline { 3 - 8 } \multicolumn{2}{|c|}{} & Total & Tower Diameters & \multicolumn{2}{c|}{ Total } & \multicolumn{2}{c|}{ Tower Diameters } \\
\cline { 3 - 8 } \multicolumn{2}{|c|}{} & Tower Mass & Base & Top & Tower Mass & Base & Top \\
\cline { 3 - 8 } & & & & & & \\
\hline \multirow{2}{*}{ Vestas } & \multirow{2}{*}{ V66 } & $145,000 \mathrm{~kg}$ & $4.3 \mathrm{~m}$ & $2.3 \mathrm{~m}$ & $120,000 \mathrm{~kg}$ & $4.8 \mathrm{~m}$ & $2.4 \mathrm{~m}$ \\
\cline { 3 - 8 } & & $320,000 \mathrm{lbs}$ & $14.1 \mathrm{ft}$ & $7.5 \mathrm{ft}$ & $263,000 \mathrm{lbs}$ & $15.75 \mathrm{ft}$ & $7.9 \mathrm{ft}$ \\
\hline \multirow{2}{*}{ Vestas } & \multirow{2}{*}{$\mathrm{V} 47$} & $50,700 \mathrm{~kg}$ & $4.0 \mathrm{~m}$ & $2.1 \mathrm{~m}$ & $56,000 \mathrm{~kg}$ & $3.6 \mathrm{~m}$ & $1.8 \mathrm{~m}$ \\
\cline { 3 - 8 } & & $112,000 \mathrm{lbs}$ & $13.1 \mathrm{ft}$ & $6.9 \mathrm{ft}$ & $123,000 \mathrm{lbs}$ & $11.8 \mathrm{ft}$ & $5.9 \mathrm{ft}$ \\
\hline \multirow{2}{*}{ Zond } & \multirow{2}{*}{$\mathrm{Z}-750$} & $59,000 \mathrm{~kg}$ & $3.7 \mathrm{~m}$ & $2.7 \mathrm{~m}$ & $35,600 \mathrm{~kg}$ & $3.3 \mathrm{~m}$ & $1.7 \mathrm{~m}$ \\
\cline { 3 - 8 } & $130,000 \mathrm{lbs}$ & $12.1 \mathrm{ft}$ & $8.9 \mathrm{ft}$ & $78,500 \mathrm{lbs}$ & $10.8 \mathrm{ft}$ & $5.6 \mathrm{ft}$ \\
\hline
\end{tabular}

\subsection{Organization of Study}

After determining the sizes and masses of the various turbine components we provided, the specifications to GEC's transportation, assembly, and crane consultants for analysis, determination of logistics, and costing. GEC developed three basic scenarios to identify specific breakpoints in the turbine specifications and costs. The scenarios were also developed to determine the impact of implementing measures not currently used by the wind energy industry to alleviate critical logistic issues that arose due to increased turbine scale. The three scenarios are described as follows:

- $\quad$ Scenario 1: This is the baseline scenario in which the current practices associated with component transportation, turbine assembly, and crane utilization are used for each WindPACT turbine without modification for increased turbine scale. Specifically, turbine components are transported and assembled in as few pieces as possible, relying upon the efficiency of preassembly to the maximum extent possible. This scenario also represents minimal field assembly and assumed that the rotors are preassembled on the ground before being placed onto the nacelle.

- Scenario 2: Scenario 2 deviates from Scenario 1 in two critical aspects. First, tower sections that present specific logistical issues due to their dimensions and/or masses are quartered lengthwise and therefore require on-site assembly. Three on-site assembly approaches were then developed and analyzed. Second, rotor assembly was assumed to be performed with the nacelle and hub installed onto the tower.

- Scenario 3: Scenario 3 corresponds to Scenario 2 except that the gearbox and generator are handled as individual components exclusive of the nacelle. This case represents the maximum on-site assembly requirements and a significant increase in the number of objects that require handling. For simplicity, the on-site tower assembly approach that appeared most practical under Scenario 2 was retained as the tower assembly approach for Scenario 3. Therefore, the impact of multiple nacelle components could be isolated.

Further clarification and assumptions used within each of these scenarios as they relate to transportation, assembly, and cranes are presented in the following sections. 
Table 2-4.Summary of Logistic Scenarios

\begin{tabular}{|c|l|}
\hline Logistic Scenarios & \multicolumn{1}{c|}{ Description } \\
\hline Scenario 1 & $\begin{array}{l}\text { Current transport and assembly techniques } \\
\text { applied to all turbine sizes without } \\
\text { modification. }\end{array}$ \\
\hline Scenario 2 & $\begin{array}{l}\text { Field-fabricate quartered tower sections, blades } \\
\text { assembled to hub in the air. }\end{array}$ \\
\hline Scenario 3 & $\begin{array}{l}\text { Same as Scenario 2, plus gearbox and } \\
\text { generators are handled as individual } \\
\text { components exclusive of the nacelle. }\end{array}$ \\
\hline
\end{tabular}




\section{Transportation Logistics}

\subsection{Background}

We evaluated logistics for various modes of transportation and included multi-modal options when required. The transportation modes evaluated were tractor-trailer, rail, steerable-dolly, barge, and chartered ocean/Great Lakes vessels. Currently, transportation of wind turbine components within the United States is generally performed with the use of trucks and, to a lesser extent, rail.

\section{Road Access}

The hypothetical project site in south-central South Dakota can only be accessed via local roads within a 80 to $95 \mathrm{~km}$ (50 to $60 \mathrm{mi}$ ) radius. U.S. Highway 18 is the primary east/west road in the area. U.S. Highways 83 and 183 are the primary north/south roads that connect U.S. 18 to Interstate 90.

\section{Railroad Access}

The Burlington Northern-Santa Fe (BNSF) railroad operates a short track line called the Dakota \& Southern Railroad that parallels Interstate 90 from Mitchell, South Dakota, to Kadoka, South Dakota. BNSF has exclusive operating rights within North and South Dakota and northern Nebraska. Maps of the Union Pacific Railroad and Burlington Northern Railroad networks are shown in Figures 3-1 and 3-2, respectively. 


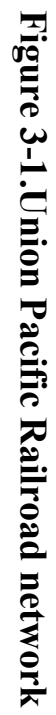

\section{Union Pacific Railroad Service Units}

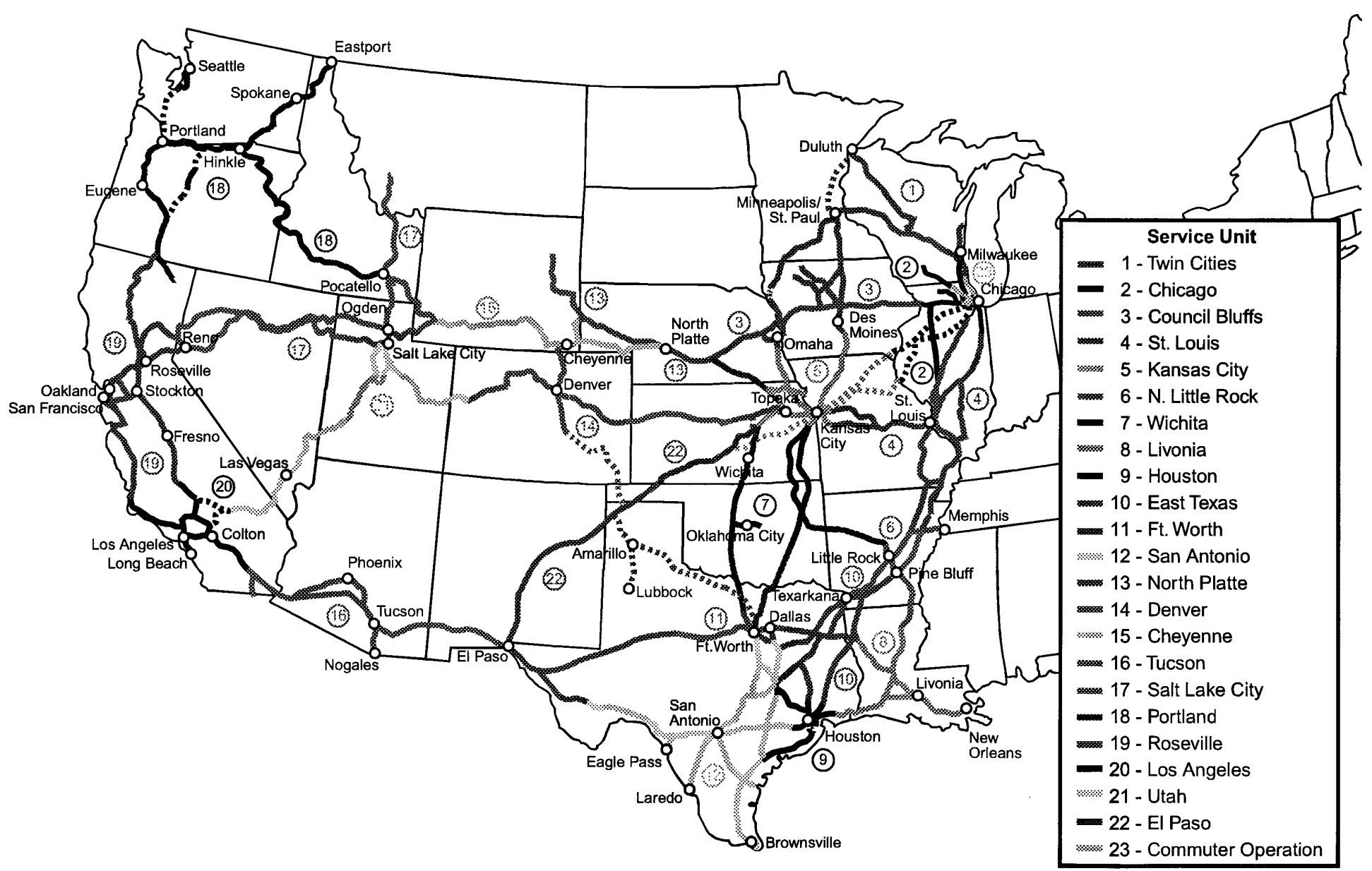

Media Graphics 9/22/19 


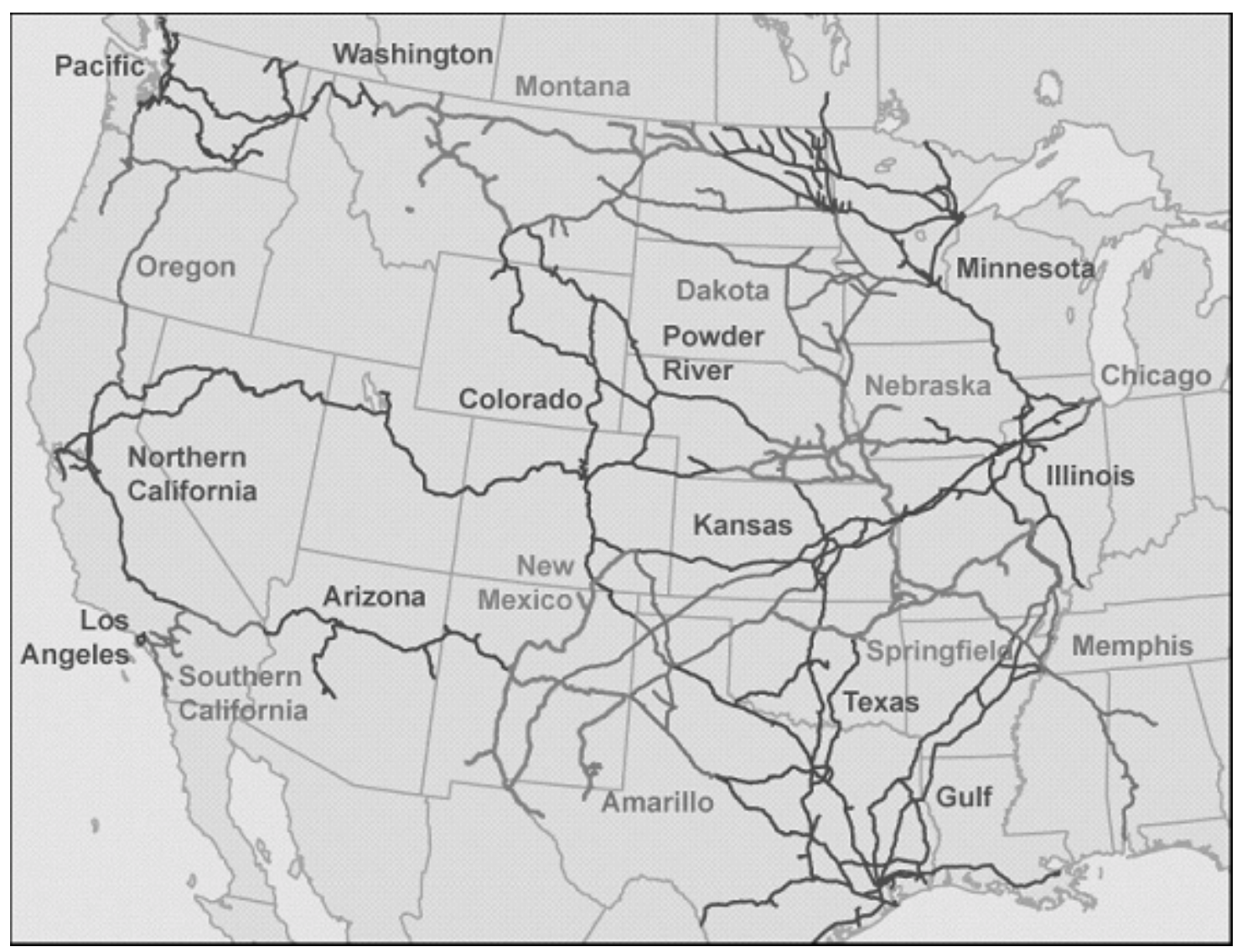

Figure 3-2.Burlington Northern-Santa Fe Railroad network

Figure 3-3 shows a more detailed view of the Dakota \& Southern Railroad area. Potentially viable debarkation towns are Presho, Murdo, and Kennebec, South Dakota. These towns are within 80 to $95 \mathrm{~km}$ (50 to $60 \mathrm{mi}$.) of the assumed project site. This area of South Dakota is significantly closer to rail lines than most favorable wind sites in the upper Midwest. Considering the remoteness of the hypothetical project site, access to a rail spur relatively close to the site is beneficial. 


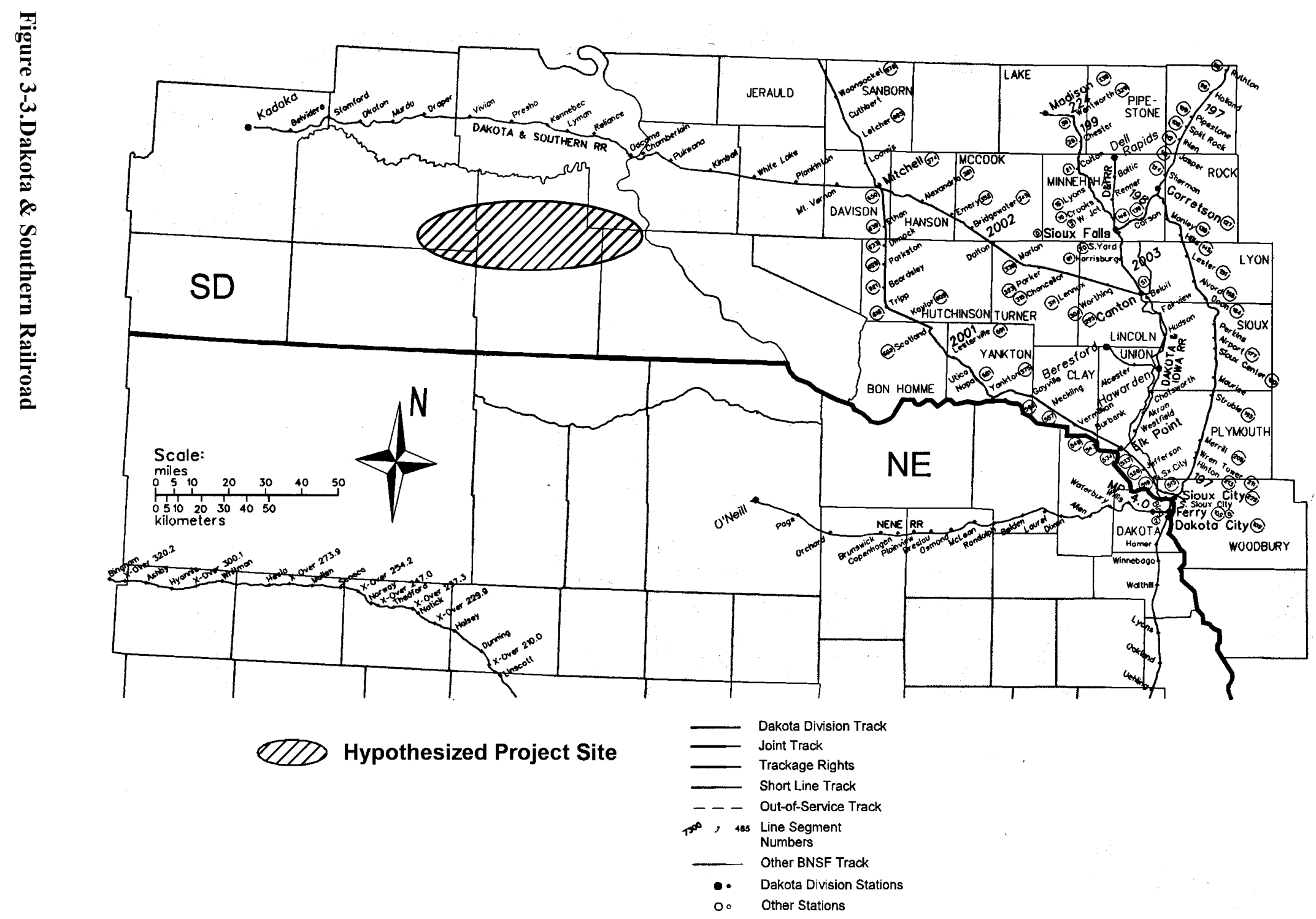




\section{Water Access}

Water access is possible by deck barge on the Missouri River up to Sioux City, Iowa. Beyond Sioux City, the Missouri River is not specifically maintained for navigation. A series of sixflood-control and power-generating dams begin near Yankton, South Dakota, that inhibit navigation up river. The Omaha District of the U.S. Army Corps of Engineers (USACE) was contacted to assess the potential for navigation between Sioux City, Iowa, and Yankton, South Dakota. The USACE stated that the Missouri River is free flowing between Yankton and Sioux City and no that navigation channel is maintained. Therefore, use of barge transport is not possible beyond Sioux City, Iowa.

The normal navigation season on the Missouri River is 8 months; however, there are specific dates that affect operations. The navigation season at Sioux City, Iowa, opens on March 23, however, access from the mouth near St. Louis, Missouri, is not possible until April 1. The season at Sioux City, Iowa closes on November 22 and on December 1 at the mouth near St. Louis. Therefore, the effective period in which wind turbine components can be delivered to Sioux City, Iowa, is between April 1 and November 22.

The next closest navigable waterway to the project site is through the Great Lakes (specifically at the Port of Duluth, Minnesota). Although primarily a bulk-commodity port, the piers have handled "project cargo" movements in the past where delivery to larger more popular ports along the lakes proved unfeasible.

\subsection{Transportation Assumptions}

We assessed transportation logistics associated with wind turbine components delivered into South Dakota for three basic travel distances: short haul, long haul, and overseas. Short hauls encompassed an area with a radius around the project site of approximately 600 to 700 miles and included areas such as Chicago, Illinois; Green Bay, Wisconsin; and Duluth, Minnesota; Sioux City, Iowa; and Denver, Colorado. Long-haul transportation distances were considered anything greater than the short-haul radius. Over short-haul distances the use of steerable dollies in addition to tractor-trailers is feasible, allowing an increase in the weight that can be transported over the road. Dollies however, are not feasible over long distances or through multiple states. Long hauls incorporated the use of all possible modes. We also evaluated ocean shipping for components manufactured in Europe assuming that chartered ships equipped with cargo cranes would be used to deliver turbine components to the ports of either Houston or Duluth.

Based on experience with oversized and overweight transportation, ATS noted that Nebraska, Kansas, and to a lesser extent Missouri and Iowa, can be difficult states to pass through with excessive loads. Being situated in the middle of the country results in a tremendous amount of transportation activity in these states and places a large (and possibly disproportional) burden on their infrastructure. In addition, because these states tend to be more agricultural as opposed to industrial areas such as the Great Lakes or Gulf Coast, their transportation regulations have evolved to be more accommodating to agriculture rather than heavy industry. Although it is not impossible to transport oversized and overweight loads through these states, it generally requires more effort and greater planning costs to obtain the necessary permits and approvals. Additional expenses incurred by traveling around these states are less than what would be spent obtaining permission to pass through. Recognizing these factors, ATS selected travel routes through Texas, Oklahoma, Missouri, Iowa, North Dakota, Illinois, and Minnesota. 
Based on their experience, ATS selected the following routes to develop estimated transportation costs for the listed components. In addition to maximizing the likelihood of permit approval, these routes were based on understanding the current source areas for existing turbine components.

\section{Blades}

L-M Glasfiber in Grand Forks, North Dakota, and Molded Fiber Glass, Inc. (MFG), in Gainesville, Texas, were used to estimate short-haul and long haul costs, respectively. Costs for blade transport from Europe were based on delivery to the ports of Duluth and Houston. Viable transport modes were chartered ocean ships, trucks, steerable dollies, and barges.

\section{Hubs}

Hubs were supplied from Chicago, Illinois for short-haul-estimating purposes and from Tehachapi, California, for long-haul-estimating purposes. In addition, transport costs from the ports of Houston and Duluth were generated to estimate costs for European-supplied components. Viable transport modes include chartered ocean ships, trucks, and rail.

\section{Nacelles}

Costs estimates for transport of nacelles used the same points of origin as the hubs. However, modifications to the routes were required due to the need for steerable dollies and barges. Viable transport modes include chartered ocean ships, trucks, rail, steerable dollies, and barges.

\section{Towers}

Three primary suppliers of tubular steel towers are based in Canutillo, Texas (near El Paso), Dallas, Texas, and Shreveport, Louisiana; therefore, tower transport costs were calculated from these areas. Although highly unlikely, tower transport costs from Europe were estimated for comparison purposes. Viable transport modes include trucks, steerable dollies, barges, and chartered ocean ships

\subsection{Oversized Load Permits}

In the United States the transportation regulation system has unique rules, regulations, and oversized permit requirements for each state. This system requires transporters such as ATS to research and determine the lowest common denominator with respect to the type of shipment being planned, its origin, and destination. Demonstrating to permit officials that all possible means have been assessed or used to either minimize travel distances, or select appropriate bypass routes, is critical in obtaining permits. Typically, detailed transportation plans are developed by the transport company that are based on specific object sizes, weights, origin, destination, and unique handling requirements. Alternative approaches are evaluated, costs are refined, and adjustments to comply with unique state requirements are made resulting in the final transportation plan. To attempt this type of detailed analysis was beyond the scope of the project; however, these plans can reduce costs or even eliminate the need for obtaining oversized permits.

The number of assumed turbines is a significant factor effecting the viability of certain transportation techniques discussed in this study. State officials are generally more accepting of one or a few oversized/overweight transport loads as opposed to 50 or 150 shipments. The longterm disruption of traffic and inconvenience to local populations would be considered intrusive. Technically, stresses placed on the infrastructure by one or a few oversized/overweight loads can be accommodated; however, the cyclical stress of multiple over-dimensioned loads could 
significantly increase the possibility of failure and is another factor making permits for large numbers of shipments very difficult to obtain.

Based on information from ATS, the 4.9-m (16-ft) loaded height is a point at which transport companies and permitting authorities become concerned about actual or potential load clearance with overhead structures and/or utilities. In addition to height, the shape of the load is also a factor. Rectangular or circular loads have larger profiles as opposed to triangular or vertical loads. It is easier to negotiate triangular or vertical loads around low-hanging objects (street lights for example) without npresenting the need for temporary removal of the utility. Based on ATS's experience, circular loads do not provide this type of flexibility. It is also possible for utilities to temporarily interrupt service on overhead utilities but not drop the line(s) to reduce the potential for injury and equipment damage in the event of an accidental strike.

The 4.9-m (16-ft) overhead utility height constraint is a larger issue in areas with older infrastructure or in rural areas; as opposed to recently built infrastructure. Careful route selection can help avoid such areas, however, it's highly probable that at least one low-utility area will be encountered during shipment. Excessive height causes considerable increases in the transport costs because local utilities are required to temporarily disconnect power, drop and protect the lines, then reinstall the wires in order for the load to pass. Utilities generally charge considerable expenses to perform this work to cover their costs including service disruption and planning costs to "permit fees" thus presenting a considerable deterrent to movement of loads with excessive height. Excessive height moves can effective, however, when all possible alternatives have been evaluated and the number of utility assist areas has been minimized. However, movement of numerous objects (50 to 350 tower sections) in this manner will not be cost effective and will likely not receive permit approval.

To underscore the difference between states, we compared South Dakota and Nebraska legal truck weights. In South Dakota, truckloads up to 70,300 kg (155,000 lbs) using 13 axles are possible without the issuance of a special permit. However, Nebraska's limit is $43,000 \mathrm{~kg}$ (95,000 lbs) using 7 axles. Therefore, a legal load in South Dakota requires an overweight permit in Nebraska.

\subsection{Equipment Capacity and Limitations}

Overweight permits usually are issued with specific dates during which transport is prohibited. These dates are state specific but tend to eliminate periods during the spring when frozen ground is thawing. Over-dimension permits are likely to have travel time limits in congested areas, limiting movement to non-rush-hour periods.

A breakdown of critical vehicle dimensions by transportation mode is presented in Table 3-1. The overall dimensions and weights correspond to the combined vehicle and load. Points at which oversize or overweight permits are required have also been noted. Application of the overall vehicle limitations to the specific wind turbine objects resulted in Table 3-2. Table 3-2 presents the breakpoint dimensions associated with wind turbine components beyond which significant increases in transportation costs occur. 
Table 3-1.Overall Dimensions

\begin{tabular}{|c|c|c|c|c|c|}
\hline MODE & EQUIPMENT & $\begin{array}{l}\text { OVERALL } \\
\text { WIDTH }\end{array}$ & $\begin{array}{l}\text { OVERALL } \\
\text { HEIGHT }\end{array}$ & $\begin{array}{l}\text { OVERALL } \\
\text { LENGTH }\end{array}$ & $\begin{array}{c}\text { OVERALL WEIGHT } \\
\text { (GVW) }\end{array}$ \\
\hline \multirow{4}{*}{$\begin{array}{l}\text { Tractor- } \\
\text { Trailer } \\
\text { Trucks }\end{array}$} & Standard Trailers & $2.6 \mathrm{~m}(8.5 \mathrm{ft})$ & $4.1 \mathrm{~m}(13.5 \mathrm{ft})$ & $\begin{array}{c}14.6-16.2 \mathrm{~m} \\
(48-53 \mathrm{ft})\end{array}$ & $\begin{array}{l}\text { up to } 36,300 \mathrm{~kg} \\
(80,000 \mathrm{lbs})\end{array}$ \\
\hline & $\begin{array}{c}\text { Special Multi-Axle } \\
\text { Drop Trailers }\end{array}$ & $2.6 \mathrm{~m}(8.5 \mathrm{ft})$ & $4.1 \mathrm{~m}(13.5 \mathrm{ft})$ & $\begin{array}{c}14.6-16.2 \mathrm{~m} \\
(48-53 \mathrm{ft})\end{array}$ & $\begin{array}{c}36,300-70,300 \mathrm{~kg} \\
(80,000-155,000 \mathrm{lbs}) \\
\text { (State dependant) }\end{array}$ \\
\hline & $\begin{array}{l}\text { Special Multi-Axle } \\
\text { Drop Trailers w/ } \\
\text { OW Permits }\end{array}$ & $2.6 \mathrm{~m}(8.5 \mathrm{ft})$ & $4.1 \mathrm{~m}(13.5 \mathrm{ft})$ & $\begin{array}{l}38.1-45.7 \mathrm{~m} \\
(125-150 \mathrm{ft})\end{array}$ & $\begin{array}{c}\text { up to } \\
102,100-106,600 \mathrm{~kg} \\
(225,000-235,000 \mathrm{lbs})\end{array}$ \\
\hline & $\begin{array}{c}\text { Special Multi-Axle } \\
\text { Drop Trailers w/ } \\
\text { OW and OD } \\
\text { Permits }\end{array}$ & $\begin{array}{c}\text { 6.1-7.6 m } \\
(20-25 \mathrm{ft}) \\
\text { possible (route } \\
\text { dependant) }\end{array}$ & $\begin{array}{c}4.83 \mathrm{~m} \\
(15.8 \mathrm{ft}) \\
\text { triggers utility } \\
\text { assistance }\end{array}$ & $\begin{array}{l}38.1-45.7 \mathrm{~m} \\
(125-150 \mathrm{ft})\end{array}$ & $\begin{array}{c}\text { up to } \\
102,100-106,600 \mathrm{~kg} \\
(225,000-235,000 \mathrm{lbs})\end{array}$ \\
\hline $\begin{array}{c}\text { Steerable } \\
\text { Dolly } \\
\text { System }\end{array}$ & $\begin{array}{l}\text { Custom-built } \\
\text { system utilizing } \\
\text { modular wheel } \\
\text { systems }\end{array}$ & $\begin{array}{c}\text { Route } \\
\text { dependant }\end{array}$ & $\begin{array}{c}4.83 \mathrm{~m} \\
(15.8 \mathrm{ft}) \\
\text { triggers utility } \\
\text { assistance }\end{array}$ & $\begin{array}{c}\text { Route } \\
\text { dependant }\end{array}$ & Route dependant \\
\hline Rail & $\begin{array}{l}\text { Standard Flat } 8 \\
\text { Axle Heavy }\end{array}$ & $3.4 \mathrm{~m}(11 \mathrm{ft})$ & $\begin{array}{c}4.0 \mathrm{~m}(13 \mathrm{ft}) \\
\text { from top of rail }\end{array}$ & $27.4 \mathrm{~m}(90 \mathrm{ft})$ & $\begin{array}{l}\text { up to } 163,300 \mathrm{~kg} \\
(360,000 \mathrm{lbs})\end{array}$ \\
\hline Barge & Deck Barge & $16.5 \mathrm{~m}(54 \mathrm{ft})$ & - & $76.2 \mathrm{~m}(250 \mathrm{ft})$ & $\begin{array}{c}217,700-272,200 \mathrm{~kg} \\
(480,000-600,000 \\
\text { lbs })\end{array}$ \\
\hline $\begin{array}{l}\text { Ocean } \\
\text { Vessel }\end{array}$ & $\begin{array}{l}\text { Chartered vessel } \\
\text { with cargo cranes }\end{array}$ & - & - & - & - \\
\hline
\end{tabular}

OW - Overweight

OD - Over-dimensioned

Table 3-2.Breakpoint Dimensions

\begin{tabular}{|c|c|c|c|c|}
\hline OBJECT & $\begin{array}{c}\text { OBJECT } \\
\text { HEIGHT }\end{array}$ & OBJECT WIDTH & OBJECT LENGTH & OBJECT WEIGHT \\
\hline Blades & $4.4 \mathrm{~m}(14.5 \mathrm{ft})$ & $7.6 \mathrm{~m}(25 \mathrm{ft})$ & $\begin{array}{c}45.7-48.8 \mathrm{~m}(150-160 \mathrm{ft}) \\
\text { (transport distance and } \\
\text { route dependant) }\end{array}$ & Not Problematic \\
\hline $\begin{array}{c}\text { Hubs } \\
\text { (w/o } \\
\text { permits) }\end{array}$ & $3.7 \mathrm{~m}(12 \mathrm{ft})$ & Not Problematic & Not Problematic & $\begin{array}{c}17,200-19,100 \mathrm{~kg} \\
(38,000-42,000 \mathrm{lbs})\end{array}$ \\
\hline $\begin{array}{c}\text { Nacelles } \\
\text { Towers } \\
\text { (w/o } \\
\text { permits) }\end{array}$ & $3.7 \mathrm{~m}(12 \mathrm{ft})$ & Not Problematic & Not Problematic & $\begin{array}{c}79,400-83,900 \mathrm{~kg} \\
(175,000-185,000 \mathrm{lbs})\end{array}$ \\
\hline $\begin{array}{c}\text { Towers } \\
\text { (w/ permits) }\end{array}$ & $4.4 \mathrm{~m}(14.5 \mathrm{ft})$ & - & $16.2 \mathrm{~m}(53 \mathrm{ft})$ & $\begin{array}{c}17,200-19,100 \mathrm{~kg} \\
(38,000-42,000 \mathrm{lbs})\end{array}$ \\
\hline
\end{tabular}




\subsection{Analysis of Scenarios}

\subsubsection{Scenario 1}

\section{Blades}

Blades for $750-\mathrm{kW}, 1500-\mathrm{kW}$, and $2500-\mathrm{kW}$ turbines can be moved with relative ease by truck. Their dimensions and masses are manageable and minimal rerouting would be necessary. Extendable flatbeds that have been modified to carry blades would be the primary technique employed. Combining numerous blades (up to six) into single transport containers (particularly the $750-\mathrm{kW}$ turbine blades), although efficient for ship transport, is illegal in certain states for road transport. According to states' jurisdictions these loads are "reducible," thus requiring the transporter to remove blades from the container before proceeding. This action indicates a wider acceptance and greater likelihood of permit approval for multiple over-dimensioned loads that are within the 36,000-kg $(80,000 \mathrm{lb})$ gross vehicle weight (GVW) limit than fewer over-dimensioned and overweight loads. Therefore for cost estimating purposes, it has been assumed that three $750-\mathrm{kW}$ blades, two $1500-\mathrm{kW}$ blades, and one $2500-\mathrm{kW}$ blade would be transported. These arrangements would stay within the 36,000-kg (80,000-lb) GVW limit, therefore requiring a permit for width and length exceptions only. For this study, we assumed that three $750-\mathrm{kW}$ blades (oriented root to tip with the leading edges perpendicular to the ground) would result in a standard load dimension of $2.6 \mathrm{~m}(8.5 \mathrm{ft})$ in width by $4.1 \mathrm{~m}(13.5 \mathrm{ft})$ in height. Two $1500-\mathrm{kW}$ blades (oriented root to tip with the leading edges parallel to the ground) would result in the oversized load dimensions of $3.3 \mathrm{~m}(10.8 \mathrm{ft})$ in width by $3.9 \mathrm{~m}(12.8 \mathrm{ft})$ in height. It was assumed that individual $2500 \mathrm{~kW}$ and larger blades would be placed on a transport trailer with their leading edges parallel to the ground. Transport costs per $\mathrm{kW}$ for the $750 \mathrm{~kW}$ to $2500 \mathrm{~kW}$ blades ranged from $\$ 2.91$ to $\$ 7.14$, depending on turbine size and origin. Transportation costs per mile ranged from $\$ 4.74$ to $\$ 5.50$.

Blades for the 3500-kW turbine can be transported over the road, however they will require a different approach. Objects with lengths exceeding approximately $45 \mathrm{~m}(150 \mathrm{ft})$ require the use of rear-steering equipment in addition to the prime mover. The blade dimensions and corresponding transport equipment will likely incur up to $25 \%$ additional miles between the shipping origin and destination due to rerouting. To reduce the overall height, it has been assumed that the blades are placed with the leading edge parallel to the ground. This orientation will require adequate support of the blade from the mid-section to the tip where bending will be the greatest. These factors increased the transport costs to between $\$ 5.51$ and $\$ 11.56$ per kW. The costs per mile for moving the $3500 \mathrm{~kW}$ blades were estimated to be $\$ 9.50$.

The $5000 \mathrm{~kW}$ blade and transport equipment will exceed the $36,000 \mathrm{~kg}(80,000 \mathrm{lb}) \mathrm{GVW}$ requiring the use of special multi-axle equipment. The total vehicle length of over 200 feet will likely cause permit difficulties for long haul scenarios since approval from multiple States would be necessary (increasing the possibility of denial), therefore this blade will only be able to travel on roads over short haul distances. In instances such as this, state official would rather see efforts put into mitigating road travel through the use of water travel. By demonstrating all reasonable efforts are being utilized to minimize land travel, acceptance and permit approval potential increases. A long haul cost scenario was estimated assuming the $5000 \mathrm{~kW}$ blades originated in Gainesville, Texas and were driven onto a deck barge at the Port of Houston. Given space limitations on the deck barge only two blades and corresponding trucks (assuming one blade per truck) could be accommodated on the barge. From the Port of Houston the barge would travel through the Mississippi and Missouri River Systems to Sioux City, Iowa. The blade/truck 
combinations would then be driven north through Iowa and South Dakota to the project site. The resulting road transport costs of $\$ 11.00$ per mile reflect the increased complexity of associated with moving these blades. The associated road transport costs for these blades range from $\$ 2.19$ to $\$ 7.29$ per $\mathrm{kW}$. However, the barge component of this scenario contributes $\$ 96.56$ per $\mathrm{kW}$. A $5000 \mathrm{~kW}$ blade manufactured in Texas would need to be transported to the Port of Houston and driven onto a deck barge. Upon arrival in Sioux City, Iowa, the vehicle would be driven off the barge and delivered to the project site. The total costs for this movement was estimated to be $\$ 100$ per $\mathrm{kW}$. Although the use of barges facilitates movement of these large blades over great distances, their dimensions adversely impact the costs since it is been estimated that only two blade/truck combinations per barge could be accommodated.

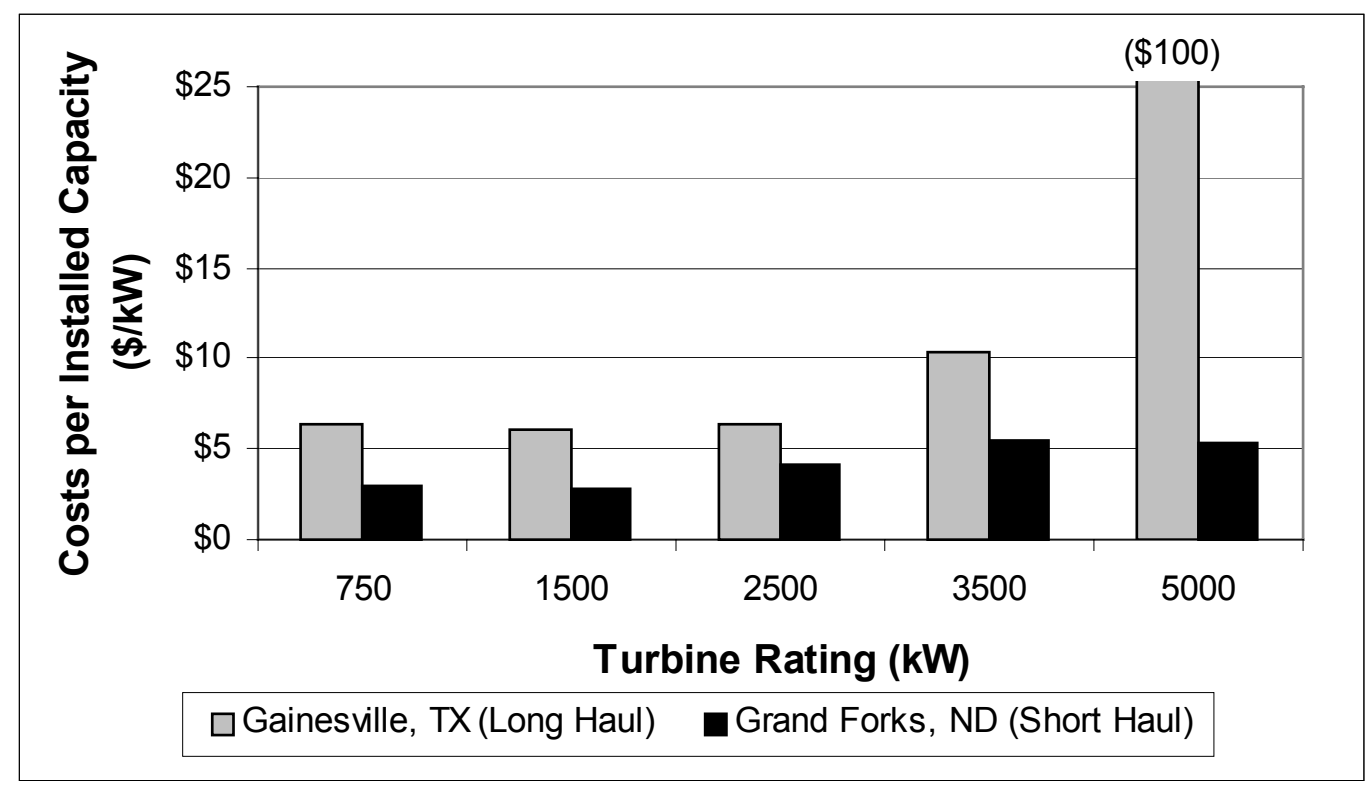

Figure 3-4.Estimated blade transport costs

(See Appendix P, Page 1)

Figure 3-4 presents truck transportation costs associated with blades manufactured in Texas and North Dakota. As would be expected, the costs from North Dakota are lower, particularly for the $5000 \mathrm{~kW}$ turbine. The largest reduction in costs is associated with not incurring barge costs from Houston, Texas to Sioux City, Iowa.

\section{Hubs}

Transportation of hubs does not appear to pose difficulty for any of the turbines. As the dimensions and masses increase they stay within a range that is easily accommodated by truck and rail transport. The hub transport costs per turbine increase as could be expected with turbine size, however, a peak in the transport costs per $\mathrm{kW}$ occurs at the $1500 \mathrm{~kW}$ turbine due a change in the transport trailer equipment. A trailer change is necessitated by the combined hub mass and vehicle mass exceeding $36,000 \mathrm{~kg}(80,000 \mathrm{lbs})$. This slight transport inefficiency for the $1500 \mathrm{~kW}$ turbine has minimal impact to the total transportation costs since hub transport costs are insignificant in comparison to the other components. 


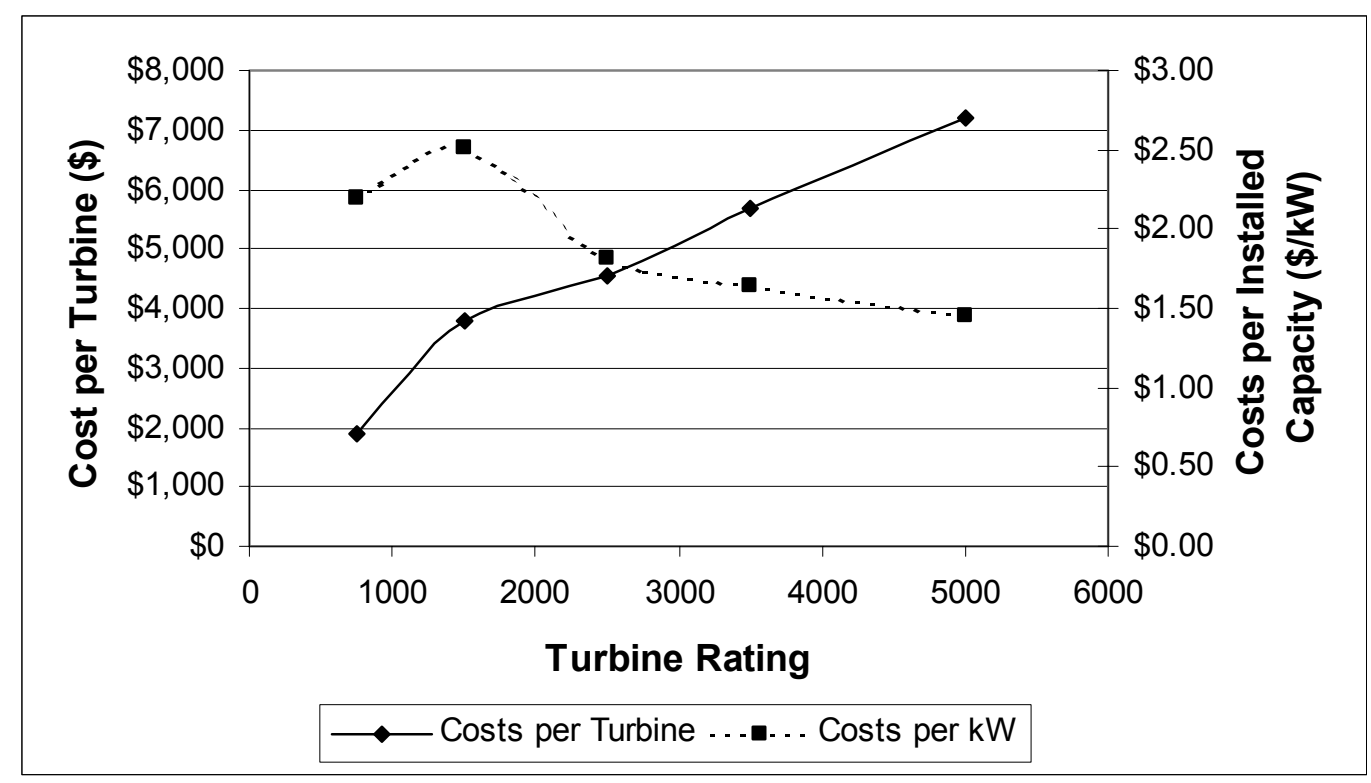

Figure 3-5.Hub transportation costs from Chicago, Illinois

(See Appendix P, Page 2)

\section{Nacelles}

Transport of $750 \mathrm{~kW}, 1500 \mathrm{~kW}$, and, $2500 \mathrm{~kW}$ nacelles can be accommodated by truck and rail with truck transport being more cost effective primarily because it does not incur a mode transfer expense. Costs to transport the $750 \mathrm{~kW}$ to $2500 \mathrm{~kW}$ nacelles via truck were estimated to range from $\$ 2.64$ to $\$ 7.32$ per installed $\mathrm{kW}$. The same costs for rail transport (including offload and final truck/dolly delivery costs) ranged from $\$ 6.07$ to $\$ 15.44$. Truck and rail costs are generally very comparable provided the rail destination is close to the project site, resulting in minimal additional road transportation costs.

Steerable dollies and rail can accommodate the $3500 \mathrm{~kW}$ nacelle. However, dolly and rail costs are very different over both short haul and long haul scenarios with rail costs being significantly lower. Dolly costs for this turbine was about $\$ 70$ per $\mathrm{kW}$ where as the rail costs (that include an offloading crane and the appropriate transport from the rail spur to the project site) were about $\$ 20$ per $\mathrm{kW}$. Based on the proximity of a rail spur within $80 \mathrm{~km}(50 \mathrm{mi})$ of the project site, rail has been determined to be the best option for the $3500 \mathrm{~kW}$ nacelle transport.

Rail transport adds an element of risk related to nacelle damage during transit. Sudden accelerations and decelerations during start-up and braking, train coupling and decoupling, rail joints, and less sophisticated rail car suspensions can induce sufficient forces and vibrations to result in damage or misalignment of components. Accelerometers are typically placed on delicate loads to monitor shipping loads.

A comparison of nacelle transport costs by mode originating from Chicago, Illinois is presented in Figure 3-6. The cost trends from Tehachapi, California are similar and differ in magnitude only. Dolly costs for the $2500 \mathrm{~kW}$ are presented to demonstrate the increase in shipping costs if the $2500 \mathrm{~kW}$ nacelle exceeds $84,000 \mathrm{~kg}(185,000 \mathrm{lbs})$. 


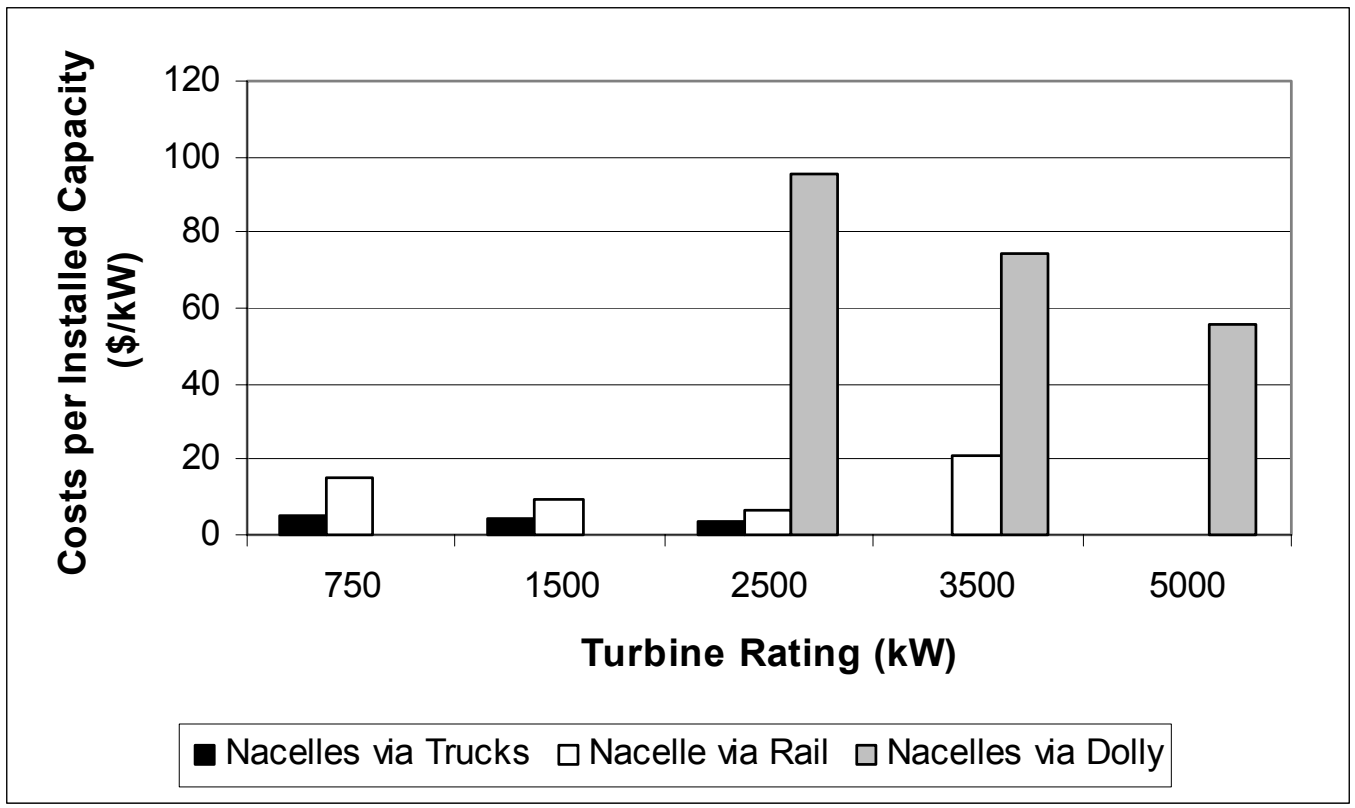

Figure 3-6.Comparison of nacelle transport modes from Chicago, Illinois (short haul) (See Appendix P, Page 3)

The mass of the $5000 \mathrm{~kW}$ nacelle results in steerable dollies being the only short haul option available. Long haul movement would require the combined use of dollies and barges because the nacelle exceeds the rail capacity of $163,000 \mathrm{~kg}(360,000 \mathrm{lbs})$. Short haul dolly costs for the $5000 \mathrm{~kW}$ turbine ranged from $\$ 50$ to $\$ 90$ per $\mathrm{kW}$ depending upon the distance. For long hauls the additional barge costs was estimated to be $\$ 45$ per $\mathrm{kW}$. In general, as the turbine sizes increased the dolly costs per $\mathrm{kW}$ decreased with the highest dolly costs being incurred for the $2,500 \mathrm{~kW}$ turbines.

The $163,000 \mathrm{~kg}(360,000 \mathrm{lb})$ limitation for rail transport of the nacelle dictates that the target turbine transportable by rail would have a $100-\mathrm{m}(328-\mathrm{ft})$ rotor diameter corresponding to the $3500 \mathrm{~kW}$ turbine.

\section{Tower}

Transport of intact tubular tower sections will rely upon trucks, steerable dollies, and barges. An unexpected result of the tower dimensions and specifications utilized in this study was that the base section of the $1500 \mathrm{~kW}$ turbine could not be transported cost effectively by truck or dolly. This is in stark contrast to current practice in which 750 and $1500 \mathrm{~kW}$ towers are being moved over the road in a relatively cost effective manner. The $1500 \mathrm{~kW}$ WindPACT turbine tower stands $86 \mathrm{~m}(282 \mathrm{ft})$ high with an estimated base diameter of $4.9 \mathrm{~m}(16 \mathrm{ft})$. The current $1500 \mathrm{~kW}$ turbines are on towers up to $80 \mathrm{~m}(262 \mathrm{ft})$ high and utilize base diameters of about 4.3 to $4.4 \mathrm{~m}$ (14.1 to $14.3 \mathrm{ft}$ ). The base diameter of the WindPACT tower results in a total vehicle height exceeding $4.83 \mathrm{~m}(15.83 \mathrm{ft})$ which exceeds the height at which utility assistance is triggered resulting is extreme costs and planning logistics.

Costs to move the intact base section of the $1500 \mathrm{~kW}$ tower with a base diameter of $4.9 \mathrm{~m} \mathrm{(16 \textrm {ft } )}$ from the Texas/Louisiana area to South Dakota were based on dolly transport to the Port of Houston (\$125 per kW, see Appendix E, page 4), barge transport to Sioux City, Iowa (\$44 per $\mathrm{kW}$, see Appendix G, page 4), then dolly transport to South Dakota (\$107 per kW, see Appendix 
E, page 4). A direct land route was not evaluated since obtaining the necessary permits and organizing all of the utilities along the route would prove to be infeasible and State authorities would likely state that a "reasonable by-pass" utilizing the Mississippi and Missouri rivers is present and should be utilized. Using this approach, the costs to transport the $1500 \mathrm{~kW}$ base section alone was estimated to be $\$ 276$ per $\mathrm{kW}$ while the total for the remaining sections was estimated at $\$ 19.50$ per $\mathrm{kW}$. The total tower transport costs for the $1500 \mathrm{~kW}$ turbine with a base diameter of $4.9 \mathrm{~m}(16 \mathrm{ft})$ was $\$ 295$ per $\mathrm{kW}$.

If the base diameter of the $1500 \mathrm{~kW}$ turbine was $4.4 \mathrm{~m}$ or less (eliminating the need for utility assistance and barge travel), the estimated transport costs for the base section alone was $\$ 8.12$ per $\mathrm{kW}$ with a resulting total turbine tower shipping cost of $\$ 27$ per $\mathrm{kW}$. Since existing tower designs for $1500 \mathrm{~kW}$ turbines employ base diameters of about 4.3 to $4.4 \mathrm{~m}$ (14.1 to $14.3 \mathrm{ft}$ ), the excessive tower shipping costs associated with a base diameter of $4.9 \mathrm{~m}(16 \mathrm{ft})$ are not being utilized further in this report. Instead, we are assuming that the base diameter of the $1500 \mathrm{~kW}$ turbine is less than $4.4 \mathrm{~m}(14.3 \mathrm{ft})$ and can be shipped without the need for utility assistance or barge transport. Tower designers can keep the base dimensions less than $4.4 \mathrm{~m}$ using a variety of options such as modifying the tower wall thickness to diameter ratio or adjusting the taper ratio.

\subsubsection{Scenario 2}

Towers

From a transportation perspective, the only impact of Scenario 2 is to mitigate tower transportation issues and costs. Under this scenario, it has been assumed that oversized tower sections are quartered lengthwise. This results in tower pieces that are dimensionally compliant with the height restrictions discussed above and in many cases results in a gross vehicle weight at or less than $36,000 \mathrm{~kg}(80,000 \mathrm{lbs})$. All transports are within the 79,400 to $83,900 \mathrm{~kg}(175,000$ to $185,000 \mathrm{lbs}$ ) range eliminating the need for high capacity dollies. It was assumed that only one quartered tower piece would be transported on a trailer to help improve permit approval potential and eliminate the possibility of a "reducible load" ruling.

This approach had a significant impact on the transportation costs. All of the tower sections and pieces could be transported for costs ranging from $\$ 1.40$ to $\$ 11.00$ per mile. For those loads that exceeded $36,000 \mathrm{~kg}(80,000 \mathrm{lb}) \mathrm{GVW}$ the costs per mile range from $\$ 6.00$ to $\$ 11.00$, demonstrating that a significant costs savings can be realized by keeping vehicle weight under $36,000 \mathrm{~kg}(80,000 \mathrm{lbs})$. Total tower short haul costs per $\mathrm{kW}$ ranged from about $\$ 16$ for the 750 $\mathrm{kW}$ turbines to about $\$ 51$ for the $5000 \mathrm{~kW}$ turbines. These same costs per $\mathrm{kW}$ under Scenario 1 were about $\$ 16$ and $\$ 692$, respectively. Figure 3-7 presents a comparison of the estimated tower transportation costs by scenario for tower sections originating from Louisiana. The significant increase in the number of loads required to deliver all of the tower pieces was more than offset by incurring lower transport costs per mile.

Section 4 of this report discusses the assembly costs in further detail, however, the additional costs incurred to assemble the tower sections were minor in comparison to the transportation costs savings. 


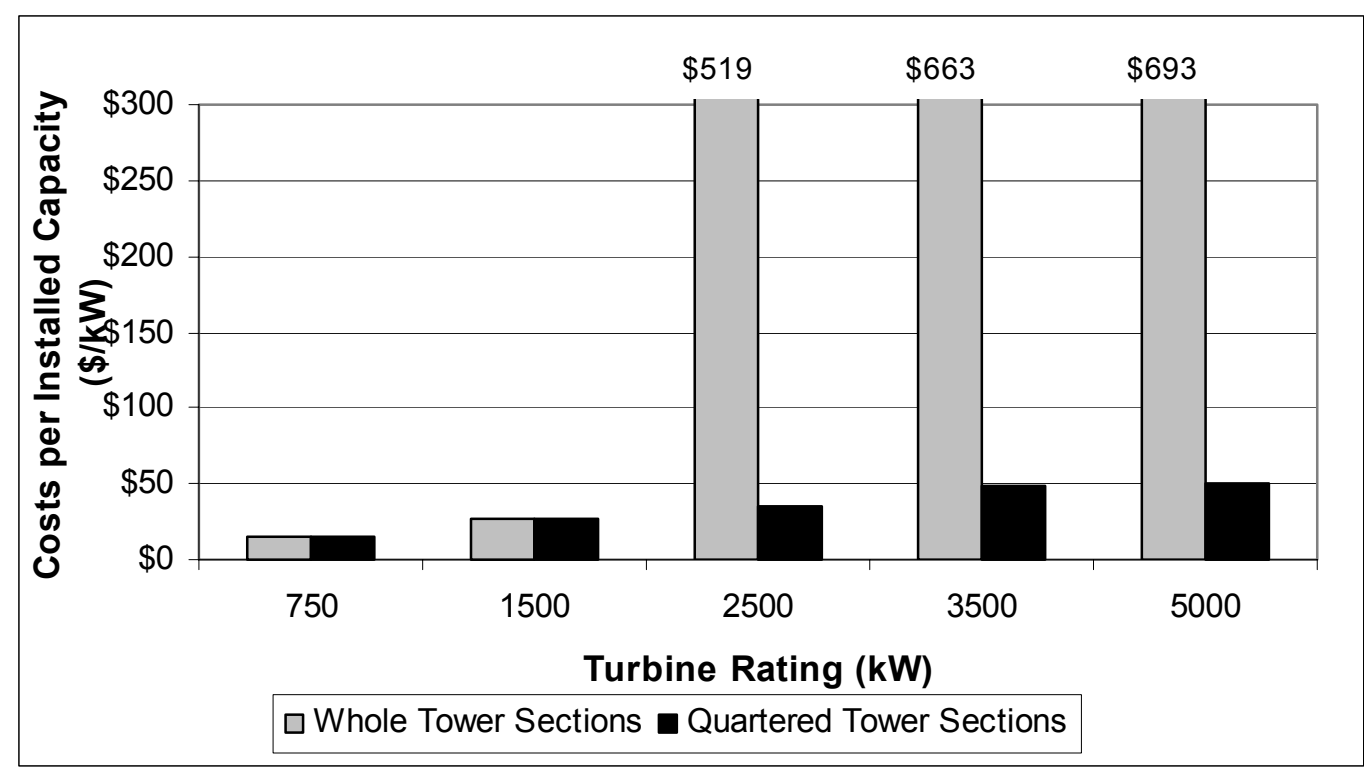

\begin{tabular}{|l|c|c|c|c|c|}
\hline TURBINE RATING (KW) & $\mathbf{7 5 0}$ & $\mathbf{1 5 0 0}$ & $\mathbf{2 5 0 0}$ & $\mathbf{3 5 0 0}$ & $\mathbf{5 0 0 0}$ \\
\hline Scenario 1 & $\$ 16$ & $\$ 27$ & $\$ 519$ & $\$ 663$ & $\$ 693$ \\
\hline Scenario 2 & $\$ 16$ & $\$ 27$ & $\$ 35$ & $\$ 48$ & $\$ 51$ \\
\hline
\end{tabular}

Figure 3-7.Scenario 1 and Scenario 2 tower transportation cost comparisons

Costs based on transport from Shreveport, Louisiana (see Appendix P, page 4)

\subsubsection{Scenario 3}

\section{Nacelle}

The third scenario in which the primary nacelle components (gearbox and generator) are removed and shipped to the site separately only had a beneficial impact on costs associated with transporting the $5000 \mathrm{~kW}$ nacelle. Under Scenario 3, the generators can be shipped on typical flat bed trailers within the $80,000 \mathrm{GVW}$ limitations to take advantage of low cost per mile rates (about \$1.50). It was estimated that the mass of the gearbox for the 3500 and $5000 \mathrm{~kW}$ turbines would require the use of double-drop type trailers resulting in cost per mile rates of about $\$ 11$.

Shipping costs for the $2500 \mathrm{~kW}$ and $3500 \mathrm{~kW}$ nacelles actually increased slightly due to requiring three shipments per nacelle as opposed to only one. A sufficient mass reduction for the $3500 \mathrm{~kW}$ and $5000 \mathrm{~kW}$ 'empty nacelles' was not realized to allow the use of tractor-trailers instead of rail or dollies. Rail costs for the nacelles remained virtually unchanged between Scenario 2 and 3. The $5000 \mathrm{~kW}$ still could not be moved by rail leaving dollies the only transport option for this nacelle. Under this scenario, truck transport was determined to be the preferred nacelle transport mode for nacelles up to $2500 \mathrm{~kW}$. The $3500 \mathrm{~kW}$ nacelle would likely be transported by rail and the $5000 \mathrm{~kW}$ nacelle would still need to be transported by dolly.

Analysis of the rail transport costs reveals a very slight decrease in the rail costs however when the truck transport costs for the generator and gearbox components are included, the total costs increase slightly. Rail shipping rates are based on the object weight and decrease as the object mass increases. Table 3-3 demonstrates the rail cost reduction with weight increase. Therefore, more favorable rail economics are realized when the nacelles shipped by rail remain intact. 


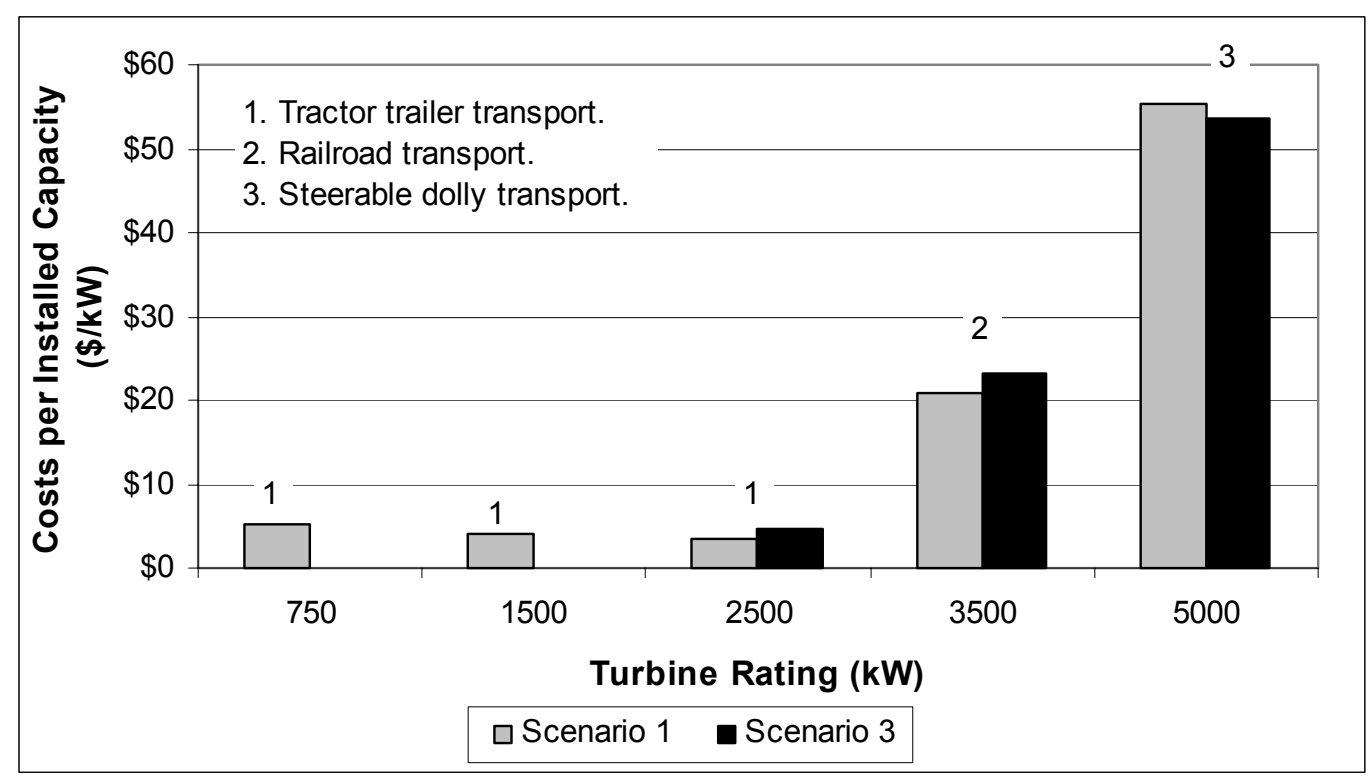

Figure 3-8. Comparison of nacelle transport approaches

Costs include truck, rail and dolly modes from Chicago, Illinois (see Appendix P, Page 5)

Table 3-3.Example Rail Costs from Houston, Texas

\begin{tabular}{|c|c|}
\hline Weight $(\mathbf{l b s})$ & $\mathbf{\$ 1 0 0} \mathbf{~ l b s}$ \\
\hline Up to 75,000 & $\$ 3.53$ \\
\hline 75,000 to 125,000 & $\$ 3.15$ \\
\hline 125,000 to 240,000 & $\$ 2.88$ \\
\hline 240,000 to 360,000 & $\$ 2.72$ \\
\hline
\end{tabular}

\subsection{Emerging Transportation Technology-Airships}

Currently, the main use of airships is advertising, however, their attributes combined with new technology and materials are expanding their commercial potential into transportation logistics. The concept is straight forward, eliminate (or significantly reduce) the planning and permitting requirements associated with oversized and overweight loads on land with airship freight that has higher capacity and lower costs than current cargo aircraft. Large, heavy objects like conventional generators, oil refinery components, and wind turbines, which must be shipped to their final destination in pieces by road, rail, and ship, could be transported directly in one piece. Worldwide, a number of companies are actively developing high capacity airships.

Three general approaches to airship design exist, non-rigid (advertising ships), semi-rigid, and rigid (classic Zeppelin design). There are 2 approaches to cargo transport by airship emerging, internal and external payloads. The internal payload approach is aimed at containerized freight and direct competition with ocean transport. External payloads are targeted at both containerized freight and the oversized object market. The external payload approach has also been referred to as a "flying crane" approach. 
The furthest-advanced airship companies are Advanced Technologies Group (ATG) and Cargo lifter. ATG has developed a flying radio controlled prototype. Their design is a semi-rigid airship that employs aerodynamic lift in addition to the buoyancy effect of helium. ATG's SkyCat has targeted internal payloads of $15 \mathrm{mt}, 200 \mathrm{mt}$, and $1000 \mathrm{mt}(33,000 \mathrm{lbs}, 440,000 \mathrm{lbs}$, and 2,200,000 lbs). Cargo lifter also employs a semi-rigid design, however, its external payload is targeted at $160 \mathrm{mt}(350,000 \mathrm{lbs})$. Cargo lifter has developed a long list of investors and is currently building a manufacturing facility in Brand, Germany. For comparison purposes, the largest cargo aircraft, the Ukrainian Antonov AN-225 has a payload capacity of $250 \mathrm{mt}$ $(550,000 \mathrm{bs})$.

Airship transport costs are being estimated as lower than aircraft cargo but higher than ocean freight. Since airships do not need to consume fuel to stay aloft, a considerable operating expense is minimized. In comparison to ocean freight, the higher velocities of airships could result in quicker transport time. Airship transport costs are targeted in the range of $\$ 0.45$ to $\$ 0.66$ per $\mathrm{kg}$. For comparison, aircraft freight ranges from $\$ 1$ to $\$ 10$ per kg and ocean freight is approximately $\$ 0.25$ per kg (or per cubic meter, which ever is greater). Current design and manufacturing activity is focused on infrastructure and prototypes. Deployment of the first commercial cargo airships is anticipated in 2003 to 2005. 


\section{Assembly and Crane Logistics}

\subsection{Assembly Assumptions}

For purposes of this study, turbine assembly was assumed to include:

- Offloading turbine components from transport vehicles, uncrating, sorting, and distribution across the site.

- Rigging and setting the tower sections including bolt inspections.

- Grouting base tower section and torque verification.

- Assembly of the blades and hub into the rotor inclusive of pitch mechanisms and alignment.

- Rigging and setting nacelles onto tower tops including alignment, application of proper bolt torque, and connection of basic electrical equipment.

- Rigging and setting the rotor assembly to the nacelle.

- Installation of climbing equipment, setting controllers, and installing power cables from nacelle to base.

- Crane relocation assistance between turbines.

Construction of the turbine foundations is not included in the turbine assembly estimates. Foundation costs are presented in WindPACT Technical Area 4 - Balance of Station Costs. Turbine commissioning activities are also excluded from the assembly estimates.

Assembly crews were based on 10 to 12 people working six days per week. The crew size was based on typical crew sizes currently being used by construction companies. Considering the remote project site, it was likely that some activities would be performed on Saturday since the work crews would not return home for the weekends. It is likely that Saturday work would be used to help offset less productive weekdays if and when weather impacts the project.

The level of effort required to perform various tasks for the $2500 \mathrm{~kW}$ and larger turbines was based on experiences gained during assembly of the current generation of $750 \mathrm{~kW}$ and $1500 \mathrm{~kW}$ turbines. Efforts were based on object sizes, work heights, assembly techniques, and the type of crane being utilized. The current practice of installing tower base sections with a relatively small capacity truck or rough terrain crane was utilized for each type of turbine. This technique allows assembly activity to be performed during inclement weather when high rotor or nacelle work cannot be performed. It also helps to minimize the time on-site for the high capacity installation crane.

In addition to estimating the levels of effort required for various activities, the 'general condition costs' were also prepared. These are construction related costs that are incurred for a variety of items. The primary general condition cost categories include:

- Professional services - consulting engineers and inspectors

- Construction management personnel

- Travel and Relocation expenses

- Field office expenses

- Temporary construction facilities and tool storage

- Mobilization and demobilization transport costs 
- Temporary construction utilities

- Construction equipment

- Worker safety

- Final site clean-up

- Project start-up and closeout

The principal general conditions expense is for the construction equipment used for a project. The following equipment was included in development of the construction equipment costs:

- 2 hydraulic truck cranes (sizes were adjusted for weights of turbine components)

- Crawler crane for offloading freight

- 2 rough terrain forklifts

- Surveying/Level equipment

- Radios/phone communications

- Bolt torque equipment

- Site pick-up trucks

- Equipment fuel and operating expenses

- Equipment repairs

- Small tools/Consumables

- Crane operator and oiler.

Costs presented in this section were based on estimates prepared by the M.A. Mortenson construction company and reflect a conservative approach. Lower costs can be realized if site conditions and weather are ideal, however, practical experience has demonstrated that these conditions cannot be relied upon.

\subsection{Crane Assumptions}

The turbine specifications presented in Table 2-1 and Table 2-2 were provided to Lampson for analysis and selection of the most cost effective crane options. Given the specified hub heights and component masses, a specific crane was selected for each turbine. Lampson prepared the drawings shown in Figures 4-1 through 4-5 to help illustrate crane setup requirements, lifting position, clearances, crane pad space requirements, and scale. Once the crane type, counterbalance mass, boom length, and jib length (if required) were selected numerous costs were estimated and have been summarized in Appendix B. Items included in the costs are monthly rental rates, stand-by rates, crew size, crane transport, crane assembly requirements, crane relocation rates (between turbines), and consumables. 


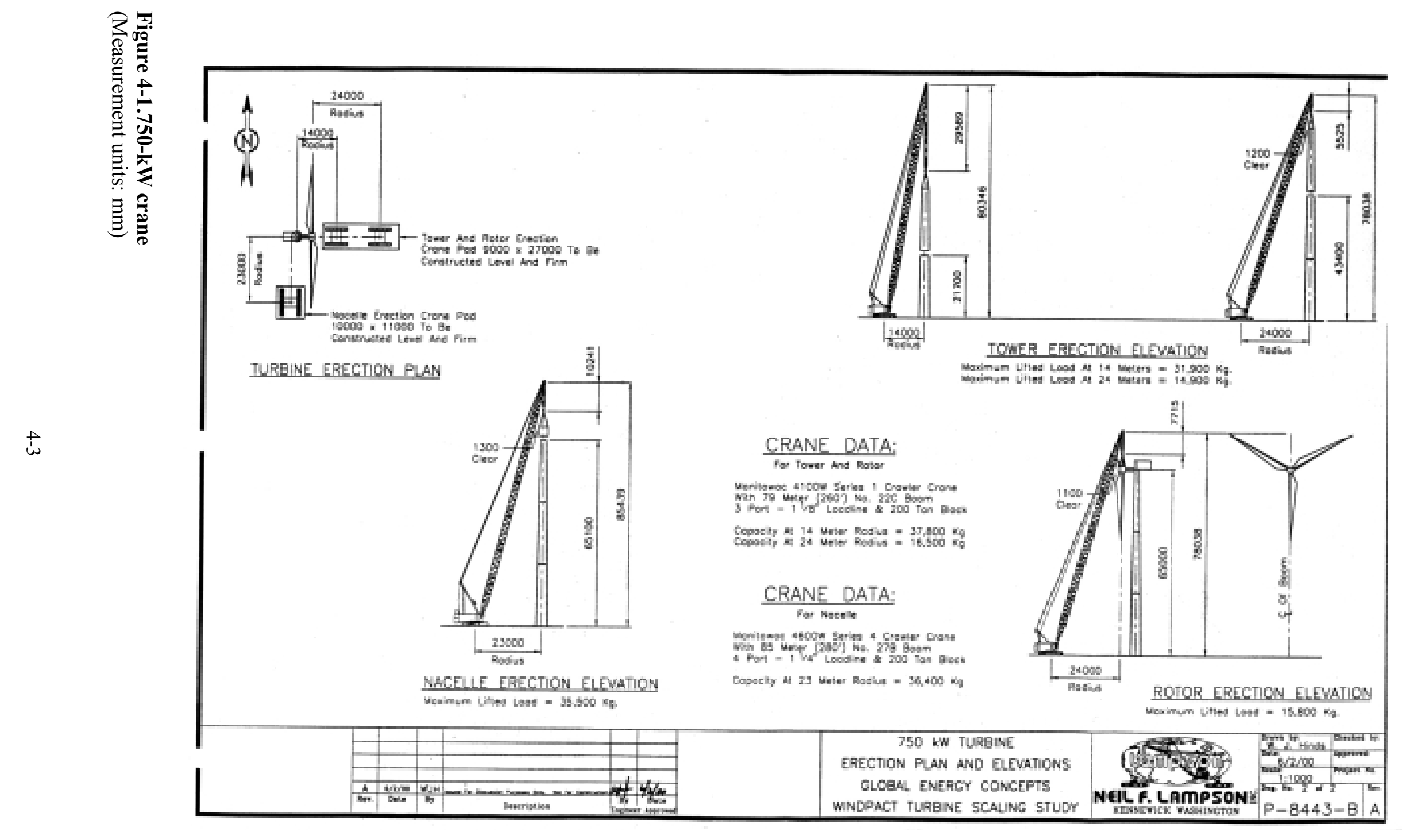




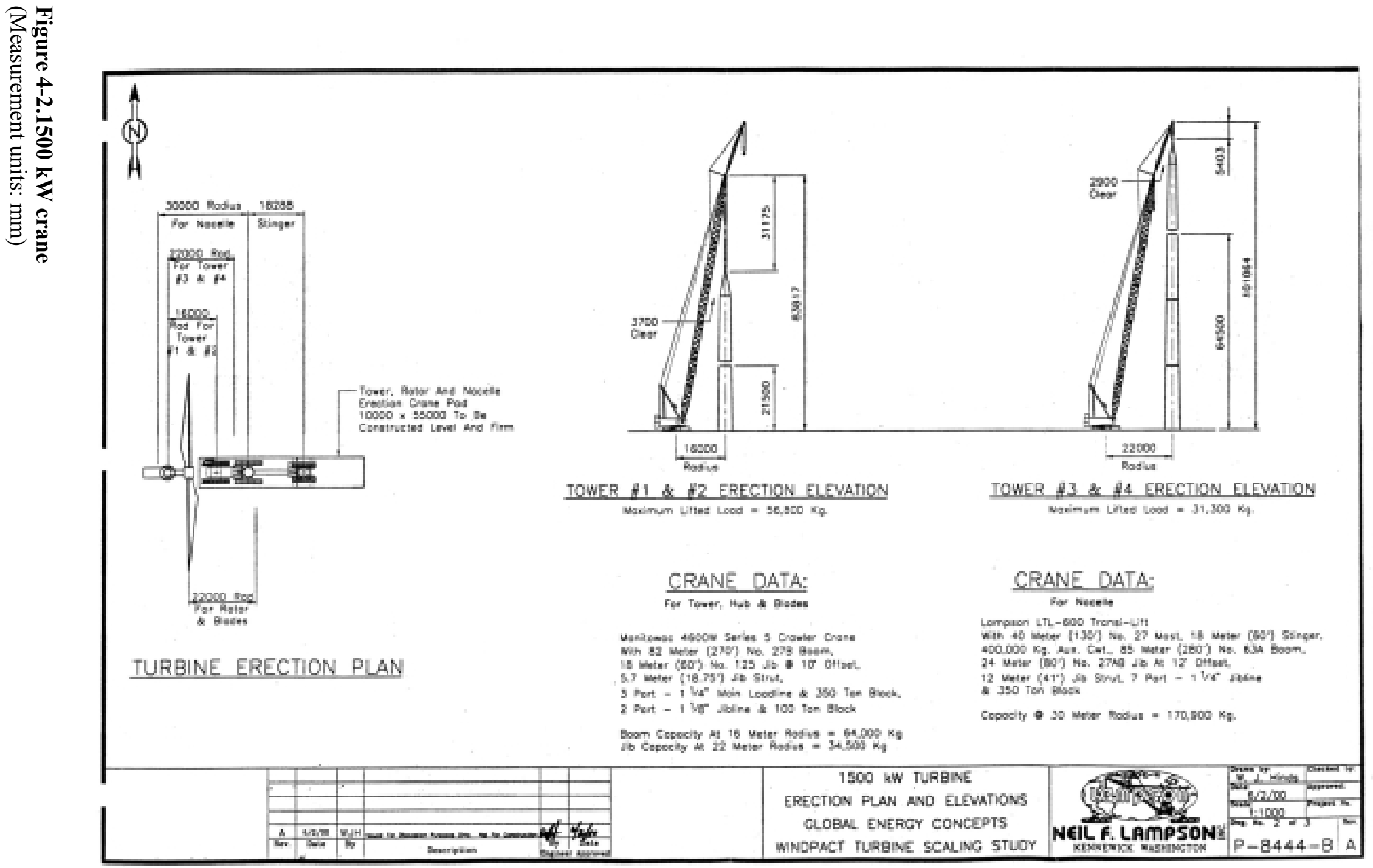




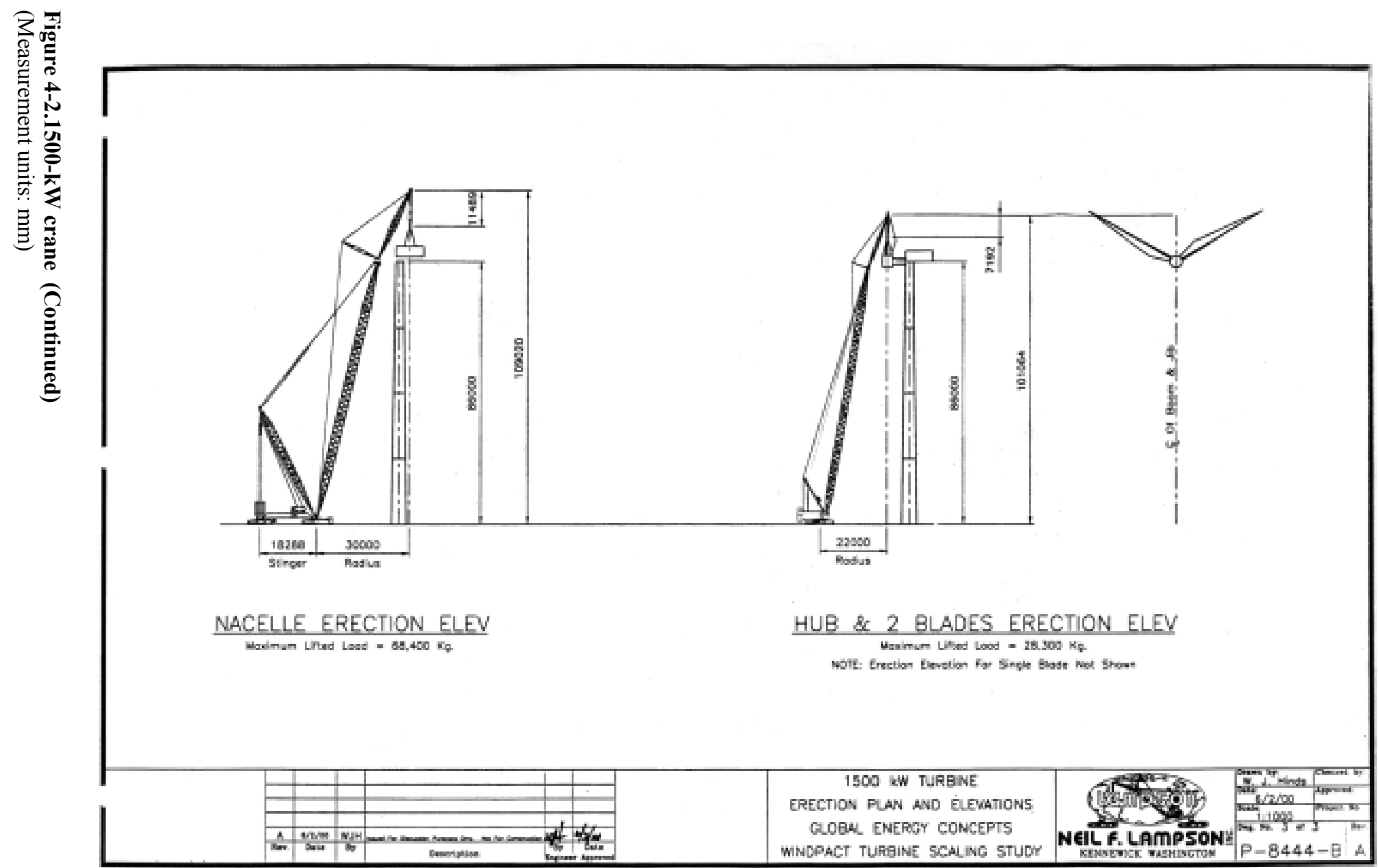




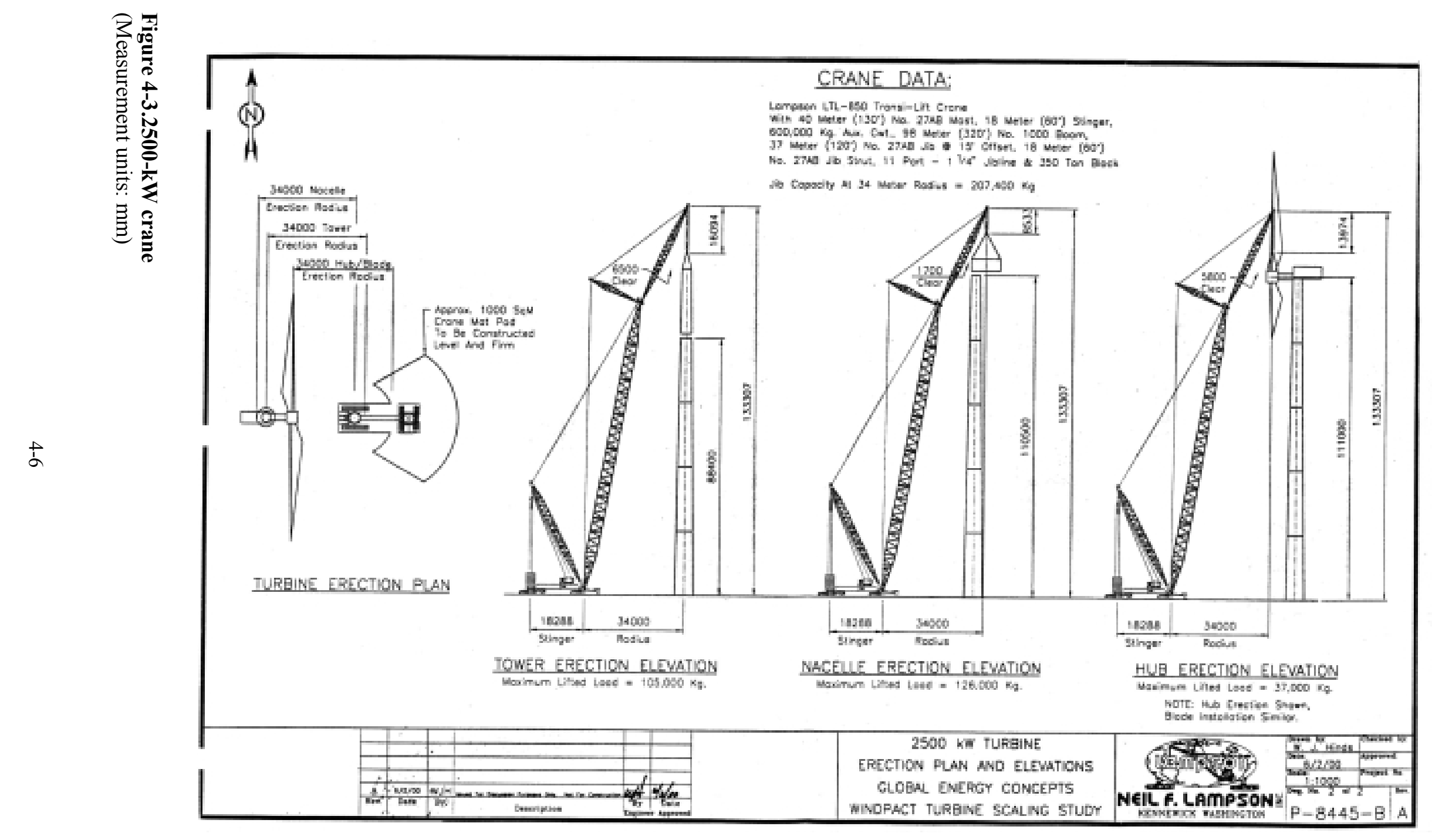




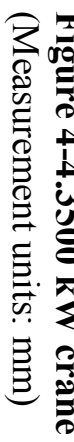

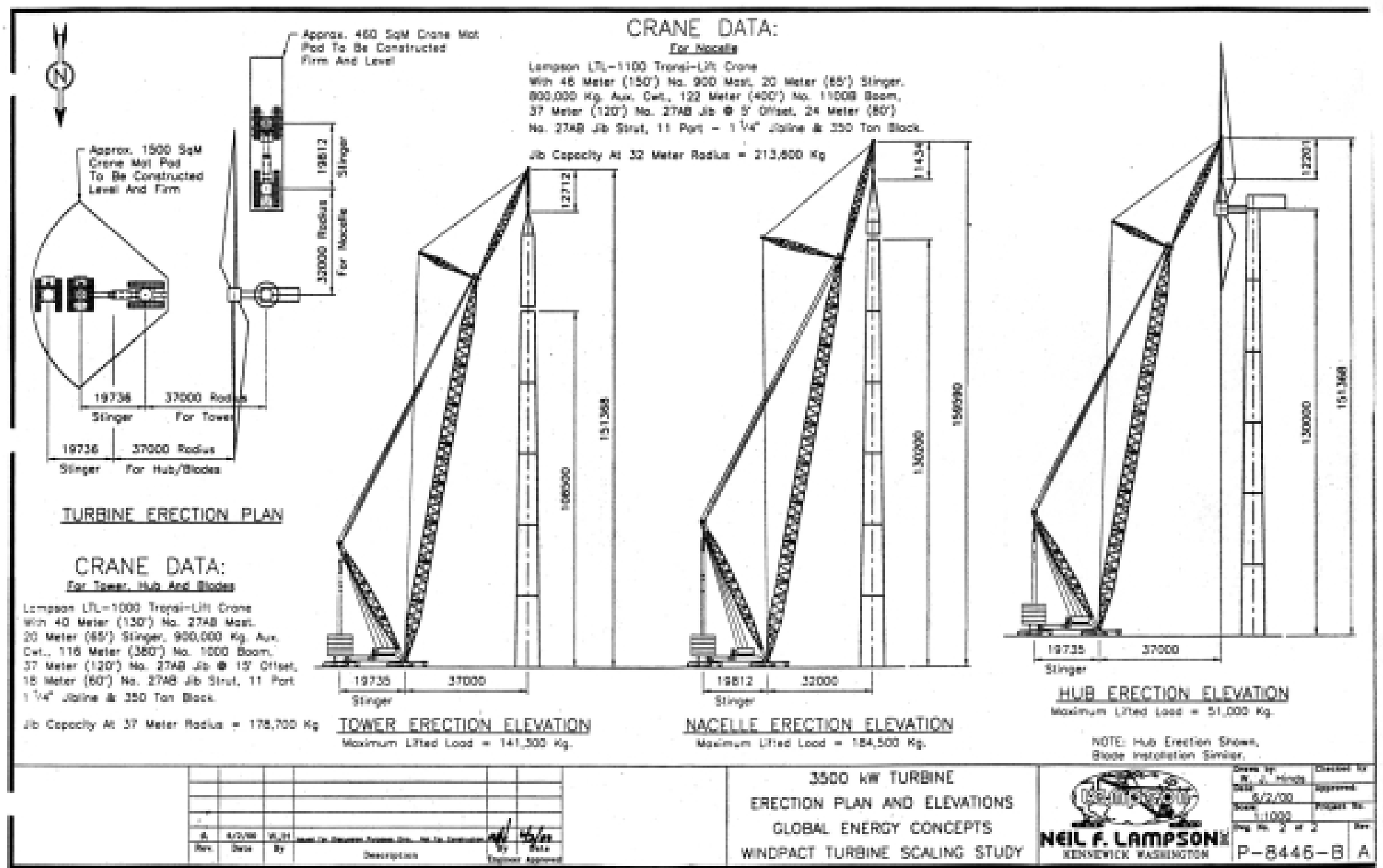




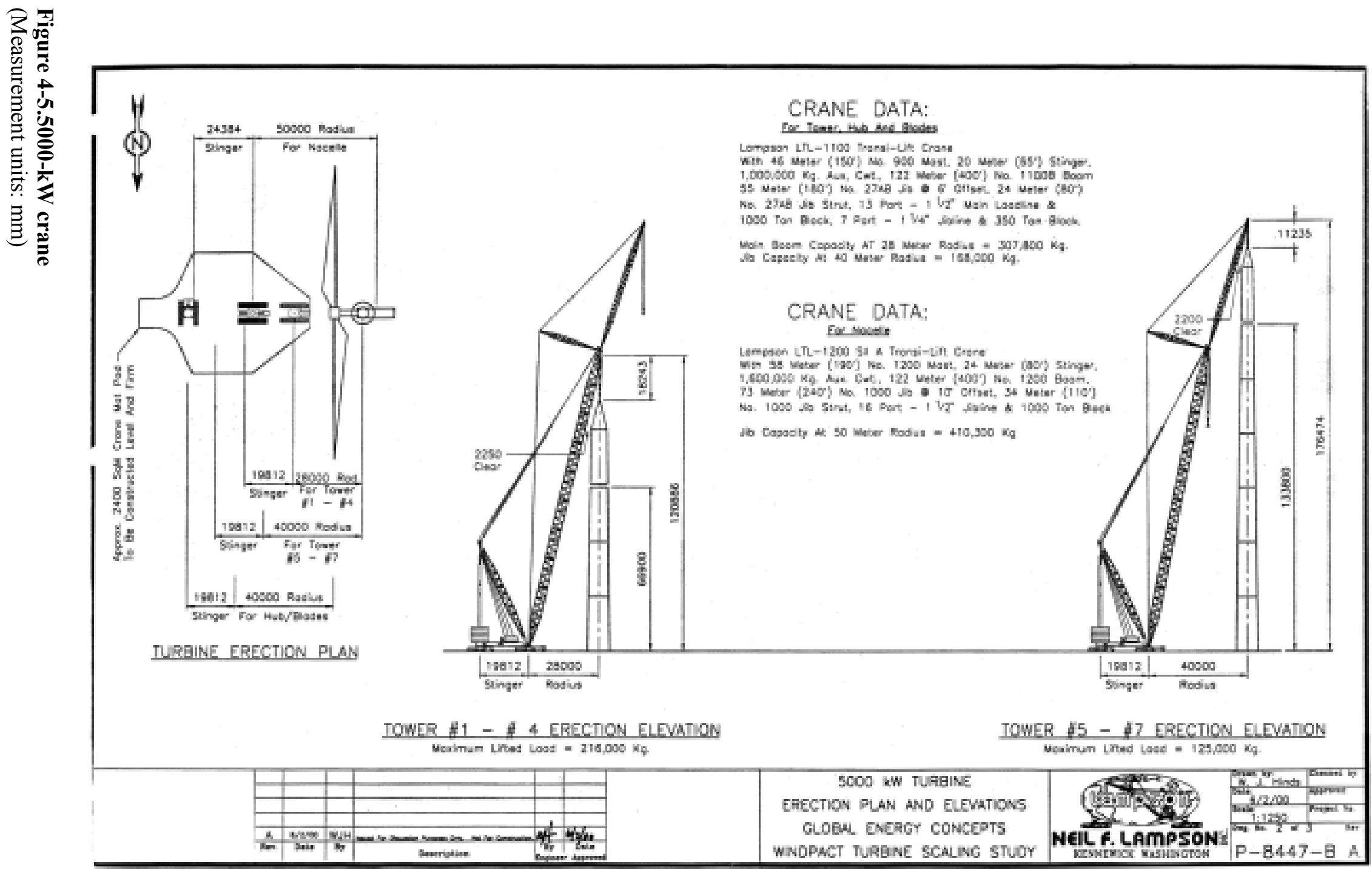




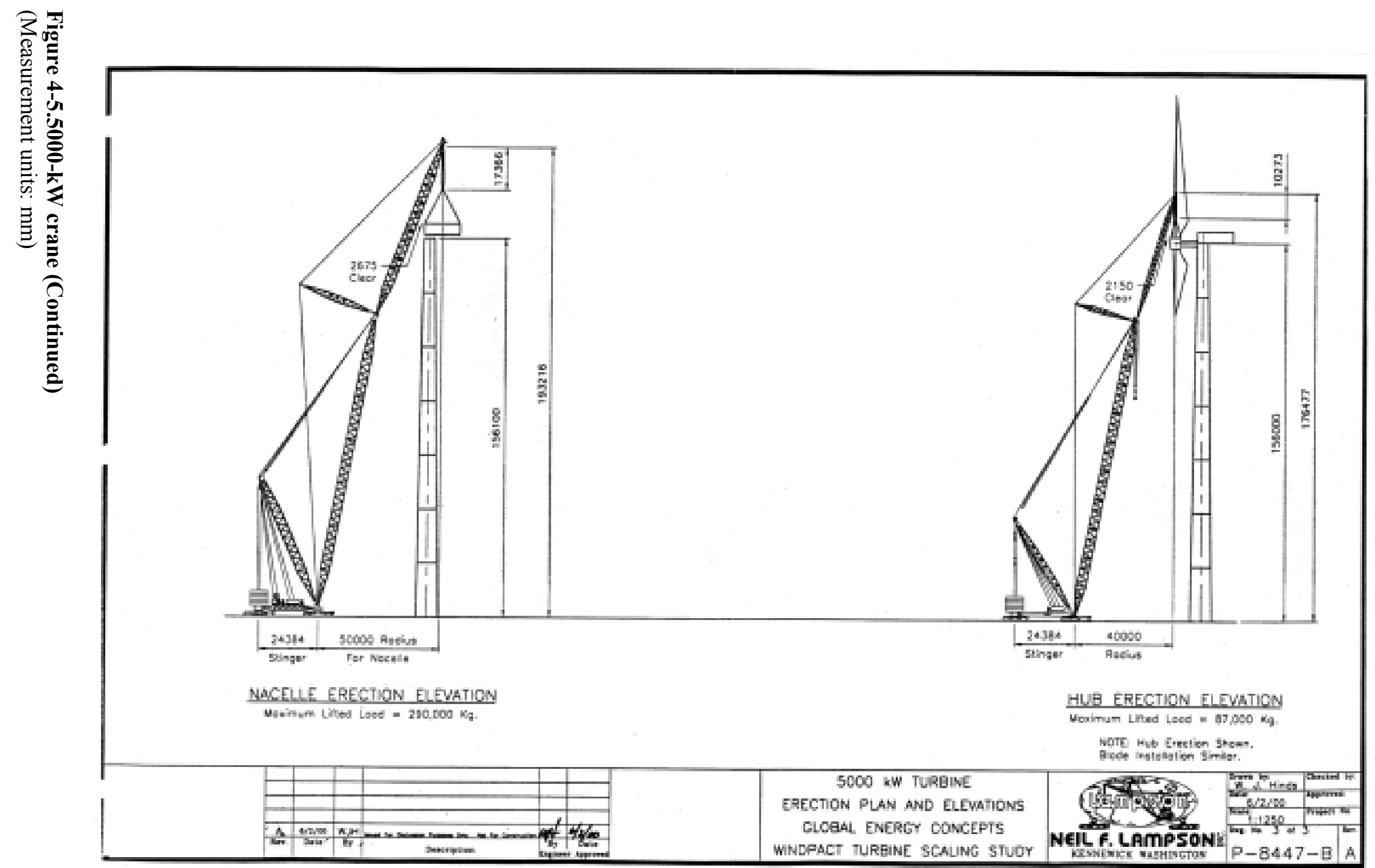


The time a crane remains on the project site will govern the crane costs. Based on input from Mortenson, the calendar time the crane would be on-site was estimated to equal the calendar time required for assembly of the towers, nacelle and rotors for all the turbines. The other turbine assembly activities can be conducted with the typical construction equipment without the high capacity crane. The total man-hours for the tower, rotor, and nacelle assembly tasks were divided by the total daily man-hours available to arrive at the estimated crane time per turbine. Then, crane relocation time between turbines was added to the crane's turbine assembly time to obtain a total crane operation time per turbine. The result of this approach was consistent with Mortenson's experience.

\subsection{Analysis of Scenarios}

The three basic scenarios discussed in Section 2.2 are analyzed in the following sections. Scenario 2 (in which the towers are sectioned lengthwise) includes analysis of three possible approaches for tower field assembly based on utilizing bolting, manual welding, and automated welding assembly techniques.

The crane costs (associated with turbine assembly) discussed in this section have been prepared under the assumption that one crane is assembling 50 turbines without being fully disassembled during relocation between turbines. This is considered optimum crane utilization. Based on actual experience, site terrain, soil conditions, and road construction significantly impact the mobility of high capacity cranes. In reality, more frequent crane disassembly and reassembly to facilitate relocation between turbines occurs during construction of wind farms. The terrain effects on the optimum crane costs discussed below are analyzed in Section 4.4.

\subsubsection{Scenario 1}

Figure 4-6 presents a summary of the general assembly and crane costs estimated for Scenario 1. Considering the turbine components are arriving on-site pre-assembled, this scenario would be expected to result in the lowest construction costs. The overall combined assembly and crane costs remain generally stable through the $2500 \mathrm{~kW}$ turbine, however, the $3500 \mathrm{~kW}$ and $5000 \mathrm{~kW}$ turbines experience increases to the combined cost per $\mathrm{kW}$. As the turbine sizes increase (in particular as the height increases) the crane costs assume a higher portion of the combined costs. 


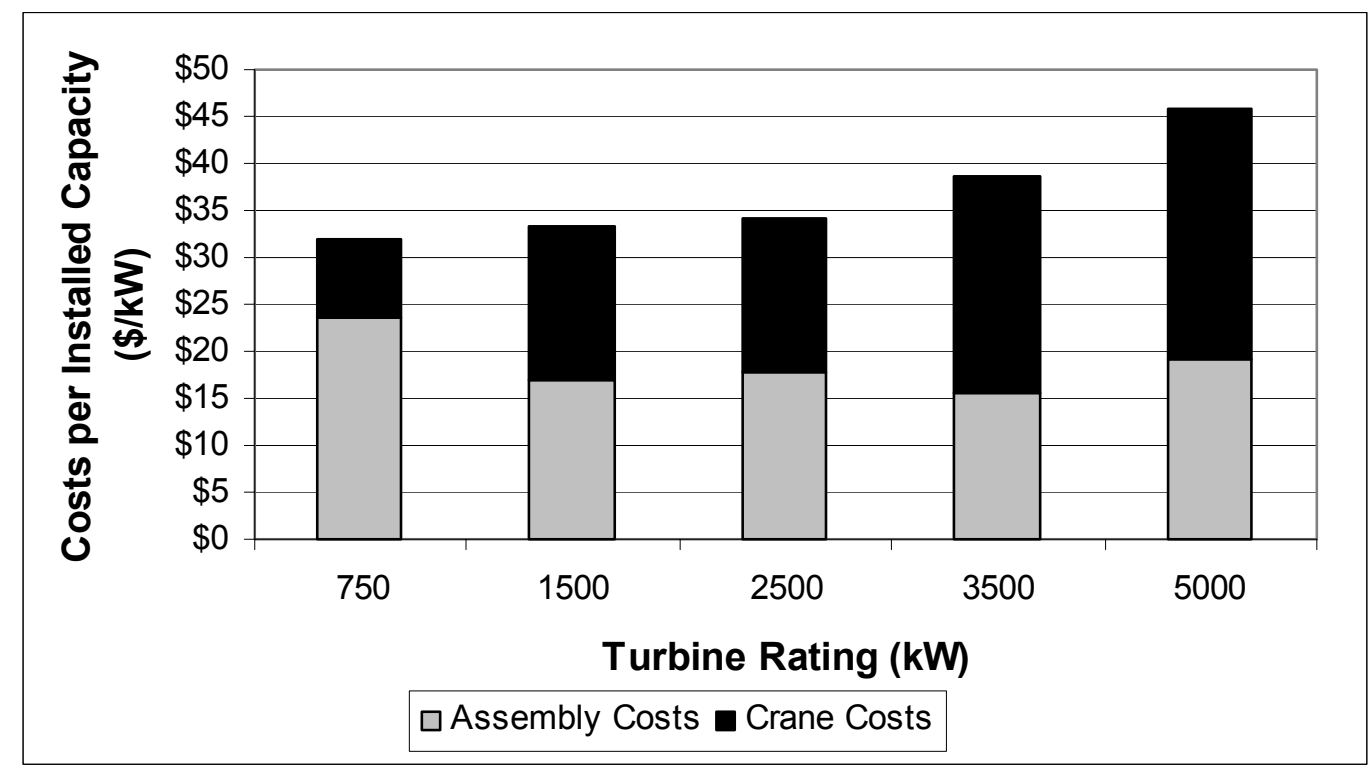

Figure 4-6.Scenario 1 assembly and crane costs

(See Appendix P, page 6)

\begin{abstract}
Assembly
Although considerable increases in the level of effort are estimated for assembling the megawatt scale turbines, with estimated assembly costs per turbine ranging from $\$ 13,000$ to $\$ 70,000$, there is a general decrease in the assembly costs per $\mathrm{kW}$ through the $3500 \mathrm{~kW}$ turbine. A slight increase is noted for the $5000 \mathrm{~kW}$ turbine. The largest decrease in assembly costs per $\mathrm{kW}$ occurs between the $750 \mathrm{~kW}$ and $1500 \mathrm{~kW}$ turbines. Experience has indicated that there is not a significant difference in the level of effort required to assemble a $1500 \mathrm{~kW}$ turbine as opposed to a $750 \mathrm{~kW}$ turbine. The largest impact is derived by the height increase and to a lesser extent the increase in component sizes, however, the increase in rated power exceeds the modest increase in assembly effort.
\end{abstract}

\title{
Cranes
}

On a cost per turbine basis, the crane costs increase as the turbine sizes increase, however, a plateau in the costs per $\mathrm{kW}$ was identified at the $1500 \mathrm{~kW}$ and $2500 \mathrm{~kW}$ turbines even though different cranes are being utilized. Analysis of the cranes' lifting capacity for each turbine was performed to determine the cause. Figures 4-7 through 4-11 demonstrate the relationships between the mass and height requirements for each turbine component with respect to potential cranes. These figures demonstrate that the nacelle is the object that ultimately determines the specified crane. Aside from the $750 \mathrm{~kW}$ turbines, the boom elevations required to lift the nacelle are essentially the maximum elevation possible for the specified cranes. Typically the boom point elevation is 15 to $20 \mathrm{~m}$ ( 50 to $65 \mathrm{ft}$ ) greater that the hub height to allow space for blocks and rigging, additional clearance of the tower, and clearance between the nacelle and boom (or jib if being utilized). 


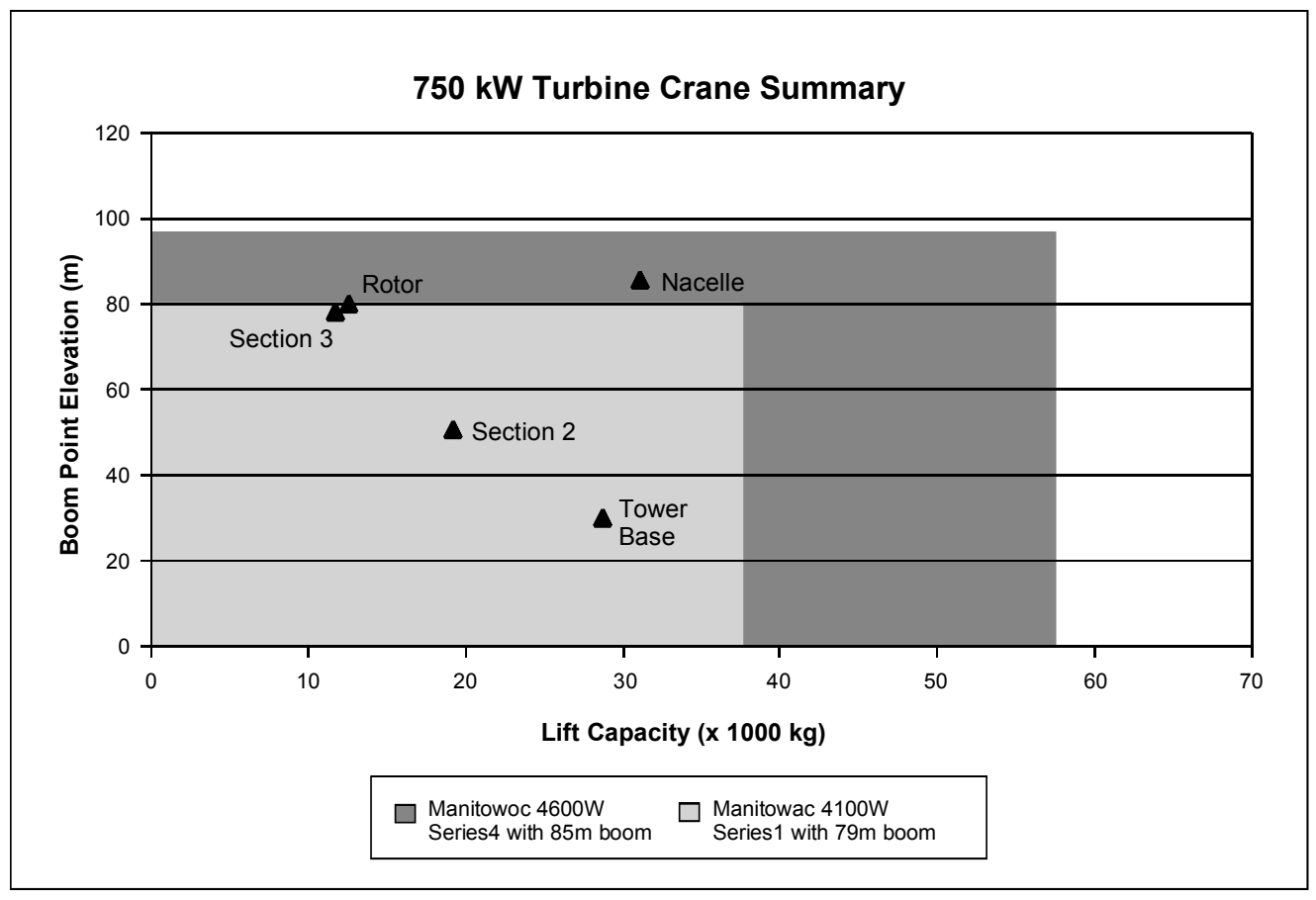

Figure 4-7.750-kW turbine crane summary

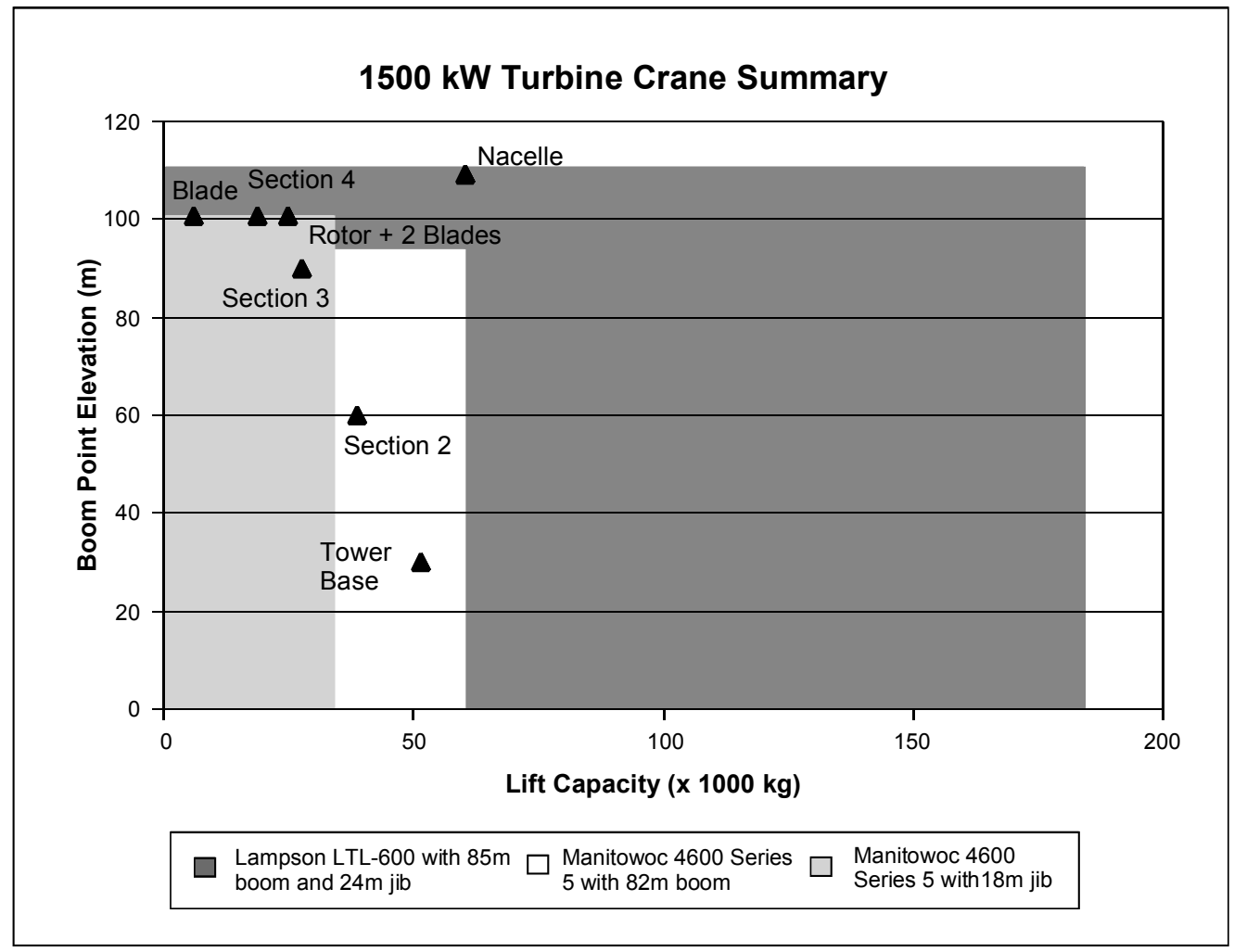

Figure 4-8.1500 kW turbine crane summary 


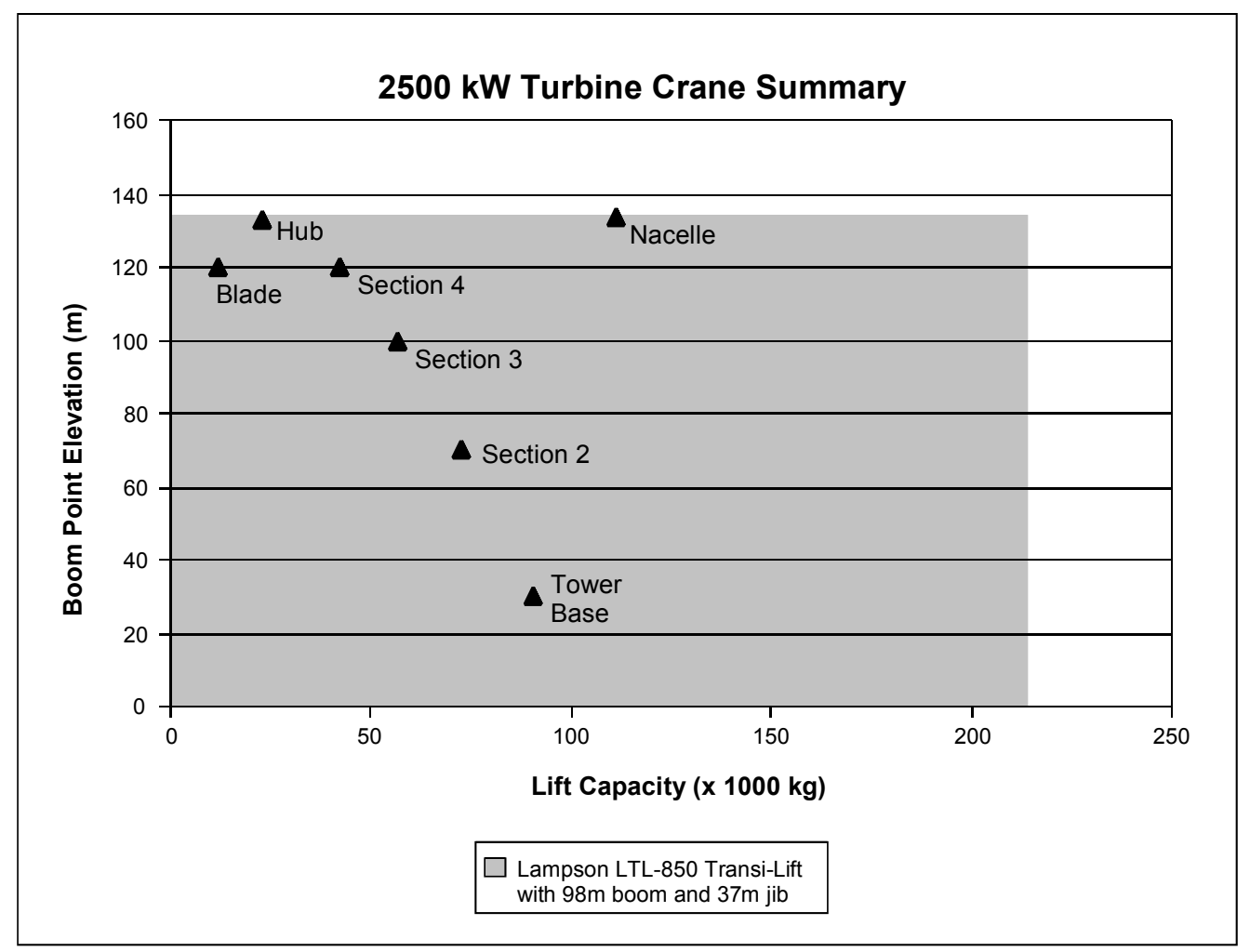

Figure 4-9.2500-kW turbine crane summary

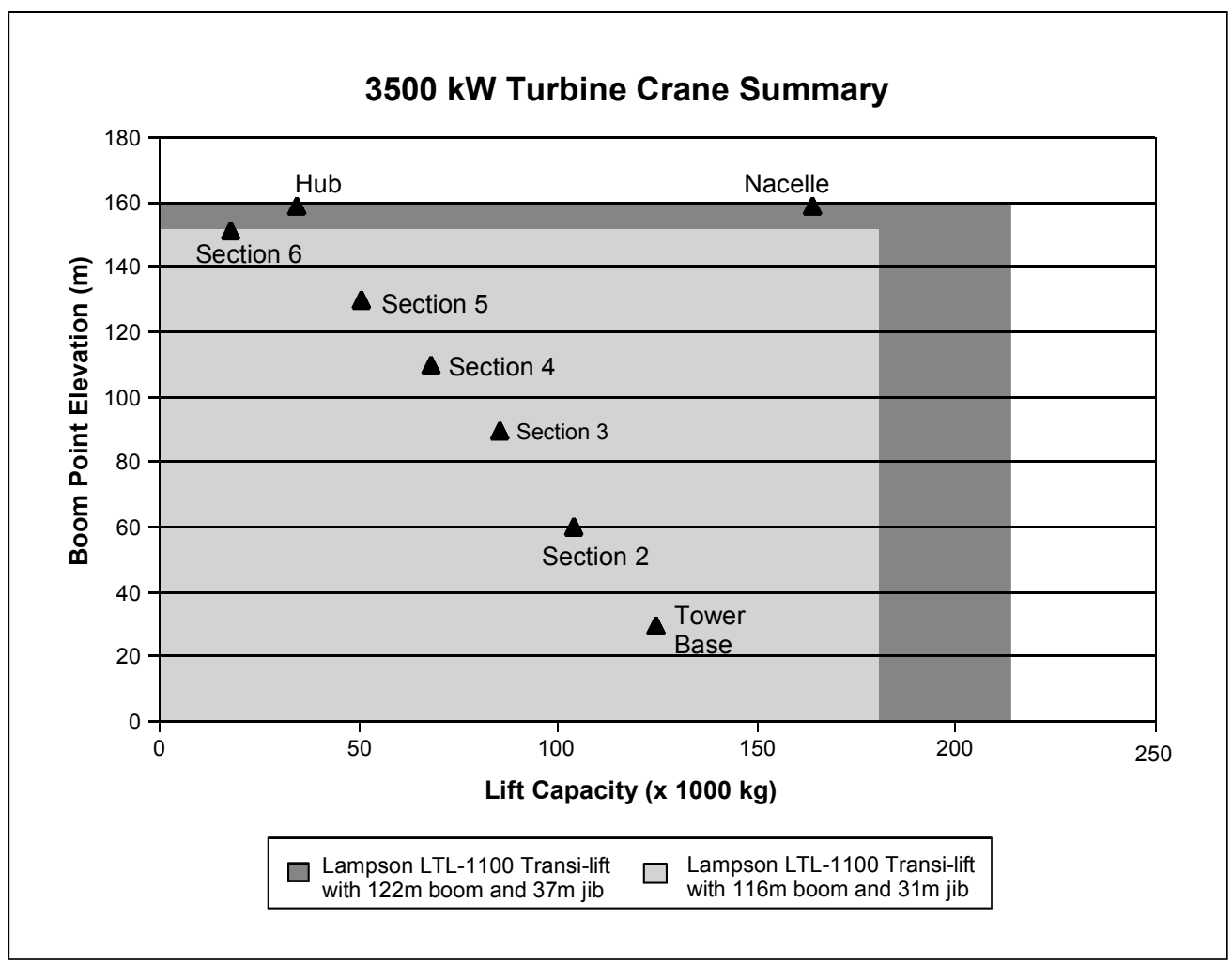

Figure 4-10.3500-kW turbine crane summary 


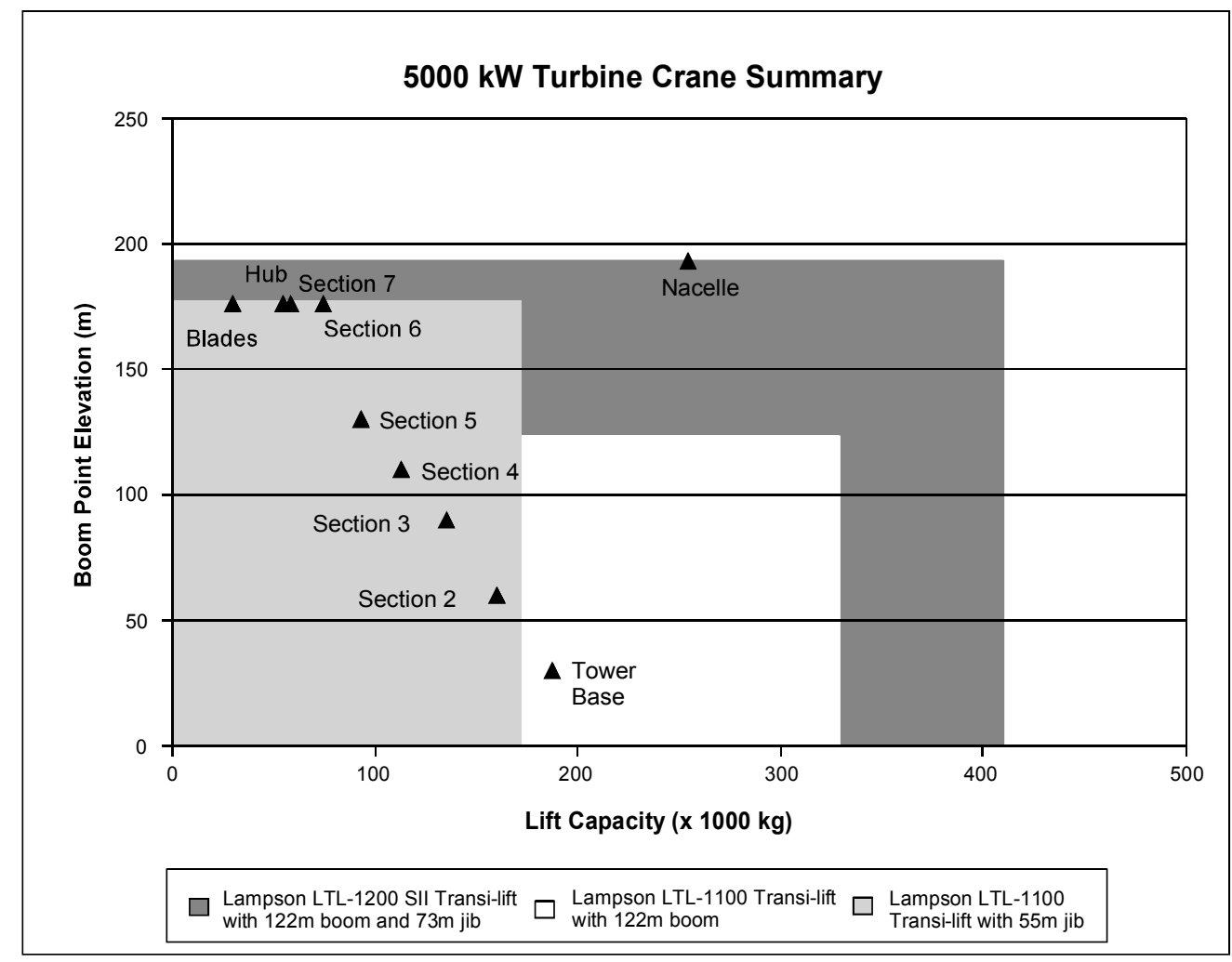

\section{Figure 4-11.5000-kW turbine crane summary}

The nacelle mass is generally at or within $50 \%$ of the total lifting capacity of the specified crane except the $1500 \mathrm{~kW}$ turbine, where the nacelle mass is only $35 \%$ of the total lifting capacity. It is apparent in Figures 4-7 through 4-11 that the crane industry generally has a greater need for lifting capacity that height. This is in sharp contrast to the wind industries need for height and.opposed to lifting capacity. The result is that cranes with significant excess capacity are used to obtain the required installation heights

The $86 \mathrm{~m}$ hub height for the $1500 \mathrm{~kW}$ turbine eliminated the possibility of utilizing a 350 -ton Manitowoc 4600 Series 5 crane with a base monthly rental of $\$ 37,500$. This resulted in the need for a 600-ton Lampson LTL-600 crane with a base monthly rental of $\$ 90,000$. Lampson noted that it might be possible to modify the Manitowoc 4600 Series 5 crane by adding a stinger attachment and a customized jib; however, the resulting monthly rental costs would be approximately $\$ 70,000$ to $\$ 80,000$. The hub height and masses of the $1500 \mathrm{~kW}$ WindPACT turbine identified a void in the crane market where a 350 -ton crane with a possible boom elevation of $110 \mathrm{~m}$ (360 ft) does not exist. In addition, there is a significant jump in the costs of cranes between the 350 ton and 600 ton capacity range.

Finally as the crane capacity increases, the number of available cranes decrease. Figure 4-12 demonstrates the sizable decrease in crane quantities as the capacities increase. This information is based on historic crane data (1997) compiled by the Chicago Bridge and Iron Company and contained data from 18 crane manufacturers. Although newer crane models produced since 1997 would likely change the absolute values at the different crane capacities, the overall trend of the data has not unchanged. 


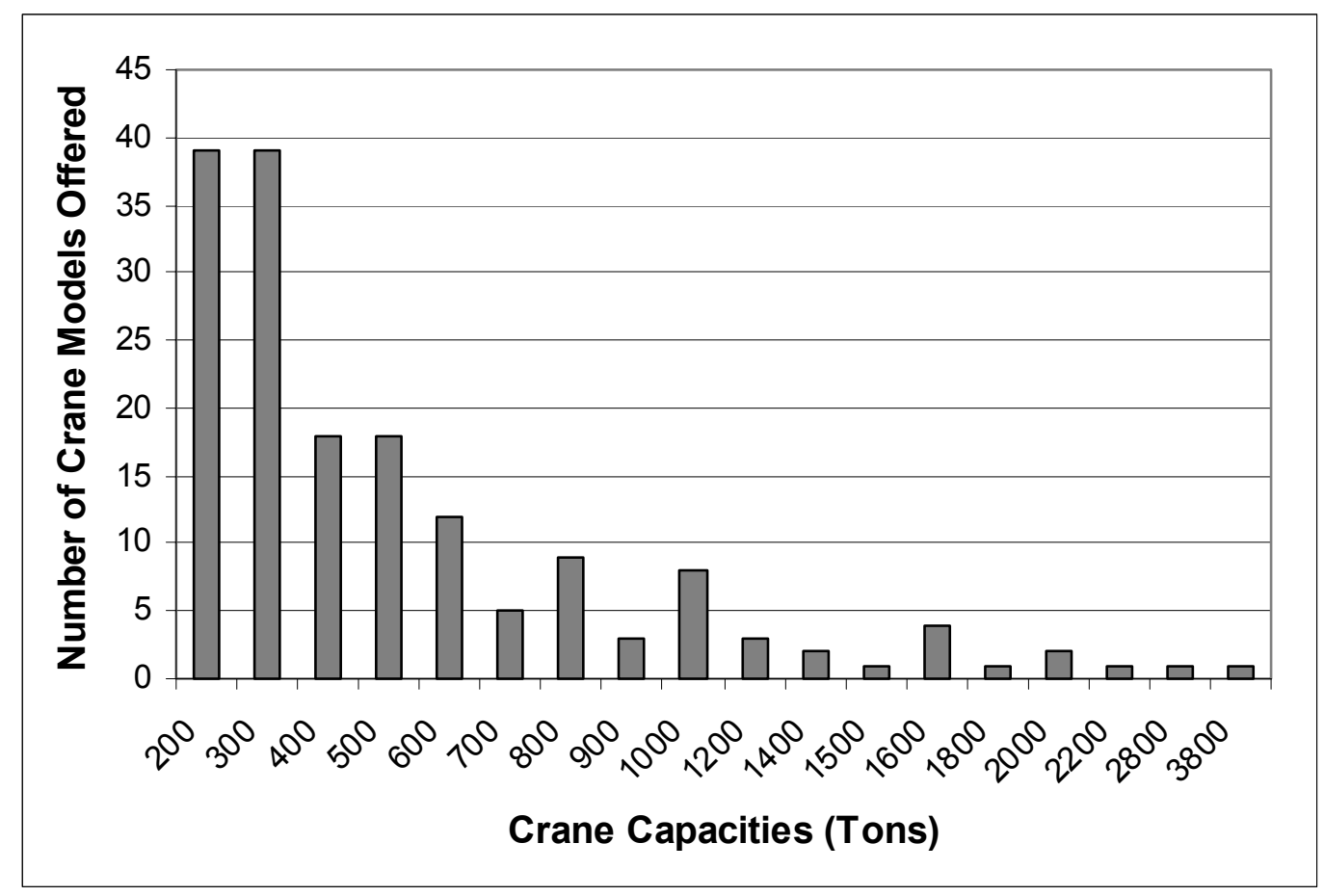

Figure 4-12.Crane capacity trends.

(See Appendix P, Page 7)

Whereas the excess crane capacity for the $1500 \mathrm{~kW}$ turbine results in the most inefficient crane usage, the $750 \mathrm{~kW}$ and $2500 \mathrm{~kW}$ turbines appears to be the most optimized. If the rotor diameter and hub height ratios for the 3500 and $5000 \mathrm{~kW}$ turbines are assumed to be close to 1 [resulting in hub heights of $100 \mathrm{~m}(328 \mathrm{ft})$ and $120 \mathrm{~m}(394 \mathrm{ft})$, respectively], then 850-ton to 1000-ton cranes could be used resulting in very efficient crane utilization.

\subsubsection{Scenario 2}

This scenario estimates the impact of assembling quartered tubular tower sections to the project costs. In comparison to Scenario 1, the combined turbine assembly and crane costs under this scenario are greater, however the decrease in transportation cost more than offsets the increase. Three separate tower fabrication approaches were analyzed with bolted connections and automated welding yielding lower costs than manual welding. The relative difference between automated welding and bolting was minor with bolted connections achieving the lowest costs. Additional material costs have been included in the estimates, however, the total impact on tower material costs was uncertain.

Figure 4-13 presents a comparison of the various assembly costs by tower assembly approach. 


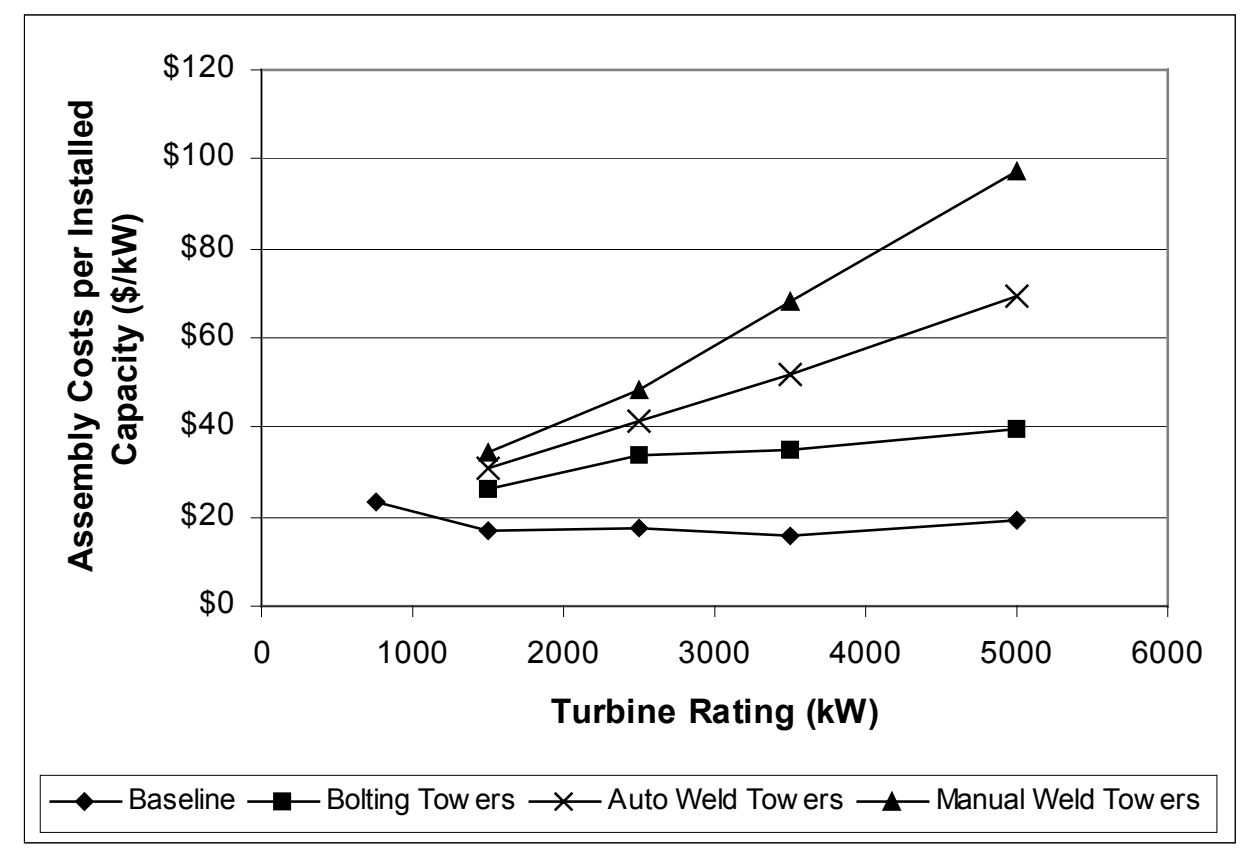

Figure 4-13.Assembly costs by scenario

(see Appendix P, page 8)

\section{Assembly}

The turbine assembly process was relatively unchanged from Scenario 1 . The only minor assumption change was that the rotor assembly would occur with the hub attached to the nacelle on the tower top. In Scenario 1, it was assumed that the rotor would be lifted in one piece to the maximum extent possible. However, Lampson believed that less time would be lost to wind by lifting individual components as opposed to an entire rotor. The rotor assembly hours between Scenario 1 and 2 were increased $10 \%$ based on Mortenson's opinions. The corresponding change to costs per turbine and costs per $\mathrm{kW}$ was negligible in comparison to the increase in assembly time required for the tower sections.

To assemble the tower sections it was assumed that four concrete pads would be built on which the fabrication would be performed. The assembly area would be located in one portion of the project site and assembled tower sections would be transported to the turbine locations. Assembly of four quartered sections into two half sections would each take place on one pad. Jigs, templates, and blocking supports would be used to accurately align and secure the sections before assembly. On a third pad, two half-tower sections would be assembled into one tower section. The forth pad would be used for final inspection, painting, and staging for transport to the turbine site. More weather protection would be required for the welding approaches as opposed to the bolting approach.

The added on-site fabrication activity changed the assembly cost per $\mathrm{kW}$ from a decreasing trend in Scenario 1 to an increasing trend. The only difference in Scenario 2 is the rate of increase. In general, the bolting approach added approximately $\$ 20$ to the Scenario 1 assembly costs per kW, whereas manual welding added $\$ 20$ to $\$ 80$ to the Scenario 1 assembly costs per kW. However, these added costs are much less than the decrease in transportation costs realized through the shipment of quartered tower sections. Figure 4-14 presents combined turbine assembly and crane costs associated with use of quartered tower sections. Table 4-1 presents the worst-case scenario 
comparing changes in short haul transportation costs with increased in on-site assembly and crane costs utilizing manual welding.

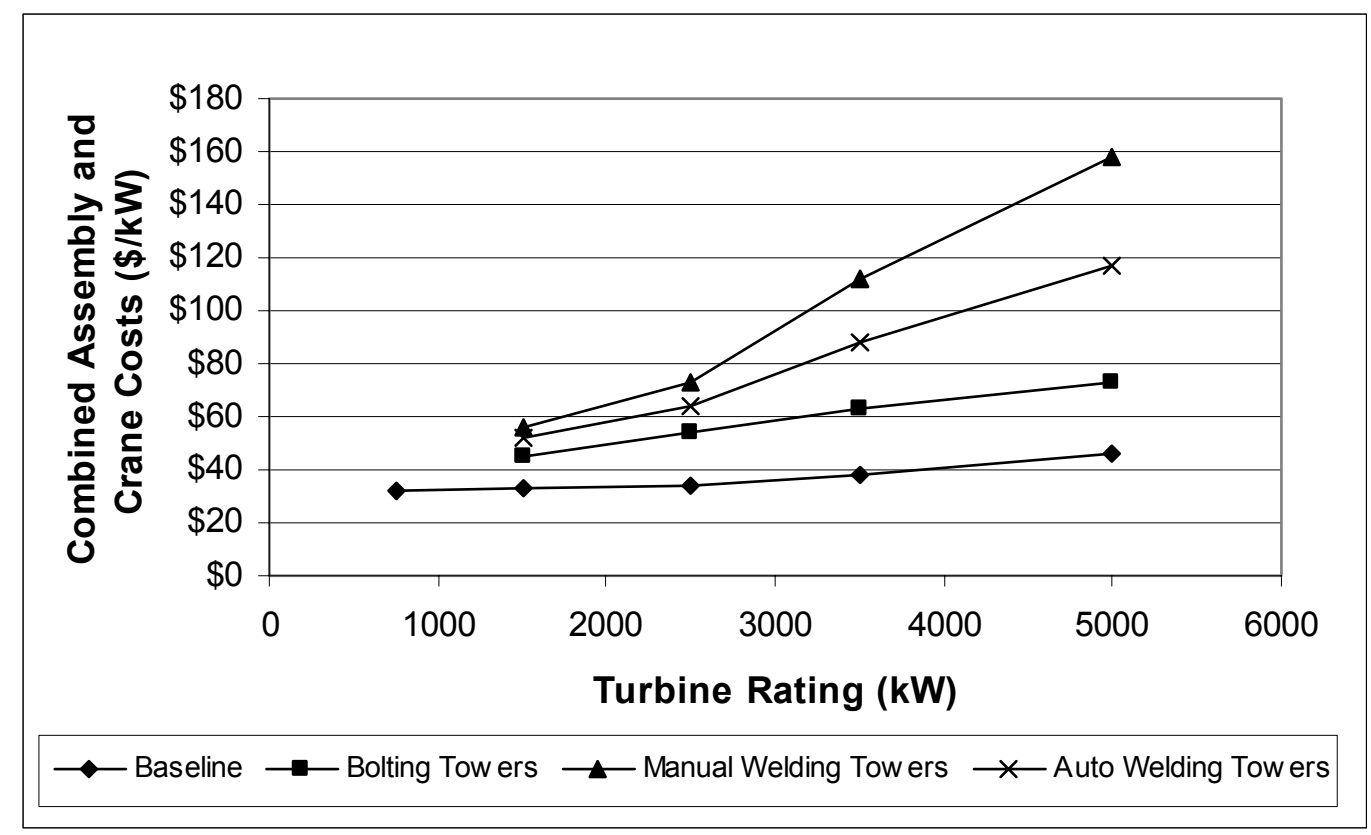

Figure 4-14.Combined assembly and crane costs by scenario (see Appendix P, page 9)

Table 4-1.Impacts of Sectional Towers on Project Costs (Units:\$/kW)

\begin{tabular}{|c|c|c|c|c|}
\hline & $2500 \mathrm{KW}$ & $3500 \mathrm{KW}$ & $5000 \mathrm{KW}$ & NOTES \\
\hline $\begin{array}{l}\text { Scenario } 1 \text { Transport Costs } \\
\text { (Short Haul) }\end{array}$ & $\$ 528$ & $\$ 691$ & $\$ 754$ & \multirow{2}{*}{$\begin{array}{c}\text { Appendix B, } \\
\text { Page } 2\end{array}$} \\
\hline Scenario 2 Transport Costs & $\$ 45$ & $\$ 76$ & $\$ 113$ & \\
\hline Cost Savings: & $\$-482$ & $\$-615$ & $\$-642$ & \\
\hline $\begin{array}{l}\text { Scenario } 1 \text { Assembly and } \\
\text { Crane Costs }\end{array}$ & $\$ 34$ & $\$ 38$ & $\$ 46$ & \multirow{2}{*}{$\begin{array}{l}\text { Appendix } P \text {, } \\
\text { Page } 9\end{array}$} \\
\hline $\begin{array}{l}\text { Scenario } 2 \text { Assembly and } \\
\text { Crane Costs (Manual Welding) }\end{array}$ & $\$ 73$ & $\$ 112$ & $\$ 158$ & \\
\hline Cost Increase: & $\$ 39$ & $\$ 74$ & $\$ 112$ & \\
\hline Net Effect: & $\$-443$ & $\$-541$ & $\$-530$ & \\
\hline
\end{tabular}

Considering the excessive transport costs incurred by attempting to move numerous intact tower sections, a significant amount of on-site assembly could be utilized. The impact that on-site tower fabrication has in reducing transportation costs is considerable and appears to be the logical approach to utilization of tubular towers greater than $85 \mathrm{~m}(279 \mathrm{ft})$ in height that utilize diameters greater than $4.4 \mathrm{~m}(14.4 \mathrm{ft})$. If tubular towers are to remain the industries preferred tower approach, then on-site fabrication will be a necessity. Although interior tower dimensions can be 
adjusted slightly, it appears that the utilizing intact tubular tower sections make sense from a logistic perspective up to hub heights of 80 to $85 \mathrm{~m}$ (262 to $279 \mathrm{ft})$.

\section{Cranes}

No change to the turbine assembly cranes selected in Scenario 1 was made. One additional crane was added to the cost estimate to account for fabricating the towers on-site. Since the mass and lifting height requirements were not significant, readily available and cost effective 200 -ton and 350 -ton cranes were selected for activity.

\subsubsection{Scenario 3}

Under this scenario, assembly and crane logistics were further analyzed to determine the impact of assembling the gearbox and generator into the nacelle while atop the turbine tower. The intent was to determine if reductions in the masses of the objects being lifted resulted in ability to use a different crane and if the added assembly costs were greater than any potential crane savings. Table 4-2 presents the lifting parameters used to evaluated Scenario 3. Gearbox and generator masses were estimated using criteria presented in reference [1]. Under Scenario 3 the 'empty' nacelle mass becomes the critical object for crane selection. Applying the combined $25 \%$ nacelle mass reduction (for the gearbox and generator) to the nacelle points shown in Figures 4-7 through 4-11 (without modifying the required boom point elevation) failed to result in the 'empty' nacelle mass point entering the capacity range of a smaller crane. The conclusion was that removal of the gearbox and generator did not sufficiently reduce the remaining mass of the nacelle to result in a crane modification. Analysis of the crane information presented in Section 4.3.1 combined with the 'empty' nacelle masses reveals that reducing the hub height (and corresponding boom point elevation) is a more effective means of reducing crane costs.

Table 4-2.Nacelles and Components Lifting Parameters

\begin{tabular}{|c|c|c|c|c|c|c|c|}
\hline & Units & \multicolumn{5}{|c|}{ Turbine Ratings } & \multirow{2}{*}{ Notes, References, Assumptions } \\
\hline & kW & 750 & 1500 & 2500 & 3500 & 5000 & \\
\hline Total Nacelle Mass & $\mathrm{kg}$ & 31,081 & 60,517 & 111,065 & 164,049 & 254,102 & $\begin{array}{l}\text { EWEA document. Figure 4.6.3 } \\
\mathrm{m}=2.60 \mathrm{D} 2.4\end{array}$ \\
\hline Gearbox Mass & $\mathrm{kg}$ & 4,662 & 9,078 & 16,660 & 24,607 & 38,115 & Estimated as $15 \%$ of Nacelle mass \\
\hline Generator Mass & $\mathrm{kg}$ & 3,108 & 5,267 & 8,567 & 11,867 & 16,817 & Estimated at $10 \%$ of Nacelle mass \\
\hline Empty Nacelle Mass & $\mathrm{kg}$ & 23,311 & 46,173 & 85,839 & 127,575 & 199,170 & \\
\hline Hub Height & $\mathrm{m}$ & 65 & 86 & 111 & 130 & 156 & $\begin{array}{l}\text { Used ratio of tower height/rotor } \\
\text { diameter of } 1.3 \text { per SOW. }\end{array}$ \\
\hline Boom Point Elevations & $\mathrm{m}$ & 85 & 109 & 133 & 159 & 193 & $\begin{array}{l}\text { Point elevations determined by } \\
\text { Lampson for load, height, } \\
\text { equipment-lifting capacity, } \\
\text { clearances, and safety margin. }\end{array}$ \\
\hline
\end{tabular}

\section{Assembly}

Disassembly of the nacelle's major components did not result in a beneficial impact to the nacelle transportation costs as discussed in Section 3.5.3 and it caused a slight increase to the assembly 
costs. The minor transportation cost savings obtained for the $5000 \mathrm{~kW}$ nacelle was offset by the increase in assembly costs resulting in an insignificant change in costs between Scenario 1 and 3.

\section{Cranes}

The only adjustment to crane costs between Scenario 2 and 3 is reflected by the increased crane time required for installing the gearbox and generator. The crane cost increased an estimated $\$ 2$ per $\mathrm{kW}$, resulting in a total increase of $\$ 4$ per $\mathrm{kW}$. Combined crane, assembly, and transportation costs for the $3500 \mathrm{~kW}$ turbine actually increase slightly under Scenario 3 while these costs remain unchanged for the $5000 \mathrm{~kW}$ turbine.

\subsection{Terrain Effects on Crane Costs}

To evaluate terrain effects on crane costs, the effort, costs and time required for crane assembly and disassembly included in crane mobilization and demobilization estimates were utilized. Fully assembled cranes do have the capability to move under optimum conditions without necessitating partial or full disassembly. However, topography at valuable wind sites with ridgelines, rolling terrain, or mesas pose significant impediments to crane movements. Turbine layout also impacts crane movement with grid configurations representing the most conducive and dispersed turbine clusters representing the most challenging.

To estimate the terrain impacts, crane disassembly and reassembly costs, in addition to those incurred during mobilization and demobilization, where calculated and added to the original crane costs. The costs include labor, crane rental, additional support cranes, and transport vehicles. Optimum crane costs (utilized in Section 4.3) represent the costs of assembling 50 turbines without any additional crane disassembly/reassembly being incurred. As the assumed number of crane disassemblies increases, the number of turbines installed between crane disassemblies decreases. The assumption is that as the terrain becomes harsher, the number of turbines that could be installed between crane disassemblies decreases. Therefore, assembly of 50 turbines per crane disassembly represents optimum crane use for the hypothetical WindPACT project. Assembly of 2 turbines per crane disassembly represents extremely harsh terrain. Based on the rolling topography of south central South Dakota, the numbers of turbines assembled per crane disassembly has been estimated to be 10 to 25 (depending upon the assumed turbine layout).

Figure 4-15 presents the increases to the optimum crane costs due to terrain effects. Incurring one crane disassembly (corresponding to 25-turbines/crane disassembly) resulted in a moderate cost increase of $12 \%$. However, incurring four crane disassemblies (corresponding to 10 turbines/crane disassembly) resulted in a 50\% cost increase. The well-matched crane capacities for the $750 \mathrm{~kW}$ and $2500 \mathrm{~kW}$ turbines (discussed at the end of Section 4.3.1) are magnified when terrain effects are accounted for. This is evident by a lower rate of cost increases for these turbines. For the cranes associated with the $1500 \mathrm{~kW}$ and $2500 \mathrm{~kW}$ turbines, there is only a modest increase in the base monthly rates and level of assembly effort, however the increased power rating of the $2500 \mathrm{~kW}$ turbine improves the cost environment. 


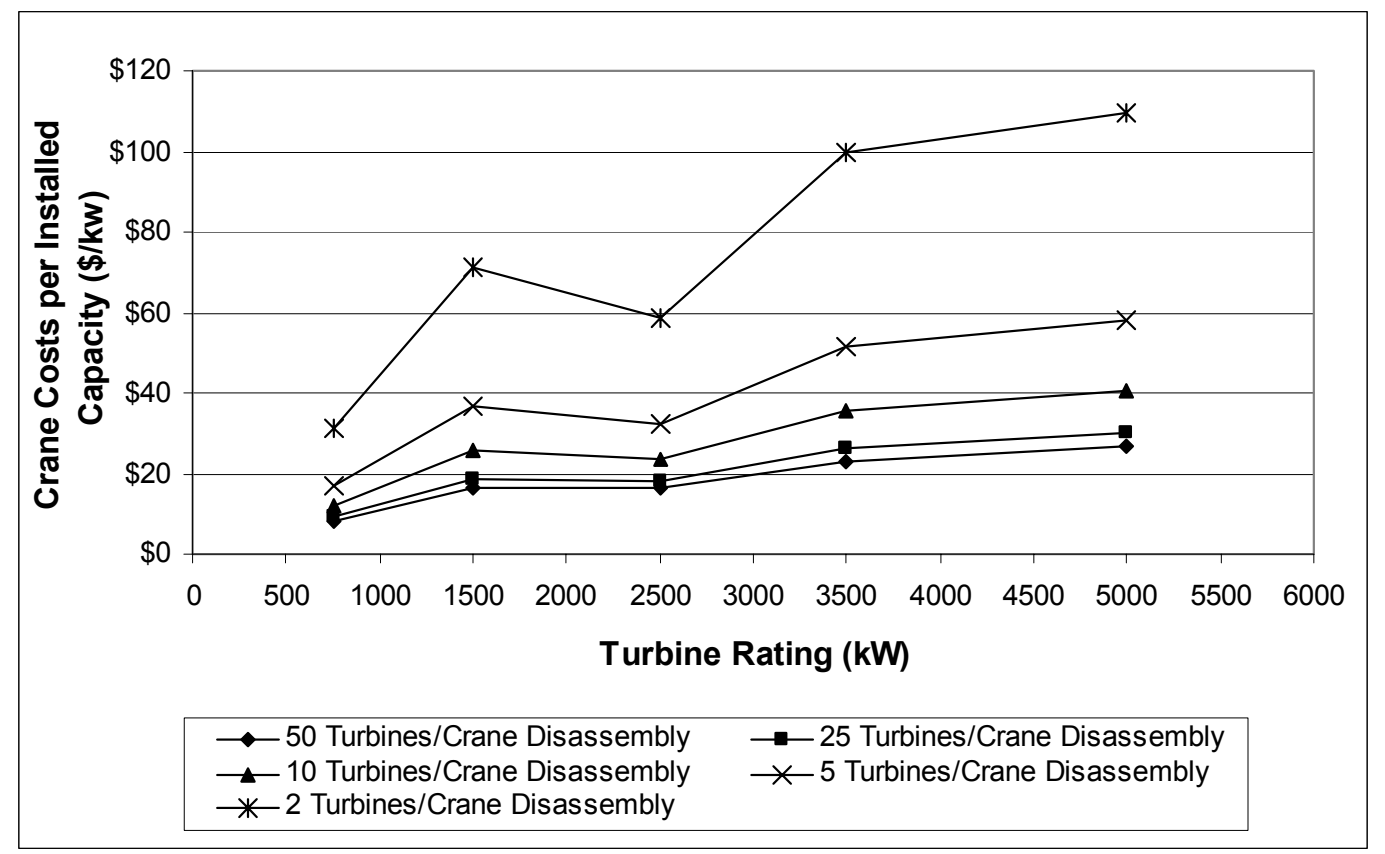

Figure 4-15. Terrain effects on crane costs

(see Appendix P, page 10)

Another area that terrain issues associated with cranes impacts a project is related to schedule. Figures 4-16 and 4-17 demonstrate increases to turbine assembly rates (days/turbine) and overall duration of assembly activities (months), respectively. The differences between a $1500 \mathrm{~kW}$ and $5000 \mathrm{~kW}$ turbines are shown for illustration purposes. Significant increases to project duration for both turbine sizes caused by terrain issues would likely have an adverse impact to project financing. 


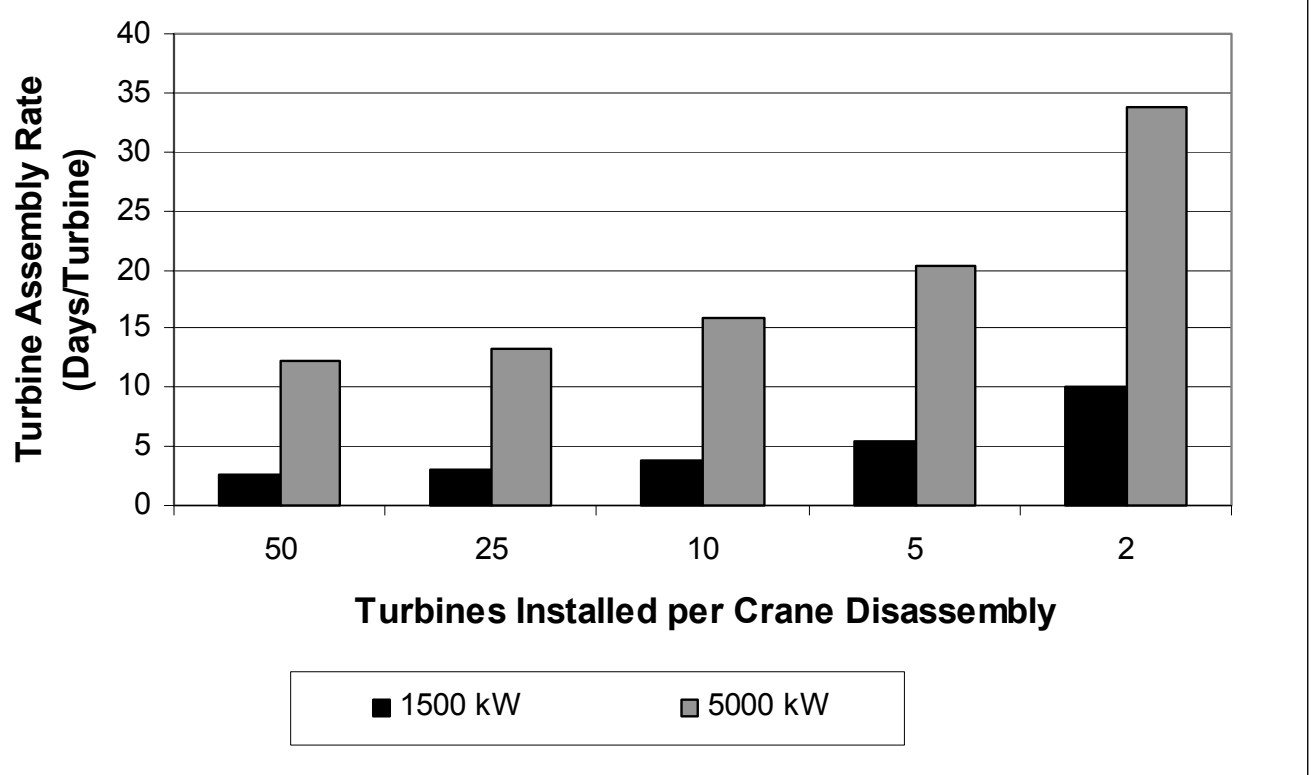

Figure 4-16.Influence of terrain impact to turbine assembly rates

(see Appendix P, page 11)

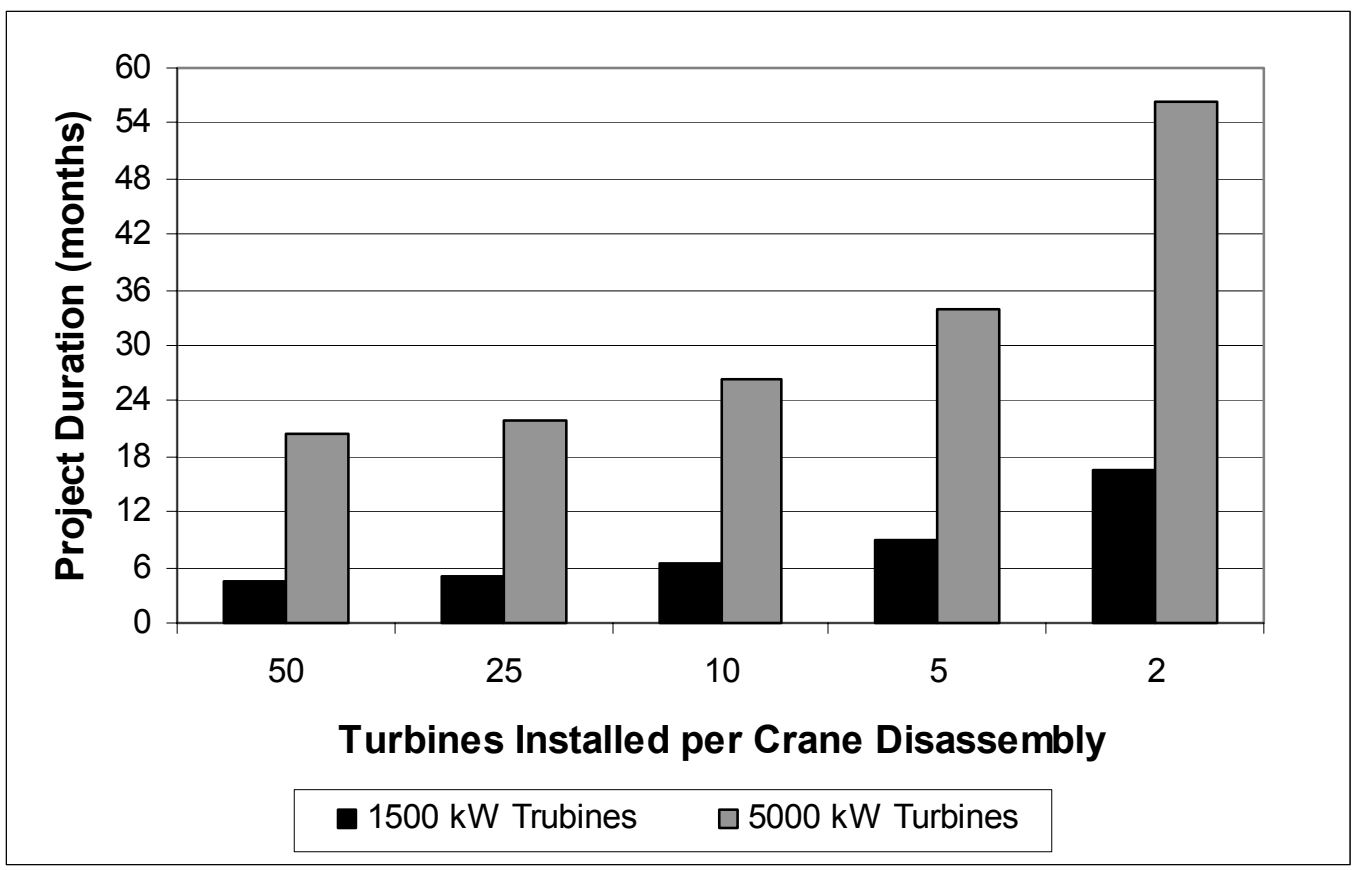

Figure 4-17.Influence of terrain impacts on project duration

(see Appendix P, page 12) 


\subsection{Crane Purchase Evaluation}

Purchase costs were evaluated to determine if a measurable benefit of crane ownership exists in comparison to crane rental. Cranes selected for the evaluation were large enough to perform individual blade, gearbox, and generator removal; however, they lacked capacity to remove the entire rotor or nacelle. These cranes were assumed to be indicative of the type of 'service cranes' that a large facility would consider owning. Purchase costs for the cranes were compared to loaded hourly rental rates using the standardized cost of energy equation in EPRI's Technical Assessment Guide [12]. The COE equation was set equal to 0 then the crane annual O \& $\mathrm{M}$ expenses were solved for based on capital crane costs and the fixed charge rate (assuming that all other cost components remained unchanged). A fixed charge rate of $10 \%$ was assumed. Using the loaded hourly crane rental rates, annual crane usage was then calculated from the annual crane $\mathrm{O} \& \mathrm{M}$ expenses. If the annual crane usage rate is low, then crane purchase costs could be recovered quickly and ownership would be favorable. Table 4-3 presents the calculations and results.

Table 4-3.Crane Purchase Evaluation

\begin{tabular}{|l|c|c|c|c|c|c|}
\hline \multicolumn{1}{|c|}{ Turbine } & $\mathrm{kW}$ & 750 & 1500 & 2500 & 3500 & 5000 \\
\hline \multicolumn{1}{|c|}{ Rotor Dia } & $\mathrm{m}$ & 50 & 66 & 85 & 100 & 120 \\
\hline O \& M Crane Type & & & & & & \\
\hline Crane Purchase Costs & & $4100-\mathrm{S} 1$ & $4600-\mathrm{S} 4$ & $\mathrm{LTL}-600$ & $\mathrm{LTL}-600$ & $\mathrm{LTL}-1000$ \\
\hline Fixed Charge Rate & & $\$ 1,150,000$ & $\$ 2,250,000$ & $\$ 3,500,000$ & $\$ 3,500,000$ & $\$ 6,500,000$ \\
\hline Capital Costs x F.C. & & 0.1 & 0.1 & 0.1 & 0.1 & 0.1 \\
\hline & & $\$ 115,000$ & $\$ 225,000$ & $\$ 350,000$ & $\$ 350,000$ & $\$ 650,000$ \\
\hline Loaded Hourly Operation Rate & & $\$ 375$ & $\$ 490$ & $\$ 920$ & $\$ 920$ & $\$ 1,030$ \\
\hline Annual Usage & $\mathrm{hrs}$ & 307 & 459 & 380 & 380 & 631 \\
\hline Annual Usage & Months & 1.7 & 2.6 & 2.2 & 2.2 & 3.6 \\
\hline & & & & & & \\
\hline Total Usage for 20 year project & hrs & 6133 & 9184 & 7609 & 7609 & 12621 \\
\hline Usage every 3 years: & Months & 5 & 8 & 6 & 6 & 11 \\
\hline Usage every 5 years: & Months & 9 & 13 & 11 & 11 & 18 \\
\hline
\end{tabular}

See Appendix S

Using the $750 \mathrm{~kW}$ turbines as an example, crane purchase would be more favorable than rental when crane usage exceeds 307 hours (or 1.7 months) for each year of the project. This usage rate would not be achievable (or desired) at one project with 50 turbines. A reasonable crane usage estimate would be 1 month or less per year. As the turbine sizes (and service crane sizes) increased, higher crane usage rates are necessary to make crane ownership cost effective.

If crane usage were distributed across 3 or 4 other projects (assuming similar number and size of turbines), then it is conceivable that the annual usage rate could be met making crane ownership more cost effective. However, a large crane purchased for $5000 \mathrm{~kW}$ turbines would not be effective at projects comprised of smaller turbines because the mobilization, assembly, and relocation time would be excessive in comparison to a rented crane properly sized for the specific turbines. Another drawback to distributing crane usage across other projects in the region is that generally small windows of low wind months exist during which $\mathrm{O} \& \mathrm{M}$ work requiring cranes is performed. It's likely that scheduling crane work for multiple projects would result in conflicts, necessitating crane rental at one or more projects, eroding the potential benefit of crane ownership. Crane ownership may be beneficial for very large projects of smaller turbines (for example $200-750 \mathrm{~kW}$ turbines) under control of one owner. However, for most projects, crane rental remains more cost effective. 


\section{Summary Analysis}

\subsection{Logistic Costs by Scenario}

The combined analyses of transportation, assembly, and crane logistics costs have been summarized in Figures 5-1, 5-2, and 5-3 below. We combined mid-range assembly and crane costs with long-haul transportation costs to generate the total values in the figures. The crane costs in these figures have not been adjusted for terrain effects; they therefore correspond to optimum crane utilization. The reduction in transportation costs associated with quartered tower sections is evident in the cost reduction between Figures 5-1 and 5-2. Figure 5-3 demonstrates that when field assembly of the gearbox and generator into the nacelle is included (in addition to quartered tower sections), there is virtually no change in total logistics costs. The latter increase with increasing turbine size, however, the rate of increase can be reduced the most by utilizing field fabrication of towers.

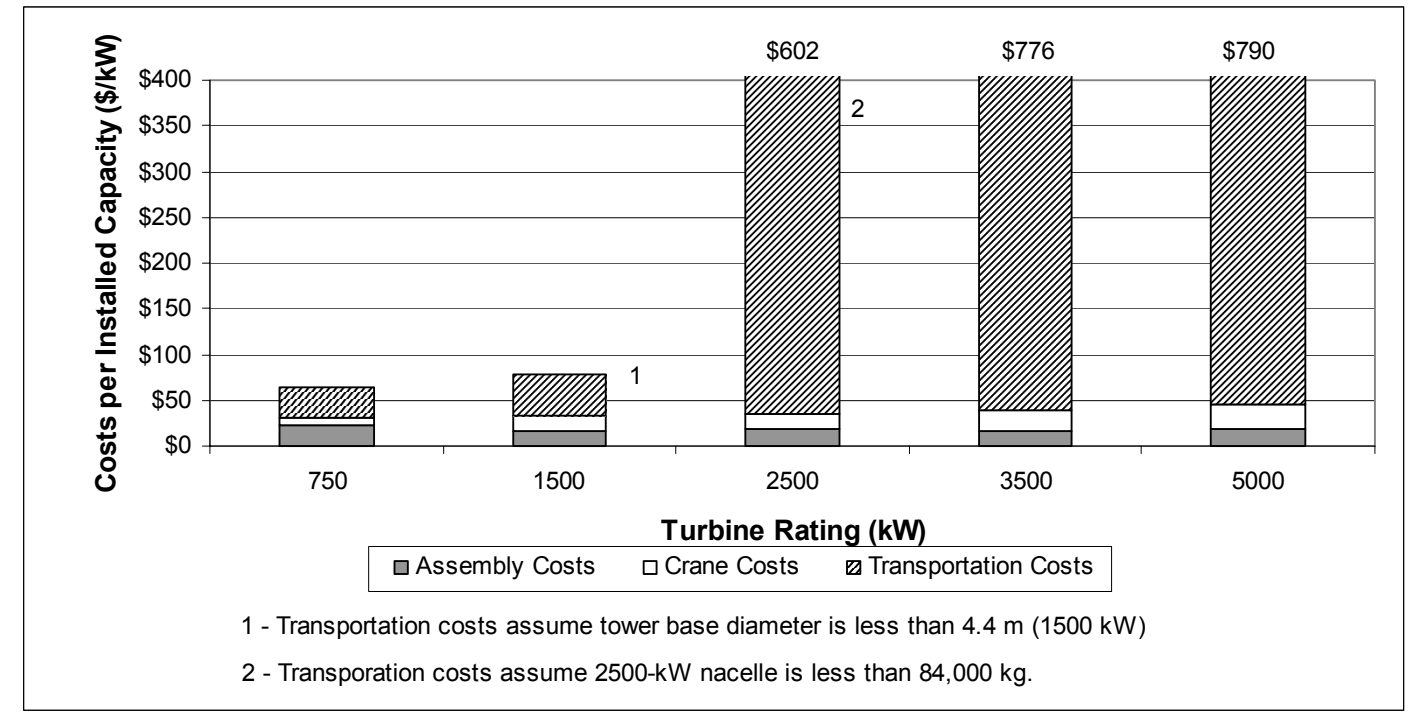

Figure 5-1.Scenario 1 cost components

(see Appendix P, page 13) 


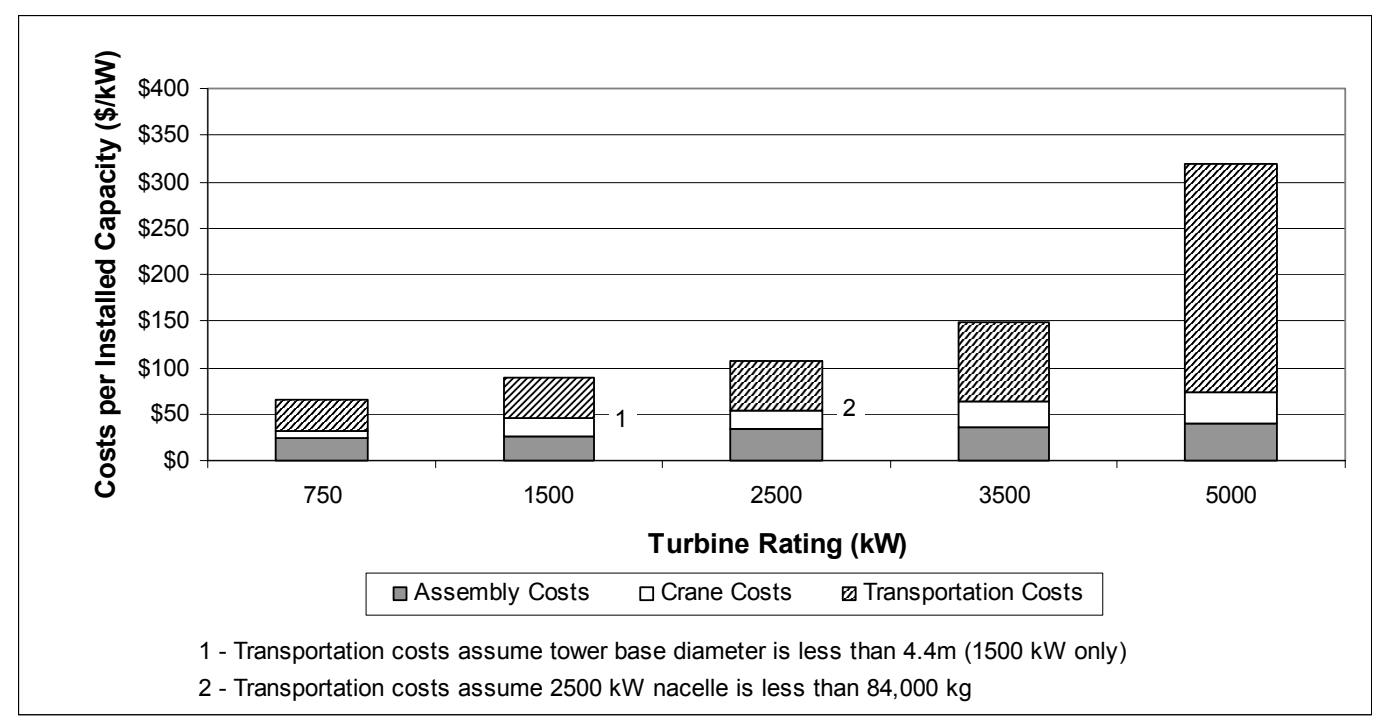

Figure 5-2.Scenario 2 cost components

$750 \mathrm{~kW}$ and $1500 \mathrm{~kW}$ costs are same as Scenario 1 (see Appendix P, page 13)

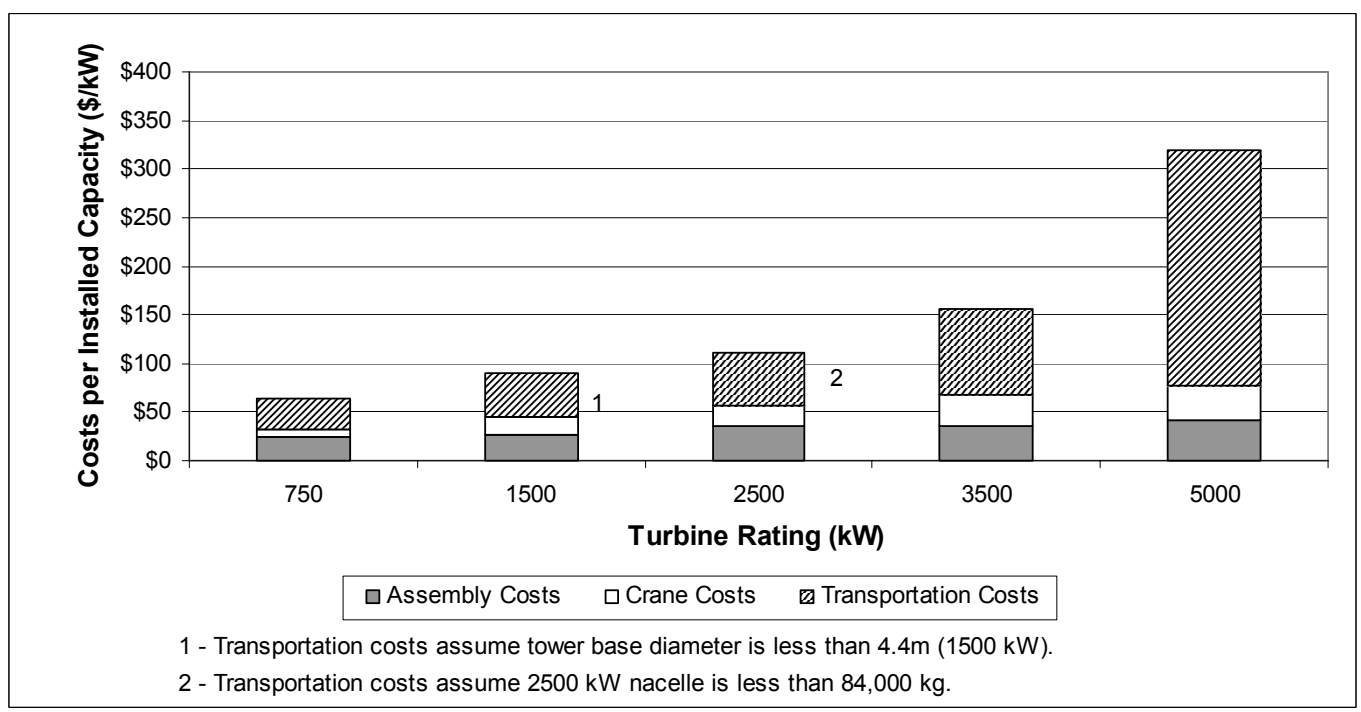

Figure 5-3.Scenario 3 cost components

$750 \mathrm{~kW}$ and $1500 \mathrm{~kW}$ costs are same as Scenario 1 (see Appendix P, page 14)

\subsection{Logistic Costs for South Dakota Site}

To provide representative costs we performed a detailed analysis of logistic costs for Scenario 2 (Figure 5-2) with respect to the hypothesized South Dakota project site. We assumed that quartered tower sections would be used for $2500 \mathrm{~kW}$ and greater turbines, and that the turbine components would be shipped to South Dakota (assuming short-haul distances). Crane costs were adjusted for terrain by assuming that 10 turbines could be assembled before complete crane disassembly would be required, based on the rolling topography of south-central South Dakota and the assumption that dispersed turbine arrays would be used. Both of these factors would increase the potential for more frequent crane disassembly during crane relocation. 
Figure 5-4 presents the representative logistics costs for 50 multi-megawatt turbines in South Dakota. Transportation costs are lower than those presented in Figure 5-2 because short-haul distances are being used. Crane costs are greater than those in Figure 5-2 due to adjustments for terrain. Figures 5-5 and 5-6 present scaling relationships of the logistic costs in Figure 5-4.

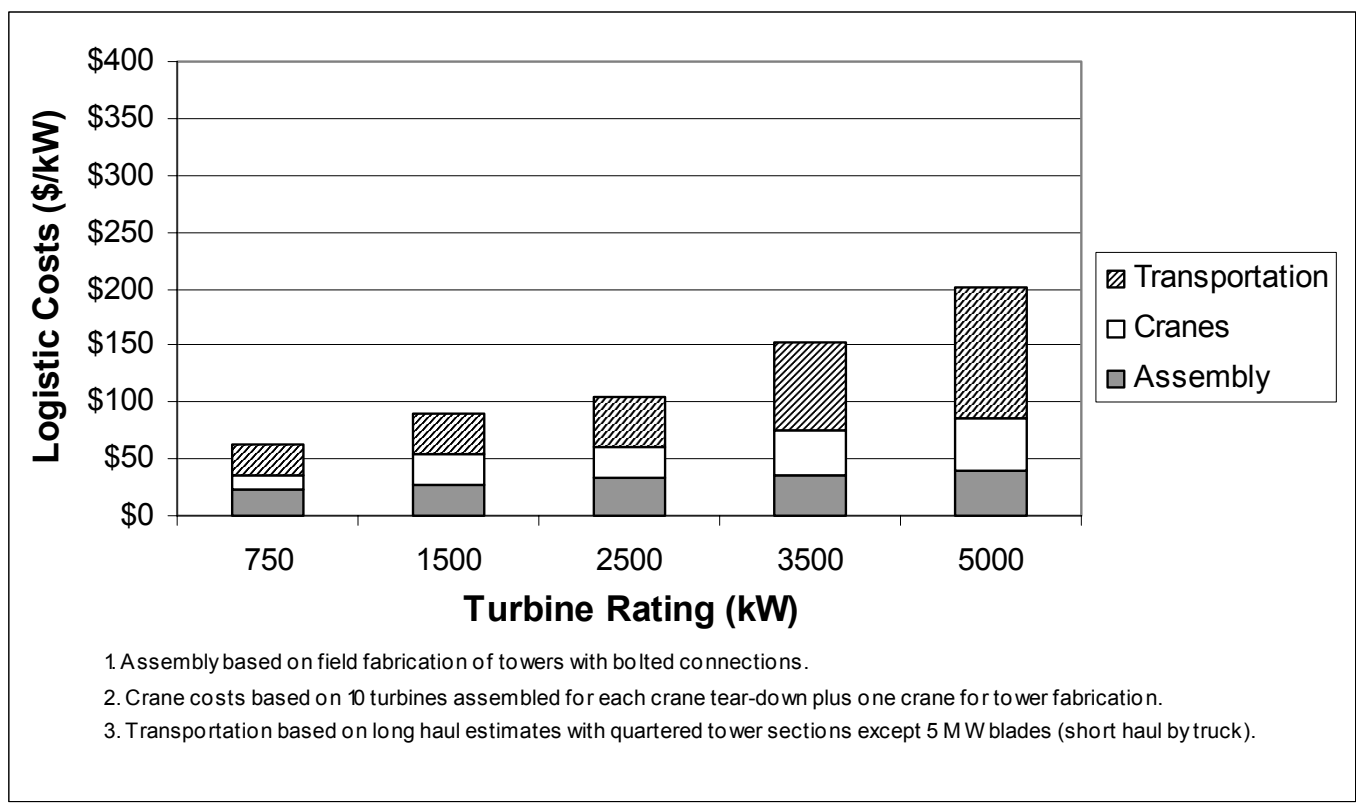

Figure 5-4.Logistic costs for multi-megawatt turbines in South Dakota (see Appendix P, page 15) 


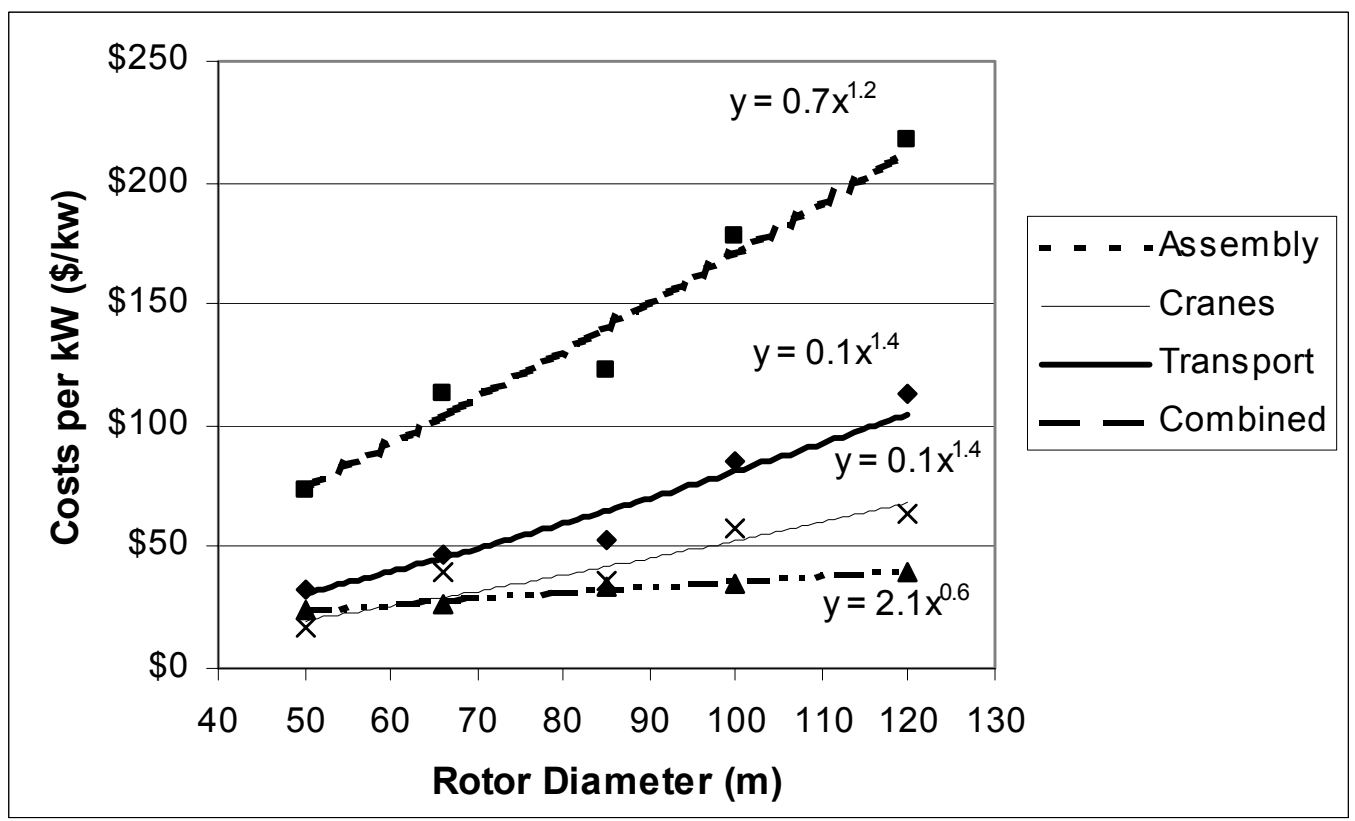

Figure 5-5.Scaling relationships by rotor diameter (see Appendix P, page 15)

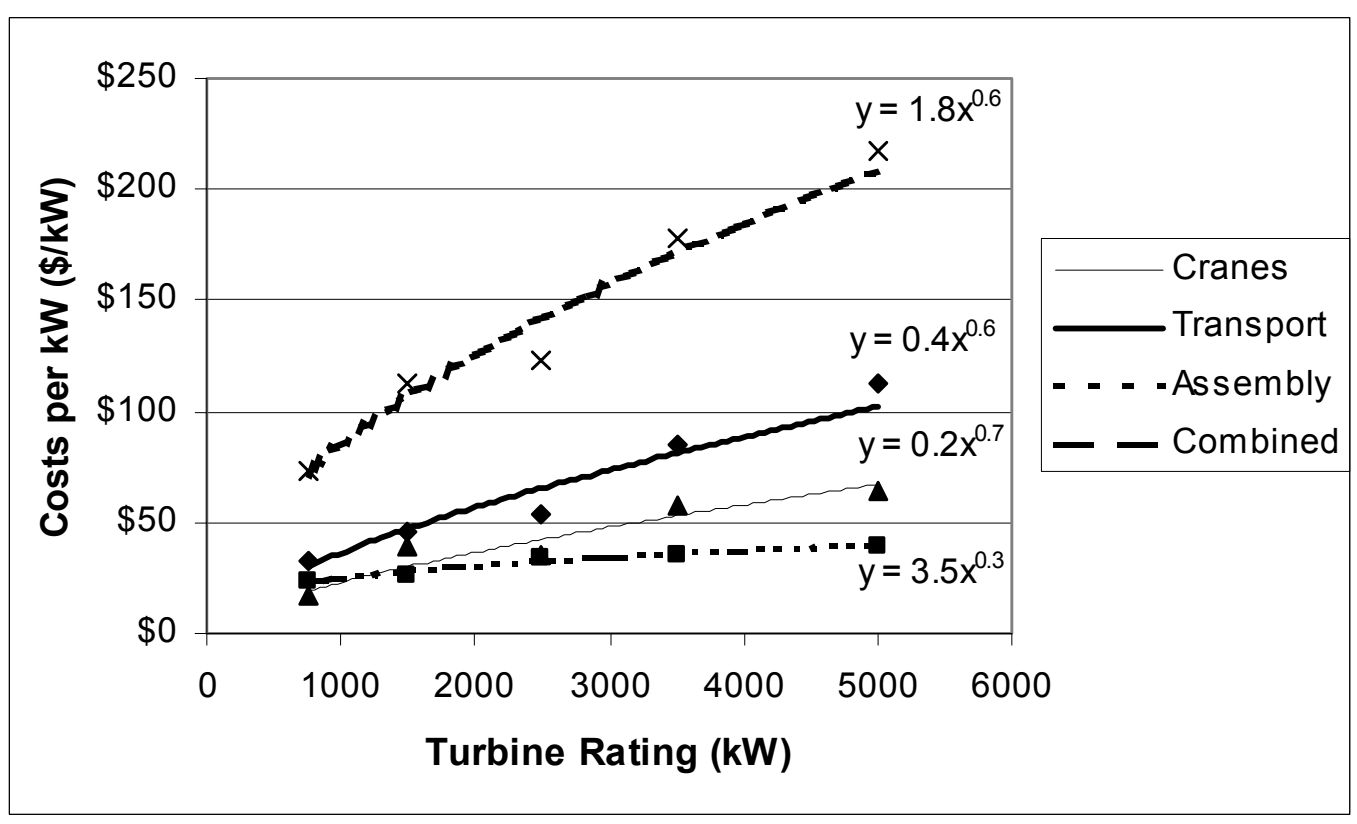

Figure 5-6.Scaling relationships by turbine rating (see Appendix P, page 15) 


\subsection{Road-Width Analysis}

On-site road widths and corresponding construction costs are determined to a large extent by the turbine transportation and assembly equipment associated with project logistics. Development of site road construction costs is a component of WindPACT Technical Area 4 - Balance of Station Costs; however, the road widths for equipment presented in this report have been summarized in Table 5-1. As indicated in this table, the road widths associated with larger cranes are significant and would be extremely expensive to construct in complex terrain.

Table 5-1.On-site Road Widths Based on Logistics Equipment

\begin{tabular}{|c|cc|cc|cc|cc|}
\hline Turbine & \multicolumn{2}{|c|}{ Nacelle } & \multicolumn{2}{|c|}{ Blades } & \multicolumn{2}{|c|}{ Tower Sections } & \multicolumn{2}{c|}{ Crane } \\
$(\mathbf{k W})$ & $(\mathbf{m})$ & $\mathbf{( f t )}$ & $\mathbf{( m )}$ & $\mathbf{( f t})$ & $(\mathbf{m})$ & $\mathbf{( f t})$ & $(\mathbf{m})$ & $(\mathbf{f t})$ \\
\hline $\mathbf{7 5 0}$ & 4.6 & 15 & 4.6 & 15 & 4.6 & 15 & 4.6 & 15 \\
\hline $\mathbf{1 5 0 0}$ & 4.6 & 15 & 4.6 & 15 & 4.6 & 15 & 9.1 & 30 \\
\hline $\mathbf{2 5 0 0}$ & 4.6 & 15 & 4.6 & 15 & 4.6 & 15 & 9.1 & 30 \\
\hline $\mathbf{3 5 0 0}$ & 6.1 & 20 & 6.1 & 20 & $4.6-6.1$ & $15-20$ & 9.8 & 32 \\
\hline $\mathbf{5 0 0 0}$ & 6.1 & 20 & 7.5 & 25 & $4.6-6.1$ & $15-20$ & 12.2 & 40 \\
\hline
\end{tabular}

\subsection{Conclusions}

The following conclusions can be made based on the combined analysis of transportation, assembly, and crane logistics:

\section{Transportation}

1. Of the three, transportation limitations and associated costs have the largest impact on total logistics costs. Different approaches used to reduce transportation costs by bringing the transported objects into common dimensional or reasonable constraints prove to be very cost effective, even when increased numbers of shipments are necessary. The U.S. transportation system is dominated by tractor-trailer transport, which results in a very competitive and efficient system. To obtain the most cost-effective transportation, movement of wind turbine components should utilize loads that remain within the standard trailer dimensions of $4.1 \mathrm{~m}(13.5 \mathrm{ft})$ high by $2.6 \mathrm{~m}(8.5 \mathrm{ft})$ wide and up to 80,000 GVW corresponding to a cargo weight of about $19,000 \mathrm{~kg}(42,000 \mathrm{lbs})$. Where this is not possible, the next most critical dimensions are height followed by weight. Loaded heights that exceed $4.83 \mathrm{~m}(15.83 \mathrm{ft})$ will trigger the need for extremely expensive utility and police assistance associated with temporary overhead utility disconnection and reconnection. These costs are extremely load and route dependant and are generally only acceptable for one-of-a-kind moves (when all alternatives have been exhausted).

Attempting to move numerous objects that require this form of assistance will most likely not be allowed by local jurisdictions due to disruption to the community. This form of disruption can also have a detrimental impact to the perceived value of wind energy. Tractor-trailer transportation with object weights up to $84,000 \mathrm{~kg}(185,000 \mathrm{lbs})$ but within the height constraint is the next most cost-effective method, although it is generally ten times the costs of standard tractor-trailer movement. These loads will necessitate 
oversized and overweight permits and come under tight scrutiny by enforcement officials. Above this weight range, dolly and rail transport become the preferred modes. Rail is best applied to dimensionally compliant and massive objects such as nacelles.

2. Alleviation of transportation issues associated with the towers results in the largest adjustment to the total logistics costs. The critical diameter dimension for transport of intact tubular tower sections is $4.4 \mathrm{~m}(14.5 \mathrm{ft})$ because diameters larger than this result in a total vehicle height exceeding $4.83 \mathrm{~m}(15.83 \mathrm{ft})$. The tower design used in this study for the $1500-\mathrm{kW}$ turbine with an $86 \mathrm{~m}(282 \mathrm{ft})$ hub height resulted in a base diameter of $4.9 \mathrm{~m}(16 \mathrm{ft})$. The unexpected result of not being able to transport this base tower section by truck helped isolate the dimension issues associated with the towers. The transportation of intact tower sections as assumed in Scenario 1 obviously cannot continue for the tubular tower design without modification of the design approach. It is likely that tower designers will be able to achieve hub heights greater that $80 \mathrm{~m}(262 \mathrm{ft})$ on tubular towers with diameters fixed at the transportation limitation; however, it will result in less efficient material usage, increased tower costs, and increased weight. The transportation costs savings may allow these inefficiencies, but only to a point.

The application of quartered tower sections demonstrated that extensive on-site assembly could be performed cost effectively because significant transportation cost savings are being attained. Tower design approaches that rely on increased field assembly appear to be the most effective technique for achieving hub heights in the range of $100 \mathrm{~m}$ with 85 $\mathrm{m}$ diameter rotors (comparable to $2500-\mathrm{kW}$ turbines). However, once designers begin to investigate field-fabricated towers, other tower configurations may prove to be more cost effective than quartered tubular towers such as truss tower, guyed towers, combined tripod and tubular towers, and cast concrete. In addition, independent pitch control systems for each blade could help mitigate thrust loads imparted to the tower under various design loading scenarios.

3. Nacelle designs should recognize that $84,000 \mathrm{~kg}(185,000 \mathrm{lbs})$ is a breakpoint and maintain an overall vehicle height below $4.83 \mathrm{~m}(15.83 \mathrm{ft})$ in order to maximize the range of cost-effective truck transportation. Based on the nacelle mass scaling Equation 2.4, the above mass limit corresponds to a rotor diameter of $84 \mathrm{~m}(275 \mathrm{ft})$. For nacelles with total masses that exceed this limit, removal of the gearbox and generator for separate shipment is cost effective until the remaining nacelle mass is at this tractor-trailer transportation limit.

For nacelles that can be fabricated within the dimensional limits of the railroad $4.2 \mathrm{~m}$ $14 \mathrm{ft}$ wide and $3.7 \mathrm{~m} 12 \mathrm{ft}$ above top of rail, rail's additional transport capacity up to $163,300 \mathrm{~kg}(360,000 \mathrm{lbs})$ could be used for turbines with 115-m (377-ft) diameter rotors.

4. The large physical dimensions of turbine components, coupled with relatively light masses, results in expensive water-based transportation costs. This mode of transport does not offer many advantages for wind turbine components other than the ability to bypass certain states through which passage by land is not allowed.

5. Proximity of manufacturing to potential wind sites is most important for the $5000-\mathrm{kW}$ turbines in order to minimize costly dolly transport distances and eliminate the use of waterway transport. 


\section{Assembly and Cranes}

The turbine assembly and crane costs were small relative to the transportation costs for each of the three development scenarios. However, within these areas we identified the following valuable information.

\section{Assembly}

1. Although total assembly costs per turbine increase as the turbine sizes increase, the assembly costs per installed kW didn't experience dramatic changes, as indicated by the constant assembly values shown in Figure 5-1. The increased assembly effort associated with larger turbines does not appear to increase faster than the power rating of the turbines.

2. When field fabrication of the towers is used to reduce transportation costs, relatively modest ( $\$ 20$ to $\$ 30)$ increases in cost per $\mathrm{kW}$ are incurred. Of the possible on-site tower fabrication approaches, use of bolted joints with overlap steel panels was demonstrated to be more cost effective than manual or automated welding. The costs for field fabrication of towers with this approach did not experience a continued increase as the turbine sizes increased. Whereas welding costs depend on the wall thickness of the towers, this dimension does not adversely affect bolted connections. Both approaches are equally affected (adversely) by increased length of tower sections and increased number of sections requiring field fabrication.

\section{Cranes}

1. Crane costs were shown to increase as turbine size increased. However, the dimensions and masses of the $750-\mathrm{kW}$ and $2500-\mathrm{kW}$ turbines resulted in the most efficient use of the selected crane capacities. Cranes are very efficient when used to assemble numerous turbines ( 50 in this example) in benign terrain where grid layouts are possible. However, sharp increases in crane costs occur when multiple crane disassembly/reassembly is required to relocate the crane between turbines. Actual crane disassembly frequency is a function of the site characteristics and project layout. Assembly of a few $3500-\mathrm{kW}$ or $5000-\mathrm{kW}$ turbines on high ridges in harsh terrain will result in excessive crane costs.

2. The best approach to decrease crane costs is to decrease lifting-height requirements. Unfortunately, this conflicts with the wind industry's need for increased heights to increase annual energy production.

3. Although crane mobilization, assembly, and demobilization costs are considerable, use of cranes is cost effective for turbine assembly, even when excess lifting capacity is being incurred to attain the required heights. 


\section{References}

1. Harrison, R.; Jenkins, G. (1994) Cost Modeling of Horizontal axis Wind Turbines. For ETSU W/34/00170/REP, University of Sunderland.

2. EWEA, European Commission, Directorate-General for Energy. (1998) "Wind energy - the facts. Volume 1: technology." (?) www.ewea.org

3. EWEA, European Commission, Directorate-General for Energy. (1998) "Wind energy - the facts. Volume 2: costs, prices and values." (?) www.ewea.org

4. EWEA, European Commission, Directorate-General for Energy. (1998) "Wind energy - the facts. Volume 3: industry and employment" (?) www.ewea.org

5. EWEA, European Commission, Directorate-General for Energy. (1998) "Wind energy - the facts. Volume 4: environment" (?) www.ewea.org

6. EWEA, European Commission, Directorate-General for Energy. (1998) "Wind energy - the facts. Volume 5: market development" (?) www.ewea.org

7. Thresher, R.W. and Dodge, D.D. (1998) "Evolution of wind turbine generator configurations," Wind Energy, Vol. 1, pp. 70-85.

8. National Aeronautics and Space Administration. (September 1982). "MOD-5 Wind Turbine System Concept and Preliminary Design Report, Volume II Detailed Report.

9. National Aeronautics and Space Administration. (September 1982). "MOD-5 Wind Turbine System Concept and Preliminary Design Report, Erection Method Trade Study (M-1).

10. National Aeronautics and Space Administration. (June 1984). "MOD-5 Wind Turbine System Detailed Design Review.

11. Blodgett, O. (1966). Design of Welded Structures. The James F. Lincoln Arc Welding Foundation.

12. Electric Power Research Institute. (December 1986) TAG-Technical Assessment Guide, Volume 1: Electricity Supply - 1986, EPRI p. 4463-SR. 
Appendix A

WindPACT Turbine 


\begin{tabular}{|c|c|c|c|c|c|c|c|c|c|c|c|c|c|c|c|c|c|c|c|c|c|c|}
\hline \multicolumn{3}{|c|}{ summary of muli MW wind turbine properties } & & & & & & & & & & & 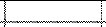 & & 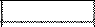 & & & & & & & \\
\hline & & & & & & & & & & & & & & & & & & & & & & \\
\hline & & & & & & & & & & & & & & & & & & tower & total & & tower & specific \\
\hline manufacturer & model & no of blades & hub type & up/downwind & rotor diameter & max power & hub heigh & $t \max$ rpm & tip speed & power/swept area & power control & drive type & blade mass: & hub mass & rotor mass & nacelle mass & head mass & \begin{tabular}{|l} 
rated mass \\
\end{tabular} & tower mass & sweptarea & a headmass & nacelle mass \\
\hline & & & & & $\mathrm{m}$ & MW & $\mathrm{m}$ & & $\mathrm{m} / \mathrm{s}$ & $\mathrm{kW} / \mathrm{m}^{2}$ & & & $\mathrm{~kg}$ & $\mathrm{~kg}$ & $\mathrm{~kg}$ & $\mathrm{~kg}$ & $\mathrm{~kg}$ & $\mathrm{~kg} / \mathrm{kw}$ & $\mathrm{kg}$ & $\mathrm{m}^{\wedge} \mathrm{2}$ & $\mathrm{kg} / \mathrm{m}^{\wedge} \mathrm{2}$ & $\mathrm{kg} / \mathrm{kw}$ \\
\hline Experimental 7 & urbines & & & & & & & & & & & & & & & & & & & & & \\
\hline Boeing & Mod-2 & 2 & teeter & up & 91.4 & 2.5 & 61 & 17 & 81 & 0.38 & ailerons & var spd & & & 89811 & 79378 & 169200 & & 115700 & 6561 & & \\
\hline $\begin{array}{l}\text { Boeng } \\
\text { Ham Std }\end{array}$ & $\begin{array}{ll}\text { Mod 5B } \\
\text { WTS-4 }\end{array}$ & 2 & teeter & up & 97.5 & 3.2 & 61 & 17 & 87 & 0.43 & ptch tip & & & & 141975 & 114760 & 256800 & 80 & 159600 & 7466 & 34 & 36 \\
\hline $\begin{array}{l}\text { Ham Std } \\
\text { GE }\end{array}$ & $\frac{\text { WTS-4 }}{\text { Mod-5A }}$ & $\frac{2}{2}$ & teeter & up & 122 & 7.3 & & 16.8 & 107 & 0.62 & & & & & & & & & & 11690 & & \\
\hline Aerodyn & multibrid & 3 & rigid & up & 100 & 5 & & 16.2 & 85 & 0.64 & stall & hybrid & 11000 & 12000 & 45000 & 110000 & 155000 & 31 & & 7854 & 20 & 22 \\
\hline Existing/Past T & urbines & & & & & & & & & & & & & & & & & & & & & \\
\hline Nordex & N80/2500 & 3 & rigid & up & 80 & 2.5 & 80 & & & 0.50 & & & & & 48300 & 82700 & 131000 & 52 & 179300 & 5027 & 26 & 33 \\
\hline$\frac{\text { Nordex }}{\text { Nordex }}$ & N 54/1000 & 3 & rigid & & 54 & 1 & 70 & 22 & 62 & 0.44 & & & & & 19000 & 46000 & 65000 & 65 & 107000 & 2290 & 28 & 46 \\
\hline $\begin{array}{l}\text { Nordex } \\
\text { Tacke }\end{array}$ & N60/1300 & $\frac{3}{3}$ & rigid & up & $\begin{array}{c}60 \\
70.5 \\
\end{array}$ & $\begin{array}{l}1.3 \\
1.5\end{array}$ & 69 & & & 0.46 & & & & & 18900 & 49200 & 68100 & 52 & 104000 & 2827 & 24 & 38 \\
\hline Tacke & TW 1.5 & 3 & rigid & up & 65 & 1.5 & 80 & 20 & 68 & 0.45 & pitch & $\operatorname{var}$ spd (1.4:1) & & & & & & & & 3318 & & \\
\hline Bonus & & 3 & rigid & up & 62 & 2 & & & & 0.66 & & $x^{\prime}=$ & & & & & & & & 3019 & & \\
\hline 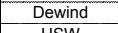 & & 3 & rigid & up & 62 & 1 & & & & 0.33 & & & & & & & & & & 3019 & & \\
\hline HSW & $1000 / 57$ & 3 & & & 57 & $\frac{1.05}{15}$ & 70 & 23 & 68 & 0.41 & pitch & 2-speed & & & & & & & & 2552 & & \\
\hline $\begin{array}{l}\text { Windtec } \\
\text { Kyazerer }\end{array}$ & & $\frac{3}{2}$ & rigid & up & 67 & 1.5 & & & & 0.43 & & & & & & & & & & 3526 & & \\
\hline $\begin{array}{l}\text { Kvaererer } \\
\text { Vestas }\end{array}$ & WTS 80 & $\frac{2}{3}$ & rigid & up & $\frac{80.5}{63}$ & $\begin{array}{r}3 \\
1.5 \\
\end{array}$ & $\frac{80}{60}$ & $\frac{21}{21}$ & 88 & 0.0 .59 & $\begin{array}{l}\text { pitch } \\
\text { pitch }\end{array}$ & $\begin{array}{l}\text { var spd (1.5:1) } \\
2 \text { speed w } 10 \% \text { sip }\end{array}$ & 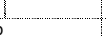 & +3 & 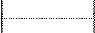 & & 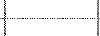 & 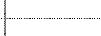 & & 5090 & 0 & \\
\hline Bonus & $1 \mathrm{MW} / 54$ & 3 & rigid & up & 54 & 1 & 60 & 22 & 62 & $\begin{array}{l}0.40 \\
0.44\end{array}$ & active stall & 2 speed & & & & & 63000 & 63 & & 2290 & 28 & \\
\hline Enercon & $E-66$ & 3 & rigid & up & 66 & 1.5 & 100 & 20 & 70 & 0.44 & pitch & var spd (2.5:1) & & & & & 99500 & 66 & & 3421 & 29 & \\
\hline & & 3 & rig & up & 54 & 1 & 59 & 21 & 59 & 14 & stall & 2 speed & & & & & 57800 & 57 & & 2290 & 25 & \\
\hline Micon & NM900/52 & 3 & rigid & up & 52 & 0.9 & & & & 0.42 & & & 2720 & 11800 & 19960 & 21800 & 41760 & 46 & & 2124 & 20 & 24 \\
\hline $\begin{array}{l}\text { Nedwind } \\
\text { Nedwind }\end{array}$ & $\begin{array}{l}\text { NW 53/2/1000-240 } \\
\text { NW 5 5/2/1000-240 }\end{array}$ & $\frac{2}{2}$ & rigid? & up & $\frac{52.6}{55}$ & $\frac{1}{1}$ & $\begin{array}{l}70 \\
70 \\
70\end{array}$ & $\frac{25}{25}$ & 68 & $\begin{array}{l}0.46 \\
0.42\end{array}$ & $\begin{array}{l}\text { active stall } \\
\text { active stall }\end{array}$ & $\begin{array}{l}2 \text { speed } \\
2 \text { speed }\end{array}$ & $x^{2}$ & & & & 65000 & 65 & & 2173 & 30 & \\
\hline$\frac{\text { Nedwind }}{\text { Nordank }}$ & $\begin{array}{l}\text { NW 5T/2/10000-240 } \\
\text { NTK } 1500 / 60\end{array}$ & $\frac{2}{3}$ & $\frac{\text { rigid? }}{\text { rigid }}$ & up & $\frac{55}{60}$ & $\frac{1}{1.5}$ & $\frac{70}{68}$ & $\frac{25}{19}$ & $\frac{71}{60}$ & $\frac{0.42}{0.53}$ & $\begin{array}{l}\text { active stall } \\
\text { stall }\end{array}$ & $\begin{array}{l}2 \text { speed } \\
\text { constant }\end{array}$ & 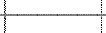 & & & & 98000 & 65 & & $\frac{2376}{2827}$ & $\frac{0}{35}$ & \\
\hline Autoflug & A1200 & 2 & $?$ & up & 61 & 1.2 & 60 & 21 & 66 & 0.41 & pitch & 2 speed & & & & & 85000 & $\frac{05}{71}$ & & $\frac{2021}{2922}$ & $\frac{50}{29}$ & \\
\hline Zond & $\mathrm{z} 8$ & 3 & rigid & up & 80 & 1.8 & 85 & 20 & 84 & 36 & pitch & var spd & & & & & & & & 27 & 0 & \\
\hline Zond & TZ-1.5 & 3 & rigid & up & 70.5 & 1.5 & 80 & & & 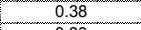 & pitch & var spd & 5600 & 15500 & 32300 & 51000 & 83300 & 56 & 109000 & 3904 & 21 & 34 \\
\hline Noridi & $Z-750$ & 3 & & & 50 & 0.75 & 50 & & & 0 & & var spd & 3780 & 4989 & 16329 & 23660 & 39989 & 53 & 58740 & 000 & 20 & 32 \\
\hline Nordic & 1000 & 2 & & & 53 & 1 & 58 & 25 & 69 & 0.45 & stall & 2 speed & & & & & & & & 2206 & 0 & 0 \\
\hline TVP T Trbine & & & & & & & & & & & & & & & & & & 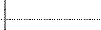 & & & & \\
\hline Vestas - BS & V66 & 3 & rigid & up & 66 & 1.65 & 80 & 19 & 66 & 0.48 & pitch & $2 \mathrm{sp}$ & 38 & 10250 & 21800 & 56000 & 78000 & 47 & 145000 & 3421 & 23 & 34 \\
\hline$v_{e}$ & V47 & 3 & rigid & up & 47 & 0.66 & 65 & 28.5 & 70 & . & pitch & constant w/ 10\% slip & & 2 & $72+3$ & 20 & $2 t$ & 42 & 50700 & $\pi$ & 16 & 31 \\
\hline Zond - & $Z-40 \mathrm{~A}$ & 3 & rigi & up & 40 & 0.0 & 40 & & & & & & 181 & 3450 & 88 & & 257 & 52 & & $\frac{1257}{1257}$ & 21 & 34 \\
\hline Zond - GMP & $z-40 \mathrm{FS}$ & $\frac{3}{3}$ & rigid & up & 40 & & 65 & & & 0. & & constant & 1610 & 4392 & & 190 & & 51 & & 1257 & 22 & 35 \\
\hline $\begin{array}{l}\text { Zond - lowa } \\
\text { AOC - KEA }\end{array}$ & $\begin{array}{c}\text { Z-50 } \\
\text { AOC } 15 / 50\end{array}$ & $\begin{array}{l}3 \\
3 \\
\end{array}$ & $\begin{array}{l}\text { rigid } \\
\text { rigid }\end{array}$ & $\begin{array}{l}\text { up } \\
\text { down }\end{array}$ & $\begin{array}{l}50 \\
15\end{array}$ & 0.75 & $\begin{array}{c}50 \\
26.5\end{array}$ & $19-32.2$ & & 0.38 & stall/tin & var spd & 3540 & & $\begin{array}{r}12250 \\
500\end{array}$ & 22220 & 34470 & $\begin{array}{r}46 \\
37\end{array}$ & & 1963 & 18 & 30 \\
\hline \begin{tabular}{|l} 
AOC-SEA \\
Zond-NE
\end{tabular} & $Z-50$ & 3 & $\begin{array}{l}\text { figla } \\
\text { rigid }\end{array}$ & down & $\frac{15}{50}$ & 0.75 & $\frac{26.5}{65}$ & & & 0.38 & stantrip & $\begin{array}{l}\text { Constant } \\
\text { var spd }\end{array}$ & 35 & & $\frac{500}{12250}$ & $\frac{1220}{2220}$ & $\frac{2440}{34470}$ & 46 & & 1963 & $\frac{14}{18}$ & $\frac{29}{30}$ \\
\hline $\begin{array}{l}\text { Tacke-WE } \\
\text { Tack }\end{array}$ & TW $600-3-3-$ CWM & 3 & $\begin{array}{l}\text { rigla } \\
\text { rigid }\end{array}$ & up & 46 & 0.6 & 60 & & & 0.36 & & $\begin{array}{l}\text { varspo } \\
\text { var spd }\end{array}$ & $\begin{array}{l}3540 \\
1900 \\
\end{array}$ & 3400 & 9100 & 292000 & $\begin{array}{l}34400 \\
38700\end{array}$ & $\frac{40}{65}$ & & $\begin{array}{l}1963 \\
1662 \\
\end{array}$ & $\frac{18}{23}$ & 49 \\
\hline & & & & & & & & & AVG & 0.44 & & & & & & & & & & & & \\
\hline
\end{tabular}


Data combined from Wind Energy Vol1 No. 2, Dec 1998 and TVP Turbine data from Page A-1.

Rotor Diam (m) Tower Top Mass $\mathrm{kg} / \mathrm{m}^{2}$

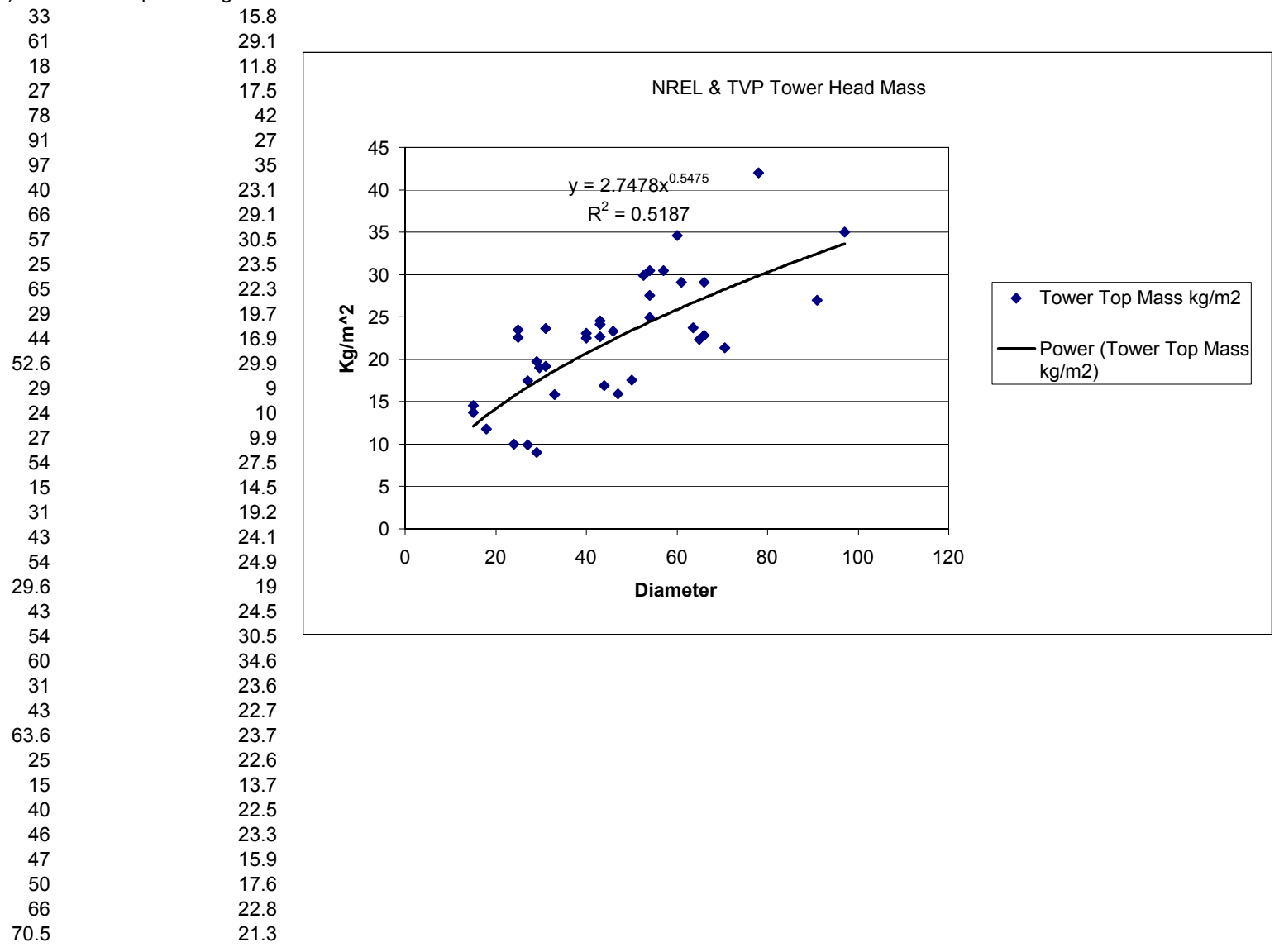


Data From Page A-2 Converted to Tower Head Mass

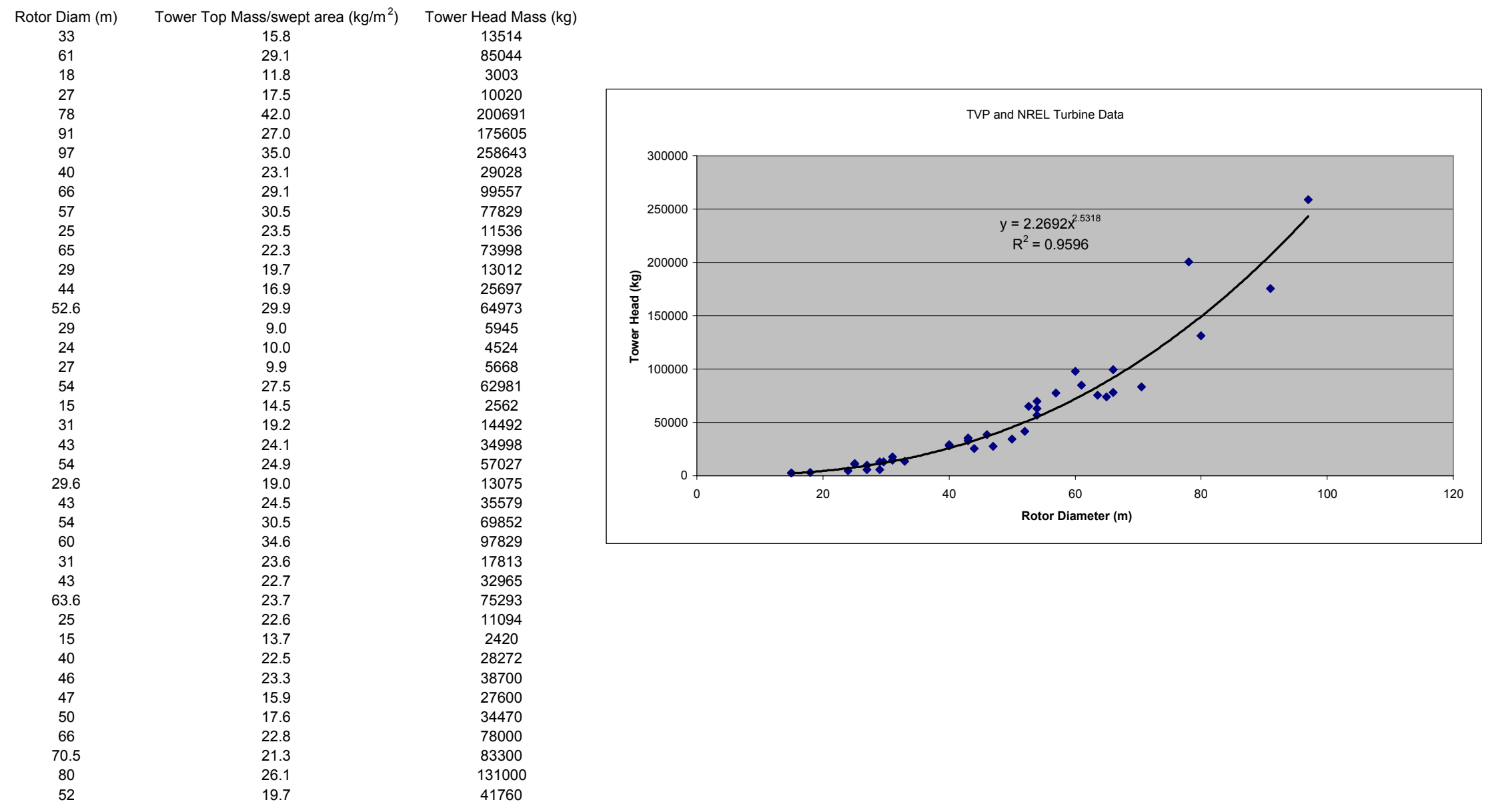


EC Equations Source: European Commission Directorate - General for Energy, Wind Energy - The Facts, Volume 1 - Technology (Appendix), EWEA Web Site, 1998

\begin{tabular}{|c|c|c|c|c|c|c|c|c|c|}
\hline \multirow{3}{*}{ Rotor Dia $(m)$} & \multicolumn{4}{|c|}{ EWEA - The Facts } & \multirow{2}{*}{$\begin{array}{c}\text { NREL and TVP Head Mass } \\
\text { Equation }\end{array}$} & \multicolumn{4}{|c|}{ Manufacturer Based Data } \\
\hline & $\begin{array}{c}\text { Nacelle Mass } \\
\text { Equation Fig 4.6.3 }\end{array}$ & \begin{tabular}{|c|}
$3 \times$ Blade Mass \\
Equation (Fig \\
$4.5 .2)$
\end{tabular} & $\begin{array}{l}\text { Manufactuer } \\
\text { Hub Mass } \\
\text { Equation }\end{array}$ & $\begin{array}{l}\text { Total Head } \\
\text { Mass }\end{array}$ & & $\begin{array}{c}3 \times \text { Blade Scaling } \\
\text { Study Mass } \\
\text { Equation }\end{array}$ & $\begin{array}{c}\text { Manufactuer } \\
\text { Hub Mass }\end{array}$ & $\begin{array}{c}\text { Manufactuer } \\
\text { Nacelle Mass }\end{array}$ & Total Head Mass \\
\hline & $\mathrm{kg}$ & $\mathrm{kg}$ & $\mathrm{kg}$ & $\mathrm{kg}$ & $\mathrm{kg}$ & $\mathrm{kg}$ & $\mathrm{kg}$ & $\mathrm{kg}$ & $\mathrm{kg}$ \\
\hline 50 & 31081 & 8819 & 5723 & 45623 & 45424 & 6150 & 5723 & 28179 & 40052 \\
\hline 66 & 60517 & 18303 & 11702 & 90522 & 91739 & 13639 & 11702 & 50622 & 75963 \\
\hline 85 & 111065 & 35604 & 22457 & 169126 & 174075 & 28184 & 22457 & 86333 & 136974 \\
\hline 100 & 164049 & 54591 & 34136 & 252776 & 262685 & 44926 & 34136 & 121648 & 200709 \\
\hline 120 & 254102 & 88180 & 54604 & 396885 & 416779 & 75800 & 54604 & 178721 & 309125 \\
\hline
\end{tabular}

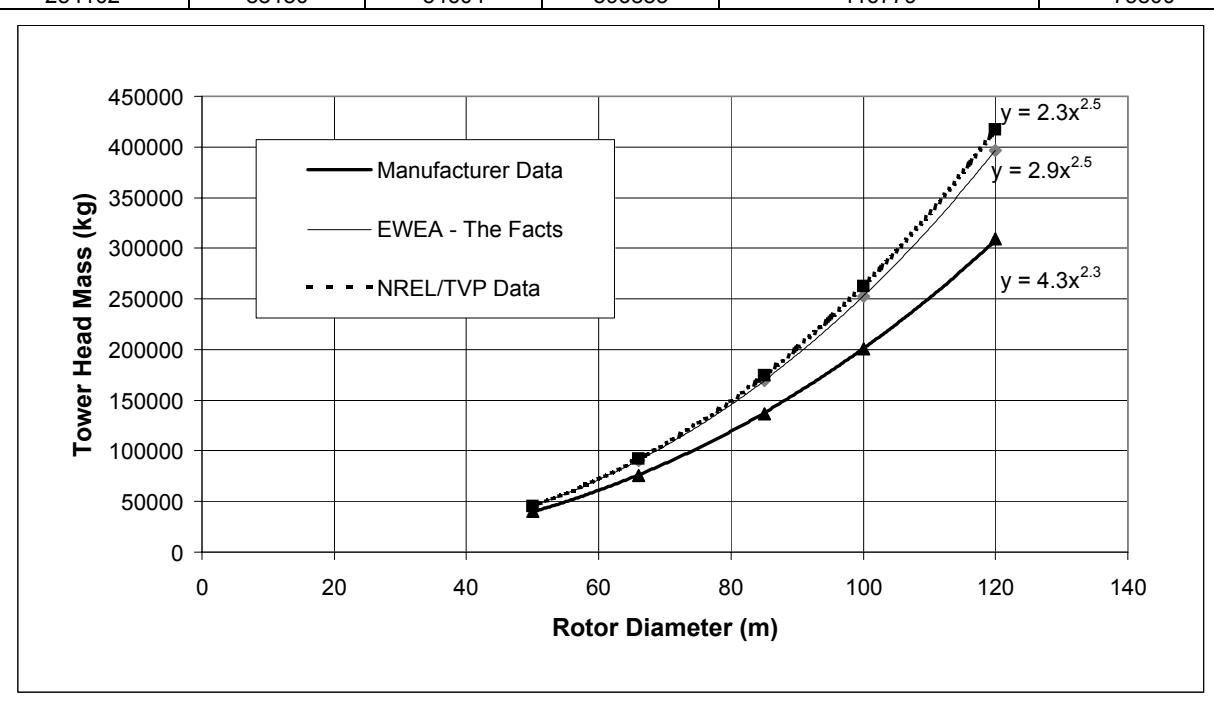


Tower design prepared by D Malcolm, May 2000

Resign or free-standing tower prepared by D Malcolm, May 2000

Assumptions:

IEC class 2 wind regime. Ve50 wind on tower, Ve50 wind on non-pitched blades. 2. Linear taper between top and base.

. Peak load governs, tower dia

5. Assumed control for pitchable blades has failed in operational position.

5. Fatigue and dynamics are not included, however, as a compromise the Ve50 wind speed is used inplace of the Ve1 value.

. Total characteristic base $\mathrm{mt}$ has been calculated to be provided as an input to the foundation design. It is assumed that the foundation design will apply a load factor to this value, therefore the tower load factor has been removed. modification has been used to allow compensation for fatigue and dynamics.

input

Ve50 wind speed $=$

Ve1 wind speed $=$

upper twr dia fraction

max dia/thickness $=$
material yield $=\mathrm{Fy}=$

material density=

wr ht / rotor diam

flapwise $\mathrm{Cd}=$

rotor solidity =

load factor =

material factor $=$

$59.5 \mathrm{~m} / \mathrm{s}$
$59.5 \mathrm{~m} / \mathrm{s}$
$0.5 *$ base diameter
$66000 / \mathrm{Fy}$
$300 \mathrm{Mpa}$
220
$7850 \mathrm{~kg} / \mathrm{m}^{\wedge} 3$
1.3
1.8
0.05
$1.225 \mathrm{~kg} / \mathrm{m}^{\wedge} 3$
1.35
1.1

$59.5 \mathrm{~m} / \mathrm{s}$
$59.5 \mathrm{~m} / \mathrm{s}$

diameter

(

$7850 \mathrm{~kg} / \mathrm{m}^{\wedge} 3$

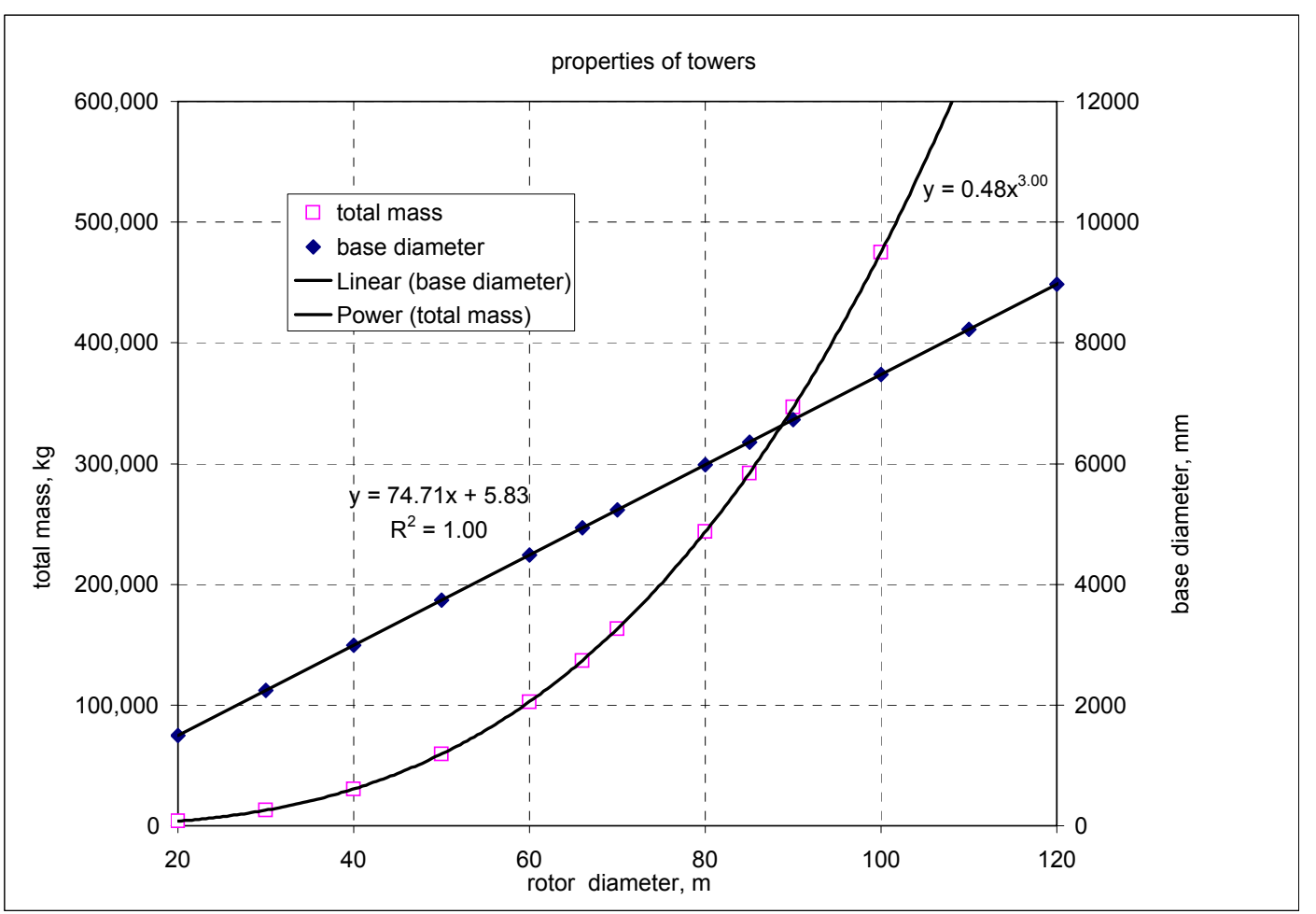

\begin{tabular}{|c|c|c|c|c|c|c|c|c|c|c|c|c|c|c|c|c|c|c|c|}
\hline & & & & & & \multicolumn{4}{|c|}{ initial design } & \multicolumn{10}{|c|}{ final design } \\
\hline $\begin{array}{l}\text { rotor } \\
\text { diam }\end{array}$ & $\begin{array}{l}\text { tower } \\
\text { height }\end{array}$ & \begin{tabular}{|c|} 
design rotor \\
thrust, Ve1 \\
\end{tabular} & $\begin{array}{c}\begin{array}{c}\text { design } \\
\text { base } \mathrm{mt} \\
\text { from thrust }\end{array} \\
\end{array}$ & $\begin{array}{l}\text { design } \\
\text { stress }\end{array}$ & $\begin{array}{c}\text { diam/ } \\
\text { thickness } \\
\text { ratio }\end{array}$ & $\begin{array}{c}\text { req'd } \\
\text { section } \\
\text { modulus }\end{array}$ & $\begin{array}{c}\text { req'd base } \\
\text { diameter } \\
\end{array}$ & $\begin{array}{c}\text { design } \\
\text { drag on } \\
\text { tower }\end{array}$ & $\begin{array}{c}\text { des base } \\
\mathrm{mt}\end{array}$ & $\begin{array}{c}\text { total } \\
\text { design } \\
\text { base } \mathrm{mt}\end{array}$ & $\begin{array}{c}\text { total } \\
\text { characteristics } \\
\text { base mt }\end{array}$ & \begin{tabular}{|c|}
$\begin{array}{c}\text { req'd } \\
\text { section } \\
\text { modulus }\end{array}$ \\
\end{tabular} & $\begin{array}{c}\text { req'd base } \\
\text { diameter }\end{array}$ & $\begin{array}{c}\text { thickness } \\
\text { at base } \\
\end{array}$ & $\begin{array}{c}\text { sect area } \\
\text { at base }\end{array}$ & $\begin{array}{c}\text { diameter at } \\
\text { top }\end{array}$ & $\begin{array}{c}\text { thickness } \\
\text { at top }\end{array}$ & $\begin{array}{c}\begin{array}{c}\text { sect area } \\
\text { at top }\end{array} \\
\end{array}$ & $\begin{array}{c}\text { total } \\
\text { mass }\end{array}$ \\
\hline 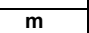 & $m$ & $\mathrm{kN}$ & $\mathrm{kN} \mathrm{m}$ & Mpa & & $\mathrm{mm}^{\wedge} 3$ & $\mathrm{~mm}$ & $\mathrm{kN}$ & $\mathrm{kN} \mathrm{m}$ & $\mathrm{kN} \mathrm{m}$ & $\mathrm{kN} \mathrm{m}$ & $\mathrm{mm}^{\wedge} \mathbf{3}$ & $\mathrm{mm}$ & $\mathrm{mm}$ & $\mathrm{mm}^{\wedge} \mathbf{2}$ & $\mathrm{mm}$ & $\mathrm{mm}$ & & $\mathbf{k g}$ \\
\hline 20 & 26.0 & 82.8 & 2152 & 272.7 & 220.0 & $7.89 \mathrm{E}+06$ & 1293 & 103.4 & 1194 & 3346 & 2479 & $1.23 \mathrm{E}+07$ & 1498 & 6.81 & $3.20 \mathrm{E}+04$ & 749 & 3.40 & $8.01 \mathrm{E}+03$ & 3816 \\
\hline 30 & 39.0 & 186.2 & 7263 & 272.7 & 220.0 & $2.66 \mathrm{E}+07$ & 1939 & 232.5 & 4029 & 11292 & 8365 & $4.14 \mathrm{E}+07$ & 2246 & 10.21 & $7.20 \mathrm{E}+04$ & 1123 & 5.10 & $1.80 \mathrm{E}+04$ & 12866 \\
\hline 40 & 52.0 & 331.1 & 17216 & 272.7 & 220.0 & $6.31 \mathrm{E}+07$ & 2585 & 413.1 & 9548 & 26764 & 19825 & $9.81 E+07$ & 2994 & 13.61 & $1.28 \mathrm{E}+05$ & 1497 & 6.80 & $3.20 \mathrm{E}+04$ & 30479 \\
\hline 50 & 65.0 & 517.3 & 33625 & 272.7 & 220.0 & $1.23 \mathrm{E}+08$ & 3230 & 645.4 & 18644 & 52269 & 38718 & $1.92 \mathrm{E}+08$ & 3741 & 17.01 & $2.00 \mathrm{E}+05$ & 1871 & 8.50 & $5.00 \mathrm{E}+04$ & 59499 \\
\hline 60 & 78.0 & 744.9 & 58104 & 272.7 & 220.0 & $2.13 \mathrm{E}+08$ & 3876 & 929.2 & 32212 & 90315 & 66900 & \begin{tabular}{|c|}
$3.31 \mathrm{E}+08$ \\
\end{tabular} & 4489 & 20.40 & $2.88 \mathrm{E}+05$ & 2244 & 10.20 & $7.19 \mathrm{E}+04$ & 102772 \\
\hline 66 & 85.8 & 901.4 & 77336 & 272.7 & 220.0 & $2.84 \mathrm{E}+08$ & 4263 & 1124.2 & 42870 & 120206 & 89041 & $4.41 \mathrm{E}+08$ & 4937 & 22.44 & $3.48 \mathrm{E}+05$ & 2469 & 11.22 & $8.70 \mathrm{E}+04$ & 136760 \\
\hline 70 & 91.0 & 1013.9 & 92266 & 272.7 & 220.0 & $3.38 \mathrm{E}+08$ & 4521 & 1264.5 & 51143 & 143410 & 106229 & $5.26 \mathrm{E}+08$ & 5236 & 23.80 & $3.92 \mathrm{E}+05$ & 2618 & 11.90 & $9.79 E+04$ & 163141 \\
\hline 80 & 104.0 & 1324.3 & 137727 & 272.7 & 220.0 & $5.05 \mathrm{E}+08$ & 5166 & 1651.4 & 76332 & 214059 & 158562 & $7.85 E+08$ & 5983 & 27.20 & $5.11 \mathrm{E}+05$ & 2992 & 13.60 & $1.28 \mathrm{E}+05$ & 243450 \\
\hline 85 & 110.5 & 1495.0 & 165199 & 272.7 & 220.0 & $6.06 \mathrm{E}+08$ & 5489 & 1864.2 & 91551 & 256750 & 190185 & $9.41 \mathrm{E}+08$ & 6357 & 28.89 & $5.77 \mathrm{E}+05$ & 3178 & 14.45 & $1.44 \mathrm{E}+05$ & 291970 \\
\hline 90 & 117.0 & 1676.1 & 196100 & 272.7 & 220.0 & $7.19 E+08$ & 5811 & 2089.8 & 108670 & 304770 & 225756 & $1.12 E+09$ & 6730 & 30.59 & $6.47 \mathrm{E}+05$ & 3365 & 15.30 & $1.62 \mathrm{E}+05$ & 346540 \\
\hline 100 & 130.0 & 2069.2 & 268998 & 272.7 & 220.0 & $9.86 \mathrm{E}+08$ & 6456 & 2579.7 & 149052 & 418050 & 309667 & $1.53 \mathrm{E}+09$ & 7477 & 33.99 & $7.98 \mathrm{E}+05$ & 3739 & 16.99 & $2.00 \mathrm{E}+05$ & 475252 \\
\hline 110 & 143.0 & 2503.8 & 358037 & 272.7 & 220.0 & $1.31 \mathrm{E}+09$ & \begin{tabular}{|l|}
7101 \\
7101
\end{tabular} & 3121.2 & 198369 & 556406 & 412153 & $2.04 \mathrm{E}+09$ & 8224 & 37.38 & $9.66 \mathrm{E}+05$ & 4112 & 18.69 & $2.41 \mathrm{E}+05$ & 632425 \\
\hline 120 & 156.0 & 2979.7 & 4829 & 272.7 & 220.0 & $1.70 E+09$ & 7746 & 3714.2 & 257515 & 722344 & 53507 & $2.65 E+09$ & 8971 & 40.78 & $1.15 \mathrm{E}+06$ & 448 & 20.39 & $2.87 \mathrm{E}+05$ & 820900 \\
\hline 130 & 169.0 & 3497.0 & 590989 & 272.7 & 220.0 & $2.17 E+09$ & 8391 & 4358.6 & 327382 & 918371 & 680275 & $3.37 \mathrm{E}+09$ & 9717 & 44.17 & $1.35 \mathrm{E}+06$ & 4859 & 22.08 & $3.37 \mathrm{E}+05$ & 1043515 \\
\hline 140 & 182.0 & 5.7 & 738131 & 272.7 & 220.0 & $2.71 \mathrm{E}+09$ & 9036 & 5054.6 & 408861 & 1146993 & 849624 & $4.21 \mathrm{E}+09$ & 10464 & 47.56 & $1.56 \mathrm{E}+06$ & 5232 & 23.78 & $3.91 \mathrm{E}+05$ & 1303109 \\
\hline 150 & 195.0 & 5.7 & 907869 & 272.7 & 220.0 & $3.33 \mathrm{E}+09$ & 9680 & 5802.1 & 502847 & 141 & 1044975 & $5.17 \mathrm{E}+09$ & 11211 & 50.96 & $1.79 \mathrm{E}+06$ & 5605 & 25.48 & $4.49 \mathrm{E}+05$ & 1602520 \\
\hline 160 & 208.0 & 5297.2 & 1101817 & 272.7 & 220.0 & $4.04 \mathrm{E}+09$ & 10325 & 6601.1 & 610231 & $\begin{array}{l}1712048 \\
\end{array}$ & 1268183 & $6.28 \mathrm{E}+09$ & 11957 & 54.35 & $2.04 \mathrm{E}+06$ & 5979 & 27.18 & $\begin{array}{l}4.45 \mathrm{~L}+05 \\
5.10 \mathrm{E}\end{array}$ & 1944585 \\
\hline 170 & 221.0 & 5980.0 & 1321589 & 272.7 & 220.0 & $4.85 \mathrm{E}+09$ & 10970 & 7451.5 & 731904 & 2053493 & 1521106 & $7.53 E+09$ & 12704 & 57.74 & $2.30 \mathrm{E}+06$ & 6352 & 28.87 & $5.76 \mathrm{E}+05$ & 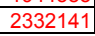 \\
\hline
\end{tabular}




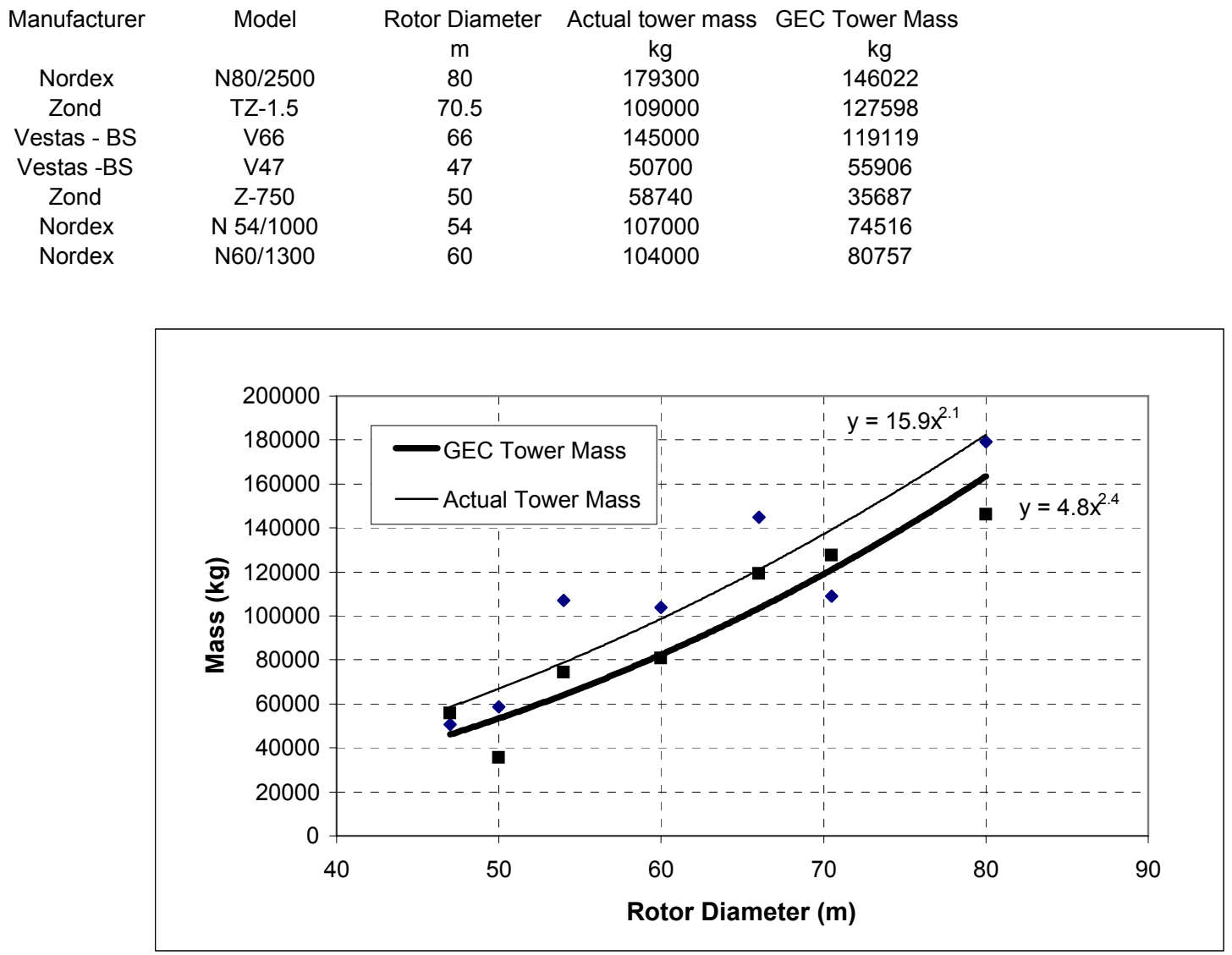




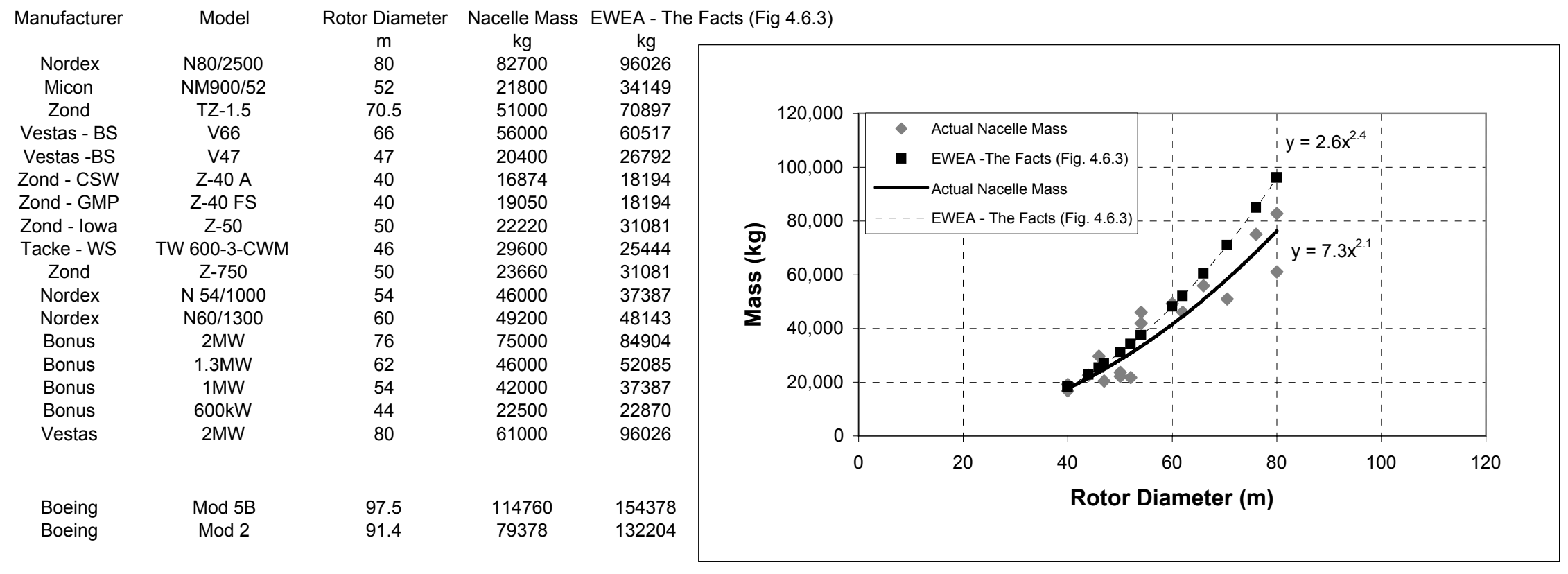




$\begin{array}{cccc}\text { manufacturer } & \text { model } & \begin{array}{c}\text { rotor diameter } \\ \text { mub mass }\end{array} & \begin{array}{c}\text { kg } \\ \text { Micon }\end{array} \\ \text { Zond } & \text { NM900/52 } & 52 & 11800 \\ \text { Vestas - BS } & \text { TZ-1.5 } & 70.5 & 15500 \\ \text { Vestas -BS } & \text { V46 } & 66 & 10250 \\ \text { Zond - CSW } & \text { Z-40 A } & 47 & 2850 \\ \text { Zond - GMP } & \text { Z-40 FS } & 40 & 3450 \\ \text { Tacke - WS } & \text { TW 600-3-CWM } & 40 & 4392 \\ \text { Zond } & \text { Z-750 } & 46 & 3400 \\ & & 50 & 4989\end{array}$

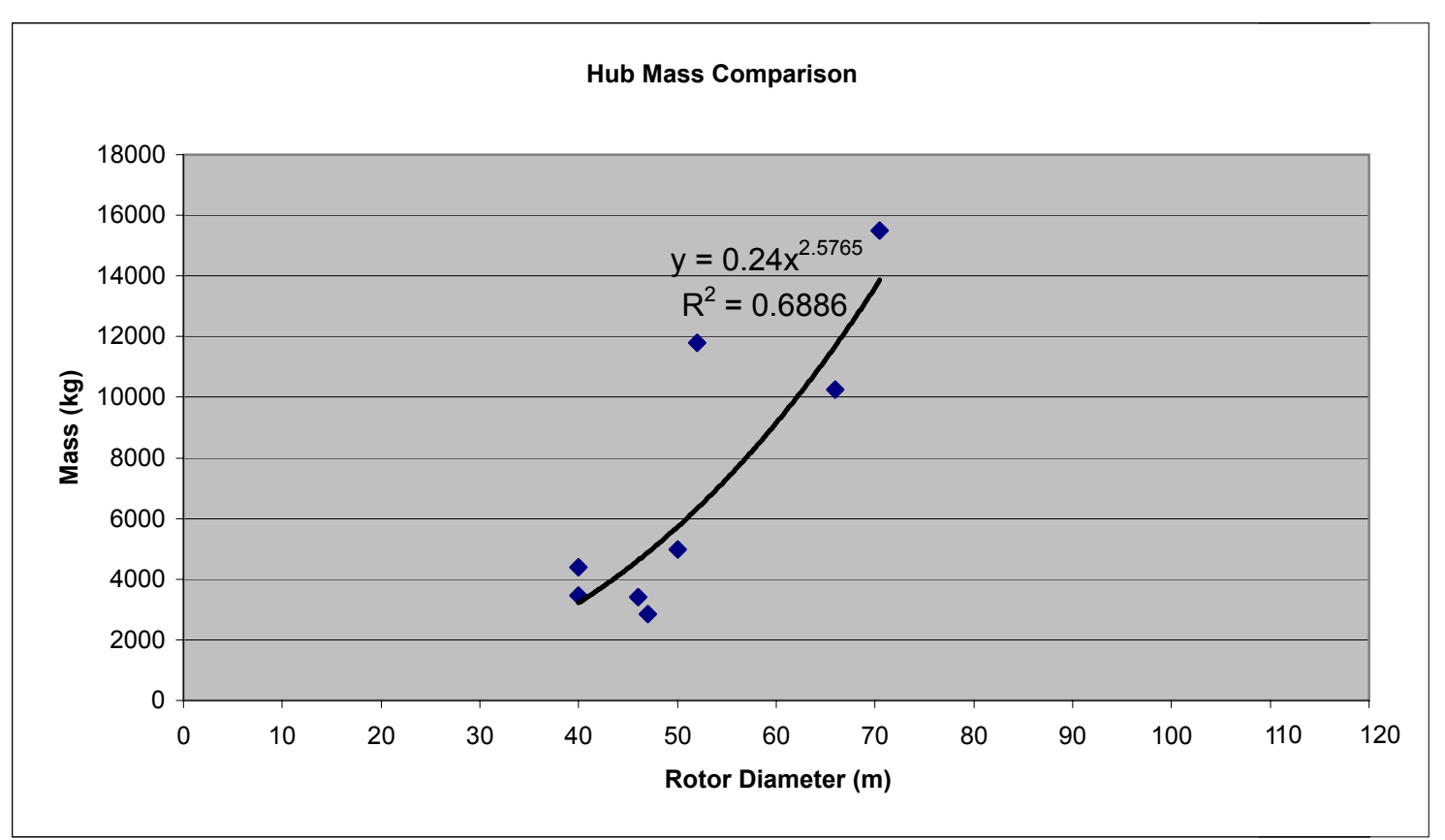




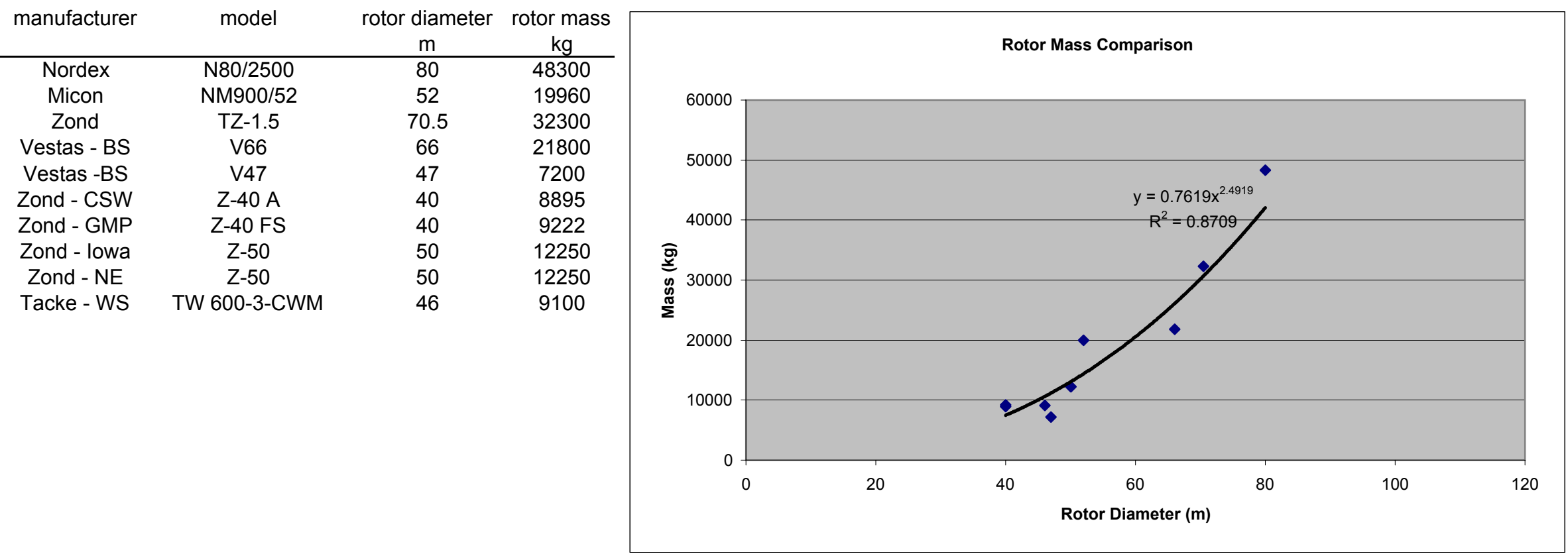


All Data for $\mathrm{TSR}_{\text {Design }}=7, \mathrm{C}_{\mathrm{Max}}=8 \% \mathrm{R}$, no parasitic weight

\begin{tabular}{|c|}
\hline Rating \\
\hline \\
\hline 1.50 \\
\hline 2.00 \\
\hline 2.30 \\
\hline 3.00 \\
\hline 4.00 \\
\hline 5.00 \\
\hline
\end{tabular}

$\begin{array}{cc}\mathrm{R} & \mathrm{L} \\ (\mathrm{m}) & (\mathrm{m}) \\ 23.3 & 22.1 \\ 32.9 & 31.3 \\ 38.0 & 36.1 \\ 40.8 & 38.8 \\ 46.6 & 44.3 \\ 53.8 & 51.1 \\ 60.2 & 57.2\end{array}$

L

$\mathrm{t} / \mathrm{c}=27 \%$ at max. chord W $(\mathrm{kg}) \quad \mathrm{W}\left(10^{3} \mathrm{~kg}\right) \quad$ Grav / Trq

$1851 \quad 1.85$

1851

5017

7597

9284
13629

13629

20685

1.85

5.02

7.60
9.28

9.28
13.63

13.63
20.69
28.63

Commercial Blade Data:

\begin{tabular}{|c|c|c|c|c|c|}
\hline $\begin{array}{l}\mathrm{L} \\
(\mathrm{m})\end{array}$ & $\begin{array}{c}R \\
(\mathrm{~m})\end{array}$ & $\begin{array}{l}\text { Rating } \\
(\mathrm{MW})\end{array}$ & $\begin{array}{l}\text { W } \\
(\mathrm{kg})\end{array}$ & $\begin{array}{c}W \\
\left(10^{3} \mathrm{~kg}\right)\end{array}$ & $\begin{array}{l}\text { Blade / } \\
\text { Turbine }\end{array}$ \\
\hline 21.0 & 22.1 & 675 & 2100 & 2.10 & LM \\
\hline 21.5 & 22.6 & 708 & 2650 & 2.65 & LM \\
\hline 22.1 & 23.3 & 748 & 1600 & 1.60 & LM \\
\hline 22.3 & 23.5 & 763 & 1450 & 1.45 & V47 \\
\hline 23.3 & 24.5 & 831 & 2800 & 2.80 & LM \\
\hline 23.8 & 25.0 & 864 & 3540 & 3.54 & Z-50 \\
\hline 24.1 & 25.4 & 889 & 2400 & 2.40 & LM \\
\hline 24.1 & 25.4 & 889 & 2600 & 2.60 & LM \\
\hline 26.1 & 27.5 & 1043 & 4000 & 4.00 & LM \\
\hline 31.4 & 33.0 & 1505 & 3850 & 3.85 & V66 \\
\hline 33.3 & 35.0 & 1692 & 5600 & 5.60 & TZ-1.6 \\
\hline 33.8 & 35.6 & 1749 & 7800 & 7.80 & LM \\
\hline 34.0 & 35.8 & 1770 & 5600 & 5.60 & LM \\
\hline 37.3 & 39.3 & 2130 & 6035 & 6.04 & LM \\
\hline 38.8 & 40.8 & 2305 & 8500 & 8.50 & LM \\
\hline
\end{tabular}

EWEA EWEA

The Facts The Facts

$\begin{array}{cccc}\mathrm{t} / \mathrm{c}=33 \% \text { at max. chord } & \text { Fig } 4.5 .2 & \text { Fig } 4.5 .2 \\ \mathrm{~W}(\mathrm{~kg}) & \mathrm{W}\left(10^{3} \mathrm{~kg}\right) & \mathrm{W}(\mathrm{kg}) & \mathrm{W}\left(10^{3} \mathrm{~kg}\right)\end{array}$

$\begin{array}{llllll}0.87 & 1.76 & 1725 & 1.73 & 2443 & 2.44\end{array}$

$\begin{array}{llllll}1.18 & 1.39 & 4651 & 4.65 & 6052 & 6.05 \\ 1.34 & 1.26 & 7029 & 7.03 & 8842 & 8.84\end{array}$

$\begin{array}{llllcc}1.34 & 1.26 & 7029 & 7.03 & 8842 & 8.84 \\ 1.43 & 1.20 & 8580 & 8.58 & 10660 & 10.66\end{array}$

$\begin{array}{llllll}1.61 & 1.08 & 12571 & 12.57 & 15120 & 15.12\end{array}$

$\begin{array}{llllll}1.83 & 0.97 & 19057 & 19.06 & 22063 & 22.06\end{array}$

$\begin{array}{llllll}2.02 & 0.89 & 26334 & 26.33 & 29652 & 29.65\end{array}$

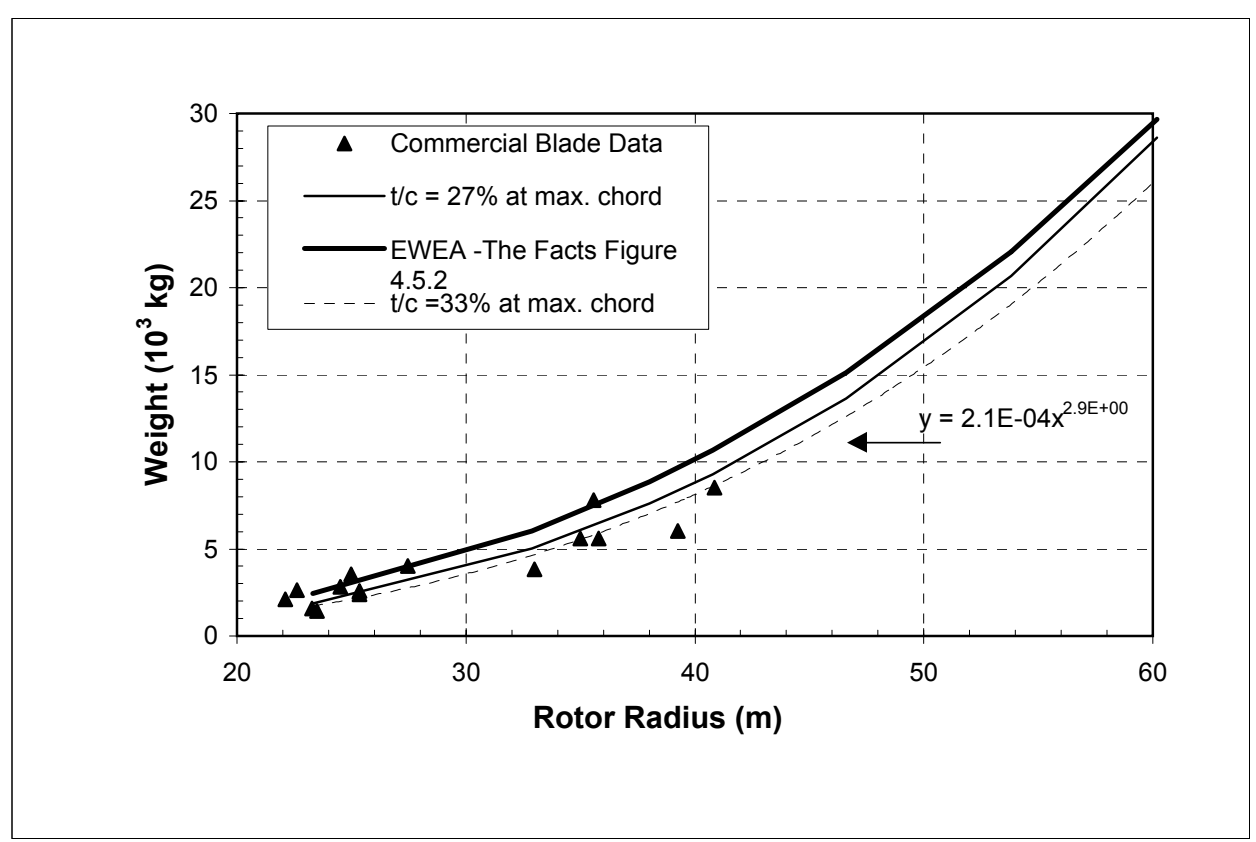


Appendix B

Scenario Summary 


\begin{tabular}{|c|c|c|c|c|c|c|}
\hline & & \multicolumn{5}{|c|}{ Summary } \\
\hline & & 750 & 1500 & 2500 & 3500 & 5000 \\
\hline & Rotor Diameter $(\mathrm{m})$ & 50 & 66 & 85 & 100 & 120 \\
\hline & Calculated Power (kW) & 864 & 1505 & 2497 & 3456 & 4976 \\
\hline \multirow{3}{*}{$\begin{array}{l}\text { Scenario 1: Baseline - all } \\
\text { Components pre-assembled to } \\
\text { maximum extent possible. }\end{array}$} & Low & $\$ 48.73$ & $\$ 59.06$ & $\$ 550.70$ & $\$ 717.75$ & $\$ 785.22$ \\
\hline & Middle & $\$ 64.58$ & $\$ 77.38$ & $\$ 601.97$ & $\$ 776.42$ & $\$ 969.63$ \\
\hline & High & $\$ 109.90$ & $\$ 151.91$ & $\$ 679.35$ & $\$ 936.06$ & $\$ 1,035.05$ \\
\hline \multirow{3}{*}{$\begin{array}{l}\text { Scenario 2: Scenario } 1 \text { except } \\
\text { rotors assembled in air and } \\
2.5+\text { MW turbine towers } \\
\text { require field assembly } \\
\text { (BOLTED). }\end{array}$} & Low & $\$ 48.73$ & $\$ 70.00$ & $\$ 84.79$ & $\$ 124.66$ & $\$ 167.93$ \\
\hline & Middle & $\$ 64.58$ & $\$ 89.48$ & $\$ 106.73$ & $\$ 148.61$ & $\$ 319.21$ \\
\hline & High & $\$ 109.90$ & $\$ 165.57$ & $\$ 186.95$ & $\$ 311.87$ & $\$ 388.57$ \\
\hline \multirow{3}{*}{$\begin{array}{l}\text { Scenario 2: Scenario 1 except } \\
\text { rotors assembled in air and } \\
2.5+\text { MW turbine towers } \\
\text { require field assembly } \\
\text { (MANUAL WELDING). }\end{array}$} & Low & $\$ 48.73$ & $\$ 79.71$ & $\$ 102.27$ & $\$ 167.91$ & $\$ 244.04$ \\
\hline & Middle & $\$ 64.58$ & $\$ 100.34$ & $\$ 126.16$ & $\$ 196.67$ & $\$ 403.77$ \\
\hline & High & $\$ 109.90$ & $\$ 178.15$ & $\$ 209.29$ & $\$ 367.13$ & $\$ 485.81$ \\
\hline \multirow{3}{*}{$\begin{array}{l}\text { Scenario 2: Scenario } 1 \text { except } \\
\text { rotors assembled in air and } \\
2.5+\text { MW turbine towers } \\
\text { require field assembly (SEMI- } \\
\text { AUTOMATED WELDING). }\end{array}$} & Low & $\$ 48.73$ & $\$ 75.37$ & $\$ 94.21$ & $\$ 146.97$ & $\$ 207.77$ \\
\hline & Middle & $\$ 64.58$ & $\$ 95.52$ & $\$ 117.20$ & $\$ 173.40$ & $\$ 363.48$ \\
\hline & High & $\$ 109.90$ & $\$ 172.61$ & $\$ 198.98$ & $\$ 340.37$ & $\$ 439.47$ \\
\hline \multirow{3}{*}{\begin{tabular}{|l} 
Scenario 3: Gearbox and \\
generator installed in nacelle \\
atop tower. Rotors assembled \\
in air. 2.5+ MW turbine towers \\
require field assembly.
\end{tabular}} & Low & $\$ 48.73$ & $\$ 70.00$ & $\$ 88.67$ & $\$ 130.33$ & $\$ 169.31$ \\
\hline & Middle & $\$ 64.58$ & $\$ 89.48$ & $\$ 111.31$ & $\$ 156.56$ & $\$ 318.76$ \\
\hline & High & $\$ 109.90$ & $\$ 165.57$ & $\$ 190.38$ & $\$ 317.10$ & $\$ 390.45$ \\
\hline
\end{tabular}

Notes:

1) Costs presented on this page correspond to combined Transportation, Assembly, and Crane costs.

2) See pages B-2 to B-4 for corresponding summaries. 


\begin{tabular}{|c|c|c|c|c|c|c|}
\hline & & \multicolumn{5}{|c|}{ Transportation } \\
\hline & & 750 & 1500 & 2500 & 3500 & 5000 \\
\hline & Rotor Diameter $(\mathrm{m})$ & 50 & 66 & 85 & 100 & 120 \\
\hline & Calculated Power (kW) & 864 & 1505 & 2497 & 3456 & 4976 \\
\hline \multirow{3}{*}{$\begin{array}{l}\text { Scenario 1: Baseline - all } \\
\text { components pre-assembled to } \\
\text { maximum extent possible. [1] }\end{array}$} & $\begin{array}{l}\text { U.S. Sourced - Short } \\
\text { Haul }\end{array}$ & $\$ 26.25$ & $\$ 35.91$ & $\$ 528.08$ & $\$ 691.42$ & $\$ 754.83$ \\
\hline & $\begin{array}{l}\text { U.S. Sourced - Long } \\
\text { Haul } \\
\end{array}$ & $\$ 32.71$ & $\$ 43.99$ & $\$ 567.92$ & $\$ 737.94$ & $\$ 923.68$ \\
\hline & European Sourced & $\$ 76.68$ & $\$ 117.08$ & $\$ 643.52$ & $\$ 895.71$ & $\$ 985.19$ \\
\hline \multirow{3}{*}{$\begin{array}{l}\text { Scenario 2: Scenario } 1 \text { except } \\
\text { rotors assembled in air and } \\
2.5+\text { MW turbine towers } \\
\text { require field assembly } \\
\text { (BOLTED). [2] }\end{array}$} & $\begin{array}{l}\text { U.S. Sourced - Short } \\
\text { Haul }\end{array}$ & $\$ 26.25$ & $\$ 35.91$ & $\$ 44.55$ & $\$ 75.91$ & $\$ 113.21$ \\
\hline & $\begin{array}{l}\text { U.S. Sourced - Long } \\
\text { Haul } \\
\end{array}$ & $\$ 32.71$ & $\$ 43.99$ & $\$ 53.02$ & $\$ 85.16$ & $\$ 246.13$ \\
\hline & European Sourced & $\$ 76.68$ & $\$ 117.08$ & $\$ 128.62$ & $\$ 242.92$ & $\$ 307.65$ \\
\hline \multirow{3}{*}{$\begin{array}{l}\text { Scenario 2: Scenario 1 except } \\
\text { rotors assembled in air and } \\
2.5+\text { MW turbine towers } \\
\text { require field assembly } \\
\text { (MANUAL WELDING). [2] }\end{array}$} & $\begin{array}{l}\begin{array}{l}\text { U.S. Sourced - Short } \\
\text { Haul }\end{array} \\
\end{array}$ & $\$ 26.25$ & $\$ 35.91$ & $\$ 44.55$ & $\$ 75.91$ & $\$ 113.21$ \\
\hline & $\begin{array}{l}\text { U.S. Sourced - Long } \\
\text { Haul }\end{array}$ & $\$ 32.71$ & $\$ 43.99$ & $\$ 53.02$ & $\$ 85.16$ & $\$ 246.13$ \\
\hline & European Sourced & $\$ 76.68$ & $\$ 117.08$ & $\$ 128.62$ & $\$ 242.92$ & $\$ 307.65$ \\
\hline \multirow{3}{*}{$\begin{array}{l}\text { Scenario 2: Scenario } 1 \text { except } \\
\text { rotors assembled in air and } \\
\text { 2.5+ MW turbine towers } \\
\text { require field assembly (SEMI- } \\
\text { AUTOMATED WELDING). [2] }\end{array}$} & $\begin{array}{l}\text { U.S. Sourced - Short } \\
\text { Haul }\end{array}$ & $\$ 26.25$ & $\$ 35.91$ & $\$ 44.55$ & $\$ 75.91$ & $\$ 113.21$ \\
\hline & $\begin{array}{l}\text { U.S. Sourced - Long } \\
\text { Haul }\end{array}$ & $\$ 32.71$ & $\$ 43.99$ & $\$ 53.02$ & $\$ 85.16$ & $\$ 246.13$ \\
\hline & European Sourced & $\$ 76.68$ & $\$ 117.08$ & $\$ 128.62$ & $\$ 242.92$ & $\$ 307.65$ \\
\hline \multirow{3}{*}{$\begin{array}{l}\text { Scenario 3: Gearbox and } \\
\text { generator installed in nacelle } \\
\text { atop tower. Rotors assembled } \\
\text { in air. 2.5+ MW turbine towers } \\
\text { require field assembly. [3] }\end{array}$} & $\begin{array}{l}\text { U.S. Sourced - Short } \\
\text { Haul }\end{array}$ & $\$ 26.25$ & $\$ 35.91$ & $\$ 45.76$ & $\$ 78.28$ & $\$ 111.38$ \\
\hline & $\begin{array}{l}\text { U.S. Sourced - Long } \\
\text { Haul }\end{array}$ & $\$ 32.71$ & $\$ 43.99$ & $\$ 54.78$ & $\$ 89.79$ & $\$ 242.44$ \\
\hline & European Sourced & $\$ 76.68$ & $\$ 117.08$ & $\$ 129.34$ & $\$ 244.77$ & $\$ 306.20$ \\
\hline
\end{tabular}

Notes:

1] See cost component summaries on pages $\mathrm{C}-1$ and $\mathrm{C}-2$.

[2] See cost component summaries on pages $\mathrm{C}-3$ and $\mathrm{C}-4$.

[3] See cost component summaries on pages C-5 and C-6. 


\begin{tabular}{|c|c|c|c|c|c|c|}
\hline & & \multicolumn{5}{|c|}{ Assembly } \\
\hline & & 750 & 1500 & 2500 & 3500 & 5000 \\
\hline & Rotor Diameter (m) & 50 & 66 & 85 & 100 & 120 \\
\hline & Calculated Power (kW) & 864 & 1505 & 2497 & 3456 & 4976 \\
\hline \multirow{3}{*}{\begin{tabular}{|l|} 
Scenario 1: Baseline - all \\
components pre-assembled to \\
maximum extent possible.
\end{tabular}} & $\begin{array}{l}\text { Minimum } \\
\text { (See Appendix I-2) }\end{array}$ & $\$ 15.11$ & $\$ 10.86$ & $\$ 11.35$ & $\$ 9.68$ & $\$ 11.85$ \\
\hline & $\begin{array}{l}\text { Average } \\
\text { (See Appendix l-1) }\end{array}$ & $\$ 23.55$ & $\$ 16.98$ & $\$ 17.70$ & $\$ 15.60$ & $\$ 19.05$ \\
\hline & \begin{tabular}{|l|} 
Maximum \\
(See Appendix I-3)
\end{tabular} & $\$ 24.57$ & $\$ 17.79$ & $\$ 18.67$ & $\$ 16.50$ & $\$ 20.73$ \\
\hline \multirow{3}{*}{\begin{tabular}{|l|} 
Scenario 2: Scenario 1 except \\
rotors assembled in air and \\
$2.5+$ MW turbine towers \\
require field assembly \\
(BOLTED).
\end{tabular}} & $\begin{array}{l}\text { Minimum } \\
\text { (See Appendix J-2) }\end{array}$ & $\$ 15.11$ & $\$ 19.32$ & $\$ 25.89$ & $\$ 27.12$ & $\$ 30.42$ \\
\hline & $\begin{array}{l}\text { Average } \\
\text { (See Appendix J-1) }\end{array}$ & $\$ 23.55$ & $\$ 26.49$ & $\$ 34.00$ & $\$ 35.12$ & $\$ 39.85$ \\
\hline & $\begin{array}{l}\text { Maximum } \\
\text { (See Appendix J-3) }\end{array}$ & $\$ 24.57$ & $\$ 28.72$ & $\$ 37.39$ & $\$ 38.94$ & $\$ 44.62$ \\
\hline \multirow{3}{*}{\begin{tabular}{|l|} 
Scenario 2: Scenario 1 except \\
rotors assembled in air and \\
$2.5+$ MW turbine towers \\
require field assembly \\
(MANUAL WELDING).
\end{tabular}} & $\begin{array}{l}\text { Minimum } \\
\text { (See Appendix K-2) }\end{array}$ & $\$ 15.11$ & $\$ 26.59$ & $\$ 38.62$ & $\$ 57.03$ & $\$ 82.33$ \\
\hline & $\begin{array}{l}\text { Average } \\
\text { (See Appendix K-1) }\end{array}$ & $\$ 23.55$ & $\$ 34.57$ & $\$ 48.16$ & $\$ 68.36$ & $\$ 97.53$ \\
\hline & $\begin{array}{l}\text { Maximum } \\
\text { (See Appendix K-3) }\end{array}$ & $\$ 24.57$ & $\$ 38.02$ & $\$ 53.67$ & $\$ 77.16$ & $\$ 110.96$ \\
\hline \multirow{3}{*}{\begin{tabular}{|l} 
Scenario 2: Scenario 1 except \\
rotors assembled in air and \\
2.5+ MW turbine towers \\
require field assembly (SEMI- \\
AUTOMATED WELDING).
\end{tabular}} & $\begin{array}{l}\text { Minimum } \\
\text { (See Appendix L-2) }\end{array}$ & $\$ 15.11$ & $\$ 23.27$ & $\$ 32.45$ & $\$ 42.11$ & $\$ 57.06$ \\
\hline & $\begin{array}{l}\text { Average } \\
\text { (See Appendix L-1) }\end{array}$ & $\$ 23.55$ & $\$ 30.89$ & $\$ 41.30$ & $\$ 51.78$ & $\$ 69.45$ \\
\hline & $\begin{array}{l}\text { Maximum } \\
\text { (See Appendix L-3) }\end{array}$ & $\$ 24.57$ & $\$ 33.78$ & $\$ 45.79$ & $\$ 58.10$ & $\$ 78.66$ \\
\hline \multirow{3}{*}{\begin{tabular}{|l} 
Scenario 3: Gearbox and \\
generator installed in nacelle \\
atop tower. Rotors assembled \\
in air. 2.5+ MW turbine towers \\
require field assembly.
\end{tabular}} & $\begin{array}{l}\text { Minimum } \\
\text { (See Appendix M-2) }\end{array}$ & $\$ 15.11$ & $\$ 19.32$ & $\$ 27.18$ & $\$ 28.53$ & $\$ 31.69$ \\
\hline & $\begin{array}{l}\text { Average } \\
\text { (See Appendix M-1) }\end{array}$ & $\$ 23.55$ & $\$ 26.49$ & $\$ 35.29$ & $\$ 36.53$ & $\$ 41.12$ \\
\hline & $\begin{array}{l}\text { Maximum } \\
\text { (See Appendix M-3) }\end{array}$ & $\$ 24.57$ & $\$ 28.72$ & $\$ 38.68$ & $\$ 40.35$ & $\$ 45.89$ \\
\hline
\end{tabular}

Notes:

1) Assembly Costs for $750 \mathrm{~kW}$ turbine presented as baseline for current construction practices. 


\begin{tabular}{|c|c|c|c|c|c|c|}
\hline & & \multicolumn{5}{|c|}{ Cranes } \\
\hline & & 750 & 1500 & 2500 & 3500 & 5000 \\
\hline & Rotor Diameter $(\mathrm{m})$ & 50 & 66 & 85 & 100 & 120 \\
\hline & Calculated Power (kW) & 864 & 1505 & 2497 & 3456 & 4976 \\
\hline \multirow{3}{*}{$\begin{array}{l}\text { Scenario 1: Baseline - all } \\
\text { components pre-assembled to } \\
\text { maximum extent possible. [1] }\end{array}$} & Minimum & $\$ 7.37$ & $\$ 12.29$ & $\$ 11.27$ & $\$ 16.65$ & $\$ 18.53$ \\
\hline & Average & $\$ 8.33$ & $\$ 16.41$ & $\$ 16.35$ & $\$ 22.88$ & $\$ 26.90$ \\
\hline & Maximum & $\$ 8.64$ & $\$ 17.03$ & $\$ 17.15$ & $\$ 23.86$ & $\$ 29.12$ \\
\hline \multirow{3}{*}{\begin{tabular}{|l} 
Scenario 2: Scenario 1 except \\
rotors assembled in air and \\
2.5+ MW turbine towers \\
require field assembly \\
(BOLTED). [2],[3]
\end{tabular}} & Minimum & $\$ 7.37$ & $\$ 14.77$ & $\$ 14.35$ & $\$ 21.63$ & $\$ 24.30$ \\
\hline & Average & $\$ 8.33$ & $\$ 19.00$ & $\$ 19.71$ & $\$ 28.32$ & $\$ 33.23$ \\
\hline & Maximum & $\$ 8.64$ & $\$ 19.76$ & $\$ 20.94$ & $\$ 30.00$ & $\$ 36.30$ \\
\hline \multirow{3}{*}{$\begin{array}{l}\text { Scenario 2: Scenario } 1 \text { except } \\
\text { rotors assembled in air and } \\
2.5+\text { MW turbine towers } \\
\text { require field assembly } \\
\text { (MANUAL WELDING). [2],[4] }\end{array}$} & Minimum & $\$ 7.37$ & $\$ 17.21$ & $\$ 19.10$ & $\$ 34.97$ & $\$ 48.49$ \\
\hline & Average & $\$ 8.33$ & $\$ 21.77$ & $\$ 24.99$ & $\$ 43.15$ & $\$ 60.11$ \\
\hline & Maximum & $\$ 8.64$ & $\$ 23.05$ & $\$ 27.00$ & $\$ 47.05$ & $\$ 67.21$ \\
\hline \multirow{3}{*}{\begin{tabular}{|l} 
Scenario 2: Scenario 1 except \\
rotors assembled in air and \\
2.5+ MW turbine towers \\
require field assembly (SEMI- \\
AUTOMATED WELDING). \\
{$[2],[5]$}
\end{tabular}} & Minimum & $\$ 7.37$ & $\$ 16.19$ & $\$ 17.21$ & $\$ 28.95$ & $\$ 37.50$ \\
\hline & Average & $\$ 8.33$ & $\$ 20.64$ & $\$ 22.88$ & $\$ 36.45$ & $\$ 47.89$ \\
\hline & Maximum & $\$ 8.64$ & $\$ 21.75$ & $\$ 24.58$ & $\$ 39.35$ & $\$ 53.17$ \\
\hline \multirow{3}{*}{\begin{tabular}{||l} 
Scenario 3: Gearbox and \\
generator installed in nacelle \\
atop tower. Rotors assembled \\
in air. 2.5+ MW turbine towers \\
require field assembly. [6]
\end{tabular}} & Minimum & $\$ 7.37$ & $\$ 14.77$ & $\$ 15.73$ & $\$ 23.53$ & $\$ 26.24$ \\
\hline & Average & $\$ 8.33$ & $\$ 19.00$ & $\$ 21.23$ & $\$ 30.24$ & $\$ 35.20$ \\
\hline & Maximum & $\$ 8.64$ & $\$ 19.76$ & $\$ 22.36$ & $\$ 31.98$ & $\$ 38.36$ \\
\hline
\end{tabular}

Notes:

[1] Scenario 1 crane costs detailed on pages I-4 to I-7.

Scenario 1), and a second crane deployed for tower fabrication. The tower fabrication crane costs are dependent on the

[3] Bolted crane costs are detailed on page $\mathrm{J}-8$.

[4] Manual welding crane costs are detalled on page K-8.

[6] Tower fabrication crane costs using the bolting fabrication technique (from page J-8)

are added to the assembly crane costs (from page M-8) 
Appendix C

Transport Summary 


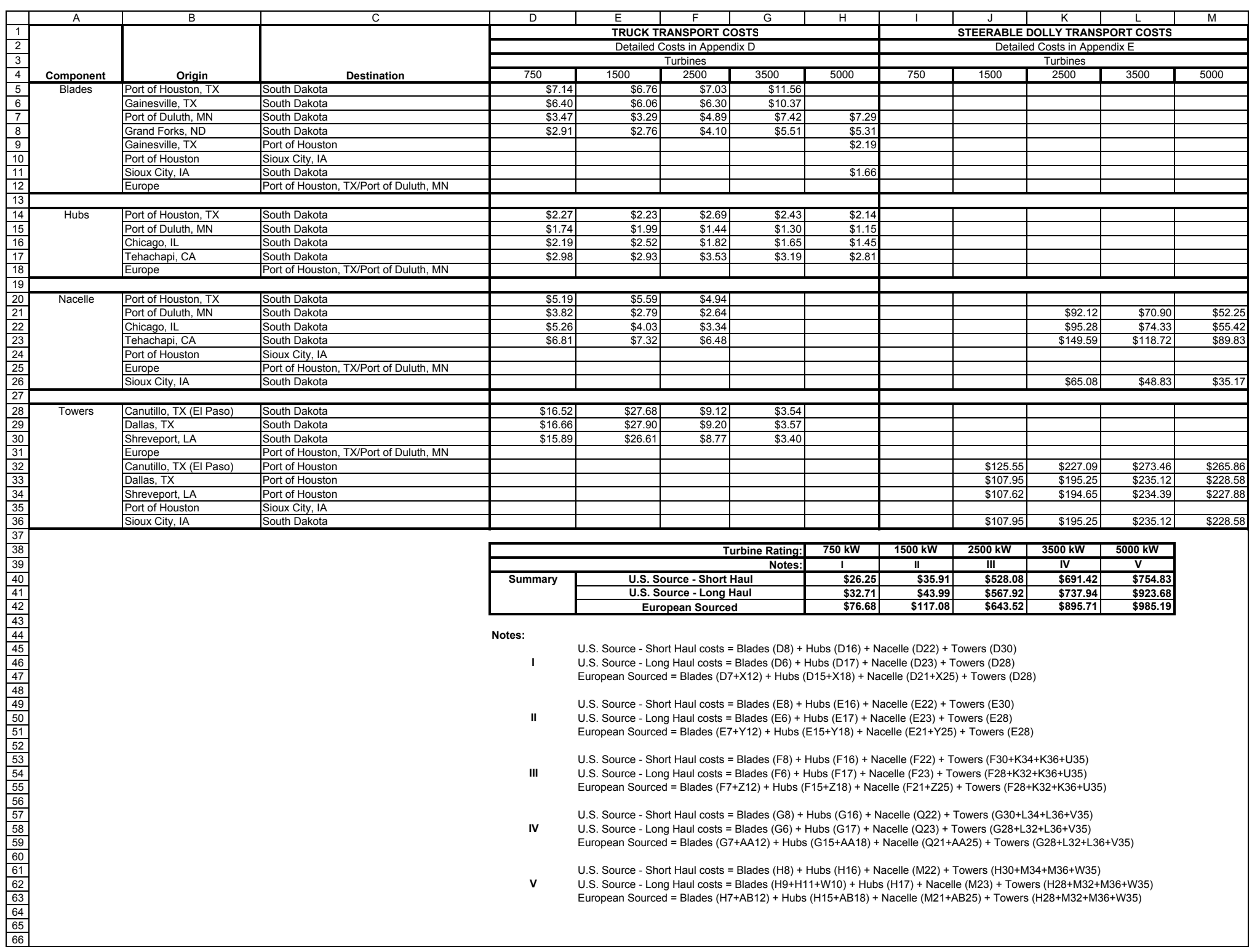




\begin{tabular}{|c|c|c|c|c|c|c|c|c|c|c|c|c|c|c|c|c|c|c|}
\hline \multirow{4}{*}{\begin{tabular}{|l|}
1 \\
2 \\
3 \\
4 \\
\end{tabular}} & \multirow[t]{3}{*}{ A } & \multirow[b]{4}{*}{ Origin } & \multirow[b]{4}{*}{ Destination } & \multirow{3}{*}{\multicolumn{5}{|c|}{$\begin{array}{c}\text { RAIL TRANSPORT COSTS } \\
\text { Detailed Costs in Appendix F }\end{array}$}} & \multirow{2}{*}{\multicolumn{5}{|c|}{\begin{tabular}{c|c|c}
$T$ & $U$ & $V$ \\
BARGE TRANSPORT COSTS \\
Detailed Costs in Appendix $G$
\end{tabular}}} & & \multirow{2}{*}{\multicolumn{4}{|c|}{ 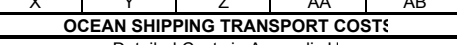 }} \\
\hline & & & & & & & & & & & & & & & & & & \\
\hline & & & & & & & & & & & & & & & & & & \\
\hline \multirow{7}{*}{\multicolumn{2}{|c|}{$\frac{\text { Component }}{\text { Blades }}$}} & & & 750 & 1500 & $\frac{2500}{2500}$ & 3500 & 5000 & 750 & 1500 & $\frac{2500}{2500}$ & 3500 & 5000 & 750 & 1500 & 2500 & 3500 & 5000 \\
\hline & & Port of Houston, TX & South Dakota & & & & & & & & & & & & & & & \\
\hline & & \begin{tabular}{|l} 
Gainesville, TX \\
Port tof Duluth MN
\end{tabular} & $\mid \frac{\text { South Dakota }}{\text { South Dakota }}$ & & & & & & & & & & & & & - & 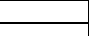 & \\
\hline & & Grand Forks, ND & South Dakota & & & & & & & & & & & & & & & \\
\hline & & Gainesville, TX & Port of Houston & & & & & & & & & & & & & & & \\
\hline & & Port of Houston & Sioux City, IA & & & & & & & & & & $\$ 96.56$ & & & & & \\
\hline & & Sioux City, IA & 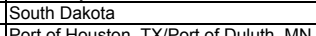 & & & & & & & & 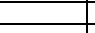 & & & (2020 & s570 & बen? & 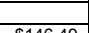 & 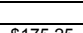 \\
\hline 13 & \multirow{5}{*}{ Hubs } & & & & & & & & & & & & & & & & & 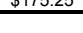 \\
\hline 14 & & Port of Houston, TX & South Dakota & & & & & & & & & & & & & & & \\
\hline$\frac{5}{16}$ & & \begin{tabular}{|l} 
Port of Duluth, MN \\
Chicago II
\end{tabular} & $\begin{array}{l}\text { South Dakota } \\
\text { South Dakota }\end{array}$ & & & & & & & & & & & & & & & \\
\hline 17 & & Tehachapi, CA & South Dakota & & & & & & & & & & & & & & & \\
\hline 18 & & Europe & Port of Houston, TX/Port of Duluth, MN & & & & & & & & & & & $\$ 2.59$ & $\$ 6.03$ & $\$ 4.78$ & $\$ 3.45$ & $\$ 3.36$ \\
\hline 19 & \multirow{7}{*}{ Nacelle } & & & & & & & & & & & & & & & & & \\
\hline 20 & & \begin{tabular}{|l} 
Port of Houston, TX \\
0
\end{tabular} & \begin{tabular}{|l} 
South Dakota \\
\end{tabular} & $\$ 15.40$ & & $\$ 7.01$ & $\$ 21.6$ & & & & & & & & & & & \\
\hline 21 & & $\begin{array}{l}\text { Port of Duluth, MN } \\
\text { Chiogon }\end{array}$ & South Dakota & $\$ 14.60$ & $\$ 8.91$ & $\$ 6.07$ & $\$ 20.5$ & & & & & & & & & & & \\
\hline$\frac{24}{23}$ & & $\frac{\text { Chicago, IL }}{\text { Tehachan CA }}$ & South Dakota & 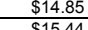 & $\begin{array}{r}\$ 9.19 \\
\$ 1022\end{array}$ & $\$ 6.37$ & \$20.92 & & & & & & & & & & & \\
\hline$\frac{30}{24}$ & & $\begin{array}{l}\text { Penachap, CA } \\
\text { Port of Houston }\end{array}$ & \begin{tabular}{|l} 
Soun Dakold \\
Sioux City, IA
\end{tabular} & $\$ 15.44$ & $\$ 10.22$ & $\$ 7.24$ & $\$ 21.84$ & & & & ens & 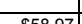 & 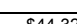 & & & & & \\
\hline 25 & & & Port of Houston. TX/Port of Duluth. MN & & & & & & & & \$ 344.60 & $\$ 38.97$ & $\$ 44.32$ & 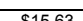 & 91831 & 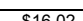 & (1290 & (950) \\
\hline 26 & & Sioux City, IA & South Dakota & & & & & & & & & & & & $\$ 18.31$ & $\$ 10.02$ & $\$ 13.89$ & $\$ 15.26$ \\
\hline 27 & \multirow{8}{*}{ Towers } & & & & & & & & & & & & & & & & & \\
\hline 28 & & $\begin{array}{l}\text { Canutillo, TX (EI Paso) } \\
\end{array}$ & South Dakota & & & & & & & & & & & & & & & \\
\hline 29 & & \begin{tabular}{|l} 
Dallas, TX \\
all
\end{tabular} & South Dakota & & & & & & & & -1 & - & & 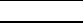 & 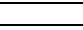 & 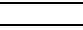 & 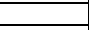 & ${ }_{-}$ \\
\hline 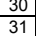 & & $\begin{array}{l}\text { Shreveport, LA } \\
\text { Europe }\end{array}$ & \begin{tabular}{|l} 
South Dakota \\
Port of Houston, TX/Port of Duluth, MN
\end{tabular} & & & & & & & & & & & $\$ 482$ & $\$ 636$ & $\$ 819$ & $\$ 963$ & $\$ 1,156$ \\
\hline 32 & & \begin{tabular}{|l} 
Canutillo, TX (EI Paso) \\
\end{tabular} & Port of Houston & & & & & & & & & & & & & & & \\
\hline 33 & & \begin{tabular}{|l} 
Dallas, TX \\
Shromont is
\end{tabular} & Port of Houston & & & & & & & & & & & & & & & \\
\hline$\frac{34}{35}$ & & $\begin{array}{l}\text { Dorreveron, }, \mathrm{A} \\
\text { Port of Houston } \\
\end{array}$ & Sioux City, IA & & & & & & & $\$ 43.90$ & $\$ 120.15$ & $\$ 190.44$ & $\$ 236.19$ & & & & & \\
\hline 36 & & Sioux City, IA & South Dakota & & & & & & & & & & & & & & & \\
\hline$\frac{37}{38}$ & & & & & & & & & & & & & & & & & & \\
\hline 39 & & & & & & & & & & & & & & & & & & \\
\hline \begin{tabular}{|l|l}
40 \\
11
\end{tabular} & & & & & & & & & & & & & & & & & & \\
\hline 42 & & & & & & & & & & & & & & & & & & \\
\hline $\begin{array}{l}43 \\
44 \\
\end{array}$ & & & & & & & & & & & & & & & & & & \\
\hline 年5 & & & & & & & & & & & & & & & & & & \\
\hline $\begin{array}{ll}47 \\
48\end{array}$ & & & & & & & & & & & & & & & & & & \\
\hline 49 & & & & & & & & & & & & & & & & & & \\
\hline $\begin{array}{l}50 \\
51 \\
\end{array}$ & & & & & & & & & & & & & & & & & & \\
\hline$\frac{52}{53}$ & & & & & & & & & & & & & & & & & & \\
\hline \begin{tabular}{|c|}
54 \\
55
\end{tabular} & & & & & & & & & & & & & & & & & & \\
\hline 56 & & & & & & & & & & & & & & & & & & \\
\hline $\begin{array}{l}58 \\
58 \\
59\end{array}$ & & & & & & & & & & & & & & & & & & \\
\hline $\begin{array}{l}59 \\
60 \\
91\end{array}$ & & & & & & & & & & & & & & & & & & \\
\hline $\begin{array}{l}61 \\
62 \\
\end{array}$ & & & & & & & & & & & & & & & & & & \\
\hline $\begin{array}{ll}63 \\
64 \\
\end{array}$ & & & & & & & & & & & & & & & & & & \\
\hline 65 & & & & & & & & & & & & & & & & & & \\
\hline
\end{tabular}




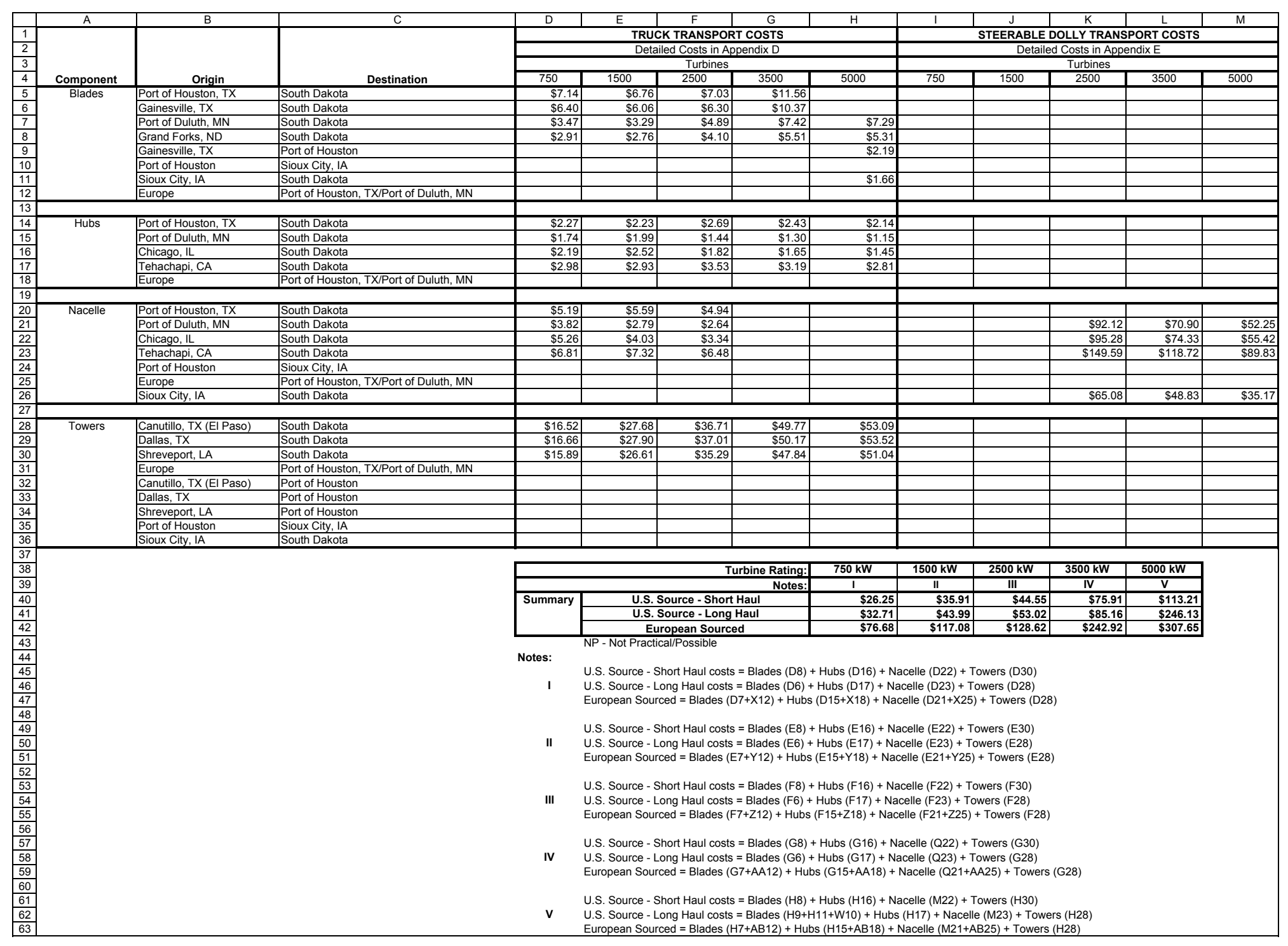




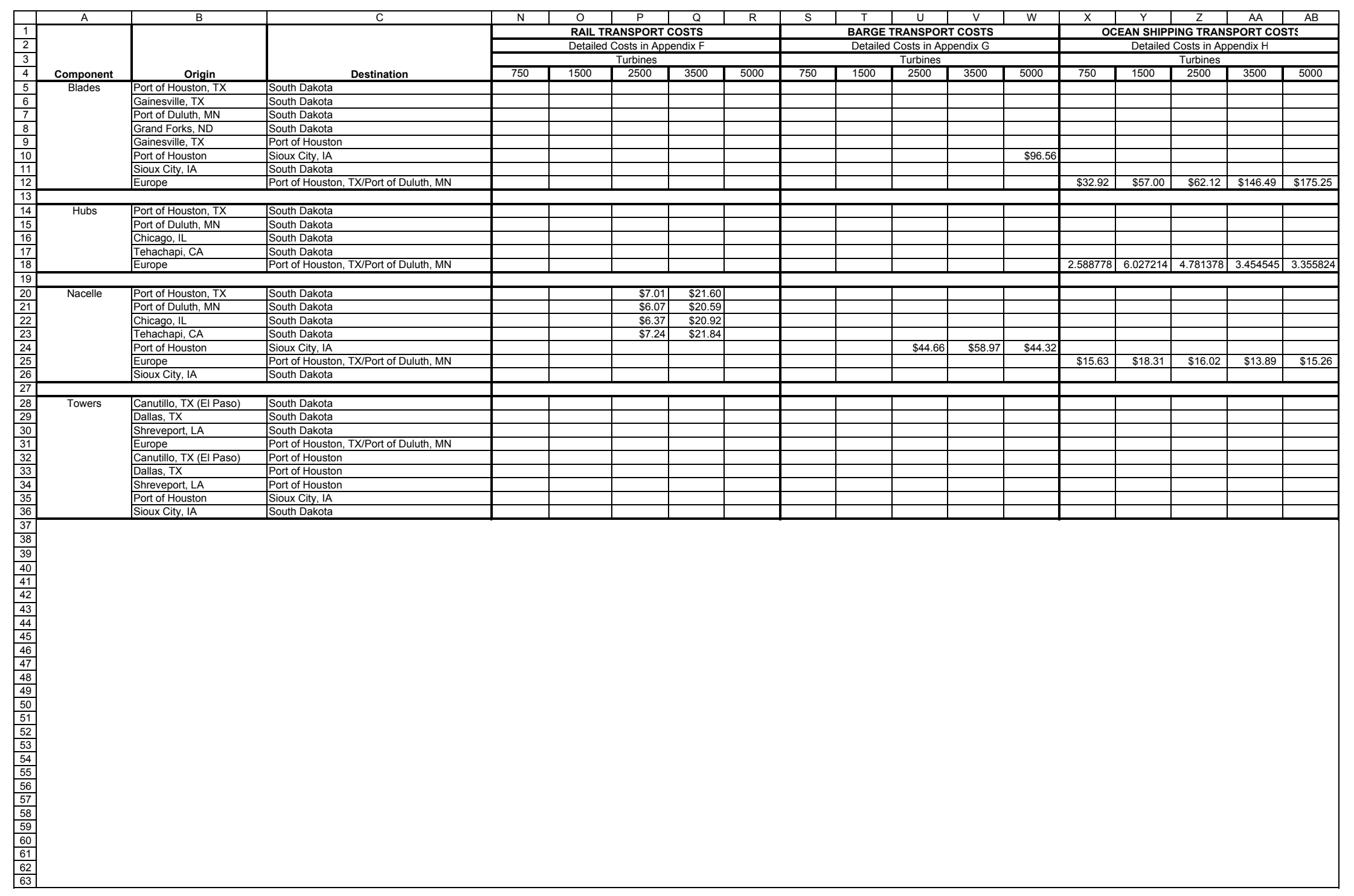




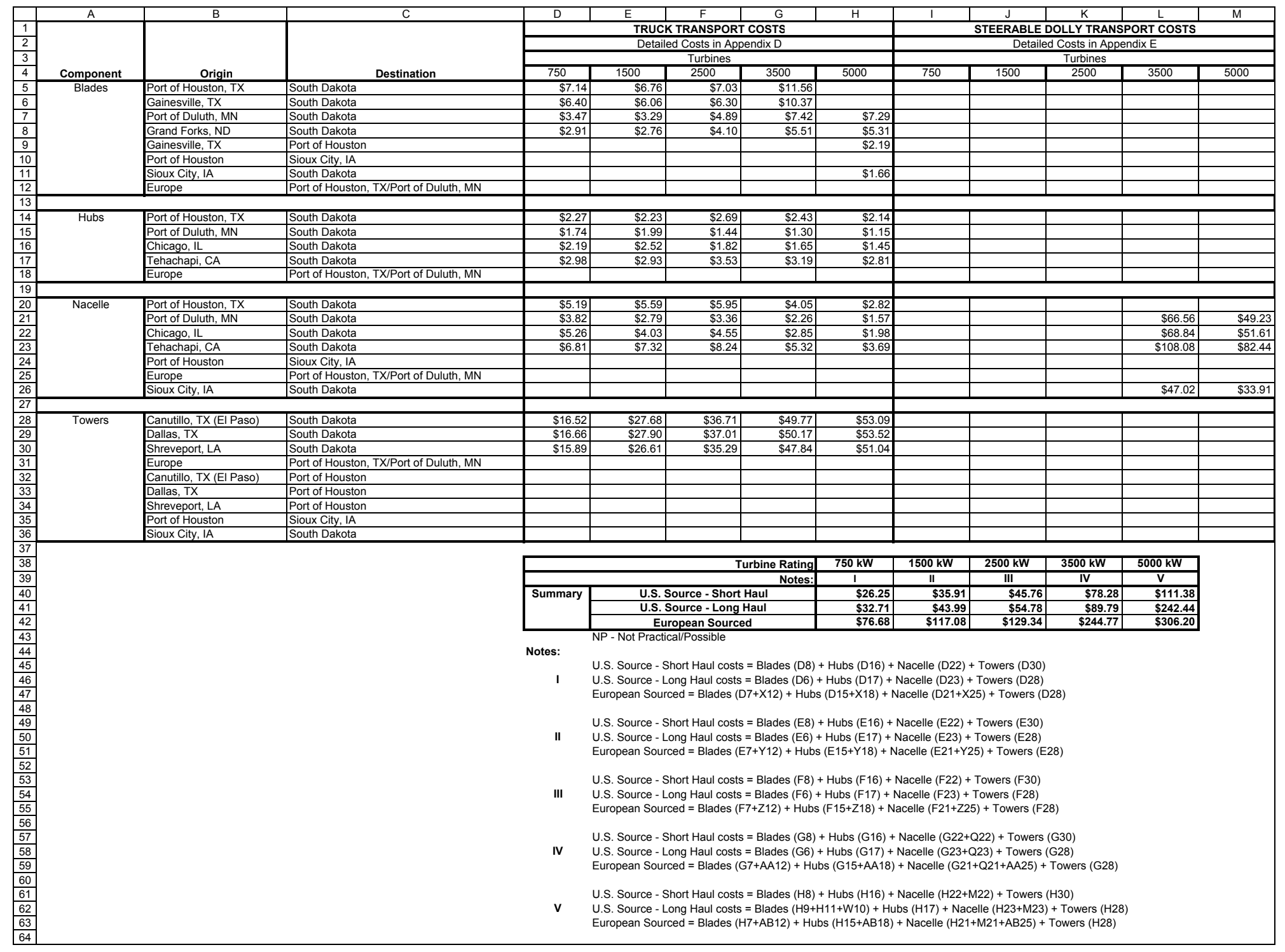




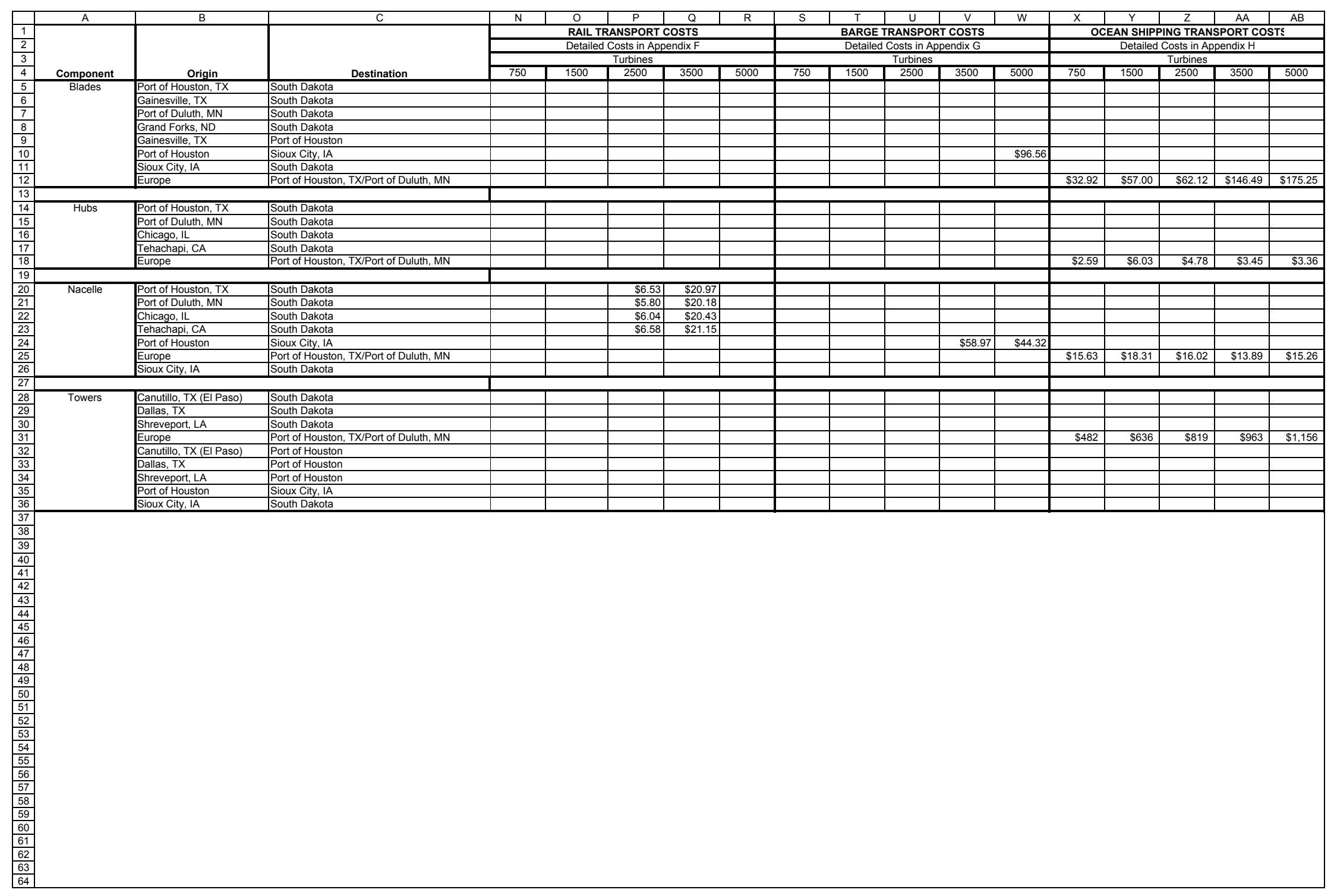


Appendix D

Truck Transport 
Rotor Diameter $(\mathrm{m})$

Turbine Class

From Port of Houston (European suppliers)

Costs per loaded-mile

Estimated mileage per load

Costs per load

Number of blades per load

Number of loads required

ad

Total costs

Cost per turbine

Costs per kW

Costs per swept area

From Gainesville, TX (Molded Fibre Glass, Inc.)

Costs per loaded-mile

Estimated mileage per load

Costs per load

Number of blades per load

Number of loads required

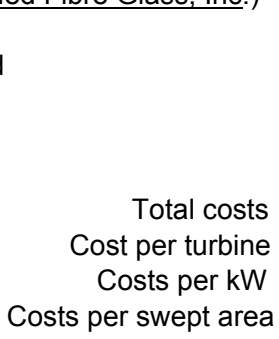

From Port of Duluth, MN (European suppliers)

Costs per loaded-mile

Estimated mileage per load

Costs per load

Number of blades per load

Number of loads required

Total costs
Cost per turbine
Costs per kW
Costs per swept area

From Grand Forks, ND (L-M Glasfiber)

Costs per loaded-mile

Estimated mileage per load

Costs per load

Number of blades per load

Number of loads required

M Glasfiber)
Total costs
Cost per turbine
Costs per kW
Costs per swept area

$\$ 5.50$
1,121
$\$ 6,166$
3
50

$\$ 308,275$

$\$ 6,166$

$\$ 7.14$

$\$ 3.14$

$\$ 5.50$

1,234

$\$ 6,787$

$\$ 4.74$

1,234

$\$ 5,849$

2
75

$\$ 509,025$

$\$ 10,181$

$\$ 6.76$

$\$ 2.98$

150
$\$ 877,374$
$\$ 17,547$
$\$ 7.03$
$\$ 3.09$

$\$ 5.50$
1005
$\$ 5,528$
3
50
$\$ 276,375$
$\$ 5,528$
$\$ 6.40$
$\$ 2.82$

$\$ 5.50$

1106

$\$ 6,083$

2

75
$\$ 456,225$

$\$ 9,125$

$\$ 6.06$

$\$ 2.67$

$\$ 5.00$

600

$\$ 3,000$

3

50

$\$ 150,000$

$\$ 3,000$

$\$ 3.47$

$\$ 1.53$

$\$ 5.00$

660

$\$ 3,300$

2

$\$ 4,950$

$\$ 3.29$

$\$ 1.45$

$\$ 5.00$

502

$\$ 2,510$

3

50

$\$ 125,500$

$\$ 2,510$

$\$ 2.91$

$\$ 1.28$

$$
\begin{array}{r}
\$ 5.00 \\
553 \\
\$ 2,765 \\
2 \\
75 \\
\$ 207,375 \\
\$ 4,148 \\
\$ 2.76
\end{array}
$$

$\$ 1.21$
$\$ 247,500$

$$
\begin{array}{r}
\$ 4.74 \\
1106 \\
\$ 5,242 \\
1 \\
150 \\
\$ 786,366 \\
\$ 15,727 \\
\$ 6.30 \\
\$ 2.77
\end{array}
$$

From Sioux City, lowa

Costs per loaded-mile

Estimated mileage per load

Costs per load

Number of blades per load

Number of loads required

Total costs

Cost per turbine

Costs per kW

Costs per swept area
$\$ 6.17$

660

$\$ 4,072$

$$
\begin{array}{r}
1 \\
150 \\
\hline
\end{array}
$$

$\$ 610,830$

$\$ 12,217$

$\$ 4.89$

$\$ 2.15$

$\begin{array}{rr}\$ 9.50 & \$ 11.00 \\ 900 & 1,100 \\ \$ 8,550 & \$ 12,100 \\ 1 & 1 \\ 150 & 150 \\ \$ 1,282,500 & \$ 1,815,000 \\ \$ 25,650 & \$ 36,300 \\ \$ 7.42 & \$ 7.29 \\ \$ 3.27 & \$ 3.21\end{array}$

$\$ 6.17$

553

$\$ 3,412$

1
150

$\$ 511,802$

$\$ 10,236$

$\$ 4.10$

$\$ 1.80$

$\begin{array}{rr}\$ 9.50 & \$ 11.00 \\ 668 & 800 \\ \$ 6,346 & \$ 8,800 \\ 1 & 1 \\ 150 & 150 \\ \$ 951,900 & \$ 1,320,000 \\ \$ 19,038 & \$ 26,400 \\ \$ 5.51 & \$ 5.31 \\ \$ 2.42 & \$ 2.33\end{array}$

11.00

100

50

300

7.29

11.00

1

000

$\$ 5.31$

$\$ 2.33$

$\begin{array}{rr}\$ 9.50 & \$ 11.00 \\ 1257 & 330 \\ \$ 11,942 & \$ 3,630 \\ 1 & 1 \\ 150 & 150 \\ \$ 1,791,225 & \$ 544,500 \\ \$ 35,825 & \$ 10,890 \\ \$ 10.37 & \$ 2.19 \\ \$ 4.56 & \$ 0.96\end{array}$

$\$ 9.50$

1,402

$\$ 13,319$

$\$ 1,997,850$

$\$ 39,957$

$\$ 11.56$

$\$ 5.09$

$\$ 4.56$

$\$ 2.19$ 
Number of Turbines

Number of Hubs per turbine

Rotor Diameter (m)

Turbine Class

Swept Area (sq m)

Rated Power kW (44\% of swept area)

$\begin{array}{rr}750 & 1500 \\ 50 & 66 \\ 1963 & 3421 \\ 864 & 1505\end{array}$

$\begin{array}{rrr}\mathbf{2 5 0 0} & \mathbf{3 5 0 0} & \mathbf{5 0 0 0} \\ 85 & 100 & 120 \\ 5675 & 7854 & 11310 \\ 2497 & 3456 & 4976 \\ & & \\ & & \\ \$ 6.00 & \$ 7.50 & \$ 9.50 \\ 1,121 & 1,121 & 1,121 \\ \$ 6,726 & \$ 8,408 & \$ 10,650 \\ 1 & 1 & 1 \\ 50 & 50 & 50 \\ \$ 336,300 & \$ 420,375 & \$ 532,475 \\ \$ 6,726 & \$ 8,408 & \$ 10,650 \\ \$ 2.69 & \$ 2.43 & \$ 2.14 \\ \$ 1.19 & \$ 1.07 & \$ 0.94\end{array}$

From Port of Houston (European suppliers)

Costs per loaded-mile

$\begin{array}{rr}\$ 1.75 & \$ 3.00 \\ 1,121 & 1,121 \\ \$ 1,962 & \$ 3,363 \\ 1 & 1 \\ 50 & 50 \\ \$ 98,088 & \$ 168,150 \\ \$ 1,962 & \$ 3,363 \\ \$ 2.27 & \$ 2.23 \\ \$ 1.00 & \$ 0.98\end{array}$

Costs per load

Number of hubs per load

Number of loads required

\author{
Total costs \\ Cost per turbine \\ Costs per kW \\ Costs per swept area
}

From Port of Duluth, MN (European suppliers)

Costs per loaded-mile

Estimated mileage per load

Costs per load

Number of hubs per load

Number of loads required

\section{Total costs \\ Cost per turbine \\ Costs per kW \\ Costs per swept area}

From Chicago, IL

Costs per loaded-mile

Estimated mileage per load

Costs per load

Number of hubs per load

Number of loads required

Total costs
Cost per turbine
Costs per kW
Costs per swept area

From Tehachapi, CA

Costs per loaded-mile

Estimated mileage per load

Costs per load

Number of hubs per load

Number of loads required

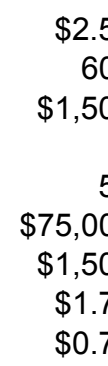

2.50
600

$\$ 1,500$

50

$\$ 75,000$

$\$ 1,500$

$\$ 1.74$

$\$ 0.76$

$\$ 2.50$
758

$\$ 1,895$

1
50

$\$ 94,750$

$\$ 1,895$

$\$ 2.19$

$\$ 0.97$

$\$ 1.75$

1470

$\$ 2,573$

Total costs

Cost per turbine

Costs per kW

Costs per swept area
1
50

$\$ 128,625$

$\$ 2,573$

$\$ 2.98$

$\$ 1.31$

$\$ 5.00$
600
$\$ 3,000$
1
50
$\$ 150,000$
$\$ 3,000$
$\$ 1.99$
$\$ 0.88$

$\$ 5.00$

758

$\$ 3,790$

1

$\$ 189,500$

$\$ 3,790$

$\$ 2.52$

$\$ 1.11$

$\$ 6.00$
600
$\$ 3,600$
1
50
$\$ 180,000$
$\$ 3,600$
$\$ 1.44$
$\$ 0.63$

$\$ 7.50$

600

$\$ 4,500$

1
50

$\$ 225,000$

$\$ 4,500$

$\$ 1.30$

$\$ 0.57$

$\$ 6.00$

758

$\$ 4,548$

1
50

$\$ 227,400$

$\$ 4,548$

$\$ 1.82$

$\$ 0.80$

$\$ 3.00$

1470

$\$ 4,410$

$$
\begin{array}{r}
1 \\
50
\end{array}
$$

$\$ 220,500$

$\$ 4,410$

$\$ 2.93$

$\$ 1.29$

$\$ 6.00$
1470
$\$ 8,820$
1
50
$\$ 441,000$
$\$ 8,820$
$\$ 3.53$
$\$ 1.55$

$\$ 6.00$

50

, 000
8,820

$\$ 3.53$

$\$ 1.55$

$\begin{array}{rr}\$ 7.50 & \$ 9.50 \\ 758 & 758 \\ \$ 5,685 & \$ 7,201 \\ 1 & 1 \\ 50 & 50 \\ \$ 284,250 & \$ 360,050 \\ \$ 5,685 & \$ 7,201 \\ \$ 1.65 & \$ 1.45 \\ \$ 0.72 & \$ 0.64\end{array}$

$\begin{array}{rr}\$ 7.50 & \$ 9.50 \\ 1470 & 1470 \\ \$ 11,025 & \$ 13,965 \\ 1 & 1 \\ 50 & 50 \\ \$ 551,250 & \$ 698,250 \\ \$ 11,025 & \$ 13,965 \\ \$ 3.19 & \$ 2.81 \\ \$ 1.40 & \$ 1.23\end{array}$


Number of nacelles

Number of nacelles per turbine

Total number of nacelles required 50

Rotor Diameter $(\mathrm{m})$

Turbine Class

$\begin{array}{rr}\mathbf{7 5 0} & \mathbf{1 5 0 0} \\ 50 & 66 \\ 1963 & 3421 \\ 864 & 1505\end{array}$

2500

85

3500

5000

Swept Area (sq m)

Rated Power kW (44\% of swept area)

5675

100

120

$7854 \quad 11310$

$2497 \quad 3456 \quad 4976$

\section{From Port of Houston (European suppliers)}

Costs per loaded-mile

Estimated mileage per load

Costs per load

Number of nacelles per load

Number of loads required

uropean suppliers)

$\$ 4.00$
1,12
$\$ 4,48$
50

$\$ 4.00$

1,121

Total costs

Cost per turbine

Costs per kW

$\$ 224,200$

$\$ 4,484$

$\$ 5.19$

Costs per swept area

$\$ 2.28$

$\$ 7.50$
1,121
$\$ 8,408$
1
50
$\$ 420,375$
$\$ 8,408$
$\$ 5.59$
$\$ 2.46$

$\$ 11.00$

1,121

$\$ 12,331$

1

50

420,375

8,408

$\$ 2.46$

$\$ 616,550$

$\$ 12,331$

$\$ 4.94$

$\$ 2.17$

From Port of Duluth, MN (European suppliers)

Costs per loaded-mile

Estimated mileage per load

Costs per load

Number of nacelles per load

Number of loads required

Total costs
Cost per turbine
Costs per kW

Costs per swept area

From Chicago, IL

Costs per loaded-mile

Estimated mileage per load

Costs per load

Number of nacelles per load

Number of loads required

Total costs

Cost per turbine

Costs per kW

Costs per swept area

From Tehachapi, CA

Costs per loaded-mile

Estimated mileage per load

Costs per load

Number of nacelles per load

Number of loads required

Total costs

Cost per turbine

Costs per kW

Costs per swept area
$\$ 5.50$

600

$\$ 3,300$

1
50
$\$ 165,000$
$\$ 3,300$
$\$ 3.82$
$\$ 1.68$

$\$ 7.00$

600

$\$ 4,200$

1
50

$\$ 210,000$

$\$ 4,200$

$\$ 2.79$

$\$ 1.23$

$\$ 6.00$

758

$\$ 4,548$

50

$\$ 227,400$

$\$ 4,548$

$\$ 5.26$

$\$ 2.32$

$\$ 8.00$

758

$\$ 6,064$

1

$\$ 303,200$

$\$ 6,064$

$\$ 4.03$

$\$ 1.77$

$\$ 4.00$

1470

$\$ 5,880$

$\$ 7.50$

1470

$\$ 11,025$

50

$\$ 294,000$

$\$ 5,880$

$\$ 6.81$

$\$ 2.99$

$\$ 551,250$

50

$\$ 11,025$

$\$ 7.32$

$\$ 3.22$
$\$ 11.00$
1470
$\$ 16,170$
1

$\$ 11.00$
1470
$\$ 16,170$
1

$\$ 11.00$
1470
$\$ 16,170$
1

$\$ 11.00$

600

$\$ 6,600$

$\$ 330,000$

$\$ 6,600$

$\$ 2.64$

$\$ 1.16$

$\$ 11.00$

758

$\$ 8,338$

$\$ 416,900$

$\$ 8,338$

$\$ 3.34$

$\$ 1.47$

$\$ 808,500$

$\$ 16,170$

$\$ 6.48$

$\$ 2.85$ 
Number of turbines

umber of nacelles per turbine

50

Total number of nacelles required

Turbine Class

Rotor Diameter $(\mathrm{m})$

Swept Area (sq m)

Rated Power kW ( $44 \%$ of swept area)

Mass (kg)

From Port of Houston (European suppliers)

\section{Costs per loaded-mile}

Costs per load

Number of units per load

Number of loads required

Total costs
Cost per turbine
Costs per kW
Costs per swept area

Costs per swept area

From Port of Duluth, MN (European suppliers)

Costs per loaded-mile

Estimated mileage per load

Costs per load

Number of units per load

Number of loads required

Total costs Cost per turbine Costs per swept area

\section{From Chicago, IL}

osts per loaded-mile

Estimated mileage per load

Costs per load

Number of nacelles per load

Number of loads required

Total costs Cost per turbine Costs per kW Costs per swept area

From Tehachapi, CA

Costs per loaded-mile

Estimated mileage per load

Costs per load

Number of nacelles per load

Total costs
Cost per turbine
Costs per kW

$\$ 2.00 \quad \$ 2.00 \quad \$ 11.00$

$600 \quad 600$

$\$ 1,200$

25

$\$ 1,200$

50

$\$ 600 \quad \$ 1,200$

$\$ 0.24 \quad \$ 0.48$

$\$ 0.48$
$\$ 0.21$

\section{$\$ 2.00 \quad \$ 2.00$}

$758 \quad 758$

$\$ 1,516$

50

$\$ 75,800$

$\$ 1,516$

$\$ 1,516$
1

50

$\$ 75,800$
$\$ 1,516$

50
$\$ 416,900$
$\$ 8,338$

$\begin{array}{lll}\$ 0.61 & \$ 0.61 & \$ 3.34 \\ \$ 0.27 & \$ 0.27 & \$ 1.47\end{array}$

$\$ 1.50$

$\$ 2,205$

50

Costs per kW

Costs per swept area
$\$ 11.00$

$\$ 2,205 \$ 16,170$

50

$\$ 110,250$

$\$ 2,205$

$\$ 2,205$
$\$ 0.88$
1
50

$\$ 808,500$

$\$ 16,170$

$\$ 6.48$
$\$ 2.85$

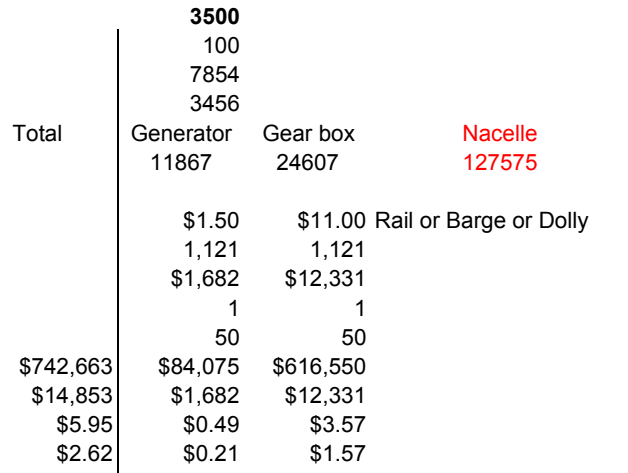

$\mid$\begin{tabular}{l|l}
$\mathbf{5 0 0 0}$ \\
120 \\
11310 \\
4976
\end{tabular}

16817

Nacelle

Total

$\$ 1.50 \$ 11.00$ Rail or Barge or Dolly

1,121
$\$ 1,682$

$\$ 12,331$

$\begin{array}{rr}1 & 1 \\ 50 & 50 \\ \$ 84,075 & \$ 616,550\end{array}$

$\$ 1,682 \quad \$ 12,331$

\begin{tabular}{r|r}
$\$ 14,013$ & $\$ 4.05$
\end{tabular}$\quad \$ 0.34 \quad \$ 2.48$

$\$ 1.78$

$\$ 0.15$

$\$ 1.09$

$\$ 14,013$
$\$ 2.82$
$\$ 1,24$

$\$ 1.24$

$\begin{array}{rr}\$ 2.00 & \$ 11.00 \\ 600 & 600\end{array}$

$\$ 2.00 \quad \$ 11.00$

$\$ 1,200 \quad \$ 6,600$

$\begin{array}{rr}1 & 1 \\ 50 & 50\end{array}$

$\$ 420,000 \quad \$ 60,000 \quad \$ 330,000$

$\$ 8,400 \quad \$ 1,200 \quad \$ 6,600$

$\$ 3.36 \quad \$ 0.35 \quad \$ 1.91$

$\$ 1.48 \quad \$ 0.15 \quad \$ 0.84$

$\begin{array}{rr}\$ 2.00 & \$ 11.00 \\ 758 & 758\end{array}$

$\begin{array}{rr}\$ 1,516 & \$ 8,338 \\ 1 & 1 \\ 50 & 50\end{array}$

\begin{tabular}{r|rr}
$\$ 568,500$ & $\$ 75,800$ & $\$ 416,900$ \\
$\$ 11,370$ & $\$ 1,516$ & $\$ 8,338$
\end{tabular}

$\$ 4.55 \quad \$ 0.44 \quad \$ 2.41$

$\$ 2.00 \quad \$ 0.19 \quad \$ 1.06$

$\begin{array}{rr}\$ 1.50 & \$ 11.00 \\ 1470 & 1470\end{array}$

$\begin{array}{rr}1470 & 1470 \\ 2,205 & \$ 16,170\end{array}$

$\begin{array}{rr}1 & 1 \\ 50 & 50\end{array}$

$\$ 1,029,000 \quad \$ 110,250 \quad \$ 808,500$

$\$ 20,580 \quad \$ 2,205 \quad \$ 16,170$

\begin{tabular}{l|ll}
$\$ 8.24$ & $\$ 0.64$ & $\$ 4.68$ \\
$\$ 3.63$ & $\$ 0.28$ & $\$ 2.06$
\end{tabular}
$\$ 1,200 \quad \$ 6,600$

$\begin{array}{rrr}1 & 1 \\ 50 & 50 \\ \$ 390,000 & \$ 60,000 & \$ 330,000\end{array}$

$\$ 7,800$
$\$ 390,000$

\begin{tabular}{l|ll}
$\$ 2.26$ & $\$ 0.24$ & $\$ 1.33$ \\
$\$ 0.99$ & $\$ 0.11$ & $\$ 0.58$
\end{tabular}

$\$ 390,000$

$\$ 7,800$

$\$ 1.57$

$\$ 11.00$

$\begin{array}{rr}\$ 2.00 & \$ 11.00 \\ 758 & 758\end{array}$

$\begin{array}{rr}758 & 758 \\ \$ 1,516 & \$ 8,338\end{array}$

$\$ 492,700$

50

$\$ 75,800$

1
50

$\$ 416,900$

$\$ 8,338$

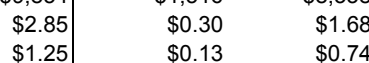

$\$ 492,700$

$\$ 9,854$

$\$ 1.98$
$\$ 0.87$

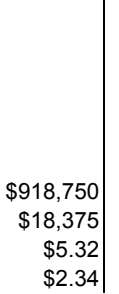

$\$ 1.50 \quad \$ 11.00$

$\begin{array}{rr}1 & 1 \\ 50 & 50\end{array}$

$\$ 808,500$

$\$ 0.44 \quad \$ 16,170$

$\$ 0.19 \$ 1.43$
$\$ 7,800 \quad \$ 1,200 \quad \$ 6,600$

$\$ 2,205 \$ \$ 16,170$ 
Number of nacelles

Number of nacelles per turbine

Total number of blades required

\section{Turbine Class}

Rotor Diameter (m)

Swept Area (sq m)

Rated Power kW (44\% of swept area)

From Canutillo, TX (EI Paso)

Costs per loaded-mile

Estimated mileage per load

Costs per load

Number of Towers per load

Number of loads required

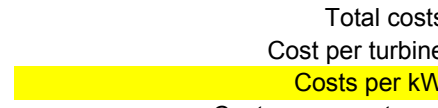
Costs per kW Costs per swept area

\section{From Dallas, TX}

Costs per loaded-mile

Estimated mileage per load

Costs per load

Number of Towers per load

Number of loads required

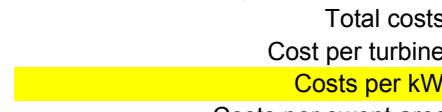

Costs per swept are

\section{From Shreveport, LA}

Estimated mileage per load

Costs per load

Number of Towers per load

Number of loads required

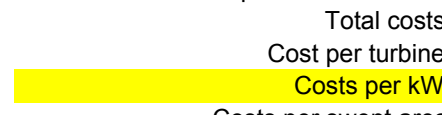

1963

$\$ 10.00 \quad \$ 1.45 \quad \$ 1.40$

$1,111 \quad 1,111 \quad 1,111$

$\$ 11,110 \quad \$ 1,611 \quad \$ 1,555$

$$
\begin{array}{r}
1 \\
50
\end{array}
$$

$\$ 555,500$

$\$ 11,110$

$\$ 12.86$

$\begin{array}{rr}1 & 1 \\ 50 & 50\end{array}$

$\begin{array}{rr}50 & 50 \\ \$ 80,548 & \$ 77,770\end{array}$

$\$ 1,611 \quad \$ 1,555$

$\$ 1.86 \quad \$ 1.80$

$\$ 1.80$
$\$ 0.79$

Section 1 Section 2 Section 3

$\$ 10.00 \quad \$ 1.45$

1,120

$\$ 11,200$

1,120

$\$ 1.40$
1.120

$\$ 1.40$
1,120
$\$ 1,568$

1
50
60,000

$\$ 560,000$

$\$ 11,200$

$\$ 12.96$

50

$\$ 81,200$

$\$ 1,624$

$\$ 1.88$

50

$\$ 78,400$
$\$ 1,568$

$\$ 1,568$
$\$ 1.81$

$\$ 1.81$
$\$ 0.80$

Section 1 Section 2 Section 3

$\$ 10.00 \quad \$ 1.45$

$1,068 \quad 1,068$

1,068
$\$ 1,549$

$\$ 1.40$
1,068
$\$ 1,495$

10,680

50

$\$ 534,000$

$\$ 10,680$

$\$ 12.36$

$50 \quad 50$

$\$ 77,430$

$\$ 77,430 \$ 74,760$

$\$ 1,549 \quad \$ 1,495$

$\begin{array}{ll}\$ 1.79 & \$ 1.73 \\ \$ 0.79 & \$ 0.76\end{array}$

$\$ 5.44$

$\$ 0.76-\$ 15.89$

1500
66

3421

1505

Section $1 \quad$ Section $2 \quad$ Section $3 \quad$ Section 4

$\begin{array}{ll}\$ 11.00 & \$ 9.50 \\ 1111 & 111\end{array}$

$\$ 6.00$

$\$ 37.50$

$\$ 12,221$

$\$ 12,221 \quad \$ 10,555$

$\$ 6,666$

$\$ 41,663$

$\begin{array}{rrrr}1 & 1 & 1 & 1\end{array}$

50

$\$ 713,818 \quad \$ 611,050 \quad \$ 611,050 \quad \$ 527,725 \quad \$ 333,300 \quad \$ 2,083,125$

$\$ 14,276 \quad \$ 12,221 \quad \$ 12,221 \quad \$ 10,555 \quad \$ 6,666 \quad \$ 41,663$

\begin{tabular}{r|rrrrr}
$\$ 16.52$ & $\$ 8.12$ & $\$ 8.12$ & $\$ 7.01$ & $\$ 4.43$ & $\$ 27.68$ \\
$\$ 7.27$ & $\$ 3.57$ & $\$ 3.57$ & $\$ 3.09$ & $\$ 1.95$ & $\$ 12.18$
\end{tabular}

Total

Section

Section 2 sectio

Section 4
$\quad \$ 6.00$

Total

1,120

$\$ 11.00 \quad \$ 11.00$

$\$ 9.50$

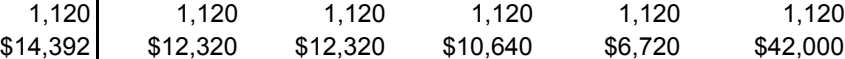

$\begin{array}{lll}1 & 1 & 1\end{array}$

50

$\$ 719,600$

$\$ 14,392$

50
$\$ 616,000$

50

50

50

$\begin{array}{lllll}\$ 616,000 & \$ 532,000 & \$ 336,000 & \$ 2,100,000\end{array}$

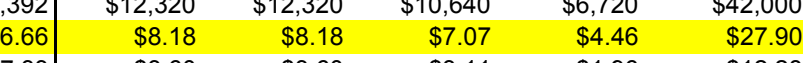

$\$ 3.60$

$\$ 3.60$

$\$ 3.11$

$\$ 1.96$

$\$ 12.28$

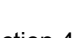

Total

\begin{tabular}{r|rrrrr}
$\$ 12.85$ & $\$ 11.00$ & $\$ 11.00$ & $\$ 9.50$ & $\$ 6.00$ & $\$ 37.50$ \\
1.068 & 1.068 & 1,068 & 1,068 & 1,068 & 1,068
\end{tabular}

$\begin{array}{rrrrrr}1,068 & 1,068 & 1,068 & 1,068 & 1,068 & 1,068\end{array}$

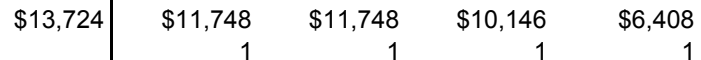

$\$ 40,050$

\begin{tabular}{r|rrrr}
50 & 50 & 50 & 50 \\
$\$ 686,190$ & $\$ 587,400$ & $\$ 587,400$ & $\$ 507,300$ & $\$ 320,400$
\end{tabular}

$\$ 13,724$

$\$ 587,400 \quad \$ 587,400 \quad \$ 507,300$

$2,002,500$

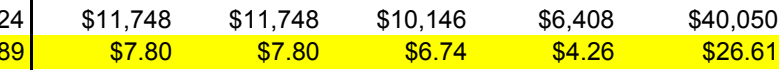

\begin{tabular}{l|lllll|}
5.89 & $\$ 7.80$ & $\$ 7.80$ & $\$ 6.74$ & $\$ 4.26$ & $\$ 26.61$ \\
6.99 & $\$ 3.43$ & $\$ 3.43$ & $\$ 2.97$ & $\$ 1.87$ & $\$ 11.71$
\end{tabular}




\begin{tabular}{|c|c|c|c|c|c|c|}
\hline \multirow{4}{*}{$\begin{array}{l}\text { Rotor Diameter }(\mathrm{m}) \quad \text { Turbine Class| } \\
\text { Swept Area }(\mathrm{sq} \mathrm{m}) \\
\text { Rated Power kW (44\% of swept area) }\end{array}$} & \multicolumn{6}{|c|}{2500} \\
\hline & 85 & & & & & \\
\hline & 5675 & & & & & \\
\hline & 2497 & & & & & \\
\hline From Canutillo, TX (EI Paso) & Section 1 & Section 2 & Section 3 & Section 4 & Section 5 & Total \\
\hline Costs per loaded-mile & $\$ 8.00$ & $\$ 6.00$ & $\$ 1.50$ & $\$ 11.00$ & $\$ 9.50$ & $\$ 36.00$ \\
\hline Estimated mileage per load & 1,111 & 1,111 & 1,111 & 1,111 & 1,111 & 1,111 \\
\hline Costs per load & $\$ 8,888$ & $\$ 6,666$ & $\$ 1,667$ & $\$ 12,221$ & $\$ 10,555$ & $\$ 39,996$ \\
\hline Number of Towers per load & 0.25 & 0.25 & 0.25 & 1 & 1 & \\
\hline Number of loads required & 200 & 200 & 200 & 50 & 50 & \\
\hline Total costs & $\$ 1,777,600$ & $\$ 1,333,200$ & $\$ 333,300$ & $\$ 611,050$ & $\$ 527,725$ & $\$ 4,582,875$ \\
\hline Cost per turbine & $\$ 35,552$ & $\$ 26,664$ & $\$ 6,666$ & $\$ 12,221$ & $\$ 10,555$ & $\$ 91,658$ \\
\hline Costs per kW & $\$ 14.24$ & $\$ 10.68$ & $\$ 2.67$ & $\$ 4.89$ & $\$ 4.23$ & $\$ 36.71$ \\
\hline Costs per swept area & $\$ 6.27$ & $\$ 4.70$ & $\$ 1.17$ & $\$ 2.15$ & $\$ 1.86$ & $\$ 16.15$ \\
\hline From Dallas, TX & Section 1 & Section 2 & Section 3 & Section 4 & Section 5 & Total \\
\hline$\overline{\text { Costs per loaded-mile }}$ & $\$ 8.00$ & $\$ 6.00$ & $\$ 1.50$ & $\$ 11.00$ & $\$ 9.50$ & $\$ 36.00$ \\
\hline Estimated mileage per load & 1,120 & 1,120 & 1,120 & 1,120 & 1,120 & 1,120 \\
\hline Costs per load & $\$ 8,960$ & $\$ 6,720$ & $\$ 1,680$ & $\$ 12,320$ & $\$ 10,640$ & $\$ 40,320$ \\
\hline Number of Towers per load & 0.25 & 0.25 & 0.25 & 1 & 1 & \\
\hline Number of loads required & 200 & 200 & 200 & 50 & 50 & \\
\hline Total costs & $\$ 1,792,000$ & $\$ 1,344,000$ & $\$ 336,000$ & $\$ 616,000$ & $\$ 532,000$ & $\$ 4,620,000$ \\
\hline Cost per turbine & $\$ 35,840$ & $\$ 26,880$ & $\$ 6,720$ & $\$ 12,320$ & $\$ 10,640$ & $\$ 92,400$ \\
\hline Costs per kW & $\$ 14.35$ & $\$ 10.77$ & $\$ 2.69$ & $\$ 4.93$ & $\$ 4.26$ & $\$ 37.01$ \\
\hline Costs per swept area & $\$ 6.32$ & $\$ 4.74$ & $\$ 1.18$ & $\$ 2.17$ & $\$ 1.88$ & $\$ 16.28$ \\
\hline From Shreveport, LA & Section 1 & Section 2 & Section 3 & Section 4 & Section 5 & Total \\
\hline Costs per loaded-mile & $\$ 8.00$ & $\$ 6.00$ & $\$ 1.50$ & $\$ 11.00$ & $\$ 9.50$ & $\$ 36.00$ \\
\hline Estimated mileage per load & 1,068 & 1,068 & 1,068 & 1,068 & 1,068 & 1,068 \\
\hline Costs per load & $\$ 8,544$ & $\$ 6,408$ & $\$ 1,602$ & $\$ 11,748$ & $\$ 10,146$ & $\$ 38,448$ \\
\hline Number of Towers per load & 0.25 & 0.25 & 0.25 & 1 & 1 & \\
\hline Number of loads required & 200 & 200 & 200 & 50 & 50 & \\
\hline Total costs & $\$ 1,708,800$ & $\$ 1,281,600$ & $\$ 320,400$ & $\$ 587,400$ & $\$ 507,300$ & $\$ 4,405,500$ \\
\hline Cost per turbine & $\$ 34,176$ & $\$ 25,632$ & $\$ 6,408$ & $\$ 11,748$ & $\$ 10,146$ & $\$ 88,110$ \\
\hline Costs per kW & $\$ 13.69$ & $\$ 10.27$ & $\$ 2.57$ & $\$ 4.71$ & $\$ 4.06$ & $\$ 35.29$ \\
\hline Costs per swept area & $\$ 6.02$ & $\$ 4.52$ & $\$ 1.13$ & $\$ 2.07$ & $\$ 1.79$ & $\$ 15.53$ \\
\hline
\end{tabular}


Total number of blades required

\begin{tabular}{|c|c|c|c|c|c|c|c|}
\hline Turbine Class & & & & 3500 & & & \\
\hline Rotor Diameter $(\mathrm{m})$ & 100 & & & & & & \\
\hline Swept Area (sq m) & 7854 & & & & & & \\
\hline Rated Power kW ( $44 \%$ of swept area) & 3456 & & & & & & \\
\hline From Canutillo, TX (El Paso) & Section 1 & Section 2 & Section 3 & Section 4 & Section 5 & Section 6 & Total \\
\hline Costs per loaded-mile & $\$ 11.00$ & $\$ 9.50$ & $\$ 8.00$ & $\$ 6.00$ & $\$ 1.45$ & $\$ 11.00$ & $\$ 46.95$ \\
\hline Estimated mileage per load & 1,111 & 1,111 & 1,111 & 1,111 & 1,111 & 1,111 & 1,111 \\
\hline Costs per load & $\$ 12,221$ & $\$ 10,555$ & $\$ 8,888$ & $\$ 6,666$ & $\$ 1,611$ & $\$ 12,221$ & $\$ 52,161$ \\
\hline Number of Towers per load & 0.25 & 0.25 & 0.25 & 0.25 & 0.25 & 1 & \\
\hline Number of loads required & 200 & 200 & 200 & 200 & 200 & 50 & \\
\hline Total costs & $\$ 2,444,200$ & $\$ 2,110,900$ & $\$ 1,777,600$ & $\$ 1,333,200$ & $\$ 322,190$ & $\$ 611,050$ & $\$ 8,599,140$ \\
\hline Cost per turbine & $\$ 48,884$ & $\$ 42,218$ & $\$ 35,552$ & $\$ 26,664$ & $\$ 6,444$ & $\$ 12,221$ & $\$ 171,983$ \\
\hline Costs per kW & $\$ 14.15$ & $\$ 12.22$ & $\$ 10.29$ & $\$ 7.72$ & $\$ 1.86$ & $\$ 3.54$ & $\$ 49.77$ \\
\hline Costs per swept area & $\$ 6.22$ & $\$ 5.38$ & $\$ 4.53$ & $\$ 3.39$ & $\$ 0.82$ & $\$ 1.56$ & $\$ 21.90$ \\
\hline From Dallas, TX & Section 1 & Section 2 & Section 3 & Section 4 & Section 5 & Section 6 & Total \\
\hline Costs per loaded-mile & $\$ 11.00$ & $\$ 9.50$ & $\$ 8.00$ & $\$ 6.00$ & $\$ 1.45$ & $\$ 11.00$ & $\$ 46.95$ \\
\hline Estimated mileage per load & 1,120 & 1,120 & 1,120 & 1,120 & 1,120 & 1,120 & 1,120 \\
\hline Costs per load & $\$ 12,320$ & $\$ 10,640$ & $\$ 8,960$ & $\$ 6,720$ & $\$ 1,624$ & $\$ 12,320$ & $\$ 52,584$ \\
\hline Number of Towers per load & 0.25 & 0.25 & 0.25 & 0.25 & 0.25 & 1 & \\
\hline Number of loads required & 200 & 200 & 200 & 200 & 200 & 50 & \\
\hline Total costs & $\$ 2,464,000$ & $\$ 2,128,000$ & $\$ 1,792,000$ & $\$ 1,344,000$ & $\$ 324,800$ & $\$ 616,000$ & $\$ 8,668,800$ \\
\hline Cost per tu & $\$ 49,280$ & $\$ 42,560$ & $\$ 35,840$ & $\$ 26,880$ & $\$ 6,496$ & $\$ 12,320$ & $\$ 173,376$ \\
\hline Costs per kW & $\$ 14.26$ & $\$ 12.32$ & $\$ 10.37$ & $\$ 7.78$ & $\$ 1.88$ & $\$ 3.57$ & $\$ 50.17$ \\
\hline Costs per swept area & $\$ 6.27$ & $\$ 5.42$ & $\$ 4.56$ & $\$ 3.42$ & $\$ 0.83$ & $\$ 1.57$ & $\$ 22.07$ \\
\hline From Shreveport, LA & Section 1 & Section 2 & Section 3 & Section 4 & Section 5 & Section 6 & Total \\
\hline Costs per loaded-mile & $\$ 11.00$ & $\$ 9.50$ & $\$ 8.00$ & $\$ 6.00$ & $\$ 1.45$ & $\$ 11.00$ & $\$ 46.95$ \\
\hline Estimated mileage per load & 1,068 & 1,068 & 1,068 & 1,068 & 1,068 & 1,068 & 1,068 \\
\hline Costs per load & $\$ 11,748$ & $\$ 10,146$ & $\$ 8,544$ & $\$ 6,408$ & $\$ 1,549$ & $\$ 11,748$ & $\$ 50,143$ \\
\hline Number of Towers per load & 0.25 & 0.25 & 0.25 & 0.25 & 0.25 & 1 & \\
\hline Number of loads required & 200 & 200 & 200 & 200 & 200 & 50 & \\
\hline Total & $\$ 2,349,600$ & $\$ 2,029,200$ & $\$ 1,708,800$ & $\$ 1,281,600$ & $\$ 309,720$ & $\$ 587,400$ & $\$ 8,266,320$ \\
\hline Cost per turbine & $\$ 46,992$ & $\$ 40,584$ & $\$ 34,176$ & $\$ 25,632$ & $\$ 6,194$ & $\$ 11,748$ & $\$ 165,326$ \\
\hline Costs per kW & $\$ 13.60$ & $\$ 11.74$ & $\$ 9.89$ & $\$ 7.42$ & $\$ 1.79$ & $\$ 3.40$ & $\$ 47.84$ \\
\hline Costs per swept area & $\$ 5.98$ & $\$ 5.17$ & $\$ 4.35$ & $\$ 3.26$ & $\$ 0.79$ & $\$ 1.50$ & $\$ 21.05$ \\
\hline
\end{tabular}


Number of nacelles

Number of nacelles per turbine

Total number of blades required

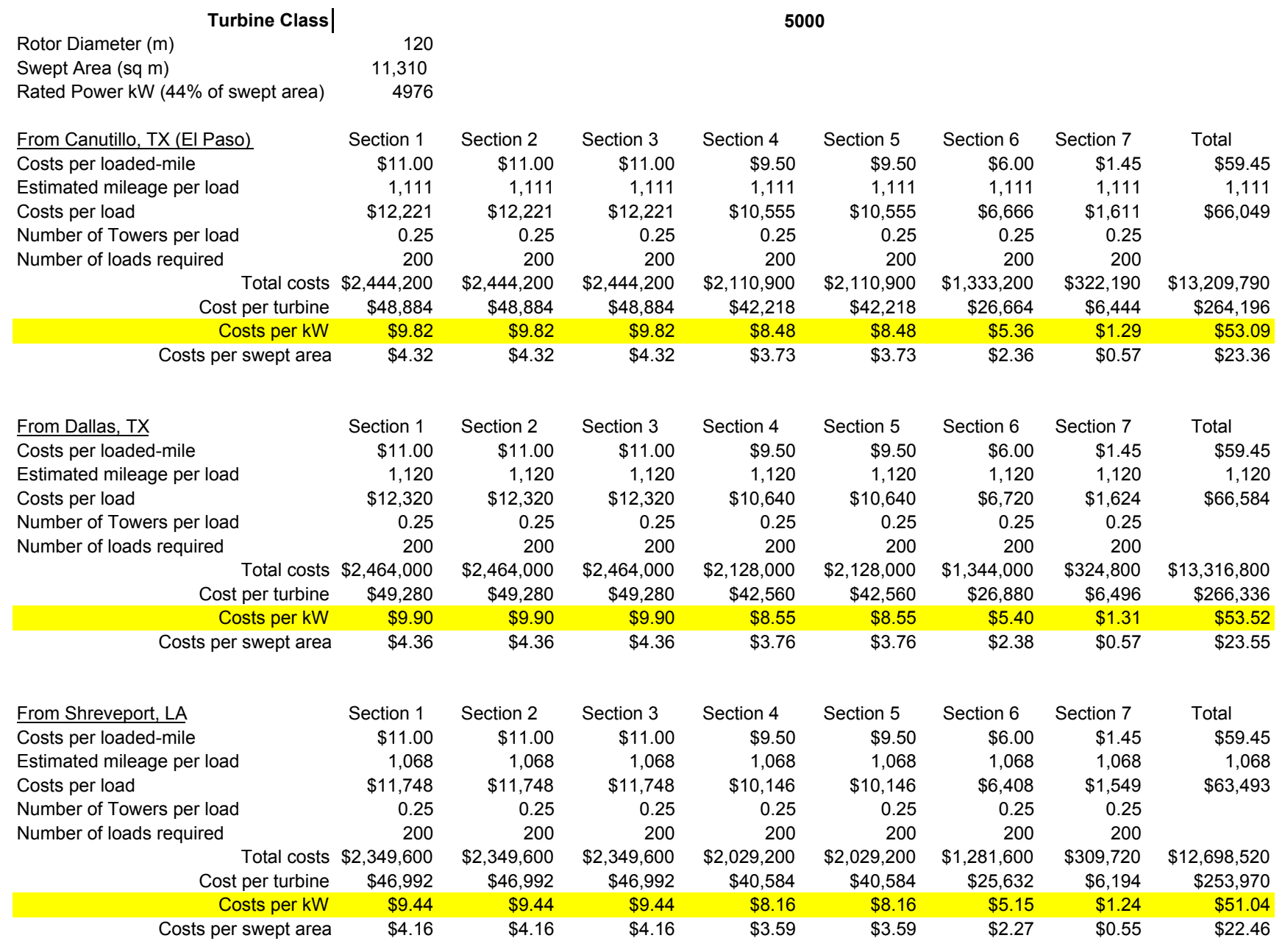


Appendix E

Dolly Transport 
Number of turbines $\quad 50$

Number of blades per turbine 3

Total number of blades required $\quad 150$

Rotor Diameter $(\mathrm{m})$

Turbine Class

750

1500

2500

3500

5000

50
1963

66

85

100

120

Swept Area (sq m)

864

3421

5675

11310

Rated Power kW (44\% of swept area)

1505

2497

7854

3456

4976

From Port of Houston (European suppliers)

Costs per loaded-mile

Estimated mileage per load

Costs per load

Number of blades per load

Number of loads required

Total costs

Cost per turbine

Costs per kW

Costs per swept area

$\$ 0.00$

1,402

From Gainesville, TX (Molded Fibre Glass, Inc.)

To Port of Houston

Costs per loaded-mile

$\$ 0.00$

1257

Estimated mileage per load

Costs per load

Number of blades per load

Number of loads required

Total costs

Cost per turbine

Costs per kW

$\$ 0.00$

Costs per swept area

$\$ 0.00$

From Port of Duluth, MN (European suppliers)

Costs per loaded-mile

$\$ 11.00$

1,100

Estimated mileage per load

Costs per load

Number of blades per load

Number of loads required

Total costs

Cost per turbine

Costs per kW

Costs per swept area

$\$ 12,100$

150

$\$ 1,815,000$

$\$ 36,300$

$\$ 7.29$

$\$ 3.21$

From Grand Forks, ND (L-M Glasfiber)

Costs per loaded-mile

$\$ 11.00$

800

Estimated mileage per load

$\$ 8,800$

Number of blades per load

Number of loads required

Total costs

150

$\$ 1,320,000$

Cost per turbine

Costs per kW

$\$ 26,400$

$\$ 5.31$

Costs per swept area

$\$ 2.33$ 
Number of nacelles

Number of nacelles per turbine

Turbine Class

Rotor Diameter (m)

2500

85

5675

2497

Rated Power kW (44\% of swept area)

From Sioux City, lowa (European suppliers)

Costs per loaded-mile

Estimated mileage per load

Costs per load

Utility, DOT, Police Assistance

Number of nacelles per load

Number of loads required

Total costs

Cost per turbine

Costs per kW

Costs per swept area

From Port of Duluth, MN (European suppliers)

Costs per loaded-mile

Estimated mileage per load

Costs per load

Utility, DOT, Police Assistance

Number of nacelles per load

Number of loads required

Total costs

Cost per turbine

Costs per kW

Costs per swept area

From Chicago, IL

Costs per loaded-mile

Estimated mileage per load

Costs per load

Utility, DOT, Police Assistance

Number of nacelles per load

Number of loads required

Total costs

Cost per turbine

Costs per kW

Costs per swept area

From Tehachapi, CA

Costs per loaded-mile

Estimated mileage per load

Costs per load

Utility, DOT, Police Assistance

Number of nacelles per load

Number of loads required

Total costs

Cost per turbine

Costs per kW

Costs per swept area
$\$ 50.00$

250

$\$ 12,500$

$\$ 150,000$

50

$\$ 8,125,000$

$\$ 162,500$

$\$ 65.08$

$\$ 28.64$

$\$ 50.00$

600

$\$ 30,000$

$\$ 200,000$

1

$\$ 11,500,000$

$\$ 230,000$

$\$ 92.12$

$\$ 40.53$

$\$ 50.00$

758

$\$ 37,900$

$\$ 200,000$

1

$\$ 11,895,000$

$\$ 237,900$

$\$ 95.28$

$\$ 41.92$

$\$ 50.00$

1470

$\$ 73,500$

$\$ 300,000$

50

$\$ 18,675,000$

$\$ 373,500$

$\$ 149.59$

$\$ 65.82$
3500

100

7854

3456

5000

120

11310

4976

$\begin{array}{rr}\$ 75.00 & \$ 100.00 \\ 250 & 250 \\ \$ 18,750 & \$ 25,000 \\ \$ 150,000 & \$ 150,000 \\ 1 & 1 \\ 50 & 50 \\ \$ 8,437,500 & \$ 8,750,000 \\ \$ 168,750 & \$ 175,000 \\ \$ 48.83 & \$ 35.17 \\ \$ 21.49 & \$ 15.47\end{array}$

$\begin{array}{rr}\$ 75.00 & \$ 100.00 \\ 600 & 600 \\ \$ 45,000 & \$ 60,000 \\ \$ 200,000 & \$ 200,000 \\ 1 & 1 \\ 50 & 50 \\ \$ 12,250,000 & \$ 13,000,000 \\ \$ 245,000 & \$ 260,000 \\ \$ 70.90 & \$ 52.25 \\ \$ 31.19 & \$ 22.99\end{array}$

$\begin{array}{rr}\$ 75.00 & \$ 100.00 \\ 758 & 758 \\ \$ 56,850 & \$ 75,800 \\ \$ 200,000 & \$ 200,000 \\ 1 & 1 \\ 50 & 50 \\ \$ 12,842,500 & \$ 13,790,000 \\ \$ 256,850 & \$ 275,800 \\ \$ 74.33 & \$ 55.42 \\ \$ 32.70 & \$ 24.39\end{array}$

$\begin{array}{rr}\$ 75.00 & \$ 100.00 \\ 1470 & 1470 \\ \$ 110,250 & \$ 147,000 \\ \$ 300,000 & \$ 300,000 \\ 1 & 1 \\ 50 & 50 \\ \$ 20,512,500 & \$ 22,350,000 \\ \$ 410,250 & \$ 447,000 \\ \$ 118.72 & \$ 89.83 \\ \$ 52.23 & \$ 39.52\end{array}$


Number of nacelles $\quad 50$

number of nacelles per turbine 1

Total number of nacelles required 50

Turbine Class

Rotor Diameter $(\mathrm{m})$

3500

100

7854

3456

Rated Power kW (44\% of swept area)

"Empty" Nacelle Mass

From Souix City, lowa (European suppliers)

Costs per loaded-mile

Estimated mileage per load

Costs per load

Utility, DOT, Police Assistance

Number of nacelles per load

Number of loads required

Total costs

Cost per turbine

Costs per kW

Costs per swept area

From Port of Duluth, MN (Eurpoean suppliers)

Costs per loaded-mile

Estimated mileage per load

Costs per load

Utility, DOT, Police Assistance

Number of nacelles per load

Number of loads required

Total costs

Cost per turbine

Costs per kW

Costs per swept area

From Chicago, IL

Costs per loaded-mile

Estimated mileage per load

Costs per load

Utility, DOT, Police Assistance

Number of nacelles per load

Number of loads required

Total costs

Cost per turbine

Costs per kW

Costs per swept area

From Tehachapi, CA

Costs per loaded-mile

Estimated mileage per load

Costs per load

Utility, DOT, Police Assistance

Number of nacelles per load

Number of loads required

Total costs

Cost per turbine

Costs per kW

Costs per swept area
127575

$\$ 50.00$

250

$\$ 12,500$

$\$ 150,000$

1

50

$\$ 8,125,000$

$\$ 162,500$

$\$ 47.02$

$\$ 20.69$

$\$ 50.00$

600

$\$ 30,000$

$\$ 200,000$

1
50

$\$ 11,500,000$

$\$ 230,000$

$\$ 66.56$

$\$ 29.28$

$\$ 50.00$

758

$\$ 37,900$

$\$ 200,000$

1

$\$ 11,895,000$

$\$ 237,900$

$\$ 68.84$

$\$ 30.29$

$\$ 50.00$

1470

$\$ 73,500$

$\$ 300,000$

1

$\$ 18,675,000$

$\$ 373,500$

$\$ 108.08$

$\$ 47.56$
5000

120

11310

4976

199170

$\$ 75.00$

250

$\$ 18,750$

$\$ 150,000$

1

$\$ 8,437,500$

$\$ 168,750$

$\$ 33.91$

$\$ 14.92$

$\$ 75.00$

600

$\$ 45,000$

$\$ 200,000$

50

$\$ 12,250,000$

$\$ 245,000$

$\$ 49.23$

$\$ 21.66$

$\$ 75.00$

758

$\$ 56,850$

$\$ 200,000$

50

$\$ 12,842,500$

$\$ 256,850$

$\$ 51.61$

$\$ 22.71$

$\$ 75.00$

1470

$\$ 110,250$

$\$ 300,000$

1

$\$ 20,512,500$

$\$ 410,250$

$\$ 82.44$

$\$ 36.27$ 


\begin{tabular}{|c|c|c|c|c|}
\hline Turbine Class & 1500 & & 2500 & \\
\hline Rotor Diameter $(\mathrm{m})$ & & 85 & & \\
\hline Swept Area (sq m) & 3421 & 5675 & & \\
\hline Rated Power kW ( $44 \%$ of swept area) & 1505 & 2497 & & \\
\hline From Canutillo, TX (EI Paso) to Port of Houston & Section 1 & Section 1 & Section 2 & Section 3 \\
\hline Costs per loaded-mile & $\$ 50.00$ & $\$ 50.00$ & $\$ 50.00$ & $\$ 50.00$ \\
\hline Estimated mileage per load & 780 & 780 & 780 & 780 \\
\hline Costs per load & $\$ 39,000$ & $\$ 39,000$ & $\$ 39,000$ & $\$ 39,000$ \\
\hline Utility, DOT, Police Assistance & $\$ 150,000$ & $\$ 150,000$ & $\$ 150,000$ & $\$ 150,000$ \\
\hline Number of Towers per load & & & 1 & 1 \\
\hline Number of loads required & 50 & & 50 & 50 \\
\hline Total costs & $\$ 9,450,000$ & $\$ 9,450,000$ & $\$ 9,450,000$ & $\$ 9,450,000$ \\
\hline Cost per turbine & $\$ 189,000$ & $\$ 189,000$ & $\$ 189,000$ & $\$ 189,000$ \\
\hline Costs per kW & $\$ 125.55$ & $\$ 75.70$ & $\$ 75.70$ & $\$ 75.70$ \\
\hline Costs per swept area & $\$ 55.24$ & $\$ 33.31$ & $\$ 33.31$ & $\$ 33.31$ \\
\hline From Dallas, $T X$ to Port of Houston & Section 1 & Section 1 & Section 2 & Section 3 \\
\hline Costs per loaded-mile & $\$ 50.00$ & $\$ 50.00$ & $\$ 50.00$ & $\$ 50.00$ \\
\hline Estimated mileage per load & 250 & 250 & 250 & 250 \\
\hline Costs per load & $\$ 12,500$ & $\$ 12,500$ & $\$ 12,500$ & $\$ 12,500$ \\
\hline Utility, DOT, Police Assistance & $\$ 150,000$ & $\$ 150,000$ & $\$ 150,000$ & $\$ 150,000$ \\
\hline Number of Towers per load & 1 & 1 & 1 & 1 \\
\hline Number of loads required & 50 & 50 & 50 & 50 \\
\hline Total costs & $\$ 8,125,000$ & $\$ 8,125,000$ & $\$ 8,125,000$ & $\$ 8,125,000$ \\
\hline Cost per turbine & $\$ 162,500$ & $\$ 162,500$ & $\$ 162,500$ & $\$ 162,500$ \\
\hline Costs per kW & $\$ 107.95$ & $\$ 65.08$ & $\$ 65.08$ & $\$ 65.08$ \\
\hline Costs per swept area & $\$ 47.50$ & $\$ 28.64$ & $\$ 28.64$ & $\$ 28.64$ \\
\hline From $\mathrm{S}$ & Section 1 & Section 1 & Section 2 & Section 3 \\
\hline Costs per loaded-mile & $\$ 50.00$ & $\$ 50.00$ & $\$ 50.00$ & $\$ 50.00$ \\
\hline Estimated mileage per load & 240 & 240 & 240 & 240 \\
\hline Costs per load & $\$ 12,000$ & $\$ 12,000$ & $\$ 12,000$ & $\$ 12,000$ \\
\hline Utility, DOT, Police Assistance & $\$ 150,000$ & $\$ 150,000$ & $\$ 150,000$ & $\$ 150,000$ \\
\hline Number of Towers per load & 1 & 1 & 1 & 1 \\
\hline Number of loads required & 50 & 50 & 50 & 50 \\
\hline Total & $\$ 8,100,000$ & $\$ 8,100,000$ & $\$ 8,100,000$ & $\$ 8,100,000$ \\
\hline Cost per turbine & $\$ 162,000$ & $\$ 162,000$ & $\$ 162,000$ & $\$ 162,000$ \\
\hline Costs per kW & $\$ 107.62$ & $\$ 64.88$ & $\$ 64.88$ & $\$ 64.88$ \\
\hline Costs per swept area & $\$ 47.35$ & $\$ 28.55$ & $\$ 28.55$ & $\$ 28.55$ \\
\hline From Sioux City, IA to South Dakota & Section 1 & Section 1 & Section 2 & Section 3 \\
\hline Costs per loaded-mile & $\$ 50.00$ & $\$ 50.00$ & $\$ 50.00$ & $\$ 50.00$ \\
\hline Estimated mileage per load & 250 & 250 & 250 & 250 \\
\hline Costs & $\$ 12,500$ & $\$ 12,500$ & $\$ 12,500$ & $\$ 12,500$ \\
\hline $\mathrm{T}$, Police Assistance & $\$ 150,000$ & $\$ 150,000$ & $\$ 150,000$ & $\$ 150,000$ \\
\hline Number of Towers per load & 1 & 1 & 1 & 1 \\
\hline Number of loads required & 50 & 50 & 50 & 50 \\
\hline Total cos & $\$ 8,12$ & 000 & 000 & $\$ 8,125,000$ \\
\hline Cost per turbine & & $\$ 162,500$ & $\$ 162,500$ & $\$ 162,500$ \\
\hline Costs per kW & $\$ 107.95$ & $\$ 65.08$ & $\$ 65.08$ & $\$ 65.08$ \\
\hline Costs per swept area & $\$ 47.50$ & $\$ 28.64$ & $\$ 28.64$ & $\$ 28.64$ \\
\hline
\end{tabular}




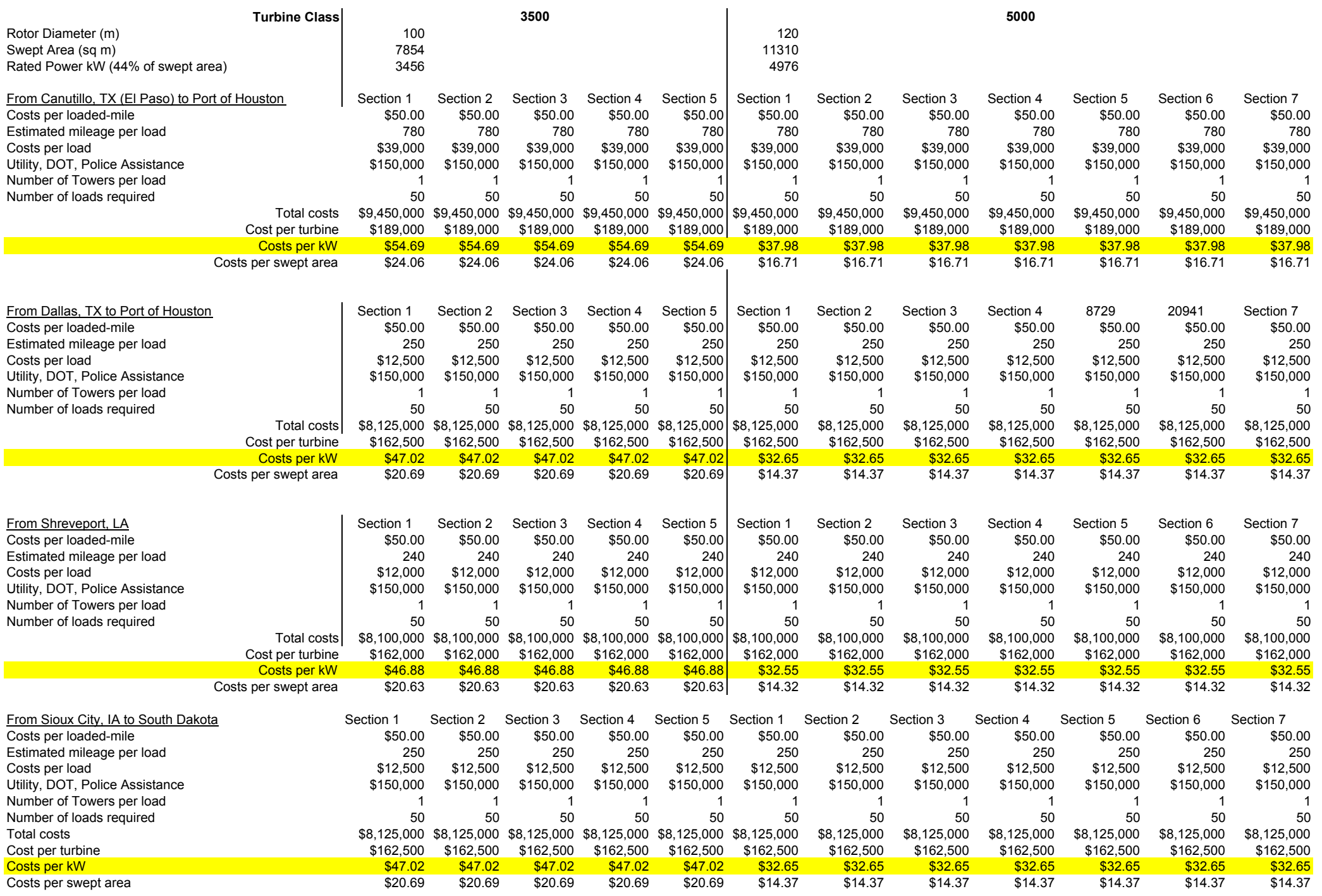


Appendix F

Rail Transport 


Rotor Diameter $(\mathrm{m})$
Swept Area (sq m)
Rated Power kW (44\% of swept area)
Nacelle Mass $(\mathrm{kg})$
From Port of Duluth, MN (European suppliers)
Costs per kg
Costs per Turbine
Rail Car Rental
Rail car use fee
50 mile Dolly Costs
Crane Rental - Offloading Misc Labor Grand total per turbine Costs per kW Costs per swept area

From Chicago, IL Costs per kg Costs per Turbine

Rail Car Rental

Rail car use fee

50 mile Dolly Costs

Total Rail Costs Crane Rental - Offloading

Misc Labor Subtotal

Grand total per turbine Costs per kW

Costs per swept area

From Port of Houston, TX

Costs per kg

Costs per Turbine

Rail Car Rental

Rail car use fee

50 mile Dolly Costs

Total Rail Costs Crane Rental - Offloading

Misc Labor Subtotal Grand total per turbine Costs per kW Costs per swept area

From Tehachapi, CA

Costs per kg

Costs per Turbine

Rail Car Rental

Rail car use fee

50 mile Dolly Costs

Total Rail Costs

Crane Rental - Offloading

Misc Labor

Subtotal

Grand total per turbine

Costs per kW

Costs per swept area

$\begin{array}{rr}\mathbf{7 5 0} & \mathbf{1 5 0 0} \\ 50 & 66 \\ 1963 & 3421 \\ 864 & 1505\end{array}$

31,081

60,517

$\$ 0.057$

$\$ 1,765$

$\$ 3,000$

$\$ 2,000$

$\$ 550$

$\$ 365,770$

$\$ 215,000$

$\$ 50,000$

$\$ 630,770$

$\$ 12,615$

$\$ 14.60$

$\$ 6.42$

$\$ 0.064$

$\$ 1,980$

$\$ 3,000$

$\$ 2,000$

$\$ 550$

$\$ 376,493$

$\$ 215,000$

$\$ 50,000$

$\$ 641,493$

$\$ 12,830$

$\$ 14.85$

$\$ 6.53$

$\$ 0.079$

$\$ 2,452$

$\$ 3,000$

$\$ 2,000$

$\$ 550$

$\$ 400,115$

$\$ 215,000$

$\$ 50,000$

$\$ 665,115$

$\$ 13,302$

$\$ 15.40$

$\$ 6.77$

$\$ 0.080$

$\$ 2,486$

$\$ 3,000$

$\$ 2,000$

$\$ 550$

$\$ 401,824$

$\$ 215,000$

$\$ 50,000$

$\$ 666,824$

$\$ 13,336$

$\$ 15.44$

$\$ 6.79$
$\$ 0.042$

$\$ 2,560$

$\$ 3,000$

$\$ 2,000$

$\$ 550$

$\$ 405,493$

$\$ 215,000$

$\$ 50,000$

$\$ 670,493$

$\$ 13,410$

$\$ 8.91$

$\$ 3.92$

$\$ 0.049$

$\$ 2,977$

$\$ 3,000$

$\$ 2,000$

$\$ 550$

$\$ 426,372$

$\$ 215,000$

$\$ 50,000$

$\$ 691,372$

$\$ 13,827$

$\$ 9.19$

$\$ 4.04$

$\$ 0.064$

$\$ 3,843$

$\$ 3,000$

$\$ 2,000$

$\$ 550$

$\$ 469,641$

$\$ 215,000$

$\$ 50,000$

$\$ 734,641$

$\$ 14,693$

$\$ 9.76$

$\$ 4.29$

$\$ 0.075$

$\$ 4,539$

$\$ 3,000$

$\$ 2,000$

$\$ 550$

$\$ 504,439$

$\$ 215,000$

$\$ 50,000$

$\$ 769,439$

$\$ 15,389$

$\$ 10.22$

$\$ 4.50$
2500

85
5675

2497

111,065

$\$ 0.039$

$\$ 4,308$

$\$ 3,000$

$\$ 2,000$

$\$ 550$

$\$ 492,911$

$\$ 215,000$

$\$ 50,000$

$\$ 757,911$

$\$ 15,158$

$\$ 6.07$

$\$ 2.67$

$\$ 0.046$

$\$ 5,065$

$\$ 3,000$

$\$ 2,000$

$\$ 550$

$\$ 530,728$

$\$ 215,000$

$\$ 50,000$

$\$ 795,728$

$\$ 15,915$

$\$ 6.37$

$\$ 2.80$

$\$ 0.060$

$\$ 6,664$

$\$ 3,000$

$\$ 2,000$

$\$ 550$

$\$ 610,695$

$\$ 215,000$

$\$ 50,000$

$\$ 875,695$

$\$ 17,514$

$\$ 7.01$

$\$ 3.09$

$\$ 0.065$

$\$ 7,219$

$\$ 3,000$

$\$ 2,000$

$\$ 550$

$\$ 638,461$

$\$ 215,000$

$\$ 50,000$

$\$ 903,461$

$\$ 18,069$

$\$ 7.24$

$\$ 3.18$

$\begin{array}{rr}\mathbf{3 5 0 0} & \mathbf{5 0 0 0} \\ 100 & 120 \\ 7854 & 11310 \\ 3456 & 4976 \\ 64,049 & 254,102\end{array}$

Too Heavy for Rail

$\$ 0.039$

$\$ 6,363$

$\$ 3,000$

$\$ 2,000$

$\$ 52,500$

$\$ 3,193,173$

$\$ 315,000$

$\$ 50,000$

$\$ 3,558,173$

$\$ 71,163$

$\$ 20.59$

$\$ 9.06$

$\$ 0.046$

$\$ 7,481$

$\$ 3,000$

$\$ 2,000$

$\$ 52,500$

$\$ 3,249,032$

$\$ 315,000$

$\$ 50,000$

$\$ 3,614,032$

$\$ 72,281$

$\$ 20.92$

$\$ 9.20$

$\$ 0.060$

$\$ 9,843$

$\$ 3,000$

$\$ 2,000$

$\$ 52,500$

$\$ 3,367,147$

$\$ 315,000$

$\$ 50,000$

$\$ 3,732,147$

$\$ 74,643$

$\$ 21.60$

$\$ 9.50$

$\$ 0.065$

$\$ 10,663$

$\$ 3,000$

$\$ 2,000$

$\$ 52,500$

$\$ 3,408,159$

$\$ 315,000$

$\$ 50,000$

$\$ 3,773,159$

$\$ 75,463$

$\$ 21.84$ 


Rotor Diameter $(\mathrm{m})$
Swept Area (sq m)
Rated Power kW (44\% of swept area)
"Empty" Nacelle Mass (kg)
From Port of Duluth, MN (European suppliers)
Costs per kg
Costs per Turbine
Rail Car Rental
Rail car use fee
50 mile Dolly Costs
Misc Labor Grand total per turbine Costs per kW Costs per swept area

From Chicago, IL Costs per kg Costs per Turbine Rail Car Rental

Rail car use fee 50 mile Dolly Costs Crane Rental - Offloading

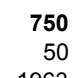

1963

864

23,311$$
\$ 0
$$$$
\$ 343,703
$$

$\$ 343,703$

$\$ 215,000$

$\$ 50,000$

$\$ 608,703$

$\$ 12,174$

$\$ 14.09$

$\$ 6.20$

1500
66
3421

1505

46,173

$\$ 0.057$

$\$ 1,324$

3,000

, 000

550

$\$ 0.048$

$\$ 2,216$

$\$ 3,000$

$\$ 2,000$

$\$ 550$

$\$ 388,315$

$\$ 215,000$

$\$ 50,000$

$\$ 653,315$

$\$ 13,066$

$\$ 8.68$

$\$ 3.82$

2500
85
5675

5675
2497

85,839

85
75
97
39

350
100
785

$3500 \quad 5000$

120

127,575

4976

199,170

Too Heavy for Rail

$\$ 0.042 \quad \$ 0.039$

$\$ 3,631 \quad \$ 4,949$

$\$ 3,000 \quad \$ 3,000$

$\$ 2,000 \quad \$ 2,000$

$\$ 550 \quad \$ 52,500$

$\$ 459,049 \quad \$ 3,122,432$

$\$ 215,000 \quad \$ 315,000$

$\$ 50,000 \quad \$ 50,000$

$\$ 724,049 \quad \$ 3,487,432$

$\$ 14,481 \quad \$ 69,749$

$\$ 5.80 \quad \$ 20.18$

$\$ 2.55 \quad \$ 8.88$

$\$ 0.064$

$\$ 1,485$

$\$ 3,000$

$\$ 2,000$

$\$ 550$

$\$ 351,746$

$\$ 215,000$

$\$ 50,000$

$\$ 616,746$

$\$ 12,335$

$\$ 14.28$

$\$ 6.28$

$\$ 0.055$

$\$ 2,540$

$\$ 3,000$

$\$ 2,000$

$\$ 550$

$\$ 404,476$

$\$ 215,000$

$\$ 50,000$

$\$ 669,476$

$\$ 13,390$

$\$ 8.89$

$\$ 3.91$

$\$ 0.049$

$\$ 4,223$

$\$ 3,000$

$\$ 2,000$

$\$ 550$

$\$ 488,664$

$\$ 215,000$

$\$ 50,000$

$\$ 753,664$

$\$ 15,073$

$\$ 6.04$

$\$ 2.66$

From Port of Houston, TX

Costs per kg

Costs per Turbine

Rail Car Rental

Rail car use fee

50 mile Dolly Costs

TX

Total Rail Costs
Crane Rental - Offloading

Misc Labor

Subtotal

Grand total per turbine

Costs per kW

Costs per swept area

From Tehachapi, CA

Costs per kg

Costs per Turbine

Rail Car Rental

Rail car use fee

50 mile Dolly Costs

Total Rail Costs

Crane Rental - Offloading

Misc Labor

Subtotal

Grand total per turbine

Costs per kW

Costs per swept area
$\$ 0.079$

$\$ 1,839$

$\$ 3,000$

$\$ 2,000$

$\$ 550$

$\$ 369,462$

$\$ 215,000$

$\$ 50,000$

$\$ 634,462$

$\$ 12,689$

$\$ 14.69$

$\$ 6.46$

$\$ 0.069$

$\$ 3,204$

$\$ 3,000$

$\$ 2,000$

$\$ 550$

$\$ 437,720$

$\$ 215,000$

$\$ 50,000$

$\$ 702,720$

$\$ 14,054$

$\$ 9.34$

$\$ 4.11$

$\$ 0.080$

$\$ 1,865$

$\$ 3,000$

$\$ 2,000$

$\$ 550$

$\$ 370,744$

$\$ 215,000$

$\$ 50,000$

$\$ 635,744$

$\$ 12,715$

$\$ 14.72$

$\$ 6.48$
$\$ 0.075$

$\$ 3,463$

$\$ 3,000$

$\$ 2,000$

$\$ 550$

$\$ 450,649$

$\$ 215,000$

$\$ 50,000$

$\$ 715,649$

$\$ 14,313$

$\$ 9.51$

$\$ 4.18$
$\$ 0.064$

$\$ 5,451$

$\$ 3,000$

$\$ 2,000$

$\$ 550$

$\$ 550,039$

$\$ 215,000$

$\$ 50,000$

$\$ 815,039$

$\$ 16,301$

$\$ 6.53$

$\$ 2.87$

$\$ 0.065$

$\$ 5,580$

$\$ 3,000$

$\$ 2,000$

$\$ 550$

$\$ 556,477$

$\$ 215,000$

$\$ 50,000$

$\$ 821,477$

$\$ 16,430$

$\$ 6.58$

$\$ 2.90$
$\$ 0.046$

$\$ 5,817$

$\$ 3,000$

$\$ 2,000$

$\$ 52,500$

$\$ 3,165,871$

$\$ 315,000$

$\$ 50,000$

$\$ 3,530,871$

$\$ 70,617$

$\$ 20.43$

$\$ 8.99$

$\$ 0.060$

$\$ 7,655$

$\$ 3,000$

$\$ 2,000$

$\$ 52,500$

$\$ 3,257,725$

$\$ 315,000$

$\$ 50,000$

$\$ 3,622,725$

$\$ 72,455$

$\$ 20.97$

$\$ 9.23$

$\$ 0.065$

$\$ 8,292$

$\$ 3,000$

$\$ 2,000$

$\$ 52,500$

$\$ 3,289,619$

$\$ 315,000$

$\$ 50,000$

$\$ 3,654,619$

$\$ 73,092$

$\$ 21.15$

$\$ 9.31$ 
Appendix G

Barge Transport 
Barge Analysis from Houston, TX to Sioux City, IA

Number of Turbines

Rotor Diameter (m)

750

1500

2500

3500

5000

Calculated Power (kW)

1505

2497

100

120

4976

\section{Blades}

Barge costs per Load

$\$ 175,000$

Number of Truck/Blade Loads per Barge Volume of Blade Load (cu. m)

Load/Lash/Release Lash Costs per cubic m

$\$ 50$

Total Load/Lash/Release per Barge

Total Barge Costs per load

$\$ 145,350$

$\$ 320,350$

Number of Turbines

Number of Blades per Turbine

Total Number of Blades Number of Barge Loads Required

Total Barge Costs

Barge Costs per Turbine

Barge Costs per kW

$\$ 24,026,250$

$\$ 480,525$

$\$ 96.56$ 


\section{Rotor Diameter $(\mathrm{m})$ Calculated Power (kW)}

\section{Nacelles}

Barge costs per Load Number of Dolly/Nacelle Loads per Barge Volume of Dolly/Nacelle Load (cu. m) Load/Lash/Release Lash Costs per cubic $m$ Total Load/Lash/Release per Barge Total Barge Costs per load

Number of Turbines Number of nacelles per Turbine

Total Number of nacelles Number of Barge Loads Required Total Barge Costs Barge Costs per Turbine Barge Costs per kW
750

1500
66
1505 2500

85

2497

$\$ 175,000$

2

480

$\$ 50$

$\$ 48,000$

$\$ 223,000$

50

1

50

25

$\$ 5,575,000$

$\$ 111,500$

$\$ 44.66$
3500

100

3456

5000

120

4976

$\$ 175,000$
1
576
$\$ 50$
$\$ 28,800$
$\$ 203,800$
50
1
50
50
$\$ 10,190,000$
$\$ 203,800$
$\$ 58.97$

$\$ 175,000$

1

911

$\$ 50$

$\$ 45,563$

$\$ 220,563$

$\begin{array}{rr}50 & 50 \\ 1 & 1 \\ 0 & 50 \\ 0 & 50 \\ 0 & \$ 11,028,125 \\ 0 & \$ 220,563 \\ 7 & \$ 44.32\end{array}$


Rotor Diameter (m)
Calculated Power (kW)
"Empty" Nacelle Mass

Barge costs per Load

Nacelles

Number of Dolly/Nacelle Loads per Barge

Volume of Dolly/Nacelle Load (cu. m)

Load/Lash/Release Lash Costs per cubic $m$

Total Load/Lash/Release per Barge

Total Barge Costs per load

Number of Turbines

Number of nacelles per Turbine

Total Number of nacelles

Number of Barge Loads Required

Total Barge Costs

Barge Costs per Turbine

Barge Costs per kW
750

1500

66

1505

2500

3500

100

3456

127,575

2497

27,575

$\$ 175,000 \quad \$ 175,000$

576

$\$ 50$

$\$ 28,800$

$\$ 203,800$

50

1

50

50

$\$ 10,190,000$

$\$ 203,800$

$\$ 58.97$
5000

120

4976

199,170

$\$ 175,000$
1

911

$\$ 50$

$\$ 45,563$

$\$ 220,563$

50
1
50
50
$\$ 11,028,125$
$\$ 220,563$
$\$ 44.32$




\begin{tabular}{|c|c|c|c|c|}
\hline Turbine Class & 1500 & \multicolumn{3}{|c|}{2500} \\
\hline Rotor Diameter (m) & 66 & 85 & & \\
\hline Swept Area (sq m) & 3421 & 5675 & & \\
\hline Rated Power kW ( $44 \%$ of swept area) & 1505 & 2497 & & \\
\hline From Houston to Sioux City, lowa & Section 1 & Section 1 & Section 2 & Section 3 \\
\hline Barge costs per Load & $\$ 175,000$ & $\$ 175,000$ & $\$ 175,000$ & $\$ 175,000$ \\
\hline Number of Dolly/Tower Section Loads per Barge & 4 & 2 & 3 & 3 \\
\hline Volume of Load (cu. m) & 447 & 794 & 633 & 490 \\
\hline Load/Lash/Release Lash Costs per cubic m & $\$ 50$ & $\$ 50$ & $\$ 50$ & $\$ 50$ \\
\hline Total Load/Lash/Release per Barge & $\$ 89,327$ & $\$ 79,415$ & $\$ 94,901$ & $\$ 73,430$ \\
\hline Total Barge Costs per load & $\$ 264,327$ & $\$ 254,415$ & $\$ 269,901$ & $\$ 248,430$ \\
\hline Number of Turbines & 50 & 50 & 50 & 50 \\
\hline Number of Sections per Turbine & 1 & 1 & 1 & 1 \\
\hline Total Number of Sections & 50 & 50 & 50 & 50 \\
\hline Number of Barge Loads Required & 12.5 & 25.0 & 16.7 & 16.7 \\
\hline Total Barge Costs & $\$ 3,304,091$ & $\$ 6,360,377$ & $\$ 4,498,351$ & $\$ 4,140,492$ \\
\hline Barge Costs per Turbine & $\$ 66,082$ & $\$ 127,208$ & $\$ 89,967$ & $\$ 82,810$ \\
\hline Barge Costs per kW & $\$ 43.90$ & $\$ 50.95$ & $\$ 36.03$ & $\$ 33.17$ \\
\hline Total Costs per kW & $\$ 43.90$ & $\$ 120.15$ & & \\
\hline
\end{tabular}




\begin{tabular}{|c|c|c|c|c|c|}
\hline $\begin{array}{l}\text { Rotor Diameter }(\mathrm{m}) \\
\text { Swept Area }(\mathrm{sq} \mathrm{m}) \\
\text { Rated Power kW ( } 44 \% \text { of swept area) }\end{array}$ & $\begin{array}{r}3500 \\
100 \\
7854 \\
3456\end{array}$ & & & & \\
\hline From Houston to Sioux City, lowa & Section 1 & Section 2 & Section 3 & Section 4 & Section 5 \\
\hline Barge costs per Load & $\$ 175,000$ & $\$ 175,000$ & $\$ 175,000$ & $\$ 175,000$ & $\$ 175,000$ \\
\hline Number of Dolly/Tower Section Loads per Barge & 1 & 2 & 2 & 3 & 3 \\
\hline Volume of Load (cu. m) & 1104 & 914 & 742 & 598 & 471 \\
\hline Load/Lash/Release Lash Costs per cubic m & $\$ 50$ & $\$ 50$ & $\$ 50$ & $\$ 50$ & $\$ 50$ \\
\hline Total Load/Lash/Release per Barge & $\$ 55,220$ & $\$ 91,399$ & $\$ 74,159$ & $\$ 89,764$ & $\$ 70,591$ \\
\hline Total Barge Costs per load & $\$ 230,220$ & $\$ 266,399$ & $\$ 249,159$ & $\$ 264,764$ & $\$ 245,591$ \\
\hline Number of Turbines & 50 & 50 & 50 & 50 & 50 \\
\hline Number of Sections per Turbine & 1 & 1 & 1 & 1 & 1 \\
\hline Total Number of Sections & 50 & 50 & 50 & 50 & 50 \\
\hline Number of Barge Loads Required & 50.0 & 25.0 & 25.0 & 16.7 & 16.7 \\
\hline Total Barge Costs & $\$ 11,510,989$ & $\$ 6,659,979$ & $\$ 6,228,974$ & $\$ 4,412,735$ & $\$ 4,093,176$ \\
\hline Barge Costs per Turbine & $\$ 230,220$ & $\$ 133,200$ & $\$ 124,579$ & $\$ 88,255$ & $\$ 81,864$ \\
\hline Barge Costs per kW & $\$ 66.62$ & $\$ 38.54$ & $\$ 36.05$ & $\$ 25.54$ & $\$ 23.69$ \\
\hline Total Costs per kW & $\$ 190.44$ & & & & \\
\hline
\end{tabular}




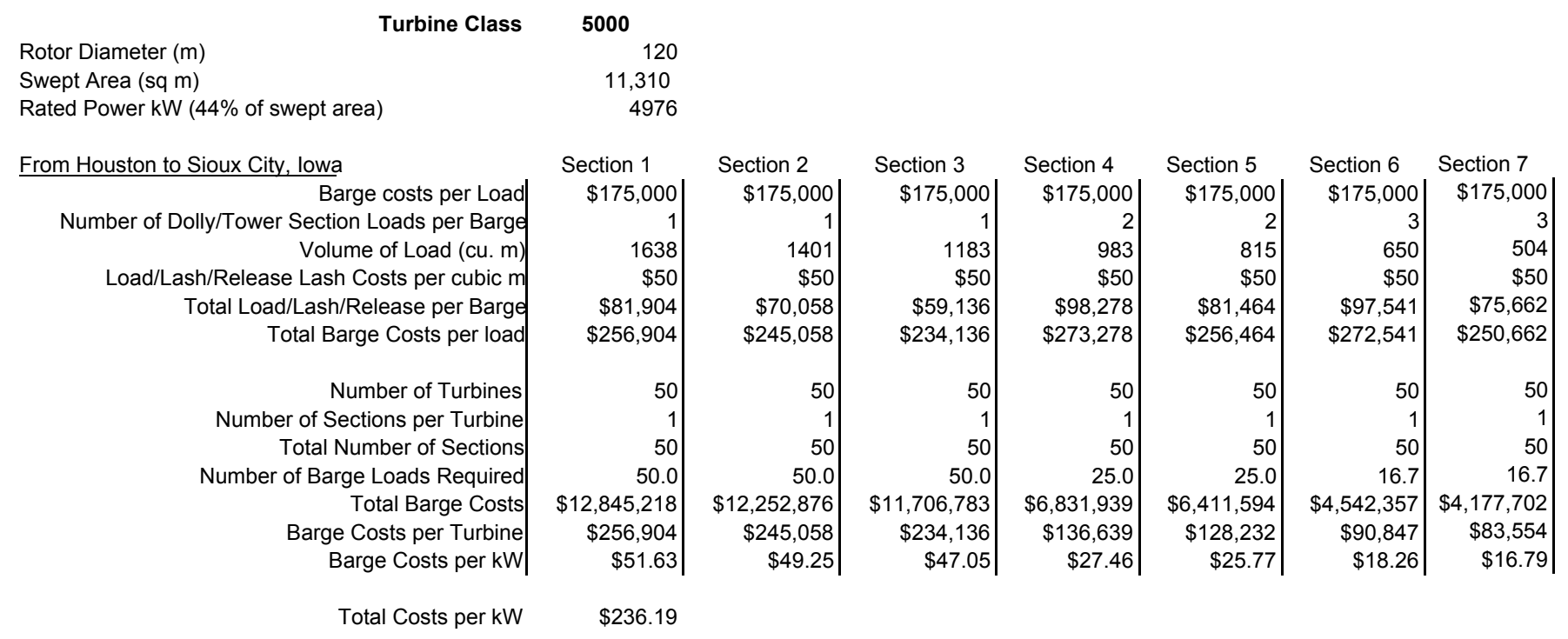


Appendix $\mathrm{H}$

Ocean Transport 


$\begin{array}{lrrrrr}\text { Name } & 750 & 1500 & 2500 & 3500 & 5000 \\ \text { Rotor Diameter } & 50 & 66 & 85 & 100 & 120 \\ \text { Area } & 1963 & 3421 & 5675 & 7854 & 11310 \\ \text { Rating } & 864 & 1505 & 2497 & 3456 & 4976 \\ & & & & & \\ \text { Blades } & \$ 32.92 & \$ 57.00 & \$ 62.12 & \$ 146.49 & \$ 175.25 \\ \text { Hub } & \$ 2.59 & \$ 6.03 & \$ 4.78 & \$ 3.45 & \$ 3.36 \\ \text { Nacelle } & \$ 15.63 & \$ 18.31 & \$ 16.02 & \$ 13.89 & \$ 15.26 \\ \text { Tower } & \$ 482 & \$ 636 & \$ 819 & \$ 963 & \$ 1,156 \\ & & & & & \\ \text { Total } & \$ 533.56 & \$ 717.67 & \$ 902.03 & \$ 1,127.23 & \$ 1,349.64\end{array}$




\begin{tabular}{|c|c|c|c|c|c|c|}
\hline Number of Turbines & 50 & & & & & \\
\hline Number of Blades per Turbine & 3 & & & & & \\
\hline \multicolumn{7}{|l|}{ Ocean Cargo Cost: } \\
\hline To Duluth, MN & $\$ 250$ & per metric ton or & ubic meter, wh & hever is greate & & \\
\hline \multirow[t]{2}{*}{ To Houston, TX } & $\$ 250$ & per metric ton or & ubic meter, wh & hever is greate & & \\
\hline & & 750 & 1500 & 2500 & 3500 & 5000 \\
\hline Rotor Diameter & $\mathrm{m}$ & 50 & 66 & 85 & 100 & 120 \\
\hline Swept Area & sq. $\mathrm{m}$ & 1963 & 3421 & 5675 & 7854 & 11310 \\
\hline Rated Power & $\mathrm{kW}$ & 864 & 1505 & 2497 & 3456 & 4976 \\
\hline \multicolumn{7}{|l|}{ Blades } \\
\hline Length & & 25 & 33 & 42.5 & 50 & 60 \\
\hline Maximum Cord (height) & & 3.5 & 4 & 4.25 & 5 & 6 \\
\hline Maximum Diameter (width) & $\mathrm{m}$ & 2.6 & 2.6 & 2.29 & 2.7 & 3.23 \\
\hline Mass & & 2940 & 6101 & 11,868 & 18,197 & 29,393 \\
\hline Transport Mass & metric tons & 2.9 & 6.1 & 11.9 & 18.2 & 29.4 \\
\hline Transport Volume & cu. M & 228 & 343 & 414 & 675 & 1163 \\
\hline Cost per Transport Unit & & $\$ 56,875$ & $\$ 85,800$ & $\$ 103,408$ & $\$ 168,750$ & $\$ 290,700$ \\
\hline Blades per Transport Unit & each & 6 & 3 & 2 & 1 & 1 \\
\hline Number of Transport Units & & 25 & 50 & 75 & 150 & 150 \\
\hline Total Transport Costs & & $\$ 1,421,875$ & $\$ 4,290,000$ & $\$ 7,755,586$ & $\$ 25,312,500$ & $\$ 43,605,000$ \\
\hline Transport Costs per Turbine & & $\$ 28,438$ & $\$ 85,800$ & $\$ 155,112$ & $\$ 506,250$ & $\$ 872,100$ \\
\hline Transport Cost per kW & $\$ / \mathrm{kW}$ & $\$ 32.92$ & $\$ 57.00$ & $\$ 62.12$ & $\$ 146.49$ & $\$ 175.25$ \\
\hline Transport Costs per Swept Area & $\$$ /sq. m & $\$ 14.48$ & $\$ 25.08$ & $\$ 27.33$ & $\$ 64.46$ & $\$ 77.11$ \\
\hline \multicolumn{7}{|l|}{ Hub } \\
\hline Height & $\mathrm{m}$ & 2.25 & 3.2 & 3.8 & 3.8 & 4.2 \\
\hline Diameter & $\mathrm{m}$ & 2.25 & 3.8 & 4 & 4 & 4.5 \\
\hline Mass & $\mathrm{kg}$ & 3816 & 12516 & 22,457 & 34,136 & 54,604 \\
\hline Transport Mass & metric tons & 4 & 13 & 22 & 34 & 55 \\
\hline Transport Volume & cu. M & 9 & 36 & 48 & 48 & 67 \\
\hline Cost per Transport Unit & & $\$ 2,237$ & $\$ 9,073$ & $\$ 11,938$ & $\$ 11,938$ & $\$ 16,700$ \\
\hline Hubs per Transport Unit & each & 1 & 1 & 1 & 1 & 1 \\
\hline Number of Transport Units & & 50 & 50 & 50 & 50 & 50 \\
\hline Total Transport Costs & & $\$ 111,827$ & $\$ 453,646$ & $\$ 596,903$ & $\$ 596,903$ & $\$ 834,976$ \\
\hline Transport Costs per Turbine & & $\$ 2,237$ & $\$ 9,073$ & $\$ 11,938$ & $\$ 11,938$ & $\$ 16,700$ \\
\hline Transport Cost per kW & $\$ / \mathrm{kW}$ & $\$ 2.59$ & $\$ 6.03$ & $\$ 4.78$ & $\$ 3.45$ & $\$ 3.36$ \\
\hline Transport Costs per Swept Area & $\$$ /sq. m & $\$ 1.14$ & $\$ 2.65$ & $\$ 2.10$ & $\$ 1.52$ & $\$ 1.48$ \\
\hline \multicolumn{7}{|l|}{ Nacelle } \\
\hline \multicolumn{2}{|c|}{ Length $\mathrm{m}$} & 6 & 9 & 10 & 12 & 15 \\
\hline \multicolumn{2}{|c|}{ Width $\mathrm{m}$} & 3 & 3.5 & 4 & 4 & 4.5 \\
\hline \multicolumn{2}{|c|}{ Height $\mathrm{m}$} & 3 & 3.5 & 4 & 4 & 4.5 \\
\hline \multicolumn{2}{|c|}{ Total Nacelle Mass kg } & 31,081 & 60,517 & 111,065 & 164,049 & 254,102 \\
\hline \multicolumn{2}{|c|}{ Transport Mass metric tons } & 31 & 61 & 111 & 164 & 254 \\
\hline \multicolumn{2}{|c|}{ Transport Volume cu. M } & 54 & 110 & 160 & 192 & 304 \\
\hline \multicolumn{2}{|c|}{ Cost per Transport Unit \$ } & $\$ 13,500$ & $\$ 27,563$ & $\$ 40,000$ & $\$ 48,000$ & $\$ 75,938$ \\
\hline \multicolumn{2}{|c|}{ Nacelles per Transport Unit each } & 1 & 1 & 1 & 1 & 1 \\
\hline \multicolumn{2}{|l|}{ Number of Transport Units } & 50 & 50 & 50 & 50 & 50 \\
\hline \multicolumn{2}{|l|}{ Total Transport Costs } & $\$ 675,000$ & $\$ 1,378,125$ & $\$ 2,000,000$ & $\$ 2,400,000$ & $\$ 3,796,875$ \\
\hline Transport Costs per Turbine & & $\$ 13,500$ & $\$ 27,563$ & $\$ 40,000$ & $\$ 48,000$ & $\$ 75,938$ \\
\hline Transport Cost per kW & $\$ / k W$ & $\$ 15.63$ & $\$ 18.31$ & $\$ 16.02$ & $\$ 13.89$ & $\$ 15.26$ \\
\hline Transport Costs per Swept Area & $\$ / \mathrm{sq} . \mathrm{m}$ & $\$ 6.88$ & $\$ 8.06$ & $\$ 7.05$ & $\$ 6.11$ & $\$ 6.71$ \\
\hline
\end{tabular}




\begin{tabular}{|c|c|}
\hline Rotor Diameter & $\mathrm{m}$ \\
\hline Swept Area & sq. $\mathrm{m}$ \\
\hline Rated Power & $\mathrm{kW}$ \\
\hline \multicolumn{2}{|l|}{ |Tower } \\
\hline \multicolumn{2}{|l|}{ Total Cost per Turbine } \\
\hline Transport Cost per kW & $\$ / \mathrm{kW}$ \\
\hline Transport Costs per Swept Area & \$/sq. m \\
\hline \multicolumn{2}{|l|}{ Section 1 (Base) } \\
\hline \multicolumn{2}{|c|}{ Length $\mathrm{m}$} \\
\hline \multicolumn{2}{|c|}{ Base Diameter m } \\
\hline \multicolumn{2}{|c|}{ Diameter $2 \mathrm{~m}$} \\
\hline \multicolumn{2}{|c|}{ Mass kg } \\
\hline \multicolumn{2}{|c|}{ Transport Volume cu. M } \\
\hline \multicolumn{2}{|c|}{ Cost per Transport Unit \$ } \\
\hline \multicolumn{2}{|l|}{ Section 2} \\
\hline \multicolumn{2}{|c|}{ Length $\mathrm{m}$} \\
\hline \multicolumn{2}{|c|}{ Diameter $1 \mathrm{~m}$} \\
\hline \multicolumn{2}{|c|}{ Diameter $2 \mathrm{~m}$} \\
\hline \multicolumn{2}{|c|}{ Mass $\mathrm{kg}$} \\
\hline \multicolumn{2}{|c|}{ Transport Mass metric tons } \\
\hline \multicolumn{2}{|c|}{ Transport Volume cu. M } \\
\hline \multicolumn{2}{|l|}{ Section 3} \\
\hline \multicolumn{2}{|c|}{ Length $\mathrm{m}$} \\
\hline \multicolumn{2}{|c|}{ Diameter $1 \mathrm{~m}$} \\
\hline \multicolumn{2}{|c|}{ Diameter 2 m } \\
\hline \multicolumn{2}{|c|}{ Mass kg } \\
\hline \multicolumn{2}{|c|}{ Transport Mass metric tons } \\
\hline \multicolumn{2}{|c|}{ Transport Volume cu. M } \\
\hline \multicolumn{2}{|c|}{ Cost per Transport Unit \$ } \\
\hline \multicolumn{2}{|l|}{ Section 4} \\
\hline \multicolumn{2}{|c|}{ Length $\mathrm{m}$} \\
\hline \multicolumn{2}{|c|}{ Diameter $1 \mathrm{~m}$} \\
\hline \multicolumn{2}{|c|}{ Diameter $2 \mathrm{~m}$} \\
\hline Mass & $\mathrm{kg}$ \\
\hline Transport Mass & metric tons \\
\hline $\begin{array}{l}\text { Transport Volume } \\
\text { Cost per Transport Uni }\end{array}$ & $\begin{array}{l}\text { cu. M } \\
\$\end{array}$ \\
\hline
\end{tabular}

Section 5

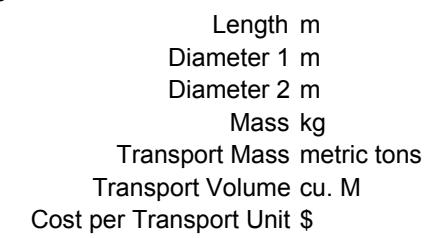

Section 6

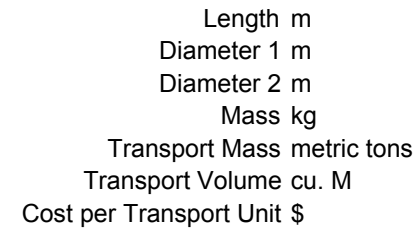

Section 7

\begin{tabular}{|c|c|c|c|c|}
\hline 750 & 1500 & 2500 & 3500 & 5 \\
\hline 50 & 66 & 85 & 100 & \\
\hline 1963 & 3421 & 5675 & 7854 & 115 \\
\hline 864 & 1505 & 2497 & 3456 & \\
\hline$\$ 416,786$ & $\$ 957,889$ & $\$ 2,045,111$ & $\$ 3,329,230$ & $\$ 5,751,4$ \\
\hline$\$ 482$ & $\$ 636$ & $\$ 819$ & $\$ 963$ & $\$ 1,1$ \\
\hline$\$ 212$ & $\$ 280$ & $\$ 360$ & $\$ 424$ & \\
\hline 21.67 & 21.45 & 22.10 & 21.67 & \\
\hline 3.74 & 4.94 & 6.36 & 7.48 & \\
\hline 3.12 & 4.32 & 5.72 & 6.85 & \\
\hline 28,642 & 51,574 & 90,403 & 124,764 & 187, \\
\hline 29 & 52 & 90 & 125 & \\
\hline 803 & 1445 & 2534 & 3497 & \\
\hline$\$ 200,675$ & $\$ 361,346$ & $\$ 633,400$ & $\$ 874,143$ & $\$ 1,310,3$ \\
\hline 21.67 & 21.45 & 22.10 & 21.67 & 2 \\
\hline 3.12 & 4.32 & 5.72 & 6.85 & \\
\hline 2.49 & 3.70 & 5.08 & 6.23 & \\
\hline 19,199 & 38,757 & 72,389 & 104,022 & 160 \\
\hline 19 & 39 & 72 & 104 & \\
\hline 538 & 1086 & 2029 & 2915 & 4 \\
\hline$\$ 134,518$ & $\$ 271,544$ & $\$ 507,188$ & $\$ 728,819$ & $\$ 1,123,4$ \\
\hline
\end{tabular}

$\begin{array}{rr}21.67 & 21.45 \\ 2.49 & 3.70 \\ 1.87 & 3.09 \\ 11,646 & 27,771 \\ 12 & 28 \\ 326 & 778 \\ \$ 81,593 & \$ 194,571\end{array}$

$\begin{array}{rr}21.67 & 22.29 \\ 6.23 & 7.69 \\ 5.61 & 7.05 \\ 85,166 & 135,732 \\ 85 & 136 \\ 2387 & 3804 \\ \$ 596,707 & \$ 950,990\end{array}$

$\begin{array}{rrrr}21.45 & 22.10 & 21.67 & 22.29 \\ 3.09 & 4.45 & 5.61 & 7.05 \\ 2.47 & 3.81 & 4.98 & 6.41 \\ 18,615 & 42,366 & 68,196 & 113,167 \\ 19 & 42 & 68 & 113 \\ 522 & 1187 & 1911 & 3172 \\ \$ 130,427 & \$ 296,833 & \$ 477,806 & \$ 792,891\end{array}$

$\begin{array}{rrr}22.10 & 21.67 & 22.29 \\ 3.81 & 4.98 & 6.41 \\ 3.18 & 4.36 & 5.77 \\ 30,357 & 53,111 & 92,653 \\ 30 & 53 & 93 \\ 851 & 1488 & 2597 \\ \$ 212,692 & \$ 372,116 & \$ 649,164\end{array}$

$\begin{array}{rr}21.67 & 22.29 \\ 4.36 & 5.77 \\ 3.74 & 5.13 \\ 39,912 & 74,191 \\ 40 & 74 \\ 1119 & 2079 \\ \$ 279,638 & \$ 519,811\end{array}$


Appendix I

Scenario 1 


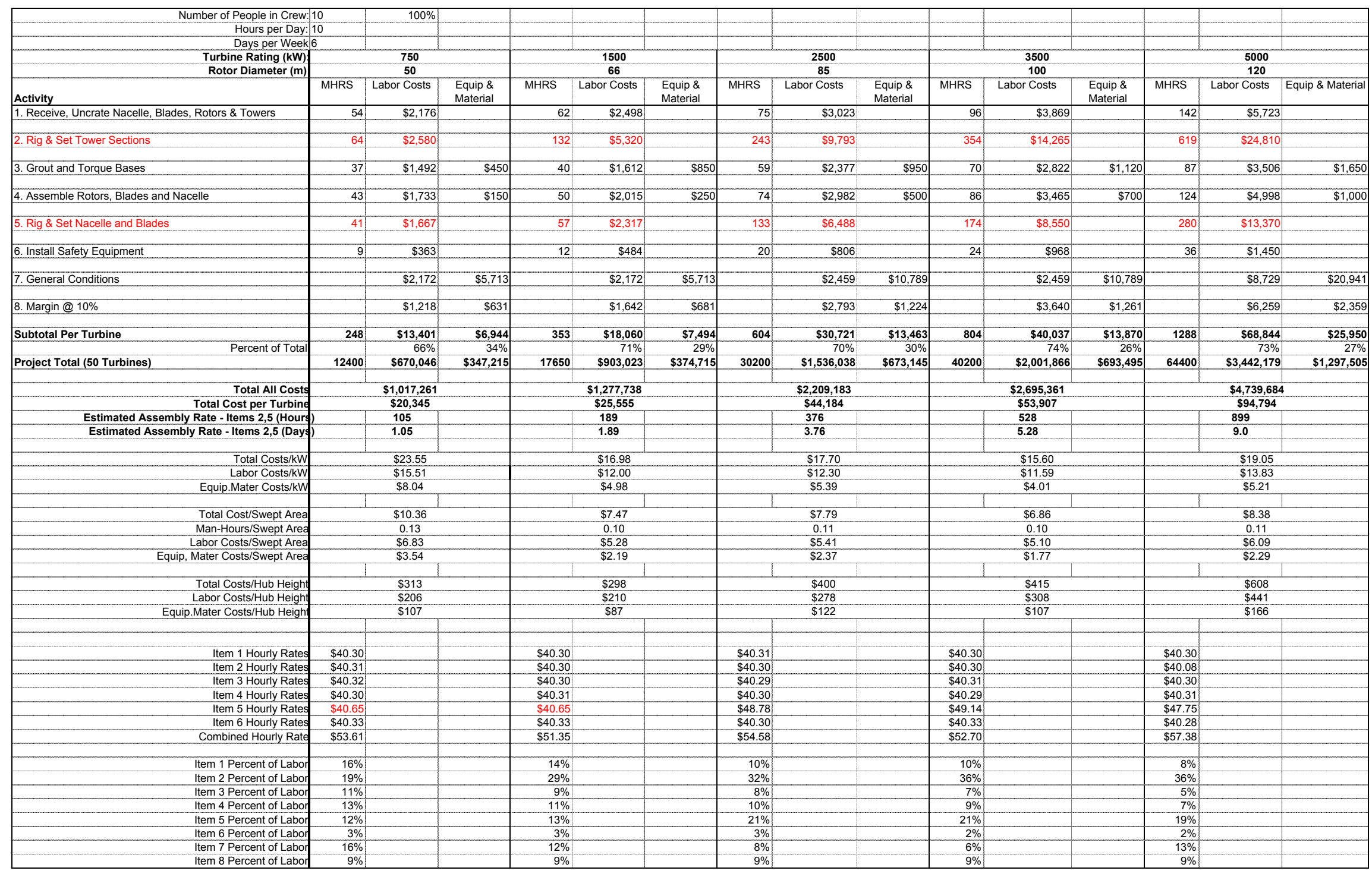




\begin{tabular}{|c|c|c|c|c|c|c|c|c|c|c|c|c|c|c|c|}
\hline $\begin{array}{c}\text { Number of People in Crew: } \\
\text { Hours per Day: }\end{array}$ & & $75 \%$ & & & & & & & & & & & & & \\
\hline Days per Week & & & & & & & & & & & & & & & \\
\hline Turbine Rating $(\mathrm{kW})$ : & & 750 & & & 1500 & & & 2500 & & & 3500 & & & 5000 & \\
\hline Rotor Diameter $(\mathbf{m})$ : & & 50 & & & 66 & & & 85 & & & 100 & & & 120 & \\
\hline Activity & MHRS & Labor Costs & $\begin{array}{l}\text { Equip \& } \\
\text { Material }\end{array}$ & MHRS & Labor Costs & $\begin{array}{l}\text { Equip \& } \\
\text { Material }\end{array}$ & MHRS & Labor Costs & $\begin{array}{l}\text { Equip \& } \\
\text { Material }\end{array}$ & MHRS & Labor Costs & $\begin{array}{l}\text { Equip \& } \\
\text { Material }\end{array}$ & MHRS & Labor Costs & Equip \& Material \\
\hline 1. Receive, Uncrate Nacelle, Blades, Rotors \& Towers & 24 & $\$ 840$ & & 40 & $\$ 1,400$ & & 75 & $\$ 2,625$ & & 96 & $\$ 3,360$ & & 142 & $\$ 4,970$ & \\
\hline 2. Rig \& Set Tower Sections & 42 & $\$ 1,470$ & & 60 & $\$ 2,100$ & & 110 & $\$ 3,866$ & & 161 & $\$ 5,645$ & & 282 & $\$ 9,878$ & \\
\hline 3. Grout and Torque Bases & 32 & $\$ 1,120$ & $\$ 450$ & 40 & $\$ 1,400$ & $\$ 850$ & 59 & $\$ 2,065$ & $\$ 950$ & 70 & $\$ 2,450$ & $\$ 1,120$ & 87 & $\$ 3,045$ & $\$ 1,650$ \\
\hline 4. Assemble Rotors, Blades and Nacelle & 30 & $\$ 1,050$ & $\$ 150$ & 50 & $\$ 1,750$ & $\$ 250$ & 74 & $\$ 2,590$ & $\$ 500$ & 86 & $\$ 3,010$ & $\$ 700$ & 124 & $\$ 4,340$ & $\$ 1,000$ \\
\hline 5. Rig \& Set Nacelle and Blades & 16 & $\$ 560$ & & 22 & $\$ 778$ & & 52 & $\$ 2,528$ & & 68 & $\$ 3,335$ & & 109 & $\$ 5,219$ & \\
\hline 6. Install Safety Equipment & 9 & $\$ 315$ & & 12 & $\$ 420$ & & 20 & $\$ 700$ & & 24 & $\$ 840$ & & 36 & $\$ 1,260$ & \\
\hline 7. General Conditions & & $\$ 1,629$ & $\$ 4,285$ & & $\$ 1,629$ & $\$ 4,285$ & & $\$ 1,844$ & $\$ 8,092$ & & $\$ 1,844$ & $\$ 8,092$ & & $\$ 6,546$ & $\$ 15,706$ \\
\hline 8. Margin@ @ 10\% & & $\$ 698$ & $\$ 488$ & & $\$ 948$ & $\$ 538$ & & $\$ 1,622$ & $\$ 954$ & & $\$ 2,048$ & $\$ 991$ & & $\$ 3,526$ & $\$ 1,836$ \\
\hline Subtotal Per Turbine & 153 & $\$ 7,682$ & $\$ 5,373$ & 224 & $\$ 10,425$ & $\$ 5,923$ & 390 & $\$ 17,840$ & $\$ 10,496$ & 505 & $\$ 22,533$ & $\$ 10,903$ & 781 & $\$ 38,783$ & $\$ 20,191$ \\
\hline \begin{tabular}{|l} 
\\
Project Total (50 Turbines)
\end{tabular} & 7650 & $59 \%$ & $\begin{aligned} 41 \% \\
\$ 268.661\end{aligned}$ & 11212 & $\begin{array}{l}64 \% \\
\$ 521.257\end{array}$ & $\begin{aligned} 36 \% \% \\
\$ 236.161\end{aligned}$ & 19514 & $\begin{aligned} 63 \% \\
889997\end{aligned}$ & $\begin{aligned} 37 \% \\
\$ 522796 \\
\end{aligned}$ & 25258 & $\begin{array}{r}67 \% \\
61.26 .632\end{array}$ & $\begin{aligned} 33 \% \\
\$ 545.146\end{aligned}$ & 39026 & $\begin{aligned} 66 \% \\
\$ 1.939 .157 \\
\end{aligned}$ & $\begin{array}{r}34 \% \\
100956\end{array}$ \\
\hline a & & & & & & & & & & & & & 00000 & & \\
\hline $\begin{array}{c}\text { Total All Costs } \\
\text { Total Cost per Turbine } \\
\end{array}$ & & $\frac{\frac{5652,781}{\$ 1,056}}{4}$ & & & $\frac{\$ 817,418}{\$ 16,348}$ & & & 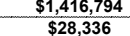 & & & 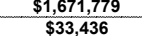 & & & 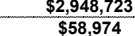 & \\
\hline Estimated Assembly Rate - Items 2,5 (Hours) & & 58 & & & & & & & & & 229 & & & & \\
\hline Estimated Assembly Rate - Items 2,5 (Days) & & 0.58 & & & 0.82 & & & 1.62 & & & 2.29 & & & 3.92 & \\
\hline Total Costs $/ \mathrm{kW}$ & 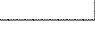 & $\$ 15.11$ & & & $\$ 10.86$ & & & $\$ 11,35$ & & & $\$ 9.68$ & & & & \\
\hline $\begin{array}{l}\text { Lotal Cosis/kW } \\
\text { Labor Costs/kW }\end{array}$ & & $\frac{\$ 15.11}{\$ 8.89}$ & & & $\$ 6.93$ & & & $\frac{\$ 11.35}{\$ 7.15}$ & & & $\begin{array}{l}\$ 9.68 \\
\$ 6.52\end{array}$ & & & $\frac{\$ 11.85}{\$ 7.79}$ & \\
\hline Equip.Mater Costs/kW & & $\$ 6.22$ & & & $\$ 3.93$ & & & $\$ 4.20$ & & & $\$ 3.16$ & & & $\$ 4.06$ & \\
\hline & & & & & & & & & & & & & & & \\
\hline Total Cost/Swept Area & & $\$ 6.65$ & & & $\$ 4.78$ & & & $\$ 4.99$ & & & $\$ 4.26$ & & & $\$ 5.21$ & \\
\hline Man-Hours/Swept Area & & 0.08 & & & 0.07 & & & 0.07 & & & 0.06 & & & 0.07 & \\
\hline Labor Costs/Swept Area & & $\$ 3.91$ & & & $\$ 3.05$ & & & $\$ 3.14$ & & & $\$ 2.87$ & & & $\$ 3.43$ & \\
\hline Equip, Mater Costs/Swept Area & & $\$ 2.74$ & & & $\$ 1.73$ & & & $\$ 1.85$ & & & $\$ 1.39$ & & & $\$ 1.79$ & \\
\hline Total Costs/Hub Height & 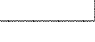 & $\$ 201$ & & & $\$ 191$ & & & $\$ 256$ & & & $\$ 257$ & & & $\$ 378$ & \\
\hline Labor Costs/Hub Height & & $\$ \$ \$ 118$ & & & $\begin{array}{l}\frac{S 191}{\$ 122} \\
\$ 1\end{array}$ & & & $\$ \$ \$ 161$ & & & $\$ \$ \$ 173$ & & & $\$ 249$ & \\
\hline Equip.Mater Costs/Hub Height & & $\$ 83$ & & & $\$ 69$ & & & $\$ 95$ & & & $\$ 84$ & & & $\$ 129$ & \\
\hline & & & & & & & & & & & & & & & \\
\hline Item 1 Hourly Rates & $\$ 35.00$ & 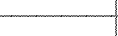 & & $\$ 35.00$ & & & $\$ 35.00$ & & & $\$ 35.00$ & & & $\$ 35.00$ & & \\
\hline Item 2 Hourly Rates & $\$ 35.00$ & & & $\$ 35.00$ & & & $\$ 35.00$ & & & $\$ 35.00$ & & & $\$ 35.00$ & & \\
\hline Item 3 Hourly Rates & $\$ 35.00$ & & & $\$ 35.00$ & & & $\$ 35.00$ & & & $\$ 35.00$ & & & $\$ 35.00$ & & \\
\hline Item 4 Hourly Rates & $\$ 35.00$ & & & $\$ 35.00$ & & & $\$ 35.00$ & & & $\$ 35.00$ & & & $\$ 35.00$ & & \\
\hline Item 5 Hourly Rates & $\$ 35.00$ & & & $\$ 35.00$ & & & $\$ 48.78$ & & & $\$ 49.14$ & & & $\$ 47.75$ & & \\
\hline Item 6 Hourly Rates & $\$ 35.00$ & & & $\$ 35.00$ & & & $\$ 35.00$ & & & $\$ 35.00$ & & & $\$ 35.00$ & & \\
\hline Combined Hourly Rate & & & & & & & & & & & & & & & \\
\hline Item 1 Percent of Labor & $11 \%$ & & & $13 \%$ & & & $15 \%$ & & & $15 \%$ & & & $13 \%$ & & \\
\hline Item 2 Percent of Labor & $19 \%$ & & & 0 & & & $22 \%$ & & & $25 \%$ & & & $25 \%$ & & \\
\hline Item 3 Percent of Labor & $15 \%$ & & & $13 \%$ & & & $12 \%$ & & & $11 \%$ & & & $8 \%$ & & \\
\hline Item 4 Percent of Labor & $14 \%$ & & & $17 \%$ & & & $15 \%$ & & & $13 \%$ & & & $11 \%$ & & \\
\hline Item 5 Percent of Labor & $7 \%$ & & & $7 \%$ & & & $14 \%$ & & & $15 \%$ & & & $13 \%$ & & \\
\hline Item 6 Percent of Labor & $4 \%$ & & & $4 \%$ & & & $4 \%$ & & & $4 \%$ & & & $3 \%$ & & \\
\hline Item 7 Percent of Labor & $21 \%$ & & & $16 \%$ & & & $10 \%$ & & & $8 \%$ & & & $17 \%$ & & \\
\hline Item 8 Percent of Labor & $9 \%$ & & & $9 \%$ & & & $9 \%$ & & & $9 \%$ & & & $9 \%$ & & \\
\hline
\end{tabular}




\begin{tabular}{|c|c|c|c|c|c|c|c|c|c|c|c|c|c|c|c|}
\hline \multirow{2}{*}{\multicolumn{16}{|c|}{\begin{tabular}{|r} 
Number of People in Crew: 10 \\
Hours per Day: 10 \\
Days per Week 6
\end{tabular}}} \\
\hline & & & & & & & & & & & & & & & \\
\hline Turbine Rating $(\mathrm{kW}):$ & & 750 & & & 1500 & & & 2500 & & & 3500 & & & 5000 & \\
\hline Rotor Diameter $(\mathbf{m})$ : & & 50 & & & 66 & & & 85 & & & 100 & & & 120 & \\
\hline Activity & MHRS & Labor Costs & $\begin{array}{l}\text { Equip \& } \\
\text { Material }\end{array}$ & MHRS & Labor Costs & $\begin{array}{l}\text { Equip \& } \\
\text { Material }\end{array}$ & MHRS & Labor Costs & $\begin{array}{l}\text { Equip \& } \\
\text { Material }\end{array}$ & MHRS & Labor Costs & $\begin{array}{l}\text { Equip \& } \\
\text { Material }\end{array}$ & MHRS & Labor Costs & Equip \& Material \\
\hline 1. Receive, Uncrate Nacelle, Blades, Rotors \& Towers & 54 & $\$ 2,176$ & & 64 & $\$ 2,579$ & & 75 & $\$ 3,023$ & & 96 & $\$ 3,869$ & & 142 & $\$ 5,723$ & \\
\hline 2. Rig \& Set Tower Sections & 67 & $\$ 2,701$ & & 138 & $\$ 5,569$ & & 254 & $\$ 10,253$ & & 371 & $\$ 14,970$ & & 687 & $\$ 27,693$ & \\
\hline 3. Grout and Torque Bases & 37 & $\$ 1,492$ & $\$ 450$ & 40 & $\$ 1,612$ & $\$ 850$ & 59 & $\$ 2,377$ & $\$ 950$ & 70 & $\$ 2,822$ & $\$ 1,120$ & 87 & $\$ 3,506$ & $\$ 1,650$ \\
\hline 4. Assemble Rotors, Blades and Nacelle & 43 & $\$ 1,733$ & $\$ 150$ & 50 & $\$ 2,015$ & $\$ 250$ & 74 & $\$ 2,982$ & $\$ 500$ & 86 & $\$ 3,465$ & $\$ 700$ & 124 & $\$ 4,998$ & $\$ 1,000$ \\
\hline 5. Rig \& Set Nacelle and Blades & 48 & $\$ 1,951$ & & 67 & $\$ 2,712$ & & 155 & $\$ 7,583$ & & 204 & $\$ 10,006$ & & 348 & $\$ 16,628$ & \\
\hline 6. Install Safety Equipment & 9 & $\$ 363$ & & 12 & $\$ 484$ & & 20 & $\$ 806$ & & 24 & $\$ 968$ & & 36 & $\$ 1,450$ & \\
\hline 7. General Conditions & & $\$ 2,281$ & $\$ 5,999$ & & $\$ 2,281$ & $\$ 5,999$ & & $\$ 2,582$ & $\$ 11,328$ & & $\$ 2,582$ & $\$ 11,328$ & & $\$ 9,165$ & $\$ 21,988$ \\
\hline 8. Margin@10\% & & $\$ 1,270$ & $\$ 660$ & & $\$ 1,725$ & $\$ 710$ & & $\$ 2,961$ & $\$ 1,278$ & & $\$ 3,868$ & $\$ 1,315$ & & $\$ 6,916$ & $\$ 2,464$ \\
\hline Subtotal Per Turbine & 258 & $\$ 13,966$ & $\$ 7,259$ & 371 & $\$ 18,978$ & $\$ 7,809$ & 638 & $\$ 32,567$ & $\$ 14,056$ & 851 & $\$ 42,550$ & $\$ 14,463$ & 1424 & $\$ 76,079$ & $\$ 27,102$ \\
\hline \begin{tabular}{|l} 
Project Total (50 Turbines) \\
\end{tabular} & 12900 & $\begin{array}{r}66 \% \\
\$ 698,321\end{array}$ & $\begin{array}{r}34 \% \\
\$ 362,926\end{array}$ & 18545.38 & $\begin{array}{r}71 \% \\
\$ 948,875\end{array}$ & $\begin{array}{r}29 \% \\
\$ 390,426\end{array}$ & 31893 & $\begin{array}{r}70 \% \\
\$ 1,628,329\end{array}$ & $\begin{array}{r}30 \% \\
\$ 702,815 \\
\end{array}$ & 42554 & $\begin{array}{r}75 \% \\
\$ 2,127,513\end{array}$ & $\begin{array}{r}25 \% \\
\$ 723,165\end{array}$ & 71219 & $\begin{array}{r}74 \% \\
\$ 3,803,962\end{array}$ & $\begin{array}{r}26 \% \\
\$ 1,355,093\end{array}$ \\
\hline 年 & & & & & & & & & & & & & & & \\
\hline $\begin{array}{r}\text { Total All Costs } \\
\text { Total Cost per Turbine }\end{array}$ & & $\frac{\$ 1,061,246}{\$ 21,225}$ & & & $\$ 1,339,301$ & & & $\frac{\$ 2,331,143}{\$ 46,623}$ & & & $\frac{\$ 2,850,678}{\$ 57014}$ & & & $\frac{\$ 5,159,055}{\$ 103,18}$ & \\
\hline $\begin{array}{l}\text { lotal Cost per lurbine } \\
\text { Estimated Assembly Rate - Items 2,5 (Hours) }\end{array}$ & & $\frac{\$ 2,1225}{115}$ & & & $\frac{\$ 26,786}{205}$ & & & $\frac{\$ 46,623}{410}$ & & & $\frac{\$ 57,014}{575}$ & & & $\frac{\$ 103,181}{1,035}$ & \\
\hline Estimated Assembly Rate - Items 2,5 (Days) & & 1.15 & & & 2.05 & & & 4.10 & & & 5.75 & & & 10.35 & \\
\hline Total Costs $/ \mathrm{kW} / \mathrm{s}$ & 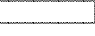 & $\$ 2457>07$ & & & $\$ 1779$ & & & 91807 & & & 9650 & & & 90073 & \\
\hline $\begin{array}{l}\text { Latal Cosis/kV } \\
\text { Labor Costs/kW }\end{array}$ & & $\$ 24.57$ & & & $\begin{array}{l}\$ 1.19 \\
\$ 12.61\end{array}$ & & & $\$ \$ \$ 13.04$ & & & $\begin{array}{l}\$ 16.50 \\
\$ 12.31\end{array}$ & & & $\$ \$ \$ 15.29$ & \\
\hline Equip.Mater Costs/kW & & $\$ 8.40$ & & & $\$ 5.19$ & & & $\$ 5.63$ & & & $\$ 4.19$ & & & $\$ 5.45$ & \\
\hline & & & & & & & & & & & & & & & \\
\hline Total Cost/Swept Area & & $\$ 10.81$ & & & $\$ 7.83$ & & & $\$ 8.22$ & & & $\$ 7.26$ & & & $\$ 9.12$ & \\
\hline Man-Hours/Swept Area & & 0.13 & & & 0.11 & & & 0.11 & & & 0.11 & & & 0.13 & \\
\hline Labor Costs/Swept Area & & $\$ 7.11$ & & & $\$ 5.55$ & & & $\$ 5.74$ & & & $\$ 5.42$ & & & $\$ 6.73$ & \\
\hline Equip, Mater Costs/Swept Area & & $\$ 3.70$ & & & $\$ 2.28$ & & & $\$ 2.48$ & & & $\$ 1.84$ & & & $\$ 2.40$ & \\
\hline Total Costs//Hub Height & - & $\$ 327$ & & & $\$ 312$ & & & $\$ 422$ & & & $\$ 439$ & & & $\$ 661$ & \\
\hline Labor Costs/Hub Height & & $\$ 215$ & & & $\$ 221$ & & & $\$ 295$ & & & $\$ 327$ & & & $\$ 488$ & \\
\hline Equip.Mater Costs/Hub Height & & $\$ 112$ & & & $\$ 91$ & & & $\$ 127$ & & & $\$ 111$ & & & $\$ 174$ & \\
\hline & & & & & & & & & & & & & & & \\
\hline Item 1 Hourly Rates & $\$ 40.30$ & -0 & & $\$ 40.30$ & & & $\$ 40.31$ & & & $\$ 40.30$ & & & $\$ 40.30$ & & \\
\hline $\begin{array}{l}\text { Item } 2 \text { Hourly Rates } \\
\end{array}$ & $\$ 40.31$ & & & $\$ 40.30$ & & & $\$ 40.30$ & & & $\$ 40.30$ & & & $\$ 40.30$ & & \\
\hline Item 3 Hourly Rates & $\$ 40.32$ & & & $\$ 40.30$ & & & $\$ 40.29$ & & & $\$ 40.31$ & & & $\$ 40.30$ & & \\
\hline Item 4 Hourly Rates & $\$ 40.30$ & & & $\$ 40.31$ & & & $\$ 40.30$ & & & $\$ 40.29$ & & & $\$ 40.31$ & & \\
\hline $\begin{array}{l}\text { Item } 5 \text { Hourly Rates } \\
\end{array}$ & $\frac{\$ 40.65}{\$ 40.33}$ & & & $\$ 40.65$ & & & $\$ 48.78$ & & & $\begin{array}{l}\$ 49.14 \\
\$ 40.33\end{array}$ & & & $\$ 47.75$ & & \\
\hline $\begin{array}{l}\text { Item } 6 \text { Hourly Rates } \\
\text { Combined Hourly Rate }\end{array}$ & $\begin{array}{l}\$ 40.33 \\
\$ 53.61\end{array}$ & & & $\begin{array}{l}\$ 40.33 \\
\$ 51.35\end{array}$ & & & $\begin{array}{l}\$ 40.30 \\
\$ 55.58\end{array}$ & & & $\$ \$ 40.33$ & & & $\begin{array}{l}\$ 40.28 \\
\$ 57.38\end{array}-10$ & & \\
\hline Combined Hourly Rate & $\$ 53.61$ & & & $\$ 51.35$ & & & $\$ 54.58$ & & & $\$ 52.70$ & & & $\$ 57.38$ & & \\
\hline Item 1 Percent of Labor & $16 \%$ & & & $14 \%$ & & & $9 \%$ & & & $9 \%$ & & & $8 \%$ & & \\
\hline Item 2 Percent of Labor & $19 \%$ & & & $29 \%$ & & & $31 \%$ & & & $35 \%$ & & & $36 \%$ & & \\
\hline Item 3 Percent of Labor & $11 \%$ & & & $8 \%$ & & & $7 \%$ & & & $7 \%$ & & & $5 \%$ & & \\
\hline Item 4 Percent of Labor & $12 \%$ & & & $11 \%$ & & & $9 \%$ & & & $8 \%$ & & & $7 \%$ & & \\
\hline Item 5 Percent of Labor & $14 \%$ & & & $14 \%$ & & & $23 \%$ & & & $24 \%$ & & & $22 \%$ & & \\
\hline Item 6 Percent of Labor & $3 \%$ & & & $3 \%$ & & & $2 \%$ & & & $2 \%$ & & & $2 \%$ & & \\
\hline Item 7 Percent of Labor & $16 \%$ & & & $12 \%$ & & & $8 \%$ & & & $6 \%$ & & & $12 \%$ & & \\
\hline Item 8 Percent of Labor & $9 \%$ & & & $9 \%$ & & & $9 \%$ & & & $9 \%$ & & & $9 \%$ & & \\
\hline
\end{tabular}




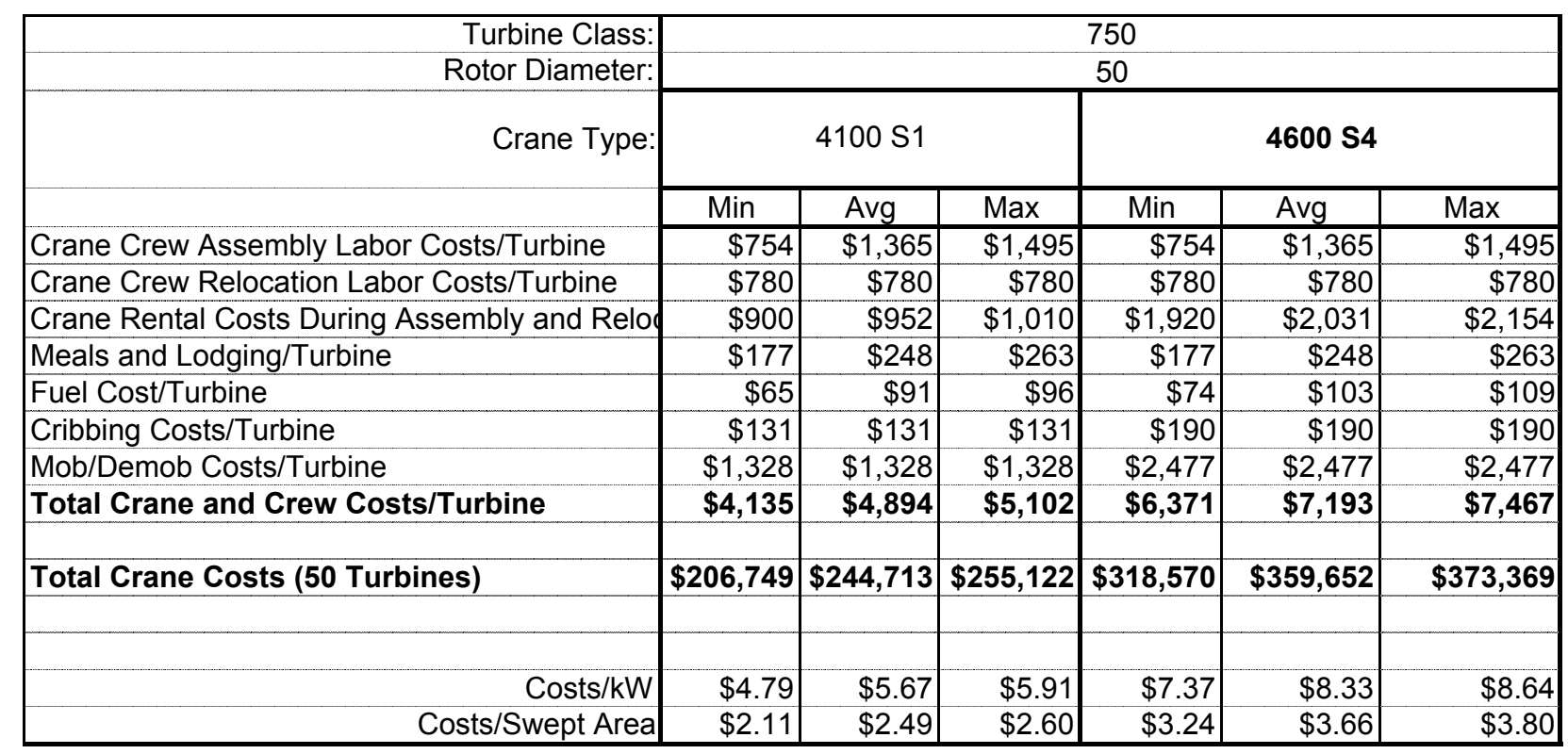




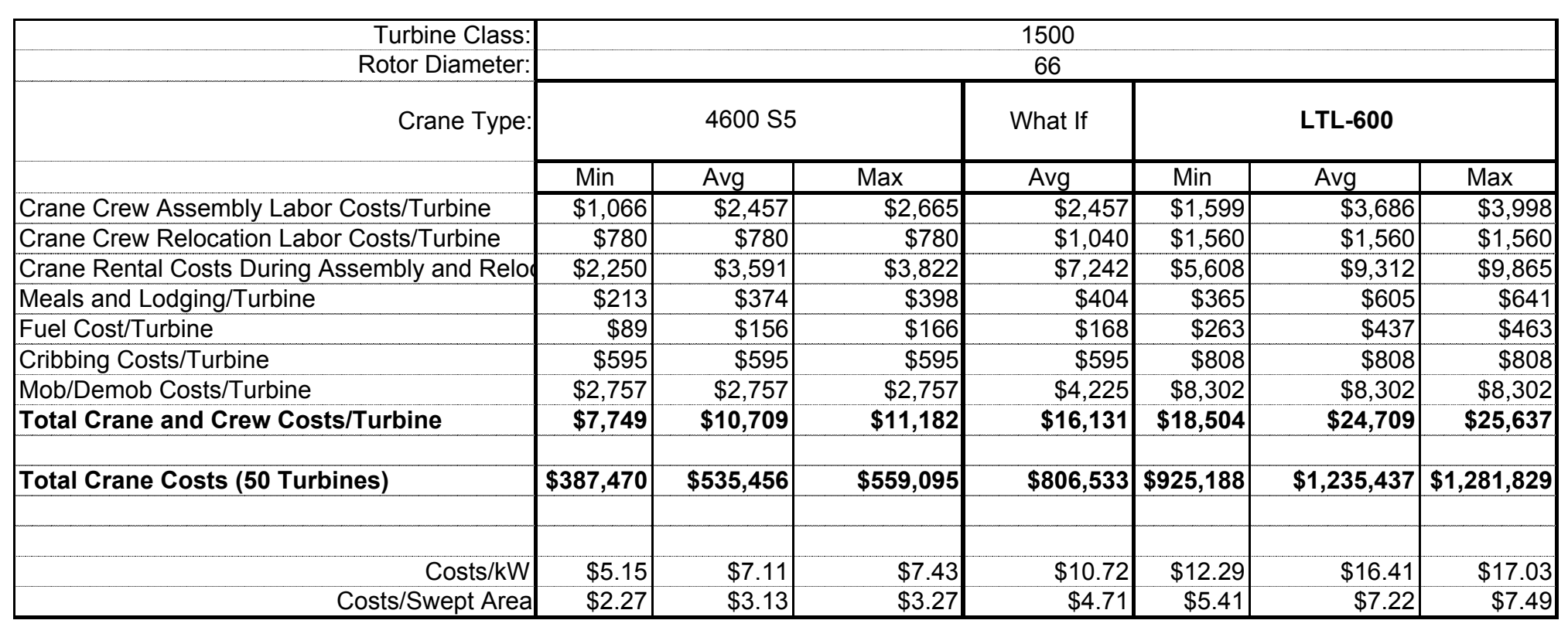




\begin{tabular}{|c|c|c|c|c|c|c|c|c|c|}
\hline Turbine Class: & \multicolumn{3}{|c|}{2500} & \multicolumn{6}{|c|}{3500} \\
\hline Rotor Diameter: & \multicolumn{3}{|c|}{85} & \multicolumn{6}{|c|}{100} \\
\hline \multirow[t]{2}{*}{ Crane Type: } & \multicolumn{3}{|c|}{ LTL-850 } & \multicolumn{3}{|c|}{ LTL-1000 } & \multicolumn{3}{|c|}{ LTL-1100 } \\
\hline & Min & Avg & Max & Min & Avg & Max & Min & Avg & Max \\
\hline Crane Crew Assembly Labor Costs/Turbine & $\$ 3,159$ & $\$ 7,332$ & $\$ 7,995$ & $\$ 4,466$ & $\$ 10,296$ & $\$ 11,213$ & $\$ 4,466$ & $\$ 10,296$ & $\$ 11,213$ \\
\hline Crane Crew Relocation Labor Costs/Turbine & $\$ 2,730$ & $\$ 2,730$ & $\$ 2,730$ & $\$ 4,095$ & $\$ 4,095$ & $\$ 4,095$ & $\$ 4,875$ & $\$ 4,875$ & $\$ 4,875$ \\
\hline Crane Rental Costs During Assembly and Relod & $\$ 10,841$ & $\$ 18,523$ & $\$ 19,744$ & $\$ 18,995$ & $\$ 31,933$ & $\$ 33,966$ & $\$ 23,213$ & $\$ 37,703$ & $\$ 39,981$ \\
\hline Meals and Lodging/Turbine & $\$ 680$ & $\$ 1,161$ & $\$ 1,238$ & $\$ 988$ & $\$ 1,661$ & $\$ 1,766$ & $\$ 1,078$ & $\$ 1,751$ & $\$ 1,856$ \\
\hline Fuel Cost/Turbine & $\$ 491$ & $\$ 839$ & $\$ 894$ & $\$ 768$ & $\$ 1,292$ & $\$ 1,374$ & $\$ 838$ & $\$ 1,362$ & $\$ 1,444$ \\
\hline Cribbing Costs/Turbine & $\$ 538$ & $\$ 538$ & $\$ 538$ & $\$ 808$ & $\$ 808$ & $\$ 808$ & $\$ 943$ & $\$ 943$ & $\$ 943$ \\
\hline Mob/Demob Costs/Turbine & $\$ 9,695$ & $\$ 9,695$ & $\$ 9,695$ & $\$ 19,522$ & $\$ 19,522$ & $\$ 19,522$ & $\$ 22,141$ & $\$ 22,141$ & $\$ 22,141$ \\
\hline Total Crane and Crew Costs/Turbine & $\$ 28,132$ & $\$ 40,817$ & $\$ 42,832$ & $\$ 49,642$ & $\$ 69,606$ & $\$ 72,744$ & $\$ 57,553$ & $\$ 79,069$ & $\$ 82,451$ \\
\hline Total Crane Costs (50 Turbines) & $\$ 1,406,616$ & $\$ 2,040,831$ & $\$ 2,141,594$ & $\$ 2,482,078$ & $\$ 3,480,278$ & $\$ 3,637,185$ & $\$ 2,877,640$ & $\$ 3,953,465$ & $\$ 4,122,575$ \\
\hline & & & & & & & & & \\
\hline Costs/kW & $\$ 11.27$ & $\$ 16.35$ & $\$ 17.15$ & $\$ 14.36$ & $\$ 20.14$ & $\$ 21.05$ & $\$ 16.65$ & $\$ 22.88$ & $\$ 23.86$ \\
\hline Costs/Swept Area & $\$ 4.96$ & $\$ 7.19$ & $\$ 7.55$ & $\$ 6.32$ & $\$ 8.86$ & $\$ 9.26$ & $\$ 7.33$ & $\$ 10.07$ & $\$ 10.50$ \\
\hline
\end{tabular}




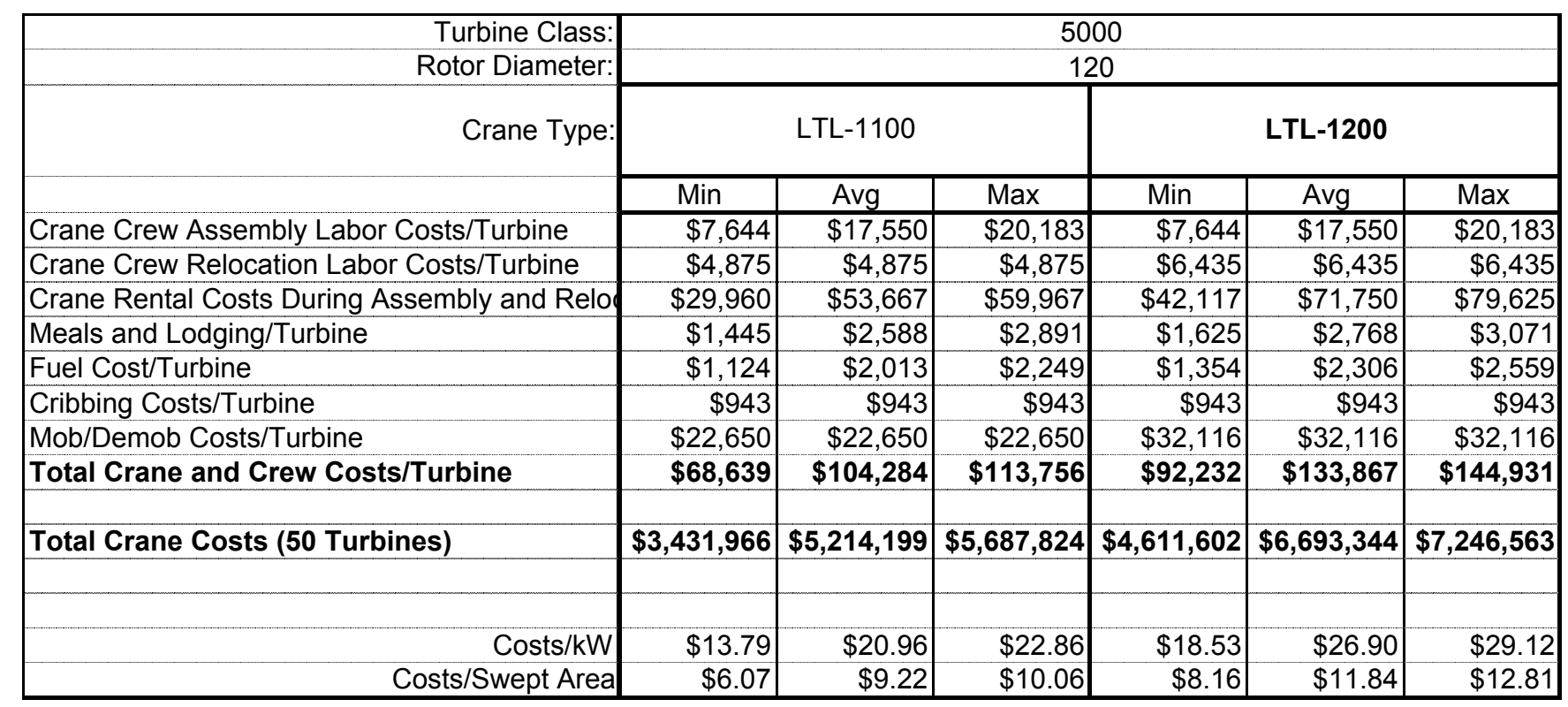




\begin{tabular}{|c|c|c|c|c|c|}
\hline Initial Assumptions & Turbine Assembly & Crane Assembly & & & \\
\hline Work Hours/Day & 10 & 8 & & & \\
\hline Number of Days/Wk & 6 & 5 & & & \\
\hline Number of Weeks/Year & 52 & 52 & & & \\
\hline Number of Weeks/Month & 4.3333 & 4.4000 & & & \\
\hline Number of Days/Month & 26 & 22 & & & \\
\hline Number of Hours/Month & 260 & 176 & & & \\
\hline Number of Turbines & 50 & & & & \\
\hline Turbine Rating (kW) & 75 & & & 1500 & \\
\hline Crane Type & 4100 S1 & $4600 \$ 4$ & 4600 S5 & what if & LTL-600 \\
\hline Monthly Crane Costs during turbine assembly (60hr wk) & $\$ 15,000$ & $\$ 32,000$ & $\$ 37,500$ & $\$ 70,000$ & $\$ 90,000$ \\
\hline Monthly crane costs other time & $\$ 15,000$ & $\$ 32,000$ & $\$ 37,500$ & $\$ 70,000$ & $\$ 90,000$ \\
\hline 6 Month Rental Costs & $\$ 14,000$ & $\$ 29,867$ & $\$ 35,000$ & $\$ 65,333$ & $\$ 84,000$ \\
\hline 9 Month Rental Costs & $\$ 13,500$ & $\$ 28,800$ & $\$ 33,750$ & $\$ 63,000$ & $\$ 81,000$ \\
\hline 12 Month Rental Costs & $\$ 13,000$ & $\$ 27,733$ & $\$ 32,500$ & $\$ 60,667$ & $\$ 78,000$ \\
\hline 1. Assembly Crew Information - (Assembly crew pro & uction rate determi & nes crane product & $\mathrm{n}$ and costs) & & \\
\hline Number of People in Crew & 10 & 10 & 10 & 10 & 10 \\
\hline Number of Crews & 1 & 1 & 1 & 1 & 1 \\
\hline Man Hours/Day & 100 & 100 & 100 & 100 & 100 \\
\hline Number of Man Hours/Wk & 600 & 600 & 600 & 600 & 600 \\
\hline Crane Assembly Rate Days/Turbine & 1.05 & 1.05 & 1.89 & 1.89 & 1.89 \\
\hline & & & & & \\
\hline 2. Crane Crew Information - During Turbine Assemb & & & & & \\
\hline Number of People in Crane Crew & 2 & 2 & 2 & 2 & 3 \\
\hline Number of Cranes and Crew & 1 & 1 & 1 & 1 & 1 \\
\hline Number of Turbines/Crane & 50 & 50 & 50 & 50 & 50 \\
\hline Man Hours/Day & 20 & 20 & 20 & 20 & 30 \\
\hline Estimated Crane Crew Man Hours/Turbine & 21 & 21 & 37.8 & 37.8 & 56.7 \\
\hline Labor Costs/Crane Crew Man Hour & $\$ 65$ & $\$ 65$ & $\$ 65$ & $\$ 65$ & $\$ 65$ \\
\hline Crane Crew Assembly Labor Costs/Turbine & $\$ 1,365$ & $\$ 1,365$ & $\$ 2,457$ & $\$ 2,457$ & $\$ 3,686$ \\
\hline 3. Crane Relocation Information & & & & 列 & \\
\hline Estimated Relocation Hours/Turbine & 6 & 6 & 6 & 8 & 8 \\
\hline Total Relocation Hours & 300 & 300 & 300 & 400 & 400 \\
\hline Total Relocation Hours/Crane & 300 & 300 & 300 & 400 & 400 \\
\hline Relocation Days/Crane & 30 & 30 & 30 & 40 & 40 \\
\hline Estimated Relocation Days/Turbine & 0.6 & 0.6 & 0.6 & 0.8 & 0.8 \\
\hline Crane Crew Relocation Man Hours/Turbine & 12.0 & 12.0 & 12.0 & 16.0 & 24.0 \\
\hline Crane Crew Relocation Labor Costs/Turbine & $\$ 780$ & $\$ 780$ & $\$ 780$ & $\$ 1,040$ & $\$ 1,560$ \\
\hline Crane Costs During Relocation/Turbine & $\$ 0$ & $\$ 0$ & $\$ 0$ & $\$ 0$ & $\$ 0$ \\
\hline & & & & & \\
\hline 3. Totals & & & & & \\
\hline Total Number of Crane Assembly Days/Turbine & 1.65 & 1.65 & 2.49 & 2.69 & 2.69 \\
\hline Total Number of Days Required: & 83 & 83 & 125 & 135 & 135 \\
\hline Total Number of Weeks Required & 13.8 & 13.8 & 20.8 & 22.4 & 22.4 \\
\hline Installed kW per Day & 455 & 455 & 602 & 0 & 558 \\
\hline & & & & & \\
\hline Total Number of Months for Assembly & 3.2 & 3.2 & 4.8 & 5.2 & 5.2 \\
\hline 3 Month Min Crane Rental Costs & $\$ 45,000$ & $\$ 96,000$ & $\$ 112,500$ & $\$ 210,000$ & $\$ 270,000$ \\
\hline Total Crane Rental Charges & $\$ 47,596$ & $\$ 101,538$ & $\$ 179,567$ & $\$ 362,115$ & $\$ 465,577$ \\
\hline Crane Rental Costs/Turbine & $\$ 952$ & $\$ 2,031$ & $\$ 3,591$ & $\$ 7,242$ & $\$ 9,312$ \\
\hline & & & & & \\
\hline 4. Material/Supplies/Incidental Crane Costs & & & & & \\
\hline Meals and Lodging/Person/Day & $\$ 75$ & $\$ 75$ & $\$ 75$ & $\$ 75$ & $\$ 75$ \\
\hline Number of Person-Days & 165 & 165 & 249 & 269 & 403.5 \\
\hline Total Meals and Lodging Costs & $\$ 12,375$ & $\$ 12,375$ & $\$ 18,675$ & $\$ 20,175$ & $\$ 30,263$ \\
\hline Meals and Lodging/Turbine & $\$ 248$ & $\$ 248$ & $\$ 374$ & $\$ 404$ & $\$ 605$ \\
\hline & & & & & \\
\hline 5. Fuel & & & & & \\
\hline Fuel Cost/Gallon & $\$ 1.50$ & $\$ 1.50$ & $\$ 1.50$ & $\$ 1.50$ & $\$ 1.50$ \\
\hline Gallons of Fuel/Week & 220 & 250 & 250 & 250 & 650 \\
\hline Total Cost of Fuel & $\$ 4,538$ & $\$ 5,156$ & $\$ 7,781$ & $\$ 8,406$ & $\$ 21,856$ \\
\hline Fuel Cost/Turbine & $\$ 91$ & $\$ 103$ & $\$ 156$ & $\$ 168$ & $\$ 437$ \\
\hline & & & & & \\
\hline 6. Cribbing & & & & & \\
\hline Cribbing Cost/sq ft & $\$ 2.50$ & $\$ 2.50$ & $\$ 2.50$ & $\$ 2.50$ & $\$ 2.50$ \\
\hline Required Cribbing sq ft/Turbine & 2615 & 3800 & 11900 & 11900 & 16150 \\
\hline Cribbing Costs/Turbine & $\$ 131$ & $\$ 190$ & $\$ 595$ & $\$ 595$ & $\$ 808$ \\
\hline & & & & & \\
\hline 7. Mobilization and Demobilization & & & & & \\
\hline Crane Assembly and Disassembly Hours & 24 & 48 & 48 & 80 & 160 \\
\hline Lampson Supervisor Hours & 24 & 48 & 48 & 80 & 160 \\
\hline Lampson Supervisor Hourly Cost & $\$ 75$ & $\$ 75$ & $\$ 75$ & $\$ 75$ & $\$ 75$ \\
\hline Number of Iron Workers & 4 & 4 & 4 & 4 & 6 \\
\hline Man Hours for Iron Workers & 96 & 192 & 192 & 320 & 960 \\
\hline Iron Worker Hourly Cost & $\$ 65$ & $\$ 65$ & $\$ 65$ & $\$ 65$ & $\$ 65$ \\
\hline Crane Rental Period (Months) During Assembly & 0.1 & 0.3 & 0.3 & 0.5 & 0.9 \\
\hline Crane Rental Cost & $\$ 2,045$ & $\$ 8,727$ & $\$ 10,227$ & $\$ 31,818$ & $\$ 81,818$ \\
\hline Total Labor Costs & $\$ 8,040$ & $\$ 16,080$ & $\$ 16,080$ & $\$ 26,800$ & $\$ 74,400$ \\
\hline Truck Crane 1 Hourly Cost & $\$ 185$ & $\$ 185$ & $\$ 185$ & $\$ 185$ & $\$ 185$ \\
\hline Truck Crane 2 Hourly Cost & $\$ 325$ & $\$ 325$ & $\$ 325$ & $\$ 325$ & $\$ 325$ \\
\hline Truck Crane 3 Hourly Costs & & & & & \\
\hline Total Truck Crane Costs & $\$ 12,240$ & $\$ 24,480$ & $\$ 24,480$ & $\$ 40,800$ & $\$ 81,600$ \\
\hline Total Transportation Freight in/out & $\$ 40,000$ & $\$ 60,000$ & $\$ 70,000$ & $\$ 80,000$ & $\$ 120,000$ \\
\hline Transport Days in/out & 6 & 10 & 10 & 10 & 14 \\
\hline Transport Hours in/out & 48 & 80 & 80 & 80 & 112 \\
\hline Crane Rental During Transport & $\$ 4,091$ & $\$ 14,545$ & $\$ 17,045$ & $\$ 31,818$ & $\$ 57,273$ \\
\hline SubTotal & $\$ 66,416$ & $\$ 123,833$ & $\$ 137,833$ & $\$ 211,236$ & $\$ 415,091$ \\
\hline Mob/Demob Costs/Turbine & $\$ 1,328$ & $\$ 2,477$ & $\$ 2,757$ & $\$ 4,225$ & $\$ 8,302$ \\
\hline & & & & & \\
\hline Crane assembly costs & $\$ 22,325$ & $\$ 49287$ & $\$ 50.787$ & $\$ 99418$ & $\$ 237818$ \\
\hline Total Crane Costs & $\$ 244,713$ & $\$ 359,652$ & $\$ 535,456$ & $\$ 806,533$ & $\$ 1,235,437$ \\
\hline Total Crane time (months) & 3.72 & 4.17 & 5.79 & 6.54 & 7.63 \\
\hline Loaded Hourly Crane Costs & $\$ 373.91$ & $\$ 489.68$ & $\$ 525.59$ & $\$ 701.05$ & $\$ 920.28$ \\
\hline
\end{tabular}




\begin{tabular}{|c|c|c|c|c|c|c|c|c|c|c|}
\hline Initial Assumptions & Turbine Assembly & Crane Assembly & & & & & & & & \\
\hline Work Hours/Day & 10 & 8 & & & & & & & & \\
\hline Number of Days/Wk & 6 & 5 & & & & & & & & \\
\hline Number of Weeks/Year & 52 & 52 & & & & & & & & \\
\hline Number of Weeks/Month & 4.3333 & 4.4000 & & & & & & & & \\
\hline Number of Days/Month & 26 & 22 & & & & & & & & \\
\hline Number of Turbines & 50 & & & & & & & & & \\
\hline Turbine Rating (kW) & 750 & & & 1500 & & 2500 & 35 & & 5000 & \\
\hline Crane Type & $4100 \mathrm{~S} 1$ & $4600 \mathrm{~S} 4$ & $4600 \$ 5$ & what if & LTL-600 & LTL-850 & LTL-1000 & LTL-1100 & LTL-1100 & LTL-1200 \\
\hline Monthly Crane Costs during turbine assembly (60hr wk) & $\$ 15,000$ & $\$ 32,000$ & $\$ 37,500$ & $\$ 70,000$ & $\$ 90,000$ & $\$ 100,000$ & $\$ 125,000$ & $\$ 140,000$ & $\$ 140,000$ & $\$ 175,000$ \\
\hline Monthly crane costs other time & $\$ 15,000$ & $\$ 32,000$ & $\$ 37,500$ & $\$ 70,000$ & $\$ 90,000$ & $\$ 100,000$ & $\$ 125,000$ & $\$ 140,000$ & $\$ 140,000$ & $\$ 175,000$ \\
\hline 6 Month Rental Costs & $\$ 14,000$ & $\$ 29,867$ & $\$ 35,000$ & $\$ 65,333$ & $\$ 84,000$ & $\$ 93,333$ & $\$ 116,667$ & $\$ 130,667$ & $\$ 130,667$ & $\$ 163,333$ \\
\hline 9 Month Rental Costs & $\$ 13,500$ & $\$ 28,800$ & $\$ 33,750$ & $\$ 63,000$ & $\$ 81,000$ & $\$ 90,000$ & $\$ 112,500$ & $\$ 126,000$ & $\$ 126,000$ & $\$ 157,500$ \\
\hline 12 Month Rental Costs & $\$ 13,000$ & $\$ 27,733$ & $\$ 32,500$ & $\$ 60,667$ & $\$ 78,000$ & $\$ 86,667$ & $\$ 108,333$ & $\$ 121,333$ & $\$ 121,333$ & $\$ 151,667$ \\
\hline \multicolumn{11}{|c|}{ 1. Assembly Crew Information - (Assembly crew production rate determines crane production and costs) } \\
\hline Number of People in Crew & 10 & 10 & 10 & 10 & 10 & 10 & 10 & 10 & 10 & 10 \\
\hline Number of Crews & 1 & 1 & 1 & 1 & 1 & 1 & 1 & 1 & 1 & \\
\hline Man Hours/Day & 100 & 100 & 100 & 100 & 100 & 100 & 100 & 100 & 100 & 100 \\
\hline Number of Man Hours/Wk & 600 & 600 & 600 & 600 & 600 & 600 & 600 & 600 & 600 & 600 \\
\hline Crane Assembly Rate Days/Turbine & 0.58 & 0.58 & 0.82 & 0.82 & 0.82 & 1.62 & 2.29 & 2.29 & 3.92 & 3.92 \\
\hline & & & & & & & & & & \\
\hline 2. Crane Crew Information - During Turbine Assemb & & & & & & & & & & \\
\hline Number of People in Crane Crew & 2 & 2 & 2 & 2 & 3 & 3 & 3 & 3 & 3 & \\
\hline Number of Turbines/Crane & 50 & 50 & 50 & 50 & 50 & 50 & 50 & 50 & 50 & 50 \\
\hline Man Hours/Day & 20 & 20 & 20 & 20 & 30 & 30 & 30 & 30 & 30 & 30 \\
\hline Estimated Crane Crew Man Hours/Turbine & 11.6 & 11.6 & 16.4 & 16.4 & 24.6 & 48.6 & 68.7 & 68.7 & 117.6 & 117.6 \\
\hline Labor Costs/Crane Crew Man Hour & $\$ 65$ & $\$ 65$ & $\$ 65$ & $\$ 65$ & $\$ 65$ & $\$ 65$ & $\$ 65$ & $\$ 65$ & $\$ 65$ & $\$ 65$ \\
\hline Crane Crew Assembly Labor Costs/Turbine & $\$ 754$ & $\$ 754$ & $\$ 1,066$ & $\$ 1,066$ & $\$ 1,599$ & $\$ 3,159$ & $\$ 4,466$ & $\$ 4,466$ & $\$ 7,644$ & $\$ 7,644$ \\
\hline 3. Crane Relocation Information & & & & & & & & & & \\
\hline Estimated Relocation Hours/Turbine & 6 & 6 & 6 & 8 & 8 & 14 & 21 & 25 & 25 & 33 \\
\hline $\begin{array}{l}\text { Total Relocation Hours } \\
\end{array}$ & 300 & 300 & 300 & 400 & 400 & 700 & 1050 & 1250 & 1250 & 1650 \\
\hline Total Relocation Hours/Crane & 300 & 300 & 300 & 400 & 400 & 700 & 1050 & 1250 & 1250 & 1650 \\
\hline Relocation Days/Crane & 30 & 30 & 30 & 40 & 40 & 70 & 105 & 125 & 125 & 165 \\
\hline Estimated Relocation Days/Turbine & 0.6 & 0.6 & 0.6 & 0.8 & 0.8 & 1.4 & 2.1 & 2.5 & 2.5 & 3.3 \\
\hline Crane Crew Relocation Man Hours/Turbine & 12.0 & 12.0 & 12.0 & 16.0 & 24.0 & 42.0 & 63.0 & 75.0 & 75.0 & 99.0 \\
\hline Crane Crew Relocation Labor Costs/Turbine & $\$ 780$ & $\$ 780$ & $\$ 780$ & $\$ 1,040$ & $\$ 1,560$ & $\$ 2,730$ & $\$ 4,095$ & $\$ 4,875$ & $\$ 4,875$ & $\$ 6,435$ \\
\hline Crane Costs During Relocation/Turbine & $\$ 0$ & $\$ 0$ & $\$ 0$ & $\$ 0$ & $\$ 0$ & $\$ 0$ & $\$ 0$ & $\$ 0$ & $\$ 0$ & $\$ 0$ \\
\hline 3. Totals & & & & & & & & & & \\
\hline Total Number of Crane Assembly Days/Turbine & 1.18 & 1.18 & 1.42 & 1.62 & 1.62 & 302 & 4.39 & 479 & 6.42 & 7.22 \\
\hline Total Number of Days Required: & 59 & 59 & 71 & 81 & 81 & 151 & 220 & 240 & 321 & $\begin{array}{ll}7.261 \\
361\end{array}$ \\
\hline Total Number of Weeks Required & 9.8 & 9.8 & 11.8 & 13.5 & 13.5 & 25.2 & 36.6 & 39.9 & 53.5 & 60.2 \\
\hline Installed kW per Day & 636 & 636 & 1056 & 0 & 926 & 828 & 797 & 731 & 545 & 693 \\
\hline & & & & & & & & & & \\
\hline Total Number of Months for Assembly & 2.3 & 2.3 & 2.7 & 3.1 & 3.1 & 5.8 & 8.4 & 9.2 & 12.3 & 13.9 \\
\hline 3 Month Min Crane Rental Costs & $\$ 45,000$ & $\$ 96,000$ & $\$ 112,500$ & $\$ 210,000$ & $\$ 270,000$ & $\$ 300,000$ & $\$ 375,000$ & $\$ 420,000$ & $\$ 420,000$ & $\$ 525,000$ \\
\hline \begin{tabular}{|l|} 
Meals and Lodging/Person/Day \\
Number of Person-Days
\end{tabular} & $\frac{\$ 15}{118}$ & $\frac{\$ 15}{118}$ & $\frac{\$ 15}{142}$ & $\frac{S 15}{162}$ & 243 & 453 & $\begin{array}{r}\$ 75 \\
658.5\end{array}$ & $\$ 7185$ & $\frac{15}{963}$ & $\frac{\$ 75}{1083}$ \\
\hline Total Meals and Lodging Costs & $\$ 8,850$ & $\$ 8,850$ & $\$ 10,650$ & $\$ 12,150$ & $\$ 18,225$ & $\$ 33,975$ & $\$ 49,388$ & $\$ 53,888$ & $\$ 72,225$ & $\$ 81,225$ \\
\hline Meals and Lodging/Turbine & $\$ 177$ & $\$ 177$ & $\$ 213$ & $\$ 243$ & $\$ 365$ & $\$ 680$ & $\$ 988$ & $\$ 1,078$ & $\$ 1,445$ & $\$ 1,625$ \\
\hline & & & & & & & & & & \\
\hline 5. Fuel & & & & & & & & & & \\
\hline Fuel Cost/Gallon & $\$ 1.50$ & $\$ 1.50$ & $\$ 1.50$ & $\$ 1.50$ & $\$ 1.50$ & $\$ 1.50$ & $\$ 1.50$ & $\$ 1.50$ & $\$ 1.50$ & $\$ 1.50$ \\
\hline Gallons of Fuel/Week & 220 & 250 & 250 & 250 & 650 & 650 & 700 & 700 & 700 & 750 \\
\hline Total Cost of Fuel & $\$ 3,245$ & $\$ 3,688$ & $\$ 4,438$ & $\$ 5,063$ & $\$ 13,163$ & $\$ 24,538$ & $\$ 38.413$ & $\$ 41.913$ & $\$ 56,175$ & $\$ 67,688$ \\
\hline Fuel Cost/Turbine & $\$ 65$ & $\$ 74$ & $\$ 89$ & $\$ 101$ & $\$ 263$ & $\$ 491$ & $\$ 768$ & $\$ 838$ & $\$ 1,124$ & $\$ 1,354$ \\
\hline & & & & & & & & & & \\
\hline 6. Cribbing & & & & & & & & & & \\
\hline Cribbing Cost/sq ft & $\$ 2.50$ & $\$ 2.50$ & $\$ 2.50$ & $\$ 2.50$ & $\$ 2.50$ & $\$ 2.50$ & $\$ 2.50$ & $\$ 2.50$ & $\$ 2.50$ & $\$ 2.50$ \\
\hline Required Cribbing sq ft/Turbine & 2615 & 3800 & 11900 & 11900 & 16150 & 10750 & 16150 & 18850 & 18850 & 18850 \\
\hline Cribbing Costs/Turbine & $\$ 131$ & $\$ 190$ & $\$ 595$ & $\$ 595$ & $\$ 808$ & $\$ 538$ & $\$ 808$ & $\$ 943$ & $\$ 943$ & $\$ 943$ \\
\hline & & & & & & & & & & \\
\hline 7. Mobilization and Demobilization & & & & & & & & & & \\
\hline Crane Assembly and Disassembly Hours & 24 & 48 & 48 & 80 & 160 & 192 & 360 & 360 & 360 & 480 \\
\hline Lampson Supervisor Hours & 24 & 48 & 48 & 80 & 160 & 192 & 360 & 360 & 360 & 480 \\
\hline Lampson Supervisor Hourly Cost & $\$ 75$ & $\$ 75$ & $\$ 75$ & $\$ 75$ & $\$ 75$ & $\$ 75$ & $\$ 75$ & $\$ 75$ & $\$ 75$ & $\$ 75$ \\
\hline Number of Iron Workers & 4 & 4 & 4 & 4 & 6 & 6 & 8 & 8 & 8 & 10 \\
\hline Man Hours for Iron Workers & 96 & 192 & 192 & 320 & 960 & 1152 & 2880 & 2880 & 2880 & 4800 \\
\hline Iron Worker Hourly Cost & $\$ 65$ & $\$ 65$ & $\$ 65$ & $\$ 65$ & $\$ 65$ & $\$ 65$ & $\$ 65$ & $\$ 65$ & $\$ 65$ & $\$ 65$ \\
\hline Crane Rental Period (Months) During Assembly & 0.1 & 0.3 & 0.3 & 0.5 & 0.9 & 1.1 & 2.0 & 2.0 & 2.0 & 2.7 \\
\hline Crane Rental Cost & $\$ 2,045$ & $\$ 8,727$ & $\$ 10,227$ & $\$ 31,818$ & $\$ 81,818$ & $\$ 109,091$ & $\$ 255,682$ & $\$ 286,364$ & $\$ 286,364$ & $\$ 477,273$ \\
\hline Total Labor Costs & $\$ 8,040$ & $\$ 16,080$ & $\$ 16,080$ & $\$ 26,800$ & $\$ 74,400$ & $\$ 89,280$ & $\$ 214,200$ & $\$ 214,200$ & $\$ 214,200$ & $\$ 348,000$ \\
\hline Truck Crane 1 Hourly Cost & $\$ 185$ & $\$ 185$ & $\$ 185$ & $\$ 185$ & $\$ 185$ & $\$ 185$ & $\$ 185$ & $\$ 185$ & $\$ 185$ & $\$ 185$ \\
\hline Truck Crane 2 Hourly Cost & $\$ 325$ & $\$ 325$ & $\$ 325$ & $\$ 325$ & $\$ 325$ & $\$ 350$ & $\$ 350$ & $\$ 350$ & $\$ 350$ & $\$ 400$ \\
\hline Truck Crane 3 Hourly Costs & & & & & & & & $\$ 185$ & $\$ 185$ & $\$ 185$ \\
\hline Total Truck Crane Costs & $\$ 12,240$ & $\$ 24,480$ & $\$ 24,480$ & $\$ 40,800$ & $\$ 81,600$ & $\$ 102,720$ & $\$ 192,600$ & $\$ 259,200$ & $\$ 259,200$ & $\$ 369,600$ \\
\hline Total Transportation Freight in/out & $\$ 40,000$ & $\$ 60,000$ & $\$ 70,000$ & $\$ 80,000$ & $\$ 120,000$ & $\$ 120,000$ & $\$ 200,000$ & $\$ 220,000$ & $\$ 220,000$ & $\$ 220,000$ \\
\hline Transport Days in/out & 6 & 10 & 10 & 10 & 14 & 14 & 20 & 20 & 24 & 24 \\
\hline Transport Hours in/out & 48 & 80 & 80 & 80 & 112 & 112 & 160 & 160 & 192 & 192 \\
\hline Crane Rental During Transport & $\$ 4,091$ & $\$ 14,545$ & $\$ 17,045$ & $\$ 31,818$ & $\$ 57,273$ & $\$ 63,636$ & $\$ 113,636$ & $\$ 127,273$ & $\$ 152,727$ & $\$ 190,909$ \\
\hline SubTotal & $\$ 66,416$ & $\$ 123,833$ & $\$ 137,833$ & $\$ 211,236$ & $\$ 415,091$ & $\$ 484,727$ & $\$ 976,118$ & $\$ 1,107,036$ & $\$ 1,132,491$ & $\$ 1,605,782$ \\
\hline Mob/Demob Costs/Turbine & $\$ 1,328$ & $\$ 2,477$ & $\$ 2,757$ & $\$ 4,225$ & $\$ 8,302$ & $\$ 9,695$ & $\$ 19,522$ & $\$ 22,141$ & $\$ 22,650$ & $\$ 32,116$ \\
\hline
\end{tabular}




\begin{tabular}{|c|c|c|c|c|c|c|c|c|c|c|}
\hline Initial Assumptions & Turbine Assembly & Crane Assembly & & & & & & & & \\
\hline Work Hours/Day & 10 & 8 & & & & & & & & \\
\hline Number of Days/Wk & 6 & 5 & & & & & & & & \\
\hline Number of Weeks/Year & 52 & 52 & & & & & & & & \\
\hline Number of Weeks/Month & 4.3333 & 4.4000 & & & & & & & & \\
\hline Number of Days/Month & 26 & 22 & & & & & & & & \\
\hline Number of Hours/Month & 260 & 176 & & & & & & & & \\
\hline Number of Turbines & 50 & & & & & & & & & \\
\hline Turbine Rating (kW) & 75 & & & 1500 & & 2500 & 35 & & 500 & \\
\hline Crane Type & 4100 S1 & $4600 \mathrm{S4}$ & $4600 \$ 5$ & what if & LTL-600 & LTL-850 & LTL-1000 & LTL-1100 & LTL-1100 & LTL-1200 \\
\hline Monthly Crane Costs during turbine assembly (60hr wk) & $\$ 15,000$ & $\$ 32,000$ & $\$ 37,500$ & $\$ 70,000$ & $\$ 90,000$ & $\$ 100,000$ & $\$ 125,000$ & $\$ 140,000$ & $\$ 140,000$ & $\$ 175,000$ \\
\hline Monthly crane costs other time & $\$ 15,000$ & $\$ 32,000$ & $\$ 37,500$ & $\$ 70,000$ & $\$ 90,000$ & $\$ 100,000$ & $\$ 125,000$ & $\$ 140,000$ & $\$ 140,000$ & $\$ 175,000$ \\
\hline 6 Month Rental Costs & $\$ 14,000$ & $\$ 29,867$ & $\$ 35,000$ & $\$ 65,333$ & $\$ 84,000$ & $\$ 93,333$ & $\$ 116,667$ & $\$ 130,667$ & $\$ 130,667$ & $\$ 163,333$ \\
\hline 9 Month Rental Costs & $\$ 13,500$ & $\$ 28,800$ & $\$ 33,750$ & $\$ 63,000$ & $\$ 81,000$ & $\$ 90,000$ & $\$ 112,500$ & $\$ 126,000$ & $\$ 126,000$ & $\$ 157,500$ \\
\hline 12 Month Rental Costs & $\$ 13,000$ & $\$ 27,733$ & $\$ 32,500$ & $\$ 60,667$ & $\$ 78,000$ & $\$ 86,667$ & $\$ 108,333$ & $\$ 121,333$ & $\$ 121,333$ & $\$ 151,667$ \\
\hline 1. Assembly Crew Information - (Assembly crew prod & ction rate determin & $s$ crane product & costs) & & & & & & & \\
\hline Number of People in Crew & 10 & 10 & 10 & 10 & 10 & 10 & 10 & 10 & 10 & 10 \\
\hline Number of Crews & 1 & 1 & 1 & 1 & 1 & 1 & 1 & 1 & 1 & \\
\hline Man Hours/Day & 100 & 100 & 100 & 100 & 100 & 100 & 100 & 100 & 100 & 100 \\
\hline Number of Man Hours/Wk & 600 & 600 & 600 & 600 & 600 & 600 & 600 & 600 & 600 & 600 \\
\hline Crane Assembly Rate Days/Turbine & 1.15 & 1.15 & 2.05 & 2.05 & 2.05 & 4.1 & 5.75 & 5.75 & 10.35 & 10.35 \\
\hline & & & & & & & & & & \\
\hline 2. Crane Crew Information - During Turbine Assembl & & & & & & & & & & \\
\hline Number of People in Crane Crew & 2 & 2 & 2 & 2 & 3 & 3 & 3 & 3 & 3 & 3 \\
\hline Number of Cranes and Crew & 1 & 1 & 1 & 1 & 1 & 1 & 1 & 1 & 1 & 1 \\
\hline Number of Turbines/Crane & 50 & 50 & 50 & 50 & 50 & 50 & 50 & 50 & 50 & 50 \\
\hline Man Hours/Day & 20 & 20 & 20 & 20 & 30 & 30 & 30 & 30 & 30 & 30 \\
\hline Estimated Crane Crew Man Hours/Turbine & 23 & 23 & 41 & 41 & 61.5 & 123 & 172.5 & 172.5 & 310.5 & 310.5 \\
\hline Labor Costs/Crane Crew Man Hour & $\$ 65$ & $\$ 65$ & $\$ 65$ & $\$ 65$ & $\$ 65$ & $\$ 65$ & $\$ 65$ & $\$ 65$ & $\$ 65$ & $\$ 65$ \\
\hline Crane Crew Assembly Labor Costs/Turbine & $\$ 1,495$ & $\$ 1,495$ & $\$ 2,665$ & $\$ 2,665$ & $\$ 3,998$ & $\$ 7,995$ & $\$ 11,213$ & $\$ 11,213$ & $\$ 20,183$ & $\$ 20,183$ \\
\hline & & & & & & & & & & \\
\hline 3. Crane Relocation Information & & & & & & & & & & \\
\hline Estimated Relocation Hours/Turbine & 6 & 6 & 6 & 8 & 8 & 14 & 21 & 25 & 25 & 33 \\
\hline Total Relocation Hours & 300 & 300 & 300 & 400 & 400 & 700 & 1050 & 1250 & 1250 & 1650 \\
\hline Total Relocation Hours/Crane & 300 & 300 & 300 & 400 & 400 & 700 & 1050 & 1250 & 1250 & 1650 \\
\hline Relocation Days/Crane & 30 & 30 & 30 & 40 & 40 & 70 & 105 & 125 & 125 & 165 \\
\hline Estimated Relocation Days/Turbine & 0.6 & 0.6 & 0.6 & 0.8 & 0.8 & 1.4 & 2.1 & 2.5 & 2.5 & 3.3 \\
\hline Crane Crew Relocation Man Hours/Turbine & 12.0 & 12.0 & 12.0 & 16.0 & 24.0 & 42.0 & 63.0 & 75.0 & 75.0 & 99.0 \\
\hline Crane Crew Relocation Labor Costs/Turbine & $\$ 780$ & $\$ 780$ & $\$ 780$ & $\$ 1,040$ & $\$ 1,560$ & $\$ 2,730$ & $\$ 4,095$ & $\$ 4,875$ & $\$ 4,875$ & $\$ 6,435$ \\
\hline Crane Costs During Relocation/Turbine & $\$ 0$ & \$o & \$o & $\$ 0$ & \$o & $\$ 0$ & $\$ 0$ & $\$ 0$ & \$o & $\$ 0$ \\
\hline & & & & & & & & & & \\
\hline 3. Totals & & & & & & & & & & \\
\hline Total Number of Crane Assembly Days/Turbine & 1.75 & 1.75 & 2.65 & 2.85 & 2.85 & 5.50 & 7.85 & 8.25 & 12.85 & 13.65 \\
\hline Total Number of Days Required: & 88 & 88 & 133 & 143 & 143 & 275 & 393 & 413 & 643 & 683 \\
\hline Total Number of Weeks Required & 14.6 & 14.6 & 22.1 & 23.8 & 23.8 & 45.8 & 65.4 & 68.8 & 107.1 & 113.8 \\
\hline Installed kW per Day & 429 & 429 & 566 & 0 & 526 & 455 & 446 & 424 & 272 & 366 \\
\hline & & & & & & & & & & \\
\hline Total Number of Months for Assembly & 3.4 & 3.4 & 5.1 & 5.5 & 5.5 & 10.6 & 15.1 & 15.9 & 24.7 & 26.3 \\
\hline 3 Month Min Crane Rental Costs & $\$ 45,000$ & $\$ 96,000$ & $\$ 112,500$ & $\$ 210,000$ & $\$ 270,000$ & $\$ 300,000$ & $\$ 375,000$ & $\$ 420,000$ & $\$ 420,000$ & $\$ 525,000$ \\
\hline Total Crane Rental Charges & $\$ 50,481$ & $\$ 107,692$ & $\$ 191,106$ & $\$ 383,654$ & $\$ 493,269$ & $\$ 987,179$ & $\$ 1,698,317$ & $\$ 1,999,038$ & $\$ 2,998,333$ & $\$ 3,981,250$ \\
\hline Crane Rental Costs/Turbine & $\$ 1,010$ & $\$ 2,154$ & $\$ 3,822$ & $\$ 7,673$ & $\$ 9,865$ & $\$ 19,744$ & $\$ 33,966$ & $\$ 39,981$ & $\$ 59,967$ & $\$ 79,625$ \\
\hline 4. Material/Supplies/Incidental Crane Costs & & & & & & & & & & \\
\hline Meals and Lodging/Person/Day & $\$ 75$ & $\$ 75$ & $\$ 75$ & $\$ 75$ & $\$ 75$ & $\$ 75$ & $\$ 75$ & $\$ 75$ & $\$ 75$ & $\$ 75$ \\
\hline Number of Person-Days & 175 & 175 & 265 & 285 & 427.5 & 825 & 1177.5 & 1237.5 & 1927.5 & 2047.5 \\
\hline Total Meals and Lodging Costs & $\$ 13,125$ & $\$ 13,125$ & $\$ 19,875$ & $\$ 21,375$ & $\$ 32,063$ & $\$ 61,875$ & $\$ 88,313$ & $\$ 92,813$ & $\$ 144,563$ & $\$ 153,563$ \\
\hline Meals and Lodging/Turbine & $\$ 263$ & $\$ 263$ & $\$ 398$ & $\$ 428$ & $\$ 641$ & $\$ 1,238$ & $\$ 1,766$ & $\$ 1,856$ & $\$ 2,891$ & $\$ 3,071$ \\
\hline 5. Fuel & & & & & & & & & & \\
\hline Fuel Cost/Gallon & $\$ 1.50$ & $\$ 1.50$ & $\$ 1.50$ & $\$ 1.50$ & $\$ 1.50$ & $\$ 1.50$ & $\$ 1,50$ & $\$ 1.50$ & $\$ 1.50$ & $\$ 1.50$ \\
\hline Gallons of Fuel/Week & 220 & 250 & 250 & 250 & 650 & 650 & 700 & 700 & 700 & 750 \\
\hline Total Cost of Fuel & $\$ 4,813$ & $\$ 5,469$ & $\$ 8,281$ & $\$ 8,906$ & $\$ 23,156$ & $\$ 44,688$ & $\$ 68,688$ & $\$ 72,188$ & $\$ 112,438$ & $\$ 127,969$ \\
\hline Fuel Cost/Turbine & $\$ 96$ & $\$ 109$ & $\$ 166$ & $\$ 178$ & $\$ 463$ & $\$ 894$ & $\$ 1,374$ & $\$ 1,444$ & $\$ 2,249$ & $\$ 2,559$ \\
\hline & & & & & & & & & & \\
\hline $\begin{array}{l}\text { 6. Cribbing } \\
\text { Cribbing Cost/sg ft }\end{array}$ & $\$ 2.50$ & $\$ 250$ & $\$ 250$ & $\$ 250$ & $\$ 250$ & $\$ 250$ & $\$ 250$ & $\$ 250$ & $\$ 250$ & $\$ 250$ \\
\hline Required Cribbing sq ft/Turbine & 2615 & 3800 & 11900 & 11900 & 16150 & 10750 & 16150 & 18850 & 18850 & 18850 \\
\hline Cribbing Costs/Turbine & $\$ 131$ & $\$ 190$ & $\$ 595$ & $\$ 595$ & $\$ 808$ & $\$ 538$ & $\$ 808$ & $\$ 943$ & $\$ 943$ & $\$ 943$ \\
\hline & & & & & & & & & & \\
\hline 7. Mobilization and Demobilization & & & & & & & & & & \\
\hline Crane Assembly and Disassembly Hours & 24 & 48 & 48 & 80 & 160 & 192 & 360 & 360 & 360 & 480 \\
\hline Lampson Supervisor Hours & 24 & 48 & 48 & 80 & 160 & 192 & 360 & 360 & 360 & 480 \\
\hline Lampson Supervisor Hourly Cost & $\$ 75$ & $\$ 75$ & $\$ 75$ & $\$ 75$ & $\$ 75$ & $\$ 75$ & $\$ 75$ & $\$ 75$ & $\$ 75$ & $\$ 75$ \\
\hline Number of Iron Workers & 4 & 4 & 4 & 4 & 6 & 6 & 8 & 8 & 8 & 10 \\
\hline Man Hours for Iron Workers & 96 & 192 & 192 & 320 & 960 & 1152 & 2880 & 2880 & 2880 & 4800 \\
\hline Iron Worker Hourly Cost & $\$ 65$ & $\$ 65$ & $\$ 65$ & $\$ 65$ & $\$ 65$ & $\$ 65$ & $\$ 65$ & $\$ 65$ & $\$ 65$ & $\$ 65$ \\
\hline Crane Rental Period (Months) During Assembly & 0.1 & 0.3 & 0.3 & 0.5 & 0.9 & 1.1 & 2.0 & 2.0 & 2.0 & 2.7 \\
\hline Crane Rental Cost & $\$ 2,045$ & $\$ 8,727$ & $\$ 10,227$ & $\$ 31,818$ & $\$ 81,818$ & $\$ 109,091$ & $\$ 255,682$ & $\$ 286,364$ & $\$ 286,364$ & $\$ 477,273$ \\
\hline Total Labor Costs & $\$ 8,040$ & $\$ 16,080$ & $\$ 16,080$ & $\$ 26,800$ & $\$ 74,400$ & $\$ 89,280$ & $\$ 214,200$ & $\$ 214,200$ & $\$ 214,200$ & $\$ 348,000$ \\
\hline Truck Crane 1 Hourly Cost & $\$ 185$ & $\$ 185$ & $\$ 185$ & $\$ 185$ & $\$ 185$ & $\$ 185$ & $\$ 185$ & $\$ 185$ & $\$ 185$ & $\$ 185$ \\
\hline Truck Crane 2 Hourly Cost & $\$ 325$ & $\$ 325$ & $\$ 325$ & $\$ 325$ & $\$ 325$ & $\$ 350$ & $\$ 350$ & $\$ 350$ & $\$ 350$ & $\$ 400$ \\
\hline Truck Crane 3 Hourly Costs & & & & & & & & $\$ 185$ & $\$ 185$ & $\$ 185$ \\
\hline Total Truck Crane Costs & $\$ 12,240$ & $\$ 24,480$ & $\$ 24,480$ & $\$ 40,800$ & $\$ 81,600$ & $\$ 102,720$ & $\$ 192,600$ & $\$ 259,200$ & $\$ 259,200$ & $\$ 369,600$ \\
\hline Total Transportation Freight in/out & $\$ 40,000$ & $\$ 60,000$ & $\$ 70,000$ & $\$ 80,000$ & $\$ 120,000$ & $\$ 120,000$ & $\$ 200,000$ & $\$ 220,000$ & $\$ 220,000$ & $\$ 220,000$ \\
\hline Transport Days in/out & 6 & 10 & 10 & 10 & 14 & 14 & 20 & 20 & 24 & 24 \\
\hline Transport Hours in/out & 48 & 80 & 80 & 80 & 112 & 112 & 160 & 160 & 192 & 192 \\
\hline Crane Rental During Transport & $\$ 4,091$ & $\$ 14,545$ & $\$ 17,045$ & $\$ 31,818$ & $\$ 57,273$ & $\$ 63,636$ & $\$ 113,636$ & $\$ 127,273$ & $\$ 152,727$ & $\$ 190,909$ \\
\hline SubTotal & $\$ 66,416$ & $\$ 123,833$ & $\$ 137,833$ & $\$ 211,236$ & $\$ 415,091$ & $\$ 484,727$ & $\$ 976,118$ & $\$ 1,107,036$ & $\$ 1,132,491$ & $\$ 1,605,782$ \\
\hline Mob/Demob Costs/Turbine & $\$ 1,328$ & $\$ 2,477$ & $\$ 2,757$ & $\$ 4,225$ & $\$ 8,302$ & $\$ 9,695$ & $\$ 19,522$ & $\$ 22,141$ & $\$ 22,650$ & $\$ 32,116$ \\
\hline
\end{tabular}




\section{Appendix J}

Scenario 2-Bolting 


\begin{tabular}{|c|c|c|c|c|c|c|c|c|c|c|c|c|c|c|c|}
\hline \multirow{2}{*}{\multicolumn{2}{|c|}{\begin{tabular}{|r|} 
Number of People in Crew: 10 \\
Hours per Day: 10 \\
Days per Week 6
\end{tabular}}} & \multicolumn{2}{|c|}{$100 \%$} & \multirow{2}{*}{\multicolumn{2}{|c|}{ 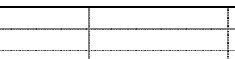 }} & \multirow{2}{*}{$x$} & \multirow{2}{*}{ 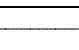 } & \multirow{2}{*}{ 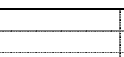 } & & \multirow{2}{*}{ 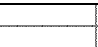 } & & & & & \multirow{2}{*}{ 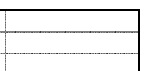 } \\
\hline & & & & & & & & & & & & & & & \\
\hline Turbine Rating $(\mathrm{kW}):$ & & 750 & & & 1500 & & & 2500 & & & 3500 & & & 5000 & \\
\hline Rotor Diameter $(m)$ : & MHRS & \begin{tabular}{|c|}
50 \\
Labor Costs
\end{tabular} & $\begin{array}{l}\text { Equip \& } \\
\text { Material } \\
\end{array}$ & MHRS & $\begin{array}{l}66 \\
\text { Labor Costs }\end{array}$ & $\begin{array}{l}\text { Equip \& } \\
\text { Material }\end{array}$ & MHRS & $\begin{array}{r}85 \\
\text { Labor Costs }\end{array}$ & Equip \& Material & MHRS & $\begin{array}{c}100 \\
\text { Labor Costs }\end{array}$ & Equip \& Material & MHRS & $\begin{array}{l}\frac{120}{\text { Labor Costs }} \\
\end{array}$ & Equip \& Material \\
\hline 1. Receive, Uncrate Nacelle, Blades, Rotors \& Towers & & & & 62. & $\$ 2,498$ & & 75 & $\$ 3,023$ & & 96 & $\$ 3,869$ & & 142 & $\$ 5,723$ & \\
\hline 2. Fabricate Tower Assembly Area & & & & 14 & $\$ 544$ & $\$ 1,289$ & 14 & $\$ 544$ & $\$ 1,289$ & 14 & $\$ 544$ & $\$ 1,289$ & 14 & $\$ 544$ & $\$ 1,289$ \\
\hline 3. Field Fabrication Tower Sections & & & & 101 & $\$ 4,135$ & $\$ 6,621$ & 333 & $\$ 13,647$ & $\$ 20,701$ & 588 & $\$ 24,142$ & $\$ 34,315$ & 952 & $\$ 39,128$ & $\$ 51,509$ \\
\hline 4. Rig \& Set Tower Sections & & & & 132 & $\$ 5,320$ & & 243 & $\$ 9,793$ & & 354 & $\$ 14,265$ & & 619 & $\$ 24,810$ & \\
\hline 5. Grout and Torque Bases & & & & 40 & $\$ 1,612$ & $\$ 850$ & 59 & $\$ 2,377$ & $\$ 950$ & 70 & $\$ 2,822$ & $\$ 1,120$ & 87 & $\$ 3,506$ & $\$ 1,650$ \\
\hline 6. Rig Blades, Assemble Rotors In Air & & & & 61 & $\$ 2,447$ & $\$ 250$ & 95 & $\$ 3,816$ & $\$ 500$ & 112 & $\$ 4,513$ & $\$ 700$ & 164 & $\$ 6,626$ & $\$ 1,000$ \\
\hline 7. Rig \& Set Nacelle & & & & 57 & $\$ 2,317$ & & 133 & $\$ 6,488$ & & 174 & $\$ 8,550$ & & 280 & $\$ 13,370$ & \\
\hline 8. Install Safety Equipment & & & & 12 & $\$ 484$ & & 20 & $\$ 806$ & & 24 & $\$ 968$ & & 36 & $\$ 1,450$ & \\
\hline 9. General Conditions & & & & & $\$ 2,172$ & $\$ 5,713$ & & $\$ 2,459$ & $\$ 10,789$ & & $\$ 2,459$ & $\$ 10,789$ & & $\$ 8,729$ & $\$ 20,941$ \\
\hline 10. Margin@ @ 10\% & & & & & $\$ 2,153$ & 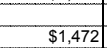 & & $\$ 4,295$ & $\$ 3,423$ & & $\$ 6,213$ & $\$ 4,821$ & & $\$ 10,389$ & $\$ 7,639$ \\
\hline Subtotal Per Turbine & & & & 479 & $\$ 23,682$ & $\$ 16,195$ & 972 & $\$ 47,248$ & $\$ 37,652$ & 1432 & $\$ 68,345$ & $\$ 53,034$ & 2294 & $\$ 114,274$ & $\$ 84,028$ \\
\hline Percent of Total & & & & & $59 \%$ & $41 \%$ & & $56 \%$ & $44 \%$ & & $56 \%$ & $44 \%$ & & & $42 \%$ \\
\hline Project Total (50 Turbines) & & & & 23947.239 & $\$ 1,184,123$ & $\$ 809,752$ & 48596 & $\$ 2,362,424$ & $\$ 1,882,619$ & 71616 & $\$ 3,417,233$ & $\$ 2,651,696$ & 114721 & $\$ 5,713,703$ & $\$ 4,201,393$ \\
\hline Total All Costs & & & & & $\$ 1,993,875$ & & & $\$ 4,245,043$ & & & $\$ 6,068,929$ & & & $\$ 9,915,096$ & \\
\hline Total cost per Turbine & & & & & $\$ 39,878$ & & & $\$ 84,901$ & & & $\$ 121,379$ & & & $\$ 198,302$ & \\
\hline Estimated Assembly Rate - Items 2,5 (Hours) & & & & & 189 & & & 376 & & & 528 & & & & \\
\hline Estimated Assembly Rate - Items 2,5 (Days) & & & & & 1.9 & & & 3.8 & & & 5.3 & & & 9.0 & \\
\hline Total Costs/kW & & 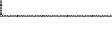 & & & $\$ 26.49$ & & & $\$ 34.00$ & & & $\$ 35.12$ & & & $\$ 39.85$ & \\
\hline Labor Costs $/ k W$ & & & & & $\$ 15.73$ & & & $\$ 18.92$ & & & $\$ 19.78$ & & & $\$ 22.96$ & \\
\hline Equip.Mater Costs/kW & & & & & $\$ 10.76$ & & & $\$ 15.08$ & & & $\$ 15.35$ & & & $\$ 16.89$ & \\
\hline & & & & & & & & & & & & & & & \\
\hline $\begin{array}{l}\text { Total CostSSwept Area } \\
\text { Man-Hours Swept Area }\end{array}$ & & 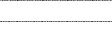 & & & $\$ 11.66$ & & & $\$ 14.96$ & & & $\$ 15.45$ & & & $\$ 17.53$ & \\
\hline $\begin{array}{l}\text { Man-lours/Sweept Area } \\
\text { Labor Costs/Swept Area }\end{array}$ & & - & - & & $\frac{0.14}{\$ 6.92}$ & & & $\frac{0.17}{\$ 8.33}$ & & & $\frac{0.18}{\$ 8.70}$ & & & $\frac{0.20}{\$ 10.10}$ & \\
\hline Equip, Mater Costs/Swept Area & & & & & $\$ 4.73$ & & & $\$ 6.64$ & & & $\$ 6.75$ & & & $\$ 7.43$ & \\
\hline Total Costs/Hub Heigh & & & & 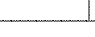 & $\$ 465$ & & & $\$ 768$ & & & 34 & & & $\$ 1,271$ & \\
\hline Labor Costs/Hub Heigh & & & & & \$476 & & & $\$ 428$ & & & $\$ 526$ & & & $\frac{9,211}{\$ 733}$ & \\
\hline Equip.Mater Costs/Hub Heigh & & & & & $\$ 189$ & & & $\$ 341$ & & & $\$ 408$ & & & $\$ 539$ & \\
\hline & & & & & & & - & & & 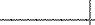 & & & 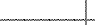 & & \\
\hline Item 1 Hourly Rates & & & & $\$ 40.30$ & & & $\$ 40.31$ & & & $\$ 40.30$ & & & $\$ 40.30$ & & \\
\hline Item 2 Hourly Rates & & & & $\$ 40.30$ & & & $\$ 40.30$ & & & $\$ 40.30$ & & & $\$ 40.08$ & & \\
\hline Item 3 Hourly Rates & & & & $\$ 40.30$ & & & $\$ 40.29$ & & & $\$ 40.31$ & & & $\$ 40.30$ & & \\
\hline Item 4 Hourly Rates & & & & $\$ 40.31$ & & & $\$ 40.30$ & & & $\$ 40.29$ & & & $\$ 40.31$ & & \\
\hline Item 5 Hourly Rates & & & & $\$ 40.65$ & & & $\$ 48.78$ & & & $\$ 49.14$ & & & $\$ 47.75$ & & \\
\hline $\begin{array}{l}\text { Item } 6 \text { Hourly Rates } \\
\text { Combined Hourly Rate }\end{array}$ & & & & $\begin{array}{l}\$ 40.33 \\
\$ 55.135\end{array}$ & & & $\begin{array}{l}\$ 40.30 \\
\$ 54.58\end{array}$ & & & $\begin{array}{l}\$ 40.33 \\
\$ 5.70\end{array}$ & & & $\begin{array}{l}\$ 40.28 \\
\$ 57.38\end{array}$ & & \\
\hline & & & & & & & & & & & & & & & \\
\hline Item 1 Percent of L & & & & $11 \%$ & & & $6 \%$ & & & $6 \%$ & & & $5 \%$ & & \\
\hline Item 2 Percent of Labor & & & & $22 \%$ & & & $21 \%$ & & & $21 \%$ & & & $22 \%$ & & \\
\hline Item 3 Percent of Labor & & & & $7 \%$ & & & $5 \%$ & & & $4 \%$ & & & $3 \%$ & & \\
\hline $\begin{array}{l}\text { Item } 4 \text { Percent of Labor } \\
\text { Item } 5 \text { Percent of Labor }\end{array}$ & & & & $\begin{array}{ll}10 \% \\
10 \%\end{array}$ & & & $8 \%$ & & & $7 \%$ & & & $6 \%$ & & \\
\hline$\frac{\text { Item } 5 \text { Percent of Labor }}{\text { Item } 6 \text { Percent of Labor }}$ & & & & $\frac{10 \%}{2 \%}$ & & & $\frac{14 \%}{2 \%}$ & & & $\frac{13 \%}{1 \%}$ & & & $\frac{12 \%}{10 \%}$ & & \\
\hline Item 7 Percent of Labor & & & & $9 \%$ & & & $\frac{20}{5 \%}$ & & & $4 \%$ & & & $8 \%$ & & \\
\hline Item 8 Percent of Labor & & & & $9 \%$ & & & $9 \%$ & & & $9 \%$ & & & $9 \%$ & & \\
\hline
\end{tabular}




\begin{tabular}{|c|c|c|c|c|c|c|c|c|c|c|c|c|c|c|c|}
\hline \multirow{2}{*}{\multicolumn{2}{|c|}{\begin{tabular}{|c|} 
Number of People in Crew: 10 \\
Hours per Day: 10 \\
Days per Week 6 \\
\end{tabular}}} & \multicolumn{2}{|c|}{$75 \%$} & & & & & & & & & & & & \\
\hline & & & & & & & & & & & & & & & \\
\hline \multirow{2}{*}{\begin{tabular}{|r|} 
Turbine Rating $(\mathrm{kW})$ \\
Rotor Diameter $(\mathrm{m})$ \\
\end{tabular}} & \multicolumn{3}{|c|}{$\begin{array}{c}750 \\
50\end{array}$} & \multicolumn{3}{|c|}{1500} & \multicolumn{3}{|c|}{2500} & \multicolumn{3}{|c|}{$\begin{array}{l}3500 \\
100\end{array}$} & \multicolumn{3}{|c|}{5000} \\
\hline & MHRS & $\begin{array}{c}50 \\
\text { Labor Costs }\end{array}$ & $\begin{array}{l}\text { Equip \& } \\
\text { Material }\end{array}$ & MHRS & \begin{tabular}{|c|}
\multicolumn{6}{c}{66} \\
Labor Costs
\end{tabular} & $\begin{array}{l}\text { Equip \& } \\
\text { Material }\end{array}$ & MHRS & $\begin{array}{r}85 \\
\text { Labor Costs }\end{array}$ & Equip \& Material & MHRS & $\begin{array}{c}100 \\
\text { Labor Costs }\end{array}$ & Equip \& Material & MHRS & $\begin{aligned} & 120 \\
& \text { Labor Costs }\end{aligned}$ & Equip \& Material \\
\hline 1. Receive, Uncrate Nacelle, Blades, Rotors \& Towers & & & & 40 & $\$ 1,400$ & & 75 & $\$ 2,625$ & & 96 & $\$ 3,360$ & & 142 & $\$ 4,970$ & \\
\hline 2. Fabricate Tower Assembly Area & & & & 12 & $\$ 490$ & $\$ 1,160$ & 12 & $\$ 490$ & $\$ 1,160$ & 12 & $\$ 490$ & $\$ 1,160$ & 12 & $\$ 490$ & $\$ 1,160$ \\
\hline 3. Field Fabrication Tower Sections & & & & 91 & $\$ 3,722$ & $\$ 5,959$ & 300 & $\$ 12,282$ & $\$ 18,631$ & 530 & $\$ 21,728$ & $\$ 30,883$ & 857 & $\$ 35,215$ & $\$ 46,358$ \\
\hline 4. Rig \& Set Tower Sections & & & & 60 & $\$ 2,100$ & & 110 & $\$ 3,850$ & & 161 & $\$ 5,635$ & & 282 & $\$ 9,870$ & \\
\hline 5. Grout and Torque Bases & & & & 40 & $\$ 1,400$ & $\$ 850$ & 59 & $\$ 2,065$ & $\$ 950$ & 70 & $\$ 2,450$ & $\$ 1,120$ & 87 & $\$ 3,045$ & $\$ 1,650$ \\
\hline 6. Rig Blades, Assemble Rotors In Air & & & & 57 & $\$ 2,002$ & $\$ 250$ & 87 & $\$ 3,031$ & $\$ 500$ & 101 & $\$ 3,549$ & $\$ 700$ & 147 & $\$ 5,156$ & $\$ 1,000$ \\
\hline 7. Rig \& Set Nacelle & & & & 22 & $\$ 770$ & & 52 & $\$ 2,537$ & & 68 & $\$ 3,341$ & & 109 & $\$ 5,205$ & \\
\hline 8. Install Safety Equipment & & & & 12 & $\$ 420$ & & 20 & $\$ 700$ & & 24 & $\$ 840$ & & 36 & $\$ 1,260$ & \\
\hline 9. General Conditions & & & & & $\$ 1,629$ & $\$ 4,285$ & & $\$ 1,844$ & $\$ 8,092$ & & $\$ 1,844$ & $\$ 8,092$ & & $\$ 6,547$ & $\$ 15,706$ \\
\hline 10. Margin @ 10\% & & & & & $\$ 1,393$ & $\$ 1,250$ & & $\$ 2,942$ & $\$ 2,933$ & & $\$ 4.324$ & $\$ 4.196$ & & $\$ 7176$ & 56587 \\
\hline & & & & & & & & & & & & & & & \\
\hline Subtotal Per Turbine & & & & 335 & $\$ 15,326$ & $\$ 13,754$ & 715 & $\$ 32,366$ & $\$ 32,266$ & 1062 & $\$ 47,561$ & $\$ 46,151$ & 1673 & $\$ 78,932$ & $\$ 72,461$ \\
\hline Percent of Total & & & & 16746.016 & $53 \%$ & $468 \%$ & & $\begin{aligned} 50 \% \\
\$ 161308\end{aligned}$ & $\begin{aligned} 50 \% \\
1.613 .33 \\
\end{aligned}$ & 53124 & $51 \%$ & $49 \%$ & 83636 & $52 \%$ & $48 \%$ \\
\hline Project Total (50 Turbines) & & & & $160 / 46.016$ & $\$ 766,296$ & $\$ 687,695$ & $30 / 55$ & $\$ 1,618,308$ & & 53124 & $\$ 2,378,047$ & $\$ 2,307,527$ & 83636 & $\$ 3,946,618$ & \\
\hline Total All Costs & & & & & $\$ 1,453,991$ & & & $\$ 3,231,632$ & & & $\$ 4,685,574$ & & & $\$ 7,569,683$ & \\
\hline Total Cost per Turbing & & & & & $\$ 29,080$ & & & $\$ 64,633$ & & & $\$ 93,711$ & & & $\$ 151,394$ & \\
\hline Estimated Assembly Rate - Items 2,5 (Hour) & & & & & 82 & & & 162 & & & 229 & & & 391 & \\
\hline Estimated Assembly Rate - Items 2,5 (Day $\$$ & & & & & 0.8 & & & 1.6 & & & 2.3 & & & 3.9 & \\
\hline Total Costs/kW & - & & & {[} & $\$ 19.32$ & & & $\$ 25.89$ & & & $\$ 27.12$ & & & $\$ 30.42$ & \\
\hline Labor Costs/kW & & & & & $\$ 10.18$ & & & $\$ 12.96$ & & & $\$ 13.76$ & & & $\$ 15.86$ & \\
\hline Equip.Mater Costs/kW & & & & & $\$ 9.14$ & & & $\$ 12.92$ & & & $\$ 13.35$ & & & $\$ 14.56$ & \\
\hline & 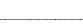 & & & & & & 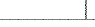 & & & 1 & & & 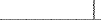 & & \\
\hline Total Cost/Swept Area & & & & & $\$ 8.50$ & & & $\$ 11.39$ & & & $\$ 11.93$ & & & $\$ 13.39$ & \\
\hline Man-Hours/Swept Area & & & & & 0.10 & & & 0.13 & & & 0.14 & & & 0.15 & \\
\hline Labor Costs/Swept Area & & & & & $\$ 4.48$ & & & $\$ 5.70$ & & & $\$ 6.06$ & & & $\$ 6.98$ & \\
\hline Equip, Mater Costs/Swept Area & & & & & $\$ 4.02$ & & & $\$ 5.69$ & & & $\$ 5.88$ & & & $\$ 6.41$ & \\
\hline & & & & & & & & & & & & & & & \\
\hline Total Costs/Hub Height & & & & & $\$ 339$ & & & $\$ 585$ & & & $\$ 721$ & & & $\$ 970$ & \\
\hline Labor Costs/Hub Height & & & & & $\$ 179$ & & & $\$ 293$ & & & $\$ 366$ & & & $\$ 506$ & \\
\hline Equip.Mater Costs/Hub Height & & & & & $\$ 160$ & & & $\$ 292$ & & & $\$ 355$ & & & $\$ 464$ & \\
\hline & (ing & & & & & & & & & & & & & & \\
\hline Item 1 Hourly Rates & & & & $\$ 35.00$ & & & $\$ 35.00$ & & & $\$ 35.00$ & & & $\$ 35.00$ & & \\
\hline Item 2 Hourly Rates & & & & $\$ 35.00$ & & & $\$ 35.00$ & & & $\$ 35.00$ & & & $\$ 35.00$ & & \\
\hline Item 3 Hourly Rates & & & & $\$ 35.00$ & & & $\$ 35.00$ & & & $\$ 35.00$ & & & $\$ 35.00$ & & \\
\hline Item 4 Hourly Rates & & & & $\$ 35.00$ & & & $\$ 35.00$ & & & $\$ 35.00$ & & & $\$ 35.00$ & & \\
\hline Item 5 Hourly Rates & & & & $\$ 35.00$ & & & $\$ 48.78$ & & & $\$ 49.14$ & & & $\$ 47.75$ & & \\
\hline Item 6 Hourly Rates & & & & $\$ 35.00$ & & & $\$ 35.00$ & & & $\$ 35.00$ & & & $\$ 35.00$ & & \\
\hline Combined Hourly Rate & & & & $\$ 51.35$ & & & & & & & & & & & \\
\hline Item 1 Percent of Labor & & & & $9 \%$ & & & $8 \%$ & & & $7 \%$ & & & $6 \%$ & & \\
\hline Item 2 Percent of Labor & & & & $14 \%$ & & & $12 \%$ & & & $12 \%$ & & & $13 \%$ & & \\
\hline Item 3 Percent of Labor & & & & $9 \%$ & & & $6 \%$ & & & $5 \%$ & & & $4 \%$ & & \\
\hline Item 4 Percent of Labor & & & & $13 \%$ & & & $9 \%$ & & & $7 \%$ & & & $7 \%$ & & \\
\hline Item 5 Percent of Labor & & & & $5 \%$ & & & $8 \%$ & & & $7 \%$ & & & $7 \%$ & & \\
\hline Item 6 Percent of Labor & & & & $3 \%$ & & & $2 \%$ & & & $2 \%$ & & & $2 \%$ & & \\
\hline \begin{tabular}{|l|} 
Item 7 Percent of Labor \\
Item 8 Percent of Labot
\end{tabular} & & & & $\frac{11 \%}{9 \%}$ & & & $\frac{6 \%}{9 \%}$ & & & $\frac{4 \%}{9 \%}$ & & & $\frac{8 \%}{9 \%}$ & & \\
\hline
\end{tabular}




\begin{tabular}{|c|c|c|c|c|c|c|c|c|c|c|c|c|c|c|c|}
\hline \multirow{2}{*}{\multicolumn{2}{|c|}{\begin{tabular}{|r} 
Number of People in Crew: 10 \\
Hours per Day: 10 \\
Days per Week 6
\end{tabular}}} & \multicolumn{2}{|c|}{$105 \%$} & \multirow{2}{*}{\multicolumn{2}{|c|}{ 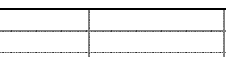 }} & - & \multirow{2}{*}{+1} & & & & & & & & \\
\hline & & & & & & & & & & & & & & & \\
\hline Turbine Rating $(\mathrm{kW}):$ & & 750 & & & 1500 & & & 2500 & & & 3500 & & & 5000 & \\
\hline Rotor Diameter $(\mathrm{m}):$ & & 50 & & & 66 & & & 85 & & & 100 & & & 120 & \\
\hline . & MHRS & Labor Costs & $\begin{array}{l}\text { Equip \& } \\
\text { Material }\end{array}$ & MHRS & Labor Costs & $\begin{array}{l}\text { Equip \& } \\
\text { Material }\end{array}$ & MHRS & Labor Costs & Equip \& Material & MHRS & Labor Costs & Equip \& Material & MHRS & Labor Costs & Equip \& Material \\
\hline 1. Receive, Uncrate Nacelle, Blades, Rotors \& Towers & & & & 64 & $\$ 2,579$ & & 75 & $\$ 3,023$ & & 96 & $\$ 3,869$ & & 142 & $\$ 5,723$ & \\
\hline 2. Fabricate Tower Assembly Area & & & & 16 & $\$ 626$ & $\$ 1,483$ & 16 & $\$ 626$ & $\$ 1,483$ & 16 & $\$ 626$ & $\$ 1,483$ & 16 & $\$ 626$ & $\$ 1,483$ \\
\hline 3. Field Fabrication Tower Sections & & & & 117 & $\$ 4,755$ & $\$ 7,614$ & 383 & $\$ 15,694$ & $\$ 23,807$ & 677 & $\$ 27,763$ & $\$ 39,462$ & 1095 & $\$ 44,997$ & $\$ 59,235$ \\
\hline 4. Rig \& Set Tower Sections & & & & 138 & $\$ 5,562$ & & 254 & $\$ 10,236$ & & 371 & $\$ 14,950$ & & 687 & $\$ 27,535$ & \\
\hline 5. Grout and Torque Bases & & & & 40 & $\$ 1,612$ & $\$ 850$ & 59 & $\$ 2,377$ & $\$ 950$ & 70 & $\$ 2,822$ & $\$ 1,120$ & 87 & $\$ 3,506$ & $\$ 1,650$ \\
\hline 6. Rig Blades, Assemble Rotors In Air & & & & 62 & $\$ 2,487$ & $\$ 250$ & 97 & $\$ 3,905$ & $\$ 500$ & 115 & $\$ 4,633$ & $\$ 700$ & 171 & $\$ 6,900$ & $\$ 1,000$ \\
\hline 7. Rig \& Set Nacelle & & & & 67 & $\$ 2,724$ & & 155 & $\$ 7,561$ & & 204 & $\$ 10,024$ & & 348 & $\$ 16,616$ & \\
\hline 8. Install Safety Equipment & & & & 12 & $\$ 484$ & & 20 & $\$ 806$ & & 24 & $\$ 968$ & & 36 & $\$ 1,450$ & \\
\hline 9. General Conditions & & & & & $\$ 2,281$ & $\$ 5,999$ & & $\$ 2,582$ & $\$ 11,328$ & & $\$ 2,582$ & $\$ 11,328$ & & $\$ 9,165$ & $\$ 21,988$ \\
\hline 10. Margin@ $10 \%$ & & & & & $\$ 2,311$ & $\$ 1,619 \mid$ & & $\$ 4,681$ & $\$ 3,807$ & & $\$ 6,824$ & $\$ 5,409$ & & $\$ 11,652$ & $\$ 8,536$ \\
\hline Subtotal Per Turbine & & & & 515 & $\$ 25,420$ & $\$ 17814$ & 1059 & $\$ 51.491$ & $\$ 41.874$ & 1573 & 875061 & 550507 & 2592 & S198, 171 $>$ > & 593891 \\
\hline Percent of Total & & & & (1) & $59 \%$ & $41 \%$ & 年 & $55 \%$ & \$4 $\$ 4,1,6 \%$ & $15 / 3$ & $56 \%$ & $\$$ & & $\$ \frac{\$ 128,171}{58 \%}$ & $\$ \$ \$ 3,891 \mid$ \\
\hline Project Total (50 Turbines) & & & & 25761.575 & $\$ 1,271,009$ & $\$ 890,719$ & 52960 & $\$ 2,574,545$ & $\$ 2,093,710$ & 78633 & $\$ 3,753,057$ & $\$ 2,975,096$ & 129106 & $\$ 6,408,555$ & $\$ 4,694,564$ \\
\hline Total All Costs & & & & & $\$ 2,161,728$ & & & $\$ 4,668,255$ & & & $\$ 6,728,153$ & & & $\$ 11,103,119$ & \\
\hline Total Cost per Turbine & & & & & $\$ 43,235$ & & & $\$ 93,365$ & & & $\$ 134,563$ & & & $\$ 222,062$ & \\
\hline Estimated Assembly Rate - Items 2,5 (Hours) & & & & & 205 & & & 409 & & & 575 & & & 1,035 & \\
\hline Estimated Assembly Rate - Items 2,5 (Days) & & & & & 2.1 & & & 4.1 & & & 5.8 & & & 10.4 & \\
\hline Total Costs/kW & & & & & $\$ 28.72$ & & & $\$ 37.39$ & & & $\$ 38.94$ & & & $\$ 44.62$ & \\
\hline Labor Costs/kW & & & & & 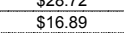 & & & $\$ 20.62$ & & & $\$ 21.72$ & & & $\$ 25.76$ & \\
\hline Equip.Mater Costs/kW & & & & & $\$ 11.83$ & & & $\$ 16.77$ & & & $\$ 17.22$ & & & $\$ 18.87$ & \\
\hline & & & & & & & & & & & & & & & \\
\hline Total Cost/Swept Area & & & & & $\$ 12.64$ & & & $\$ 16.45$ & & & $\$ 17.13$ & & & $\$ 19.63$ & \\
\hline Man-Hours/Swept Area & & & & & 0.15 & & & 0.19 & & & 0.20 & & & 0.23 & \\
\hline Labor Costs/Swept Area & & & & & $\$ 7.43$ & & & $\$ 9.07$ & & & $\$ 9.56$ & & & $\$ 11.33$ & \\
\hline Equip, Mater Costs/Swept Area & & & & & $\$ 5.21$ & & & $\$ 7.38$ & & & $\$ 7.58$ & & & $\$ 8.30$ & \\
\hline Total Costs//Hub Heigh & & & & & $\$ 504$ & & & $\$ 845$ & & & $\$ 1,035$ & & & $\$ 1,423$ & \\
\hline Labor Costs/Hub Heigh & & & & & $\$ 296$ & & & $\$ 466$ & & & $\$ 577$ & & & $\$ 822$ & \\
\hline Equip.Mater Costs/Hub Heigh & & & & & $\$ 208$ & & & $\$ 379$ & & & $\$ 458$ & & & $\$ 602$ & \\
\hline & & & & & & & $\Pi$ & & & & & & & & \\
\hline Item 1 Hourly Rates & & & & $\$ 40.30$ & & & $\$ 40.31$ & & & $\$ 40.30$ & & & $\$ 40.30$ & & \\
\hline Item 2 Hourly Rates & & & & $\$ 40.30$ & & & $\$ 40.30$ & & & $\$ 40.30$ & & & $\$ 40.08$ & & \\
\hline Item 3 Hourly Rates & & & & $\$ 40.30$ & & & $\$ 40.29$ & & & $\$ 40.31$ & & & $\$ 40.30$ & & \\
\hline Item 4 Hourly Rates & & & & $\$ 40.31$ & & & $\$ 40.30$ & & & $\$ 40.29$ & & & $\$ 40.31$ & & \\
\hline Item 5 Hourly Rates & & & & $\$ 40.65$ & & & $\$ 48.78$ & & & $\$ 49.14$ & & & $\$ 47.75$ & & \\
\hline $\begin{array}{l}\text { Item } 6 \text { Hourly Rates } \\
\text { Combined Hourly Rate }\end{array}$ & & & & 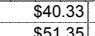 & & & $\$ 40.30$ & & & $\$ 40.33$ & & & $\$ 40.28$ & & \\
\hline - & & & & & & & & & & & & & & & \\
\hline Item 1 Percent of Labor & & & & $10 \%$ & & & $6 \%$ & & & $5 \%$ & & & $4 \%$ & & \\
\hline Item 2 Percent of Labor & & & & $22 \%$ & & & $20 \%$ & & & $20 \%$ & & & $21 \%$ & & \\
\hline Item 3 Percent of Labor & & & & $6 \%$ & & & $5 \%$ & & & $4 \%$ & & & $3 \%$ & & \\
\hline Item 4 Percent of Labor & & & & $10 \%$ & & & $8 \%$ & & & $6 \%$ & & & $5 \%$ & & \\
\hline Item 5 Percent of Labor & & & & $11 \%$ & & & $15 \%$ & & & $13 \%$ & & & $13 \%$ & & \\
\hline $\begin{array}{l}\text { Item } 6 \text { Percent of Labor } \\
\text { temem } 7 \text { Percent of Labor }\end{array}$ & & & & $\frac{2 \%}{9 \%}$ & & & $\frac{2 \%}{5 \%}$ & & & $\frac{1 \%}{3 \%}$ & & & $\frac{1 \%}{7 \%}$ & & \\
\hline Item 8 Percent of Labor & & & & $9 \%$ & & & $9 \%$ & & & $9 \%$ & & & $9 \%$ & & \\
\hline
\end{tabular}


BOLTED CONNECTIONS

1. Tower section properties based on data in Table 2-2.

2. See Page J-12 for determination of bolt count.

3. Overlap steel plate costs based on 18-inch wide by 1 -inch thick sheet steel.

$$
\begin{array}{lr}
\text { Tower Length } & 21.5 \mathrm{~m} \\
\text { Volume of } 1 \text { plate } & 8.8 \mathrm{cu} \mathrm{ft} \\
\text { Steel density } & 490 \mathrm{lb} / \mathrm{cu} \mathrm{ft} \\
\text { Steel cost } & \$ 0.35 \mathrm{llb} \\
\text { Plate cost } & \$ 1,512
\end{array}
$$

\begin{tabular}{|c|c|c|c|c|c|c|c|c|c|}
\hline Description & QTY. & UNITS & $\begin{array}{l}\text { UNITS/ } \\
\text { MHR }\end{array}$ & $\begin{array}{l}\text { TOTAL } \\
\text { MHRS }\end{array}$ & $\begin{array}{c}\text { COST/ } \\
\text { MHR }\end{array}$ & $\begin{array}{l}\text { TOTAL } \\
\text { LABOR }\end{array}$ & $\begin{array}{l}\text { MAT'L. } \\
\text { UNIT \$\$ }\end{array}$ & $\begin{array}{l}\text { TOTAL } \\
\text { MAT'L. }\end{array}$ & $\begin{array}{l}\text { TOTAL } \\
\text { L \& M }\end{array}$ \\
\hline Screw Jacks & 20 & EA & 5 & 4 & $\$ 40$ & $\$ 160$ & $\$ 0$ & $\$ 0$ & $\$ 160$ \\
\hline Quarter-Section Bolts & 119 & BOLTS & 10.5 & 11 & $\$ 40$ & $\$ 453$ & $\$ 1.50$ & $\$ 179$ & $\$ 632$ \\
\hline Half-Section Bolts & 119 & BOLTS & 10.5 & 11 & $\$ 40$ & $\$ 453$ & $\$ 1.50$ & $\$ 179$ & $\$ 632$ \\
\hline Steel Overlap Plates & 4 & EA & 0 & 0 & $\$ 40$ & $\$ 0$ & $\$ 1,512$ & $\$ 6,049$ & $\$ 6,049$ \\
\hline Top \& Bottom Template & 10.67 & EA & 0.25 & 43 & $\$ 40$ & $\$ 1,707$ & $\$ 0$ & $\$ 0$ & $\$ 1,707$ \\
\hline Move Cradles & 16 & EA & 2 & 8 & $\$ 40$ & $\$ 320$ & $\$ 0$ & $\$ 0$ & $\$ 320$ \\
\hline Lifting Eyes & 6 & EA & 1 & 6 & $\$ 40$ & $\$ 240$ & $\$ 35$ & $\$ 210$ & $\$ 450$ \\
\hline Paint & 10 & SF & 5 & 2 & $\$ 40$ & $\$ 80$ & $\$ 0.50$ & $\$ 5$ & $\$ 85$ \\
\hline Non-Productive Time & $3 \%$ & hours & & 3 & $\$ 40$ & $\$ 102$ & $\$ 0$ & $\$ 0$ & $\$ 102$ \\
\hline Torque Test Bolts & 238 & BOLTS & 30 & 8 & $\$ 50$ & $\$ 397$ & & $\$ 0$ & $\$ 397$ \\
\hline Load \& Transport Towers & 1 & SECTIONS & 0.18 & 6 & $\$ 40$ & $\$ 222$ & $\$ 0.00$ & $\$ 0$ & $\$ 222$ \\
\hline \multicolumn{4}{|l|}{ TOTALS (PER SECTION) } & \multicolumn{2}{|l|}{101} & \multicolumn{2}{|l|}{$\$ 4,135$} & $\$ 6,621$ & $\$ 10,756$ \\
\hline Minimum & \multicolumn{3}{|l|}{$-10 \%$} & \multicolumn{2}{|l|}{91} & \multicolumn{2}{|l|}{$\$ 3,722$} & $\$ \$ \$ 5,959$ & $\$ \$ \$ 9,680$ \\
\hline Maximum & \multicolumn{3}{|l|}{$15 \%$} & \multicolumn{2}{|l|}{117} & \multicolumn{2}{|l|}{$\$ 4,755$} & $\$ 7,614$ & $\$ 12,369$ \\
\hline \multicolumn{3}{|l|}{ Total Number of Sections in Turbine: } & 1 & \multicolumn{2}{|l|}{101} & \multicolumn{2}{|l|}{$\$ 4,135$} & $\$ 6,621$ & $\$ 10,756$ \\
\hline \multicolumn{3}{|l|}{ Minimum } & 1 & \multicolumn{2}{|l|}{91} & \multicolumn{2}{|l|}{$\$ 3,722$} & $\$ 5,959$ & $\$ 9,680$ \\
\hline \multirow[t]{2}{*}{ Maximum } & & & 1 & 117 & & \multicolumn{2}{|l|}{$\$ 4,755$} & $\$ 7,614$ & $\$ 12,369$ \\
\hline & \multicolumn{2}{|l|}{ Avg } & \multicolumn{3}{|c|}{ Max } & & & & \\
\hline 10 People, 10 hrs/day & 100 & 100 & 10 & & & & & & \\
\hline Days per Tower Section & 1.0 & 0.9 & 1. & & & & & & \\
\hline Total number of sections for assembly & 50 & 50 & 5 & & & & & & \\
\hline Number of days for assembly & 51 & 46 & 5 & & & & & & \\
\hline Number of assembly days per turbine & 1.0 & 0.9 & 1. & & & & & & \\
\hline Number of 6-day weeks & 7.2 & 7.6 & 9 . & & & & & & \\
\hline
\end{tabular}

Tower Section Assembly 1500 kW 
BOLTED CONNECTIONS

1. Tower section properties based on data in Table 2-2.

2. See Page J-12 for determination of bolt count.

3. Overlap steel plate costs based on 18-inch wide by 1 -inch thick sheet steel.

$$
\begin{array}{lr}
\text { Tower Length } & 22.1 \mathrm{~m} \\
\text { Volume of } 1 \text { plate } & 9.1 \mathrm{cu} \mathrm{ft} \\
\text { Steel density } & 490 \mathrm{lb} / \mathrm{cu} \mathrm{ft} \\
\text { Steel cost } & \$ 0.35 \mathrm{llb} \\
\text { Plate cost } & \$ 1,554
\end{array}
$$

Tower Section Assembly 2500 kW

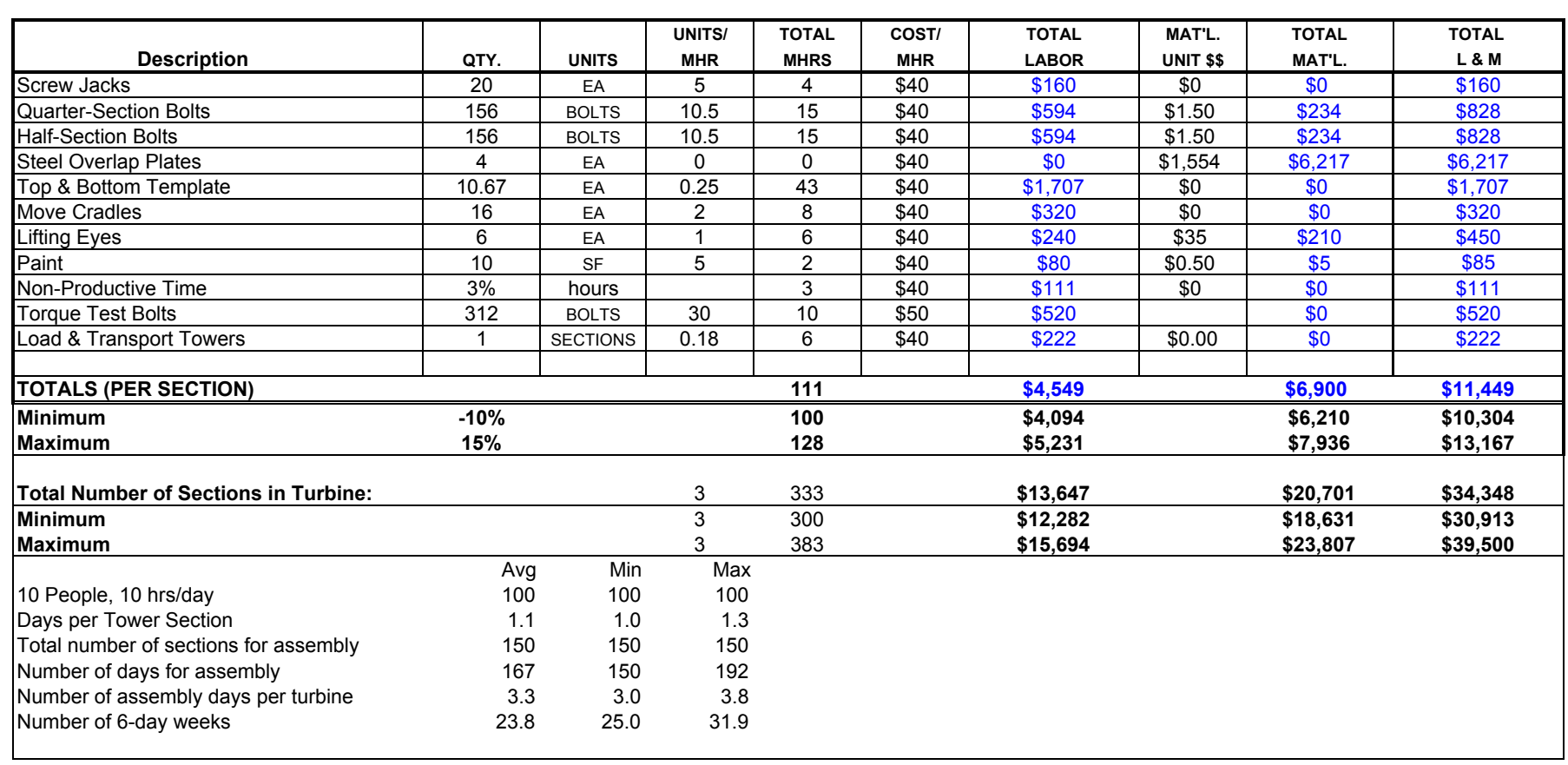


BOLTED CONNECTIONS

1. Tower section properties based on data in Table 2-2.

2. See Page J-12 for determination of bolt count.

3. Overlap steel plate costs based on 18-inch wide by 1 -inch thick sheet steel.

$$
\begin{array}{lr}
\text { Tower Length } & 21.7 \mathrm{~m} \\
\text { Volume of } 1 \text { plate } & 8.9 \mathrm{cu} \mathrm{ft} \\
\text { Steel density } & 490 \mathrm{lb} / \mathrm{cu} \mathrm{ft} \\
\text { Steel cost } & \$ 0.35 \mathrm{lb} \\
\text { Plate cost } & \$ 1,526
\end{array}
$$

\begin{tabular}{|c|c|c|c|c|c|c|c|c|c|}
\hline Description & QTY. & UNITS & $\begin{array}{c}\text { UNITS/ } \\
\text { MHR }\end{array}$ & $\begin{array}{l}\text { TOTAL } \\
\text { MHRS }\end{array}$ & $\begin{array}{c}\text { COST/ } \\
\text { MHR }\end{array}$ & $\begin{array}{l}\text { TOTAL } \\
\text { LABOR }\end{array}$ & $\begin{array}{l}\text { MAT'L. } \\
\text { UNIT \$\$ }\end{array}$ & $\begin{array}{l}\text { TOTAL } \\
\text { MAT'L. }\end{array}$ & $\begin{array}{c}\text { TOTAL } \\
\text { L \& M }\end{array}$ \\
\hline Screw Jacks & 20 & EA & 5 & 4 & $\$ 40$ & $\$ 160$ & $\$ 0$ & $\$ 0$ & $\$ 160$ \\
\hline Quarter-Section Bolts & 181 & BOLTS & 10.5 & 17 & $\$ 40$ & $\$ 690$ & $\$ 1.50$ & $\$ 272$ & $\$ 961$ \\
\hline Half-Section Bolts & 181 & BOLTS & 10.5 & 17 & $\$ 40$ & $\$ 690$ & $\$ 1.50$ & $\$ 272$ & $\$ 961$ \\
\hline Steel Overlap Plates & 4 & EA & 0 & 0 & $\$ 40$ & $\$ 0$ & $\$ 1,526$ & $\$ 6,105$ & $\$ 6,105$ \\
\hline Top \& Bottom Template & 10.67 & EA & 0.25 & 43 & $\$ 40$ & $\$ 1,707$ & $\$ 0$ & $\$ 0$ & $\$ 1,707$ \\
\hline Move Cradles & 16 & EA & 2 & 8 & $\$ 40$ & $\$ 320$ & $\$ 0$ & $\$ 0$ & $\$ 320$ \\
\hline Lifting Eyes & 6 & EA & 1 & 6 & $\$ 40$ & $\$ 240$ & $\$ 35$ & $\$ 210$ & $\$ 450$ \\
\hline Paint & 10 & $\mathrm{SF}$ & 5 & 2 & $\$ 40$ & $\$ 80$ & $\$ 0.50$ & $\$ 5$ & $\$ 85$ \\
\hline Non-Productive Time & $3 \%$ & hours & & 3 & $\$ 40$ & $\$ 117$ & $\$ 0$ & $\$ 0$ & $\$ 117$ \\
\hline Torque Test Bolts & 362 & BOLTS & 30 & 12 & $\$ 50$ & $\$ 603$ & & $\$ 0$ & $\$ 603$ \\
\hline Load \& Transport Towers & 1 & SECTIONS & 0.18 & 6 & $\$ 40$ & $\$ 222$ & $\$ 0.00$ & $\$ 0$ & $\$ 222$ \\
\hline TOTALS (PER SECTION) & & & & 118 & & $\$ 4,828$ & & $\$ 6,863$ & $\$ 11,691$ \\
\hline Minimum & $-10 \%$ & & & 106 & & $\$ \$ \$ 4,346$ & & $\$ \$ \$ 6,177$ & $\overline{\$ 10,522}$ \\
\hline Maximum & $15 \%$ & & & 135 & & $\$ 5,553$ & & $\$ 7,892$ & $\$ 13,445$ \\
\hline Total Number of Sections in Turbine: & & & 5 & 588 & & $\$ 24,142$ & & $\$ 34,315$ & $\$ 58,456$ \\
\hline Minimum & & & 5 & 530 & & $\$ 21,728$ & & $\$ 30,883$ & $\$ 52,611$ \\
\hline Maximum & & & 5 & 677 & & $\$ 27,763$ & & $\$ 39,462$ & $\$ 67,225$ \\
\hline
\end{tabular}

Tower Section Assembly 3500 kW

10 People, $10 \mathrm{hrs} /$ day

Days per Tower Section

Total number of sections for assembly

Number of days for assembly

Number of assembly days per turbine

Number of 6-day weeks

$\begin{array}{rrr}\text { Avg } & \text { Min } & \text { Max } \\ 100 & 100 & 100 \\ 1.2 & 1.1 & 1.4 \\ 250 & 250 & 250 \\ 294 & 265 & 338 \\ 5.9 & 5.3 & 6.8 \\ 49.0 & 44.1 & 56.4\end{array}$


BOLTED CONNECTIONS

1. Tower section properties based on data in Table 2-2.

2. See Page J-12 for determination of bolt count.

3. Overlap steel plate costs based on 18-inch wide by 1 -inch thick sheet steel.

Tower Length $22.3 \mathrm{~m}$

Volume of 1 plate $\quad 9.1 \mathrm{cu} \mathrm{ft}$

Steel density $\quad 490 \mathrm{lb} / \mathrm{cu} \mathrm{ft}$

Steel cost $\quad \$ 0.35 / \mathrm{lb}$

Plate cost $\$ 1,568$

Tower Section Assembly 5000 kW

\begin{tabular}{|c|c|c|c|c|c|c|c|c|c|}
\hline Descriptior & QTY. & UNITS & $\begin{array}{l}\text { UNITS/ } \\
\text { MHR }\end{array}$ & $\begin{array}{l}\text { TOTAL } \\
\text { MHRS }\end{array}$ & $\begin{array}{l}\text { COST/ } \\
\text { MHR }\end{array}$ & $\begin{array}{l}\text { TOTAL } \\
\text { LABOR }\end{array}$ & $\begin{array}{l}\text { MAT'L. } \\
\text { UNIT \$\$ }\end{array}$ & $\begin{array}{l}\text { TOTAL } \\
\text { MAT'L. }\end{array}$ & $\begin{array}{l}\text { TOTAL } \\
\text { L \& M }\end{array}$ \\
\hline Screw Jacks & 20 & EA & 5 & 4 & $\$ 40$ & $\$ 160$ & $\$ 0$ & $\$ 0$ & $\$ 160$ \\
\hline Quarter-Section Bolts & 223 & BOLTS & 9 & 25 & $\$ 40$ & $\$ 991$ & $\$ 1.95$ & $\$ 435$ & $\$ 1,426$ \\
\hline Half-Section Bolts & 223 & BOLTS & 9 & 25 & $\$ 40$ & $\$ 991$ & $\$ 1.95$ & $\$ 435$ & $\$ 1,426$ \\
\hline Steel Overlap Plates & 4 & EA & 0 & 0 & $\$ 40$ & $\$ 0$ & $\$ 1,568$ & $\$ 6,274$ & $\$ 6,274$ \\
\hline Top \& Bottom Template & 10.67 & EA & 0.25 & 43 & $\$ 40$ & $\$ 1,707$ & $\$ 0$ & $\$ 0$ & $\$ 1,707$ \\
\hline Move Cradles & 16 & EA & 2 & 8 & $\$ 40$ & $\$ 320$ & $\$ 0$ & $\$ 0$ & $\$ 320$ \\
\hline Lifting Eyes & 6 & EA & 1 & 6 & $\$ 40$ & $\$ 240$ & $\$ 35$ & $\$ 210$ & $\$ 450$ \\
\hline Paint & 10 & $\mathrm{SF}$ & 5 & 2 & $\$ 40$ & $\$ 80$ & $\$ 0.50$ & $\$ 5$ & $\$ 85$ \\
\hline Non-Productive Time & $3 \%$ & hours & & 3 & $\$ 40$ & $\$ 135$ & $\$ 0$ & $\$ 0$ & $\$ 135$ \\
\hline Torque Test Bolts & 446 & BOLTS & 30 & 15 & $\$ 50$ & $\$ 743$ & & $\$ 0$ & $\$ 743$ \\
\hline Load \& Transport Towers & 1 & SECTIONS & 0.18 & 6 & $\$ 40$ & $\$ 222$ & $\$ 0.00$ & $\$ 0$ & $\$ 222$ \\
\hline TOTALS (PER SECTION) & & & & 136 & & $\$ 5,590$ & & $\$ 7,358$ & $\$ 12,948$ \\
\hline Minimum & $-10 \%$ & & & 122 & & $\$ 5,031$ & & $\$ 6,623$ & $\$ 11,653$ \\
\hline Maximum & $15 \%$ & & & 156 & & $\$ 6,428$ & & $\$ 8,462$ & $\$ 14,890$ \\
\hline Total Number of Sections in Turbine: & & & 7 & 952 & & $\$ 39,128$ & & $\$ 51,509$ & $\$ 90,636$ \\
\hline Minimum & & & 7 & 857 & & $\$ 35,215$ & & $\$ 46,358$ & $\$ 81,573$ \\
\hline Maximum & & & 7 & 1095 & & $\$ 44,997$ & & $\$ 59,235$ & $\$ 104,232$ \\
\hline
\end{tabular}

10 People, 10 hrs/day

Days per Tower Section

Total number of sections for assembly

Number of days for assembly

Number of assembly days per turbine

Number of 6-day weeks

$\begin{array}{rrr}\text { Avg } & \text { Min } & \text { Max } \\ 100 & 100 & 100 \\ 1.4 & 1.2 & 1.6 \\ 350 & 350 & 350 \\ 476 & 428 & 547 \\ 9.5 & 8.6 & 10.9 \\ 79.3 & 71.4 & 91.2\end{array}$




\begin{tabular}{|c|c|c|c|c|c|c|c|c|c|c|c|c|}
\hline $\begin{array}{l}\text { Turbine Class: } \\
\text { Rotor Diameter: }\end{array}$ & \multicolumn{3}{|c|}{$\frac{1,500}{66}$} & \multicolumn{3}{|c|}{$\frac{2.500}{85}$} & \multicolumn{3}{|c|}{$\begin{array}{l}3,500 \\
100\end{array}$} & \multicolumn{3}{|c|}{5000} \\
\hline Crane Type: & & $4100 \mathrm{~s} 1$ & & & $4100 \mathrm{~s} 1$ & & & 4600 s4 & & & 4600 s5 & \\
\hline & $\frac{\operatorname{Min}}{8186}$ & $\begin{array}{ll}\text { Avg } \\
\$ 1318 \\
\end{array}$ & $\begin{array}{ll}\operatorname{Max} \\
51516\end{array}$ & $\begin{array}{ccc}\text { Min } \\
3900\end{array}$ & 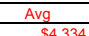 & $\frac{\operatorname{Max}}{5498}$ & 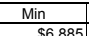 & $\frac{\text { Avg }}{57650}$ & \begin{tabular}{c|cl}
$\operatorname{Max}$ \\
8898
\end{tabular} & $\frac{\operatorname{Min}}{811400}$ & $\frac{\mathrm{Avg}}{912378}$ & $\frac{\operatorname{Max}}{\$ \$ 1 / 2}$ \\
\hline $\begin{array}{l}\text { Crane Crew WAssembly Labor Costs/furbine } \\
\text { Crane Crew Relocation Labor Costs/Turbine }\end{array}$ & & & & $\begin{array}{r}\$ 3,900 \\
\$ 0\end{array}$ & $\begin{array}{r}\$ 4,334 \\
\$ 0\end{array}$ & $\$ 4,9840$ & $\begin{array}{rll}\$ 6,885 \\
\$ 0\end{array}$ & $\frac{57,650}{\$ 0}$ & $\begin{array}{rll}\$ 8,798 \\
\$ 0\end{array}$ & $\begin{array}{llll}\$ 11,140 \\
\$ 0\end{array}$ & & \\
\hline Crane Rental Costs During Assembly and Relocation/Turbine & $\$ 900$ & $\$ 900$ & $\$ 900$ & $\$ 1,731$ & $\$ 1,923$ & $\$ 2,212$ & $\frac{86,518}{804}$ & $\$ 7,243$ & \$8,329 & $\$ 12,360$ & 1,733 & $\$ 15,793$ \\
\hline $\begin{array}{l}\text { Meals and Lodging/Turbine } \\
\text { Fuel CostItTurbine }\end{array}$ & $\frac{\$ 137}{\$ 50}$ & $\frac{\$ 152}{\$ 56}$ & $\frac{\$ 175}{\$ 64}$ & $\frac{\$ 400}{\$ 165}$ & $\frac{\$ 500}{\$ 183}$ & $\frac{\$ 575}{\$ 211}$ & $\frac{\$ 794}{\$ 331}$ & $\frac{\$ 883}{\$ 688}$ & $1 \frac{1,015}{\$ 423}$ & $\frac{\$ 1,285}{\$ 536}$ & $\frac{428}{5295}$ & $\$ 1,642$ \\
\hline Cribbing & $\$ 131$ & $\$ 131$ & & $\$ 131$ & $\$ 131$ & $\$ 131$ & 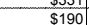 & $\frac{506}{\$ 190}$ & 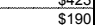 & \$5055 & $\frac{5350}{5955}$ & 845 \\
\hline MobiDemob Costs Turbin & $\$ 1,328$ & $\begin{array}{ll}\$ 1,328 & \end{array}$ & $\$ 1,328$ & $\$ 1,328$ & $\$ 1,328$ & $\$ 1,328>$ & $\$ 2,477$ & $\$ 2,477 \mathrm{~s}-\mathrm{s}$ & $\$ 2,477$ & $\$ 2,757$ & $\$ 2,757$ & 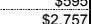 \\
\hline Total Crane and Crew Costs/Turbine & $\$ 3,732$ & $\$ 3,885[$ & $\$ 4,114$ & $\$ 7,705$ & $\$ 8,399$ & $\$ 9,441, \quad>1$ & $\$ 17,196$ & $\$ 18,810$ & $\$ 21,231$ & $\$ 28,673$ & $\$ 31,487$ & S35,707 \\
\hline Total Crane Costs (50 Turbines) & $\$ 186,624$ & $\$ 194,254$ & $\$ 205,699$ & $\$ 385,272$ & $\$ 419,974$ & $\$ 472,027$ & $\$ 859,776$ & $\$ 940,492$ & $\$ 1,061,566$ & $\$ 1,433,654$ & $\$ 1,574,328$ & $\$ 1,785,340$ \\
\hline & & & & & & & & 8544 & & & & \\
\hline Costs/Swept Area & $\$ \$ \$ .09$ & \$1.1.14 & $\$ 1.20$ & $\$ 1.36$ & $\$ \$ \$ .36$ & $\$ \frac{\$ 3.66}{\$ 1.66}$ & $\$ \frac{\$ 4.98}{\$ 2.19}$ & $\$ \frac{\$ 5.43}{\$ 2.39}$ & 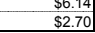 & 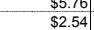 & (56.33 & 83.1.16 \\
\hline
\end{tabular}




\begin{tabular}{|c|c|c|c|c|}
\hline Initial Assumptions & & Tower Fabrication & Crane Assembly & \\
\hline Work Hours/Day & & 10 & 8 & \\
\hline Number of Days/Wk & & 6 & 5 & \\
\hline Number of Weeks/Year & & 52 & 52 & \\
\hline Number of Weeks/Month & & 4.3333 & 4.4000 & \\
\hline Number of Days/Month & & 26 & 22 & \\
\hline Number of Hours/Month & & 260 & 176 & \\
\hline Number of Turbines & & 50 & & \\
\hline Turbine Rating (kW) & 1500 & 2500 & 3500 & 5000 \\
\hline Crane Type & 4100 S1 & 4100 S1 & 4600 S4 & 4600 S5 \\
\hline Monthly Crane Costs during turbine assembly (60hr week) & $\$ 15,000$ & $\$ 15,000$ & $\$ 32,000$ & $\$ 37,500$ \\
\hline Monthly crane costs other time & $\$ 15,000$ & $\$ 15,000$ & $\$ 32,000$ & $\$ 37,500$ \\
\hline 6 Month Rental Costs & $\$ 14,000$ & $\$ 14,000$ & $\$ 29,867$ & $\$ 35,000$ \\
\hline 9 Month Rental Costs & $\$ 13,500$ & $\$ 13,500$ & $\$ 28,800$ & $\$ 33,750$ \\
\hline 12 Month Rental Costs & $\$ 13,000$ & $\$ 13,000$ & $\$ 27,733$ & $\$ 32,500$ \\
\hline \multicolumn{5}{|c|}{ 1. Tower Assembly Crew Information - (Assembly crew production rate determines crane production and costs) } \\
\hline Number of People in Crew & 10 & 10 & 10 & 10 \\
\hline Number of Crews & 1 & 1 & 1 & 1 \\
\hline Man Hours/Day & 100 & 100 & 100 & 100 \\
\hline Number of Man Hours/Wk & 600 & 600 & 600 & 600 \\
\hline Tower Fabrication Assembly Rate Days/Turbine & 1.0 & 3.3 & 5.9 & 9.5 \\
\hline \multicolumn{5}{|l|}{ 2. Crane Crew Information - During Tower Assembly } \\
\hline Number of People in Crane Crew & 2 & 2 & 2 & 2 \\
\hline Number of Cranes and Crew & 1 & 1. & 1 & 1 \\
\hline Number of Turbines/Crane & 50 & 50 & 50 & 50 \\
\hline Man Hours/Day & 20 & 20 & 20 & 20 \\
\hline Estimated Crane Crew Man Hours/Turbine & 20 & 67 & 118 & 190 \\
\hline Labor Costs/Crane Crew Man Hour & $\$ 65$ & $\$ 65$ & $\$ 65$ & $\$ 65$ \\
\hline Crane Crew Assembly Labor Costs/Turbine & $\$ 1,318$ & $\$ 4,334$ & $\$ 7,650$ & $\$ 12,378$ \\
\hline \multicolumn{5}{|l|}{ 3. Crane Relocation Information } \\
\hline Estimated Relocation Hours/Turbine & 0 & 0 & 0 & 0 \\
\hline Total Relocation Hours & 0 & 0 & 0 & 0 \\
\hline Total Relocation Hours/Crane & 0 & 0 & 0 & 0 \\
\hline Relocation Days/Crane & 0 & 0 & 0 & 0 \\
\hline Estimated Relocation Days/Turbine & 0 & 0 & 0 & 0 \\
\hline Crane Crew Relocation Man Hours/Turbine & 0.0 & 0.0 & 0.0 & 0.0 \\
\hline Crane Crew Relocation Labor Costs/Turbine & $\$ 0$ & \$0, & $\$ 0$ & $\$ 0$ \\
\hline Crane Costs During Relocation/Turbine & $\$ 0$ & $\$ 0$ & $\$ 0$ & $\$ 0$ \\
\hline \multicolumn{5}{|l|}{ 3. Totals } \\
\hline Total Number of Tower Fabrication Days/Turbine & 1.0 & 3.3 & 5.9 & 9.5 \\
\hline Total Number of Days Required: & 51 & 167 & 294 & 476 \\
\hline Total Number of Weeks Required & 8.4 & 27.8 & 49.0 & 79.3 \\
\hline & & & & \\
\hline Total Number of Months for Assembly & 1.9 & 6.4 & 11.3 & 18.3 \\
\hline 3 Month Min Crane Rental Costs & $\$ 45,000$ & $\$ 45,000$ & $\$ 96,000$ & $\$ 112,500$ \\
\hline Total Crane Rental Charges & $\$ 29,249$ & $\$ 96,163$ & $\$ 362,133$ & $\$ 686,664$ \\
\hline Crane Rental Costs/Turbine & $\$ 900$ & $\$ 1,923$ & $\$ 7,243$ & $\$ 13,733$ \\
\hline \multicolumn{5}{|l|}{ 4. Material/Supplies/Incidental Crane Costs } \\
\hline Meals and Lodging/Person/Day & $\$ 75$ & $\$ 75$ & $\$ 75$ & $\$ 75$ \\
\hline Number of Person-Days & 101.3959556 & 333.3650095 & 588.4654921 & 952.1739111 \\
\hline Total Meals and Lodging Costs & $\$ 7,605$ & $\$ 25,002$ & $\$ 44,135$ & $\$ 71,413$ \\
\hline Meals and Lodging/Turbine & $\$ 152$ & $\$ 500$ & $\$ 883$ & $\$ 1,428$ \\
\hline \multicolumn{5}{|l|}{ 5. Fuel } \\
\hline Fuel Cost/Gallon & $\$ 1.50$ & $\$ 1.50$ & $\$ 1.50$ & $\$ 1.50$ \\
\hline Gallons of Fuel/Week & 220 & 220 & 250 & 250 \\
\hline Total Cost of Fuel & $\$ 2,788$ & $\$ 9,168$ & $\$ 18,390$ & $\$ 29,755$ \\
\hline Fuel Cost/Turbine & $\$ 56$ & $\$ 183$ & $\$ 368$ & $\$ 595$ \\
\hline 6. Cribbing & & & & \\
\hline Cribbing Cost/sq ft & $\$ 2.50$ & $\$ 2.50$ & $\$ 2.50$ & $\$ 2.50$ \\
\hline Required Cribbing sq ft/Turbine & 2615 & 2615 & 3800 & 11900 \\
\hline Cribbing Costs/Turbine & $\$ 131$ & $\$ 131$ & $\$ 190$ & $\$ 595$ \\
\hline 7. Mobilization and Demobilization & & & & \\
\hline Crane Assembly and Disassembly Hours & 24 & 24 & 48 & 48 \\
\hline Lampson Supervisor Hours & 24 & 24 & 48 & 48 \\
\hline Lampson Supervisor Hourly Cost & $\$ 75$ & $\$ 75$ & $\$ 75$ & $\$ 75$ \\
\hline Number of Iron Workers & 4 & 4 & 4 & 4 \\
\hline Man Hours for Iron Workers & 96 & 96 & 192 & 192 \\
\hline Iron Worker Hourly Cost & $\$ 65$ & $\$ 65$ & $\$ 65$ & $\$ 65$ \\
\hline Crane Rental Period (Months) During Assembly & 0.1 & 0.1 & 0.3 & 0.3 \\
\hline Crane Rental Cost & $\$ 2,045$ & $\$ 2,045$ & $\$ 8,727$ & $\$ 10,227$ \\
\hline Total Labor Costs & $\$ 8,040$ & $\$ 8,040$ & $\$ 16,080$ & $\$ 16,080$ \\
\hline Truck Crane 1 Hourly Cost & $\$ 185$ & $\$ 185$ & $\$ 185$ & $\$ 185$ \\
\hline Truck Crane 2 Hourly Cost & $\$ 325$ & $\$ 325$ & $\$ 325$ & $\$ 325$ \\
\hline Truck Crane 3 Hourly Costs & & & & \\
\hline Total Truck Crane Costs & $\$ 12,240$ & $\$ 12,240$ & $\$ 24,480$ & $\$ 24,480$ \\
\hline Total Transportation Freight in/out & $\$ 40,000$ & $\$ 40,000$ & $\$ 60,000$ & $\$ 70,000$ \\
\hline Transport Days in/out & 6 & 6 & 10 & 10 \\
\hline Transport Hours in/out & 48 & 48 & 80 & 80 \\
\hline Crane Rental During Transport & $\$ 4,091$ & $\$ 4,091$ & $\$ 14,545$ & $\$ 17,045$ \\
\hline SubTotal & $\$ 66,416$ & $\$ 66,416$ & $\$ 123,833$ & $\$ 137,833$ \\
\hline Mob/Demob Costs/Turbine & $\$ 1,328$ & $\$ 1,328$ & $\$ 2,477$ & $\$ 2,757$ \\
\hline
\end{tabular}




\begin{tabular}{|c|c|c|c|c|}
\hline Initial Assumptions & & \begin{tabular}{l|l} 
Tower Fabrication \\
\end{tabular} & Crane Assembly & \\
\hline Work Hours/Day & & 10 & 8 & \\
\hline Number of Days/Wk & & 6 & 5 & \\
\hline Number of Weeks/Year & & 52 & 52 & \\
\hline Number of Weeks/Month & & 4.3333 & 4.4000 & \\
\hline Number of Days/Month & & 26 & 22 & \\
\hline Number of Hours/Month & & 260 & 176 & \\
\hline Number of Turbines & & 50 & & \\
\hline Turbine Rating (kW) & 1500 & 2500 & 3500 & 5000 \\
\hline Crane Type & 4100 S1 & 4100 S1 & $4600 \$ 4$ & 4600 S5 \\
\hline Monthly Crane Costs during turbine assembly (60hr week) & $\$ 15,000$ & $\$ 15,000$ & $\$ 32,000$ & $\$ 37,500$ \\
\hline Monthly crane costs other time & $\$ 15,000$ & $\$ 15,000$ & $\$ 32,000$ & $\$ 37,500$ \\
\hline 6 Month Rental Costs & $\$ 14,000$ & $\$ 14,000$ & $\$ 29,867$ & $\$ 35,000$ \\
\hline 9 Month Rental Costs & $\$ 13,500$ & $\$ 13,500$ & $\$ 28,800$ & $\$ 33,750$ \\
\hline 12 Month Rental Costs & $\$ 13,000$ & $\$ 13,000$ & $\$ 27,733$ & $\$ 32,500$ \\
\hline \multicolumn{5}{|c|}{ 1. Assembly Crew Information - (Assembly crew production rate determines crane production and costs) } \\
\hline Number of People in Crew & 10 & 10 & 10 & 10 \\
\hline Number of Crews & 1 & 1 & 1 & 1 \\
\hline Man Hours/Day & 100 & 100 & 100 & 100 \\
\hline Number of Man Hours/Wk & 600 & 600 & 600 & 600 \\
\hline Tower Fabrication Assembly Rate Days/Turbine & 0.9 & 3.0 & 5.3 & 8.6 \\
\hline \multicolumn{5}{|l|}{ 2. Crane Crew Information - During Tower Assembly } \\
\hline Number of People in Crane Crew & 2 & 2 & 2 & 2 \\
\hline Number of Cranes and Crew & 1. & 1 & 1 & 1 \\
\hline Number of Turbines/Crane & 50 & 50 & 50 & 50 \\
\hline Man Hours/Day & 20 & 20 & 20 & 20 \\
\hline Estimated Crane Crew Man Hours/Turbine & 18 & 60 & 106 & 171 \\
\hline Labor Costs/Crane Crew Man Hour & $\$ 65$ & $\$ 65$ & $\$ 65$ & $\$ 65$ \\
\hline Crane Crew Assembly Labor Costs/Turbine & $\$ 1,186$ & $\$ 3,900$ & $\$ 6,885$ & $\$ 11,140$ \\
\hline \multicolumn{5}{|l|}{ 3. Crane Relocation Information } \\
\hline Estimated Relocation Hours/Turbine & 0 & 0 & 0 & 0 \\
\hline Total Relocation Hours & 0 & 0 & 0 & 0 \\
\hline Total Relocation Hours/Crane & 0 & 0 & 0 & 0 \\
\hline Relocation Days/Crane & 0 & 0 & 0 & 0 \\
\hline Estimated Relocation Days/Turbine & 0 & 0 & 0 & 0 \\
\hline Crane Crew Relocation Man Hours/Turbine & 0.0 & 0.0 & 0.0 & 0.0 \\
\hline Crane Crew Relocation Labor Costs/Turbine & $\$ 0$ & $\$ 0$ & $\$ 0$ & $\$ 0$ \\
\hline Crane Costs During Relocation/Turbine & $\$ 0$ & $\$ 0$ & $\$ 0$ & $\$ 0$ \\
\hline \multicolumn{5}{|l|}{ 3. Totals } \\
\hline Total Number of Tower Fabrication Days/Turbine & 0.9 & 3.0 & 5.3 & 8.6 \\
\hline Total Number of Days Required: & 46 & 150 & 265 & 428 \\
\hline Total Number of Weeks Required & 7.6 & 25.0 & 44.1 & 71.4 \\
\hline & & & & \\
\hline Total Number of Months for Assembly & 1.8 & 5.8 & 10.2 & 16.5 \\
\hline 3 Month Min Crane Rental Costs & $\$ 45,000$ & $\$ 45,000$ & $\$ 96,000$ & $\$ 112,500$ \\
\hline Total Crane Rental Charges & $\$ 26,324$ & $\$ 86,547$ & $\$ 325,919$ & $\$ 617,997$ \\
\hline Crane Rental Costs/Turbine & $\$ 900$ & $\$ 1,731$ & $\$ 6,518$ & $\$ 12,360$ \\
\hline \multicolumn{5}{|l|}{ 4. Material/Supplies/Incidental Crane Costs } \\
\hline Meals and Lodging/Person/Day & $\$ 75$ & $\$ 75$ & $\$ 75$ & $\$ 75$ \\
\hline Number of Person-Days & 91.25636 & 300.0285086 & 529.6189429 & 856.95652 \\
\hline Total Meals and Lodging Costs & $\$ 6,844$ & $\$ 22,502$ & $\$ 39,721$ & $\$ 64,272$ \\
\hline Meals and Lodging/Turbine & $\$ 137$ & $\$ 450$ & $\$ 794$ & $\$ 1,285$ \\
\hline \multicolumn{5}{|l|}{ 5. Fuel } \\
\hline Fuel Cost/Gallon & $\$ 1.50$ & $\$ 1.50$ & $\$ 1.50$ & $\$ 1.50$ \\
\hline Gallons of Fuel/Week & 220 & 220 & 250 & 250 \\
\hline Total Cost of Fuel & $\$ 2,510$ & $\$ 8,251$ & $\$ 16,551$ & $\$ 26,780$ \\
\hline Fuel Cost/Turbine & $\$ 50$ & $\$ 165$ & $\$ 331$ & $\$ 536$ \\
\hline 6. Cribbing & & & & \\
\hline Cribbing Cost/sq ft & $\$ 2.50$ & $\$ 2.50$ & $\$ 2.50$ & $\$ 2.50$ \\
\hline Required Cribbing sq ft/Turbine & 2615 & 2615 & 3800 & 11900 \\
\hline Cribbing Costs/Turbine & $\$ 131$ & $\$ 131$ & $\$ 190$ & $\$ 595$ \\
\hline & & & & \\
\hline 7. Mobilization and Demobilization & & & & \\
\hline Crane Assembly and Disassembly Hours & 24 & 24 & 48 & 48 \\
\hline Lampson Supervisor Hours & 24 & 24 & 48 & 48 \\
\hline Lampson Supervisor Hourly Cost & $\$ 75$ & $\$ 75$ & $\$ 75$ & $\$ 75$ \\
\hline Number of Iron Workers & 4 & 4 & 4 & 4 \\
\hline Man Hours for Iron Workers & 96 & 96 & 192 & 192 \\
\hline Iron Worker Hourly Cost & $\$ 65$ & $\$ 65$ & $\$ 65$ & $\$ 65$ \\
\hline Crane Rental Period (Months) During Assembly & 0.1 & 0.1 & 0.3 & 0.3 \\
\hline Crane Rental Cost & $\$ 2,045$ & $\$ 2,045$ & $\$ 8,727$ & $\$ 10,227$ \\
\hline Total Labor Costs & $\$ 8,040$ & $\$ 8,040$ & $\$ 16,080$ & $\$ 16,080$ \\
\hline Truck Crane 1 Hourly Cost & $\$ 185$ & $\$ 185$ & $\$ 185$ & $\$ 185$ \\
\hline Truck Crane 2 Hourly Cost & $\$ 325$ & $\$ 325$ & $\$ 325$ & $\$ 325$ \\
\hline Truck Crane 3 Hourly Costs & & & & \\
\hline Total Truck Crane Costs & $\$ 12,240$ & $\$ 12,240$ & $\$ 24,480$ & $\$ 24,480$ \\
\hline Total Transportation Freight in/out & $\$ 40,000$ & $\$ 40,000$ & $\$ 60,000$ & $\$ 70,000$ \\
\hline Transport Days in/out & 6 & 6 & 10 & 10 \\
\hline Transport Hours in/out & 48 & 48 & 80 & 80 \\
\hline Crane Rental During Transport & $\$ 4,091$ & $\$ 4,091$ & $\$ 14,545$ & $\$ 17,045$ \\
\hline SubTotal & $\$ 66,416$ & $\$ 66,416$ & $\$ 123,833$ & $\$ 137,833$ \\
\hline Mob/Demob Costs/Turbine & $\$ 1,328$ & $\$ 1,328$ & $\$ 2,477$ & $\$ 2,757$ \\
\hline
\end{tabular}




\begin{tabular}{|c|c|c|c|c|}
\hline Initial Assumptions & & Tower Fabrication & Crane Assembly & \\
\hline Work Hours/Day & & 10 & 8 & \\
\hline Number of Days/Wk & & 6 & 5 & \\
\hline Number of Weeks/Year & & 52 & 52 & \\
\hline Number of Weeks/Month & & 4.3333 & 4.4000 & \\
\hline Number of Days/Month & & 26 & 22 & \\
\hline Number of Hours/Month & & 260 & 176 & \\
\hline Number of Turbines & & 50 & & \\
\hline Turbine Rating $(\mathrm{kW})$ & 1500 & 2500 & 3500 & 5000 \\
\hline Crane Type & $4100 \mathrm{~S} 1$ & $4100 \mathrm{~S} 1$ & $4600 \mathrm{~S} 4$ & $4600 \mathrm{~S} 5$ \\
\hline Monthly Crane Costs during turbine assembly (60hr week) & $\$ 15,000$ & $\$ 15,000$ & $\$ 32,000$ & $\$ 37,500$ \\
\hline Monthly crane costs other time & $\$ 15,000$ & $\$ 15,000$ & $\$ 32,000$ & $\$ 37,500$ \\
\hline 6 Month Rental Costs & $\$ 14,000$ & $\$ 14,000$ & $\$ 29,867$ & $\$ 35,000$ \\
\hline 9 Month Rental Costs & $\$ 13,500$ & $\$ 13,500$ & $\$ 28,800$ & $\$ 33,750$ \\
\hline 12 Month Rental Costs & $\$ 13,000$ & $\$ 13,000$ & $\$ 27,733$ & $\$ 32,500$ \\
\hline \multicolumn{5}{|c|}{ 1. Assembly Crew Information - (Assembly crew production rate determines crane production and costs) } \\
\hline Number of People in Crew & 10 & 10 & 10 & 10 \\
\hline Number of Crews & 1 & 1 & 1 & 1 \\
\hline Man Hours/Day & 100 & 100 & 100 & 100 \\
\hline Number of Man Hours/Wk & 600 & 600 & 600 & 600 \\
\hline Tower Fabrication Assembly Rate Days/Turbine & 1.2 & 3.8 & 6.8 & 10.9 \\
\hline \multicolumn{5}{|l|}{ 2. Crane Crew Information - During Tower Assembly } \\
\hline Number of People in Crane Crew & 2 & 2 & 2 & 2 \\
\hline Number of Cranes and Crew & 1 & 1 & 1 & 1 \\
\hline Number of Turbines/Crane & 50 & 50 & 50 & 50 \\
\hline Man Hours/Day & 20 & 20 & 20 & 20 \\
\hline Estimated Crane Crew Man Hours/Turbine & 23 & 77 & 135 & 219 \\
\hline Labor Costs/Crane Crew Man Hour & $\$ 65$ & $\$ 65$ & $\$ 65$ & $\$ 65$ \\
\hline Crane Crew Assembly Labor Costs/Turbine & $\$ 1,516$ & $\$ 4,984$ & $\$ 8,798$ & $\$ 14,235$ \\
\hline \multicolumn{5}{|l|}{ 3. Crane Relocation Information } \\
\hline Estimated Relocation Hours/Turbine & 0 & 0 & 0 & 0 \\
\hline Total Relocation Hours & 0 & 0 & 0 & 0 \\
\hline Total Relocation Hours/Crane & 0 & 0 & 0 & 0 \\
\hline Relocation Days/Crane & 0 & 0 & 0 & 0 \\
\hline Estimated Relocation Days/Turbine & 0 & 0 & 0 & 0 \\
\hline Crane Crew Relocation Man Hours/Turbine & 0.0 & 0.0 & 0.0 & 0.0 \\
\hline Crane Crew Relocation Labor Costs/Turbine & $\$ 0$ & $\$ 0$ & $\$ 0$ & $\$ 0$ \\
\hline Crane Costs During Relocation/Turbine & $\$ 0$ & $\$ 0$ & $\$ 0$ & $\$ 0$ \\
\hline \multicolumn{5}{|l|}{ 3. Totals } \\
\hline Total Number of Tower Fabrication Days/Turbine & 1.2 & 3.8 & 6.8 & 10.9 \\
\hline Total Number of Days Required: & 58 & 192 & 338 & 547 \\
\hline Total Number of Weeks Required & 9.7 & 31.9 & 56.4 & 91.2 \\
\hline & & & & \\
\hline Total Number of Months for Assembly & 2.2 & 7.4 & 13.0 & 21.1 \\
\hline 3 Month Min Crane Rental Costs & $\$ 45,000$ & $\$ 45,000$ & $\$ 96,000$ & $\$ 112,500$ \\
\hline Total Crane Rental Charges & $\$ 33,636$ & $\$ 110,587$ & $\$ 416,453$ & $\$ 789,663$ \\
\hline Crane Rental Costs/Turbine & $\$ 900$ & $\$ 2,212$ & $\$ 8,329$ & $\$ 15,793$ \\
\hline \multicolumn{5}{|l|}{ 4. Material/Supplies/Incidental Crane Costs } \\
\hline Meals and Lodging/Person/Day & $\$ 75$ & $\$ 75$ & $\$ 75$ & $\$ 75$ \\
\hline Number of Person-Days & 116.6053489 & 383.369761 & 676.7353159 & 1094.999998 \\
\hline Total Meals and Lodging Costs & $\$ 8,745$ & $\$ 28,753$ & $\$ 50,755$ & $\$ 82,125$ \\
\hline Meals and Lodging/Turbine & $\$ 175$ & $\$ 575$ & $\$ 1,015$ & $\$ 1,642$ \\
\hline \multicolumn{5}{|l|}{ 5. Fuel } \\
\hline Fuel Cost/Gallon & $\$ 1.50$ & $\$ 1.50$ & $\$ 1.50$ & $\$ 1.50$ \\
\hline Gallons of Fuel/Week & 220 & 220 & 250 & 250 \\
\hline Total Cost of Fuel & $\$ 3,207$ & $\$ 10,543$ & $\$ 21,148$ & $\$ 34,219$ \\
\hline Fuel Cost/Turbine & $\$ 64$ & $\$ 211$ & $\$ 423$ & $\$ 684$ \\
\hline 6. Cribbing & & & & \\
\hline Cribbing Cost/sq ft & $\$ 2.50$ & $\$ 2.50$ & $\$ 2.50$ & $\$ 2.50$ \\
\hline Required Cribbing sq ft/Turbine & 2615 & 2615 & 3800 & 11900 \\
\hline Cribbing Costs/Turbine & $\$ 131$ & $\$ 131$ & $\$ 190$ & $\$ 595$ \\
\hline 7. Mobilization and Demobilization & & & & \\
\hline Crane Assembly and Disassembly Hours & 24 & 24 & 48 & 48 \\
\hline Lampson Supervisor Hours & 24 & 24 & 48 & 48 \\
\hline Lampson Supervisor Hourly Cost & $\$ 75$ & $\$ 75$ & $\$ 75$ & $\$ 75$ \\
\hline Number of Iron Workers & 4 & 4 & 4 & 4 \\
\hline Man Hours for Iron Workers & 96 & 96 & 192 & 192 \\
\hline Iron Worker Hourly Cost & $\$ 65$ & $\$ 65$ & $\$ 65$ & $\$ 65$ \\
\hline Crane Rental Period (Months) During Assembly & 0.1 & 0.1 & 0.3 & 0.3 \\
\hline Crane Rental Cost & $\$ 2,045$ & $\$ 2,045$ & $\$ 8,727$ & $\$ 10,227$ \\
\hline Total Labor Costs & $\$ 8,040$ & $\$ 8,040$ & $\$ 16,080$ & $\$ 16,080$ \\
\hline Truck Crane 1 Hourly Cost & $\$ 185$ & $\$ 185$ & $\$ 185$ & $\$ 185$ \\
\hline Truck Crane 2 Hourly Cost & $\$ 325$ & $\$ 325$ & $\$ 325$ & $\$ 325$ \\
\hline Truck Crane 3 Hourly Costs & & & & \\
\hline Total Truck Crane Costs & $\$ 12,240$ & $\$ 12,240$ & $\$ 24,480$ & $\$ 24,480$ \\
\hline Total Transportation Freight in/out & $\$ 40,000$ & $\$ 40,000$ & $\$ 60,000$ & $\$ 70,000$ \\
\hline Transport Days in/out & 6 & 6 & 10 & 10 \\
\hline Transport Hours in/out & 48 & 48 & 80 & 80 \\
\hline Crane Rental During Transport & $\$ 4,091$ & $\$ 4,091$ & $\$ 14,545$ & $\$ 17,045$ \\
\hline SubTotal & $\$ 66,416$ & $\$ 66,416$ & $\$ 123,833$ & $\$ 137,833$ \\
\hline Mob/Demob Costs/Turbine & $\$ 1,328$ & $\$ 1,328$ & $\$ 2,477$ & $\$ 2,757$ \\
\hline
\end{tabular}




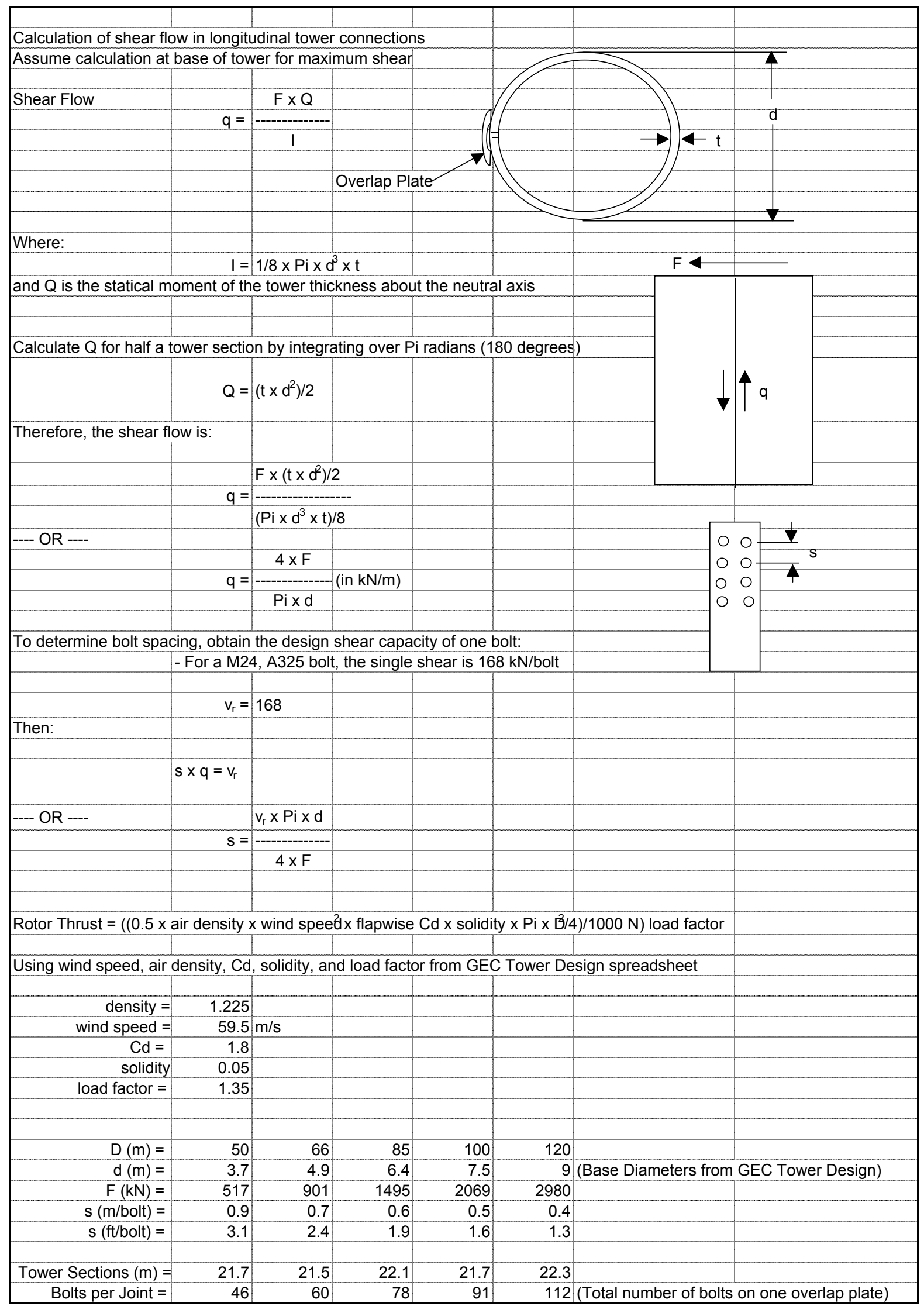




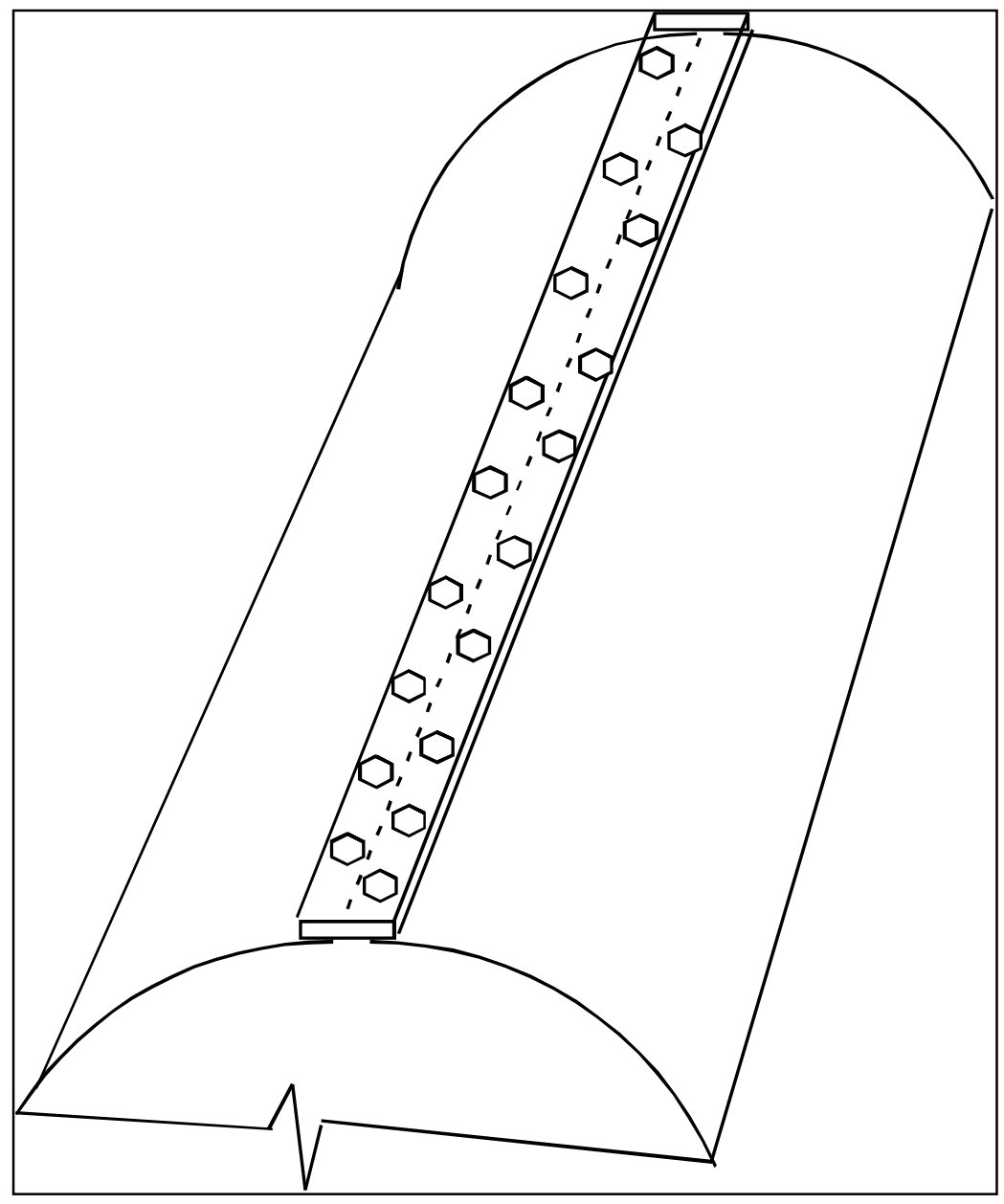

Illustration of bolted tower overlap joint. Overlap joint runs the entire length of the tower section. This view shows the connection between 2 quartered tower sections. 
Appendix K

Scenario 2-Welding 


\begin{tabular}{|c|c|c|c|c|c|c|c|c|c|c|c|c|c|c|c|}
\hline Number of People in Crew: & & $100 \%$ & & & & & & & & & & & & & \\
\hline $\begin{array}{l}\text { Hours per Day: } \\
\text { Dass per Week } \\
\end{array}$ & & & & & & & & & & & & & & & \\
\hline Turbine Rating (kW): & & 750 & & & 1500 & & & 2500 & & & 3500 & & & 5000 & \\
\hline Rotor Diameter $(m)$ : & & 50 & & & 66 & & & 85 & & & 100 & & & 120 & \\
\hline Activity & MHRS & Labor Costs & $\begin{array}{l}\text { Equip \& } \\
\text { Material }\end{array}$ & MHRS & Labor Costs & $\begin{array}{l}\text { Equip \& } \\
\text { Material }\end{array}$ & MHRS & Labor Costs & Equip \& Material & MHRS & Labor Costs & Equip \& Material & MHRS & Labor Costs & Equip \& Material \\
\hline 1. Receive, Uncrate Nacelle, Blades, Rotors \& Towers & & & & 62 & $\$ 2,498$ & & 75 & $\$ 3,023$ & & 96 & $\$ 3,869$ & & 142 & $\$ 5,723$ & \\
\hline 2. Fabricate Tower Assembly Area & & & & 14 & $\$ 544$ & $\$ 1,289$ & 14 & $\$ 544$ & $\$ 1,289$ & 14 & $\$ 544$ & $\$ 1,289$ & 14 & $\$ 544$ & $\$ 1,289$ \\
\hline 3. Field Fabrication Tower Sections & & & & 317 & $\$ 13,193$ & $\$ 8,625$ & 966 & $\$ 40,134$ & $\$ 26,339$ & 2456 & $\$ 100,739$ & $\$ 62,121$ & 5479 & $\$ 222,673$ & $\$ 128,899$ \\
\hline 4. Rig \& Set Tower Sections & & & & 132 & $\$ 5,320$ & & 243 & $\$ 9,793$ & & 354 & $\$ 14,265$ & & 619 & $\$ 24,810$ & \\
\hline 5. Grout and Torque Bases & & & & 40 & $\$ 1,612$ & $\$ 850$ & 59 & $\$ 2,377$ & $\$ 950$ & 70 & $\$ 2,822$ & $\$ 1,120$ & 87 & $\$ 3,506$ & $\$ 1,650$ \\
\hline 6. Rig Blades, Assemble Rotors In Air & & & & 61 & $\$ 2,447$ & $\$ 250$ & 95 & $\$ 3,816$ & $\$ 500$ & 112 & $\$ 4,513$ & $\$ 700$ & 164 & $\$ 6,626$ & $\$ 1,000$ \\
\hline 7. Rig \& Set Nacelle & & & & 57 & $\$ 2,317$ & & 133 & $\$ 6,488$ & & 174 & $\$ 8,550$ & & 280 & $\$ 13,370$ & \\
\hline 8. Install Safety Equipment & & & & 12. & $\$ 484$ & & 20 & $\$ 806$ & & 24 & $\$ 968$ & & 36 & $\$ 1,450$ & \\
\hline 9. General Conditions & & & & & $\$ 2,172$ & $\$ 5,713$ & & $\$ 2,459$ & $\$ 10,789$ & & $\$ 2,459$ & $\$ 10,789$ & & $\$ 8,729$ & $\$ 20,941$ \\
\hline 10. Margin @ $10 \%$ & & & & 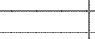 & $\$ 3,059$ & $\$ 1,673$ & & $\$ 6,944$ & $\$ 3,987$ & & $\$ 13,873$ & $\$ 7,602$ & 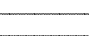 & $\$ 28,743$ & $\$ 15,378$ \\
\hline Subtotal Per Turbine & & & & 695 & $\$ 33,646$ & $\$ 18,400$ & 1604 & $\$ 76,385$ & $\$ 43,854$ & 3300 & $\$ 152,601$ & $\$ 83,621$ & 6822 & $\$ 316,174$ & $\$ 169,158$ \\
\hline Project Total (50 Turbines) & & & & 34744 & $\begin{array}{r}65 \% \\
\$ 1.682312 \\
\end{array}$ & $\begin{array}{r}35 \% \\
\$ 9919.997\end{array}$ & 80220 & \begin{tabular}{r|}
$64 \%$ \\
$\$ 3.819 .231$ \\
\end{tabular} & $\begin{array}{r}36 \% \\
\$ 2,192,689\end{array}$ & 164991 & $\begin{aligned} 656 \% \\
\$ \$ 7,630,070\end{aligned}$ & $\begin{array}{r}35 \% \\
\$ 4,181,1,72\end{array}$ & 341079 & $\begin{array}{r}65 \% \\
\$ 15.80 .693 \\
\end{array}$ & $\begin{array}{r}35 \% \\
\$ 8.457 .89 \\
\end{array}$ \\
\hline 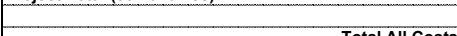 & & & & & $0,002,012$ & & & 每 & & & & & 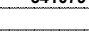 & & \\
\hline $\begin{array}{l}\text { Total All Costs } \\
\text { Total Cost per Turbine }\end{array}$ & & & & & 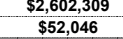 & & & $\begin{array}{l}\$, 011,920 \\
\$ 120,238\end{array}$ & & $x_{0}$ & 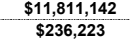 & & & 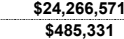 & \\
\hline Estimated Assembly Rate - Items 2,5 (Hours) & & & & & 189 & & & & & & 528 & & & & \\
\hline Estimated Assembly Rate - Items 2,5 (Days) & & & & & 1.9 & & & 3.8 & & & 5.3 & & & 9.0 & \\
\hline Total Costs/kW & & 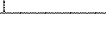 & & & $\$ 34.57$ & & & $\$ 48.16$ & & & $\$ 68.36$ & & & $\$ 97.53$ & \\
\hline Labor Costss/kW & & & & & $\$ 22.35$ & & & $\$ 30.59$ & & & $\$ 44.16$ & & & $\$ 63.54$ & \\
\hline Equip.Mater Costs/kW & & & & & $\$ 12.22$ & & & $\$ 17.56$ & & & $\$ 24.20$ & & & $\$ 33.99$ & \\
\hline & & & & & & & & & & & & & & & \\
\hline Total Cost/Swept Area & & & & & $\$ 15.21$ & & & $\$ 21.19$ & & & $\$ 30.08$ & & & $\$ 42.91$ & \\
\hline Man-Hours/Swept Area & & & & & 0.20 & & & 0.28 & & & 0.42 & & & 0.60 & \\
\hline Labor Costs/Swept Area & & & & & $\$ 9.83$ & & & $\$ 13.46$ & & & $\$ 19.43$ & & & $\$ 27.96$ & \\
\hline Equip, Mater Costs/Swept Area & & & & & $\$ 5.38$ & & & $\$ 7.73$ & & & $\$ 10.65$ & & & $\$ 14.96$ & \\
\hline Total Costs/Hub Heigh & & & & & $\$ 607$ & & & $\$ 1,088$ & & & $\$ 1,817$ & & & $\$ 3,111$ & \\
\hline Labor Costs//Hub Heigh & & & & & $\$ 392$ & & & $\$ 691$ & & & $\$ 1,174$ & & & $\$ 2,027$ & \\
\hline Equip.Mater Costs/Hub Heigh & & & & & $\$ 214$ & & & $\$ 397$ & & & $\$ 643$ & & & $\$ 1,084$ & \\
\hline & & & & & & & & & & & & & & & \\
\hline Item 1 Hourly Rates & & & & $\$ 40.30$ & & & $\$ 40.31$ & & & $\$ 40.30$ & & & $\$ 40.30$ & & \\
\hline Item 2 Hourly Rates & & & & $\$ 40.30$ & & & $\$ 40.30$ & & & $\$ 40.30$ & & & $\$ 40.08$ & & \\
\hline Item 3 Hourly Rates & & & & $\$ 40.30$ & & & $\$ 40.29$ & & & $\$ 40.31$ & & & $\$ 40.30$ & & \\
\hline Item 4 Hourly Rates & & & & $\$ 40.31$ & & & $\$ 40.30$ & & & $\$ 40.29$ & & & $\$ 40.31$ & & \\
\hline Item 5 Hourly Rates & & & & $\$ 40.65$ & & & $\$ 48.78$ & & & $\$ 49.14$ & & & $\$ 47.75$ & & \\
\hline $\begin{array}{l}\text { Itemb } 6 \text { Hourly Rates } \\
\text { Combined Hourly Rate }\end{array}$ & & & & $\begin{array}{l}\$ 40.33 \\
\$ \$ 51.35\end{array}$ & & & $\begin{array}{l}\$ 40.30 \\
\$ 54.58\end{array}$ & & & $\begin{array}{l}\$ 40.33 \\
\$ 52.70\end{array}$ & & & $\begin{array}{l}\$ 40.28 \\
\$ 57.38\end{array}$ & & \\
\hline & & & & $\$ 51.35$ & & & $\$ 54.58$ & & & $\$ 52.70$ & & & $\$ 57.38$ & & \\
\hline Item 1 Percent of Labor & & & & $7 \%$ & & & $4 \%$ & & & $3 \%$ & & & $2 \%$ & & \\
\hline Item 2 Percent of Labor & & & & $16 \%$ & & & $13 \%$ & & & $9 \%$ & & & $8 \%$ & & \\
\hline Item 3 Percent of Labor & & & & $5 \%$ & & & $3 \%$ & & & $2 \%$ & & & $1 \%$ & & \\
\hline Item 4 Percent of Labor & & & & $7 \%$ & & & $5 \%$ & & & $3 \%$ & & & $2 \%$ & & \\
\hline Item 5 Percent of Labor & & & & $7 \%$ & & & $8 \%$ & & & $6 \%$ & & & $4 \%$ & & \\
\hline $\begin{array}{l}\text { Item } 6 \text { Percent of Labor } \\
\text { Ittem } 7 \text { Percent of Labor }\end{array}$ & & & & $\frac{1 \%}{6 \%}$ & & & $\frac{1 \%}{3 \%}$ & & & $\frac{1 \%}{2 \%}$ & & & $0 \%$ & & \\
\hline Item 8 Percent of Labor & & & & $9 \%$ & & & $9 \%$ & & & $\frac{2 \%}{9 \%}$ & & & $\frac{3 \%}{9 \%}$ & & \\
\hline
\end{tabular}




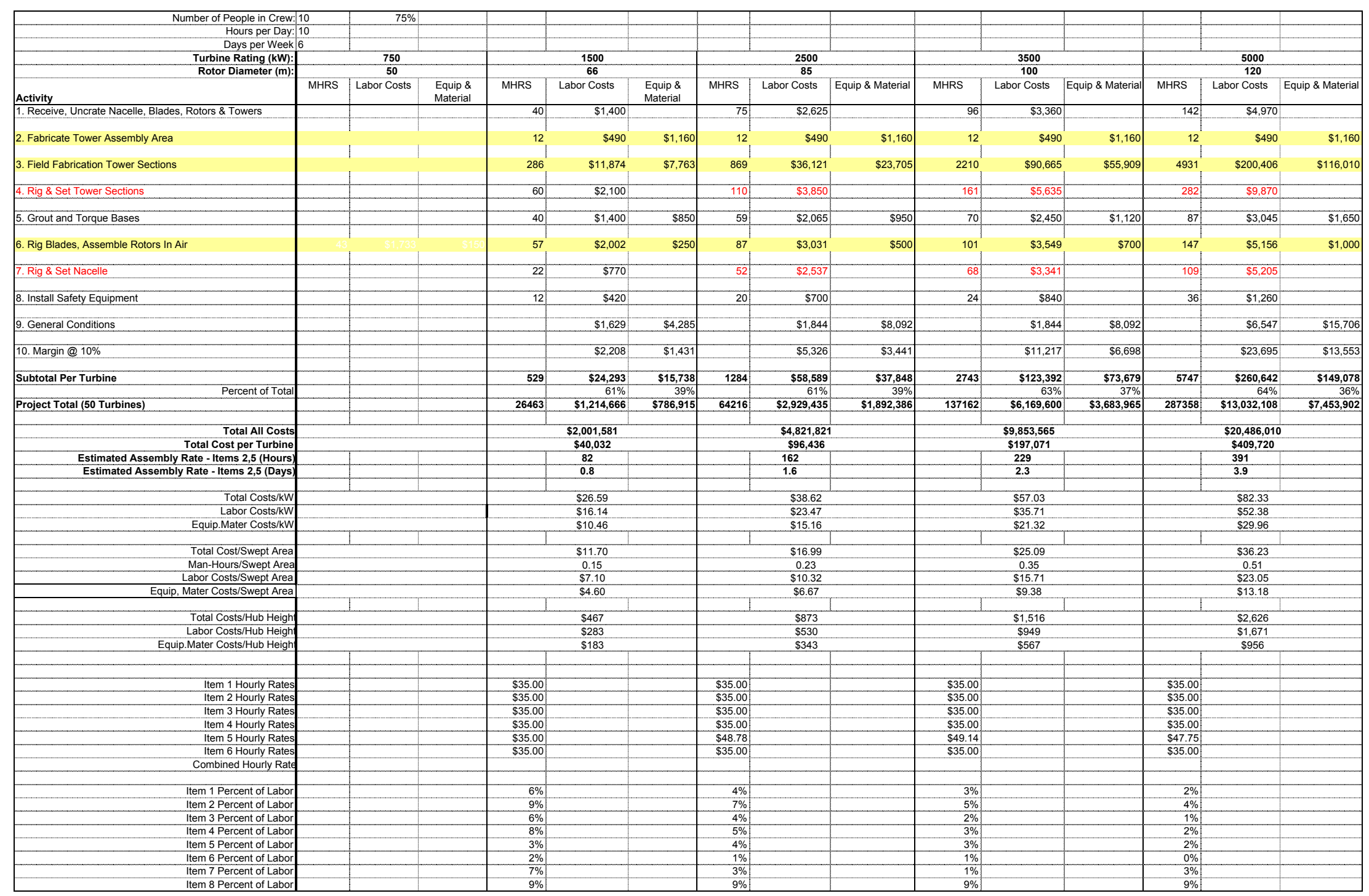




\begin{tabular}{|c|c|c|c|c|c|c|c|c|c|c|c|c|c|c|c|}
\hline $\begin{array}{l}\text { Number of People in Crew: } \\
\text { Hours per Day: }\end{array}$ & & $105 \%$ & & & & & & & & & & & & & \\
\hline $\begin{array}{l}\text { Hours per day: } \\
\text { Days per Week } \\
\end{array}$ & & & & & & & & & & & & & & & \\
\hline Turbine Rating $(\mathrm{kW}):$ & & 750 & & & 1500 & & & 2500 & & & 3500 & & & 5000 & \\
\hline Rotor Diameter $(m)$ : & & 50 & & & 66 & & & 85 & & & 100 & & & 120 & \\
\hline Activity & MHRS & Labor Costs & $\begin{array}{l}\text { Equip \& } \\
\text { Material }\end{array}$ & MHRS & Labor Costs & Equip \& Material & MHRS & Labor Costs & Equip \& Material & MHRS & Labor Costs & Equip \& Material & MHRS & Labor Costs & Equip \& Material \\
\hline 1. Receive, Uncrate Nacelle, Blades, Rotors \& Towers & & & & 64 & $\$ 2,579$ & & 75 & $\$ 3,023$ & & 96 & $\$ 3,869$ & & 142 & $\$ 5,723$ & \\
\hline 2. Fabricate Tower Assembly Area & & & & 16 & $\$ 626$ & $\$ 1,483$ & 16 & $\$ 626$ & $\$ 1,483$ & 16 & $\$ 626$ & $\$ 1,483$ & 16 & $\$ 626$ & $\$ 1,483$ \\
\hline 3. Field Fabrication Tower Sections & & & & 365 & $\$ 15,172$ & $\$ 9,919$ & 1111 & $\$ 46,154$ & $\$ 30,290$ & 2824 & $\$ 115,850$ & $\$ 71,440$ & 6301 & $\$ 256,074$ & $\$ 148,234$ \\
\hline 4. Rig \& Set Tower Sections & & & & 138 & $\$ 5,562$ & & 254 & $\$ 10,236$ & & 371 & $\$ 14,950$ & & 687 & $\$ 27,535$ & \\
\hline 5. Grout and Torque Bases & & & & 40 & $\$ 1,612$ & $\$ 850$ & 59 & $\$ 2,377$ & $\$ 950$ & 70 & $\$ 2,822$ & $\$ 1,120$ & 87 & $\$ 3,506$ & $\$ 1,650$ \\
\hline 6. Rig Blades, Assemble Rotors In Air & & & & 62 & $\$ 2,487$ & $\$ 250$ & 97 & $\$ 3,905$ & $\$ 500$ & 115 & $\$ 4,633$ & $\$ 700$ & 171 & $\$ 6,900$ & $\$ 1,000$ \\
\hline 7. Rig \& Set Nacelle & & & & 67 & $\$ 2,724$ & & 155 & $\$ 7,561$ & & 204 & $\$ 10,024$ & & 348 & $\$ 16,616$ & \\
\hline 8. Install Safety Equipment & & & & 12 & $\$ 484$ & & 20 & $\$ 806$ & & 24 & $\$ 968$ & & 36 & $\$ 1,450$ & \\
\hline 9. General Conditions & & & & & $\$ 2,281$ & $\$ 5,999$ & 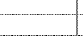 & $\$ 2,582$ & $\$ 11,328$ & & $\$ 2,582$ & $\$ 11,328$ & & $\$ 9,165$ & $\$ 21,988$ \\
\hline 10. Margin @ $10 \%$ & & & & & $\$ 3,353$ & $\$ 1,850$ & $\longrightarrow$ & $\$ \$ \$ 7,727 \mid$ & $\$ 4,455$. & & $\$ 15,632$ & $\$ 8,607$ & & $\$ 32,760$ & $\$ 17,435$ \\
\hline Subtotal Per Turbine & & & & & & & & & & & & & & & \\
\hline Subtotal Per Turbine & & & & 764 & $\$ 36,879$ & $\$ 20,350$ & 1787 & $\$ 84,997$ & $\$ 49,006$ & 3720 & $\$ 171,956$ & $\$ 94,678$ & 7788 & $\$ 360,356$ & $\$ 191,790$ \\
\hline Project Total ( 50 Turbines) & & & & 38,178 & \begin{tabular}{rl|l} 
& $64 \%$ \\
$\$ 1,843,927$
\end{tabular} & $\begin{array}{r}31,017,500 \\
\end{array}$ & 89,328 & $\$ 4,249,873$ & $\$ 2,450,291$ & 186,015 & $\$ 8,597,819$ & $\begin{array}{r}36 \% \\
\$ 4,733,878\end{array}$ & 389,417. & $\$ 18,017,793$ & $\$ 9,589,522$ \\
\hline Total All Costs & & 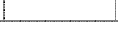 & 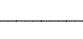 & & $\$ 2,861,427$ & & & $\$ 6,700,164$ & & & $\$ 13,331,697$ & & & $\$ 27,607,315$ & \\
\hline Total Cost per Turbine & & & 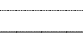 & $\omega_{0}$ & $\begin{array}{l}-12,001,421 \\
\$ 57,229\end{array}$ & & & $\frac{\$ 0,100,134,003}{\$ 13}$ & & & $\$$ & & & $\frac{\$ 21,000,1910}{\$ 552,146}$ & \\
\hline Estimated Assembly Rate - Items 2,5 (Hours) & & & & & 205 & & & 409 & & & 575 & & & 1,035 & \\
\hline Estimated Assembly Rate - Items 2,5 (Days) & & & & & 2.1 & & & 4.1 & & & 5.8 & & & 10.4 & \\
\hline Total Costs/kW & & & & & $\$ 38.02$ & & & $\$ 53.67$ & & & $\$ 77.16$ & & & $\$ 110.96$ & \\
\hline Labor Costs/kW & & & & & $\$ 24.50$ & & & $\$ 34.04$ & & & $\$ 49.76$ & & & $\$ 72.41$ & \\
\hline Equip.Mater Costs/kW & & & & & $\$ 13.52$ & & & $\$ 19.63$ & & & $\$ 27.40$ & & & $\$ 38.54$ & \\
\hline & & & & & & & & & & & & & & & \\
\hline Total Cost/Swept Area & & & & & $\$ 16.73$ & & & $\$ 23.61$ & & & $\$ 33.95$ & & & $\$ 48.82$ & \\
\hline Man-Hours//swept Area & & & & & 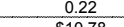 & & & 0.31 & & & 52100 & & & 0.69 & \\
\hline $\begin{array}{l}\text { Labor Costs//Swept Area } \\
\text { Equip Mater Costs/Swat Area }\end{array}$ & & & & & $\frac{\$ 10.78}{\$ 5.95}$ & & & $\frac{\$ 14.98}{\$ 8.64}$ & & & $\$ \$ \$ 21.89$ & & & $\begin{array}{l}\$ 31.86 \\
\$ 1.696\end{array}-20$ & \\
\hline Equip, Mater Cosis/swept Area & & & & 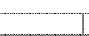 & $\$ 5.95$ & & & $\$ 8.64$ & & & $\$ 12.05$ & & & $\$ 16.96$ & \\
\hline Total Costs/Hub Heigh & & & & & $\$ 667$ & & & $\$ 1,213$ & & & $\$ 2,051$ & & & $\$ 3,539$ & \\
\hline Labor Costs/Hub Heigh & & & & & $\$ 430$ & & & $\$ 769$ & & & $\$ 1,3$, & & & $\$ 2,310$ & \\
\hline Equip.Mater Costs/Hub Heigh & & & & & $\$ 237$ & & & $\$ 443$ & & & $\$ 728$ & & & $\$ 1,229$ & \\
\hline & & & & & & & & & & & & & & & \\
\hline Item 1 Hourly Rates & & & & $\$ 40.30$ & & & $\$ 40.31$ & & & $\$ 40.30$ & & & $\$ 40.30$ & & \\
\hline Item 2 Hourly F & & & & $\$ 4$ & & & $\$ 4$ & & & $\$ 40.30$ & & & $\$ 40.08$ & & \\
\hline Iter & & & & $\$ 40.30$ & & & $\$ 40.29$ & & & $\$ 40.31$ & & & $\$ 40.30$ & & \\
\hline Hourly Rates & & & & & & & & & & $\$ 40.29$ & & & $\$ 40.31$ & & \\
\hline Item 5 Hourly Rates & & & & $\$ 40.65$ & & & $\$ 48.78$ & & & $\$ 49.14$ & & & $\$ 47.75$ & & \\
\hline $\begin{array}{l}\text { Item } 6 \text { Hourly Rates } \\
\text { Combined Hourly Rate }\end{array}$ & & & & $\begin{array}{l}\$ 40.33 \\
\$ 51.35\end{array}$ & & & $\begin{array}{l}\$ 40.30 \\
\$ 54.58\end{array}$ & & & 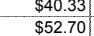 & & & $\begin{array}{l}\$ 40.28 \\
\$ 57.38\end{array}$ & & \\
\hline & & & & & & & & & & & & & & & \\
\hline $\begin{array}{l}\text { Item } 1 \mathrm{Pe} \\
\text { Iteme } 2 \mathrm{Pe}\end{array}$ & & & & $\frac{7 \%}{15 \%}$ & & & $\frac{4 \%}{12 \%}$ & & & $2 \%$ & & & $2 \%$ & & \\
\hline Item $2 \mathrm{Pe}$ & & & & $15 \%$ & & & $12 \%$ & & & $9 \%$ & & & $8 \%$ & & \\
\hline at of Labor & & & & $4 \%$ & & & $3 \%$ & & & $2 \%$ & & & $\%$ & & \\
\hline Item 4 Percent of Labor & & & & $7 \%$ & & & $\%$ & & & $3 \%$ & & & $2 \%$ & & \\
\hline$\frac{\text { Item } 5 \text { Percent of Labor }}{\text { Item } 6 \text { Percent of Labor }}$ & & & & $\frac{7 \%}{1 \%}$ & & & $\frac{9 \%}{1 \%}$ & & & $\frac{6 \%}{1 \%}$ & & & $5 \%$ & & \\
\hline $\begin{array}{l}\text { temem } 6 \text { Percent of Labor } \\
\text { Item } 7 \text { Percent of Labor }\end{array}$ & & & & $\frac{1 \%}{6 \%}$ & & & $\frac{1 \%}{3 \%}$ & & & $\frac{1 \%}{2 \%}$ & & & $3 \%$ & & \\
\hline Item 8 Percent of Labor & & & & $9 \%$ & & & $9 \%$ & & & $9 \%$ & & & $9 \%$ & & \\
\hline
\end{tabular}


WELDED QUARTER SECTIONS OF TOWERS

1. Tower section properties based on data in Table 2-2.

2. See Page N-3 for weight of weld.

3. Assumed manual weld rate:

$4 \mathrm{lbs} / \mathrm{hr}$

Weld weight - 1 inch steel $\quad 2.25 \mathrm{lb} / \mathrm{ft}$

Welding rate $21.3 \mathrm{in} / \mathrm{hr}$

Tower section length $\quad 21.5 \mathrm{~m}$

Tower section length 846 in

\begin{tabular}{|c|c|c|c|c|c|c|c|c|c|}
\hline \multicolumn{3}{|l|}{ Tower Section Assembly 1500 kW } & \multirow[b]{2}{*}{$\begin{array}{c}\text { UNITSI } \\
\text { MHR }\end{array}$} & \multirow[b]{2}{*}{$\begin{array}{l}\text { TOTAL } \\
\text { MHRS }\end{array}$} & \multirow[b]{2}{*}{$\begin{array}{c}\text { COSTI } \\
\text { MHR }\end{array}$} & \multirow[b]{2}{*}{$\begin{array}{l}\text { TOTAL } \\
\text { LABOR }\end{array}$} & \multirow[b]{2}{*}{$\begin{array}{l}\text { MAT'L. } \\
\text { UNIT \$\$ }\end{array}$} & \multirow[b]{2}{*}{$\begin{array}{l}\text { TOTAL } \\
\text { MAT'L. }\end{array}$} & \multirow[b]{2}{*}{$\begin{array}{r}\text { TOTAL } \\
\text { L \& M }\end{array}$} \\
\hline Description & QTY. & UNITS & & & & & & & \\
\hline Screw Jacks & 20 & EA & 5 & 4 & $\$ 40$ & $\$ 160$ & $\$ 0.00$ & $\$ 0$ & $\$ 160$ \\
\hline Quarter-Section Weld & 1693 & INCHES & 21.3 & 79 & $\$ 40$ & $\$ 3,174$ & $\$ 0.58$ & $\$ 982$ & $\$ 4,156$ \\
\hline Half-Section Weld & 1693 & INCHES & 21.3 & 79 & $\$ 40$ & $\$ 3,174$ & $\$ 0.58$ & $\$ 982$ & $\$ 4,156$ \\
\hline Top \& Bottom Template & 10.67 & EA & 0.25 & 43 & $\$ 40$ & $\$ 1,707$ & $\$ 0.00$ & $\$ 0$ & $\$ 1,707$ \\
\hline Move Cradles & 16 & EA & 2 & 8 & $\$ 40$ & $\$ 320$ & $\$ 0.00$ & $\$ 0$ & $\$ 320$ \\
\hline Lifting Eyes & 5.33 & $\mathrm{EA}$ & 1 & 5.33 & $\$ 40$ & $\$ 213$ & $\$ 35.00$ & $\$ 187$ & $\$ 400$ \\
\hline Paint & 5000 & SF & 80 & 63 & $\$ 40$ & $\$ 2,500$ & $\$ 0.50$ & $\$ 2,500$ & $\$ 5,000$ \\
\hline Non-Productive Time & $3 \%$ & MHRS & & 8 & $\$ 40$ & $\$ 337$ & $\$ 0.00$ & $\$ 0$ & $\$ 337$ \\
\hline X-RAY TEST & 20 & EA & 1 & 20 & $\$ 65$ & $\$ 1,300$ & $\$ 20.00$ & $\$ 400$ & $\$ 1,700$ \\
\hline Weld Correction (1\%) & 34 & INCHES & 16 & 2 & $\$ 40$ & $\$ 85$ & $\$ 0.58$ & $\$ 20$ & $\$ 104$ \\
\hline Load \& Transport Towers & 1 & SECTIONS & 0.18 & 6 & $\$ 40$ & $\$ 222$ & $\$ 0.00$ & $\$ 0$ & $\$ 222$ \\
\hline Welding Equipment/Operating Costs & 282 & LF & & & & $\$ 0$ & $\$ 12.60$ & $\$ 3,555$ & $\$ 3,555$ \\
\hline TOTALS (PER SECTION) & & & & 317 & & $\$ 13,193$ & & $\$ 8,625$ & $\$ 21,818$ \\
\hline Minimum & $-10 \%$ & & & 286 & & $\$ 11,874$ & & $\$ 7,763$ & $\$ 19,636$ \\
\hline Maximum & $15 \%$ & & & 365 & & $\$ 15,172$ & & $\$ 9,919$ & $\$ 25,091$ \\
\hline Total Number of Sections in Turbine: & & & 1 & 317 & & $\$ 13,193$ & & $\$ 8,625$ & $\$ 21,818$ \\
\hline Minimum & & & 1 & 286 & & $\$ 11,874$ & & $\$ 7,763$ & $\$ 19,636$ \\
\hline Maximum & & & 1 & 365 & & $\$ 15,172$ & & $\$ 9,919$ & $\$ 25,091$ \\
\hline & Avg & Min & Max & & & & & & \\
\hline 10 People, 10 hrs/day & 100 & 100 & 100 & & & & & & \\
\hline Days per Tower Section & 3.2 & 2.9 & 3.6 & & & & & & \\
\hline Total number of sections for assembly & 50 & 50 & 50 & & & & & & \\
\hline Number of days for assembly & 159 & 143 & 182 & & & & & & \\
\hline Number of assembly days per turbine & 3.2 & 2.9 & 3.6 & & & & & & \\
\hline Number of 6 day weeks & 26.4 & 23.8 & 30.4 & & & & & & \\
\hline
\end{tabular}


WELDED QUARTER SECTIONS OF TOWERS

1. Tower section properties based on data in Table 2-2.

2. See Page N-3 for weight of weld.

3. Assumed manual weld rate:

$4 \mathrm{lbs} / \mathrm{hr}$

Weld weight - 1 inch steel $\quad 2.25 \mathrm{lb} / \mathrm{ft}$

Welding rate $21.3 \mathrm{in} / \mathrm{hr}$

Tower section length $\quad 22.1 \mathrm{~m}$

Tower section length 870 in

\begin{tabular}{|c|c|c|c|c|c|c|c|c|c|}
\hline \multicolumn{10}{|l|}{ Tower Section Assembly 2500 kW } \\
\hline Description & QTY. & UNITS & $\begin{array}{c}\text { UNITS/ } \\
\text { MHR }\end{array}$ & $\begin{array}{c}\text { TOTAL } \\
\text { MHRS }\end{array}$ & $\begin{array}{c}\text { COST/ } \\
\text { MHR }\end{array}$ & $\begin{array}{l}\text { TOTAL } \\
\text { LABOR } \\
\end{array}$ & $\begin{array}{c}\text { MAT'L. } \\
\text { UNIT \$\$ }\end{array}$ & $\begin{array}{l}\text { TOTAL } \\
\text { MAT'L. }\end{array}$ & $\begin{array}{r}\text { TOTAL } \\
\text { L \& M } \\
\end{array}$ \\
\hline Screw Jacks & 20 & EA & 5 & 4 & $\$ 40$ & $\$ 160$ & $\$ 0.00$ & $\$ 0$ & $\$ 160$ \\
\hline Quarter-Section Weld & 1740 & INCHES & 21.3 & 82 & $\$ 40$ & $\$ 3,263$ & $\$ 0.58$ & $\$ 1,009$ & $\$ 4,272$ \\
\hline Half-Section Weld & 1740 & INCHES & 21.3 & 82 & $\$ 40$ & $\$ 3,263$ & $\$ 0.58$ & $\$ 1,009$ & $\$ 4,272$ \\
\hline Top \& Bottom Template & 10.67 & EA & 0.25 & 43 & $\$ 40$ & $\$ 1,707$ & $\$ 0.00$ & $\$ 0$ & $\$ 1,707$ \\
\hline Move Cradles & 16 & EA & 2 & 8 & $\$ 40$ & $\$ 320$ & $\$ 0.00$ & $\$ 0$ & $\$ 320$ \\
\hline Lifting Eyes & 5.33 & EA & 1 & 5.33 & $\$ 40$ & $\$ 213$ & $\$ 35.00$ & $\$ 187$ & $\$ 400$ \\
\hline Paint & 5000 & SF & 80 & 63 & $\$ 40$ & $\$ 2,500$ & $\$ 0.50$ & $\$ 2,500$ & $\$ 5,000$ \\
\hline Non-Productive Time & $3 \%$ & MHRS & & 9 & $\$ 40$ & $\$ 343$ & $\$ 0.00$ & $\$ 0$ & $\$ 343$ \\
\hline X-RAY TEST & 20 & EA & 1 & 20 & $\$ 65$ & $\$ 1,300$ & $\$ 20.00$ & $\$ 400$ & $\$ 1,700$ \\
\hline Weld Correction (1\%) & 35 & INCHES & 16 & 2 & $\$ 40$ & $\$ 87$ & $\$ 0.58$ & $\$ 20$ & $\$ 107$ \\
\hline Load \& Transport Towers & 1 & SECTIONS & 0.18 & 6 & $\$ 40$ & $\$ 222$ & $\$ 0.00$ & $\$ 0$ & $\$ 222$ \\
\hline Welding Equipment/Operating Costs & 290 & $\begin{array}{ll}\text { LF } \\
\end{array}$ & & & & $\$ 0$ & $\$ 12.60$ & $\$ 3,654$ & $\$ 3,654$ \\
\hline \multicolumn{4}{|l|}{ TOTALS (PER SECTION) } & \multicolumn{2}{|l|}{322} & \multicolumn{2}{|l|}{$\$ 13,378$} & $\$ 8,780$ & $\$ 22,158$ \\
\hline Minimum & \multicolumn{3}{|l|}{$-10 \%$} & 290 & & \multicolumn{2}{|l|}{$\$ 12,040$} & $\$ 7,902$ & \multirow{2}{*}{$\begin{array}{l}\$ 19,942 \\
\$ 25,481\end{array}$} \\
\hline Maximum & $15 \%$ & & & 370 & & $\$ 15,385$ & & $\$ 10,097$ & \\
\hline \multicolumn{3}{|l|}{ Total Number of Sections in Turbine: } & 3 & 966 & & \multicolumn{2}{|l|}{$\$ 40,134$} & $\$ 26,339$ & $\$ 66,473$ \\
\hline \multirow{2}{*}{\multicolumn{3}{|c|}{$\begin{array}{l}\text { Minimum } \\
\text { Maximum }\end{array}$}} & 3 & 869 & & \multicolumn{2}{|l|}{$\$ 36,121$} & \multirow{3}{*}{$\begin{array}{l}\$ 23,705 \\
\$ 30,290 \\
\end{array}$} & \multirow{2}{*}{$\begin{array}{l}\$ 59,826 \\
\$ 76,444\end{array}$} \\
\hline & & & 3 & 1111 & & \multicolumn{2}{|l|}{$\$ 46,154$} & & \\
\hline & \multicolumn{2}{|c|}{ Avg } & Max & & & & & & \\
\hline 10 People, 10 hrs/day & 100 & 100 & 100 & & & & & & \\
\hline Days per Tower Section & 3.2 & 2.9 & 3.7 & & & & & & \\
\hline Total number of sections for assembly & 150 & 150 & 150 & & & & & & \\
\hline Number of days for assembly & 483 & 435 & 555 & & & & & & \\
\hline Number of assembly days per turbine & 9.7 & 8.7 & 11.1 & & & & & & \\
\hline Number of 6-day weeks & 80.5 & 72.4 & 92.6 & & & & & & \\
\hline
\end{tabular}


WELDED QUARTER SECTIONS OF TOWERS

1. Tower section properties based on data in Table 2-2.

2. See Page N-3 for weight of weld.

3. Assumed manual weld rate:

-1.5 inch steel

Tower section length $\quad 21.7 \mathrm{~m}$

Tower section length 854 in

\begin{tabular}{|c|c|c|c|c|c|c|c|c|c|}
\hline \multicolumn{3}{|l|}{ Tower Section Assembly 3500 MW } & \multirow[b]{2}{*}{ UNITS/ } & \multirow[b]{2}{*}{ TOTAL } & \multirow[b]{2}{*}{$\cos \mathrm{T} /$} & \multirow[b]{2}{*}{ TOTAL } & \multirow[b]{2}{*}{ MAT'L. } & \multirow[b]{2}{*}{ TOTAL } & \multirow[b]{2}{*}{ TOTAL } \\
\hline & & & & & & & & & \\
\hline Description & QTY. & UNITS & MHR & MHRS & MHR & LABOR & UNIT \$\$ & MAT'L. & L\& $\&$ \\
\hline Screw Jacks & 20 & $\mathrm{EA}$ & 5 & 4 & $\$ 40$ & $\$ 160$ & $\$ 0.00$ & $\$ 0$ & $\$ 160$ \\
\hline Quarter-Section Weld & 1709 & INCHES & 10.4 & 164 & $\$ 40$ & $\$ 6,550$ & $\$ 0.58$ & $\$ 991$ & $\$ 7,541$ \\
\hline Half-Section Weld & 1709 & INCHES & 10.4 & 164 & $\$ 40$ & $\$ 6,550$ & $\$ 0.58$ & $\$ 991$ & $\$ 7,541$ \\
\hline Top \& Bottom Template & 10.67 & EA & 0.25 & 43 & $\$ 40$ & $\$ 1,707$ & $\$ 0.00$ & $\$ 0$ & $\$ 1,707$ \\
\hline Move Cradles & 16 & EA & 2 & 8 & $\$ 40$ & $\$ 320$ & $\$ 0.00$ & $\$ 0$ & $\$ 320$ \\
\hline Lifting Eyes & 5.33 & EA & 1 & 5.33 & $\$ 40$ & $\$ 213$ & $\$ 35.00$ & $\$ 187$ & $\$ 400$ \\
\hline Paint & 5000 & SF & 80 & 63 & $\$ 40$ & $\$ 2,500$ & $\$ 0.50$ & $\$ 2,500$ & $\$ 5,000$ \\
\hline Non-Productive Time & $3 \%$ & MHRS & & 14 & $\$ 40$ & $\$ 540$ & $\$ 0.00$ & $\$ 0$ & $\$ 540$ \\
\hline X-RAY TEST & 20 & EA & 1 & 20 & $\$ 65$ & $\$ 1,300$ & $\$ 20.00$ & $\$ 400$ & $\$ 1,700$ \\
\hline Weld Correction (1\%) & 34 & INCHES & 16 & 2 & $\$ 40$ & $\$ 85$ & $\$ 0.58$ & $\$ 20$ & $\$ 105$ \\
\hline Load \& Transport Towers & 1 & SECTIONS & 0.18 & 6 & $\$ 40$ & $\$ 222$ & $\$ 0.00$ & $\$ 0$ & $\$ 222$ \\
\hline Welding Equipment/Operating Costs & 285 & LF & & & & $\$ 0$ & $\$ 25.76$ & $\$ 7,336$ & $\$ 7,336$ \\
\hline TOTALS (PER SECTION) & & & & 491 & & $\$ 20,148$ & & $\$ 12,424$ & $\$ 32,572$ \\
\hline Minimum & $-10 \%$ & & & 442 & & $\$ 18,133$ & & $\$ 11,182$ & $\$ 29,315$ \\
\hline Maximum & $15 \%$ & & & 565 & & $\$ 23,170$ & & $\$ 14,288$ & $\$ 37,458$ \\
\hline Total Number of Sections in Turbine: & & & 5 & 2456 & & $\$ 100,739$ & & $\$ 62,121$ & $\$ 162,860$ \\
\hline Minimum & & & 5 & 2210 & & $\$ 90,665$ & & $\$ 55,909$ & $\$ 146,574$ \\
\hline Maximum & & & 5 & 2824 & & $\$ 115,850$ & & $\$ 71,440$ & $\$ 187,289$ \\
\hline & Avg & Min & Ma & & & & & & \\
\hline 10 People, 10 hrs/day & 100 & 100 & 10 & & & & & & \\
\hline Days per Tower Section & 4.9 & 4.4 & 5. & & & & & & \\
\hline Total number of sections for assembly & 250 & 250 & 25 & & & & & & \\
\hline Number of days for assembly & 1228 & 1105 & 141 & & & & & & \\
\hline Number of assembly days per turbine & 24.6 & 22.1 & 28. & & & & & & \\
\hline Number of 6-day weeks & 204.7 & 184.2 & 235. & & & & & & \\
\hline
\end{tabular}


WELDED QUARTER SECTIONS OF TOWERS

1. Tower section properties based on data in Table 2-2.

2. See Page N-3 for weight of weld.

3. Assumed manual weld rate:

$\begin{array}{lr}\text { Weld weight - 2 inch steel } & 4 \mathrm{lbs} / \mathrm{hr} \\ \text { Welding rate } & 7.8 \mathrm{Ib} / \mathrm{ft} \\ \text { Tower section length } & 6.2 \mathrm{in} / \mathrm{hr} \\ \text { Tower section length } & 22.3 \mathrm{~m} \\ & 878 \mathrm{in}\end{array}$

\begin{tabular}{|c|c|c|c|c|c|c|c|c|c|}
\hline \multicolumn{10}{|l|}{ Tower Section Assembly 5000 kW } \\
\hline Description & QTY. & UNITS & $\begin{array}{c}\text { UNITS/ } \\
\text { MHR }\end{array}$ & $\begin{array}{l}\text { TOTAL } \\
\text { MHRS } \\
\end{array}$ & $\begin{array}{c}\cos / 1 \\
\text { MHR }\end{array}$ & $\begin{array}{l}\text { TOTAL } \\
\text { LABOR } \\
\end{array}$ & $\begin{array}{c}\text { MAT'L. } \\
\text { UNIT \$\$ }\end{array}$ & $\begin{array}{l}\text { TOTAL } \\
\text { MAT'L. }\end{array}$ & $\begin{array}{r}\text { TOTAL } \\
\text { L \& M }\end{array}$ \\
\hline Screw Jacks & 24 & EA & 4 & 6 & $\$ 40$ & $\$ 240$ & $\$ 0.00$ & $\$ 0$ & $\$ 240$ \\
\hline Quarter-Section Weld & 1756 & INCHES & 6.2 & 285 & $\$ 40$ & $\$ 11,413$ & $\$ 0.58$ & $\$ 1,018$ & $\$ 12,432$ \\
\hline Half-Section Weld & 1756 & INCHES & 6.2 & 285 & $\$ 40$ & $\$ 11,413$ & $\$ 0.58$ & $\$ 1,018$ & $\$ 12,432$ \\
\hline Top \& Bottom Template & 16 & EA & 0.25 & 64 & $\$ 40$ & $\$ 2,560$ & $\$ 0.00$ & $\$ 0$ & $\$ 2,560$ \\
\hline Move Cradles & 24 & $E A$ & 2 & 12 & $\$ 40$ & $\$ 480$ & $\$ 0.00$ & $\$ 0$ & $\$ 480$ \\
\hline Lifting Eyes & 8 & EA & 1 & 8 & $\$ 40$ & $\$ 320$ & $\$ 35.00$ & $\$ 280$ & $\$ 600$ \\
\hline Paint & 5788 & SF & 80 & 72 & $\$ 40$ & $\$ 2,894$ & $\$ 0.50$ & $\$ 2,894$ & $\$ 5,788$ \\
\hline Non-Productive Time & $3 \%$ & MHRS & & 22 & $\$ 40$ & $\$ 880$ & $\$ 0.00$ & $\$ 0$ & $\$ 880$ \\
\hline X-RAY TEST & 20 & EA & 1 & 20 & $\$ 65$ & $\$ 1,300$ & $\$ 20.00$ & $\$ 400$ & $\$ 1,700$ \\
\hline Weld Correction (1\%) & 35 & INCHES & 16 & 2 & $\$ 40$ & $\$ 88$ & $\$ 0.58$ & $\$ 20$ & $\$ 108$ \\
\hline Load \& Transport Towers & 1 & SECTIONS & 0.18 & 6 & $\$ 40$ & $\$ 222$ & $\$ 0.00$ & $\$ 0$ & $\$ 222$ \\
\hline Welding Equipment/Operating Costs & 293 & LF & & & & $\$ 0$ & $\$ 43.68$ & $\$ 12,783$ & $\$ 12,783$ \\
\hline TOTALS (PER SECTION) & & & & 783 & & $\$ 31,810$ & & $\$ 18,414$ & $\$ 50,225$ \\
\hline Minimum & $-10 \%$ & & & 704 & & $\$ 28,629$ & & $\$ 16,573$ & $\$ 45,202$ \\
\hline Maximum & $15 \%$ & & & 900 & & $\$ 36,582$ & & $\$ 21,176$ & $\$ 57,758$ \\
\hline Total Number of Sections in Turbine: & & & 7 & 5479 & & $\$ 222,673$ & & $\$ 128,899$ & $\$ 351,572$ \\
\hline Minimum & & & 7 & 4931 & & $\$ 200,406$ & & $\$ 116,010$ & $\$ 316,415$ \\
\hline \multirow[t]{2}{*}{ Maximum } & & & 7 & 6301 & & $\$ 256,074$ & & $\$ 148,234$ & $\$ 404,308$ \\
\hline & Avg & Min & Max & & & & & & \\
\hline 10 People, 10 hrs/day & 100 & 100 & 100 & & & & & & \\
\hline Days per Tower Section & 7.8 & 7.0 & 9.0 & & & & & & \\
\hline Total number of sections for assembly & 350 & 350 & 350 & & & & & & \\
\hline Number of days for assembly & 2740 & 2466 & 3151 & & & & & & \\
\hline Number of assembly days per turbine & 54.8 & 49.3 & 63.0 & & & & & & \\
\hline Number of 6-day weeks & 456.6 & 410.9 & 525.1 & & & & & & \\
\hline
\end{tabular}




\begin{tabular}{|c|c|c|c|c|c|c|c|c|c|c|c|c|}
\hline $\begin{array}{l}\text { Turbine Class } \\
\text { Rotor Diameter: }\end{array}$ & \multicolumn{3}{|c|}{$\begin{array}{l}1,500 \\
66 \\
\end{array}$} & \multicolumn{3}{|c|}{$\begin{array}{l}2,500 \\
85 \\
\end{array}$} & \multicolumn{3}{|c|}{$\begin{array}{l}3,500 \\
100 \\
\end{array}$} & \multicolumn{3}{|c|}{$\frac{5000}{120}$} \\
\hline Crane Type: & \multicolumn{3}{|c|}{$4100 \mathrm{~S} 1$} & \multicolumn{3}{|c|}{$4100 \mathrm{~S} 1$} & \multicolumn{3}{|c|}{$4600 \$ 4$} & \multicolumn{3}{|c|}{4600 S5 } \\
\hline & Min & Avg & Max & Min & Avg & $\operatorname{Max}$ & $\operatorname{Min}$ & Avg & $\operatorname{Max}$ & Min & Avg & Max \\
\hline Crane Crew Assembly Labor Costs/Turbine & $\$ 3,713$ & $\$ 4,125$ & $\$ 4,744$ & $\$ 11,300$ & $\$ 12,556$ & $\$ 14,439$ & $\$ 28,735$ & $\$ 31,928$ & $\$ 36,717$ & $\$ 64,108$ & $\$ 71,231$ & $\$ 81,916$ \\
\hline Crane Crew Relocation Labor Costs/Turbine & & & & \$0 & \$0 & $\$ 0$ & \$0 & $\$ 0$ & \$0 & $\$ 0$ & $\$ 0$ & $\$ 0$ \\
\hline Crane Rental Costs During Assembly and Relocation/Turbine & $\$ 1,648$ & $\$ 1,831$ & $\$ 2,105$ & $\$ 5,015$ & $\$ 5,572$ & $\$ 6,408$ & $\$ 27,205$ & $\$ 30,227$ & $\$ 34,761$ & $\$ 71,126$ & $\$ 79,029$ & $\$ 90,883$ \\
\hline Meals and Lodging/Turbine & $\$ 428$ & $\$ 476$ & $\$ 547$ & $\$ 1,304$ & $\$ 1,449$ & $\$ 1,666$ & $\$ 3,316$ & $\$ 3,684$ & $\$ 4,237$ & $\$ 7,397$ & $\$ 8,219$ & $\$ 9,452$ \\
\hline Fuel CostTurbine & $\$ 157$ & $\$ 175$ & $\$ 201$ & $\$ 478$ & $\$ 531$ & $\$ 611$ & $\$ 1,381$ & $\$ 1,535$ & $\$ 1,765$ & $\$ 3,082$ & $\$ 3,425$ & $\$ 3,938$ \\
\hline Cribbing Costs/Turbine & $\$ 131$ & $\$ 131$ & $\$ 131$ & $\$ 131$ & $\$ 131$ & $\$ 131$ & $\$ 190$ & $\$ 190 \mid$ & $\$ 190$ & $\$ 595$ & $\$ 595$ & $\$ 595$ \\
\hline Mob/Demob Costs/Turbine & $\$ 1,328$ & $\$ 1,328$ & $\$ 1,328$ & $\$ 1,328$ & $\$ 1,328$ & $\$ 1,328$ & $\$ 2,477$ & $\$ 2,477$ & $\$ 2,477$ & $\$ 2,757$ & $\$ 2,757$ & $\$ 2,757$ \\
\hline Total Crane and Crew Costs/Turbine & $\$ 7,405$ & $\$ 8,066$ & $\$ 9,057$ & $\$ 19,557$ & $\$ 21,567$ & $\$ 24,584$ & $\$ 63,303$ & $\$ 70,041$ & $\$ 80,147$ & $\$ 149,065$ & $\$ 165,255$ & $\$ 189,541$ \\
\hline Total Crane Costs (50 Turbines) & $\$ 370,248$ & $\$ 403,281$ & $\$ 452,830$ & $\$ 977,825$ & $\$ 1,078,367$ & $\$ 1,229,179$ & $\$ 3,165,163$ & $\$ 3,502,033$ & $\$ 4,007,337$ & $\$ 7,453,237$ & \begin{tabular}{|l|}
$\$ 8,262,754$ \\
\end{tabular} & $\$ 9,477,030$ \\
\hline & & & & & & & & & & & & \\
\hline Costs/kW & $\$ 4.92$ & $\$ 5.36$ & $\$ 6.02$ & $\$ 7.83$ & $\$ 8.64$ & $\$ 9.85$ & \begin{tabular}{|l|l|l} 
&
\end{tabular} & $\$ 20.27$ & $\$ 23.19$ & $\$ 29.96$ & $\$ 33.21$ & $\$ 38.09$ \\
\hline Costs/Swept Area & $\$ 2.16$ & $\$ 2.36$ & $\$ 2.65$ & $\$ 3.45$ & $\$ 3.80$ & $\$ 4.33$ & $\$ 8.06$ & $\$ 8.92$ & $\$ 10.20$ & $\$ 13.18$ & $\$ 14.61$ & $\$ 16.76$ \\
\hline
\end{tabular}




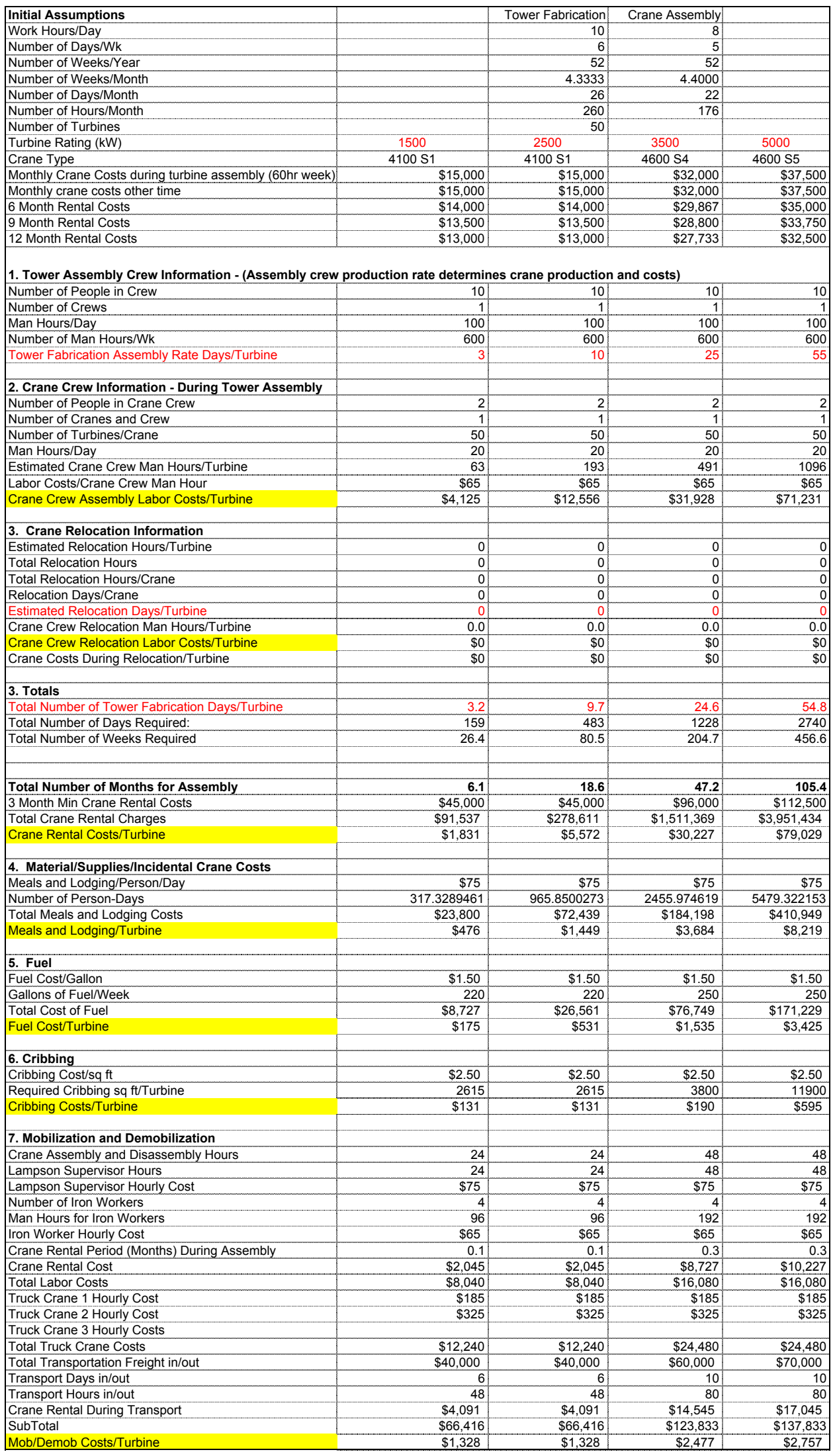




\begin{tabular}{|c|c|c|c|c|}
\hline Initial Assumptions & & Tower Fabrication & Crane Assembly & \\
\hline Work Hours/Day & & 10 & 8 & \\
\hline Number of Days/Wk & & 6 & 5 & \\
\hline Number of Weeks/Year & & 52 & 52 & \\
\hline Number of Weeks/Month & & 4.3333 & 4.4000 & \\
\hline Number of Days/Month & & 26 & 22 & \\
\hline Number of Hours/Month & & 260 & 176 & \\
\hline Number of Turbines & & 50 & & \\
\hline Turbine Rating (kW) & 1500 & 2500 & 3500 & 5000 \\
\hline Crane Type & 4100 S1 & 4100 S1 & 4600 S4 & $4600 \mathrm{~S} 5$ \\
\hline Monthly Crane Costs during turbine assembly (60hr week) & $\$ 15,000$ & $\$ 15,000$ & $\$ 32,000$ & $\$ 37,500$ \\
\hline Monthly crane costs other time & $\$ 15,000$ & $\$ 15,000$ & $\$ 32,000$ & $\$ 37,500$ \\
\hline 6 Month Rental Costs & $\$ 14,000$ & $\$ 14,000$ & $\$ 29,867$ & $\$ 35,000$ \\
\hline 9 Month Rental Costs & $\$ 13,500$ & $\$ 13,500$ & $\$ 28,800$ & $\$ 33,750$ \\
\hline 12 Month Rental Costs & $\$ 13,000$ & $\$ 13,000$ & $\$ 27,733$ & $\$ 32,500$ \\
\hline \multicolumn{5}{|c|}{ 1. Assembly Crew Information - (Assembly crew production rate determines crane production and costs) } \\
\hline Number of People in Crew & 10 & 10 & 10 & 10 \\
\hline Number of Crews & 1 & 1 & 1 & 1 \\
\hline Man Hours/Day & 100 & 100 & 100 & 100 \\
\hline Number of Man Hours/Wk & 600 & 600 & 600 & 600 \\
\hline Tower Fabrication Assembly Rate Days/Turbine & 2.9 & 8.7 & 22.1 & 49.3 \\
\hline \multicolumn{5}{|l|}{ 2. Crane Crew Information - During Tower Assembly } \\
\hline Number of People in Crane Crew & 2 & 2 & 2 & 2 \\
\hline Number of Cranes and Crew & 1 & 1 & 1 & 1 \\
\hline Number of Turbines/Crane & 50 & 50 & 50 & 50 \\
\hline Man Hours/Day & 20 & 20 & 20 & 20 \\
\hline Estimated Crane Crew Man Hours/Turbine & 57 & 174 & 442 & 986 \\
\hline Labor Costs/Crane Crew Man Hour & $\$ 65$ & $\$ 65$ & $\$ 65$ & $\$ 65$ \\
\hline Crane Crew Assembly Labor Costs/Turbine & $\$ 3,713$ & $\$ 11,300$ & $\$ 28,735$ & $\$ 64,108$ \\
\hline \multicolumn{5}{|l|}{ 3. Crane Relocation Information } \\
\hline Estimated Relocation Hours/Turbine & 0 & 0 & 0 & 0 \\
\hline Total Relocation Hours & 0 & 0 & 0 & 0 \\
\hline Total Relocation Hours/Crane & 0 & 0 & 0 & 0 \\
\hline Relocation Days/Crane & 0 & 0 & 0 & 0 \\
\hline Estimated Relocation Days/Turbine & 0 & 0 & 0 & 0 \\
\hline Crane Crew Relocation Man Hours/Turbine & 0.0 & 0.0 & 0.0 & 0.0 \\
\hline Crane Crew Relocation Labor Costs/Turbine & $\$ 0$ & $\$ 0$ & $\$ 0$ & $\$ 0$ \\
\hline Crane Costs During Relocation/Turbine & $\$ 0$ & \$0 & $\$ 0$ & $\$ 0$ \\
\hline \multicolumn{5}{|l|}{ 3. Totals } \\
\hline Total Number of Tower Fabrication Days/Turbine & 2.9 & 8.7 & 22.1 & 49.3 \\
\hline Total Number of Days Required: & 143 & 435 & 1105 & 2466 \\
\hline Total Number of Weeks Required & 23.8 & 72.4 & 184.2 & 410.9 \\
\hline & & & & \\
\hline Total Number of Months for Assembly & 5.5 & 16.7 & 42.5 & 94.8 \\
\hline 3 Month Min Crane Rental Costs & $\$ 45,000$ & $\$ 45,000$ & $\$ 96,000$ & $\$ 112,500$ \\
\hline Total Crane Rental Charges & $\$ 82,383$ & $\$ 250,750$ & $\$ 1,360,232$ & $\$ 3,556,291$ \\
\hline Crane Rental Costs/Turbine & $\$ 1,648$ & $\$ 5,015$ & $\$ 27,205$ & $\$ 71,126$ \\
\hline \multicolumn{5}{|l|}{ 4. Material/Supplies/Incidental Crane Costs } \\
\hline Meals and Lodging/Person/Day & $\$ 75$ & $\$ 75$ & $\$ 75$ & $\$ 75$ \\
\hline Number of Person-Days & 285.5960515 & 869.2650246 & 2210.377157 & 4931.389937 \\
\hline Total Meals and Lodging Costs & $\$ 21,420$ & $\$ 65,195$ & $\$ 165,778$ & $\$ 369,854$ \\
\hline Meals and Lodging/Turbine & $\$ 428$ & $\$ 1,304$ & $\$ 3,316$ & $\$ 7,397$ \\
\hline \multirow{2}{*}{\multicolumn{5}{|c|}{ 5. Fuel }} \\
\hline & & & & \\
\hline Fuel Cost/Gallon & $\$ 1.50$ & $\$ 1.50$ & $\$ 1.50$ & $\$ 1.50$ \\
\hline Gallons of Fuel/Week & 220 & 220 & 250 & 250 \\
\hline Total Cost of Fuel & $\$ 7,854$ & $\$ 23,905$ & $\$ 69,074$ & $\$ 154,106$ \\
\hline Fuel Cost/Turbine & $\$ 157$ & $\$ 478$ & $\$ 1,381$ & $\$ 3,082$ \\
\hline 6. Cribbing & & & & \\
\hline Cribbing Cost/sq ft & $\$ 2.50$ & $\$ 2.50$ & $\$ 2.50$ & $\$ 2.50$ \\
\hline Required Cribbing sq ft/Turbine & 2615 & 2615 & 3800 & 11900 \\
\hline Cribbing Costs/Turbine & $\$ 131$ & $\$ 131$ & $\$ 190$ & $\$ 595$ \\
\hline 7. Mobilization and Demobilization & & & & \\
\hline Crane Assembly and Disassembly Hours & 24 & 24 & 48 & 48 \\
\hline Lampson Supervisor Hours & 24 & 24 & 48 & 48 \\
\hline Lampson Supervisor Hourly Cost & $\$ 75$ & $\$ 75$ & $\$ 75$ & $\$ 75$ \\
\hline Number of Iron Workers & 4 & 4 & 4 & 4 \\
\hline Man Hours for Iron Workers & 96 & 96 & 192 & 192 \\
\hline Iron Worker Hourly Cost & $\$ 65$ & $\$ 65$ & $\$ 65$ & $\$ 65$ \\
\hline Crane Rental Period (Months) During Assembly & 0.1 & 0.1 & 0.3 & 0.3 \\
\hline Crane Rental Cost & $\$ 2,045$ & $\$ 2,045$ & $\$ 8,727$ & $\$ 10,227$ \\
\hline Total Labor Costs & $\$ 8,040$ & $\$ 8,040$ & $\$ 16,080$ & $\$ 16,080$ \\
\hline Truck Crane 1 Hourly Cost & $\$ 185$ & $\$ 185$ & $\$ 185$ & $\$ 185$ \\
\hline Truck Crane 2 Hourly Cost & $\$ 325$ & $\$ 325$ & $\$ 325$ & $\$ 325$ \\
\hline Truck Crane 3 Hourly Costs & & & & \\
\hline Total Truck Crane Costs & $\$ 12,240$ & $\$ 12,240$ & $\$ 24,480$ & $\$ 24,480$ \\
\hline Total Transportation Freight in/out & $\$ 40,000$ & $\$ 40,000$ & $\$ 60,000$ & $\$ 70,000$ \\
\hline Transport Days in/out & 6 & 6 & 10 & 10 \\
\hline Transport Hours in/out & 48 & 48 & 80 & 80 \\
\hline Crane Rental During Transport & $\$ 4,091$ & $\$ 4,091$ & $\$ 14,545$ & $\$ 17,045$ \\
\hline SubTotal & $\$ 66,416$ & $\$ 66,416$ & $\$ 123,833$ & $\$ 137,833$ \\
\hline Mob/Demob Costs/Turbine & $\$ 1,328$ & $\$ 1,328$ & $\$ 2,477$ & $\$ 2,757$ \\
\hline
\end{tabular}




\begin{tabular}{|c|c|c|c|c|}
\hline Initial Assumptions & & Tower Fabrication & Crane Assembly & \\
\hline Work Hours/Day & & 10 & 8 & \\
\hline Number of Days/Wk & & 6 & 5 & \\
\hline Number of Weeks/Year & & 52 & 52 & \\
\hline Number of Weeks/Month & & 4.3333 & 4.4000 & \\
\hline Number of Days/Month & & 26 & 22 & \\
\hline Number of Hours/Month & & 260 & 176 & \\
\hline Number of Turbines & & 50 & & \\
\hline Turbine Rating (kW) & 1500 & 2500 & 3500 & 5000 \\
\hline Crane Type & $4100 \mathrm{S1}$ & $4100 \mathrm{~S} 1$ & $4600 \mathrm{~S} 4$ & 4600 S5 \\
\hline Monthly Crane Costs during turbine assembly (60hr week) & $\$ 15,000$ & $\$ 15,000$ & $\$ 32,000$ & $\$ 37,500$ \\
\hline Monthly crane costs other time & $\$ 15,000$ & $\$ 15,000$ & $\$ 32,000$ & $\$ 37,500$ \\
\hline 6 Month Rental Costs & $\$ 14,000$ & $\$ 14,000$ & $\$ 29,867$ & $\$ 35,000$ \\
\hline 9 Month Rental Costs & $\$ 13,500$ & $\$ 13,500$ & $\$ 28,800$ & $\$ 33,750$ \\
\hline 12 Month Rental Costs & $\$ 13,000$ & $\$ 13,000$ & $\$ 27,733$ & $\$ 32,500$ \\
\hline \multicolumn{5}{|c|}{ 1. Assembly Crew Information - (Assembly crew production rate determines crane production and costs) } \\
\hline Number of People in Crew & 10 & 10 & 10 & 10 \\
\hline Number of Crews & 1 & 1 & 1 & 1 \\
\hline Man Hours/Day & 100 & 100 & 100 & 100 \\
\hline Number of Man Hours/Wk & 600 & 600 & 600 & 600 \\
\hline Tower Fabrication Assembly Rate Days/Turbine & 3.6 & 11.1 & 28.2 & 63.0 \\
\hline \multicolumn{5}{|l|}{ 2. Crane Crew Information - During Tower Assembly } \\
\hline Number of People in Crane Crew & 2 & 2 & 2 & 2 \\
\hline Number of Cranes and Crew & 1 & 1 & 1 & 1 \\
\hline Number of Turbines/Crane & 50 & 50 & 50 & 50 \\
\hline Man Hours/Day & 20 & 20 & 20 & 20 \\
\hline Estimated Crane Crew Man Hours/Turbine & 73 & 222 & 565 & 1260 \\
\hline Labor Costs/Crane Crew Man Hour & $\$ 65$ & $\$ 65$ & $\$ 65$ & $\$ 65$ \\
\hline Crane Crew Assembly Labor Costs/Turbine & $\$ 4,744$ & $\$ 14,439$ & $\$ 36,717$ & $\$ 81,916$ \\
\hline \multicolumn{5}{|l|}{ 3. Crane Relocation Information } \\
\hline Estimated Relocation Hours/Turbine & 0 & 0 & 0 & 0 \\
\hline Total Relocation Hours & 0 & 0 & 0 & 0 \\
\hline Total Relocation Hours/Crane & 0 & 0 & 0 & 0 \\
\hline Relocation Days/Crane & 0 & 0 & 0 & 0 \\
\hline Estimated Relocation Days/Turbine & 0 & 0 & 0 & 0 \\
\hline Crane Crew Relocation Man Hours/Turbine & 0.0 & 0.0 & 0.0 & 0.0 \\
\hline Crane Crew Relocation Labor Costs/Turbine & $\$ 0$ & $\$ 0$ & $\$ 0$ & $\$ 0$ \\
\hline Crane Costs During Relocation/Turbine & $\$ 0$ & $\$ 0$ & $\$ 0$ & $\$ 0$ \\
\hline \multicolumn{5}{|l|}{ 3. Totals } \\
\hline Total Number of Tower Fabrication Days/Turbine & 3.6 & 11.1 & 28.2 & 63.0 \\
\hline Total Number of Days Required: & 182 & 555 & 1412 & 3151 \\
\hline Total Number of Weeks Required & 30.4 & 92.6 & 235.4 & 525.1 \\
\hline & & & & \\
\hline Total Number of Months for Assembly & 7.0 & 21.4 & 54.3 & 121.2 \\
\hline 3 Month Min Crane Rental Costs & $\$ 45,000$ & $\$ 45,000$ & $\$ 96,000$ & $\$ 112,500$ \\
\hline Total Crane Rental Charges & $\$ 105,268$ & $\$ 320,402$ & $\$ 1,738,074$ & $\$ 4,544,149$ \\
\hline Crane Rental Costs/Turbine & $\$ 2,105$ & $\$ 6,408$ & $\$ 34,761$ & $\$ 90,883$ \\
\hline \multicolumn{5}{|l|}{ 4. Material/Supplies/Incidental Crane Costs } \\
\hline Meals and Lodging/Person/Day & $\$ 75$ & $\$ 75$ & $\$ 75$ & $\$ 75$ \\
\hline Number of Person-Days & 364.928288 & 1110.727531 & 2824.370812 & 6301.220476 \\
\hline Total Meals and Lodging Costs & $\$ 27,370$ & $\$ 83,305$ & $\$ 211,828$ & $\$ 472,592$ \\
\hline Meals and Lodging/Turbine & $\$ 547$ & $\$ 1,666$ & $\$ 4,237$ & $\$ 9,452$ \\
\hline \multicolumn{5}{|l|}{ 5. Fuel } \\
\hline Fuel Cost/Gallon & $\$ 1.50$ & $\$ 1.50$ & $\$ 1.50$ & $\$ 1.50$ \\
\hline Gallons of Fuel/Week & 220 & 220 & 250 & 250 \\
\hline Total Cost of Fuel & $\$ 10,036$ & $\$ 30,545$ & $\$ 88,262$ & $\$ 196,913$ \\
\hline Fuel Cost/Turbine & $\$ 201$ & $\$ 611$ & $\$ 1,765$ & $\$ 3,938$ \\
\hline 6. Cribbing & & & & \\
\hline Cribbing Cost/sg ft & $\$ 2.50$ & $\$ 2.50$ & $\$ 2.50$ & $\$ 2.50$ \\
\hline Required Cribbing sq ft/Turbine & 2615 & 2615 & 3800 & 11900 \\
\hline Cribbing Costs/Turbine & $\$ 131$ & $\$ 131$ & $\$ 190$ & $\$ 595$ \\
\hline 7. Mobilization and Demobilization & & & & \\
\hline Crane Assembly and Disassembly Hours & 24 & 24 & 48 & 48 \\
\hline Lampson Supervisor Hours & 24 & 24 & 48 & 48 \\
\hline Lampson Supervisor Hourly Cost & $\$ 75$ & $\$ 75$ & $\$ 75$ & $\$ 75$ \\
\hline Number of Iron Workers & 4 & 4 & 4 & 4 \\
\hline Man Hours for Iron Workers & 96 & 96 & 192 & 192 \\
\hline Iron Worker Hourly Cost & $\$ 65$ & $\$ 65$ & $\$ 65$ & $\$ 65$ \\
\hline Crane Rental Period (Months) During Assembly & 0.1 & 0.1 & 0.3 & 0.3 \\
\hline Crane Rental Cost & $\$ 2,045$ & $\$ 2,045$ & $\$ 8,727$ & $\$ 10,227$ \\
\hline Total Labor Costs & $\$ 8,040$ & $\$ 8,040$ & $\$ 16,080$ & $\$ 16,080$ \\
\hline Truck Crane 1 Hourly Cost & $\$ 185$ & $\$ 185$ & $\$ 185$ & $\$ 185$ \\
\hline Truck Crane 2 Hourly Cost & $\$ 325$ & $\$ 325$ & $\$ 325$ & $\$ 325$ \\
\hline Truck Crane 3 Hourly Costs & & & & \\
\hline Total Truck Crane Costs & $\$ 12,240$ & $\$ 12,240$ & $\$ 24,480$ & $\$ 24,480$ \\
\hline Total Transportation Freight in/out & $\$ 40,000$ & $\$ 40,000$ & $\$ 60,000$ & $\$ 70,000$ \\
\hline Transport Days in/out & 6 & 6 & 10 & 10 \\
\hline Transport Hours in/out & 48 & 48 & 80 & 80 \\
\hline Crane Rental During Transport & $\$ 4,091$ & $\$ 4,091$ & $\$ 14,545$ & $\$ 17,045$ \\
\hline SubTotal & $\$ 66,416$ & $\$ 66,416$ & $\$ 123,833$ & $\$ 137,833$ \\
\hline Mob/Demob Costs/Turbine & $\$ 1,328$ & $\$ 1,328$ & $\$ 2,477$ & $\$ 2,757$ \\
\hline
\end{tabular}




\section{Appendix L \\ Scenario 2-Automated Welding}




\begin{tabular}{|c|c|c|c|c|c|c|c|c|c|c|c|c|c|c|c|}
\hline \multirow{2}{*}{\multicolumn{2}{|c|}{\begin{tabular}{|r} 
Number of People in Crew: 10 \\
Hours per Day: 10 \\
Days per Week 6
\end{tabular}}} & \multicolumn{2}{|c|}{$100 \%$} & \multirow{2}{*}{\multicolumn{2}{|c|}{ 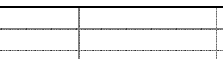 }} & 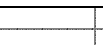 & -1 & & & & & & & & \\
\hline & & & & & & & & & & & & & & & \\
\hline Turbine Rating $(\mathrm{kW}):$ & & 750 & & & 1500 & & & 2500 & & & 3500 & & & 5000 & \\
\hline Rotor Diameter $(\mathrm{m}):$ & & 50 & & & 66 & & & 85 & & & 100 & & & 120 & \\
\hline Activity & MHRS & Labor Costs & $\begin{array}{l}\text { Equip \& } \\
\text { Material }\end{array}$ & MHRS & Labor Costs & $\begin{array}{l}\text { Equip \& } \\
\text { Material }\end{array}$ & MHRS & Labor Costs & Equip \& Material & MHRS & Labor Costs & Equip \& Material & MHRS & Labor Costs & Equip \& Material \\
\hline 1. Receive, Uncrate Nacelle, Blades, Rotors \& Towers & & & & 62. & $\$ 2,498$ & & 75 & $\$ 3,023$ & & 96 & $\$ 3,869$ & & 142 & $\$ 5,723$ & \\
\hline 2. Fabricate Tower Assembly Area & & & & 14 & $\$ 544$ & $\$ 1,289$ & 14 & $\$ 544$ & $\$ 1,289$ & 14 & $\$ 544$ & $\$ 1,289$ & 14 & $\$ 544$ & $\$ 1,289$ \\
\hline 3. Field Fabrication Tower Sections & & & & 236 & $\$ 9,924$ & $\$ 6,848$ & 714 & $\$ 30,052$ & $\$ 20,857$ & 1613 & $\$ 67,007$ & $\$ 43,782$ & 3422 & $\$ 140,382$ & $\$ 84,159$ \\
\hline 4. Rig \& Set Tower Sections & & & & 132 & $\$ 5,320$ & & 243 & $\$ 9,793$ & & 354 & $\$ 14,265$ & & 619 & $\$ 24,810$ & \\
\hline 5. Grout and Torque Bases & & & & 40 & $\$ 1,612$ & $\$ 850$ & 59 & $\$ 2,377$ & $\$ 950$ & 70 & $\$ 2,822$ & $\$ 1,120$ & 87 & $\$ 3,506$ & $\$ 1,650$ \\
\hline 6. Rig Blades, Assemble Rotors In Air & & & & 61 & $\$ 2,447$ & $\$ 250$ & 95 & $\$ 3,816$ & $\$ 500$ & 112 & $\$ 4,513$ & $\$ 700$ & 164 & $\$ 6,626$ & $\$ 1,000$ \\
\hline 7. Rig \& Set Nacelle & & & & 57 & $\$ 2,317$ & & 133 & $\$ 6,488$ & & 174 & $\$ 8,550$ & & 280 & $\$ 13,370$ & \\
\hline 8. Install Safety Equipment & & & & 12 & $\$ 484$ & & 20 & $\$ 806$ & & 24 & $\$ 968$ & & 36 & $\$ 1,450$ & \\
\hline 9. General Conditions & & & & & $\$ 2,172$ & $\$ 5,713$ & & $\$ 2,459$ & $\$ 10,789$ & & $\$ 2,459$ & $\$ 10,789$ & & $\$ 8,729$ & $\$ 20,941$ \\
\hline 10. Margin@ @10\% & & & & & $\$ 2,732$ & $\$ 1,495$ & & $\$ 5,936$ & $\$ 3,439$ & & $\$ 10,500$ & $\$ 5,768$ & & $\$ 20,514$ & $\$ 10,904$ \\
\hline Subtotal Per Turbine & & & & 613 & $\$ 30,050$ & $\$ 16,445$ & 1352 & $\$ 65,294$ & $\$ 37,824$ & 2457 & $\$ 115,496$ & $\$ 63,448$ & 4764 & $\$ 225,654$ & $\$ 119,943$ \\
\hline Percent of Total & & & & 200 & $65 \%$ & $35 \%$ & & $63 \%$ & $37 \%$ & & $65 \%$ & $35 \%$ & & $65 \%$ & (07) \\
\hline Project Total (50 Turbines) & & & & 30657.09 & $\$ 1,502,493$ & $\$ 822,231$ & 67617 & $\$ 3,264,719$ & $\$ 1,891,207$ & 122826 & $\$ 5,774,820$ & $\$ 3,172,392$ & 238215 & $\$ 11,282,715$ & $\$ 5,997,153$ \\
\hline Total All Costs & & & & & $\$ 2,324,724$ & & & $\$ 5,155,926$ & & & $\$ 8,947,211$ & & & $\$ 17,279,867$ & \\
\hline Total cost per Turbine & & & & & $\$ 46,494$ & & & $\$ 103,119$ & & & $\$ 178,944$ & & & $\$ 345,597$ & \\
\hline Estimated Assembly Rate - Items 2,5 (Hours) & & & & & 189 & & & & & & 528 & & & 899 & \\
\hline Estimated Assembly Rate - Items 2,5 (Days) & & & & & 1.9 & & & 3.8 & & & 5.3 & & & 9.0 & \\
\hline Total Costs/kW & & & & & $\$ 30.89$ & & & $\$ 41.30$ & & & $\$ 51.78$ & & & $\$ 69.45$ & \\
\hline Labor Costs/kW & & & & & $\$ 30.996$ & & & $\$ 26.15$ & & & $\$ 33.42$ & & & $\$ 45.35$ & \\
\hline Equip.Mater Costs/kW & & & & & $\$ 10.92$ & & & $\$ 15.15$ & & & $\$ 18.36$ & & & $\$ 24.10$ & \\
\hline & & & & & & & & & & & & & & & \\
\hline Total Cost/Swept Area & & & & & $\$ 13.59$ & & & $\$ 18.17$ & & & $\$ 22.78$ & & & $\$ 30.56$ & \\
\hline Man-Hours/Swept Area & & & & & 0.18 & & & 0.24 & & & 0.31 & & & 0.42 & \\
\hline $\begin{array}{l}\text { Labor Cossts/Swept Area } \\
\text { Equip, Mater Costs/Swept Area }\end{array}$ & & & & & & & & $\$ 11.51$ & & & $\$ 14.71$ & & & $\$ 19.95$ & \\
\hline Equip, Mater Costs/Swept Area & & & & & 54.81 & & & $\$ 6.67$ & & & $\$ 8.08$ & & & $\$ 10.61$ & \\
\hline Total Costs/Hub Heigh & & & & & $\$ 542$ & & & $\$ 933$ & & & $\$ 1,376$ & & & $\$ 2,215$ & \\
\hline Labor Costs/Hub Heigh & & & & & $\$ 350$ & & & $\$ 591$ & & & $\$ 888$ & & & $\$ 1,447$ & \\
\hline Equip.Mater Costs/Hub Heigh & & & & & $\$ 192$ & & & $\$ 342$ & & & $\$ 488$ & & & $\$ 769$ & \\
\hline & & & & & & & & & & & & & & & \\
\hline Item 1 Hourly Rates & & & & $\$ 40.30$ & & & $\$ 40.31$ & & & $\$ 40.30$ & & & $\$ 40.30$ & & \\
\hline Item 2 Hourly Rates & & & & $\$ 40.30$ & & & $\$ 40.30$ & & & $\$ 40.30$ & & & $\$ 40.08$ & & \\
\hline Item 3 Hourly Rates & & & & $\$ 40.30$ & & & $\$ 40.29$ & & & $\$ 40.31$ & & & $\$ 40.30$ & & \\
\hline Item 4 Hourly Rates & & & & $\$ 40.31$ & & & $\$ 40.30$ & & & $\$ 40.29$ & & & $\$ 40.31$ & & \\
\hline Item 5 Hourly Rates & & & & $\$ 40.65$ & & & $\$ 48.78$ & & & $\$ 49.14$ & & & $\$ 47.75$ & & \\
\hline $\begin{array}{l}\text { Item } 6 \text { Hourly Rates } \\
\text { Combined Hourly Rate } \\
\end{array}$ & & & & $\begin{array}{l}\$ 40.33 \\
\$ 5.135\end{array}$ & & & $\$ 40.30$ & & & 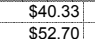 & & & $\$ 44.28$ & & \\
\hline & & & & & & & & & & & & & & & \\
\hline Item 1 Percent of Labor & & & & $8 \%$ & & & $5 \%$ & & & $3 \%$ & & & $3 \%$ & & \\
\hline Item 2 Percent of Labor & & & & $18 \%$ & & & $15 \%$ & & & $12 \%$ & & & $11 \%$ & & \\
\hline Item 3 Percent of Labor & & & & $5 \%$ & & & $4 \%$ & & & $2 \%$ & & & $2 \%$ & & \\
\hline Item 4 Percent of Labor & & & & $8 \%$ & & & $6 \%$ & & & $4 \%$ & & & $3 \%$ & & \\
\hline Item 5 Percent of Labor & & & & $8 \%$ & & & $10 \%$ & & & $7 \%$ & & & $6 \%$ & & \\
\hline $\begin{array}{l}\text { Item } 6 \text { Percent of Labor } \\
\text { temem } 7 \text { Percent of Laborot }\end{array}$ & & & & $\frac{2 \%}{7 \%}$ & & & $\frac{1 \%}{4 \%}$ & & & $\frac{1 \%}{2 \%}$ & & & $\frac{1 \%}{4 \%}$ & & \\
\hline Item 8 Percent of Labor & & & & $9 \%$ & & & $9 \%$ & & & $9 \%$ & & & $9 \%$ & & \\
\hline
\end{tabular}




\begin{tabular}{|c|c|c|c|c|c|c|c|c|c|c|c|c|c|c|c|}
\hline \multirow{2}{*}{\multicolumn{2}{|c|}{\begin{tabular}{|r|} 
Number of People in Crew: 10 \\
Hours per Day: 10 \\
Days per Week 6 \\
\end{tabular}}} & \multicolumn{2}{|c|}{$75 \%$} & & & & & & & & & & & & \\
\hline & & & & & & & & & & & & & & & \\
\hline \multirow{2}{*}{\begin{tabular}{|r|} 
Turbine Rating $(\mathrm{kW})$ \\
Rotor Diameter $(\mathrm{m})$ \\
\end{tabular}} & \multicolumn{3}{|c|}{$\begin{array}{c}750 \\
50\end{array}$} & \multicolumn{3}{|c|}{1500} & \multicolumn{3}{|c|}{2500} & \multicolumn{3}{|c|}{$\begin{array}{l}3500 \\
100\end{array}$} & \multicolumn{3}{|c|}{5000} \\
\hline & MHRS & $\begin{array}{c}50 \\
\text { Labor Costs }\end{array}$ & $\begin{array}{l}\text { Equip \& } \\
\text { Material }\end{array}$ & MHRS & \begin{tabular}{c|} 
Labor Costs \\
\end{tabular} & $\begin{array}{l}\text { Equip \& } \\
\text { Material }\end{array}$ & MHRS & $\begin{array}{r}85 \\
\text { Labor Costs }\end{array}$ & Equip \& Material & MHRS & $\begin{array}{c}100 \\
\text { Labor Costs }\end{array}$ & Equip \& Material & MHRS & $\begin{aligned} & 120 \\
& \text { Labor Costs }\end{aligned}$ & Equip \& Material \\
\hline 1. Receive, Uncrate Nacelle, Blades, Rotors \& Towers & & & & 40 & $\$ 1,400$ & & 75 & $\$ 2,625$ & & 96 & $\$ 3,360$ & & 142 & $\$ 4,970$ & \\
\hline 2. Fabricate Tower Assembly Area & & & & 12 & $\$ 490$ & $\$ 1,160$ & 12 & $\$ 490$ & $\$ 1,160$ & 12 & $\$ 490$ & $\$ 1,160$ & 12 & $\$ 490$ & $\$ 1,160$ \\
\hline 3. Field Fabrication Tower Sections & & & & 212 & $\$ 8,931$ & $\$ 6,163$ & 642 & $\$ 27,047$ & $\$ 18,772$ & 1451 & $\$ 60,306$ & $\$ 39,404$ & 3080 & $\$ 126,344$ & $\$ 75,743$ \\
\hline 4. Rig \& Set Tower Sections & & & & 60 & $\$ 2,100$ & & 110 & $\$ 3,850$ & & 161 & $\$ 5,635$ & & 282 & $\$ 9,870$ & \\
\hline 5. Grout and Torque Bases & & & & 40 & $\$ 1,400$ & $\$ 850$ & 59 & $\$ 2,065$ & $\$ 950$ & 70 & $\$ 2,450$ & $\$ 1,120$ & 87 & $\$ 3,045$ & $\$ 1,650$ \\
\hline 6. Rig Blades, Assemble Rotors In Air & & & & 57 & $\$ 2,002$ & $\$ 250$ & 87 & $\$ 3,031$ & $\$ 500$ & 101 & $\$ 3,549$ & $\$ 700$ & 147 & $\$ 5,156$ & $\$ 1,000$ \\
\hline 7. Rig \& Set Nacelle & & & & 22 & $\$ 770$ & & 52 & $\$ 2,537$ & & 68 & $\$ 3,341$ & & 109 & $\$ 5,205$ & \\
\hline 8. Install Safety Equipment & & & & 12 & $\$ 420$ & & 20 & $\$ 700$ & & 24 & $\$ 840$ & & 36 & $\$ 1,260$ & \\
\hline 9. General Conditions & & & & & $\$ 1,629$ & $\$ 4,285$ & & $\$ 1,844$ & $\$ 8,092$ & & $\$ 1,844$ & $\$ 8,092$ & & $\$ 6,547$ & $\$ 15,706$ \\
\hline 10. Margin@ @10\% & & & & & $\$ 1,914$ & $\$ 1,271$ & & $\$ 4,419$ & $\$ 2,947$ & & $\$ 8,182$ & $\$ 5,048$ & & $\$ 16,289$ & $\$ 9,526$ \\
\hline Subtotal Per Turbine & & & & 456 & $\$ 21,057$ & $\$ 13,979$ & 1057 & $\$ 48,607$ & $\$ 32.421$ & 1984 & $\$ 89.997$ & $\$ 55.523$ & 3896 & $\$ 179.175$ & \\
\hline Subtotalper lurbine & & & & & $60 \%$ & $40 \%$ & (0) & $60 \%$ & $40 \%$ & nom & $62 \%$ & $38 \%$ & 3000 & $63 \%$ & $37 \%$ \\
\hline Project Total (50 Turbines) & & & & 22784.881 & $\$ 1,052,829$ & $\$ 698,926$ & 52874 & $\$ 2,430,374$ & $\$ 1,621,052$ & 99214 & $\$ 4,499,875$ & $\$ 2,776,153$ & 194781 & $\$ 8,958,728$ & $\$ 5,239,249$ \\
\hline Total All Costs & 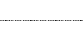 & -1 & & (3) & $\$ 1,751,755$ & & & $\$ 4051.426$ & & & $\$ 7.276 .028$ & & & $\$ 14197.977$ & \\
\hline Total cost per Turbine & & & & & $\$ 35,035$ & & & $\$ 81,029$ & & & $\$ 145,521$ & & & $\$ 283,960$ & \\
\hline Estimated Assembly Rate - Items 2,5 (Hour) & & & & & 82 & & & 162 & & & 229 & & & & \\
\hline Estimated Assembly Rate - Items 2,5 (Day $\$$ & & & & & 0.8 & & & 1.6 & & & 2.3 & & & 3.9 & \\
\hline Total Costs/kW & -1 & & & {[} & $\$ 23.27$ & & & $\$ 32.45$ & & & $\$ 42.11$ & & & $\$ 57.06$ & \\
\hline Labor Costs/kW & & & & & $\$ 13.99$ & & & $\$ 19.47$ & & & $\$ 26.04$ & & & $\$ 36.01$ & \\
\hline Equip.Mater Costs/kW & & & & & $\$ 9.29$ & & & $\$ 12.99$ & & & $\$ 16.07$ & & & $\$ 21.06$ & \\
\hline & 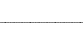 & & & & & & & & & $\square$ & & & 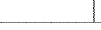 & & \\
\hline Total Cost/Swept Area & & & & & $\$ 10.24$ & & & $\$ 14.28$ & & & $\$ 18.53$ & & & $\$ 25.11$ & \\
\hline Man-Hours/Swept Area & & & & & 0.13 & & & 0.19 & & & 0.25 & & & 0.34 & \\
\hline Labor Costs/Swept Area & & & & & $\$ 6.15$ & & & $\$ 8.57$ & & & $\$ 11.46$ & & & $\$ 15.84$ & \\
\hline Equip, Mater Costs/Swept Area & & & & & $\$ 4.09$ & & & $\$ 5.71$ & & & $\$ 7.07$ & & & $\$ 9.27$ & \\
\hline & & & & & & & & & & & & & & & \\
\hline Total Costs/Hub Height & & & & & $\$ 408$ & & & $\$ 733$ & & & $\$ 1,119$ & & & $\$ 1,820$ & \\
\hline Labor Costs/Hub Height & & & & & $\$ 245$ & & & $\$ 440$ & & & $\$ 692$ & & & $\frac{\$ 1,149}{\$ 672}$ & \\
\hline Equip.Mater Costs/Hub Height & & & & & $\$ 163$ & & & $\$ 293$ & & & $\$ 427$ & & & $\$ 672$ & \\
\hline & & & & & & & & & & & & & & & \\
\hline Item 1 Hourly Rates & & & & $\$ 35.00$ & & & $\$ 35.00$ & & & $\$ 35.00$ & & & $\$ 35.00$ & & \\
\hline Item 2 Hourly Rates & & & & $\$ 35.00$ & & & $\$ 35.00$ & & & $\$ 35.00$ & & & $\$ 35.00$ & & \\
\hline Item 3 Hourly Rates & & & & $\$ 35.00$ & & & \$35.00 & & & $\$ 35.00$ & & & $\$ 35.00$ & & \\
\hline Item 4 Hourly Rates & & & & $\$ 35.00$ & & & $\$ 35.00$ & & & $\$ 35.00$ & & & $\$ 35.00$ & & \\
\hline Item 5 Hourly Rates & & & & $\$ 35.00$ & & & $\$ 48.78$ & & & $\$ 49.14$ & & & $\$ 47.75$ & & \\
\hline Item 6 Hourly Rates & & & & $\$ 35.00$ & & & $\$ 35.00$ & & & $\$ 35.00$ & & & $\$ 35.00$ & & \\
\hline Combined Hourly Rate & & & & $\$ 51.35$ & & & & & & & & & & & \\
\hline Item 1 Percent of Labor & & & & $7 \%$ & & & $5 \%$ & & & $4 \%$ & & & $3 \%$ & & \\
\hline Item 2 Percent of Labor & & & & $10 \%$ & & & $8 \%$ & & & $6 \%$ & & & $6 \%$ & & \\
\hline Item 3 Percent of Labor & & & & $7 \%$ & & & $4 \%$ & & & $3 \%$ & & & $2 \%$ & & \\
\hline Item 4 Percent of Labor & & & & $10 \%$ & & & $6 \%$ & & & $4 \%$ & & & $3 \%$ & & \\
\hline Item 5 Percent of Labor & & & & $4 \%$ & & & $5 \%$ & & & $4 \%$ & & & $3 \%$ & & \\
\hline Item 6 Percent of Labor & & & & $2 \%$ & & & $1 \%$ & & & $1 \%$ & & & $\frac{1 \%}{4 \%}$ & & \\
\hline \begin{tabular}{|l|} 
Item 7 Percent of Labor \\
Item 8 Percent of Labot
\end{tabular} & & & & $8 \%$ & & & $\frac{4 \%}{9 \%}$ & & & $\frac{2 \%}{9 \%}$ & & & $\frac{4 \%}{9 \%}$ & & \\
\hline
\end{tabular}




\begin{tabular}{|c|c|c|c|c|c|c|c|c|c|c|c|c|c|c|c|}
\hline \multirow{2}{*}{\multicolumn{2}{|c|}{\begin{tabular}{|r|} 
Number of People in Crew: 10 \\
Hours per Day: 10 \\
Days per Week 6 \\
\end{tabular}}} & \multicolumn{2}{|c|}{$105 \%$} & \multirow{2}{*}{\multicolumn{2}{|c|}{ 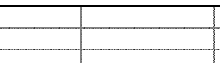 }} & \multirow{2}{*}{-1} & \multirow{2}{*}{-1} & & & -1 & & & & & \\
\hline & & & & & & & & & & & & & & & \\
\hline Turbine Rating $(\mathrm{kW}):$ & & 750 & & & 1500 & & & 2500 & & & 3500 & & & 5000 & \\
\hline Rotor Diameter $(\mathrm{m})$ & MHRS & \begin{tabular}{|c|}
50 \\
Labor Costs
\end{tabular} & $\begin{array}{l}\text { Equip \& } \\
\text { Material } \\
\end{array}$ & MHRS & $\begin{array}{l}66 \\
\text { Labor Costs }\end{array}$ & $\begin{array}{l}\text { Equip \& } \\
\text { Material }\end{array}$ & MHRS & $\begin{array}{r}85 \\
\text { Labor Costs }\end{array}$ & Equip \& Material & MHRS & $\begin{array}{l}100 \\
\text { Labor Costs }\end{array}$ & Equip \& Material & MHRS & $\begin{array}{l}120 \\
\text { Labor Costs }\end{array}$ & Equip \& Material \\
\hline 1. Receive, Uncrate Nacelle, Blades, Rotors \& Towers & & & & 64 & $\$ 2,579$ & & 75 & $\$ 3,023$ & & 96 & $\$ 3,869$ & & 142 & $\$ 5,723$ & \\
\hline 2. Fabricate Tower Assembly Area & & & & 16 & $\$ 626$ & $\$ 1,483$ & 16 & $\$ 626$ & $\$ 1,483$ & 16 & $\$ 626$ & $\$ 1,483$ & 16 & $\$ 626$ & $\$ 1,483$ \\
\hline 3. Field Fabrication Tower Sections & & & & 271 & $\$ 11,412$ & $\$ 7,875$ & 821 & $\$ 34,560$ & $\$ 23,986$ & 1855 & $\$ 77,058$ & $\$ 50,349$ & 3935 & $\$ 161,440$ & $\$ 96,783$ \\
\hline 4. Rig \& Set Tower Sections & & & & 138 & $\$ 5,562$ & & 254 & $\$ 10,236$ & & 371 & $\$ 14,950$ & & 687 & $\$ 27,535$ & \\
\hline 5. Grout and Torque Bases & & & & 40 & $\$ 1,612$ & $\$ 850$ & 59 & $\$ 2,377$ & $\$ 950$ & 70 & $\$ 2,822$ & $\$ 1,120$ & 87 & $\$ 3,506$ & $\$ 1,650$ \\
\hline 6. Rig Blades, Assemble Rotors In Air & & & & 62 & $\$ 2,487$ & $\$ 250$ & 97 & $\$ 3,905$ & $\$ 500$ & 115 & $\$ 4,633$ & $\$ 700$ & 171 & $\$ 6,900$ & $\$ 1,000$ \\
\hline 7. Rig \& Set Nacelle & & & & 67 & $\$ 2,724$ & & 155 & $\$ 7,561$ & & 204 & $\$ 10,024$ & & 348 & $\$ 16,616$ & \\
\hline 8. Install Safety Equipment & & & & 12 & $\$ 484$ & & 20 & $\$ 806$ & & 24 & $\$ 968$ & & 36 & $\$ 1,450$ & \\
\hline 9. General Conditions & & & & & $\$ 2,281$ & $\$ 5,999$ & & $\$ 2,582$ & $\$ 11,328$ & & $\$ 2,582$ & $\$ 11,328$ & & $\$ 9,165$ & $\$ 21,988$ \\
\hline 10. Margin@ 10\% & & & & & $\$ 2,977$ & $\$ 1,646$ & & $\$ 6,568$ & $\$ 3,825$ & & $\$ 11,753$ & $\$ 6,498$ & & $\$ 23,296$ & $\$ 12,290$ \\
\hline Subtotal Per Turbine & & & & 670 & $\$ 32,743$ & $\$ 18,101$ & 1497 & $\$ 72,244$ & $\$ 42,072$ & 2751 & $\$ 129,286$ & $\$ 71,478$ & 5422 & $\$ 256,258$ & $\$ 135,194$ \\
\hline Project Total (50 Turbines) & & & & 33.478 & $\begin{aligned} 64 \% \\
\$ \$ 1,637,135 \\
\end{aligned}$ & $\begin{array}{r}36 \% \\
\$ 905,069 \\
\end{array}$ & 74,835 & $\begin{aligned} 63 \% \\
3,612,184\end{aligned}$ & $\begin{array}{r}37 \% \\
\$ 2,103,586\end{array}$ & 137.525 & $\begin{array}{r}64 \% \\
\$ 6,464,281\end{array}$ & $\begin{array}{r}36 \% \\
\$ 3,573,896\end{array}$ & 271,125 & $\begin{array}{r}65 \% \\
\$ 12,812,918\end{array}$ & $\begin{array}{r}35 \% \\
\$ 66,759,687\end{array}$ \\
\hline Total All Costs & & & & & $\$ 2,542,204$ & & & $\$ 5,715,770$ & & & $\$ 10,038.177$ & & & $\$ 19.572 .606$ & \\
\hline $\begin{array}{l}\text { Total Cost per Turbine } \\
\text { Tosts }\end{array}$ & & & & & $\begin{array}{l}\$ 2,54,204 \\
\$ 50,844\end{array}$ & & & 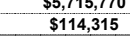 & & & $\frac{\$ 10,038,17 / 7}{\$ 200,764}$ & & & $\begin{array}{l}\$ 19,552,6606 \\
\$ 391,452\end{array}$ & \\
\hline Estimated Assembly Rate - Items 2,5 (Hours) & & & & & 205 & & & & & & 575 & & & 1,035 & \\
\hline Estimated Assembly Rate - Items 2,5 (Days) & & & & & 2.1 & & & 4.1 & & & 5.8 & & & 10.4 & \\
\hline Total Costs/kW & & 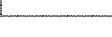 & & & $\$ 33.78$ & & & $\$ 45.79$ & & & $\$ 58.10$ & & & $\$ 78.66$ & \\
\hline Labor Costs/kW & & & & & $\$ 321.75$ & & & $\$ 28.93$ & & & $\$ 37.41$ & & & $\$ 51.50$ & \\
\hline Equip.Mater Costs/kW & & & & & $\$ 12.02$ & & & $\$ 16.85$ & & & $\$ 20.68$ & & & $\$ 27.17$ & \\
\hline & & & & & & & & & & & & & & & \\
\hline $\begin{array}{l}\text { Total CostSWwept Area } \\
\text { Man-Hours Swept Area }\end{array}$ & & 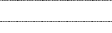 & & & $\$ 14.86$ & & & $\$ 20.15$ & & & $\$ 25.56$ & & & $\$ 34.61$ & \\
\hline Labor Costs/Swept Area & & & & & $\$ 9.20$ & & & $\frac{0.26}{\$ 12.73}$ & & & $\frac{0.35}{\$ 1646}$ & & & $\frac{0.48}{\$ 2266}$ & \\
\hline Equip, Mater Costs/Swept Area & & & & & $\$ 5.29$ & & & \$1.41 & & & $\frac{910.40}{\$ 9.10}$ & & & $\$ 111.95$ & \\
\hline & & & & & & & & & & & & & & & \\
\hline $\begin{array}{l}\text { Total Costs/Hub Heigh } \\
\text { Labor Costs/Hub Heigh }\end{array}$ & & +5 & & & $\$ 593$ & & & $\frac{\$ 1,035}{\$ 654}$ & & & $\frac{\$ 1,544}{\$ 995}$ & & & $\begin{array}{l}\$ 2,509 \\
\$ 1,643\end{array}$ & \\
\hline Equip.Mater Costs/l/Hub Heigh & & & & & $\$ 211$ & & & $\$ 381$ & & & $\$ 5550$ & & & $\begin{array}{l}\$ 1,067 \\
\$ 867\end{array}$ & \\
\hline & & & & & & & & & & & & & & & \\
\hline Item 1 Hourly Rates & & & & $\$ 40.30$ & & & $\$ 40.31$ & & & $\$ 40.30$ & & & $\$ 40.30$ & & \\
\hline Item 2 Hourly Rates & & & & $\$ 40.30$ & & & $\$ 40.30$ & & & $\$ 40.30$ & & & $\$ 40.08$ & & \\
\hline Item 3 Hourly Rates & & & & $\$ 40.30$ & & & $\$ 40.29$ & & & $\$ 40.31$ & & & $\$ 40.30$ & & \\
\hline Item 4 Hourly Rates & & & & $\$ 40.31$ & & & $\$ 40.30$ & & & $\$ 40.29$ & & & $\$ 40.31$ & & \\
\hline Item 5 Hourly Rates & & & & $\$ 40.65$ & & & $\$ 48.78$ & & & $\$ 49.14$ & & & $\$ 47.75$ & & \\
\hline $\begin{array}{l}\text { Itemb } 6 \text { Hourly Rates } \\
\text { Combined Hourry }\end{array}$ & & & & $\begin{array}{l}\frac{S 40.33}{\$ 51.35} \\
\$ 51\end{array}$ & & & $\begin{array}{l}\$ 40.30 \\
\$ 54.58\end{array}$ & & & $\$ \$ \$ 4.33$ & & & $\begin{array}{l}\$ 40.28 \\
\$ 57.38\end{array}-10$ & & \\
\hline & & & & & & & & & & & & & & & \\
\hline Item 1 Percent of $L$ & & & & $8 \%$ & & & $4 \%$ & & & $3 \%$ & & & $2 \%$ & & \\
\hline Item 2 Percent of Labor & & & & $17 \%$ & & & $14 \%$ & & & $12 \%$ & & & $11 \%$ & & \\
\hline Item 3 Percent of Labor & & & & $5 \%$ & & & $3 \%$ & & & $2 \%$ & & & $1 \%$ & & \\
\hline Item 4 Percent of Labor & & & & $8 \%$ & & & $5 \%$ & & & $4 \%$ & & & $3 \%$ & & \\
\hline Item 5 Percent of Labor & & & & $8 \%$ & & & $10 \%$ & & & $8 \%$ & & & $6 \%$ & & \\
\hline $\begin{array}{l}\text { Item } 6 \text { Percent of Labor } \\
\text { Item } 7 \text { Percent of Labor }\end{array}$ & & & & $7 \%$ & & & $4 \%$ & & & $2 \%$ & & & $\frac{1 \%}{4 \%}$ & & \\
\hline Item 8 Percent of Labor & & & & $9 \%$ & & & $9 \%$ & & & $9 \%$ & & & $9 \%$ & & \\
\hline
\end{tabular}


WELDED QUARTER SECTIONS OF TOWERS

1. Tower section properties based on data in Table 2-2.

2. See Page N-3 for weight of weld.

3. Assumed manual weld rate:

$\begin{array}{lr}\text { Weld weight - } 1 \text { inch steel } & 2.25 \mathrm{lb} / \mathrm{ft} \\ \text { Welding rate } & 42.7 \mathrm{in} / \mathrm{hr} \\ \text { Tower section length } & 21.5 \mathrm{~m} \\ \text { Tower section length } & 846 \mathrm{in}\end{array}$

Tower Section Assembly 1500 kW

\begin{tabular}{|c|c|c|c|c|c|c|c|c|c|}
\hline Description & QTY. & UNITS & $\begin{array}{c}\text { UNITS/ } \\
\text { MHR }\end{array}$ & $\begin{array}{l}\text { TOTAL } \\
\text { MHRS }\end{array}$ & $\begin{array}{c}\text { COST/ } \\
\text { MHR }\end{array}$ & $\begin{array}{l}\text { TOTAL } \\
\text { LABOR }\end{array}$ & $\begin{array}{l}\text { MAT'L. } \\
\text { UNIT \$\$ }\end{array}$ & $\begin{array}{l}\text { TOTAL } \\
\text { MAT'L. }\end{array}$ & $\begin{array}{r}\text { TOTAL } \\
\text { L \& M }\end{array}$ \\
\hline Screw Jacks & 20 & EA & 5 & 4 & $\$ 40$ & $\$ 160$ & $\$ 0.00$ & $\$ 0$ & $\$ 160$ \\
\hline Quarter-Section Weld & 1693 & INCHES & 43 & 40 & $\$ 40$ & $\$ 1,587$ & $\$ 0.58$ & $\$ 982$ & $\$ 2,569$ \\
\hline Half-Section Weld & 1693 & INCHES & 43 & 40 & $\$ 40$ & $\$ 1,587$ & $\$ 0.58$ & $\$ 982$ & $\$ 2,569$ \\
\hline Top \& Bottom Template & 10.67 & EA & 0.25 & 43 & $\$ 40$ & $\$ 1,707$ & $\$ 0.00$ & $\$ 0$ & $\$ 1,707$ \\
\hline Move Cradles & 16 & EA & 2 & 8 & $\$ 40$ & $\$ 320$ & $\$ 0.00$ & $\$ 0$ & $\$ 320$ \\
\hline Lifting Eyes & 5.33 & EA & 1 & 5.33 & $\$ 40$ & $\$ 213$ & $\$ 35.00$ & $\$ 187$ & $\$ 400$ \\
\hline Paint & 5000 & SF & 80 & 63 & $\$ 40$ & $\$ 2,500$ & $\$ 0.50$ & $\$ 2,500$ & $\$ 5,000$ \\
\hline \begin{tabular}{|l|} 
Non-Productive Time \\
\end{tabular} & $3 \%$ & MHRS & & 6 & $\$ 40$ & $\$ 242$ & $\$ 0.00$ & $\$ 0$ & $\$ 242$ \\
\hline X-RAY TEST & 20 & EA & 1 & 20 & $\$ 65$ & $\$ 1,300$ & $\$ 20.00$ & $\$ 400$ & $\$ 1,700$ \\
\hline Weld Correction (1\%) & 34 & INCHES & 16 & 2 & $\$ 40$ & $\$ 85$ & $\$ 0.58$ & $\$ 20$ & $\$ 104$ \\
\hline Load \& Transport Towers & 1 & SECTIONS & 0.18 & 6 & $\$ 40$ & $\$ 222$ & $\$ 0.00$ & $\$ 0$ & $\$ 222$ \\
\hline Welding Equipment/Operating Costs & 282 & LF & & & & $\$ 0$ & $\$ 6.30$ & $\$ 1,778$ & $\$ 1,778$ \\
\hline TOTALS (PER SECTION) & & 236 & \multicolumn{3}{|c|}{$\$ 9,924$} & $\$ 6,848$ & $\$ 16,771$ \\
\hline Minimum & \multicolumn{3}{|l|}{$-10 \%$} & 212 & \multicolumn{3}{|c|}{$\$ 8,931$} & $\$ \$ \$ 6,163$ & $\$ \$ \$ 15,094$ \\
\hline Maximum & \multicolumn{3}{|l|}{$15 \%$} & 271 & \multicolumn{3}{|c|}{$\$ 11,412$} & $\$ 7,875$ & $\$ 19,287$ \\
\hline Total Number of Sections in Turbine: & & & 1 & 236 & \multicolumn{3}{|c|}{$\$ 9,924$} & $\$ 6,848$ & $\$ 16,771$ \\
\hline Minimum & & & 1 & 212 & \multicolumn{3}{|c|}{$\$ 8,931$} & $\$ 6,163$ & $\$ 15,094$ \\
\hline \multirow[t]{2}{*}{ Maximum } & & 1 & 271 & \multicolumn{3}{|c|}{$\$ 11,412$} & $\$ 7,875$ & $\$ 19,287$ \\
\hline & Avg & Min & Max & & & & & & \\
\hline 10 People, 10 hrs/day & 100 & 100 & 100 & & & & & & \\
\hline Days per Tower Section & 2.4 & 2.1 & 2.7 & & & & & & \\
\hline Total number of sections for assembly & 50 & 50 & 50 & & & & & & \\
\hline Number of days for assembly & 118 & 106 & 135 & & & & & & \\
\hline Number of assembly days per turbine & 2.4 & 2.1 & 2.7 & & & & & & \\
\hline Number of 6 day weeks & 19.6 & 17.7 & 22.6 & & & & & & \\
\hline
\end{tabular}


WELDED QUARTER SECTIONS OF TOWERS

1. Tower section properties based on data in Table 2-2.

2. See Page N-3 for weight of weld.

3. Assumed manual weld rate:

$8 \mathrm{lbs} / \mathrm{hr}$

Weld weight - 1 inch steel $\quad 2.25 \mathrm{lb} / \mathrm{ft}$

Welding rate $\quad 42.7 \mathrm{in} / \mathrm{hr}$

Tower section length $\quad 22.1 \mathrm{~m}$

Tower section length 870 in

\begin{tabular}{|c|c|c|c|c|c|c|c|c|c|}
\hline \multicolumn{3}{|l|}{ Tower Section Assembly 2500 kW } & \multirow[b]{2}{*}{$\begin{array}{c}\text { UNITS/ } \\
\text { MHR }\end{array}$} & \multirow[b]{2}{*}{$\begin{array}{l}\text { TOTAL } \\
\text { MHRS }\end{array}$} & \multirow[b]{2}{*}{$\begin{array}{c}\text { COST/ } \\
\text { MHR }\end{array}$} & \multirow[b]{2}{*}{$\begin{array}{l}\text { TOTAL } \\
\text { LABOR }\end{array}$} & \multirow[b]{2}{*}{$\begin{array}{l}\text { MAT'L. } \\
\text { UNIT \$\$ }\end{array}$} & \multirow[b]{2}{*}{$\begin{array}{l}\text { TOTAL } \\
\text { MAT'L. }\end{array}$} & \multirow[b]{2}{*}{$\begin{array}{r}\text { TOTAL } \\
\text { L \& M }\end{array}$} \\
\hline Description & QTY. & UNITS & & & & & & & \\
\hline Screw Jacks & 20 & EA & 5 & 4 & $\$ 40$ & $\$ 160$ & $\$ 0.00$ & $\$ 0$ & $\$ 160$ \\
\hline Quarter-Section Weld & 1740 & INCHES & 42.7 & 41 & $\$ 40$ & $\$ 1,631$ & $\$ 0.58$ & $\$ 1,009$ & $\$ 2,641$ \\
\hline Half-Section Weld & 1740 & INCHES & 42.7 & 41 & $\$ 40$ & $\$ 1,631$ & $\$ 0.58$ & $\$ 1,009$ & $\$ 2,641$ \\
\hline Top \& Bottom Template & 10.67 & EA & 0.25 & 43 & $\$ 40$ & $\$ 1,707$ & $\$ 0.00$ & $\$ 0$ & $\$ 1,707$ \\
\hline Move Cradles & 16 & EA & 2 & 8 & $\$ 40$ & $\$ 320$ & $\$ 0.00$ & $\$ 0$ & $\$ 320$ \\
\hline Lifting Eyes & 5.33 & EA & 1 & 5.33 & $\$ 40$ & $\$ 213$ & $\$ 35.00$ & $\$ 187$ & $\$ 400$ \\
\hline Paint & 5000 & $\mathrm{SF}$ & 80 & 63 & $\$ 40$ & $\$ 2,500$ & $\$ 0.50$ & $\$ 2,500$ & $\$ 5,000$ \\
\hline Non-Productive Time & $3 \%$ & MHRS & & 6 & $\$ 40$ & $\$ 245$ & $\$ 0.00$ & $\$ 0$ & $\$ 245$ \\
\hline X-RAY TEST & 20 & EA & 1 & 20 & $\$ 65$ & $\$ 1,300$ & $\$ 20.00$ & $\$ 400$ & $\$ 1,700$ \\
\hline Weld Correction (1\%) & 35 & INCHES & 16 & 2 & $\$ 40$ & $\$ 87$ & $\$ 0.58$ & $\$ 20$ & $\$ 107$ \\
\hline Load \& Transport Towers & 1 & SECTIONS & 0.18 & 6 & $\$ 40$ & $\$ 222$ & $\$ 0.00$ & $\$ 0$ & $\$ 222$ \\
\hline Welding Equipment/Operating Costs & 290 & \begin{tabular}{|l|l} 
LF & \\
\end{tabular} & & & & $\$ 0$ & $\$ 6.30$ & $\$ 1,827$ & $\$ 1,827$ \\
\hline TOTALS (PER SECTION) & & & & 238 & & $\$ 10,017$ & & $\$ 6,952$ & $\$ 16,970$ \\
\hline Minimum & $-10 \%$ & & & 214 & & $\$ 9,016$ & & $\$ 6,257$ & $\$ 15,273$ \\
\hline Maximum & $15 \%$ & & & 274 & & $\$ 11,520$ & & $\$ 7,995$ & $\$ 19,515$ \\
\hline Total Number of Sections in Turbine: & & & 3 & 714 & & $\$ 30,052$ & & $\$ 20,857$ & $\$ 50,909$ \\
\hline Minimum & & & 3 & 642 & & $\$ 27,047$ & & $\$ 18,772$ & $\$ 45,818$ \\
\hline Maximum & & & 3 & 821 & & $\$ 34,560$ & & $\$ 23,986$ & $\$ 58,546$ \\
\hline & Avg & Min & Max & & & & & & \\
\hline 10 People, 10 hrs/day & 100 & 100 & 100 & & & & & & \\
\hline Days per Tower Section & 2.4 & 2.1 & 2.7 & & & & & & \\
\hline Total number of sections for assembly & 150 & 150 & 150 & & & & & & \\
\hline Number of days for assembly & 357 & 321 & 410 & & & & & & \\
\hline Number of assembly days per turbine & 7.1 & 6.4 & 8.2 & & & & & & \\
\hline Number of 6 day weeks & 59.5 & 53.5 & 68.4 & & & & & & \\
\hline
\end{tabular}


WELDED QUARTER SECTIONS OF TOWERS

1. Tower section properties based on data in Table 2-2.

2. See Page N-3 for weight of weld.

3. Assumed manual weld rate:

- 1.5 inch steel

Welding rate

$20.9 \mathrm{in} / \mathrm{hr}$

Tower section length $\quad 21.7 \mathrm{~m}$

Tower section length 854 in

\begin{tabular}{|c|c|c|c|c|c|c|c|c|c|}
\hline \multicolumn{3}{|c|}{ Tower Section Assembly 3500 MW } & \multirow[b]{2}{*}{$\begin{array}{c}\text { UNITS/ } \\
\text { MHR }\end{array}$} & \multirow[b]{2}{*}{$\begin{array}{l}\text { TOTAL } \\
\text { MHRS }\end{array}$} & \multirow[b]{2}{*}{$\begin{array}{c}\text { COST/ } \\
\text { MHR }\end{array}$} & \multirow[b]{2}{*}{$\begin{array}{l}\text { TOTAL } \\
\text { LABOR }\end{array}$} & \multirow[b]{2}{*}{$\begin{array}{l}\text { MAT'L. } \\
\text { UNIT \$\$ }\end{array}$} & \multirow[b]{2}{*}{$\begin{array}{l}\text { TOTAL } \\
\text { MAT'L. }\end{array}$} & \multirow[b]{2}{*}{$\begin{array}{l}\text { TOTAL } \\
\text { L \& M }\end{array}$} \\
\hline Description & QTY. & UNITS & & & & & & & \\
\hline Screw Jacks & 20 & EA & 5 & 4 & $\$ 40$ & $\$ 160$ & $\$ 0.00$ & $\$ 0$ & $\$ 160$ \\
\hline Quarter-Section Weld & 1709 & INCHES & 20.9 & 82 & $\$ 40$ & $\$ 3,275$ & $\$ 0.58$ & $\$ 991$ & $\$ 4,266$ \\
\hline Half-Section Weld & 1709 & INCHES & 20.9 & 82 & $\$ 40$ & $\$ 3,275$ & $\$ 0.58$ & $\$ 991$ & $\$ 4,266$ \\
\hline Top \& Bottom Template & 10.67 & EA & 0.25 & 43 & $\$ 40$ & $\$ 1,707$ & $\$ 0.00$ & $\$ 0$ & $\$ 1,707$ \\
\hline Move Cradles & 16 & EA & 2 & 8 & $\$ 40$ & $\$ 320$ & $\$ 0.00$ & $\$ 0$ & $\$ 320$ \\
\hline Lifting Eyes & 5.33 & EA & 1 & 5.33 & $\$ 40$ & $\$ 213$ & $\$ 35.00$ & $\$ 187$ & $\$ 400$ \\
\hline Paint & 5000 & $\mathrm{SF}$ & 80 & 63 & $\$ 40$ & $\$ 2,500$ & $\$ 0.50$ & $\$ 2,500$ & $\$ 5,000$ \\
\hline Non-Productive Time & $3 \%$ & MHRS & & 9 & $\$ 40$ & $\$ 344$ & $\$ 0.00$ & $\$ 0$ & $\$ 344$ \\
\hline X-RAY TEST & 20 & EA & 1 & 20 & $\$ 65$ & $\$ 1,300$ & $\$ 20.00$ & $\$ 400$ & $\$ 1,700$ \\
\hline Weld Correction (1\%) & 34 & INCHES & 16 & 2 & $\$ 40$ & $\$ 85$ & $\$ 0.58$ & $\$ 20$ & $\$ 105$ \\
\hline Load \& Transport Towers & 1 & SECTIONS & 0.18 & 6 & $\$ 40$ & $\$ 222$ & $\$ 0.00$ & $\$ 0$ & $\$ 222$ \\
\hline Welding Equipment/Operating Costs & 285 & LF & & & & $\$ 0$ & $\$ 12.88$ & $\$ 3,668$ & $\$ 3,668$ \\
\hline TOTALS (PER SECTION) & & & & 323 & & $\$ 13,401$ & & $\$ 8,756$ & $\$ 22,158$ \\
\hline Minimum & $-10 \%$ & & & 290 & & $\$ 12,061$ & & $\$ 7,881$ & $\$ 19,942$ \\
\hline Maximum & $15 \%$ & & & 371 & & $\$ 15,412$ & & $\$ 10,070$ & $\$ 25,481$ \\
\hline Total Number of Sections in Turbine: & & & 5 & 1613 & & $\$ 67,007$ & & $\$ 43,782$ & $\$ 110,789$ \\
\hline Minimum & & & 5 & 1451 & & $\$ 60,306$ & & $\$ 39,404$ & $\$ 99,710$ \\
\hline Maximum & & & 5 & 1855 & & $\$ 77,058$ & & $\$ 50,349$ & $\$ 127,407$ \\
\hline & Avg & Min & Max & & & & & & \\
\hline 10 People, 10 hrs/day & 100 & 100 & 100 & & & & & & \\
\hline Days per Tower Section & 3.2 & 2.9 & 3.7 & & & & & & \\
\hline Total number of sections for assembly & 250 & 250 & 250 & & & & & & \\
\hline Number of days for assembly & 806 & 726 & 927 & & & & & & \\
\hline Number of assembly days per turbine & 16.1 & 14.5 & 18.5 & & & & & & \\
\hline Number of 6-day weeks & 134.4 & 121.0 & 154.5 & & & & & & \\
\hline
\end{tabular}


WELDED QUARTER SECTIONS OF TOWERS

1. Tower section properties based on data in Table 2-2.

2. See Page N-3 for weight of weld.

3. Assumed manual weld rate:

inch steel

$8 \mathrm{lbs} / \mathrm{h}$
$7.8 \mathrm{lb} / \mathrm{ft}$

$12.3 \mathrm{in} / \mathrm{hr}$

$22.3 \mathrm{~m}$

Tower section length

878 in

\begin{tabular}{|c|c|c|c|c|c|c|c|c|c|}
\hline \multicolumn{10}{|l|}{ Tower Section Assembly 5000 kW } \\
\hline Description & QTY. & UNITS & $\begin{array}{l}\text { UNITS/ } \\
\text { MHR }\end{array}$ & $\begin{array}{l}\text { TOTAL } \\
\text { MHRS }\end{array}$ & $\begin{array}{c}\text { COST/ } \\
\text { MHR }\end{array}$ & $\begin{array}{l}\text { TOTAL } \\
\text { LABOR }\end{array}$ & $\begin{array}{l}\text { MAT'L. } \\
\text { UNIT \$\$ }\end{array}$ & $\begin{array}{l}\text { TOTAL } \\
\text { MAT'L. }\end{array}$ & $\begin{array}{l}\text { TOTAL } \\
\text { L \& M }\end{array}$ \\
\hline Screw Jacks & 24 & EA & 4 & 6 & $\$ 40$ & $\$ 240$ & $\$ 0.00$ & $\$ 0$ & $\$ 240$ \\
\hline Quarter-Section Weld & 1756 & INCHES & 12.3 & 143 & $\$ 40$ & $\$ 5,707$ & $\$ 0.58$ & $\$ 1,018$ & $\$ 6,725$ \\
\hline Half-Section Weld & 1756 & INCHES & 12.3 & 143 & $\$ 40$ & $\$ 5,707$ & $\$ 0.58$ & $\$ 1,018$ & $\$ 6,725$ \\
\hline Top \& Bottom Template & 16 & EA & 0.25 & 64 & $\$ 40$ & $\$ 2,560$ & $\$ 0.00$ & $\$ 0$ & $\$ 2,560$ \\
\hline Move Cradles & 24 & EA & 2 & 12 & $\$ 40$ & $\$ 480$ & $\$ 0.00$ & $\$ 0$ & $\$ 480$ \\
\hline Lifting Eyes & 8 & EA & 1 & 8 & $\$ 40$ & $\$ 320$ & $\$ 35.00$ & $\$ 280$ & $\$ 600$ \\
\hline Paint & 5788 & SF & 80 & 72 & $\$ 40$ & $\$ 2,894$ & $\$ 0.50$ & $\$ 2,894$ & $\$ 5,788$ \\
\hline Non-Productive Time & $3 \%$ & MHRS & & 13 & $\$ 40$ & $\$ 537$ & $\$ 0.00$ & $\$ 0$ & $\$ 537$ \\
\hline X-RAY TEST & 20 & EA & 1 & 20 & $\$ 65$ & $\$ 1,300$ & $\$ 20.00$ & $\$ 400$ & $\$ 1,700$ \\
\hline Weld Correction (1\%) & 35 & INCHES & 16 & 2 & $\$ 40$ & $\$ 88$ & $\$ 0.58$ & $\$ 20$ & $\$ 108$ \\
\hline \multirow[t]{2}{*}{ Load \& Transport Towers } & 1 & SECTIONS & 0.18 & 6 & $\$ 40$ & $\$ 222$ & $\$ 0.00$ & $\$ 0$ & $\$ 222$ \\
\hline & 293 & LF & & \multirow{2}{*}{\multicolumn{2}{|c|}{489}} & $\$ 0$ & $\$ 21.84$ & $\$ 6,391$ & $\$ 6,391$ \\
\hline TOTALS (PER SECTION) & & & & & & \multicolumn{2}{|l|}{$\$ 20,055$} & $\$ 12,023$ & $\$ 32,077$ \\
\hline Minimum & \multirow{2}{*}{\multicolumn{2}{|c|}{$\begin{array}{c}-10 \% \\
15 \% \\
\end{array}$}} & & \multicolumn{2}{|l|}{440} & \multicolumn{2}{|l|}{$\$ 18,049$} & $\$ 10,820$ & $\$ 28,870$ \\
\hline Maximum & & & & \multicolumn{2}{|l|}{562} & \multicolumn{2}{|l|}{$\$ 23,063$} & $\$ 13,826$ & $\$ 36,889$ \\
\hline \multicolumn{3}{|l|}{ Total Number of Sections in Turbine: } & 7 & \multicolumn{2}{|l|}{3422} & \multicolumn{2}{|l|}{$\$ 140,382$} & $\$ 84,159$ & $\$ 224,541$ \\
\hline \multirow{2}{*}{\multicolumn{3}{|c|}{$\begin{array}{l}\text { Minimum } \\
\text { Maximum }\end{array}$}} & 7 & \multicolumn{2}{|l|}{3080} & \multicolumn{2}{|l|}{$\$ 126,344$} & $\$ 75,743$ & $\$ 202,087$ \\
\hline & & & 7 & \multicolumn{2}{|l|}{3935} & \multicolumn{2}{|l|}{$\$ 161,440$} & $\$ 96,783$ & $\$ 258,223$ \\
\hline & Avg & Min & Max & & & & & & \\
\hline 10 People, 10 hrs/day & 100 & 100 & 100 & & & & & & \\
\hline Days per Tower Section & 4.9 & 4.4 & 5.6 & & & & & & \\
\hline Total number of sections for assembly & 350 & 350 & 350 & & & & & & \\
\hline Number of days for assembly & 1711 & 1540 & 1968 & & & & & & \\
\hline Number of assembly days per turbine & 34.2 & 30.8 & 39.4 & & & & & & \\
\hline Number of 6-day weeks & 285.2 & 256.7 & 327.9 & & & & & & \\
\hline
\end{tabular}




\begin{tabular}{|c|c|c|c|c|c|c|c|c|c|c|c|c|}
\hline $\begin{array}{c}\text { Turbine Class: } \\
\text { Rotor Diameter: }\end{array}$ & \multicolumn{3}{|c|}{$\begin{array}{c}1,500 \\
66 \\
\end{array}$} & \multicolumn{3}{|c|}{$\begin{array}{c}2,500 \\
85 \\
\end{array}$} & \multicolumn{3}{|c|}{$\begin{array}{l}3,500 \\
100 \\
\end{array}$} & \multicolumn{3}{|c|}{$\begin{array}{c}5000 \\
120\end{array}$} \\
\hline Crane Type: & \multicolumn{3}{|c|}{$4100 \mathrm{~S} 1$} & \multicolumn{3}{|c|}{$4100 \mathrm{~S} 1$} & \multicolumn{3}{|c|}{$4600 \mathrm{~S} 4$} & \multicolumn{3}{|c|}{4600 S5 } \\
\hline & Min & Avg & Max & Min & Avg & Max & $\operatorname{Min}$ & Avg & $\operatorname{Max}$ & $\operatorname{Min}$ & Avg & $\operatorname{Max}$ \\
\hline Crane Crew Assembly Labor Costs/Turbine & \begin{tabular}{|l|l}
2,756 \\
\end{tabular} & $\$ 3,063$ & $\$ 3,522$ & $\$ 8,351$ & $\$ 9,279$ & $\$ 10,671$ & $\$ 18,868$ & $\$ 20,965$ & $\$ 24,110$ & $\$ 40,038$ & $\$ 44,487$ & $\$ 51,160$ \\
\hline Crane Crew Relocation Labor Costs/Turbine & & & & \$0 & $\$ 0$ & $\$ 0$ & $\$ 0$ & $\$ 0$ & $\$ 0$ & $\$ 0$ & $\$ 0$ & \\
\hline Crane Rental Costs During Assembly and Relocation/Turbine & $\$ 1,223$ & $\$ 1,359$ & $\$ 1,563$ & $\$ 3,706$ & $\$ 4,118$ & $\$ 4,736$ & $\$ 17,864$ & $\$ 19,848$ & $\$ 22,826$ & $\$ 44,421$ & $\$ 49,357$ & $\frac{\$ 56,760}{65002}$ \\
\hline Meals and Lodging/Turbine & $\$ 318$ & $\$ 353$ & $\$ 406$ & $\$ 964$ & $\$ 1,071$ & $\$ 1,231$ & $\$ 2,177$ & $\$ 2,419$ & $\$ 2,782$ & $\$ 4,620$ & $\$ 5,133$ & $\$ 5,903$ \\
\hline Fuel Cost/Turbine & $\$ 117$ & $\$ 130$ & $\$ 149$ & $\$ 353$ & $\$ 393$ & $\$ 451$ & $\$ 907$ & $\$ 1,008$ & $\$ 1,159$ & $\$ 1,925$ & $\$ 2,139$ & $\$ 2,460$ \\
\hline Cribbing Costs/Turbine & $\$ 131$ & $\$ 131$ & $\$ 131$ & $\$ 131$ & $\$ 131$ & $\$ 131$ & $\$ 190$ & $\$ 190$ & $\$ 190$ & $\$ 595$ & $\$ 595$ & $\$ 595$ \\
\hline Mob/Demob Costs/Turbine & $\$ 1,328$ & $\$ 1,328$ & $\$ 1,328$ & $\$ 1,328$ & $\$ 1,328$ & $\$ 1,328$ & $\$ 2,477$ & $\$ 2,477$ & $\$ 2,477$ & $\$ 2,757$ & $\$ 2,757$ & $\$ 2,757$ \\
\hline Total Crane and Crew Costs/Turbine & $\$ 5,873$ & $\$ 6,364$ & $\$ 7,100$ & $\$ 14,834$ & $\$ 16,320$ & $\$ 18,549$ & $\$ 42,483$ & $\$ 46,907$ & $\$ 53,543$ & $\$ 94,355$ & $\$ 104,467$ & $\$ 119,634$ \\
\hline Total Crane Costs (50 Turbines) & $\$ 293,673$ & $\$ 318,197$ & $\$ 354,984$ & $\$ 741,688$ & $\$ 815,991$ & $\$ 927,447$ & $\$ 2,124,138$ & $\$ 2,345,339$ & $\$ 2,677,140$ & $\$ 4,717,770$ & $\$ 5,223,346$ & $\$ 5,981,711$ \\
\hline & & & & & & & & & & & & \\
\hline Costs $/ \mathrm{kW}$ & $\$ 3.90$ & $\$ 4.23$ & $\$ 4.72$ & $\$ 5.94$ & $\$ 6.54$ & $\$ 7.43$ & $\$ 12.29$ & $\$ 13.57$ & $\$ 15.49$ & $\$ 18.96$ & $\$ 20.99$ & $\$ 24.04$ \\
\hline Costs/Swept Areal & $\$ 1.72$ & $\$ 1.86$ & $\$ 2.08$ & $\$ 2.61$ & $\$ 2.88$ & $\$ 3.27$ & $\$ 5.41$ & $\$ 5.97$ & $\$ 6.82$ & $\$ 8.34$ & $\$ 9.24$ & $\$ 10.58$ \\
\hline
\end{tabular}




\begin{tabular}{|c|c|c|c|c|}
\hline Initial Assumptions & & Tower Fabrication & Crane Assembly & \\
\hline Work Hours/Day & & 10 & 8 & \\
\hline Number of Days/Wk & & 6 & 5 & \\
\hline Number of Weeks/Year & & 52 & 52 & \\
\hline Number of Weeks/Month & & 4.3333 & 4.4000 & \\
\hline Number of Days/Month & & 26 & 22 & \\
\hline Number of Hours/Month & & 260 & 176 & \\
\hline Number of Turbines & & 50 & & \\
\hline Turbine Rating (kW) & 1500 & 2500 & 3500 & 5000 \\
\hline Crane Type & $4100 \mathrm{~S} 1$ & $4100 \mathrm{~S} 1$ & $4600 \mathrm{~S} 4$ & $4600 \mathrm{~S} 5$ \\
\hline Monthly Crane Costs during turbine assembly (60hr week) & $\$ 15,000$ & $\$ 15,000$ & $\$ 32,000$ & $\$ 37,500$ \\
\hline Monthly crane costs other time & $\$ 15,000$ & $\$ 15,000$ & $\$ 32,000$ & $\$ 37,500$ \\
\hline 6 Month Rental Costs & $\$ 14,000$ & $\$ 14,000$ & $\$ 29,867$ & $\$ 35,000$ \\
\hline 9 Month Rental Costs & $\$ 13,500$ & $\$ 13,500$ & $\$ 28,800$ & $\$ 33,750$ \\
\hline 12 Month Rental Costs & $\$ 13,000$ & $\$ 13,000$ & $\$ 27,733$ & $\$ 32,500$ \\
\hline \multicolumn{5}{|c|}{ 1. Tower Assembly Crew Information - (Assembly crew production rate determines crane production and costs) } \\
\hline Number of People in Crew & 10 & 10 & 10 & 10 \\
\hline Number of Crews & 1 & 1 & 1 & 1 \\
\hline Man Hours/Day & 100 & 100 & 100 & 100 \\
\hline Number of Man Hours/Wk & 600 & 600 & 600 & 600 \\
\hline Tower Fabrication Assembly Rate Days/Turbine & 2.4 & 7.1 & 16.1 & 34.2 \\
\hline \multicolumn{5}{|l|}{ 2. Crane Crew Information - During Tower Assembly } \\
\hline Number of People in Crane Crew & 2 & 2 & 2 & 2 \\
\hline Number of Cranes and Crew & 1 & 1 & 1 & 1 \\
\hline Number of Turbines/Crane & 50 & 50 & 50 & 50 \\
\hline Man Hours/Day & 20 & 20 & 20 & 20 \\
\hline Estimated Crane Crew Man Hours/Turbine & 47.1 & 142.8 & 322.5 & 684.4 \\
\hline Labor Costs/Crane Crew Man Hour & $\$ 65$ & $\$ 65$ & $\$ 65$ & $\$ 65$ \\
\hline Crane Crew Assembly Labor Costs/Turbine & $\$ 3,063$ & $\$ 9,279$ & $\$ 20,965$ & $\$ 44,487$ \\
\hline \multicolumn{5}{|l|}{ 3. Crane Relocation Information } \\
\hline Estimated Relocation Hours/Turbine & 0 & 0 & 0 & 0 \\
\hline Total Relocation Hours & 0 & 0 & 0 & 0 \\
\hline Total Relocation Hours/Crane & 0 & 0 & 0 & 0 \\
\hline Relocation Days/Crane & 0 & 0 & 0 & 0 \\
\hline Estimated Relocation Days/Turbine & 0 & 0 & 0 & 0 \\
\hline Crane Crew Relocation Man Hours/Turbine & 0.0 & 0.0 & 0.0 & 0.0 \\
\hline Crane Crew Relocation Labor Costs/Turbine & $\$ 0$ & \$o & $\$ 0$ & $\$ 0$ \\
\hline Crane Costs During Relocation/Turbine & $\$ 0$ & $\$ 0$ & $\$ 0$ & $\$ 0$ \\
\hline & & & & \\
\hline \multicolumn{5}{|l|}{ 3. Totals } \\
\hline Total Number of Tower Fabrication Days/Turbine & 2.4 & 7.1 & 16.1 & 34.2 \\
\hline Total Number of Days Required: & 118 & 357 & 806 & 1711 \\
\hline Total Number of Weeks Required & 19.6 & 59.5 & 134.4 & 285.2 \\
\hline & & & & \\
\hline Total Number of Months for Assembly & 4.5 & 13.7 & 31.0 & 65.8 \\
\hline 3 Month Min Crane Rental Costs & $\$ 45,000$ & $\$ 45,000$ & $\$ 96,000$ & $\$ 112,500$ \\
\hline Total Crane Rental Charges & $\$ 67,960$ & $\$ 205,904$ & $\$ 992,418$ & $\$ 2,467,831$ \\
\hline Crane Rental Costs/Turbine & $\$ 1,359$ & $\$ 4,118$ & $\$ 19,848$ & $\$ 49,357$ \\
\hline \multicolumn{5}{|l|}{ 4. Material/Supplies/Incidental Crane Costs } \\
\hline Meals and Lodging/Person/Day & $\$ 75$ & $\$ 75$ & $\$ 75$ & $\$ 75$ \\
\hline Number of Person-Days & 235.5929717 & 713.7990923 & 1612.679015 & 3422.059357 \\
\hline Total Meals and Lodging Costs & $\$ 17,669$ & $\$ 53,535$ & $\$ 120,951$ & $\$ 256,654$ \\
\hline Meals and Lodging/Turbine & $\$ 353$ & $\$ 1,071$ & $\$ 2,419$ & $\$ 5,133$ \\
\hline \multicolumn{5}{|l|}{ 5. Fuel } \\
\hline Fuel Cost/Gallon & $\$ 1.50$ & $\$ 1.50$ & $\$ 1.50$ & $\$ 1.50$ \\
\hline Gallons of Fuel/Week & 220 & 220 & 250 & 250 \\
\hline Total Cost of Fuel & $\$ 6,479$ & $\$ 19,629$ & $\$ 50,396$ & $\$ 106,939$ \\
\hline Fuel Cost/Turbine & $\$ 130$ & $\$ 393$ & $\$ 1,008$ & $\$ 2,139$ \\
\hline 6. Cribbing & & & & \\
\hline Cribbing Cost/sq ft & $\$ 2.50$ & $\$ 2.50$ & $\$ 2.50$ & $\$ 2.50$ \\
\hline Required Cribbing sq ft/Turbine & 2615 & 2615 & 3800 & 11900 \\
\hline Cribbing Costs/Turbine & $\$ 131$ & $\$ 131$ & $\$ 190$ & $\$ 595$ \\
\hline 7. Mobilization and Demobilization & & & & \\
\hline Crane Assembly and Disassembly Hours & 24 & 24 & 48 & 48 \\
\hline Lampson Supervisor Hours & 24 & 24 & 48 & 48 \\
\hline Lampson Supervisor Hourly Cost & $\$ 75$ & $\$ 75$ & $\$ 75$ & $\$ 75$ \\
\hline Number of Iron Workers & 4 & 4 & 4 & 4 \\
\hline Man Hours for Iron Workers & 96 & 96 & 192 & 192 \\
\hline Iron Worker Hourly Cost & $\$ 65$ & $\$ 65$ & $\$ 65$ & $\$ 65$ \\
\hline Crane Rental Period (Months) During Assembly & 0.1 & 0.1 & 0.3 & 0.3 \\
\hline Crane Rental Cost & $\$ 2,045$ & $\$ 2,045$ & $\$ 8,727$ & $\$ 10,227$ \\
\hline Total Labor Costs & $\$ 8,040$ & $\$ 8,040$ & $\$ 16,080$ & $\$ 16,080$ \\
\hline Truck Crane 1 Hourly Cost & $\$ 185$ & $\$ 185$ & $\$ 185$ & $\$ 185$ \\
\hline Truck Crane 2 Hourly Cost & $\$ 325$ & $\$ 325$ & $\$ 325$ & $\$ 325$ \\
\hline Truck Crane 3 Hourly Costs & & & & \\
\hline Total Truck Crane Costs & $\$ 12,240$ & $\$ 12,240$ & $\$ 24,480$ & $\$ 24,480$ \\
\hline Total Transportation Freight in/out & $\$ 40,000$ & $\$ 40,000$ & $\$ 60,000$ & $\$ 70,000$ \\
\hline Transport Days in/out & 6 & 6 & 10 & 10 \\
\hline Transport Hours in/out & 48 & 48 & 80 & 80 \\
\hline Crane Rental During Transport & $\$ 4,091$ & $\$ 4,091$ & $\$ 14,545$ & $\$ 17,045$ \\
\hline SubTotal & $\$ 66,416$ & $\$ 66,416$ & $\$ 123,833$ & $\$ 137,833$ \\
\hline Mob/Demob Costs/Turbine & $\$ 1,328$ & $\$ 1,328$ & $\$ 2,477$ & $\$ 2,757$ \\
\hline
\end{tabular}




\begin{tabular}{|c|c|c|c|c|}
\hline Initial Assumptions & & Tower Fabrication & Crane Assembly & \\
\hline Work Hours/Day & & 10 & 8 & \\
\hline Number of Days/Wk & & 6 & 5 & \\
\hline Number of Weeks/Year & & 52 & 52 & \\
\hline Number of Weeks/Month & & 4.3333 & 4.4000 & \\
\hline Number of Days/Month & & 26 & 22 & \\
\hline Number of Hours/Month & & 260 & 176 & \\
\hline Number of Turbines & & 50 & & \\
\hline Turbine Rating (kW) & 1500 & 2500 & 3500 & 5000 \\
\hline Crane Type & 4100 S1 & 4100 S1 & 4600 S4 & 4600 S5 \\
\hline Monthly Crane Costs during turbine assembly (60hr week) & $\$ 15,000$ & $\$ 15,000$ & $\$ 32,000$ & $\$ 37,500$ \\
\hline Monthly crane costs other time & $\$ 15,000$ & $\$ 15,000$ & $\$ 32,000$ & $\$ 37,500$ \\
\hline 6 Month Rental Costs & $\$ 14,000$ & $\$ 14,000$ & $\$ 29,867$ & $\$ 35,000$ \\
\hline 9 Month Rental Costs & $\$ 13,500$ & $\$ 13,500$ & $\$ 28,800$ & $\$ 33,750$ \\
\hline 12 Month Rental Costs & $\$ 13,000$ & $\$ 13,000$ & $\$ 27,733$ & $\$ 32,500$ \\
\hline \multicolumn{5}{|c|}{ 1. Assembly Crew Information - (Assembly crew production rate determines crane production and costs) } \\
\hline Number of People in Crew & 10 & 10 & 10 & 10 \\
\hline Number of Crews & 1 & 1 & 1 & 1 \\
\hline Man Hours/Day & 100 & 100 & 100 & 100 \\
\hline Number of Man Hours/Wk & 600 & 600 & 600 & 600 \\
\hline Tower Fabrication Assembly Rate Days/Turbine & 2.1 & 6.4 & 14.5 & 30.8 \\
\hline \multicolumn{5}{|l|}{ 2. Crane Crew Information - During Tower Assembly } \\
\hline Number of People in Crane Crew & 2 & 2 & 2 & 2 \\
\hline Number of Cranes and Crew & 1 & 1 & 1 & 1 \\
\hline Number of Turbines/Crane & 50 & 50 & 50 & 50 \\
\hline Man Hours/Day & 20 & 20 & 20 & 20 \\
\hline Estimated Crane Crew Man Hours/Turbine & 42.4 & 128.5 & 290.3 & 616.0 \\
\hline Labor Costs/Crane Crew Man Hour & $\$ 65$ & $\$ 65$ & $\$ 65$ & $\$ 65$ \\
\hline Crane Crew Assembly Labor Costs/Turbine & $\$ 2,756$ & $\$ 8,351$ & $\$ 18,868$ & $\$ 40,038$ \\
\hline \multicolumn{5}{|l|}{ 3. Crane Relocation Information } \\
\hline Estimated Relocation Hours/Turbine & 0 & 0 & 0 & 0 \\
\hline Total Relocation Hours & 0 & 0 & 0 & 0 \\
\hline Total Relocation Hours/Crane & 0 & 0 & 0 & 0 \\
\hline Relocation Days/Crane & 0 & 0 & 0 & 0 \\
\hline Estimated Relocation Days/Turbine & 0 & 0 & 0 & 0 \\
\hline Crane Crew Relocation Man Hours/Turbine & 0.0 & 0.0 & 0.0 & 0.0 \\
\hline Crane Crew Relocation Labor Costs/Turbine & $\$ 0$ & $\$ 0$ & $\$ 0$ & $\$ 0$ \\
\hline Crane Costs During Relocation/Turbine & \$0, & $\$ 0$ & $\$ 0$ & $\$ 0$ \\
\hline & & & & \\
\hline \multicolumn{5}{|l|}{ 3. Totals } \\
\hline Total Number of Tower Fabrication Days/Turbine & 2.1 & 6.4 & 14.5 & 30.8 \\
\hline Total Number of Days Required: & 106 & 321 & 726 & 1540 \\
\hline Total Number of Weeks Required & 17.7 & 53.5 & 121.0 & 256.7 \\
\hline & & & & \\
\hline Total Number of Months for Assembly & 4.1 & 12.4 & 27.9 & 59.2 \\
\hline 3 Month Min Crane Rental Costs & $\$ 45,000$ & $\$ 45,000$ & $\$ 96,000$ & $\$ 112,500$ \\
\hline Total Crane Rental Charges & $\$ 61,164$ & $\$ 185,313$ & $\$ 893,176$ & $\$ 2,221,048$ \\
\hline Crane Rental Costs/Turbine & $\$ 1,223$ & $\$ 3,706$ & $\$ 17,864$ & $\$ 44,421$ \\
\hline \multicolumn{5}{|l|}{ 4. Material/Supplies/lncidental Crane Costs } \\
\hline Meals and Lodging/Person/Day & $\$ 75$ & $\$ 75$ & $\$ 75$ & $\$ 75$ \\
\hline Number of Person-Days & 212.0336745 & 642.419183 & 1451.411114 & 3079.853422 \\
\hline Total Meals and Lodging Costs & $\$ 15,903$ & $\$ 48,181$ & $\$ 108,856$ & $\$ 230,989$ \\
\hline Meals and Lodging/Turbine & $\$ 318$ & $\$ 964$ & $\$ 2,177$ & $\$ 4,620$ \\
\hline \multicolumn{5}{|l|}{ 5. Fuel } \\
\hline Fuel Cost/Gallon & $\$ 1.50$ & $\$ 1.50$ & $\$ 1.50$ & $\$ 1.50$ \\
\hline Gallons of Fuel/Week & 220 & 220 & 250 & 250 \\
\hline Total Cost of Fuel & $\$ 5,831$ & $\$ 17,667$ & $\$ 45,357$ & $\$ 96,245$ \\
\hline Fuel Cost/Turbine & $\$ 117$ & $\$ 353$ & $\$ 907$ & $\$ 1,925$ \\
\hline 6. Cribbing & & & & \\
\hline Cribbing Cost/sq ft & $\$ 2.50$ & $\$ 2.50$ & $\$ 2.50$ & $\$ 2.50$ \\
\hline Required Cribbing sq ft/Turbine & 2615 & 2615 & 3800 & 11900 \\
\hline Cribbing Costs/Turbine & $\$ 131$ & $\$ 131$ & $\$ 190$ & $\$ 595$ \\
\hline 7. Mobilization and Demobilization & & & & \\
\hline Crane Assembly and Disassembly Hours & 24 & 24 & 48 & 48 \\
\hline Lampson Supervisor Hours & 24 & 24 & 48 & 48 \\
\hline Lampson Supervisor Hourly Cost & $\$ 75$ & $\$ 75$ & $\$ 75$ & $\$ 75$ \\
\hline Number of Iron Workers & 4 & 4 & 4 & 4 \\
\hline Man Hours for Iron Workers & 96 & 96 & 192 & 192 \\
\hline Iron Worker Hourly Cost & $\$ 65$ & $\$ 65$ & $\$ 65$ & $\$ 65$ \\
\hline Crane Rental Period (Months) During Assembly & 0.1 & 0.1 & 0.3 & 0.3 \\
\hline Crane Rental Cost & $\$ 2,045$ & $\$ 2,045$ & $\$ 8,727$ & $\$ 10,227$ \\
\hline Total Labor Costs & $\$ 8,040$ & $\$ 8,040$ & $\$ 16,080$ & $\$ 16,080$ \\
\hline Truck Crane 1 Hourly Cost & $\$ 185$ & $\$ 185$ & $\$ 185$ & $\$ 185$ \\
\hline Truck Crane 2 Hourly Cost & $\$ 325$ & $\$ 325$ & $\$ 325$ & $\$ 325$ \\
\hline Truck Crane 3 Hourly Costs & & & & \\
\hline Total Truck Crane Costs & $\$ 12,240$ & $\$ 12,240$ & $\$ 24,480$ & $\$ 24,480$ \\
\hline Total Transportation Freight in/out & $\$ 40,000$ & $\$ 40,000$ & $\$ 60,000$ & $\$ 70,000$ \\
\hline Transport Days in/out & 6 & 6 & 10 & 10 \\
\hline Transport Hours in/out & 48 & 48 & 80 & 80 \\
\hline Crane Rental During Transport & $\$ 4,091$ & $\$ 4,091$ & $\$ 14,545$ & $\$ 17,045$ \\
\hline SubTotal & $\$ 66,416$ & $\$ 66,416$ & $\$ 123,833$ & $\$ 137,833$ \\
\hline Mob/Demob Costs/Turbine & $\$ 1,328$ & $\$ 1,328$ & $\$ 2,477$ & $\$ 2,757$ \\
\hline
\end{tabular}




\begin{tabular}{|c|c|c|c|c|}
\hline Initial Assumptions & & Tower Fabrication & Crane Assembly & \\
\hline Work Hours/Day & & 10 & 8 & \\
\hline Number of Days/Wk & & 6 & 5 & \\
\hline Number of Weeks/Year & & 52 & 52 & \\
\hline Number of Weeks/Month & & 4.3333 & 4.4000 & \\
\hline Number of Days/Month & & 26 & 22 & \\
\hline Number of Hours/Month & & 260 & 176 & \\
\hline Number of Turbines & & 50 & & \\
\hline Turbine Rating (kW) & 1500 & 2500 & 3500 & 5000 \\
\hline Crane Type & $4100 \mathrm{S1}$ & 410051 & $4600 S 4$ & 4600 S5 \\
\hline Monthly Crane Costs during turbine assembly (60hr week) & $\$ 15,000$ & $\$ 15,000$ & $\$ 32,000$ & $\$ 37,500$ \\
\hline Monthly crane costs other time & $\$ 15,000$ & $\$ 15,000$ & $\$ 32,000$ & $\$ 37,500$ \\
\hline 6 Month Rental Costs & $\$ 14,000$ & $\$ 14,000$ & $\$ 29,867$ & $\$ 35,000$ \\
\hline 9 Month Rental Costs & $\$ 13,500$ & $\$ 13,500$ & $\$ 28,800$ & $\$ 33,750$ \\
\hline 12 Month Rental Costs & $\$ 13,000$ & $\$ 13,000$ & $\$ 27,733$ & $\$ 32,500$ \\
\hline \multicolumn{5}{|c|}{ 1. Assembly Crew Information - (Assembly crew production rate determines crane production and costs) } \\
\hline Number of People in Crew & 10 & 10 & 10 & 10 \\
\hline Number of Crews & 1 & 1 & 1 & 1 \\
\hline Man Hours/Day & 100 & 100 & 100 & 100 \\
\hline Number of Man Hours/Wk & 600 & 600 & 600 & 600 \\
\hline Tower Fabrication Assembly Rate Days/Turbine & 2.7 & 8.2 & 18.5 & 39.4 \\
\hline \multicolumn{5}{|l|}{ 2. Crane Crew Information - During Tower Assembly } \\
\hline Number of People in Crane Crew & 2 & 2 & 2 & 2 \\
\hline Number of Cranes and Crew & 1 & 1 & 1 & 1 \\
\hline Number of Turbines/Crane & 50 & 50 & 50 & 50 \\
\hline Man Hours/Day & 20 & 20 & 20 & 20 \\
\hline Estimated Crane Crew Man Hours/Turbine & 54.2 & 164.2 & 370.9 & 787.1 \\
\hline Labor Costs/Crane Crew Man Hour & $\$ 65$ & $\$ 65$ & $\$ 65$ & $\$ 65$ \\
\hline Crane Crew Assembly Labor Costs/Turbine & $\$ 3,522$ & $\$ 10,671$ & $\$ 24,110$ & $\$ 51,160$ \\
\hline \multicolumn{5}{|l|}{ 3. Crane Relocation Information } \\
\hline Estimated Relocation Hours/Turbine & 0 & 0 & 0 & 0 \\
\hline Total Relocation Hours & 0 & 0 & 0 & 0 \\
\hline Total Relocation Hours/Crane & 0 & 0 & 0 & 0 \\
\hline Relocation Days/Crane & 0 & 0 & 0 & 0 \\
\hline Estimated Relocation Days/Turbine & 0 & 0 & 0 & 0 \\
\hline Crane Crew Relocation Man Hours/Turbine & 0.0 & 0.0 & 0.0 & 0.0 \\
\hline Crane Crew Relocation Labor Costs/Turbine & $\$ 0$ & $\$ 0$ & $\$ 0$ & $\$ 0$ \\
\hline Crane Costs During Relocation/Turbine & $\$ 0$ & $\$ 0$ & \$o & $\$ 0$ \\
\hline \multicolumn{5}{|l|}{ 3. Totals } \\
\hline Total Number of Tower Fabrication Days/Turbine & 2.7 & 8.2 & 18.5 & 39.4 \\
\hline Total Number of Days Required: & 135 & 410 & 927 & 1968 \\
\hline Total Number of Weeks Required & 22.6 & 68.4 & 154.5 & 327.9 \\
\hline & & & & \\
\hline Total Number of Months for Assembly & 5.2 & 15.8 & 35.7 & 75.7 \\
\hline 3 Month Min Crane Rental Costs & $\$ 45,000$ & $\$ 45,000$ & $\$ 96,000$ & $\$ 112,500$ \\
\hline Total Crane Rental Charges & $\$ 78,153$ & $\$ 236,789$ & $\$ 1,141,281$ & $\$ 2,838,006$ \\
\hline Crane Rental Costs/Turbine & $\$ 1,563$ & $\$ 4,736$ & $\$ 22,826$ & $\$ 56,760$ \\
\hline \multicolumn{5}{|l|}{ 4. Material/Supplies/Incidental Crane Costs } \\
\hline Meals and Lodging/Person/Day & $\$ 75$ & $\$ 75$ & $\$ 75$ & $\$ 75$ \\
\hline Number of Person-Days & 270.9319175 & 820.8689561 & 1854.580868 & 3935.368261 \\
\hline Total Meals and Lodging Costs & $\$ 20,320$ & $\$ 61,565$ & $\$ 139,094$ & $\$ 295,153$ \\
\hline Meals and Lodging/Turbine & $\$ 406$ & $\$ 1,231$ & $\$ 2,782$ & $\$ 5,903$ \\
\hline 5. Fuel & & & & \\
\hline Fuel Cost/Gallon & $\$ 1.50$ & $\$ 1.50$ & $\$ 1.50$ & $\$ 1.50$ \\
\hline Gallons of Fuel/Week & 220 & 220 & 250 & 250 \\
\hline Total Cost of Fuel & $\$ 7,451$ & $\$ 22,574$ & $\$ 57,956$ & $\$ 122,980$ \\
\hline Fuel Cost/Turbine & $\$ 149$ & $\$ 451$ & $\$ 1,159$ & $\$ 2,460$ \\
\hline 6. Cribbing & & & & \\
\hline Cribbing Cost/sq ft & $\$ 2.50$ & $\$ 2.50$ & $\$ 2.50$ & $\$ 2.50$ \\
\hline Required Cribbing sq ft/Turbine & 2615 & 2615 & 3800 & 11900 \\
\hline Cribbing Costs/Turbine & $\$ 131$ & $\$ 131$ & $\$ 190$ & $\$ 595$ \\
\hline 7. Mobilization and Demobilization & & 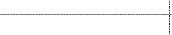 & & \\
\hline Crane Assembly and Disassembly Hours & 24 & 24 & 48 & 48 \\
\hline Lampson Supervisor Hours & 24 & 24 & 48 & 48 \\
\hline Lampson Supervisor Hourly Cost & $\$ 75$ & $\$ 75$ & $\$ 75$ & $\$ 75$ \\
\hline Number of Iron Workers & 4 & 4 & 4 & 4 \\
\hline Man Hours for Iron Workers & 96 & 96 & 192 & 192 \\
\hline Iron Worker Hourly Cost & $\$ 65$ & $\$ 65$ & $\$ 65$ & $\$ 65$ \\
\hline Crane Rental Period (Months) During Assembly & 0.1 & 0.1 & 0.3 & 0.3 \\
\hline Crane Rental Cost & $\$ 2,045$ & $\$ 2,045$ & $\$ 8,727$ & $\$ 10,227$ \\
\hline Total Labor Costs & $\$ 8,040$ & $\$ 8,040$ & $\$ 16,080$ & $\$ 16,080$ \\
\hline Truck Crane 1 Hourly Cost & $\$ 185$ & $\$ 185$ & $\$ 185$ & $\$ 185$ \\
\hline Truck Crane 2 Hourly Cost & $\$ 325$ & $\$ 325$ & $\$ 325$ & $\$ 325$ \\
\hline Truck Crane 3 Hourly Costs & & & & \\
\hline Total Truck Crane Costs & $\$ 12,240$ & $\$ 12,240$ & $\$ 24,480$ & $\$ 24,480$ \\
\hline Total Transportation Freight in/out & $\$ 40,000$ & $\$ 40,000$ & $\$ 60,000$ & $\$ 70,000$ \\
\hline Transport Days in/out & 6 & 6 & 10 & 10 \\
\hline Transport Hours in/out & 48 & 48 & 80 & 80 \\
\hline Crane Rental During Transport & $\$ 4,091$ & $\$ 4,091$ & $\$ 14,545$ & $\$ 17,045$ \\
\hline SubTotal & $\$ 66,416$ & $\$ 66,416$ & $\$ 123,833$ & $\$ 137,833$ \\
\hline Mob/Demob Costs/Turbine & $\$ 1,328$ & $\$ 1,328$ & $\$ 2,477$ & $\$ 2,757$ \\
\hline
\end{tabular}


Appendix M

Scenario 3 


\begin{tabular}{|c|c|c|c|c|c|c|c|c|c|c|c|c|c|c|c|}
\hline \multirow{2}{*}{\multicolumn{2}{|c|}{\begin{tabular}{|r|} 
Number of People in Crew: 10 \\
Hours per Day: 10 \\
Days per Week 6
\end{tabular}}} & \multicolumn{2}{|l|}{$100 \%$} & \multirow{2}{*}{ 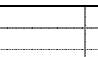 } & \multirow{2}{*}{ 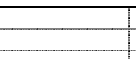 } & \multirow{2}{*}{-1} & \multirow{2}{*}{ 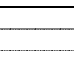 } & \multirow{2}{*}{+2} & \multirow[t]{2}{*}{$x^{2}$} & \multirow{2}{*}{ 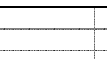 } & \multirow{2}{*}{ 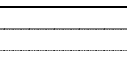 } & & 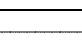 & \multirow{2}{*}{+2} & \\
\hline & & & & & & & & & & & & & & & \\
\hline & & 750 & & & 1500 & & & 2500 & & & 3500 & & & 5000 & \\
\hline Rotor Diameter (m): & & 50 & & & 66 & & & 85 & & & 100 & & & 120 & \\
\hline Activity & MHRS & Labor Costs & $\begin{array}{l}\text { Equip \& } \\
\text { Material }\end{array}$ & MHRS & Labor Costs & $\begin{array}{l}\text { Equip \& } \\
\text { Material }\end{array}$ & MHRS & Labor Costs & Equip \& Material & MHRS & Labor Costs & Equip \& Material & MHRS & Labor Costs & Equip \& Material \\
\hline 1. Receive, Uncrate Nacelle, Blades, Rotors \& Towers & & & & 62 & $\$ 2,498$ & & 75 & $\$ 3,023$ & & 96. & $\$ 3,869$ & & 142 & $\$ 5,723$ & \\
\hline 2. Fabricate Tower Assembly Area & & & & 14 & $\$ 544$ & $\$ 1,289$ & 14 & $\$ 544$ & $\$ 1,289$ & 14 & $\$ 544$ & $\$ 1,289$ & 14 & $\$ 544$ & $\$ 1,289$ \\
\hline 3. Field Fabrication Tower Sections & & & & 101 & $\$ 4,135$ & $\$ 6,621$ & 333 & $\$ 13,647$ & $\$ 20,701$ & 588 & $\$ 24,142$ & $\$ 34,315$ & 952 & $\$ 39,128$ & $\$ 51,509$ \\
\hline 4. Rig \& Set Tower Sections & & & & 132 & $\$ 5,320$ & & 243 & $\$ 9,793$ & & 354 & $\$ 14,265$ & & 619 & $\$ 24,810$ & \\
\hline 5. Grout and Torque Bases & & & & 40 & $\$ 1,612$ & $\$ 850$ & 59 & $\$ 2,377$ & $\$ 950$ & 70 & $\$ 2,822$ & $\$ 1,120$ & 87 & $\$ 3,506$ & $\$ 1,650$ \\
\hline 6. Rig Blades, Assemble Rotors In Air & & & & 61 & $\$ 2,447$ & $\$ 250$ & 95 & $\$ 3,816$ & $\$ 500$ & 112 & $\$ 4,513$ & $\$ 700$ & 164 & $\$ 6,626$ & $\$ 1,000$ \\
\hline 7. Rig \& Set Nacelle & & & & 57 & $\$ 2,317$ & & 133 & $\$ 6,4881$ & & 174 & $\$ 8,550 \mid$ & & 280 & $\$ 13,370$ & \\
\hline 8. Install Gearbox and Generator in Nacelle - on tower & & & & - & & & 60 & $\$ 2.927$ & & 90 & $\$ 4.422$ & & 120 & $\$ 5,730$ & \\
\hline 9. Install Safety Equipment & & & & 12 & $\$ 484$ & & 20 & $\$ 806$ & & 24 & $\$ 968$ & & 36 & $\$ 1,450$ & \\
\hline 10 cepera conditiong & & & & - & S0170 & & & 80150 & & & S20150 & & & 68700 & $\$ 20.941$, \\
\hline 10. General Conditions & & & & & $\$ 2,172$ & $\$ 5,713$ & & $\$ 2,459$ & $\$ 10,789$ & & $\$ 2,459$ & $\$ 10,789$ & & $\$ 8,729$ & $\$ 20,941$ \\
\hline 11. Margin @ 10\% & & & & I & $\$ 2,153$ & $\$ 1,472$ & & $\$ 4,588$ & $\$ 3,423$ & & $\$ 6,655$ & $\$ 4,821$ & & $\$ 10,962$ & $\$ 7,639$ \\
\hline Subtotal Per Turbine & & & & 479 & $\$ 23,682$ & $\$ 16,195$ & 1032 & $\$ 50,468$ & $\$ 37,652$ & 1522 & $\$ 73,209$ & $\$ 53,034$ & 2414 & $\$ 120,577$ & $\$ 84,028$ \\
\hline Percent of Total & & & & & $59 \%$ & & & $57 \%$ & & & $58 \%$ & $42 \%$ & & & $41 \%$ \\
\hline Project Total (50 Turbines) & & & & 23947 & $\$ 1,184,123$ & $\$ 809,752$ & 51596 & $\$ 2,523,401$ & $\$ 1,882,619$ & 76116 & $\$ 3,660,453$ & $\$ 2,651,696$ & 120721 & $\$ 6,028,843$ & $\$ 4,201,393$ \\
\hline Total All Costs & & & & & $\$ 1,993,875$ & & & $\$ 4,406,020$ & & & $\$ 6,312,150$ & & & $\$ 10,230,235$ & \\
\hline Total cost per Turbine & & & & & $\$ 39,878$ & & & $\$ 88,120$ & & & $\$ 126,243$ & & & $\$ 204,605$ & \\
\hline Estimated Assembly Rate - Items 2,5 (Hours & & & & & 189 & & & & & & 618 & & & 1,019 & \\
\hline Estimated Assembly Rate - Items 2,5 (Days) & & & & & 1.9 & & & & & & 6.2 & & & 10.2 & \\
\hline Total Costs/kW & & & & & $\$ 26.49$ & & & $\$ 35.29$ & & & $\$ 36.53$ & & & $\$ 41.12$ & \\
\hline Labor Costs/kW & & & & & $\$ 15.73$ & & & $\$ 20.21$ & & & $\$ 21.18$ & & & $\$ 24.23$ & \\
\hline Equip.Mater Costs/kw & & & & & $\$ 10.76$ & & & $\$ 15.08$ & & & $\$ 15.35$ & & & $\$ 16.89$ & \\
\hline & & & & I & & & & & & & & & & & \\
\hline Total Cost/Swept Area & & & & & $\$ 11.66$ & & & $\$ 15.53$ & & & $\$ 16.07$ & & & $\$ 18.09$ & \\
\hline Man-Hours/Swept Area & & 型 & ${ }^{2}$ & & 0.14 & & & 0.18 & & & 0.19 & & & 0.21 & \\
\hline Labor Costs/Swept Area & & & & & $\$ 6.92$ & & & $\$ 8.89$ & & & $\$ 9.32$ & & & $\$ 10.66$ & \\
\hline Equip, Mater Costs/Swept Area & & & & & $\$ 4.73$ & & & $\$ 6.64$ & & & $\$ 6.75$ & & & $\$ 7.43$ & \\
\hline Total Costs/Hub Heigh & & & & & $\$ 465$ & & & $\$ 797$ & & & $\$ 971$ & & & $\$ 1,312$ & \\
\hline Labor Costs/Hub Heigh & & & & & $\$ 276$ & & & $\$ 457$ & & & $\$ 563$ & & & $\$ 773$ & \\
\hline Equip.Mater Costs/Hub Heigh & & & & & $\$ 189$ & & & $\$ 341$ & & & $\$ 408$ & & & $\$ 539$ & \\
\hline & & & & & & & & & & & & & & & \\
\hline Item 1 Hourly Rates & & & & $\$ 40.30$ & & & $\$ 40.31$ & & & $\$ 40.30$ & & & $\$ 40.30$ & & \\
\hline Item 2 Hourly Rates & & & & $\$ 40.30$ & & & $\$ 40.30$ & & & $\$ 40.30$ & & & $\$ 40.08$ & & \\
\hline Item 3 Hourly Rates & & & & $\$ 40.30$ & & & $\$ 40.29$ & & & $\$ 40.31$ & & & $\$ 40.30$ & & \\
\hline Item 4 Hourly Rates & & & & $\$ 40.31$ & & & $\$ 40.30$ & & & $\$ 40.29$ & & & $\$ 40.31$ & & \\
\hline Item 5 Hourly Rates & & & & $\$ 40.65$ & & & $\$ 48.78$ & & & $\$ 49.14$ & & & $\$ 47.75$ & & \\
\hline Item 6 Hourly Rates & & & & $\$ 40.33$ & & & $\$ 40.30$ & & & $\$ 40.33$ & & & $\$ 40.28$ & & \\
\hline Combined Hourly Rate & & & & $\$ 51.35$ & & & $\$ 54.58$ & & & $\$ 52.70$ & & & $\$ 57.38$ & & \\
\hline Item 1 Percent of Labor & & & & $11 \%$ & & & $6 \%$ & & & $5 \%$ & & & $5 \%$ & & \\
\hline Item 2 Percen & & & & $22 \%$ & & & $19 \%$ & & & $19 \%$ & & & $21 \%$ & & \\
\hline Item 3 Percent of Labor & & & & $7 \%$ & & & $5 \%$ & & & $4 \%$ & & & $3 \%$ & & \\
\hline Item 4 Percent of Labor & & & & $10 \%$ & & & $8 \%$ & & & $6 \%$ & & & $5 \%$ & & \\
\hline Item 5 Percent of Labor & & & & $10 \%$ & & & $13 \%$ & & & $12 \%$ & & & $11 \%$ & & \\
\hline $\begin{array}{l}\text { them } 6 \text { Percent of Labor } \\
\text { Item } 7 \text { Percent of Labort }\end{array}$ & & & & $2 \%$ & & & $\frac{2 \%}{5 \%}$ & & & $\frac{1 \%}{3 \%}$ & & & $\frac{1 \%}{7 \%}$ & & \\
\hline Item 8 Percent of Labort & & & & $9 \%$ & & & $9 \%$ & & & $9 \%$ & & & $9 \%$ & & \\
\hline
\end{tabular}




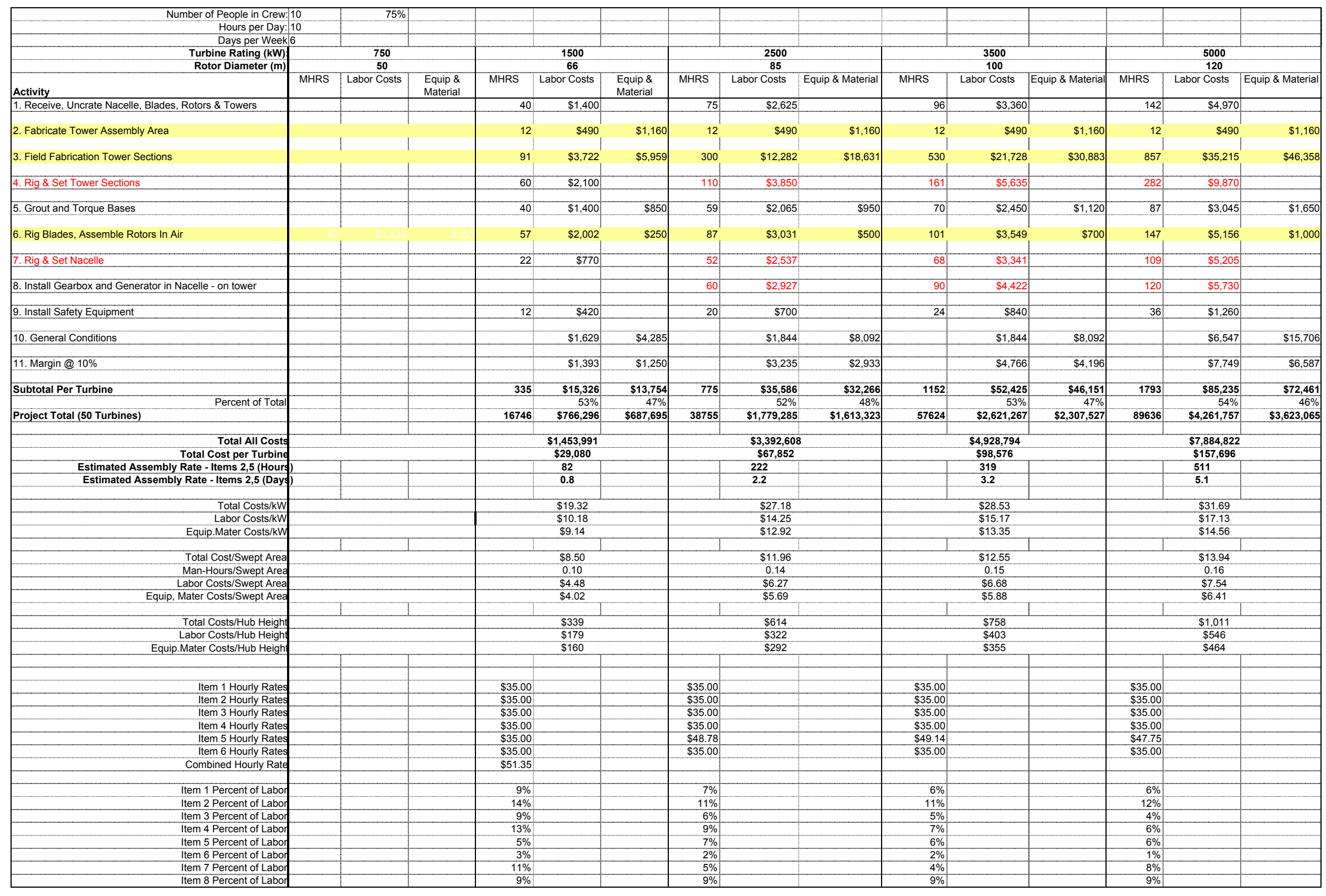




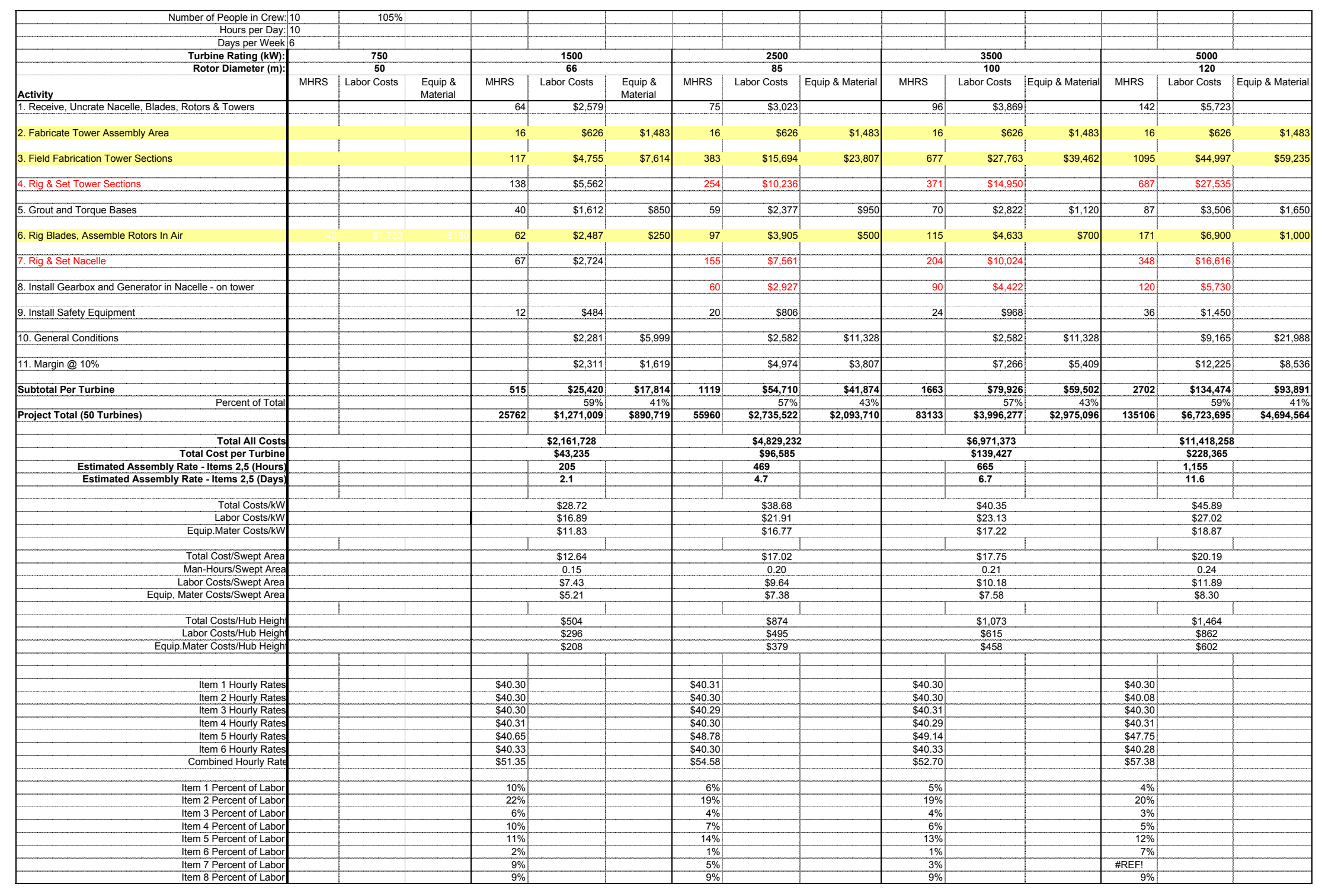




\begin{tabular}{|c|c|c|c|c|c|c|c|c|c|}
\hline Turbine Class: & \multicolumn{3}{|c|}{2500} & 3500 & & & \multicolumn{3}{|c|}{5000} \\
\hline Rotor Diameter: & & 85 & & 100 & & & 120 & & \\
\hline \multirow[t]{2}{*}{ Crane Type: } & \multicolumn{3}{|c|}{ LTL-850 } & \multicolumn{3}{|c|}{ LTL-1100 } & \multicolumn{3}{|c|}{ LTL-1200 } \\
\hline & Min & Avg & Max & Min & Avg & $\operatorname{Max}$ & Min & Avg & $\operatorname{Max}$ \\
\hline Crane Crew Assembly Labor Costs/Turbine & $\$ 4,290$ & $\$ 8,580$ & $\$ 9,165$ & $\$ 6,240$ & $\$ 12,090$ & $\$ 13,065$ & $\$ 9,945$ & $\$ 19,890$ & $\$ 22,620$ \\
\hline Crane Crew Relocation Labor Costs/Turbine & $\$ 2,730$ & $\$ 2,730$ & $\$ 2,730$ & $\$ 4,875$ & $\$ 4,875$ & $\$ 4,875$ & $\$ 6,435$ & $\$ 6,435$ & $\$ 6,435$ \\
\hline Crane Rental Costs During Assembly and Reloc & $\$ 12,923$ & $\$ 20,821$ & $\$ 21,897$ & $\$ 27,623$ & $\$ 42,162$ & $\$ 44,585$ & $\$ 49,000$ & $\$ 78,750$ & $\$ 86,917$ \\
\hline Meals and Lodging/Turbine & $\$ 810$ & $\$ 1,305$ & $\$ 1,373$ & $\$ 1,283$ & $\$ 1,958$ & $\$ 2,070$ & $\$ 1,890$ & $\$ 3,038$ & $\$ 3,353$ \\
\hline Fuel Cost/Turbine & $\$ 585$ & $\$ 943$ & $\$ 991$ & $\$ 998$ & $\$ 1,523$ & $\$ 1,610$ & $\$ 1,575$ & $\$ 2,531$ & $\$ 2,794$ \\
\hline Cribbing Costs/Turbine & $\$ 538$ & $\$ 538$ & $\$ 538$ & $\$ 943$ & $\$ 943$ & $\$ 943$ & $\$ 943$ & $\$ 943$ & $\$ 943$ \\
\hline Mob/Demob Costs/Turbine & $\$ 9,695$ & $\$ 9,695$ & $\$ 9,695$ & $\$ 22,141$ & $\$ 22,141$ & $\$ 22,141$ & $\$ 32,116$ & $\$ 32,116$ & $\$ 32,116$ \\
\hline Total Crane and Crew Costs/Turbine & $\$ 31,570$ & $\$ 44,610$ & $\$ 46,388$ & $\$ 64,101$ & $\$ 85,690$ & $\$ 89,288$ & $\$ 101,903$ & $\$ 143,702$ & $\$ 155,176$ \\
\hline Total Crane Costs (50 Turbines) & $\$ 1,578,506$ & $\$ 2,230,503$ & $\$ 2,319,411$ & $\$ 3,205,065$ & $\$ 4,284,488$ & $\$ 4,464,392$ & $\$ 5,095,157$ & $\$ 7,185,094$ & $\$ 7,758,802$ \\
\hline & & & & & & & & & \\
\hline & & & & & & & & & \\
\hline Costs/kW & $\$ 12.64$ & $\$ 17.87$ & $\$ 18.58$ & $\$ 18.55$ & $\$ 24.80$ & $\$ 25.84$ & $\$ 20.48$ & $\$ 28.88$ & $\$ 31.18$ \\
\hline Costs/Swept Area & $\$ 5.56$ & $\$ 7.86$ & $\$ 8.17$ & $\$ 8.16$ & $\$ 10.91$ & $\$ 11.37$ & $\$ 9.01$ & $\$ 12.71$ & $\$ 13.72$ \\
\hline
\end{tabular}




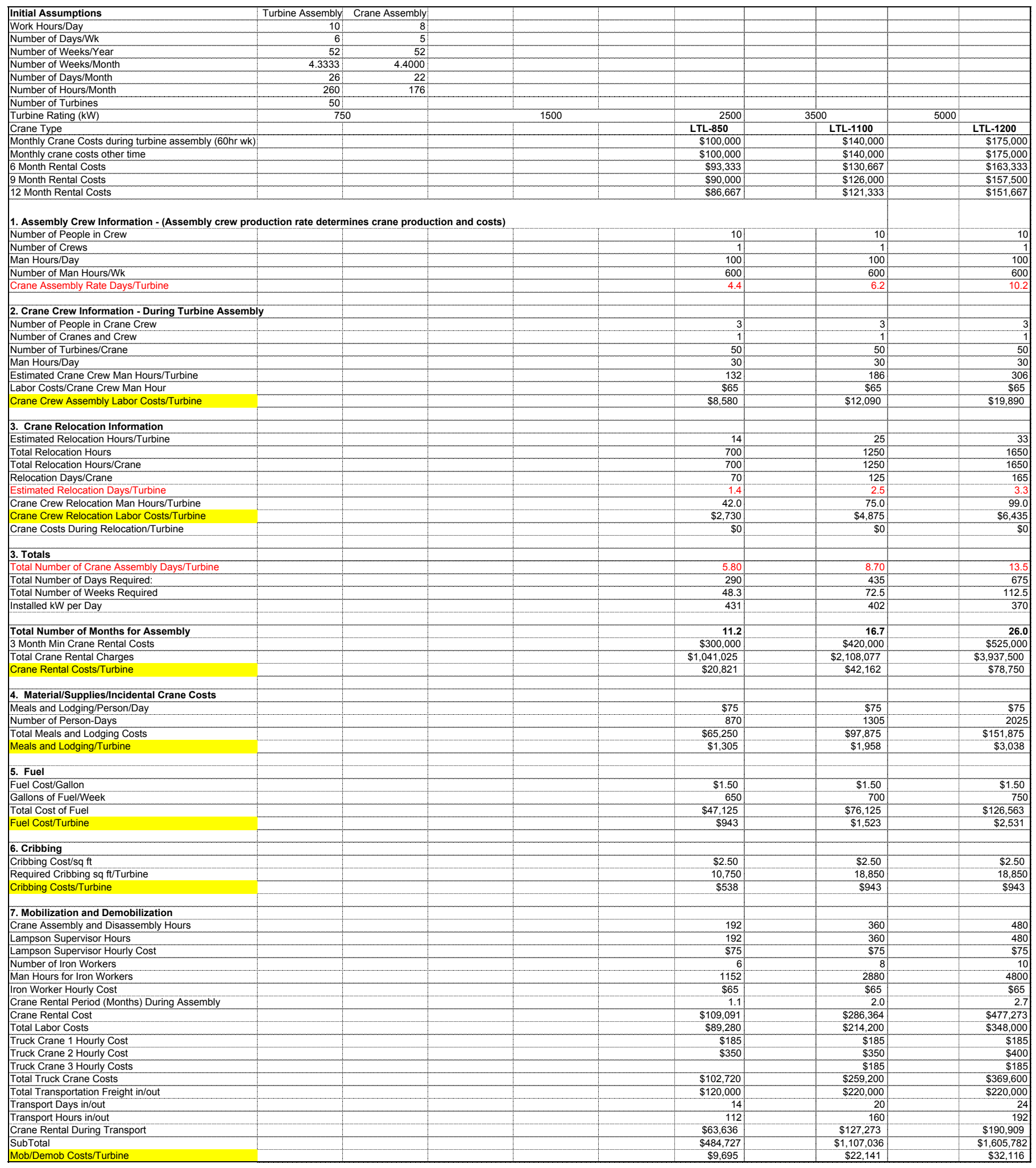




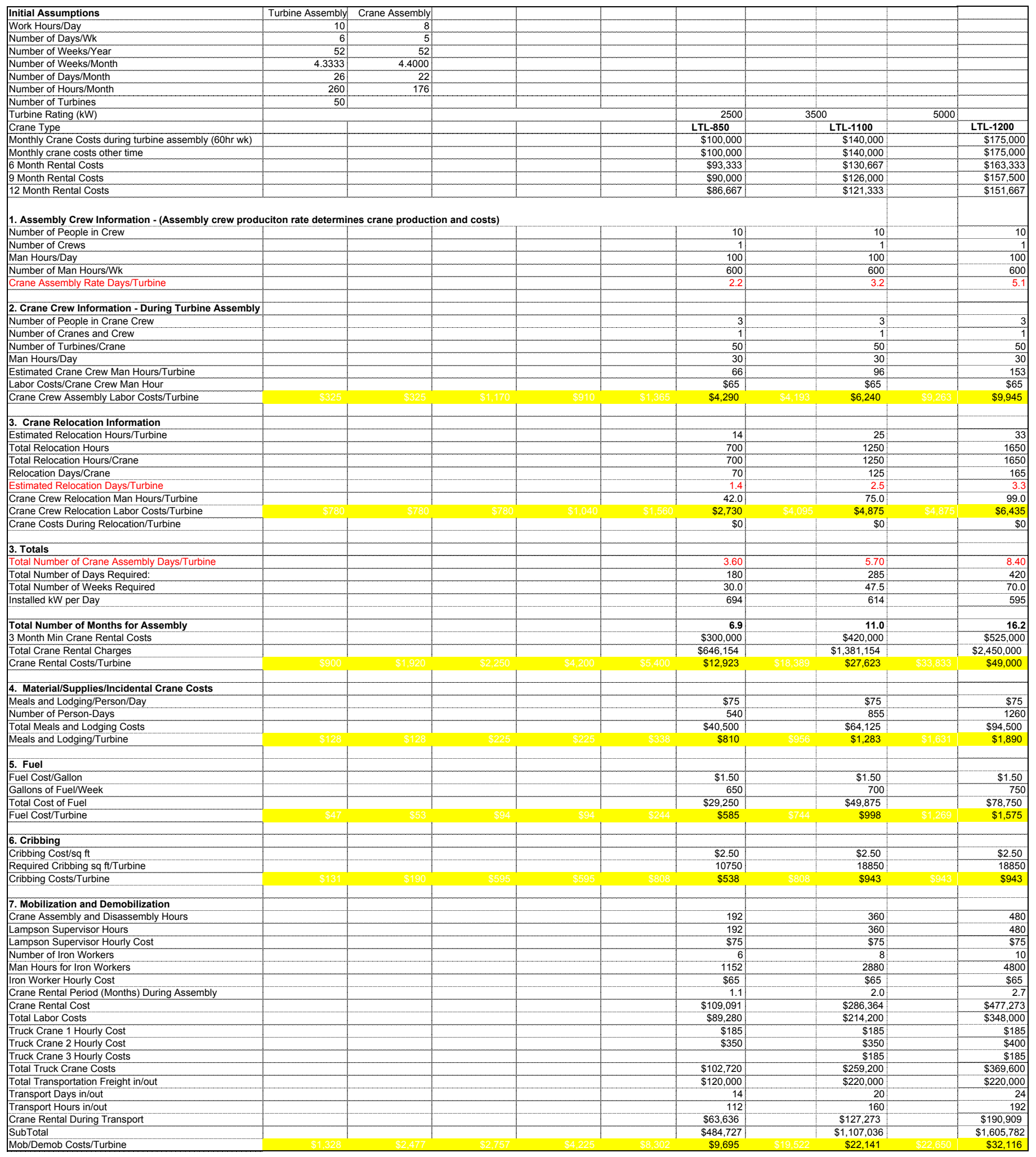




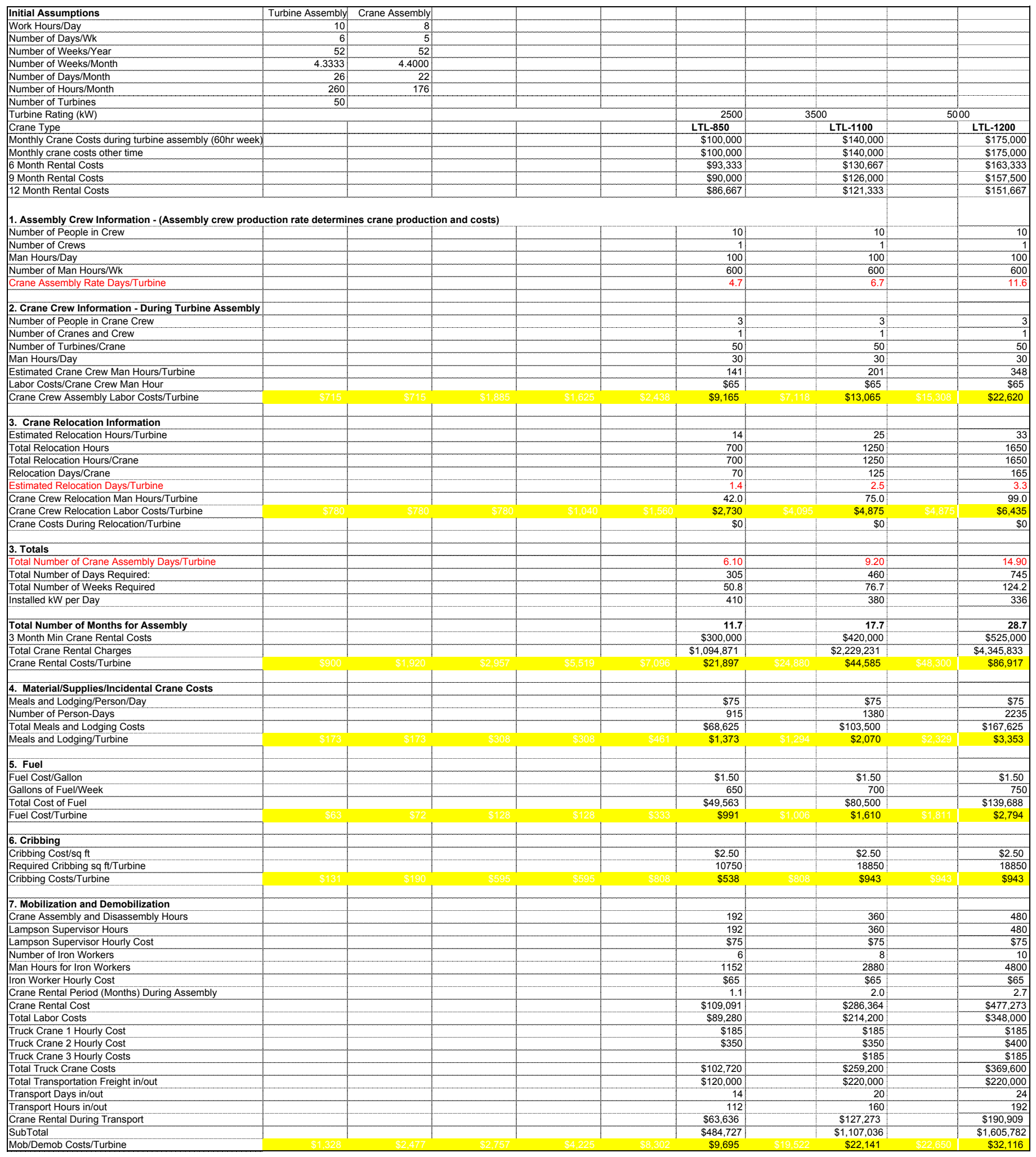


Appendix $\mathbf{N}$

Loaded Crane Rates and Assembly Work Pads 


\section{Crane Cost Periods

Hrs/month
Hrs/Year
Weeks/Year Weeks/Year \\ Bare operation Rate Standby Rate OT Rate (for hrs over 40/wk)}

$60 \mathrm{hr}$ week:

\section{Hrs/Week}

Weeks/yr

Hrs/y

O.T. Hrs/year

O.T. Hrs/week

\section{Bare operation Rate}

Standby Rate

OT Rate (for hrs over 40/wk)

Total Rental Costs for $60 \mathrm{hr}$ week

Hourly rate for $60 \mathrm{hr}$ week

Total Yearly Costs for $60 \mathrm{hr}$ week

Monthly Rate for $60 \mathrm{hr}$ week

176
2112
52
40.6

52
40.6

40.6

$\begin{array}{ll}\$ 85 & \$ 182 \\ \$ 57 & \$ 136 \\ \$ 57 & \$ 121\end{array}$

40.6

3120.0

1008.0
19.4

$100 \%$

$\begin{array}{rrr}\$ 3,461.54 & \$ 7,385 & \$ 8,654 \\ \$ 0 & \$ 0 & \$ 0 \\ \$ 0 & \$ 0 & \$ 0 \\ \$ 3,462 & \$ 7,385 & \$ 8,654 \\ \$ 58 & \$ 123 & \$ 144 \\ \$ 180,000 & \$ 384,000 & \$ 450,000 \\ \$ 15,000 & \$ 32,000 & \$ 37,500\end{array}$

$\begin{array}{rr}\$ 16,154 & \$ 20,769 \\ \$ 0 & \$ 0 \\ \$ 0 & \$ 0 \\ \$ 16,154 & \$ 20,769 \\ \$ 269 & \$ 346 \\ \$ 840,000 & \$ 1,080,000 \\ \$ 70,000 & \$ 90,000\end{array}$

$\$ 28,846$
$\$ 0$
$\$ 0$
$\$ 28,846$
$\$ 481$
$\$ 1,500,000$
$\$ 125,000$

$\$ 32,308$
$\$ 0$
$\$ 0$
$\$ 32,308$
$\$ 538$
$\$ 1,680,000$
$\$ 140,000$

$\begin{array}{rr}\$ 32,308 & \$ 40,385 \\ \$ 0 & \$ 0 \\ \$ 0 & \$ 0 \\ \$ 32,308 & \$ 40,385 \\ \$ 538 & \$ 673 \\ \$ 1,680,000 & \$ 2,100,000 \\ \$ 140,000 & \$ 175,000\end{array}$




\section{Assembly Work Pads}

36'-0" x 83'-0" x 1'-0" - 4 req'd

\begin{tabular}{|c|c|c|c|c|c|c|c|c|c|}
\hline Description & QTY. & UNITS & $\begin{array}{c}\text { UNITS/ } \\
\text { MHR }\end{array}$ & $\begin{array}{l}\text { TOTAL } \\
\text { MHRS }\end{array}$ & $\begin{array}{c}\text { COST/ } \\
\text { MHR }\end{array}$ & $\begin{array}{l}\text { TOTAL } \\
\text { LABOR }\end{array}$ & $\begin{array}{l}\text { MAT'L. } \\
\text { UNIT \$\$ }\end{array}$ & $\begin{array}{l}\text { TOTAL } \\
\text { MAT'L. }\end{array}$ & $\begin{array}{r}\text { TOTAL } \\
\text { L \& M }\end{array}$ \\
\hline Concrete & 444 & $\mathrm{CY}$ & 3 & 148 & $\$ 40$ & $\$ 5,964$ & $\$ 80$ & $\$ 35,520$ & $\$ 41,484$ \\
\hline Reinforcing steel & 43,373 & LBS & 120 & 361 & $\$ 40$ & $\$ 14,458$ & & $\$ 0$ & $\$ 14,458$ \\
\hline Concrete forming & 238 & SF & 8 & 30 & $\$ 40$ & $\$ 1,200$ & $\$ 3$ & $\$ 714$ & $\$ 1,914$ \\
\hline Concrete Curing & 3226 & SF & 250 & 13 & $\$ 0$ & $\$ 0$ & $\$ 0.10$ & $\$ 323$ & $\$ 323$ \\
\hline Concrete Finishing & 3226 & SF & 70 & 46 & $\$ 40$ & $\$ 1,840$ & & $\$ 0$ & $\$ 1,840$ \\
\hline Embedded Plates & 3312 & LBS & 100 & 34 & $\$ 40$ & $\$ 1,360$ & $\$ 1.25$ & $\$ 4,140$ & $\$ 5,500$ \\
\hline Cradle Section & 48 & EA & 1 & 48 & $\$ 40$ & $\$ 1,920$ & $\$ 200$ & $\$ 9,600$ & $\$ 11,520$ \\
\hline Center Cradle Section & 24 & EA & 2 & 12 & $\$ 40$ & $\$ 480$ & $\$ 15$ & $\$ 360$ & $\$ 840$ \\
\hline Top \& Bottom Templates & 8 & EA & 0 & 0 & $\$ 0$ & $\$ 0$ & $\$ 1,500$ & $\$ 12,000$ & $\$ 12,000$ \\
\hline Screw Jacks & 60 & EA & 0 & 0 & $\$ 0$ & $\$ 0$ & $\$ 30$ & $\$ 1,800$ & $\$ 1,800$ \\
\hline TOTALS & & & & 692 & & $\$ 27,22$ & & $\$ 64,457$ & $\$ 91,679$ \\
\hline Cost per Turbine & & & & 14 & & $\$ 54$ & & $\$ 1,289$ & $\$ 1,834$ \\
\hline Minimum & $-10 \%$ & & & 12 & & $\$ 49$ & & $\$ 1,160$ & $\$ 1,650$ \\
\hline Maximum & $15 \%$ & & & 16 & & $\$ 62$ & & $\$ 1,483$ & $\$ 2,109$ \\
\hline
\end{tabular}




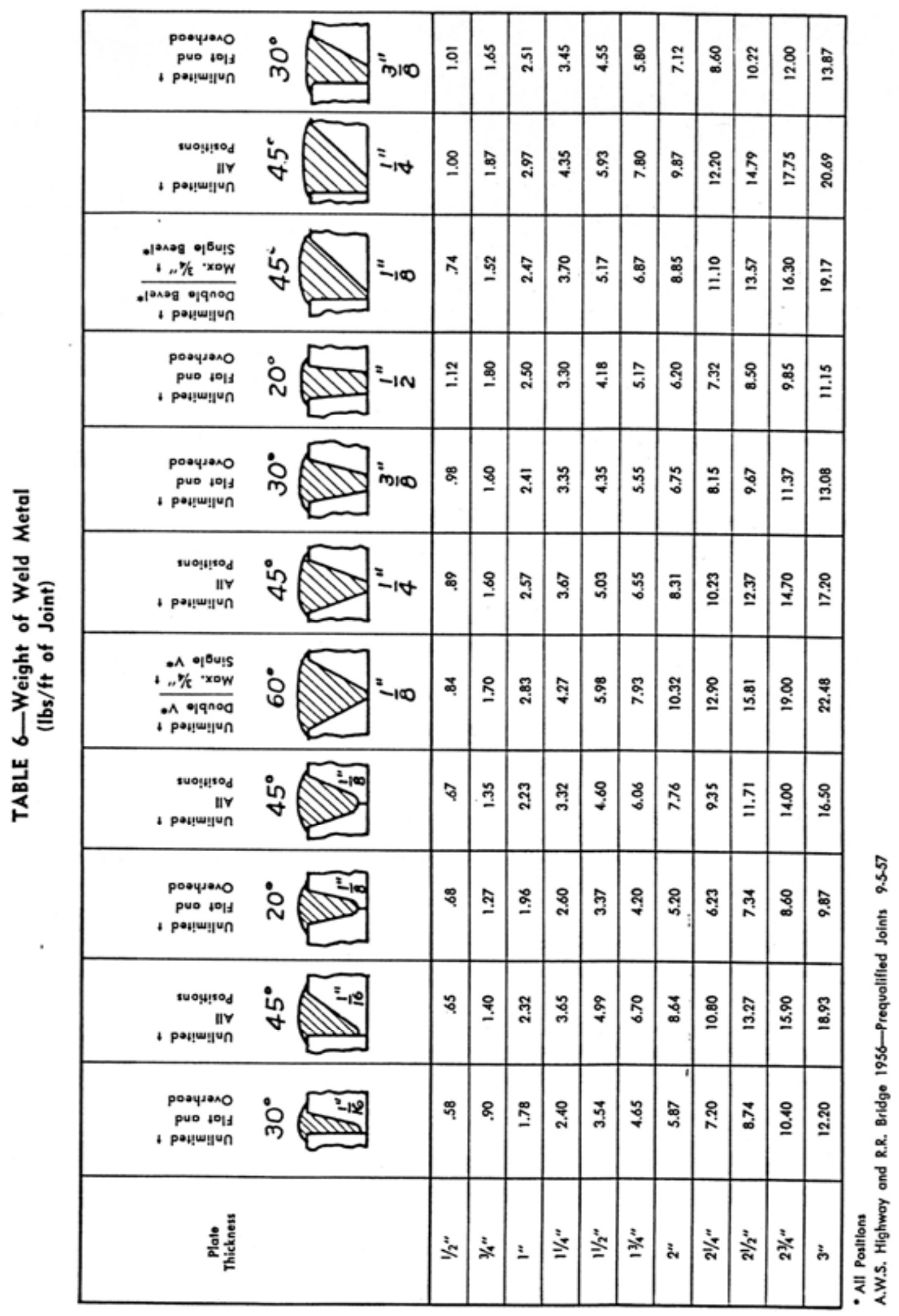


Appendix 0

Terrain Effects on Cranes 
Assumptions:

Terrain effects on crane costs are being estimated by determining the cost impacts of multiple crane dissassembly and reassembly events.

2) Flat terrain with turbines aligned in a $2 \mathrm{D}$ by $10 \mathrm{D}$ grid represents ideal crane conditions in which the crane is assembled once during mobilization and dissassembled during demobilization. Relocation between turbines occurs without dissassembling the crane.

3) Sites with varying terrain, or those not aligned in a simplistic grid pose crane relocation challenges that result in more frequent disassembly of the crane to facilitate relocation.

4) The costs associated with crane disassembly/reassembly include crew labor, crane rental, and additional support cranes. The support cranes are necessary to assist the disassembly and reassembly process.

5) A conservative estimating approach is being applied that assumes the cranes need to be fully disassembled for relocation. It is being assumed that this approach will compensate for minor miscellaneous costs not contained in the labor and crane rental costs.

Since there is no obvious relationship between terrain and the number of crane disassemblies required, this analysis will determine the costs based on different values for the number of cran disassemblies per turbine.

From S1 - Detailed Crane Costs:

\begin{tabular}{|c|c|c|c|c|c|c|c|c|c|c|}
\hline Crane Type & $4100 S 1$ & $4600 S 4$ & $4600 \mathrm{~S} 5$ & what if & LTL-600 & LTL-850 & LTL-1000 & LTL-1100 & LTL-1100 & LTL-1200 \\
\hline Monthly Crane Costs during turbine assembly (60hr wk) & $\$ 15,000$ & $\$ 32,000$ & $\$ 37,500$ & $\$ 70,000$ & $\$ 90,000$ & $\$ 100,000$ & $\$ 125,000$ & $\$ 140,000$ & $\$ 140,000$ & $\$ 175,000$ \\
\hline Monthly crane costs other time & $\$ 15,000$ & $\$ 32,000$ & $\$ 37,500$ & $\$ 70,000$ & $\$ 90,000$ & $\$ 100,000$ & $\$ 125,000$ & $\$ 140,000$ & $\$ 140,000$ & $\$ 175,000$ \\
\hline 6 Month Rental Costs & $\$ 14,000$ & $\$ 29,867$ & $\$ 35,000$ & $\$ 65,333$ & $\$ 84,000$ & $\$ 93,333$ & $\$ 116,667$ & $\$ 130,667$ & $\$ 130,667$ & $\$ 163,333$ \\
\hline 9 Month Rental Costs & $\$ 13,500$ & $\$ 28,800$ & $\$ 33,750$ & $\$ 63,000$ & $\$ 81,000$ & $\$ 90,000$ & $\$ 112,500$ & $\$ 126,000$ & $\$ 126,000$ & $\$ 157,500$ \\
\hline 12 Month Rental Costs & $\$ 13,000$ & $\$ 27,733$ & $\$ 32,500$ & $\$ 60,667$ & $\$ 78,000$ & $\$ 86,667$ & $\$ 108,333$ & $\$ 121,333$ & $\$ 121,333$ & $\$ 151,667$ \\
\hline \multicolumn{11}{|l|}{ 7. Mobilization and Demobilization } \\
\hline Crane Assembly and Disassembly Hours & 24 & 48 & 48 & 80 & 160 & 192 & 360 & 360 & 360 & 480 \\
\hline Lampson Supervisor Hours & 24 & 48 & 48 & 80 & 160 & 192 & 360 & 360 & 360 & 480 \\
\hline Lampson Supervisor Hourly Cost & $\$ 75$ & $\$ 75$ & $\$ 75$ & $\$ 75$ & $\$ 75$ & $\$ 75$ & $\$ 75$ & $\$ 75$ & $\$ 75$ & $\$ 75$ \\
\hline Number of Iron Workers & 4 & 4 & 4 & 4 & 6 & 6 & 8 & 8 & 8 & \\
\hline Man Hours for Iron Workers & 96 & 192 & 192 & 320 & 960 & 1152 & 2880 & 2880 & 2880 & 4800 \\
\hline Iron Worker Hourly Cost & $\$ 65$ & $\$ 65$ & $\$ 65$ & $\$ 65$ & $\$ 65$ & $\$ 65$ & $\$ 65$ & $\$ 65$ & $\$ 65$ & $\$ 65$ \\
\hline Crane Rental Period (Months) During Assembly & 0.1 & 0.3 & 0.3 & 0.5 & 0.9 & 1.1 & 2.0 & 2.0 & 2.0 & 2.7 \\
\hline Crane Rental Cost & $\$ 2,045$ & $\$ 8,727$ & $\$ 10,227$ & $\$ 31,818$ & $\$ 81,818$ & $\$ 109,091$ & $\$ 255,682$ & $\$ 286,364$ & $\$ 286,364$ & $\$ 477,273$ \\
\hline Total Labor Costs & $\$ 8,040$ & $\$ 16,080$ & $\$ 16,080$ & $\$ 26,800$ & $\$ 74,400$ & $\$ 89,280$ & $\$ 214,200$ & $\$ 214,200$ & $\$ 214,200$ & $\$ 348,000$ \\
\hline Truck Crane 1 Hourly Cost & $\$ 185$ & $\$ 185$ & $\$ 185$ & $\$ 185$ & $\$ 185$ & $\$ 185$ & $\$ 185$ & $\$ 185$ & $\$ 185$ & $\$ 185$ \\
\hline Truck Crane 2 Hourly Cost & $\$ 325$ & $\$ 325$ & $\$ 325$ & $\$ 325$ & $\$ 325$ & $\$ 350$ & $\$ 350$ & $\$ 350$ & $\$ 350$ & \\
\hline Truck Crane 3 Hourly Costs & $\$ 0$ & $\$ 0$ & $\$ 0$ & \$0 & \$0 & $\$ 0$ & $\$ 0$ & $\$ 185$ & $\$ 185$ & $\$ 185$ \\
\hline Total Truck Crane Costs & $\$ 12,240$ & $\$ 24,480$ & $\$ 24,480$ & $\$ 40,800$ & $\$ 81,600$ & $\$ 102,720$ & $\$ 192,600$ & $\$ 259,200$ & $\$ 259,200$ & $\$ 369,600$ \\
\hline Total Transportation Freight in/out & $\$ 40,000$ & $\$ 60,000$ & $\$ 70,000$ & $\$ 80,000$ & $\$ 120,000$ & $\$ 120,000$ & $\$ 200,000$ & $\$ 220,000$ & $\$ 220,000$ & $\$ 220,000$ \\
\hline Transport Days in/out & 6 & 10 & 10 & 10 & 14 & 14 & 20 & 20 & 24 & 24 \\
\hline Transport Hours in/out & 48 & 80 & 80 & 80 & 112 & 112 & 160 & 160 & 192 & 192 \\
\hline Crane Rental During Transport & $\$ 4,091$ & $\$ 14,545$ & $\$ 17,045$ & $\$ 31,818$ & $\$ 57,273$ & $\$ 63,636$ & $\$ 113,636$ & $\$ 127,273$ & $\$ 152,727$ & $\$ 190,909$ \\
\hline SubTotal & $\$ 66,416$ & $\$ 123,833$ & $\$ 137,833$ & $\$ 211,236$ & $\$ 415,091$ & $\$ 484,727$ & $\$ 976,118$ & $\$ 1,107,036$ & $\$ 1,132,491$ & $\$ 1,605,782$ \\
\hline Mob/Demob Costs/Turbine & $\$ 1,328$ & $\$ 2,477$ & $\$ 2,757$ & $\$ 4,225$ & $\$ 8,302$ & $\$ 9,695$ & $\$ 19,522$ & $\$ 22,141$ & $\$ 22,650$ & $\$ 32,116$ \\
\hline
\end{tabular}

Mob/Demob Costs/Turbine

$\$ 1,328$

\begin{tabular}{ll|l}
$\$ 2,477$ & $\$ 2,757$ & $\$ 4,225$
\end{tabular}

$\$ 9,695$

$\$ 19,522$

$\$ 22,650$ 
The existing crane costs include one crane assembly/disassembly as part of mobilization and demobilization. These costs have been specifically shown below:

\begin{tabular}{|c|c|c|c|c|c|c|c|c|c|c|}
\hline & $4100 \mathrm{~S} 1$ & $4600 \mathrm{~S} 4$ & $4600 \mathrm{~S} 5$ & what if & LTL-600 & LTL-850 & LTL-1000 & LTL-1100 & LTL-1100 & LTL-1200 \\
\hline Total Labor Costs & $\$ 8,040$ & $\$ 8,040$ & $\$ 8,040$ & $\$ 8,040$ & $\$ 8,040$ & $\$ 8,040$ & $\$ 8,040$ & $\$ 8,040$ & $\$ 8,040$ & $\$ 8,040$ \\
\hline Crane Rental Cost & $\$ 2,045$ & $\$ 8,727$ & $\$ 10,227$ & $\$ 31,818$ & $\$ 81,818$ & $\$ 109,091$ & $\$ 255,682$ & $\$ 286,364$ & $\$ 286,364$ & $\$ 477,273$ \\
\hline Total Truck Crane Costs & $\$ 12,240$ & $\$ 24,480$ & $\$ 24,480$ & $\$ 40,800$ & $\$ 81,600$ & $\$ 102,720$ & $\$ 192$ & $\$ 259,200$ & $\$ 259,200$ & $\$ 369,600$ \\
\hline & $\$ 22,325$ & $\$ 41,247$ & $\$ 42,747$ & & $\$ 171$ & & $\$ 456$ & $\$ 553,604$ & $\$ 553,604$ & $\$ 854,913$ \\
\hline Subtotal per Turbine: & $\$ 447$ & $\$ 825$ & $\$ 855$ & $\$ 1,613$ & $\$ 3,429$ & $\$ 4,397$ & $\$ 9,126$ & $\$ 11,072$ & $\$ 11,072$ & $\$ 17,098$ \\
\hline
\end{tabular}

\begin{tabular}{|c|c|c|c|c|c|c|c|c|c|c|}
\hline $\begin{array}{l}\text { Number of additional disassembly and reassembly events (during } \\
\text { construction of a } 50 \text { turbine facility): }\end{array}$ & \multicolumn{10}{|c|}{ Additional costs per turbine for varying frequency of crane disassembly/reassembly } \\
\hline 1 & $\$ 447$ & $\$ 825$ & $\$ 855$ & $\$ 1,613$ & $\$ 3,429$ & $\$ 4,397$ & $\$ 9,126$ & $\$ 11,072$ & $\$ 11,072$ & $\$ 17,098$ \\
\hline 4 & $\$ 1,786$ & $\$ 3,300$ & $\$ 3,420$ & $\$ 6,453$ & $\$ 13,717$ & $\$ 17,588$ & $\$ 36,5$ & $\$ 44,288$ & $\$ 44$ & 668,393 \\
\hline 9 & $\$ 4,019$ & $\$ 7,425$ & $\$ 7,695$ & $\$ 14,518$ & $\$ 30,862$ & $\$ 39,573$ & $\$ 82,138$ & $\$ 99,649$ & $\$ 99,649$ & $\$ 153,884$ \\
\hline
\end{tabular}

Calculating the impact of additional crane disassembly/reassembly events on the total crane costs $/ \mathrm{kW}$ is presented below. Since the existing costs/kW include one crane assembly/disassembly, only the costs associated with ADDITIONAL events are being added.

From S1 Summarized Crane Costs:

\begin{tabular}{|c|c|c|c|c|c|c|c|c|c|c|}
\hline Turbine Class: & 750 & & & 1,500 & & 2,500 & & 500 & & 000 \\
\hline Rotor Diameter: & 50 & 50 & 66 & 66 & 66 & 85 & 100 & 100 & 120 & 120 \\
\hline Crane Type: & $4100 \mathrm{~S} 1$ & $4600 S 4$ & 4600 S5 & what if & LTL-600 & LTL-850 & LTL-1000 & LTL-1100 & LTL-1100 & LTL-1200 \\
\hline Crane Crew Assembly Labor Costs/Turbine & $\$ 1,365$ & $\$ 1,365$ & $\$ 2,457$ & $\$ 2,457$ & $\$ 3,686$ & $\$ 7,332$ & $\$ 10,296$ & $\$ 10,296$ & $\$ 17,550$ & $\$ 17,550$ \\
\hline Crane Crew Relocation Labor Costs/Turbine & $\$ 780$ & $\$ 780$ & $\$ 780$ & $\$ 1,040$ & $\$ 1,560$ & $\$ 2,730$ & $\$ 4,095$ & $\$ 4,875$ & $\$ 4,875$ & $\$ 6,435$ \\
\hline Crane Rental Costs During Assembly and Relocation/Turbine & $\$ 952$ & $\$ 2,031$ & $\$ 3,591$ & $\$ 7,242$ & $\$ 9,312$ & $\$ 18,523$ & $\$ 31,933$ & $\$ 37,703$ & $\$ 53,667$ & $\$ 71,750$ \\
\hline Meals and Lodging/Turbine & $\$ 248$ & $\$ 248$ & $\$ 374$ & $\$ 404$ & $\$ 605$ & $\$ 1,161$ & $\$ 1,661$ & $\$ 1,751$ & $\$ 2,588$ & $\$ 2,768$ \\
\hline Fuel Cost/Turbine & $\$ 91$ & $\$ 103$ & $\$ 156$ & $\$ 168$ & $\$ 437$ & $\$ 839$ & $\$ 1,292$ & $\$ 1,362$ & $\$ 2,013$ & $\$ 2,306$ \\
\hline Cribbing Costs/Turbine & $\$ 131$ & $\$ 190$ & $\$ 595$ & $\$ 595$ & $\$ 808$ & $\$ 538$ & $\$ 808$ & $\$ 943$ & $\$ 943$ & $\$ 943$ \\
\hline Mob/Demob Costs/Turbine & $\$ 1,328$ & $\$ 2,477$ & $\$ 2,757$ & $\$ 4,225$ & $\$ 8,302$ & $\$ 9,695$ & $\$ 19,522$ & $\$ 22,141$ & $\$ 22,650$ & $\$ 32,116$ \\
\hline Total Crane and Crew Costs/Turbine & $\$ 4,894$ & $\$ 7,193$ & $\$ 10,709$ & $\$ 16,131$ & $\$ 24,709$ & $\$ 40,817$ & $\$ 69,606$ & $\$ 79,069$ & $\$ 104,284$ & $\$ 133,867$ \\
\hline Total Crane Costs (50 Turbines) & $\$ 244,713$ & $\$ 359,652$ & $\$ 535,456$ & $\$ 806,533$ & $\$ 1,235,437$ & $\$ 2,040,831$ & $\$ 3,480,278$ & $\$ 3,953,465$ & $\$ 5,214,199$ & $\$ 6,693,344$ \\
\hline Costs/kW & $\$ 5.67$ & $\$ 8.33$ & $\$ 7.11$ & $\$ 10.72$ & $\$ 16.41$ & $\$ 16.35$ & $\$ 20.14$ & $\$ 22.88$ & $\$ 20.96$ & $\$ 26.90$ \\
\hline $\begin{array}{l}\text { Number of Turbines Assembled per Crane } \\
\text { Assembly/Disassembly }\end{array}$ & & & djusted $\mathrm{C}$ - & e Costs & W based & dditiona & disassemb & nd reassembly e & events & \\
\hline 50 & $\$ 5.67$ & $\$ 8.33$ & $\$ 7.11$ & $\$ 10$ & $\$$ & $\$ 16$ & $\$ 20.14$ & $\$ 2$ & $\$ 20.96$ & $\$ 26.90$ \\
\hline 25 & $\$ 6.18$ & $\$ 9.28$ & $\$ 7.68$ & $\$ 11.79$ & $\$ 1 \varepsilon$ & $\$ 18.11$ & $\$ 22.78$ & $\$ 2$ & $\$ 23.18$ & $\$ 30.34$ \\
\hline 10 & & 2.15 & $\$ 9.39$ & $\$ 1$ & & $\$ 23.39$ & $\$ 30.71$ & & 29.86 & $\$ 40.64$ \\
\hline 5 & $\$ 10.32$ & 92 & $\$ 12.23$ & 36 & $\$ 3$ & $\$ 32.20$ & & & $\$ 40.98$ & $\$ 57.82$ \\
\hline & 77 & $\$ 3124$ & $\$ 20.74$ & $\$ 36.44$ & $\$ 71.09$ & $\$ 58.61$ & 3.52 & 5997 & $\$ 74.36$ & \\
\hline
\end{tabular}


4600 S4 LTL-600 LTL-850 LTL-1100 LTL-1200

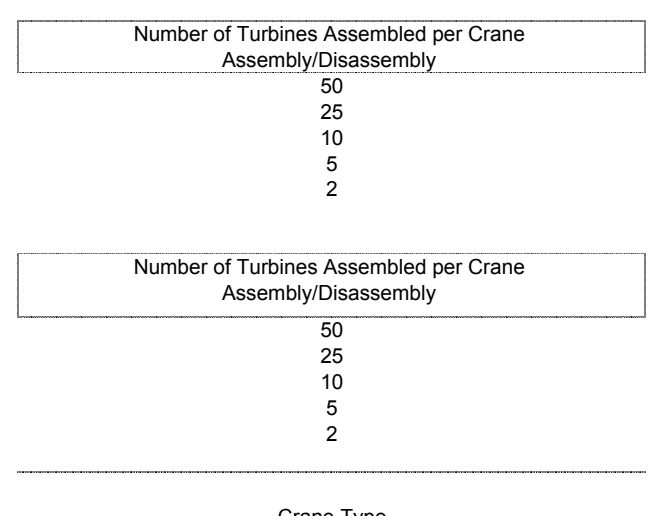

$\begin{array}{rrrrr}\mathbf{7 5 0} & \mathbf{1 5 0 0} & \mathbf{2 5 0 0} & \mathbf{3 5 0 0} & \mathbf{5 0 0 0} \\ \$ 8.33 & \$ 16.41 & \$ 16.35 & \$ 22.88 & \$ 26.90 \\ \$ 1.28 & \$ 18.69 & \$ 18.11 & \$ 26.08 & \$ 30.34 \\ \$ 12.15 & \$ 25.53 & \$ 23.39 & \$ 35.70 & \$ 40.64 \\ \$ 16.92 & \$ 36.92 & \$ 32.20 & \$ 51.72 & \$ 57.82 \\ \$ 31.24 & \$ 71.09 & \$ 58.61 & \$ 99.78 & \$ 109.36\end{array}$

Crane Assembly Rate Days/Turbine

Crane Assembly and Disassembly Hours

Number of Turbines:

Hours per day

Number of Turbines Assembled per Crane Assembly/Disassembly

50
25
10
5
2

\section{Number of Extra Cran}

Relocations

50

49

41
26

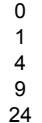

$4100 \mathrm{~S} 14600 \mathrm{~S} 4 \quad 4600 \mathrm{~S} 5$ what if LTL-600 LTL-850 LTL-1000 LTL-1100 LTL-1100 LTL-1200

\begin{tabular}{|c|c|c|c|c|c|c|c|c|c|}
\hline 1.05 & 1.05 & 1.89 & 1.89 & 1.89 & 3.76 & 5.28 & 5.28 & 9 & 9 \\
\hline 6 & 6 & 6 & 8 & 8 & 14 & 21 & 25 & 25 & \\
\hline 24 & 48 & 48 & 80 & 160 & 192 & 360 & 360 & 360 & 480 \\
\hline
\end{tabular}

50
10

Combined Normal Crane Relocation Time AND Extra Crane Disassembly Time (Days/Turbine)

\begin{tabular}{|c|c|c|c|c|c|c|c|c|}
\hline 0.6 & 0.6 & 0.8 & 0.8 & 1.4 & 2.1 & 2.5 & 2.5 & 3.3 \\
\hline 0.6 & 0.7 & 0.9 & 1.1 & 1.8 & 2.8 & 3.2 & 3.2 & 4.2 \\
\hline 0.7 & 0.9 & 1.4 & 2.0 & 2.8 & 4.8 & 5.2 & 5.2 & 6.9 \\
\hline 1.4 & 1.4 & 2.1 & 3.5 & 4.6 & 8.2 & 8.5 & 8.5 & 11.3 \\
\hline 2.6 & 2.6 & 4.3 & 8.1 & 9.9 & 18.4 & 18.6 & 18.6 & 24.8 \\
\hline
\end{tabular}

Total Number of Assembly Days per Turbine

Number of Turbines Assembled per Crane $4100 \mathrm{~S} 1 \quad 4600 \mathrm{~S} 4 \quad 4600 \mathrm{~S} 5$ what if Assembly/Disassembly 50
25
10 10
5

$\begin{array}{ll} & \mathbf{4 6 0 0} \mathbf{S 4} \\ 1.7 & 1.7 \\ 1.7 & 1.7 \\ 1.8 & 2.0 \\ 2.0 & 2.4 \\ 2.5 & 3.7\end{array}$

\begin{tabular}{lr}
.7600 & $\mathbf{4} 5$ \\
1.7 & 2.5 \\
2.0 & 2.6 \\
2.4 & 2.8 \\
3.7 & 3.2 \\
\hline & 4.5
\end{tabular}
$2.7^{\text {LTL-600 }}$ $27^{\text {LTL-850 }}$

\begin{tabular}{|c|c|c|c|c|}
\hline & & & & LTL-1200 \\
\hline 5.2 & 7.4 & 7.8 & 11.5 & 12.3 \\
\hline 5.5 & 8.1 & 8.5 & 12.2 & 13.2 \\
\hline 6.6 & 10.1 & 10.5 & 14.2 & 15.9 \\
\hline 8.4 & 13.5 & 13.8 & 17.5 & 20.3 \\
\hline 13.7 & 23.7 & 23.9 & 27.6 & 33.8 \\
\hline
\end{tabular}

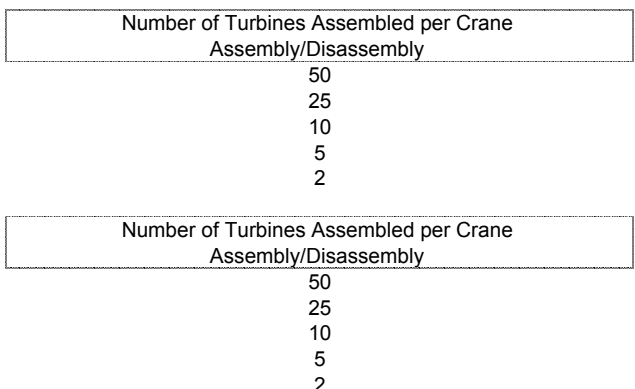

$\begin{array}{rrrrr}750 & 1500 & 2500 & 3500 & 5000 \\ 1.7 & 2.7 & 5.2 & 7.8 & 12.3 \\ 1.7 & 3.0 & 5.5 & 8.5 & 13.2 \\ 2.0 & 3.9 & 6.6 & 10.5 & 15.9 \\ 2.4 & 5.4 & 8.4 & 13.8 & 20.3 \\ 3.7 & 10.0 & 13.7 & 23.9 & 33.8 \\ & & & & \\ 750 & 1500 & 2500 & 3500 & 5000 \\ 3 & 4 & 9 & 13 & 21 \\ 3 & 5 & 9 & 14 & 22 \\ 3 & 7 & 11 & 17 & 26 \\ 4 & 9 & 14 & 23 & 34 \\ 6 & 17 & 23 & 40 & 56\end{array}$




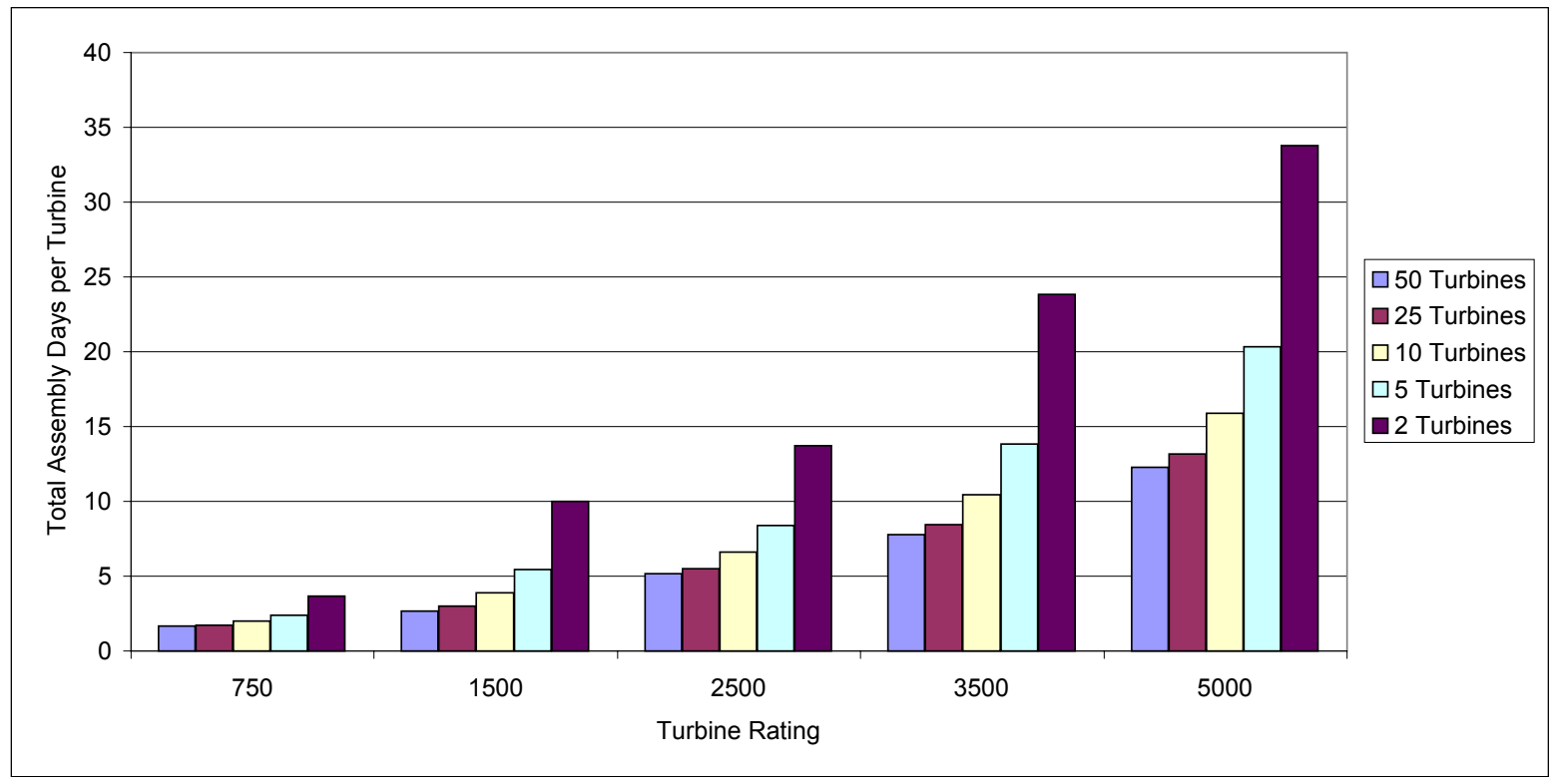




\section{Appendix $\mathbf{P}$}

Report Figures and Road-Width Analysis 
Data from Scenario 1 - Costs per kW page C-1.

750

Gainesville, TX South Dakota

Grand Forks, ND South Dakota
$\$ 6.40$

$\$ 2.91$
1500

$\$ 6.06$

$\$ 2.76$
2500

$\$ 6.30$

$\$ 4.10$
$3500 \quad 5000$

$\$ 10.37 \quad \$ 100.41$

$\$ 5.51 \quad \$ 5.31$

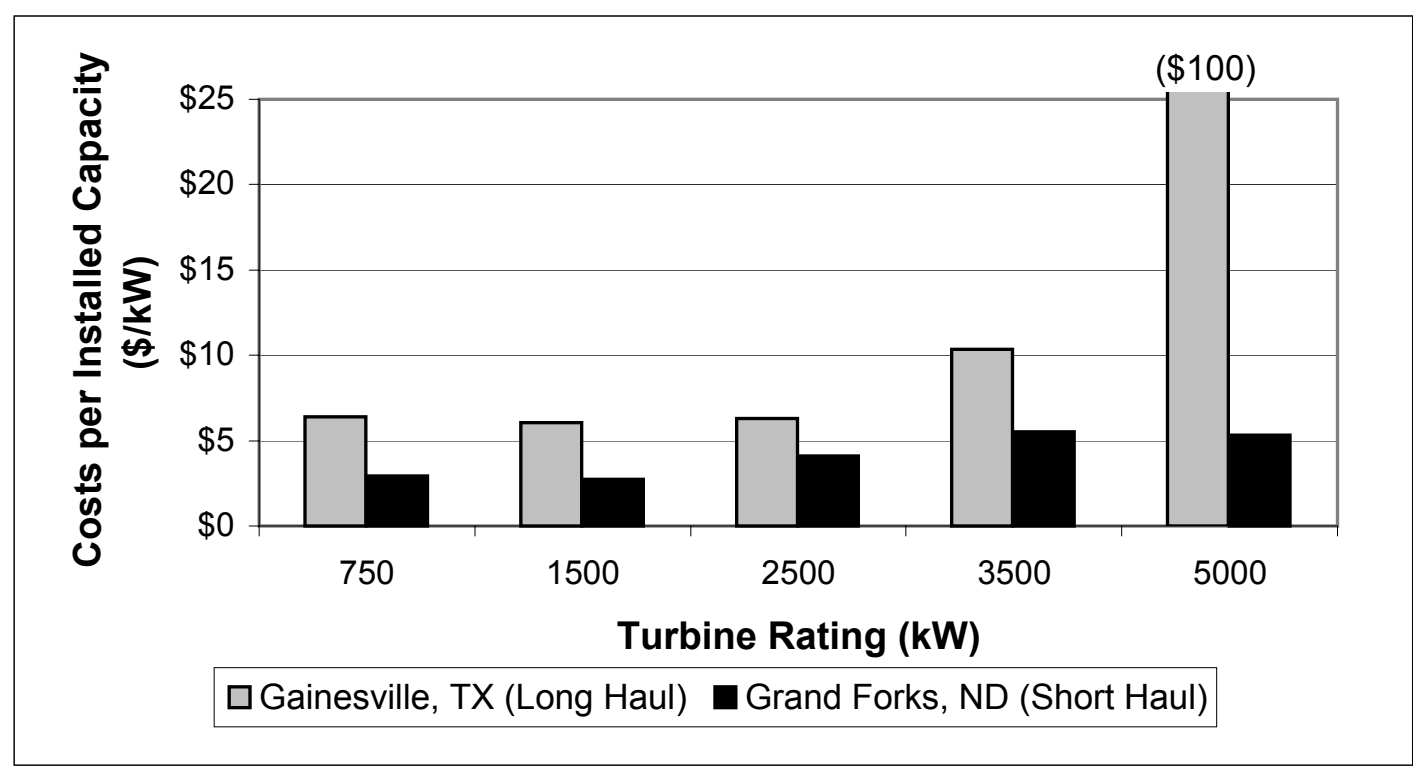


Data From Page D-2 "Hubs via Trucks"

From Chicago, IL

$\begin{array}{lrrrrr}\text { Turbine Rating (kW) } & 750 & 1500 & 2500 & 3500 & 5000 \\ \text { Costs per Turbine } & \$ 1,895 & \$ 3,790 & \$ 4,548 & \$ 5,685 & \$ 7,201 \\ \text { Costs per kW } & \$ 2.19 & \$ 2.52 & \$ 1.82 & \$ 1.65 & \$ 1.45\end{array}$

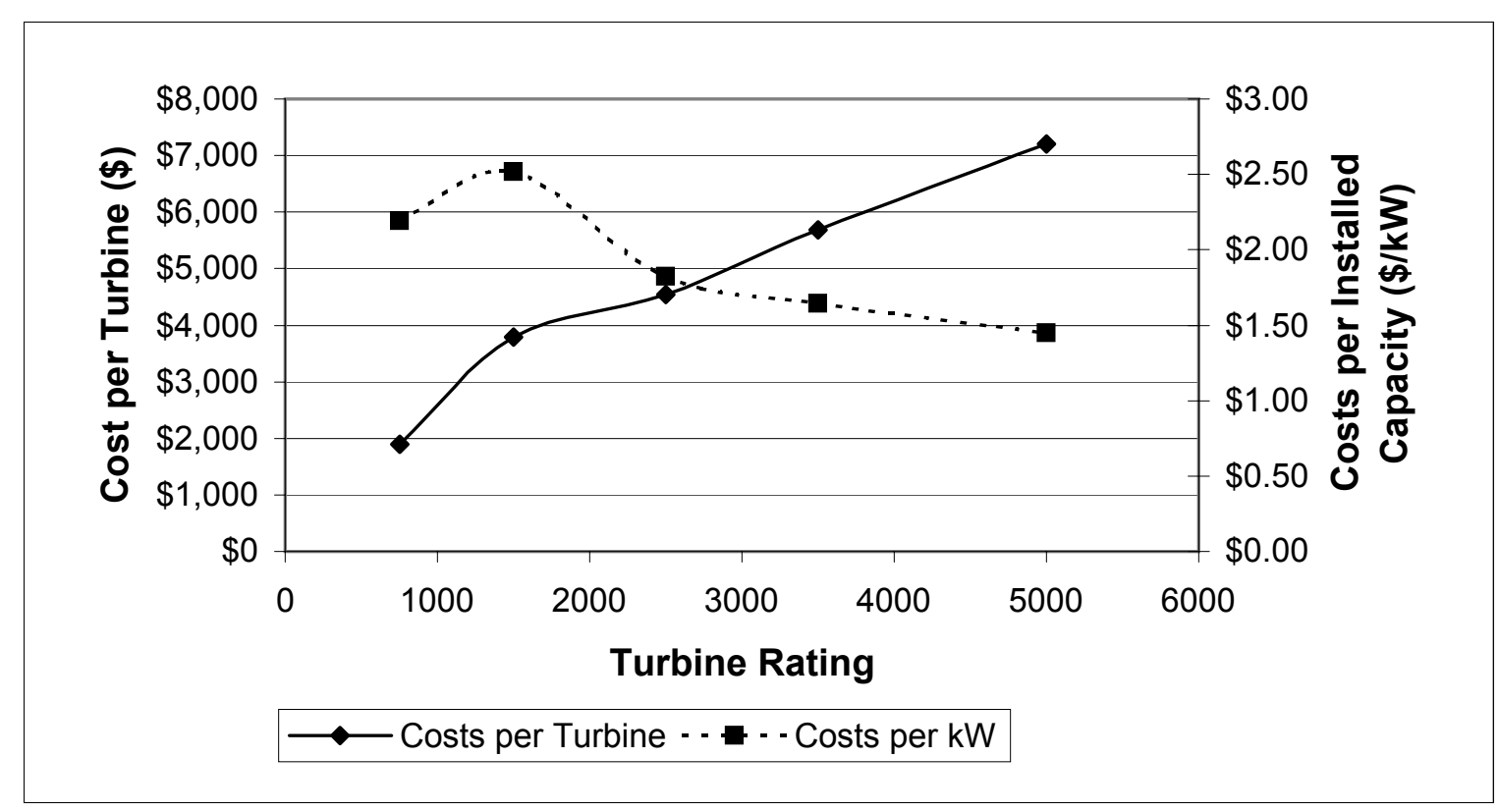


Key:

Trucks

Rail

Dolly
Chicago, IL South Dakota

Chicago, IL South Dakota

Chicago, IL South Dakota

\begin{tabular}{|rrrrr}
750 & 1500 & 2500 & 3500 & 5000 \\
$\$ 5.26$ & $\$ 4.03$ & $\$ 3.34$ & & \\
$\$ 14.85$ & $\$ 9.19$ & $\$ 6.37$ & $\$ 20.92$ & \\
\hline & & $\$ 95.28$ & $\$ 74.33$ & $\$ 55.42$
\end{tabular}

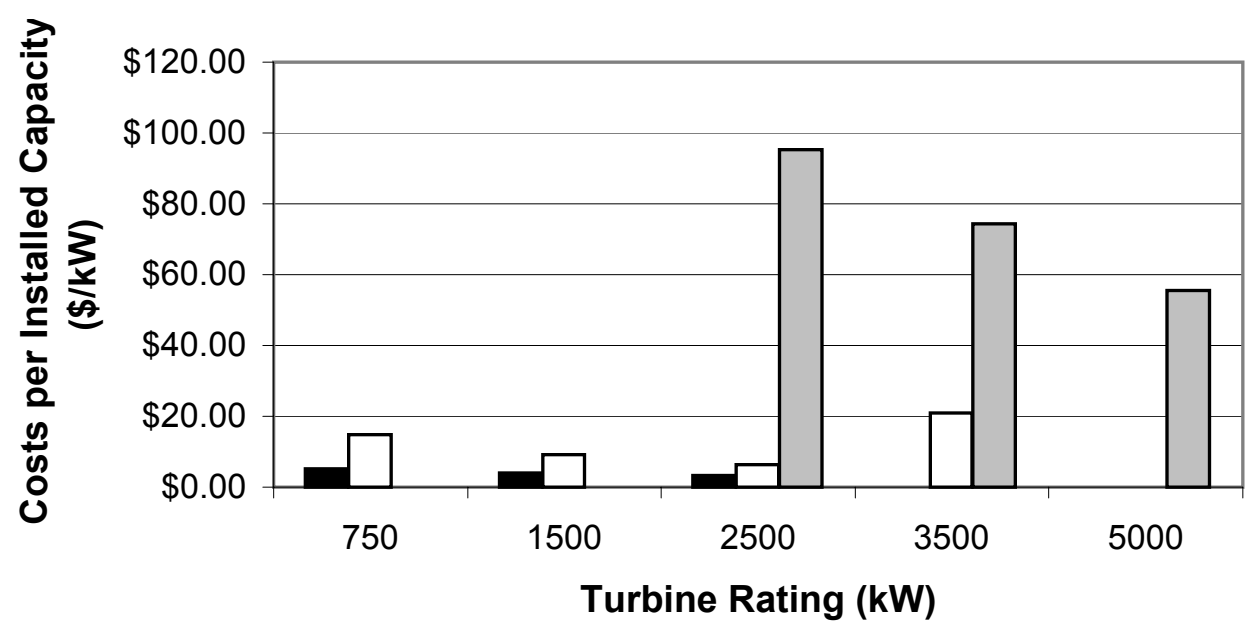

Nacelles via Trucks Nacelles via Dolly 
Data from Scenario 1 - Costs per kW page C-1 and C-2

\section{Scenario 1}

$\begin{array}{llrrrrc} & & 750 & 1500 & 2500 & 3500 & 5000 \\ \text { Shreveport, LA } & \text { South Dakota } & \$ 15.89 & \$ 26.61 & \$ 8.77 & \$ 3.40 & \$ 0.00 \text { Truck } \\ \text { Shreveport, LA } & \text { Port of Houston } & \$ 0.00 & \$ 0.00 & \$ 194.65 & \$ 234.39 & \$ 227.88 \text { Dolly } \\ \text { Port of Houston } & \text { Sioux City, IA } & \$ 0.00 & \$ 0.00 & \$ 120.15 & \$ 190.44 & \$ 236.19 \text { Barge } \\ \text { Sioux City, IA } & \text { South Dakota } & \$ 0.00 & \$ 0.00 & \$ 195.25 & \$ 235.12 & \$ 228.58 \text { Dolly } \\ & \text { Total } & \$ 16 & \$ 27 & \$ 519 & \$ 663 & \$ 693\end{array}$

Data from Scenario 2 - Costs per kW page C-3 and C-4

Scenario 2

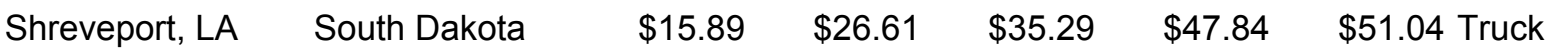

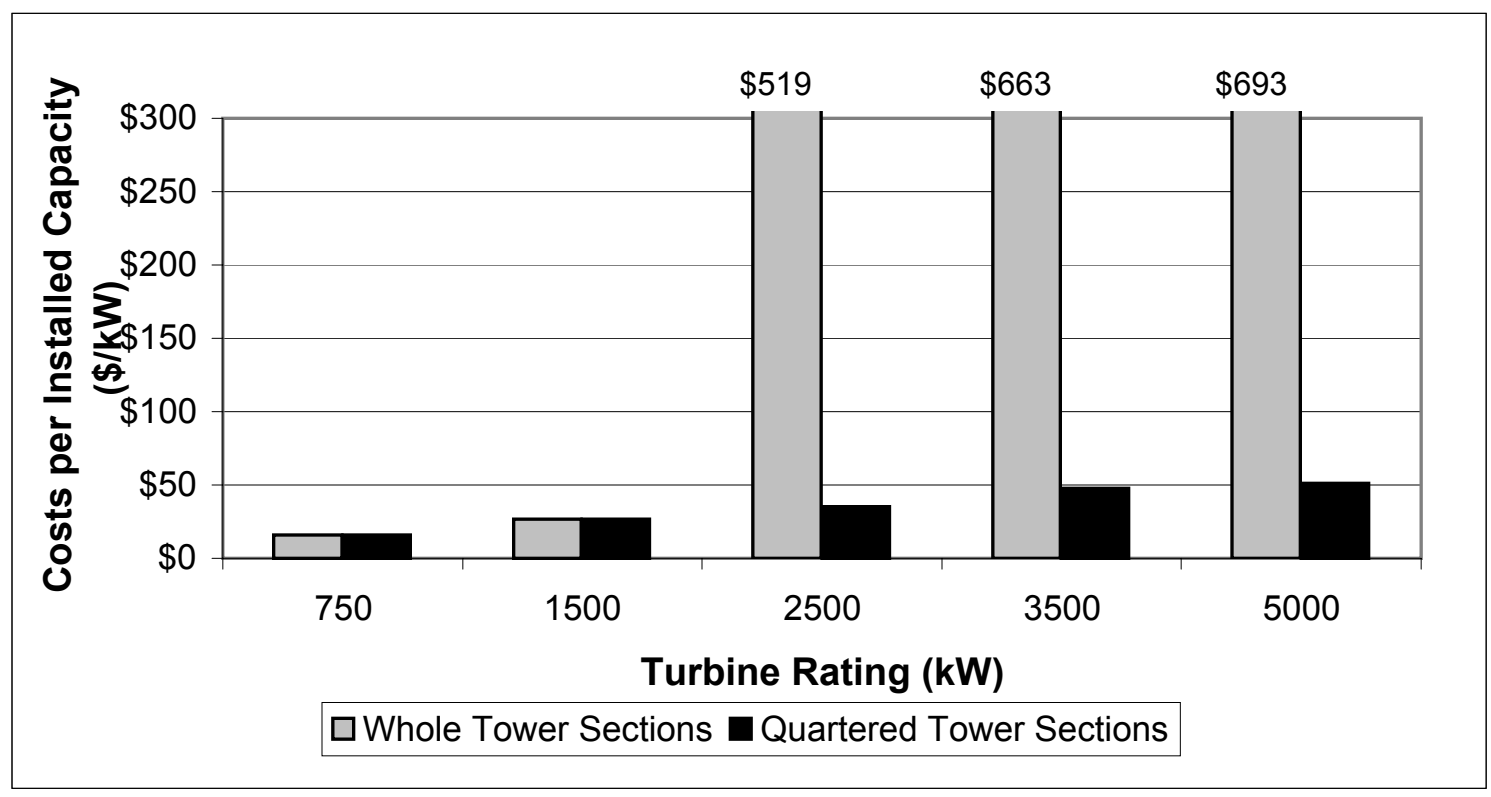


Data from Scenario 1 - Costs per kW page C-1 and C-2

Scenario 1

$750 \quad 1500$

Chicago, IL

South Dakota

$\$ 5.26 \quad \$ 4.03$

$\$ 0.00$

$\$ 4.03$

3500

5000

$\$ 14.85$

$\$ 9.19$

$\$ 3.34$

$\$ 95.28$

$\$ 6.37$

$\$ 0.00$

$\$ 74.33$

$\$ 20.92$

$\$ 0.00$ Truck

$\$ 55.42$ Dolly

$\$ 0.00$ Rail

Data from Scenario 3 - Costs per kW page C-5 and C-6

Scenario 3

Chicago, IL

South Dakota

$\$ 5.26$
$\$ 0.00$
$\$ 14.28$
$\$ 0.00$
$\$ 14.28$

$\$ 4.03$

$\$ 0.00$

$\$ 8.89$

$\$ 0.00$

$\$ 8.89$

= generator and gear box costs

$\begin{array}{lrr}\$ 4.55 & \$ 2.85 & \$ 1.98 \text { Truck } \\ \$ 0.00 & \$ 68.84 & \$ 51.61 \text { Dolly } \\ \$ 6.04 & \$ 20.43 & \$ 0.00 \text { Rail } \\ \$ 0.00 & \$ 71.69 & \$ 53.60 \text { Adjusted Dolly } \\ \$ 6.04 & \$ 23.29 & \$ 0.00 \text { Adjusted Rail }\end{array}$

\section{Lowest Cost Options}

Scenario 1

$\$ 5.26 \quad \$ 4.03$

$\$ 0.00$

$\$ 0.00$

$\$ 3.34 \quad \$ 20.92$

$\$ 55.42$

$\$ 53.60$

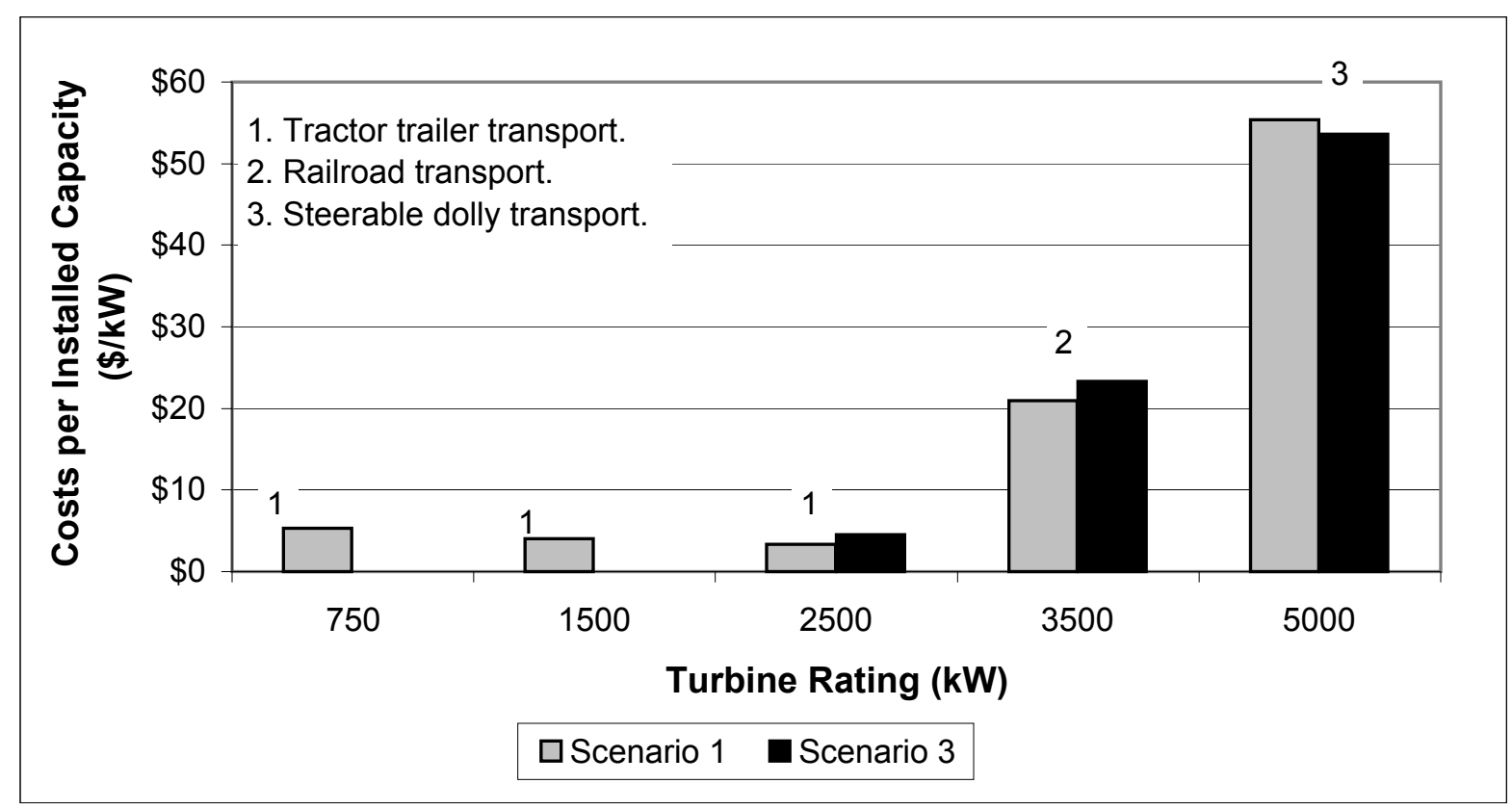


See 'Cost per kW' page B-3 and B-4

$\begin{array}{lrrrrr} & 750 & 1500 & 2500 & 3500 & 5000 \\ \text { Assembly } & \$ 23.55 & \$ 16.98 & \$ 17.70 & \$ 15.60 & \$ 19.05 \\ \text { Crane } & \$ 8.33 & \$ 16.41 & \$ 16.35 & \$ 22.88 & \$ 26.90\end{array}$

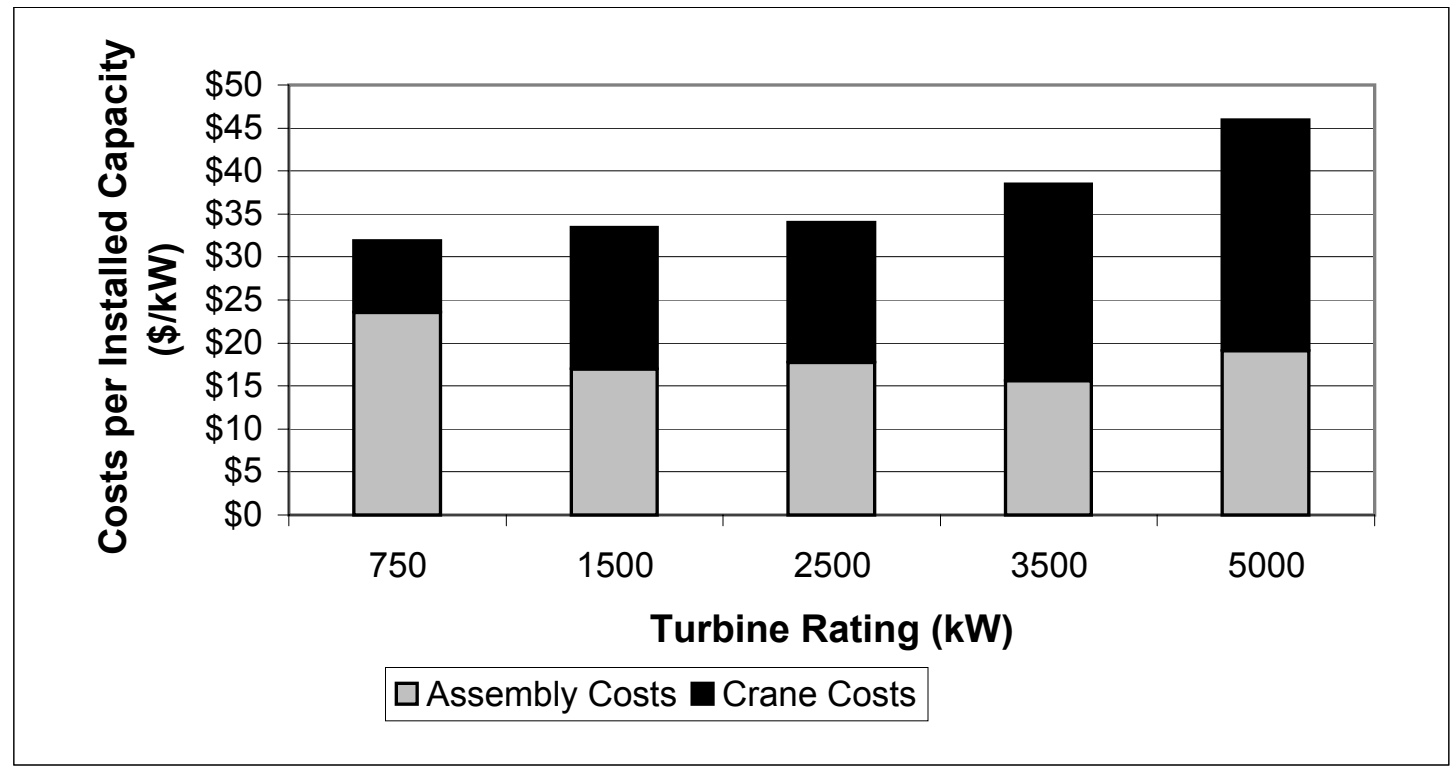


Source: Chicago Bridge and Iron Co.

\begin{tabular}{|r|r|}
\hline Capacity group & CountOfCapacity \\
\hline 100 & 3 \\
\hline 200 & 39 \\
\hline 300 & 39 \\
\hline 400 & 18 \\
\hline 500 & 18 \\
\hline 600 & 12 \\
\hline 700 & 5 \\
\hline 800 & 9 \\
\hline 900 & 3 \\
\hline 1000 & 8 \\
\hline 1200 & 3 \\
\hline 1400 & 2 \\
\hline 1500 & 1 \\
\hline 1600 & 4 \\
\hline 1800 & 1 \\
\hline 2000 & 2 \\
\hline 2200 & 1 \\
\hline 2800 & 1 \\
\hline 3800 & \\
\hline
\end{tabular}

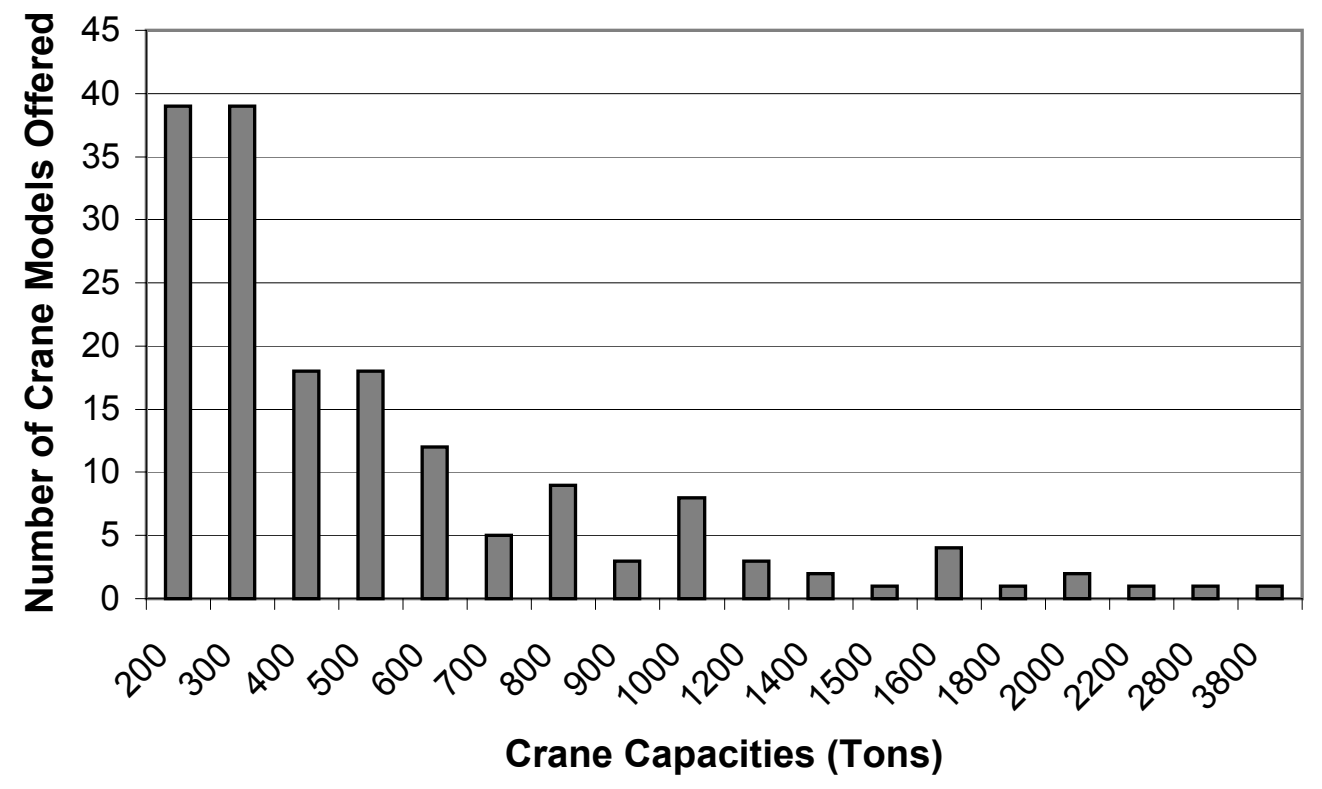


See 'Cost per kW' data on pages B-3 and B-4

\begin{tabular}{|c|c|c|c|c|c|}
\hline & 750 & 1500 & 2500 & 3500 & 5000 \\
\hline Assembly & $\$ 23.55$ & $\$ 16.98$ & $\$ 17.70$ & $\$ 15.60$ & \$19.05 S1 \\
\hline \multirow[t]{2}{*}{ Crane } & $\$ 8.33$ & $\$ 16.41$ & $\$ 16.35$ & $\$ 22.88$ & $\$ 26.90 \mathrm{~S} 1$ \\
\hline & $\$ 31.88$ & $\$ 33.39$ & $\$ 34.04$ & $\$ 38.48$ & $\$ 45.95$ \\
\hline Assembly & $\$ 23.55$ & $\$ 26.49$ & $\$ 34.00$ & $\$ 35.12$ & $\$ 39.85$ S2 Bolt \\
\hline \multirow[t]{2}{*}{ Crane } & $\$ 8.33$ & $\$ 19.00$ & $\$ 19.71$ & $\$ 28.32$ & $\$ 33.23$ S2 Bolt \\
\hline & $\$ 31.88$ & $\$ 45.49$ & $\$ 53.72$ & $\$ 63.45$ & $\$ 73.08$ \\
\hline Assembly & $\$ 23.55$ & $\$ 34.57$ & $\$ 48.16$ & $\$ 68.36$ & $\$ 97.53$ S2 Man \\
\hline \multirow[t]{2}{*}{ Crane } & $\$ 8.33$ & $\$ 21.77$ & $\$ 24.99$ & $\$ 43.15$ & $\$ 60.11$ S2 Man \\
\hline & $\$ 31.88$ & $\$ 56.35$ & $\$ 73.14$ & $\$ 111.50$ & $\$ 157.64$ \\
\hline Assembly & $\$ 23.55$ & $\$ 30.89$ & $\$ 41.30$ & $\$ 51.78$ & $\$ 69.45$ S2 Auto \\
\hline \multirow[t]{2}{*}{ Crane } & $\$ 8.33$ & $\$ 20.64$ & $\$ 22.88$ & $\$ 36.45$ & $\$ 47.89$ S2 Auto \\
\hline & $\$ 31.88$ & $\$ 51.53$ & $\$ 64.18$ & $\$ 88.24$ & $\$ 117.34$ \\
\hline Assembly & $\$ 23.55$ & $\$ 26.49$ & $\$ 35.29$ & $\$ 36.53$ & $\$ 41.12$ S3 \\
\hline \multirow[t]{2}{*}{ Crane } & $\$ 8.33$ & $\$ 19.00$ & $\$ 21.23$ & $\$ 30.24$ & $\$ 35.20$ S3 \\
\hline & $\$ 31.88$ & $\$ 45.49$ & $\$ 56.52$ & $\$ 66.77$ & $\$ 76.32$ \\
\hline
\end{tabular}

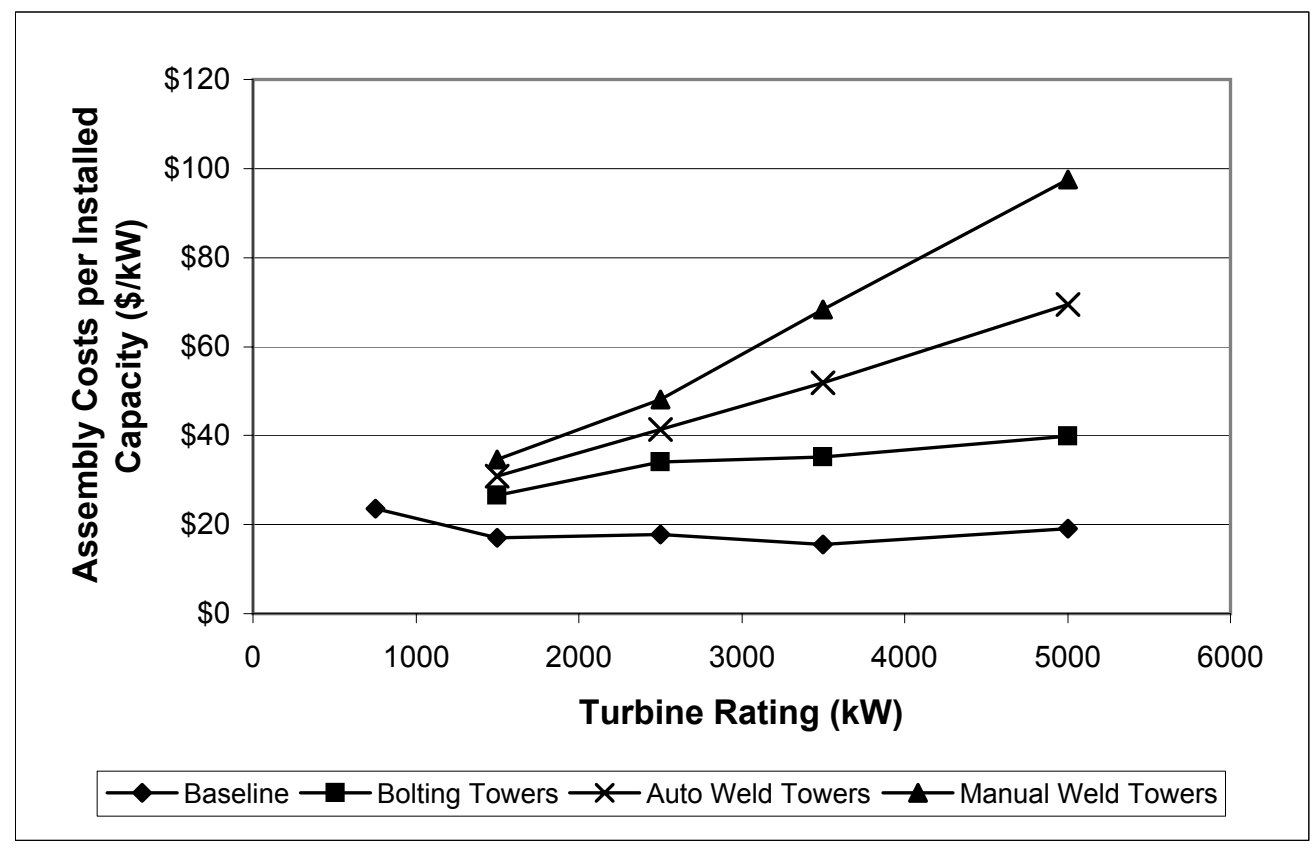


See 'Cost per kW' data on pages B-3 and B-4

\begin{tabular}{|c|c|c|c|c|c|}
\hline & 750 & 1500 & 2500 & 3500 & 5000 \\
\hline Assembly & $\$ 23.55$ & $\$ 16.98$ & $\$ 17.70$ & $\$ 15.60$ & $\$ 19.05$ S1 \\
\hline \multirow[t]{2}{*}{ Crane } & $\$ 8.33$ & $\$ 16.41$ & $\$ 16.35$ & $\$ 22.88$ & $\$ 26.90 \mathrm{~S} 1$ \\
\hline & $\$ 31.88$ & $\$ 33.39$ & $\$ 34.04$ & $\$ 38.48$ & $\$ 45.95$ Combined \\
\hline Assembly & $\$ 23.55$ & $\$ 26.49$ & $\$ 34.00$ & $\$ 35.12$ & $\$ 39.85$ S2 Bolt \\
\hline \multirow[t]{2}{*}{ Crane } & $\$ 8.33$ & $\$ 19.00$ & $\$ 19.71$ & $\$ 28.32$ & $\$ 33.23$ S2 Bolt \\
\hline & $\$ 31.88$ & $\$ 45.49$ & $\$ 53.72$ & $\$ 63.45$ & $\$ 73.08$ Combined \\
\hline Assembly & $\$ 23.55$ & $\$ 34.57$ & $\$ 48.16$ & $\$ 68.36$ & $\$ 97.53$ S2 Man \\
\hline \multirow[t]{2}{*}{ Crane } & $\$ 8.33$ & $\$ 21.77$ & $\$ 24.99$ & $\$ 43.15$ & $\$ 60.11$ S2 Man \\
\hline & $\$ 31.88$ & $\$ 56.35$ & $\$ 73.14$ & $\$ 111.50$ & \$157.64 Combined \\
\hline Assembly & $\$ 23.55$ & $\$ 30.89$ & $\$ 41.30$ & $\$ 51.78$ & $\$ 69.45$ S2 Auto \\
\hline \multirow[t]{2}{*}{ Crane } & $\$ 8.33$ & $\$ 20.64$ & $\$ 22.88$ & $\$ 36.45$ & $\$ 47.89$ S2 Auto \\
\hline & $\$ 31.88$ & $\$ 51.53$ & $\$ 64.18$ & $\$ 88.24$ & \$117.34 Combined \\
\hline Assembly & $\$ 23.55$ & $\$ 26.49$ & $\$ 35.29$ & $\$ 36.53$ & $\$ 41.12$ S3 \\
\hline \multirow[t]{2}{*}{ Crane } & $\$ 8.33$ & $\$ 19.00$ & $\$ 21.23$ & $\$ 30.24$ & $\$ 35.20$ S3 \\
\hline & $\$ 31.88$ & $\$ 45.49$ & $\$ 56.52$ & $\$ 66.77$ & $\$ 76.32$ Combined \\
\hline
\end{tabular}

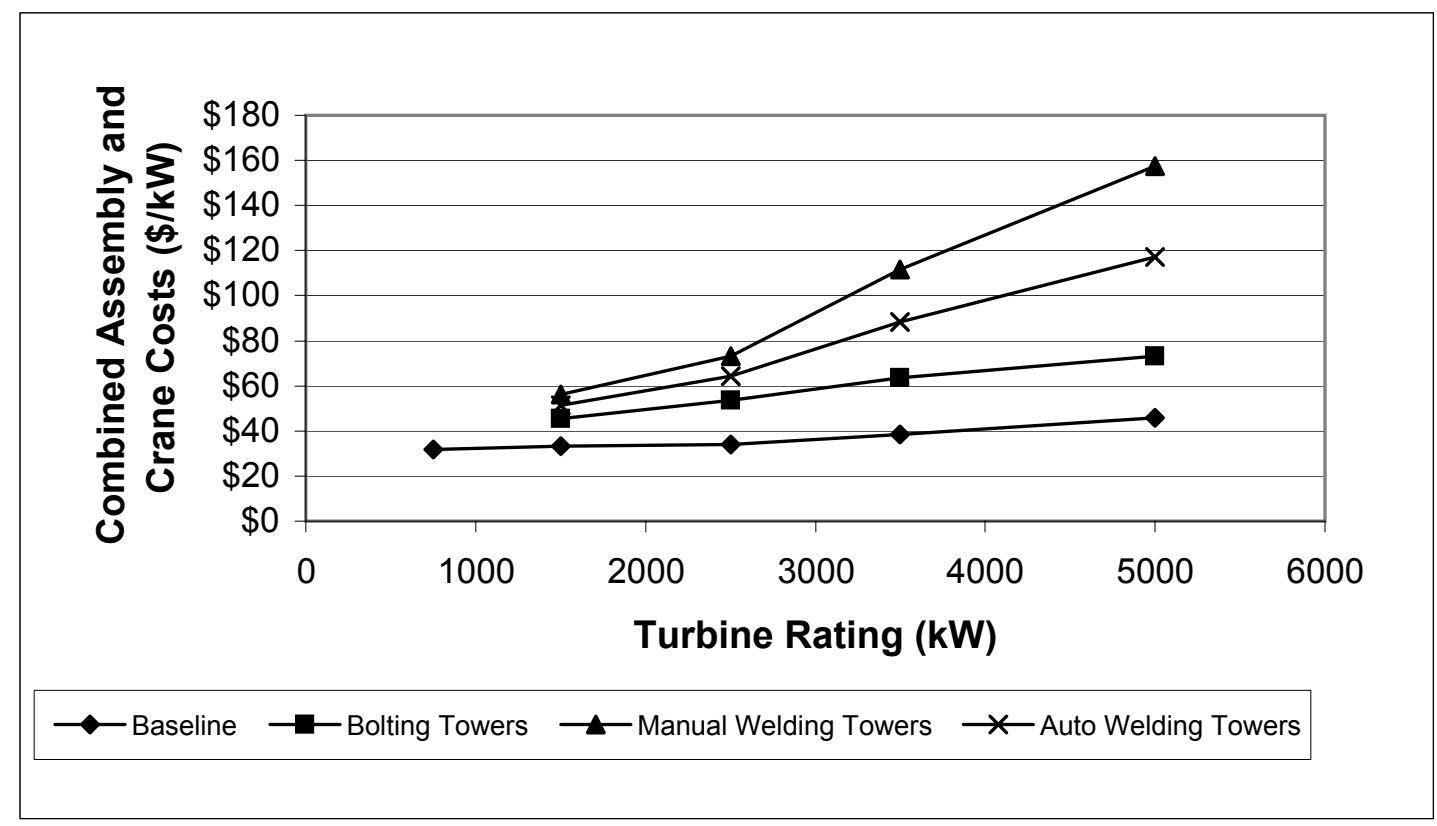


Data From Appendix O

$\begin{array}{cccccc}\text { Number of Turbines Assembled per } & 4600 \mathrm{S4} & \text { LTL-600 } & \text { LTL-850 } & \text { LTL-1100 } & \text { LTL-1200 } \\ \text { Crane Assembly/Disassembly } & 750 & 1500 & 2500 & 3500 & 5000 \\ 50 & \$ 8.33 & \$ 16.41 & \$ 16.35 & \$ 22.88 & \$ 26.90 \\ 25 & \$ 9.28 & \$ 18.69 & \$ 18.11 & \$ 26.08 & \$ 30.34 \\ 10 & \$ 12.15 & \$ 25.53 & \$ 23.39 & \$ 35.70 & \$ 40.64 \\ 5 & \$ 16.92 & \$ 36.92 & \$ 32.20 & \$ 51.72 & \$ 57.82 \\ 2 & \$ 31.24 & \$ 71.09 & \$ 58.61 & \$ 99.78 & \$ 109.36\end{array}$

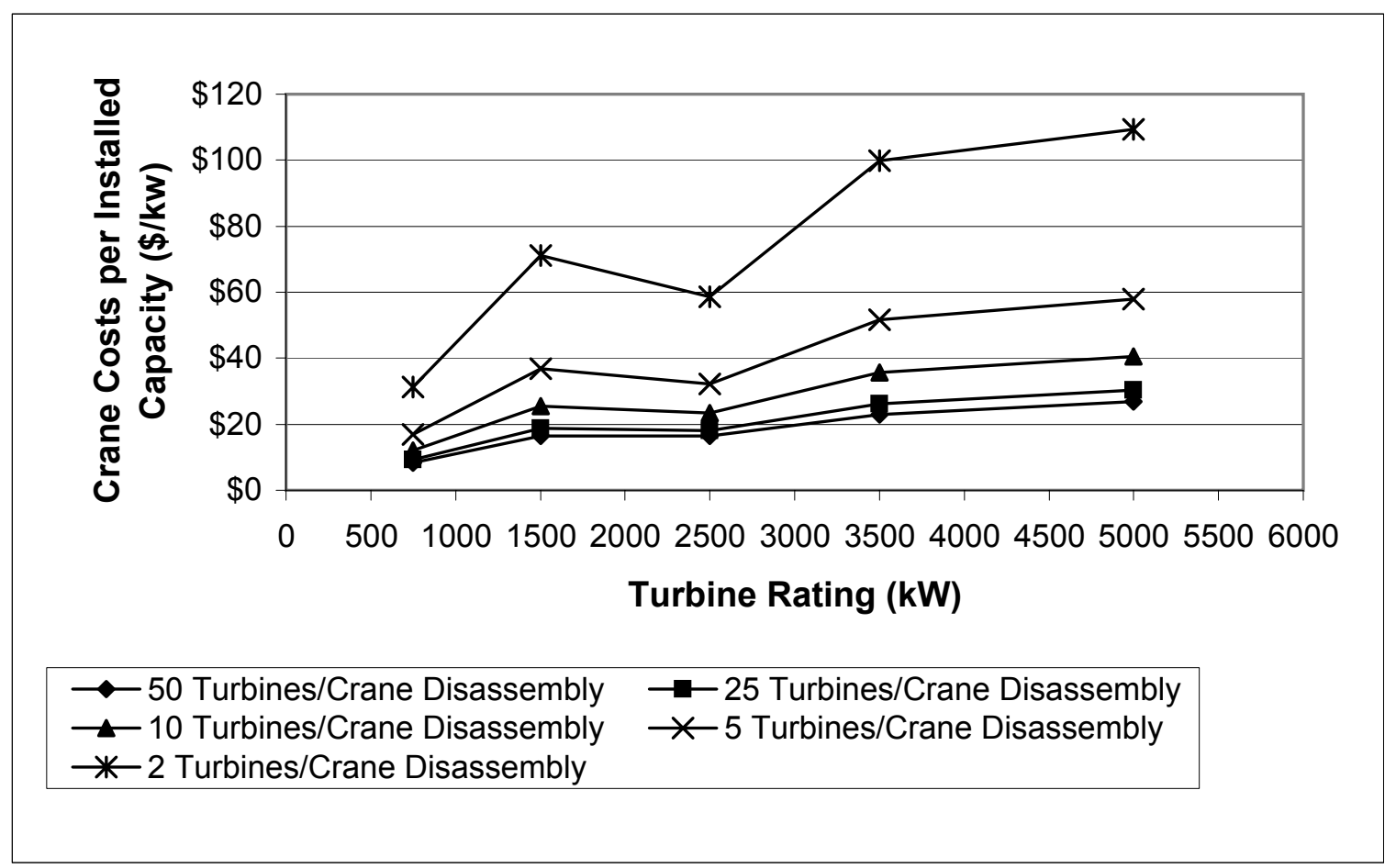


Data From Appendix O

$\begin{array}{cccccc}\text { Number of Turbines Assembled per } & & & & & \\ \text { Crane Assembly/Disassembly } & \mathbf{7 5 0} & \mathbf{1 5 0 0} & \mathbf{2 5 0 0} & \mathbf{3 5 0 0} & \mathbf{5 0 0 0} \\ 50 & 1.65 & 2.69 & 5.16 & 7.78 & 12.3 \\ 25 & 1.734 & 2.994 & 5.516 & 8.45 & 13.194 \\ 10 & 1.986 & 3.906 & 6.584 & 10.46 & 15.876 \\ 5 & 2.406 & 5.426 & 8.364 & 13.81 & 20.346 \\ 2 & 3.666 & 9.986 & 13.704 & 23.86 & 33.756\end{array}$

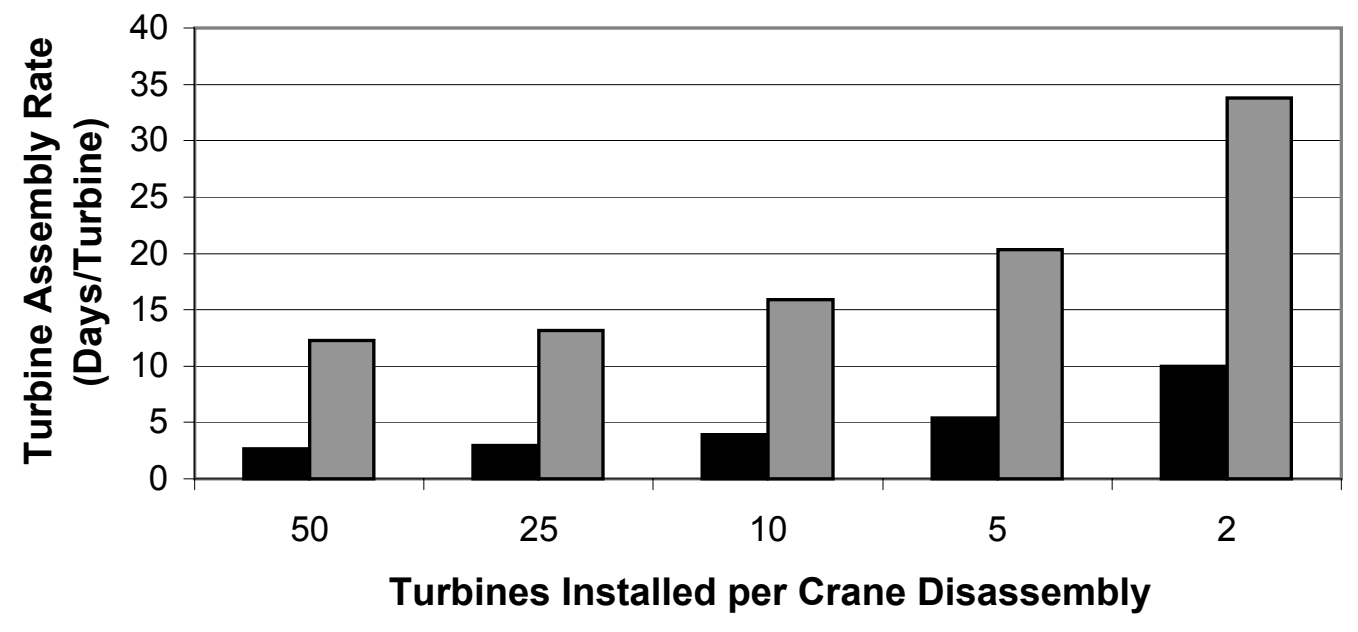

1500 kW $\square 5000 \mathrm{~kW}$ 
Data From Appendix O

$\begin{array}{cccccc}\begin{array}{c}\text { Number of } \\ \text { Turbines }\end{array} & \mathbf{7 5 0} & \mathbf{1 5 0 0} & \mathbf{2 5 0 0} & \mathbf{3 5 0 0} & \mathbf{5 0 0 0} \\ 50 & 3 & 4 & 9 & 13 & 21 \\ 25 & 3 & 5 & 9 & 14 & 22 \\ 10 & 3 & 7 & 11 & 17 & 26 \\ 5 & 4 & 9 & 14 & 23 & 34 \\ 2 & 6 & 17 & 23 & 40 & 56\end{array}$

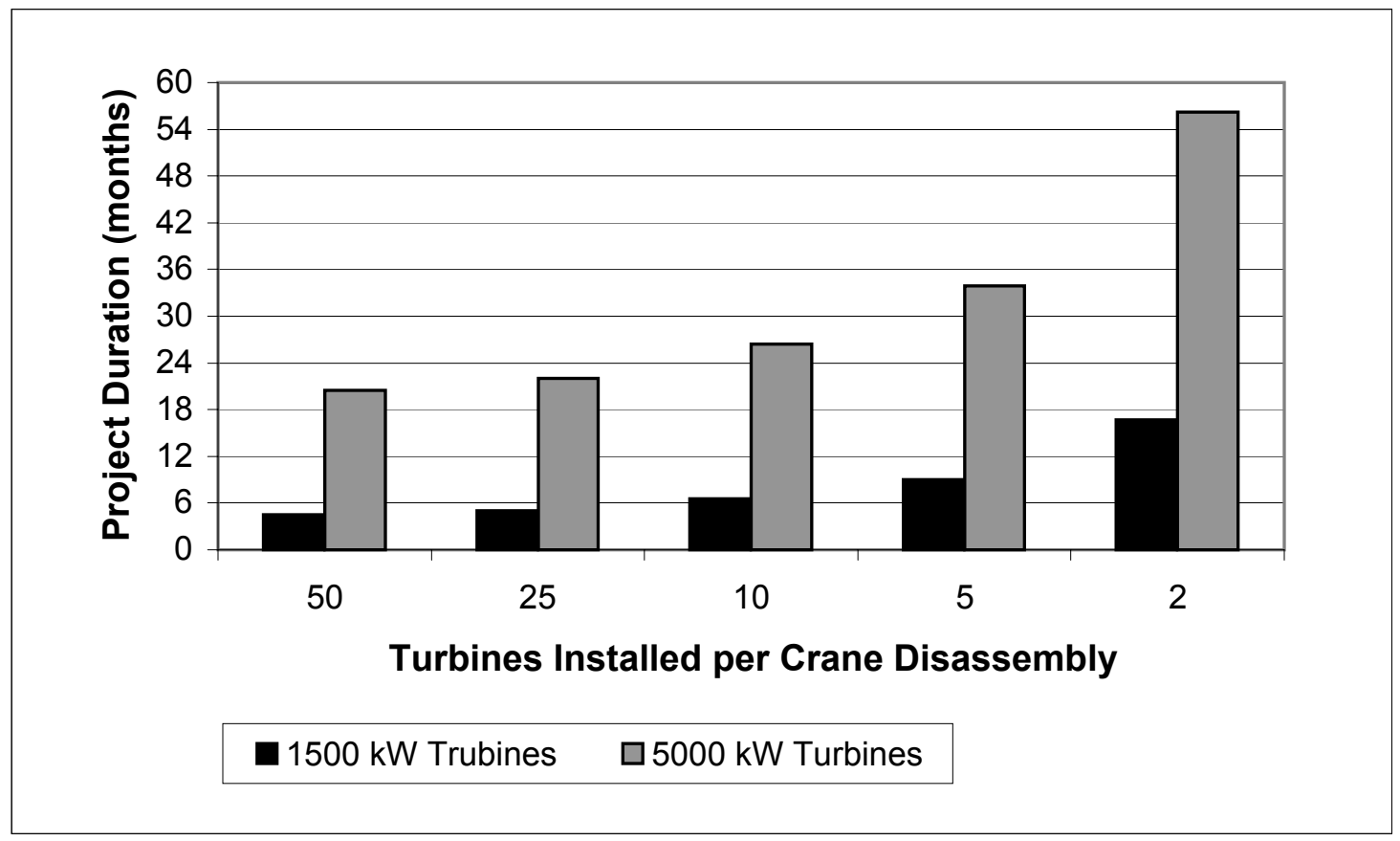


Mid-range (typical or average) Costs by Scenario from Appendix B

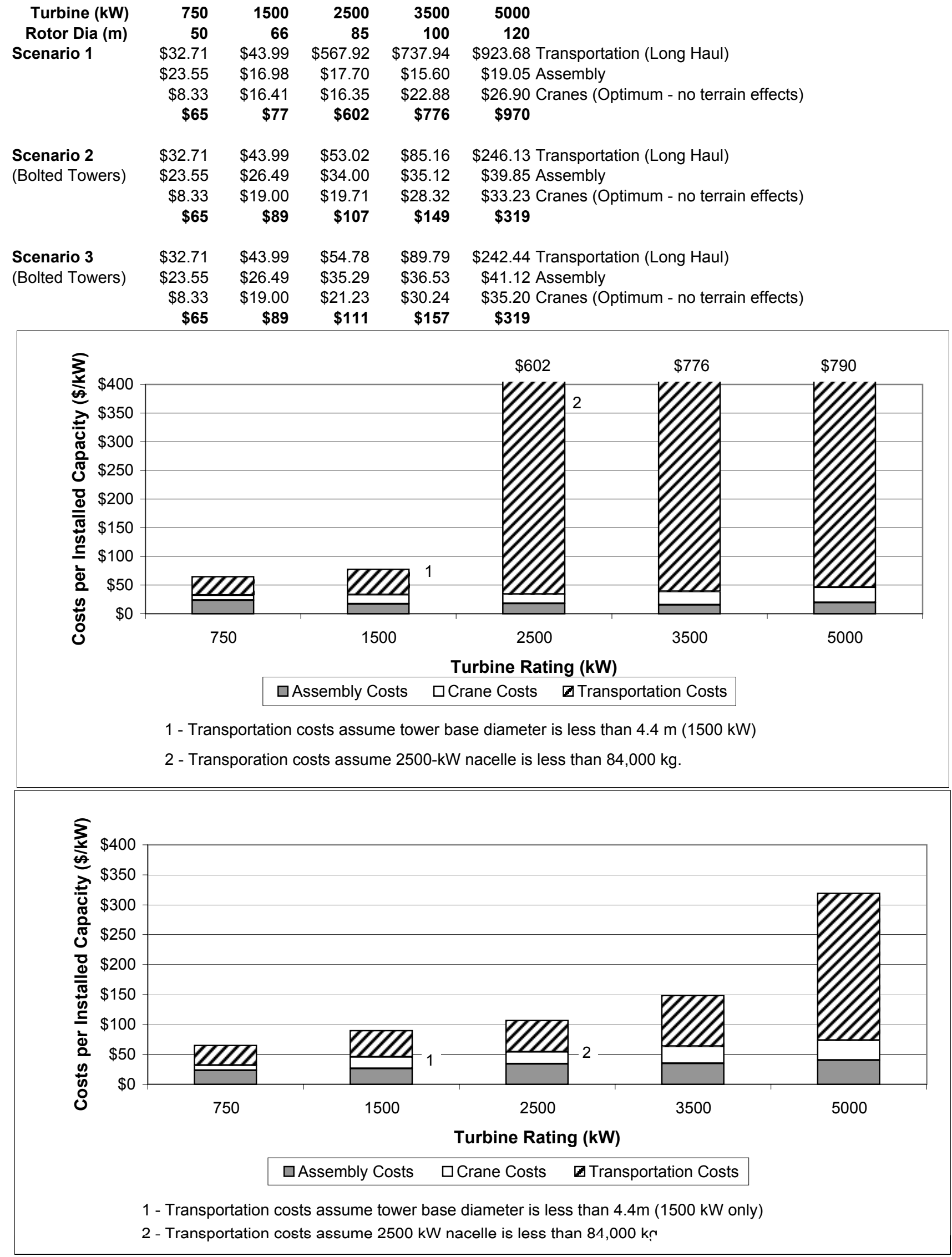




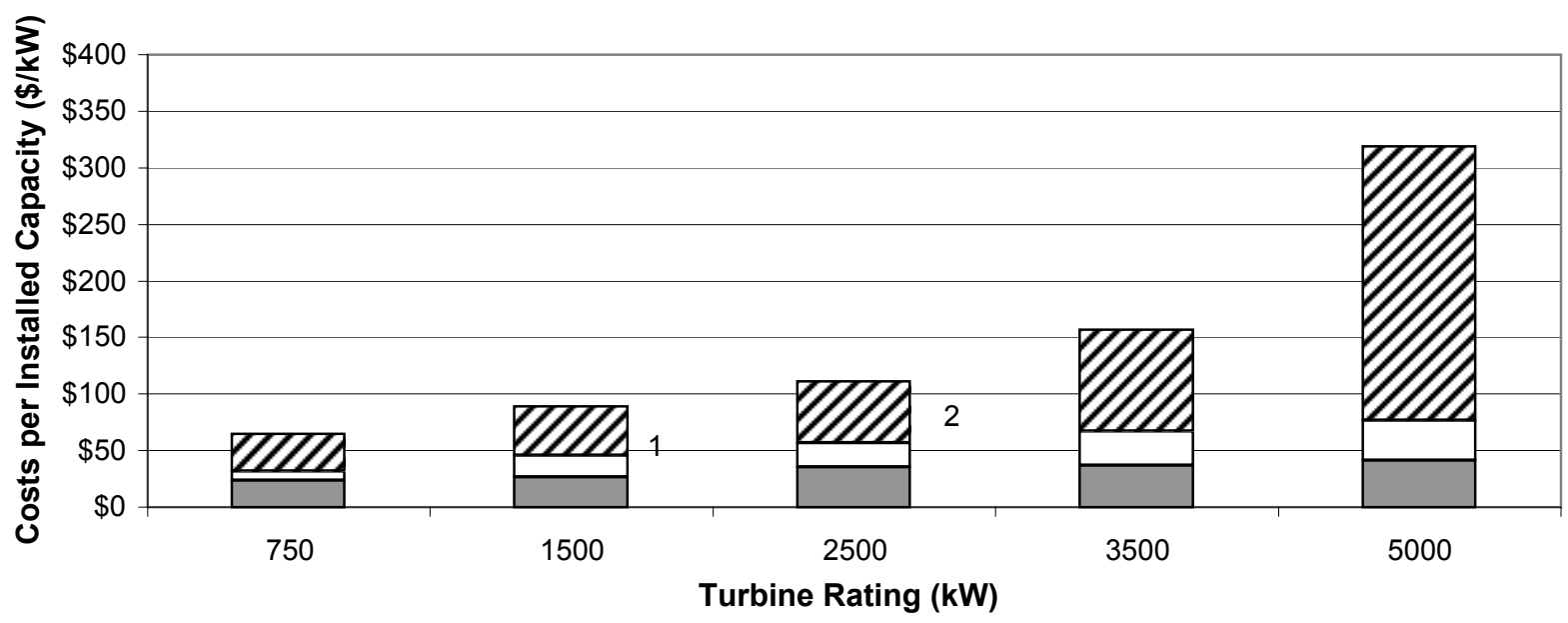

$\square$ Assembly Costs $\square$ Crane Costs $\quad \boldsymbol{V}$ Transportation Costs

1 - Transportation costs assume tower base diameter is less than $4.4 \mathrm{~m}$ (1500 kW).

2 - Transportation costs assume $2500 \mathrm{~kW}$ nacelle is less than $84,000 \mathrm{~kg}$. 


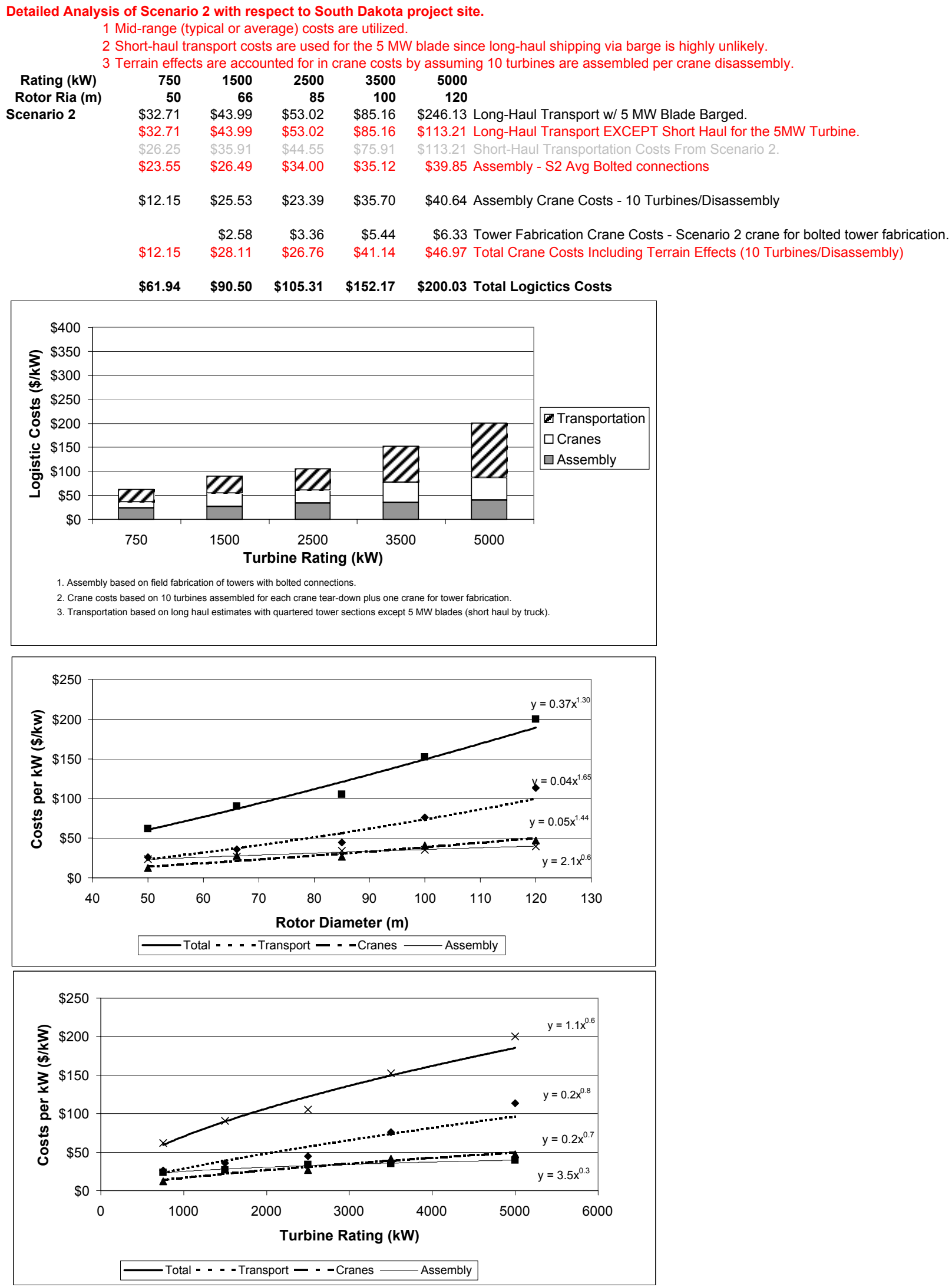


Appendix Q

Mortenson Estimates 
GLOBAL ENERGY CONCEPTS, LLC.

MEGA - WATT SCALE WIND TURBINE CONSTRUCTION

REVISED JULY 12, 2000

\begin{tabular}{|c|c|c|c|c|c|c|c|c|c|c|c|c|c|c|c|}
\hline \multirow[b]{2}{*}{$\begin{array}{l}\text { Activity } \\
\text { Description }\end{array}$} & \multicolumn{3}{|c|}{$750 \mathrm{KW}$} & \multicolumn{3}{|c|}{$1500 \mathrm{KW}$} & \multicolumn{3}{|c|}{$2500 \mathrm{KW}$} & \multicolumn{3}{|c|}{$3500 \mathrm{KW}$} & \multicolumn{3}{|c|}{$5000 \mathrm{KW}$} \\
\hline & MHRS & \begin{tabular}{|c} 
Labor \\
Cost \\
(Loaded) \\
\end{tabular} & $\begin{array}{c}\text { Equip. \& } \\
\text { Material } \\
\text { Cost } \\
\end{array}$ & MHRS & $\begin{array}{c}\text { Labor } \\
\text { Cost } \\
\text { (Loaded) }\end{array}$ & $\begin{array}{c}\text { Equip. \& } \\
\text { Material } \\
\text { Cost }\end{array}$ & MHRS & $\begin{array}{c}\text { Labor } \\
\text { Cost } \\
\text { (Loaded) }\end{array}$ & $\begin{array}{c}\text { Equip. \& } \\
\text { Material } \\
\text { Cost } \\
\end{array}$ & MHRS & \begin{tabular}{|c} 
Labor \\
Cost \\
(Loaded)
\end{tabular} & \begin{tabular}{|c} 
Equip. \& \\
Material \\
Cost \\
\end{tabular} & MHRS & $\begin{array}{c}\text { Labor } \\
\text { Cost } \\
\text { (Loaded) }\end{array}$ & \begin{tabular}{|c} 
Equip. \& \\
Material \\
Cost \\
\end{tabular} \\
\hline \begin{tabular}{|l} 
Receive/Uncrate Nacelle,Blades, \\
Rotors \& Towers
\end{tabular} & 54 & $\$ 2,176$ & & 62 & $\$ 2,499$ & & 75 & $\$ 3,023$ & & 96 & $\$ 3,869$ & & 142 & 5,723 & \\
\hline Rig \& Set Tower Sections & 64 & $\$ 2,580$ & & 132 & $\$ 5,320$ & & 243 & $\$ 9,792$ & & 354 & $\$ 14,265$ & & 619 & 24,810 & \\
\hline Grout \& Torque Bases & 37 & $\$ 1,492$ & $\$ 450$ & 40 & $\$ 1,612$ & $\$ 850$ & 59 & $\$ 2,377$ & $\$ 950$ & 70 & $\$ 2,822$ & $\$ 1,120$ & 87 & 3,506 & 1,650 \\
\hline Assemble Rotors/Blades \& Nacelle & 43 & $\$ 1,733$ & $\$ 150$ & 50 & $\$ 2,015$ & $\$ 250$ & 74 & $\$ 2,982$ & $\$ 500$ & 86 & $\$ 3,465$ & $\$ 700$ & 124 & 4,998 & 1,000 \\
\hline Rig \& Set Nacelle \& Blades & 41 & $\$ 2,248$ & & 57 & $\$ 2,317$ & & 133 & $\$ 6,488$ & & 174 & $\$ 8,549$ & & 280 & 13,370 & \\
\hline Install Safety Equipment & 9 & $\$ 363$ & & 12 & $\$ 484$ & & 20 & $\$ 806$ & & 24 & $\$ 968$ & & 36 & 1,450 & \\
\hline General Conditions & & $\$ 2,172$ & $\$ 5,713$ & & $\$ 2,172$ & $\$ 5,713$ & & $\$ 2,459$ & $\$ 10,789$ & & $\$ 2,459$ & $\$ 10,789$ & & 8,728 & 20,941 \\
\hline Margin @ 10\% & & $\$ 1,276$ & $\$ 631$ & & $\$ 1,642$ & $\$ 681$ & & $\$ 2,793$ & $\$ 1,224$ & & $\$ 3,640$ & $\$ 1,261$ & & $\$ 6,259$ & $\$ 2,359$ \\
\hline Subtotal Per Turbine & 248 & $\$ 14,040$ & $\$ 6,944$ & 353 & $\$ 18,061$ & $\$ 7,494$ & 604 & $\$ 30,720$ & $\$ 13,463$ & 804 & $\$ 40,037$ & $\$ 13,870$ & 1288 & $\$ 68,844$ & $\$ 25,950$ \\
\hline Project Total (50 turbines) & 12.400 & $\$ 702,020$ & $\$ 347,215$ & 17,650 & $\$ 903,045$ & $\$ 374,715$ & 30,200 & $\$ 1,535,985$ & $\$ 673,145$ & 40,200 & $\$ 2,001,835$ & $\$ 693.495$ & 64,400 & $\$ 3.442 .175$ & $\$ \$ 1,297,505$ \\
\hline MAGNITUDE OF UNCERTAINTY & & $\begin{array}{l}\text { plus } \\
3 \%\end{array}$ & $\begin{array}{l}\text { minus } \\
5 \%\end{array}$ & & $\begin{array}{l}\text { plus } \\
5 \%\end{array}$ & $\begin{array}{c}\text { minus } \\
10 \%\end{array}$ & & $\begin{array}{l}\text { plus } \\
8 \%\end{array}$ & $\begin{array}{l}\text { minus } \\
12 \%\end{array}$ & & $\begin{array}{l}\text { plus } \\
10 \%\end{array}$ & $\begin{array}{l}\text { minus } \\
15 \%\end{array}$ & & $\begin{array}{l}\text { plus } \\
15 \%\end{array}$ & $\begin{array}{l}\text { minus } \\
10 \%\end{array}$ \\
\hline
\end{tabular}

1) Above cost do not include Bond's, Insurances or Taxes

2) No winter work assumed
All heavy cranes not in above prices.

\begin{tabular}{|c|c|}
\hline $\begin{array}{l}\text { Activity } \\
\text { Description }\end{array}$ & ACTIVITIES INCLUDED UNDER DESCRIPTION \\
\hline $\begin{array}{l}\text { Receive/Uncrate Nacelle, Blades, } \\
\text { Rotors \& Towers }\end{array}$ & Uncrate Nacelles, Rotors \& Blades, Unloading \& Sorting, Site distribution \\
\hline Rig \& Set Tower Sections & Rig, Set, \& Torque all Tower Sections, Non Productive Time \\
\hline Grout \& Torque Bases & Grout Base Sections,Tension Anchor Bolts,Anchor Bolt Tension Verification \\
\hline Assemble Rotors/Blades \& Nacelle & Assemble Blades to Rotors,Assemble \& Prep Nacelle, Hay bales \& dunnage \\
\hline Rig \& Set Nacelle \& Blades & Rig \& set Nacelle, Torque Nacelle,Rig \& set Rotor assembly, Torque Rotor Assembly to Spindle, Premium Time (Labor) \\
\hline Install Safety Equipment & $\begin{array}{l}\text { Install Safety climbing devices,Set Controller \& Lower power cable from Nacelle to Control Cabinet, } \\
\text { Complete Ladder \& Platform Assemblies }\end{array}$ \\
\hline General Conditions & See attached worksheet for break down on whats included \\
\hline
\end{tabular}




\section{GENERAL CONDITIONS}

GLOBAL ENERGY CONCEPTS, LLC.

750 \& 1500 MW UNITS

\begin{tabular}{|c|c|c|c|c|c|}
\hline \multirow[b]{2}{*}{ DESCRIPTION } & \multicolumn{4}{|c|}{ TOTAL COST } & \multirow[b]{2}{*}{ TOTAL } \\
\hline & LABOR & MAT'L. & EQUIP. & SUB. & \\
\hline $\begin{array}{l}\text { PROFESSIONAL SERVICES } \\
\text { CONSULTING ENGINEER } \\
\text { TECH.SERVICES SAFETY }\end{array}$ & & & & & \\
\hline $\begin{array}{l}\text { "**TOTAL PROFESSIONAL SERVICES } \\
\text { PERMITS \& FEES }\end{array}$ & 0 & 70 & 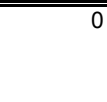 & 24 & $\overline{994}$ \\
\hline $\begin{array}{l}\text { **TOTAL PERMITS \& FEES } \\
\text { CONST. SERVICES \& MISC. } \\
\text { PHOTOGRAPHS-MAM }\end{array}$ & 0 & 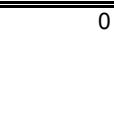 & 0 & 0 & 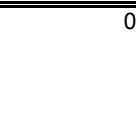 \\
\hline "**TOTAL CONST.SERVICES \& MISC. & 0 & 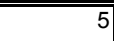 & 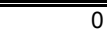 & 0 & 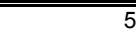 \\
\hline DESCRIPTION & LABOR & $\frac{\text { TOTAL CO }}{\text { MAT'L. }}$ & $\begin{array}{l}\text { ST } \\
\text { EQUIP. }\end{array}$ & SUB. & TOTAL \\
\hline $\begin{array}{l}\text { ON-SITE PERSONNEL } \\
\text { PROJECT MANAGER } \\
\text { GENERAL SUPERINTENDENT } \\
\text { ASSISTANT SUPERINTENDENT } \\
\text { FIELD ENGINEER \#1 } \\
\text { SECRETARY } \\
\text { FOREMAN SUPPLEMENT }\end{array}$ & & & & & \\
\hline $\begin{array}{l}* \text { TOTAL ON-SITE PERSONNEL } \\
\text { RELOCATION \& TRAVEL } \\
\text { OFFICE TRAVEL-GRAND RAPIDS } \\
\text { TRADES TRAVEL SUBSISTANCE } \\
\text { EMPLOYEE RELATIONS }\end{array}$ & 1061 & 257 & 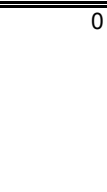 & 0 & 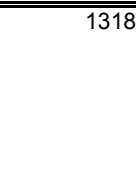 \\
\hline **TOTAL RELOCATION \& TRAVEL & 0 & 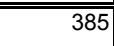 & 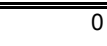 & 0 & 385 \\
\hline DESCRIPTION & LABOR & $\frac{\text { TOTAL CO }}{\text { MAT'L. }}$ & EQT & SUB. & TOTAL \\
\hline $\begin{array}{l}\text { FIELD OFFICE EXPENSES } \\
\text { OFFICE EQUIPMENT-MAM } \\
\text { DUPLICATING MACHINE-MAM } \\
\text { FAX MACHINE-MAM } \\
\text { COMPUTER-MAM } \\
\text { COMPUTER SOFTWARE } \\
\text { OFFICE SUPPLIES } \\
\text { POSTAGE/UPS } \\
\text { OVERNIGHT MAIL }\end{array}$ & & & & & \\
\hline $\begin{array}{l}\text { **TOTAL FIELD OFFICE EXPENSES } \\
\text { TEMPORARY FACILITIES } \\
\text { MAM OFFICE > } 12 \text { X } 50 \\
\text { SET UP OFFICE TRAILERS } \\
\text { OFFICE LINK } \\
\text { TOOL TRAILER } \\
\text { SET UP TOOL TRAILER } \\
\text { WORK SHACK (SKID VAN) } \\
\text { PROJECT SIGN }\end{array}$ & 0 & 102 & $\overline{0}$ & 0 & 102 \\
\hline "**TOTAL TEMPORARY FACILITIES & 268 & 1114 & 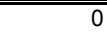 & 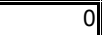 & 182 \\
\hline
\end{tabular}




\begin{tabular}{|c|c|c|c|c|c|}
\hline \multirow{2}{*}{ DESCRIPTION } & \multicolumn{4}{|c|}{ TOTAL COST } & \multirow[b]{2}{*}{ TOTAL } \\
\hline & LABOR & MAT'L. & EQUIP. & SUB. & \\
\hline $\begin{array}{l}\text { MOVING \& TRANSPORTATION } \\
\text { FREIGHT } \\
\text { MOVE OFFICE TRAILERS } \\
\text { MOVE TOOL TRAILERS } \\
\text { LOAD \& UNLOAD }\end{array}$ & & & & & \\
\hline $\begin{array}{l}\text { **TOTAL MOVING \& TRANSPORTATION } \\
\text { CONSTRUCTION UTILITIES } \\
\text { ELECTRICAL SERVICE } \\
\text { TEMPORARY LIGHT/POWER DIST. } \\
\text { ELECTRICAL ENERGY } \\
\text { TELEPHONE-MAM } \\
\text { PHONE LINE INSTALLATION } \\
\text { TELE.-DATA COMM'TNS. } \\
\text { DRINKING WATER } \\
\text { SANITATION }\end{array}$ & 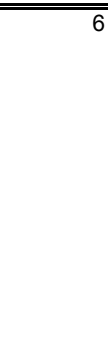 & 160 & 0 & 0 & 166 \\
\hline $\begin{array}{l}\text { **TOTAL CONSTRUCTION UTILITIES } \\
\text { HEATING \& VENTILATION }\end{array}$ & 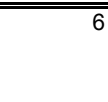 & 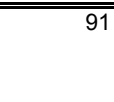 & $\overline{0}$ & 94 & 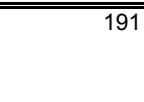 \\
\hline "**TOTAL HEATING \& VENTILATION & 0 & 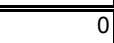 & 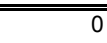 & 0 & $\overline{0}$ \\
\hline DESCRIPTION & LABOR & $\frac{\text { TOTAL CO }}{\text { MAT'L. }}$ & ST & SUB. & TOTAL \\
\hline $\begin{array}{l}\text { CONSTRUCTION EQUIPMENT } \\
45 \text { TON HYDRO } \\
100 \text { TON HYDRO } \\
\text { CRAWLER CRANE FREIGHT } \\
\text { ROUGH TERRAIN FORKLIFT } \\
\text { AUTOMATIC LEVEL } \\
\text { RADIOS } \\
\text { BOLT TORQUE EQUIP. } \\
\text { PICKUP O.S. LEASE } \\
\text { PICKUP JOB } \\
\text { EQUIPMENT OPERATING EXPENSE } \\
\text { EQUIPMENT REPAIRS } \\
\text { SMALL TOOLS/CONSUMABLES } \\
\text { EQUIPMENT OPERATOR } \\
\text { MOBILE CRANE OPERATOR } \\
\text { OILER }\end{array}$ & & & & & \\
\hline $\begin{array}{l}\text { **TOTAL CONSTRUCTION EQUIPMENT } \\
\text { QUALITY }\end{array}$ & "523 & 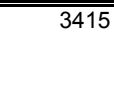 & 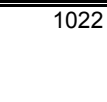 & (378 & (5338 \\
\hline $\begin{array}{l}\text { "*TOTAL QUALITY } \\
\text { SAFETY } \\
\text { PRE-EMPLOYMENT PHYSICAL } \\
\text { SAFETY INCENTIVE }\end{array}$ & 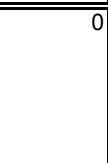 & 0 & 0 & 0 & 0 \\
\hline "**TOTAL SAFETY & 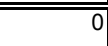 & 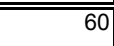 & 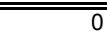 & 0 & 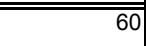 \\
\hline DESCRIPTION & LABOR & $\frac{\text { TOTAL CO }}{\text { MAT'L. }}$ & EQUIP. & SUB. & TOTAL \\
\hline $\begin{array}{l}\text { CLEAN-UP } \\
\text { RUBBISH REMOVAL } \\
\text { FINAL CLEAN UP }\end{array}$ & & & & & \\
\hline $\begin{array}{l}\text { **TOTAL CLEAN-UP } \\
\text { PROJECT STARTUP \& CLOSEOU }\end{array}$ & 12 & 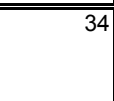 & 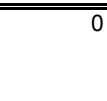 & 0 & 46 \\
\hline "*TOTAL PROJ. STARTUP \& CLEANUP & $\overline{0}$ & 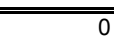 & $\overline{0}$ & 0 & $=$ \\
\hline ***TOTAL GENERAL REQUIREMENTS & $\$ 1,676$ & $\$ 4,693$ & $\$ 1,022$ & $\$ 496$ & $\$ 7,887$ \\
\hline
\end{tabular}




\section{GENERAL CONDITIONS}

GLOBAL ENERGY CONCEPTS, LLC.

$2500 \& 3500$ MW UNITS

\begin{tabular}{|c|c|c|c|c|c|}
\hline \multirow[b]{2}{*}{ DESCRIPTION } & \multicolumn{4}{|c|}{ TOTAL COST } & \multirow[b]{2}{*}{ TOTAL } \\
\hline & LABOR & MAT'L. & EQUIP. & SUB. & \\
\hline \multicolumn{6}{|l|}{$\begin{array}{l}\text { PROFESSIONAL SERVICES } \\
\text { CONSULTING ENGINEER } \\
\text { TECH.SERVICES SAFETY }\end{array}$} \\
\hline $\begin{array}{l}\text { **TOTAL PROFESSIONAL SERVICES } \\
\text { PERMITS \& FEES }\end{array}$ & 0 & 158 & \begin{tabular}{l|l}
0 \\
\end{tabular} & 54 & 212 \\
\hline $\begin{array}{l}\text { **TOTAL PERMITS \& FEES } \\
\text { CONST. SERVICES \& MISC. } \\
\text { PHOTOGRAPHS-MAM }\end{array}$ & 0 & 0 & 0 & 0 & 0 \\
\hline $\begin{array}{l}\text { **TOTAL CONST.SERVICES \& MISC. } \\
\text { ON-SITE PERSONNEL } \\
\text { PROJECT MANAGER } \\
\text { GENERAL SUPERINTENDENT } \\
\text { ASSISTANT SUPERINTENDENT } \\
\text { FIELD ENGINEER \#1 } \\
\text { SECRETARY } \\
\text { FOREMAN SUPPLEMENT }\end{array}$ & 0 & 11 & 0 & 0 & 11 \\
\hline $\begin{array}{l}\text { **TOTAL ON-SITE PERSONNEL } \\
\text { RELOCATION \& TRAVEL } \\
\text { OFFICE TRAVEL-GRAND RAPIDS } \\
\text { TRADES TRAVEL SUBSISTANCE } \\
\text { EMPLOYEE RELATIONS }\end{array}$ & 384 & (58 & 0 & 0 & 442 \\
\hline **TOTAL RELOCATION \& TRAVEL & 0 & 866 & 0 & 0 & 866 \\
\hline DESCRIPTION & LABOR & $\begin{array}{l}\text { OTAL COS } \\
\text { MAT'L. }\end{array}$ & $\begin{array}{l}\text { T } \\
\text { EQUIP. }\end{array}$ & SUB. & TOTAL \\
\hline $\begin{array}{l}\text { FIELD OFFICE EXPENSES } \\
\text { OFFICE EQUIPMENT-MAM } \\
\text { DUPLICATING MACHINE-MAM } \\
\text { FAX MACHINE-MAM } \\
\text { COMPUTER-MAM } \\
\text { COMPUTER SOFTWARE } \\
\text { OFFICE SUPPLIES } \\
\text { POSTAGE/UPS } \\
\text { OVERNIGHT MAIL }\end{array}$ & & & & & \\
\hline $\begin{array}{l}* * \text { TOTAL FIELD OFFICE EXPENSES } \\
\text { TEMPORARY FACILITIES } \\
\text { MAM OFFICE > } 12 \text { X } 50 \\
\text { SET UP OFFICE TRAILERS } \\
\text { OFFICE LINK } \\
\text { TOOL TRAILER } \\
\text { SET UP TOOL TRAILER } \\
\text { WORK SHACK (SKID VAN ) } \\
\text { PROJECT SIGN }\end{array}$ & 0 & 228 & 0 & 0 & 228 \\
\hline **TOTAL TEMPORARY FACILITIES & 167 & 269 & 0 & 0 & 436 \\
\hline
\end{tabular}




\begin{tabular}{|c|c|c|c|c|c|}
\hline & & TOTAL CO & & & \\
\hline DESCRIPTION & LABOR & MAT'L. & EQUIP. & SUB. & TOTAL \\
\hline \begin{tabular}{|l} 
MOVING \& TRANSPORTATION \\
FREIGHT \\
MOVE OFFICE TRAILERS \\
MOVE TOOL TRAILERS \\
LOAD \& UNLOAD
\end{tabular} & & & & & \\
\hline $\begin{array}{l}\text { **TOTAL MOVING \& TRANSPORTATION } \\
\text { CONSTRUCTION UTILITIES } \\
\text { ELECTRICAL SERVICE } \\
\text { TEMPORARY LIGHT/POWER DIST. } \\
\text { ELECTRICAL ENERGY } \\
\text { TELEPHONE-MAM } \\
\text { PHONE LINE INSTALLATION } \\
\text { TELE.-DATA COMM'TNS. } \\
\text { DRINKING WATER } \\
\text { SANITATION }\end{array}$ & 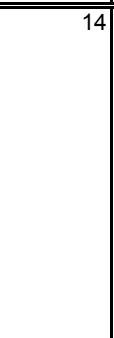 & 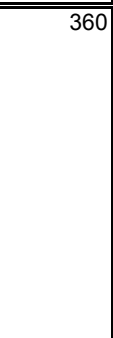 & 0 & 0 & 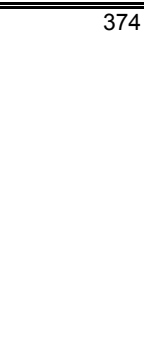 \\
\hline $\begin{array}{l}{ }^{* *} \text { TOTAL CONSTRUCTION UTILITIES } \\
\text { HEATING \& VENTILATION }\end{array}$ & 14 & 202 & 0 & 212 & 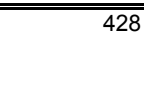 \\
\hline "**TOTAL HEATING \& VENTILATION & 0 & 0 & 0 & 0 & 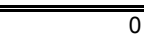 \\
\hline DESCRIPTION & LABOR & $\begin{array}{c}\text { TOTAL CO } \\
\text { MAT'L. }\end{array}$ & $\begin{array}{l}\text { ST } \\
\text { EQUIP. }\end{array}$ & SUB. & TOTAL \\
\hline $\begin{array}{l}\text { CONSTRUCTION EQUIPMENT } \\
\text { 45 TON HYDRO } \\
100 \text { TON HYDRO } \\
\text { CRAWLER CRANE FREIGHT } \\
\text { ROUGH TERRAIN FORKLIFT } \\
\text { AUTOMATIC LEVEL } \\
\text { RADIOS } \\
\text { BOLT TORQUE EQUIP. } \\
\text { PICKUP O.S. LEASE } \\
\text { PICKUP JOB } \\
\text { EQUIPMENT OPERATING EXPENSE } \\
\text { EQUIPMENT REPAIRS } \\
\text { SMALL TOOLS/CONSUMABLES } \\
\text { EQUIPMENT OPERATOR } \\
\text { MOBILE CRANE OPERATOR } \\
\text { OILER }\end{array}$ & & & & & \\
\hline $\begin{array}{l}\text { **TOTAL CONSTRUCTION EQUIPMENT } \\
\text { QUALITY }\end{array}$ & 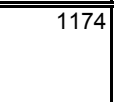 & 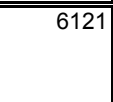 & 2307 & "515 & 10117 \\
\hline $\begin{array}{l}\text { "*TOTAL QUALITY } \\
\text { SAFETY } \\
\text { PRE-EMPLOYMENT PHYSICAL } \\
\text { SAFETY INCENTIVE }\end{array}$ & 0 & 0 & 0 & 0 & 0 \\
\hline "**TOTAL SAFETY & 0 & 135 & 0 & 0 & 135 \\
\hline DESCRIPTION & LABOR & $\begin{array}{c}\text { TOTAL CO } \\
\text { MAT'L. }\end{array}$ & ST & SUB. & TOTAL \\
\hline $\begin{array}{l}\text { CLEAN-UP } \\
\text { RUBBISH REMOVAL } \\
\text { FINAL CLEAN UP }\end{array}$ & & & & & \\
\hline $\begin{array}{l}\text { **TOTAL CLEAN-UP } \\
\text { PROJECT STARTUP \& CLOSEOUT }\end{array}$ & 27 & 77 & 0 & 0 & 104 \\
\hline "*TOTAL PROJ. STARTUP \& CLEANUP & 0 & 0 & 0 & 0 & 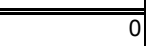 \\
\hline 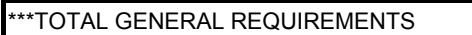 & 1780 & 8485 & 2307 & 781 & $\begin{array}{l}\$ 13,353.00 \\
\end{array}$ \\
\hline
\end{tabular}




\section{GENERAL CONDITIONS}

GLOBAL ENERGY CONCEPTS, LLC.

5000 MW UNITS

\begin{tabular}{|c|c|c|c|c|c|}
\hline \multirow[b]{2}{*}{ DESCRIPTION } & \multicolumn{4}{|c|}{ TOTAL COST } & \multirow[b]{2}{*}{ TOTAL } \\
\hline & LABOR & MAT'L. & EQUIP. & SUB. & \\
\hline $\begin{array}{l}\text { PROFESSIONAL SERVICES } \\
\text { CONSULTING ENGINEER } \\
\text { TECH.SERVICES SAFETY }\end{array}$ & & & & & \\
\hline $\begin{array}{l}\text { **TOTAL PROFESSIONAL SERVICES } \\
\text { PERMITS \& FEES }\end{array}$ & 0 & 294 & 0 & 96 & 390 \\
\hline $\begin{array}{l}\text { **TOTAL PERMITS \& FEES } \\
\text { CONST. SERVICES \& MISC. } \\
\text { PHOTOGRAPHS-MAM }\end{array}$ & 0 & 0 & 0 & 0 & $\overline{0}$ \\
\hline 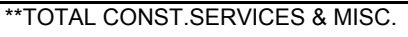 & 0 & 18 & 0 & 0 & 18 \\
\hline DESCRIPTION & LABOR & $\frac{\text { OTAL CO }}{\text { MAT'L. }}$ & $\frac{S T}{E Q U I P .}$ & SUB. & TOTAL \\
\hline $\begin{array}{l}\text { ON-SITE PERSONNEL } \\
\text { PROJECT MANAGER } \\
\text { GENERAL SUPERINTENDENT } \\
\text { ASSISTANT SUPERINTENDENT } \\
\text { FIELD ENGINEER \#1 } \\
\text { SECRETARY } \\
\text { FOREMAN SUPPLEMENT }\end{array}$ & & & & & \\
\hline $\begin{array}{l}{ }^{* *} \text { TOTAL ON-SITE PERSONNEL } \\
\text { RELOCATION \& TRAVEL } \\
\text { OFFICE TRAVEL-GRAND RAPIDS } \\
\text { TRADES TRAVEL SUBSISTANCE } \\
\text { EMPLOYEE RELATIONS }\end{array}$ & 4265 & 1033 & 0 & 0 & 5298 \\
\hline$\overline{~ * * \text { TOTAL RELOCATION \& TRAVEL }}$ & 0 & 1552 & 0 & 0 & 1552 \\
\hline DESCRIPTION & LABOR & $\begin{array}{l}\text { OTAL CO } \\
\text { MAT'L. }\end{array}$ & $\begin{array}{l}\text { EQTIP. } \\
\text { EQI }\end{array}$ & SUB. & TOTAL \\
\hline $\begin{array}{l}\text { FIELD OFFICE EXPENSES } \\
\text { OFFICE EQUIPMENT-MAM } \\
\text { DUPLICATING MACHINE-MAM } \\
\text { FAX MACHINE-MAM } \\
\text { COMPUTER-MAM } \\
\text { COMPUTER SOFTWARE } \\
\text { OFFICE SUPPLIES } \\
\text { POSTAGE/UPS } \\
\text { OVERNIGHT MAIL }\end{array}$ & & & & & \\
\hline $\begin{array}{l}\text { **TOTAL FIELD OFFICE EXPENSES } \\
\text { TEMPORARY FACILITIES } \\
\text { MAM OFFICE > } 12 \text { X } 50 \\
\text { SET UP OFFICE TRAILERS } \\
\text { OFFICE LINK } \\
\text { TOOL TRAILER } \\
\text { SET UP TOOL TRAILER } \\
\text { WORK SHACK (SKID VAN) } \\
\text { PROJECT SIGN }\end{array}$ & 0 & 409 & 0 & 0 & 409 \\
\hline$\overline{\text { **TOTAL TEMPORARY FACILITIES }}$ & 273 & 454 & 0 & 0 & 727 \\
\hline
\end{tabular}




\begin{tabular}{|c|c|c|c|c|c|}
\hline \multirow[b]{2}{*}{ DESCRIPTION } & \multicolumn{4}{|c|}{ TOTAL COST } & \multirow[b]{2}{*}{ TOTAL } \\
\hline & LABOR & MAT'L. & EQUIP. & SUB. & \\
\hline \multicolumn{6}{|l|}{$\begin{array}{l}\text { MOVING \& TRANSPORTATION } \\
\text { FREIGHT } \\
\text { MOVE OFFICE TRAILERS } \\
\text { MOVE TOOL TRAILERS }\end{array}$} \\
\hline $\begin{array}{l}\text { "*TOTAL MOVING \& TRANSPORTATION } \\
\text { CONSTRUCTION UTILITIES } \\
\text { ELECTRICAL SERVICE } \\
\text { TEMPORARY LIGHT/POWER DIST. } \\
\text { ELECTRICAL ENERGY } \\
\text { TELEPHONE-MAM } \\
\text { PHONE LINE INSTALLATION } \\
\text { TELE.-DATA COMM'TNS. } \\
\text { DRINKING WATER } \\
\text { SANITATION }\end{array}$ & 24 & $\overline{640}$ & 0 & 0 & $\overline{664}$ \\
\hline $\begin{array}{l}\text { "**OTAL CONSTRUCTION UTILITIES } \\
\text { HEATING \& VENTILATION }\end{array}$ & 24 & 361 & 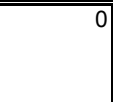 & 376 & $\overline{7761}$ \\
\hline$\overline{\text { **TOTAL HEATING \& VENTILATION }}$ & 0 & 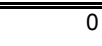 & 0 & 0 & 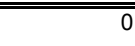 \\
\hline DESCRIPTION & LABOR & $\begin{array}{l}\text { OTAL CO } \\
\text { MAT'L. }\end{array}$ & $\begin{array}{l}\text { ST } \\
\text { EQUIP. }\end{array}$ & SUB. & TOTAL \\
\hline $\begin{array}{l}\text { CONSTRUCTION EQUIPMENT } \\
45 \text { TON HYDRO } \\
100 \text { TON HYDRO } \\
\text { CRAWLER CRANE FREIGHT } \\
\text { ROUGH TERRAIN FORKLIFT } \\
\text { AUTOMATIC LEVEL } \\
\text { RADIOS } \\
\text { BOLT TORQUE EQUIP. } \\
\text { PICKUP O.S. LEASE } \\
\text { PICKUP JOB } \\
\text { EQUIPMENT OPERATING EXPENSE } \\
\text { EQUIPMENT REPAIRS } \\
\text { SMALL TOOLS/CONSUMABLES } \\
\text { EQUIPMENT OPERATOR } \\
\text { MOBILE CRANE OPERATOR } \\
\text { OILER }\end{array}$ & & & & & \\
\hline $\begin{array}{l}\text { "*TOTAL CONSTRUCTION EQUIPMENT } \\
\text { QUALITY }\end{array}$ & 2103 & 11666 & 4138 & 1520 & 19427 \\
\hline $\begin{array}{l}{ }^{* *} \text { TOTAL QUALITY } \\
\text { SAFETY } \\
\text { PRE-EMPLOYMENT PHYSICAL } \\
\text { SAFETY INCENTIVE }\end{array}$ & 0 & 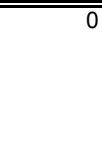 & 0 & 0 & 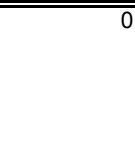 \\
\hline$\overline{~ * \star * O T A L ~ S A F E T Y ~}$ & 0 & 240 & 0 & 0 & 240 \\
\hline DESCRIPTION & LABOR & $\begin{array}{l}\text { OTAL CO } \\
\text { MAT'L. }\end{array}$ & $\frac{S T}{E Q U I P .}$ & SUB. & TOTAL \\
\hline $\begin{array}{l}\text { CLEAN-UP } \\
\text { RUBBISH REMOVAL } \\
\text { FINAL CLEAN UP }\end{array}$ & & & & & \\
\hline $\begin{array}{l}\text { "*TOTAL CLEAN-UP } \\
\text { PROJECT STARTUP \& CLOSEOU }\end{array}$ & 48 & $\overline{136}$ & 0 & 0 & 184 \\
\hline $\begin{array}{l}\text { **TOTAL PROJ. STARTUP \& CLEANUP } \\
\end{array}$ & 0 & $\overline{0}$ & 0 & 0 & 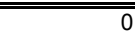 \\
\hline 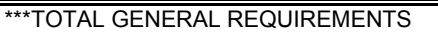 & $\mid 6737$ & 16803 & (4138 & |1992| & $\$ 29,670.00$ \\
\hline
\end{tabular}


GLOBAL ENERGY CONCEPTS, LLC.

MEGAWATT-SCALE WIND TURBINE CONSTRUCTION

Tower section assembly for 3500 \& 5000 MW Units

Assembly Work Pads

36'-0" x 83'-0" x 1'-0" - 4 req'd.

\begin{tabular}{|c|c|c|c|c|c|c|c|c|c|}
\hline Description & QTY. & UNITS & $\begin{array}{c}\text { UNITS/ } \\
\text { MHR }\end{array}$ & $\begin{array}{l}\text { TOTAL } \\
\text { MHRS } \\
\end{array}$ & $\begin{array}{c}\text { COST/ } \\
\text { MHR }\end{array}$ & $\begin{array}{l}\text { TOTAL } \\
\text { LABOR }\end{array}$ & $\begin{array}{c}\text { MAT'L. } \\
\text { UNIT \$\$ }\end{array}$ & $\begin{array}{l}\text { TOTAL } \\
\text { MAT'L. }\end{array}$ & $\begin{array}{c}\text { TOTAL } \\
\text { L \& M } \\
\end{array}$ \\
\hline Concrete & 444 & CY & 3 & 148 & $\$ 40$ & $\$ 5,964$ & $\$ 80$ & $\$ 35,520$ & $\$ 41,484$ \\
\hline Reinforcing steel & 43,373 & LBS & 120 & 361 & $\$ 40$ & $\$ 14,458$ & & $\$ 0$ & $\$ 14,458$ \\
\hline Concrete forming & 238 & SF & 8 & 30 & $\$ 40$ & $\$ 1,200$ & $\$ 3$ & $\$ 714$ & $\$ 1,914$ \\
\hline Concrete Curing & 3226 & SF & 250 & 13 & $\$ 0$ & $\$ 0$ & $\$ 0.10$ & $\$ 323$ & $\$ 323$ \\
\hline Concrete Finishing & 3226 & SF & 70 & 46 & $\$ 40$ & $\$ 1,840$ & & $\$ 0$ & $\$ 1,840$ \\
\hline Embedded Plates & 3312 & LBS & 100 & 34 & $\$ 40$ & $\$ 1,360$ & $\$ 1.25$ & $\$ 4,140$ & $\$ 5,500$ \\
\hline Cradle Section & 48 & EA & 1 & 48 & $\$ 40$ & $\$ 1,920$ & $\$ 200$ & $\$ 9,600$ & $\$ 11,520$ \\
\hline Center Cradle Section & 24 & EA & 2 & 12 & $\$ 40$ & $\$ 480$ & $\$ 15$ & $\$ 360$ & $\$ 840$ \\
\hline Top \& Bottom Templates & 8 & EA & 0 & 0 & $\$ 0$ & $\$ 0$ & $\$ 1,500$ & $\$ 12,000$ & $\$ 12,000$ \\
\hline Screw Jacks & 60 & EA & 0 & 0 & $\$ 0$ & $\$ 0$ & $\$ 30$ & $\$ 1,800$ & $\$ 1,800$ \\
\hline TOTALS & & & & 692 & \multicolumn{3}{|c|}{$\$ 27,222$} & $\$ 64,45$ & $\$ 91,679$ \\
\hline
\end{tabular}


WELDED QUARTER SECTIONS OF TOWERS

(ONLY THREE TOWER SECTIONS TO BE QUARTERED,OTHER TWO CAN BE SHIPPED AS

WHOLE SECTIONS)

Tower Section Assembly 3500 MW

\begin{tabular}{|c|c|c|c|c|c|c|c|c|c|}
\hline Description & QTY. & UNITS & $\begin{array}{c}\text { UNITSI } \\
\text { MHR }\end{array}$ & $\begin{array}{l}\text { TOTAL } \\
\text { MHRS }\end{array}$ & $\begin{array}{l}\text { COSTI } \\
\text { MHR }\end{array}$ & $\begin{array}{l}\text { TOTAL } \\
\text { LABOR }\end{array}$ & $\begin{array}{l}\text { MAT'L. } \\
\text { UNIT \$\$ }\end{array}$ & $\begin{array}{l}\text { TOTAL } \\
\text { MAT'L. }\end{array}$ & $\begin{array}{c}\text { TOTAL } \\
\text { L \& M }\end{array}$ \\
\hline Screw Jacks & 60 & EA & 4 & 12 & $\$ 40$ & $\$ 480$ & $\$ 0.00$ & $\$ 0$ & $\$ 480$ \\
\hline Quarter-Section Weld & 5041 & INCHES & 16 & 315 & $\$ 40$ & $\$ 12,600$ & $\$ 0.58$ & $\$ 2,924$ & $\$ 15,524$ \\
\hline Half-Section Weld & 5041 & INCHES & 16 & 315 & $\$ 40$ & $\$ 12,600$ & $\$ 0.58$ & $\$ 2,924$ & $\$ 15,524$ \\
\hline Top \& Bottom Template & 32 & EA & 0.25 & 128 & $\$ 40$ & $\$ 5,120$ & $\$ 0.00$ & $\$ 0$ & $\$ 5,120$ \\
\hline Move Cradles & 48 & EA & 2 & 24 & $\$ 40$ & $\$ 960$ & $\$ 0.00$ & $\$ 0$ & $\$ 960$ \\
\hline Lifting Eyes & 16 & EA & 1 & 16 & $\$ 40$ & $\$ 640$ & $\$ 35.00$ & $\$ 560$ & $\$ 1,200$ \\
\hline Paint (section 1) & 5316 & $\mathrm{SF}$ & 80 & 67 & $\$ 40$ & $\$ 2,680$ & $\$ 0.50$ & $\$ 2,658$ & $\$ 5,338$ \\
\hline Paint (section 2) & 5006 & $\mathrm{SF}$ & 80 & 63 & $\$ 40$ & $\$ 2,520$ & $\$ 0.50$ & $\$ 2,503$ & $\$ 5,023$ \\
\hline Paint (section 3) & 4496 & $\mathrm{SF}$ & 80 & 56 & $\$ 40$ & $\$ 2,240$ & $\$ 0.50$ & $\$ 2,248$ & $\$ 4,488$ \\
\hline Non-Productive Time & $3 \%$ & MHRS & & 30 & $\$ 40$ & $\$ 1,195$ & $\$ 0.00$ & $\$ 0$ & $\$ 1,195$ \\
\hline X-RAY TEST & 60 & $\mathrm{EA}$ & 1 & 60 & $\$ 65$ & $\$ 3,900$ & $\$ 20.00$ & $\$ 1,200$ & $\$ 5,100$ \\
\hline Weld Correction (1\%) & 68 & INCHES & 16 & 5 & $\$ 40$ & $\$ 200$ & $\$ 0.58$ & $\$ 39$ & $\$ 239$ \\
\hline \multirow{2}{*}{ Load \& Transport Towers } & 3 & SECTIONS & 0.18 & 16 & $\$ 40$ & $\$ 640$ & $\$ 0.00$ & $\$ 0$ & $\$ 640$ \\
\hline & & & & & $\$ 0$ & $\$ 0$ & $\$ 0.00$ & $\$ 0$ & $\$ 0$ \\
\hline \multicolumn{4}{|l|}{ TOTALS } & 1107 & & $\$ 45,775$ & & $\$ 15,056$ & $\$ 60,831$ \\
\hline \multicolumn{4}{|c|}{ Project Cost (Based on 50 towers) } & $\overline{55,344}$ & & $\overline{\$ 2,288,760}$ & & 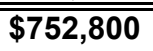 & $\$ \$ \$ 3,041,560$ \\
\hline
\end{tabular}


WELDED QUARTER SECTIONS OF TOWERS

(ONLY FIVE TOWER SECTIONS TO BE QUARTERED,OTHER TWO CAN BE SHIPPED AS

WHOLE SECTIONS)

Tower Section Assembly 5000 MW

\begin{tabular}{|c|c|c|c|c|c|c|c|c|c|}
\hline Description & QTY. & UNITS & $\begin{array}{c}\text { UNITSI } \\
\text { MHR }\end{array}$ & $\begin{array}{l}\text { TOTAL } \\
\text { MHRS }\end{array}$ & $\begin{array}{l}\text { COSTI } \\
\text { MHR }\end{array}$ & $\begin{array}{l}\text { TOTAL } \\
\text { LABOR }\end{array}$ & $\begin{array}{l}\text { MAT'L. } \\
\text { UNIT \$\$ }\end{array}$ & $\begin{array}{l}\text { TOTAL } \\
\text { MAT'L. }\end{array}$ & $\begin{array}{l}\text { TOTAL } \\
\text { L \& M }\end{array}$ \\
\hline Screw Jacks & 120 & EA & 4 & 30 & $\$ 40$ & $\$ 1,200$ & $\$ 0$ & $\$ 0$ & $\$ 1,200$ \\
\hline Quarter-Section Weld & 8780 & INCHES & 16 & 549 & $\$ 40$ & $\$ 21,960$ & $\$ 0.58$ & $\$ 5,092$ & $\$ 27,052$ \\
\hline Half-Section Weld & 8780 & INCHES & 16 & 549 & $\$ 40$ & $\$ 21,960$ & $\$ 0.58$ & $\$ 5,092$ & $\$ 27,052$ \\
\hline Top \& Bottom Template & 80 & EA & 0.25 & 280 & $\$ 40$ & $\$ 11,200$ & $\$ 0$ & $\$ 0$ & $\$ 11,200$ \\
\hline Move Cradles & 120 & EA & 2 & 60 & $\$ 40$ & $\$ 2,400$ & $\$ 0$ & $\$ 0$ & $\$ 2,400$ \\
\hline Lifting Eyes & 40 & EA & 1 & 40 & $\$ 40$ & $\$ 1,600$ & $\$ 35$ & $\$ 1,400$ & $\$ 3,000$ \\
\hline Paint (section 1) & 6784 & $\mathrm{SF}$ & 80 & 85 & $\$ 40$ & $\$ 3,392$ & $\$ 0.50$ & $\$ 3,392$ & $\$ 6,784$ \\
\hline Paint (section 2) & 6256 & $\overline{S F}$ & 80 & 78 & $\$ 40$ & $\$ 3,128$ & $\$ 0.50$ & $\$ 3,128$ & $\$ 6,256$ \\
\hline Paint (section 3) & 5803 & $\mathrm{SF}$ & 80 & 73 & $\$ 40$ & $\$ 2,902$ & $\$ 0.50$ & $\$ 2,902$ & $\$ 5,803$ \\
\hline Paint (section 4) & 5276 & SF & 80 & 66 & $\$ 40$ & $\$ 2,638$ & $\$ 0.50$ & $\$ 2,638$ & $\$ 5,276$ \\
\hline Paint (section 5) & 4824 & SF & 80 & 60 & $\$ 40$ & $\$ 2,412$ & $\$ 0.00$ & $\$ 0$ & $\$ 2,412$ \\
\hline Non-Productive Time & $3 \%$ & MHRS & & 56 & $\$ 40$ & $\$ 2,244$ & $\$ 0.00$ & $\$ 0$ & $\$ 2,244$ \\
\hline X-RAY TEST & 100 & EA & 1 & 100 & $\$ 65$ & $\$ 6,500$ & $\$ 0.00$ & $\$ 0$ & $\$ 6,500$ \\
\hline Weld Correction (1\%) & 175 & INCHES & 16 & 11 & $\$ 40$ & $\$ 440$ & $\$ 0.50$ & $\$ 88$ & $\$ 528$ \\
\hline Load \& Transport Towers & 5 & SECTIONS & 0.18 & 28 & $\$ 40$ & $\$ 1,120$ & $\$ 20.00$ & $\$ 100$ & $\$ 1,220$ \\
\hline \multicolumn{4}{|l|}{ TOTALS } & \multicolumn{2}{|l|}{2065} & \multicolumn{2}{|l|}{$\$ 85,095$} & $\$ 23,832$ & $\$ 108,927$ \\
\hline \multicolumn{4}{|c|}{ Project Cost (Based on 50 towers) } & 10103,244 & \multicolumn{3}{|c|}{$\$ 4,254,762$} & $\$ 1,191,590$ & $\$ \$ \$ 5,446,352$ \\
\hline
\end{tabular}


BOLTED CONNECTIONS

(ONLY THREE TOWER SECTIONS TO BE QUARTERED,OTHER TWO CAN BE SHIPPED AS

WHOLE SECTIONS)

Tower Section Assembly 3500 MW

\begin{tabular}{|c|c|c|c|c|c|c|c|c|c|}
\hline Description & QTY. & UNITS & $\begin{array}{l}\text { UNITSI } \\
\text { MHR }\end{array}$ & $\begin{array}{l}\text { TOTAL } \\
\text { MHRS }\end{array}$ & $\begin{array}{l}\text { COSTI } \\
\text { MHR }\end{array}$ & $\begin{array}{l}\text { TOTAL } \\
\text { LABOR }\end{array}$ & $\begin{array}{l}\text { MAT'L. } \\
\text { UNIT \$\$ }\end{array}$ & $\begin{array}{l}\text { TOTAL } \\
\text { MAT'L. }\end{array}$ & $\begin{array}{l}\text { TOTAL } \\
\text { L \& M }\end{array}$ \\
\hline Screw Jacks & 60 & EA & 4 & 12 & $\$ 40$ & $\$ 480$ & $\$ 0$ & $\$ 0$ & $\$ 480$ \\
\hline Quarter-Section Bolts & 330 & BOLTS & 10 & 30 & $\$ 40$ & $\$ 1,200$ & $\$ 0.00$ & $\$ 0$ & $\$ 1,200$ \\
\hline Half-Section Bolts & 330 & BOLTS & 10 & 30 & $\$ 40$ & $\$ 1,200$ & $\$ 0.00$ & $\$ 0$ & $\$ 1,200$ \\
\hline Top \& Bottom Template & 32 & EA & 0.25 & 128 & $\$ 40$ & $\$ 5,120$ & $\$ 0$ & $\$ 0$ & $\$ 5,120$ \\
\hline Move Cradles & 48 & EA & 2 & 24 & $\$ 40$ & $\$ 960$ & $\$ 0$ & $\$ 0$ & $\$ 960$ \\
\hline \begin{tabular}{|l|} 
Lifting Eyes \\
\end{tabular} & 16 & EA & 1 & 16 & $\$ 40$ & $\$ 640$ & $\$ 35$ & $\$ 560$ & $\$ 1,200$ \\
\hline Paint (section 1) & 10 & $\mathrm{SF}$ & 5 & 2 & $\$ 40$ & $\$ 80$ & $\$ 0.50$ & $\$ 5$ & $\$ 85$ \\
\hline Paint (section 2) & 10 & SF & 5 & 2 & $\$ 40$ & $\$ 80$ & $\$ 0.50$ & $\$ 5$ & $\$ 85$ \\
\hline Paint (section 3) & 10 & $\mathrm{SF}$ & 5 & 2 & $\$ 40$ & $\$ 80$ & $\$ 0.50$ & $\$ 5$ & $\$ 85$ \\
\hline Non-Productive Time & & & & 45 & $\$ 40$ & $\$ 1,800$ & $\$ 0$ & $\$ 0$ & $\$ 1,800$ \\
\hline Torqoe Test Bolts & 330 & BOLTS & 30 & 11 & $\$ 50$ & $\$ 550$ & & $\$ 0$ & $\$ 550$ \\
\hline \multirow{2}{*}{\multicolumn{4}{|c|}{$\begin{array}{l}\text { TOTALS } \\
\text { Project Cost (Based on } 50 \text { towers) }\end{array}$}} & 302 & \multicolumn{3}{|c|}{$\$ 12,190$} & $\$ 575$ & $\$ 12,765$ \\
\hline & & & & $\overline{15,100}$ & \multicolumn{3}{|c|}{$\$ 609,500$} & $\$ \$ 28,750$ & $\$ \$ \$ 638,250$ \\
\hline
\end{tabular}


BOLTED CONNECTIONS

(ONLY FIVE TOWER SECTIONS TO BE QUARTERED,OTHER TWO CAN BE SHIPPED AS

WHOLE SECTIONS)

Tower Section Assembly 5000 MW

\begin{tabular}{|c|c|c|c|c|c|c|c|c|c|}
\hline Description & QTY. & UNITS & $\begin{array}{l}\text { UNITS/ } \\
\text { MHR }\end{array}$ & $\begin{array}{l}\text { TOTAL } \\
\text { MHRS }\end{array}$ & $\begin{array}{l}\text { COST/ } \\
\text { MHR }\end{array}$ & $\begin{array}{l}\text { TOTAL } \\
\text { LABOR }\end{array}$ & $\begin{array}{l}\text { MAT'L. } \\
\text { UNIT \$\$ }\end{array}$ & $\begin{array}{l}\text { TOTAL } \\
\text { MAT'L. }\end{array}$ & $\begin{array}{l}\text { TOTAL } \\
\text { L \& M }\end{array}$ \\
\hline Screw Jacks & 120 & EA & 4 & 30 & $\$ 40$ & $\$ 1,200$ & $\$ 0$ & $\$ 0$ & $\$ 1,200$ \\
\hline Quarter-Section Bolt & 558 & BOLTS & 10 & 58 & $\$ 40$ & $\$ 2,320$ & $\$ 0.00$ & $\$ 0$ & $\$ 2,320$ \\
\hline Half-Section Bolt & 558 & BOLTS & 10 & 58 & $\$ 40$ & $\$ 2,320$ & $\$ 0.00$ & $\$ 0$ & $\$ 2,320$ \\
\hline Top \& Bottom Template & 80 & EA & 0.25 & 280 & $\$ 40$ & $\$ 11,200$ & $\$ 0$ & $\$ 0$ & $\$ 11,200$ \\
\hline Move Cradles & 120 & EA & 2 & 60 & $\$ 40$ & $\$ 2,400$ & $\$ 0$ & $\$ 0$ & $\$ 2,400$ \\
\hline \begin{tabular}{|l|} 
Lifting Eyes \\
\end{tabular} & 40 & EA & 1 & 40 & $\$ 40$ & $\$ 1,600$ & $\$ 35$ & $\$ 1,400$ & $\$ 3,000$ \\
\hline Paint (section 1) & 10 & $\mathrm{SF}$ & 5 & 2 & $\$ 40$ & $\$ 80$ & $\$ 0.50$ & $\$ 5$ & $\$ 85$ \\
\hline Paint (section 2) & 10 & SF & 5 & 2 & $\$ 40$ & $\$ 80$ & $\$ 0.50$ & $\$ 5$ & $\$ 85$ \\
\hline Paint (section 3) & 10 & $\mathrm{SF}$ & 5 & 2 & $\$ 40$ & $\$ 80$ & $\$ 0.50$ & $\$ 5$ & $\$ 85$ \\
\hline Paint (section 4) & 10 & SF & 5 & 2 & $\$ 40$ & $\$ 80$ & $\$ 0.50$ & $\$ 5$ & $\$ 85$ \\
\hline Paint (section 5) & 10 & SF & 5 & 2 & $\$ 40$ & $\$ 80$ & $\$ 0.50$ & $\$ 5$ & $\$ 85$ \\
\hline Non-Productive Time & & & & 60 & $\$ 40$ & $\$ 2,400$ & 0 & $\$ 0$ & $\$ 2,400$ \\
\hline \multirow[t]{3}{*}{ Torque Test Bolts } & 1116 & BOLTS & 30 & 37 & $\$ 40$ & $\$ 1,480$ & $\$ 0$ & $\$ 0$ & $\$ 1,480$ \\
\hline & & & & & & $\$ 0$ & 0 & $\$ 0$ & $\$ 0$ \\
\hline & & & & & & $\$ 0$ & 0 & $\$ 0$ & $\$ 0$ \\
\hline \multicolumn{4}{|l|}{ TOTALS } & 633 & & $\$ 25,320$ & & $\$ 1,425$ & $\$ 26,745$ \\
\hline \multicolumn{4}{|c|}{ Project Cost (Based on 50 towers) } & 31,650 & & $\$ 1,266,000$ & & $\$ 71,250$ & $\$ 1,337,250$ \\
\hline
\end{tabular}


Appendix $\mathbf{R}$

Lampson Estimates 
OPTION \# 1: This option will make use of 2 cranes per turbine. The first crane is assumed to start erecting the tower sections sufficiently ahead of the nacelle installation so as to produce a smooth schedule. At the client's option either the first crane can continue around behind the nacelle installation crane and ere the hub and blades or the nacelle erection crane can remain at each turbine until installation of the hub and blades is complete or some combination of each

Option \# 2: This option will make use of 1 crane per turbine. The crane will either fully complete each turbine before moving to the next or move from turbine to turbine after completing only a portion of each (l.e., erect the towers) before returning to the start and erecting the next items (I.e., erect the nacelle and rotor)

750 kW --- OPTION \# 1

1) Brief Description Of Lifting Approach:

$1 \mathrm{a}$ - Number of cranes required per turbine

1c - Alternative lifting approach, if applicable

\begin{tabular}{|c|c|}
\hline \multicolumn{2}{|c|}{2} \\
\hline \#1 - Tower \& Rotor & $\# 2$ - Nacelle \\
\hline (3) & - \\
\hline
\end{tabular}

2) For Each Type Of Crane Identified Provide:

$2 \mathrm{a}$ - Crane type, manufacturer \& model

$2 \mathrm{~b}$ - Boom length, capacity \& reach

2c - Description of crane operation

$2 \mathrm{~d}$ - Monthly Rental Costs (Bare rates - no labor)

1 - Operating Rates (based on $176 \mathrm{hrs} / \mathrm{month})$

2 - Stand-by Rates

3- Overtime Rates (per hour)

$2 e$ - Manufactures specification sheets, tables or graphs

2f - Availability of equipment, scheduling requirements, lead time

$2 \mathrm{~g}$ - Crane pad/Working area dimensions

$2 \mathrm{~h}$ - Weather operations limits - temperature, wind, visibility, precipitation

2i - Other operation limits

2) Crane Crew For Each Turbine Case:

2a - Crew size and responsibilities

$2 \mathrm{~b}$ - Estimated hourly crew costs (straight time)

2c - Estimated lodging/per diem costs (per person)

$2 \mathrm{~d}$ - Travel costs $(\$ / \mathrm{mile})$

\begin{tabular}{|c|c|}
\hline Manitowoc-4100 S1 & Manitowoc - 4600 S4 \\
\hline 79 Meter ( $\left.260^{\prime}\right)$ & 85 Meter $\left(280^{\prime}\right)$ \\
\hline 37,800 Kg @ 14 Meters (Boom) & 36,400 Kg @ 23 Meter (Boom) \\
\hline $16,500 \mathrm{Kg} @ 24$ Meters (Boom) & \\
\hline Tracked & Tracked \\
\hline$\$ 15,000$ & $\$ 32,000$ \\
\hline$\$ 10,000$ & $\$ 24,000$ \\
\hline$\$ 57$ & $\$ 121$ \\
\hline See Attachment 10.1.1 & See Attachment 10.1.1 \\
\hline 30 days & 60 days \\
\hline $243 \mathrm{SqM}$ & $110 \mathrm{SqM}$ \\
\hline Min. Temp. $-30 \mathrm{~F}$, Wind up to & $\mathrm{m} / \mathrm{s}(20 \mathrm{MPH})$, Good visibility \\
\hline
\end{tabular}

Min. Temp. $-30 \mathrm{~F}$, Wind up to $9 \mathrm{~m} / \mathrm{s}(20 \mathrm{MPH})$, Good visibility

2 Total - 1 Operator \& 1 Oiler $\quad 2$ Total - 1 Operator \& 1 Oiler

\begin{tabular}{|c|c|}
\hline$\$ 130$ & $\$ 130$ \\
\hline$\$ 75$ & $\$ 75$ \\
\hline
\end{tabular}

3) Crane Assembly:

\begin{tabular}{|l|c|c|}
\hline 3a - Estimated crane assembly cost & $\$ 10,000$ & $\$ 18,000$ \\
\hline $3 b-$ Ond & 4 Hours @ Operating Rate & 2 Hours @ Operating Rate \\
\hline
\end{tabular}

4) Mobilization/Demobilization

\begin{tabular}{|c|c|c|}
\hline 4a - Brief Description Of Logistics & $\begin{array}{c}\begin{array}{c}\text { Deliver carbody \& house, install } \\
\text { side frames, install boom, wire \& } \\
\text { block - boom up }\end{array} \\
12 \text { Hours Up \& } 12 \text { Hours Down }\end{array}$ & \begin{tabular}{|c|}
$\begin{array}{c}\text { Deliver carbody \& house, install } \\
\text { side frames, install boom, wire } \& \\
\text { block - boom up }\end{array}$ \\
24 Hours UP \& 24 Hours Down \\
\end{tabular} \\
\hline \multicolumn{3}{|l|}{ 4c - Identify costs separately } \\
\hline 1 - Lampson Supervisor & 24 hrs @ \$75/hr & 48 hrs @ \$75/hr \\
\hline 2 - Iron Workers (4) & 96 hrs @ \$65/hr & 192 hrs @ \$65/hr \\
\hline $3-50$ ton hydraulic crane & 32 hrs @ \$370/hr (2 cranes) & 24 hrs @ \$185/hr \\
\hline 4 - (1) 150 ton truck crane & - & 24 hrs @ \$325/hr \\
\hline $5-$ & & \\
\hline $4 d$ - Fixed Costs - Freight Estimate to and from project - Total & $\$ 40,000$ & $\$ 60,000$ \\
\hline
\end{tabular}

$4 \mathrm{e}$ - Mileage or time dependent cos

\section{5) Materials And Consumables:}

\begin{tabular}{|l|c|c|}
\hline $5 \mathrm{a}-$ Cribbing (One Layer Crane Mat Pad) @ \$2.50/sq ft & 1 Ea. @ 243 SqM (2615 SqFt) & 1 Ea. @ 110 SqM (1185 SqFt) \\
\hline $5 \mathrm{~b}-$ Fuel @ \$1.50/gal & $220 \mathrm{gal} / \mathrm{wk}$ & $250 \mathrm{gal} / \mathrm{wk}$ \\
\hline
\end{tabular}

\section{6) Off-site And On-site Road Requirements:}

$6 a$ - Road geometry

$$
1 \text { - Maximum grade, } \%
$$

2 - Maximum road crown, $M$

3-Minimum curve radius

4 - Minimum road width, $M$

6b - Surface limitations (gravel, asphalt)

$6 \mathrm{c}$ - General road design/loading requirements

$6 \mathrm{~d}$ - Off-site equipment transportation requiremen

1- Oversized load permits required

2- Route planning required

3 - Escorts required

ments

\begin{tabular}{|c|c|c|}
\hline & $8 \%$ & $8 \%$ \\
\hline & Empty $2 \%$, loaded $0 \%$ & Empty $2 \%$, loaded $0 \%$ \\
\hline & $50-100 \mathrm{ft}$ & $50-100 \mathrm{ft}$ \\
\hline & $26 \mathrm{ft}$ & $30 \mathrm{ft}$ \\
\hline & gravel & gravel \\
\hline & $100 \%$ compaction & $100 \%$ compaction \\
\hline
\end{tabular}

\begin{tabular}{|c|c|}
\hline$y$ & $y$ \\
$y$ & $y$
\end{tabular}

\begin{tabular}{|c|}
$\mathrm{y}$ \\
$\mathrm{y}$ \\
$\mathrm{y}$
\end{tabular}

\section{7) Job Site Facility Needs:}

$7 a$ - Site Office/storage

$$
\begin{array}{|l|}
\hline 1 \text { - Site Office } \\
\hline 2 \text { - Site Storage } \\
\hline 3-\text { Site Amenies }
\end{array}
$$

2 - Site Storage

\begin{tabular}{|c|c|c|}
\hline share & share \\
\hline$y$ & $y$ \\
\hline$y$ & $y$ \\
\hline
\end{tabular}

8) General Industry Overhead, Fees and Profit:

1 - Overheads

2 - Fees

9) Overall Project Crane Costs Per Facility:

9 a - Combine crew, equipment, material, expendables, mob/demob, overhead,

profit, etc. into total costs per facility and per turbine

1 - Total cost per facility (50 turbines farm)

2 - Total cost per turbine

$9 \mathrm{~b}$ - Provide total schedule per facility

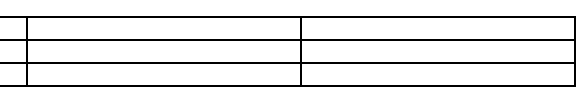

$750 \mathrm{~kW}$--- OPTION \# 2
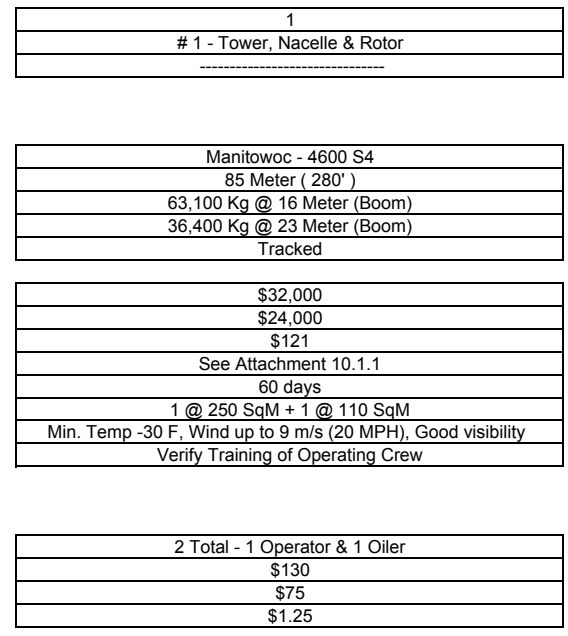

$\$ 18,000$

6 Hours @ Operating Rate

Deliver carbody \& house, install side frames, install boom, wire \& block - boom up

24 Hours Up \& 24 Hours Down

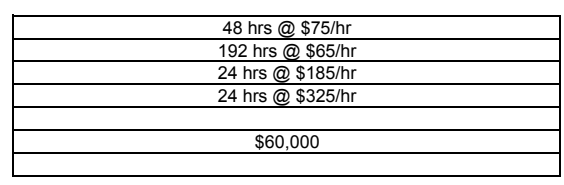

\begin{tabular}{|c|}
\hline 1 Ea @ $243 \mathrm{SqM}+1$ Ea. @ $110 \mathrm{SqM}=353 \mathrm{SqM}(3800 \mathrm{SqFt})$ \\
\hline $250 \mathrm{gal} / \mathrm{wk}$ \\
\hline
\end{tabular}
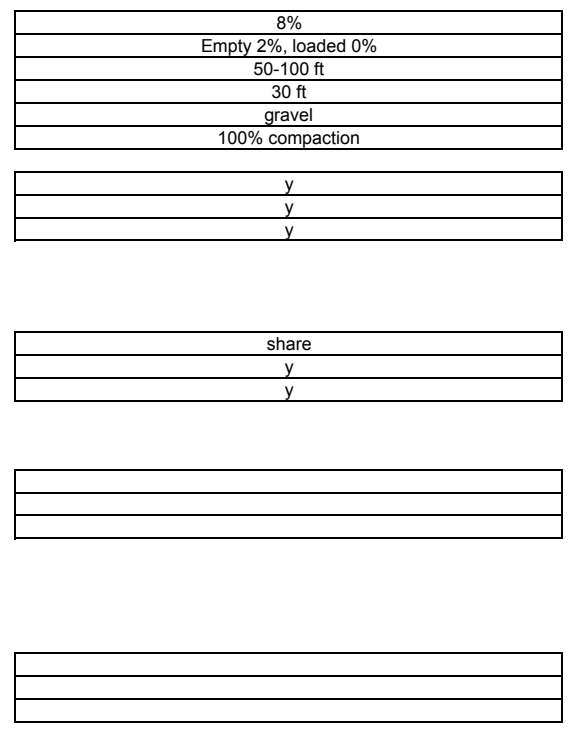
OPTION \# 1: This option will make use of 2 cranes per turbine. The first crane is assumed to start erecting the tower sections sufficiently ahead of the nacelle installation so as to produce a smooth schedule. At the client's option either the first crane can continue around behind the nacelle installation crane and ere the hub and blades or the nacelle erection crane can remain at each turbine until installation of the hub and blades is complete or some combination of each

Option \# 2: This option will make use of 1 crane per turbine. The crane will either fully complete each turbine before moving to the next or move from turbine to turbine after completing only a portion of each (l.e., erect the towers) before returning to the start and erecting the next items (I.e., erect the nacelle and rotor)

$1500 \mathrm{~kW}$--. OPTION\# 1

1) Brief Description Of Lifting Approach:

$1 \mathrm{a}$ - Number of cranes required per turbine

1c - Crane assignment

2) For Each Type Of Crane Identified Provide:

\begin{tabular}{|c|c|c|}
\hline 2a - Crane type, manufacturer \& model & Manitowoc - 4600 S5 & Lampson LTL - 600 \\
\hline \multirow{2}{*}{$2 b$ - Boom length, capacity \& reach } & $64,000 \mathrm{Kg} @ 16$ Meters (Boom) & 170,900 Kg @ 30 Meters (Jib) \\
\hline & 34,500 Kg @ 22 Meters (Jib) & \\
\hline \multirow{2}{*}{\multicolumn{3}{|c|}{$\frac{2 \mathrm{C} \text { - Description of crane operation }}{2 \mathrm{~d} \text { - Monthly Rental Costs (Bare rates - no labor) }}$}} \\
\hline & & \\
\hline 1 - Operating Rates (based on $176 \mathrm{hrs} / \mathrm{month}$ ) & $\$ 37,500$ & $\$ 90,000$ \\
\hline 2 - Stand-by Rates & $\$ 25,000$ & $\$ 60,000$ \\
\hline 3 - Overtime Rates (per hour) & $\$ 142$ & $\$ 341$ \\
\hline 2e - Manufactures specification sheets, tables or graphs & See Attachment 10.1.2 & See Attachment 10.1.2 \\
\hline 2f - Availability of equipment, scheduling requirements, lead time & 60 days & 120 days \\
\hline $2 \mathrm{~g}-$ Crane pad/Working area dimensions & \multicolumn{2}{|c|}{550 SqM -- shared crane pad } \\
\hline $2 \mathrm{~h}$ - Weather operations limits - temperature, wind, visibility, & Min. Temp. $-30 \mathrm{~F}$, Wind up to $\subseteq$ & $\mathrm{m} / \mathrm{s}(20 \mathrm{MPH})$, Good visibility \\
\hline
\end{tabular}

2) Crane Crew For Each Turbine Case:

2a - Crew size and responsibilities

$2 \mathrm{~b}$ - Estimated hourly crew costs (straight time)

2c - Estimated lodging/per diem costs (per diem)

$2 \mathrm{~d}$ - Travel costs (\$/mile)

\begin{tabular}{|c|c|}
\hline 2 Total - 1 Operator \& 1 Oiler & 3 Total - 3 Operators \\
\hline$\$ 130$ & $\$ 195$ \\
\hline$\$ 75$ & $\$ 75$ \\
\hline$\$ 1.25$ & $\$ 1.25$ \\
\hline
\end{tabular}

3) Crane Assembly:

3a - Estimated crane assembly costs

\begin{tabular}{|l|c|c|}
\hline 3b - On-site relocation time/logistics (Includes One Layer Crane Mat Pad) & $\$ 18,000$ & $\$ 65,000$ \\
\hline
\end{tabular}

4) Mobilization/Demobilization:

\begin{tabular}{|c|c|c|}
\hline 4 a - Brief Description Of Logistics & $\begin{array}{c}\text { Deliver carbody \& house, install } \\
\text { side frames, install boom/jib, wire } \\
\text { \& block \& boom up }\end{array}$ & $\begin{array}{c}\text { Deliver components, assemble } \\
\text { crawlers, assemble mast, strut } \\
\text { and stinger into triangle, install } \\
\text { boom/jib, wire \& block \& boom up }\end{array}$ \\
\hline $\begin{array}{l}\text { 4b-Schedule/time requirements } \\
4 \mathrm{c}-\text { Identify costs separately }\end{array}$ & 24 Hours UP \& 24 Hours Down & 80 Hours Up \& 80 Hours Down \\
\hline \multicolumn{3}{|l|}{ 4c - Identify costs separately } \\
\hline 1 - Lampson Supervisor & 48 hrs @ \$75/hr & $160 \mathrm{hrs} @ \$ 75 / \mathrm{hr}$ \\
\hline 2 - Iron Workers (number shown) & 192 hrs @ \$65/hr (4) & 960 hrs @ \$65/hr (6) \\
\hline $3-$ & & 年 \\
\hline 4 - (1) 150 ton truck crane & 48 hrs @ \$325/hr & 160 hrs @ \$325/hr \\
\hline $5-$ & & \\
\hline 4d - Fixed Costs - Transporation Freight To and From Project & $\$ 70,000$ & $\$ 120,000$ \\
\hline $4 \mathrm{e}$ - Mileage or time dependent costs & & \\
\hline
\end{tabular}

5) Materials And Consumables:

\begin{tabular}{|l|l|r|}
\hline $5 \mathrm{a}-$ Cribbing (One Layer Crane Mat Pad) @ \$2.50/sq ft & $2 \mathrm{Ea}$ @ @ 550 SqM = 1100 SqM (11,900 SqFt) \\
\hline $5 \mathrm{~b}-$ Fuel @ \$1.50/gal & $250 \mathrm{gal} / \mathrm{wk}$ & $650 \mathrm{gal} / \mathrm{wk}$ \\
\hline
\end{tabular}

6) Off-site And On-site Road Requirements:

$6 a$ - Road geometry

\begin{tabular}{|c|c|c|}
\hline 1 - Maximum grade, $\%$ & $8 \%$ & $8 \%$ \\
\hline 2 - Maximum road crown, $M$ & Empty $2 \%$, loaded $0 \%$ & Empty $2 \%$, loaded $0 \%$ \\
\hline 3- Minimum curve radius & $50-100 \mathrm{ft}$ & $50-100 \mathrm{ft}$ \\
\hline 4 - Minimum road width, $M$ & $30 \mathrm{ft}$ & $30 \mathrm{ft}$ \\
\hline 6b - Surface limitations (gravel, asphalt) & gravel & gravel \\
\hline 6c - General road design/loading requirements & $100 \%$ compaction & $100 \%$ compaction \\
\hline \multicolumn{3}{|l|}{$6 \mathrm{~d}$ - Off-site equipment transportation requirements } \\
\hline 1- Oversized load permits required & $y$ & $y$ \\
\hline 2- Route planning required & $\mathrm{y}$ & $\mathrm{y}$ \\
\hline 3 - Escorts required & $y$ & $\mathrm{y}$ \\
\hline
\end{tabular}

7) Job Site Facility Needs

$7 a-$ Site Office/storage

1 - Site Office

2 - Site Storage

3 - Site Amenities (Lunchroom, Toilets, etc.)
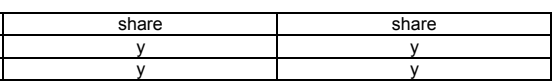

8) General Industry Overhead, Fees and Profit:

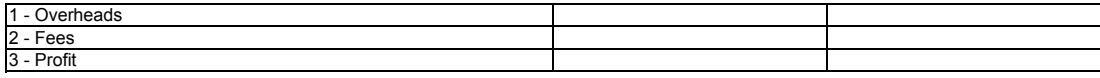

9) Overall Project Crane Costs Per Facility:

9a - Combine crew, equipment, material, expendables, mob/demob, overhead,

profit, etc. into total costs per facility and per turbine

1 - Total cost per facility (50 turbines farm)

$9 b$ - Provide total sch

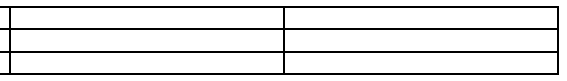

$1500 \mathrm{~kW}$--- OPTION \# 2
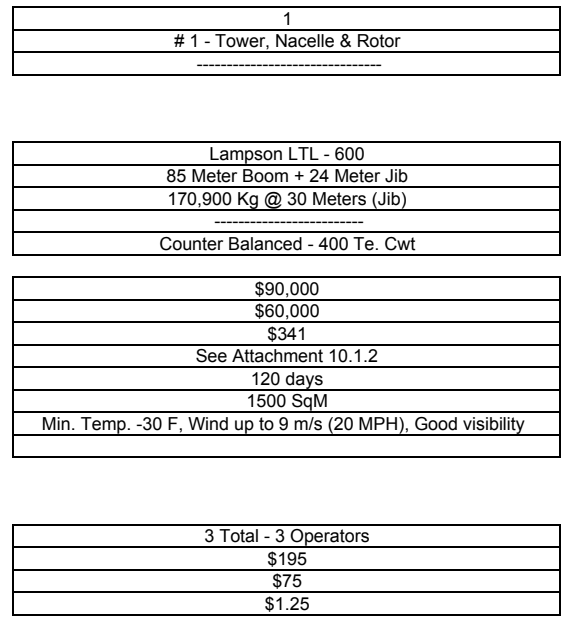

$\$ 50,000$

21 Hours @ Operating Rate

\begin{tabular}{|c|}
\hline $\begin{array}{l}\text { Deliver components, assemble crawlers, assemble mast, strut and } \\
\text { stinger into triangle, install boom/jib, wire \& block \& boom up }\end{array}$ \\
\hline 80 Hours UP \& 80 Hours Down \\
\hline $160 \mathrm{hrs} @ \$ 75 / \mathrm{hr}$ \\
\hline $960 \mathrm{hrs} @ \$ 75 / \mathrm{hr}(6)$ \\
\hline- \\
\hline $160 \mathrm{hrs} @ \$ 325 / \mathrm{hr}$ \\
\hline \\
\hline \\
\hline
\end{tabular}
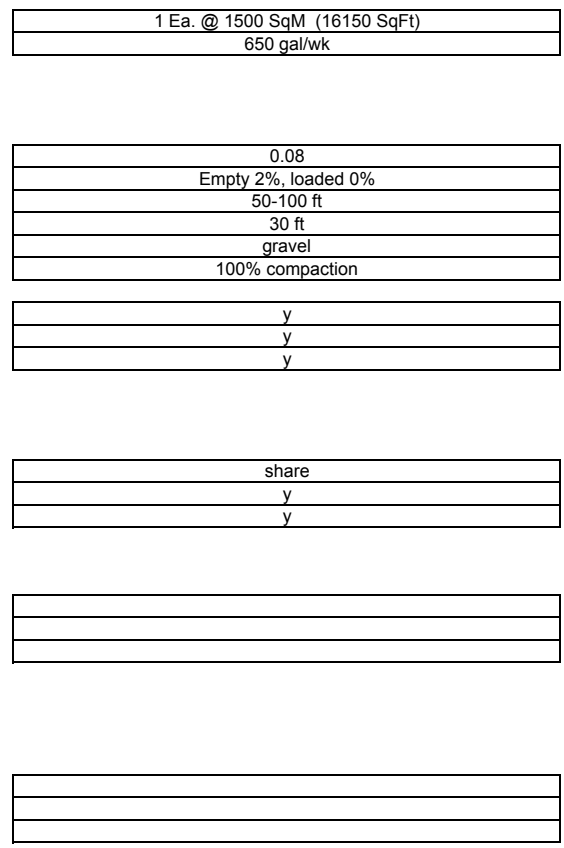
OPTION \# 1: This option will make use of 2 cranes per turbine. The first crane is assumed to start erecting the tower sections sufficiently ahead of the nacelle installation so as to produce a smooth schedule. At the client's option either the first crane can continue around behind the nacelle installation crane and erect the hub and blades or the nacelle erection crane can remain at each turbine until installation of the hub and blades is complete or some combination of each.

Option \# 2: This option will make use of 1 crane per turbine. The crane will either fully complete each turbine before moving to the next or move from turbine to turbine after completing only a portion of each (I.e., erect the towers) before returning to the start and erecting the next items (I.e., erect the nacelle and rotor).

$2500 \mathrm{~kW}$--- OPTION \# 1

1) Brief Description Of Lifting Approach:

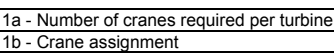

1c - Alternative lifting approach, if applicable

2) For Each Type Of Crane Identified Provide:

\begin{tabular}{|c|c|c|}
\hline 2a - Crane type, manufacturer \& model & Lampson LTL - 850 & Lampson LTL - 850 \\
\hline \multirow[t]{4}{*}{ 2b- Boom length, capacity \& reach } & 98 Meter Boom + 37 Meter Jib & 98 Meter Boom +37 Meter Jib \\
\hline & $207,400 \mathrm{Kg} @ 34$ Meters (Jib) & $207,400 \mathrm{Kg} @ 34$ Meters (Jib) \\
\hline & & \\
\hline & Counter-Balanced - 600 Te. Cwt. & Counter-Balanced - 600 Te. Cwt. \\
\hline \multicolumn{3}{|l|}{ 2d - Monthly Rental Costs (Bare rates - no labor) } \\
\hline 1 - Operating Rates (based on 176 hrs/month) & $\$ 100,000$ & $\$ 100,000$ \\
\hline 2 - Stand-by Rates & $\begin{array}{lll}\$ 100,6006 \\
\$ 66,666\end{array}$ & $\begin{array}{lll}\$ 100,6006 \\
\$ 66,666\end{array}$ \\
\hline 3 - Overtime Rates (per hour) & $\$ 379$ & $\$ 379$ \\
\hline $2 \mathrm{e}-$ Manufactures specification sheets, tables or graphs & See Attachment 10.1.3 & See Attachment 10.1.3 \\
\hline $2 f$ - Availability of equipment, scheduling requirements, lead time & 120 days & 120 days \\
\hline $2 \mathrm{~g}$ - Crane pad/Working area dimensions & \multicolumn{2}{|c|}{$1000 \mathrm{SqM}$} \\
\hline 2h - Weather operations limits - temperature, wind, visibility, preci & Min. Temp. - $30 \mathrm{~F}$, Wind up & $\overline{\mathrm{MM}}$ \\
\hline $2 i$ - Other operation limits & Verify Training of Operatin & Verify Training \\
\hline
\end{tabular}

2) Crane Crew For Each Turbine Case:

2a - Crew size and responsibilities

$2 \mathrm{~b}$ - Estimated hourly crew costs (straight time)

2c - Estimated lodging/per diem costs (per person)

$2 \mathrm{~d}$ - Travel costs (\$/mile)

3) Crane Assembly:

3a- Estimated crane assembly costs 3b - On-site relocation time/logistics (Includes One Layer Crane Mat Pad)

4) Mobilization/Demobilization:

\begin{tabular}{|c|c|c|}
\hline 4a - Brief Description Of Logistics & $\begin{array}{c}\text { Deliver components, assemble } \\
\text { crawlers, assemble mast, strut and } \\
\text { stinger into triangle, install boom/jib, } \\
\text { wire \& block \& boom up }\end{array}$ & \begin{tabular}{|c|} 
Deliver components, assemble \\
crawlers, assemble mast, strut and \\
stinger into triangle, install \\
boom/jib, wire \& block \& boom up
\end{tabular} \\
\hline $4 \mathrm{~b}-$ Schedule/time requirements & 96 Hours UP \& 96 Hours Down & 96 Hours Up \& 96 Hours Down \\
\hline \multicolumn{3}{|l|}{ 4c - Identify costs separately } \\
\hline 1 - Lampson Supervisor & 192 hrs @ \$75/hr & 192 hrs @ \$75/hr \\
\hline 2 - Iron Workers & 1152 hrs @ \$65/hr & $1152 \mathrm{hrs} @ \$ 65 / \mathrm{hr}$ \\
\hline $3-$ & & \\
\hline 4 - (1) 175 ton truck crane & 144 hrs @ \$350/hr & 144 hrs @ \$350/hr \\
\hline $5-50$ ton hydraulic crane & $52 \mathrm{hrs} @ \$ 185 / \mathrm{hr}$ & 52 hrs @ \$185/hr \\
\hline 4d - Fixed Costs - Transporation Freight To and From Project & $\$ 120,000$ & $\$ 120,000$ \\
\hline $4 \mathrm{e}$ - Mileage or time dependent costs & & \\
\hline
\end{tabular}

5) Materials And Consumables:

5a - Cribbing (One Layer Crane Mat Pad) @ \$2.50/sq ft $5 \mathrm{~b}$ - Fuel @ \$1.50/gal

6) Off-site And On-site Road Requirements:

$6 a$ - Road geometr

1 - Maximum grade, $\%$

2 - Maximum road crown, $M$

3- Minimum vertical curve, $M$

4-Minimum road width, $M$

$6 \mathrm{~b}$ - Surface limitations (gravel, asphalt)

6c - General road design/loading requirements

6d - Off-site equipment transportation requiremen

1- Oversized load permits require

2- Route planning required

3 - Escorts required

7) Job Site Facility Needs:

7 a - Site Office/storag

\begin{tabular}{|l|}
\hline - Site Office \\
\hline 2 - Site Storage \\
\hline - Sit Amanits
\end{tabular}

3 - Site Amenities (Lunchroom, Toilets, etc.)
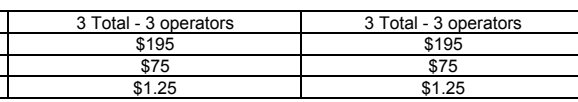

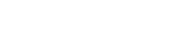

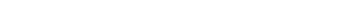

\begin{tabular}{|l|l|}
\hline$\$ 75,000$ & $\$ 75,000$ \\
\hline 7 Hours & 7 Hours \\
\hline
\end{tabular}

General Industry Overhead, Fees and Profit:

\begin{tabular}{|l|}
\hline 1 - Overheads \\
\hline 2 - Fees \\
\hline 3 -Profit \\
\hline
\end{tabular}

9) Overall Project Crane Costs Per Facility:

9 a - Combine crew, equipment, material, expendables, mob/demob,

overhead, profit, etc. into total costs per facility and per turbine

1 - Total cost per facility (50 turbines farm)

2 - Total cost per turbine

$9 \mathrm{~b}$ - Provide total schedule per facility

\section{$2500 \mathrm{~kW}$--- OPTION \# 2}
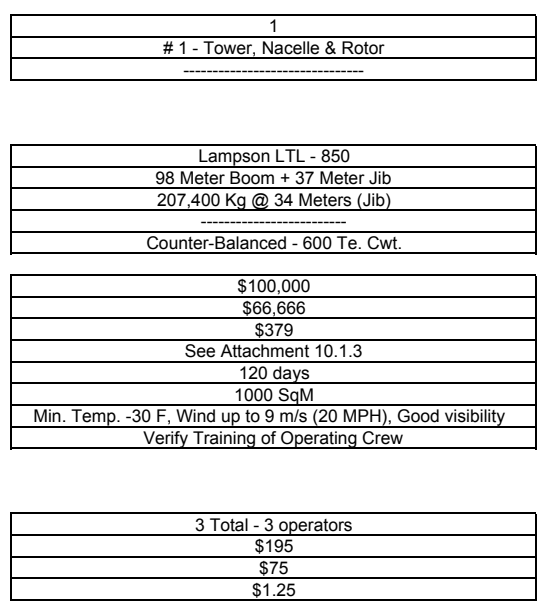

$\$ 75,000$
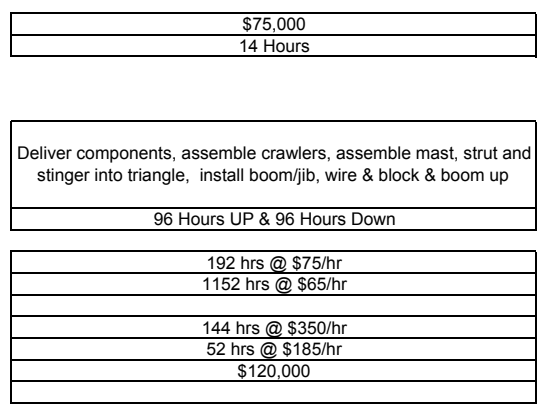

1 Ea. @ $1000 \mathrm{SqM}(10750 \mathrm{SqFt})$
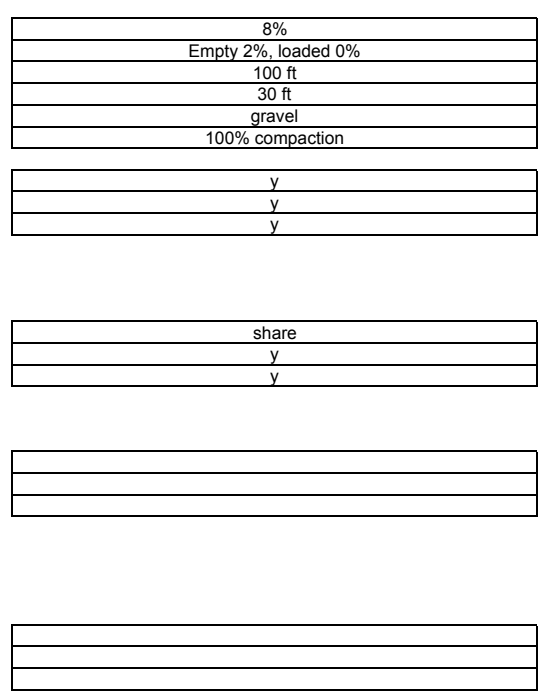
OPTION \# 1: This option will make use of 2 cranes per turbine. The first crane is assumed to start erecting the tower sections sufficiently ahead of the nacelle installation so as to produce a smooth schedule. At the client's option either the first crane can continue around behind the nacelle installation crane and erec the hub and blades or the nacelle erection crane can remain at each turbine until installation of the hub and blades is complete or some combination of each

Option \# 2: This option will make use of 1 crane per turbine. The crane will either fully complete each turbine before moving to the next or move from turbine to turbine after completing only a portion of each (l.e., erect the towers) before returning to the start and erecting the next items (I.e., erect the nacelle and rotor)

3500 kW --- OPTION \# 1

1) Brief Description Of Lifting Approach:

$1 \mathrm{a}$ - Number of cranes required per turbine 1b - Crane assignment

1c- Alternative lifting approach, if applicable

2) For Each Type Of Crane Identified Provide:

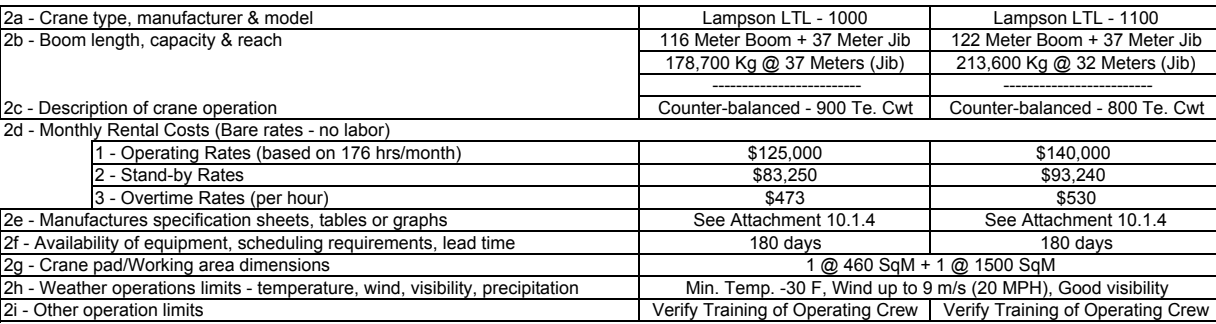

2) Crane Crew For Each Turbine Case:

2a - Crew size and responsibilities

$2 \mathrm{~b}$ - Estimated hourly crew costs (straight time)

2c - Estimated lodging/per diem costs (per person)

$2 \mathrm{~d}$ - Travel costs $(\$ / \mathrm{mile})$

\section{3) Crane Assembly:} \begin{tabular}{l}
$3 \mathrm{a}$ - Estimated crane assembly costs \\
\hline 3b - On-site relocation time/logistics (Includes One Layer Crane Mat Pad)
\end{tabular}

\begin{tabular}{|c|c|}
\hline \multicolumn{3}{|c|}{2} \\
\hline \# 1 - Tower \& Rotor & \# 2 - Nacelle \\
\hline-------- \\
\hline
\end{tabular}

3500 kW --- OPTION \# 2
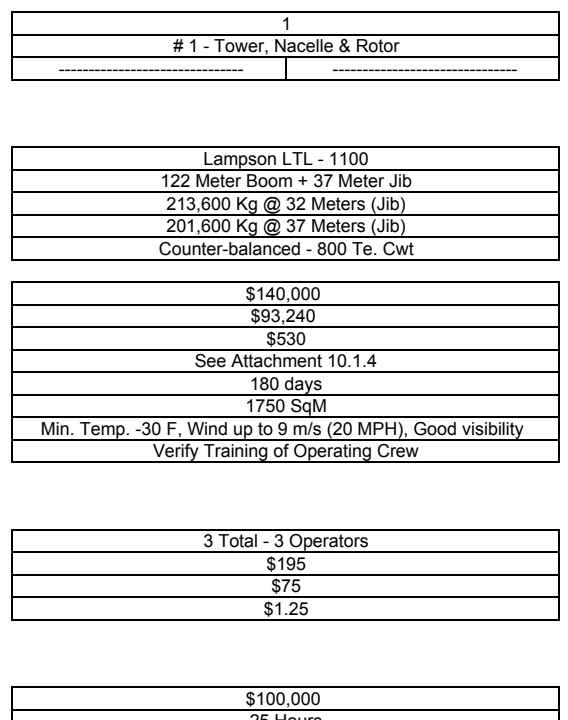

25 Hours

4) Mobilization/Demobilization:

\begin{tabular}{|c|c|c|}
\hline $4 a$ - Brief Description Of Logistics & $\begin{array}{c}\text { Deliver components, assemble } \\
\text { crawlers, assemble mast, strut and } \\
\text { stinger into triangle, install } \\
\text { boom/jib, wire \& block \& boom up } \\
180 \text { Hours UP \& } 180 \text { Hours Down }\end{array}$ & \begin{tabular}{|c|} 
Deliver components, assemble \\
crawlers, assemble mast, strut and \\
stinger into triangle, install \\
boom/jib, wire \& block \& boom up \\
180 Hours UP \& 180 Hours Down \\
\end{tabular} \\
\hline \multicolumn{3}{|l|}{ 4c - Identify costs separately } \\
\hline 1 - Lampson Supervisor & 360 hrs@ @ $75 / \mathrm{hr}$ & 360 hrs @ \$75/hr \\
\hline 2 - Iron Workers (8) & $2880 \mathrm{hrs} @ \$ 65 / \mathrm{hr}$ & $2880 \mathrm{hrs} @ \$ 65 / \mathrm{hr}$ \\
\hline $3-$ & & \\
\hline 4 - (1) 200 ton crawler crane & $120 \mathrm{hrs} @ \$ 350 / \mathrm{hr}$ & $120 \mathrm{hrs} @ \$ 350 / \mathrm{hr}$ \\
\hline 5 - (2) 50 ton hydraulic truck cranes & $240 \mathrm{hrs} @ \$ 185 / \mathrm{hr}$ & $240 \mathrm{hrs} @ \$ 185 / \mathrm{hr}$ \\
\hline $4 \mathrm{~d}$ - Fixed Costs - Transporation Freight To and From Project & $\$ 200,000$ & $\$ 220,000$ \\
\hline
\end{tabular}

$4 \mathrm{e}$ - Mileage or time dependent costs

5) Materials And Consumables:

\begin{tabular}{|l|c|c|}
\hline $5 a-$ Cribbing (One Layer Crane Mat Pad) @ \$2.50/sq ft & 1 Ea. @ 460 SqM + 1 Ea. @ 1500 SqM = 1960 SqM (21,100 SqFt) \\
\hline $5 \mathrm{~b}-$ Fuel @ \$1.50/gal & $700 \mathrm{gal} / \mathrm{wk}$ & $700 \mathrm{gal} / \mathrm{wk}$ \\
\hline
\end{tabular}

\section{6) Off-site And On-site Road Requirements:}

$6 a$ - Road geometry

\begin{tabular}{|c|c|c|}
\hline 1 - Maximum grade, $\%$ & $8 \%$ & $8 \%$ \\
\hline 2 - Maximum road crown, $M$ & Empty $2 \%$, loaded $0 \%$ & Empty $2 \%$, loaded $0 \%$ \\
\hline 3- Minimum vertical curve, $M$ & $120 \mathrm{ft}$ & $120 \mathrm{ft}$ \\
\hline 4 - Minimum road width, $M$ & $32 \mathrm{ft}$ & $32 \mathrm{ft}$ \\
\hline 6b - Surface limitations (gravel, asphalt) & gravel & gravel \\
\hline $6 \mathrm{c}$ - General road design/loading requirements & $100 \%$ compaction & $100 \%$ compaction \\
\hline \multicolumn{3}{|l|}{$6 \mathrm{~d}$ - Off-site equipment transportation requirements } \\
\hline 1- Oversized load permits required & $y$ & $y$ \\
\hline 2-Route planning required & $\mathrm{y}$ & $\mathrm{y}$ \\
\hline 3 - Escorts required & $\mathrm{y}$ & $\mathrm{y}$ \\
\hline
\end{tabular}

\section{7) Job Site Facility Needs:}

7 a - Site Office/storage

1 - Site Office

2 - Site Storage

3 - Site Amenities (Lunchroom, Toilets, etc.)

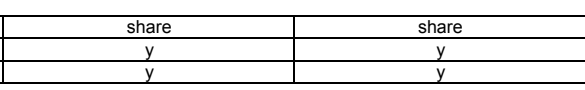

8) General Industry Overhead, Fees and Profit:

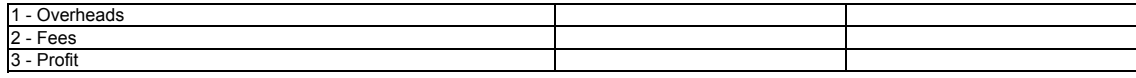

9) Overall Project Crane Costs Per Facility:

$9 a$ - Combine crew, equipment, material, expendables, mob/demob, overhead,

profit, etc. into total costs per facility and per turbine

1 - Total cost per facility (50 turbines farm)

$9 b-$ Provide tolalschedule perfacily

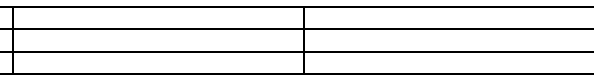

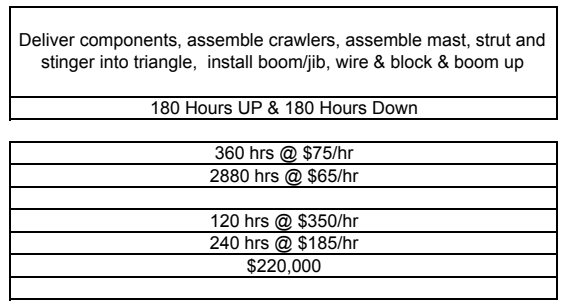
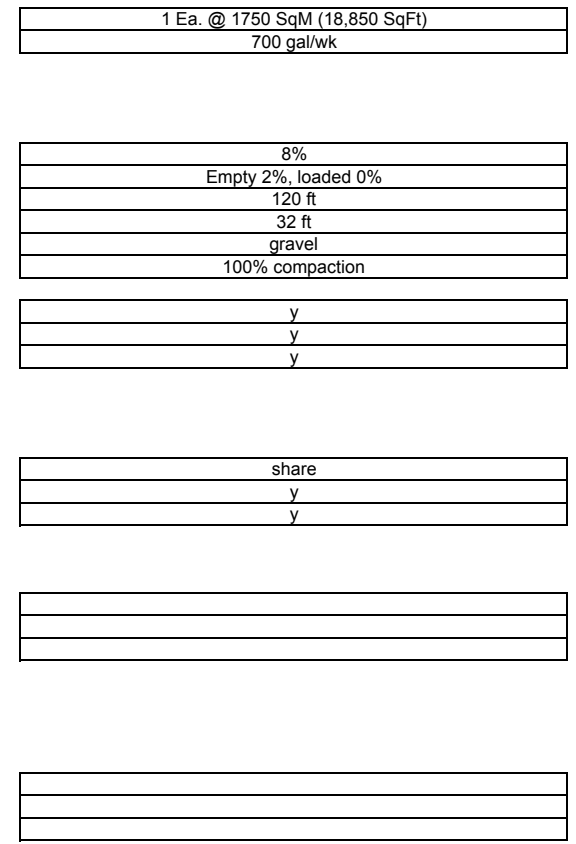
OPTION \# 1: This option will make use of 2 cranes per turbine. The first crane is assumed to start erecting the tower sections sufficiently ahead of the nacelle installation so as to produce a smooth schedule. At the client's option either the first crane can continue around behind the nacelle installation crane and erect the hub and blades or the nacelle erection crane can remain at each turbine until installation of the hub and blades is complete or some combination of each.

Option \# 2: This option will make use of 1 crane per turbine. The crane will either fully complete each turbine before moving to the next or move from turbine to turbine after completing only a portion of each (l.e., erect the towers) before returning to the start and erecting the next items (l.e., erect the nacelle and rotor).

$5000 \mathrm{~kW}---$ OPTION \# 1

1) Brief Description Of Lifting Approach

$1 \mathrm{a}$ - Number of cranes required per turbine 1b - Crane assignment

1c - Alternative lifting approach, if applicable

5000 kW --- OPTION \#

5000 kW --- OPTION \# 2

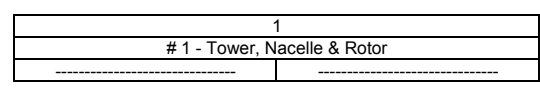

2) For Each Type Of Crane Identified Provide:

2a - Crane type, manufacturer \& model

$2 \mathrm{~b}$ - Boom length, capacity \& reach

2c - Description of crane operation

$2 \mathrm{~d}$ - Monthly Rental Costs (Bare rates - no labor)

1 - Operating Rates (based on $176 \mathrm{hrs} / \mathrm{month}$ )

2 - Stand-by Rates

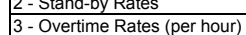

$2 \mathrm{e}$ - Manufactures specification sheets, tables or graphs

2f - Availability of equipment, scheduling requirements, lead time

$2 \mathrm{~g}$ - Crane pad/Working area dimensions

$2 \mathrm{~h}$ - Weather operations limits - temperature, wind, visibility, precipitation

$2 i$ - Other operation limits

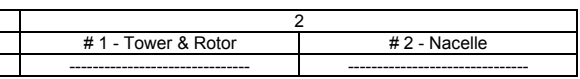

2) Crane Crew For Each Turbine Case:

$2 a$ - Crew size and responsibilities

$2 \mathrm{~b}$ - Estimated hourly crew costs (straight time)

$2 \mathrm{c}$ - Estimated lodging/per diem costs (per person)

$2 \mathrm{~d}$ - Travel costs (\$/mile)

3) Crane Assembly:

\begin{tabular}{l} 
3a - Estimated crane assembly costs \\
\hline 3b - On-site relocation time/logistics (Includes One Layer Crane Mat Pad)
\end{tabular}

$\$ 190,000$
33 Hours

\begin{tabular}{c|c|}
\hline Lampson LTL - 1100 & Lampson LTL - 1200 SII A \\
\hline
\end{tabular} 122 Meter Boom + 55 Meter Jib 122 Meter Boom + 73 Meter Jib $307,800 \mathrm{Kg} @ 28$ Meters (Boom) 410,300 Kg @ 50 Meters (Jib) $168,000 \mathrm{Kg} @ 40$ Meters (Jib) 410,300 Kg @ 50 Meters (Jib) Counter-balanced - 1000 Te. Cwt. Counter-balanced - 1600 Te. Cwt.

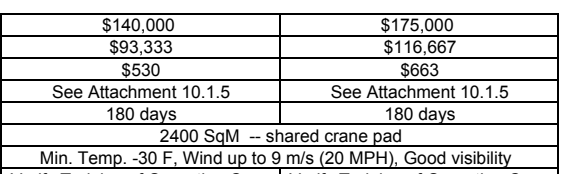
\begin{tabular}{|l|l|l|}
\hline & Verify Training of Operating Crew & Verify Training of Operating Crew \\
\hline
\end{tabular}

4) Mobilization/Demobilization:

\begin{tabular}{|c|c|c|}
\hline 4a - Brief Description Of Logistics & $\begin{array}{l}\text { Deliver components, assemble } \\
\text { crawlers, assemble mast, strut and } \\
\text { stinger into triangle, install } \\
\text { boom/jib, wire \& block \& boom up } \\
\end{array}$ & \begin{tabular}{|c|} 
Deliver components, assemble \\
crawlers, assemble mast, strut and \\
stinger into triangle, install \\
boom/jib, wire \& block \& boom up \\
\end{tabular} \\
\hline $4 \mathrm{~b}-$ Schedule/time requirements & 180 Hours UP \& 180 Hours Down & 240 Hours Up $\& 240$ Hours Down \\
\hline \multicolumn{3}{|l|}{ 4c - Identify costs separately } \\
\hline 1 - Lampson Supervisor & 360 hrs @ \$75/hr & $480 \mathrm{hrs} @ \$ 75 / \mathrm{hr}$ \\
\hline 2 - Iron Workers (8 to 10$)$ & 2880 hrs @ \$65/hr (8) & 4800 hrs @ \$65/hr (10) \\
\hline \multicolumn{3}{|l|}{$3-$} \\
\hline 4 - (1) 200 ton crawler crane & 80 hrs @ \$350/hr & - \\
\hline 5 - (1) 230 ton crawler crane & & $100 \mathrm{hrs} @ \$ 400 / \mathrm{hr}$ \\
\hline $6-50$ ton hydraulic crane (1 to 2 ) & $160 \mathrm{hrs} @ \$ 185 / \mathrm{hr}$ & 280 hrs @ \$185 \\
\hline $4 \mathrm{~d}$ - Fixed Costs - Transporation Freight To and From Project & $\$ 220,000$ & $\$ 250,000$ \\
\hline $4 \mathrm{e}-$ Mileage or time depend & & \\
\hline
\end{tabular}

5) Materials And Consumables:

5 a - Cribbing (One Layer Crane Mat Pad) @ \$2.50/sq ft $5 \mathrm{~b}$ - Fuel @ \$1.50/gal 2 Ea. @ $2400 \mathrm{SqM}=4800 \mathrm{SqM}(51,700 \mathrm{SqFt})$
\begin{tabular}{l|}
$750 \mathrm{gal} / \mathrm{wk}$ \\
$750 \mathrm{gal} / \mathrm{wk}$
\end{tabular}

6) Off-site And On-site Road Requirements:

6 - Road geometry

1 - Maximum grade, $\%$

2 - Maximum road crown, $M$

3- Minimum vertical curve, $M$

4 - Minimum road width, $M$

$6 \mathrm{~b}$ - Surface limitations (gravel, asphalt

6c - General road design/loading requirements

$6 \mathrm{~d}$ - Off-site equipment transportation requirements

1- Oversized load permits required

1- Oversized load permits requte planning required

3 - Escorts required

7) Job Site Facility Needs:

7a - Site Office/storage

1 - Site Office

\begin{tabular}{l}
1 - Site Office \\
\hline 2 - Site Storage \\
\hline 3 - Site Amenies
\end{tabular}

3 - Site Amenities (Lunchroom, Toilets, etc.)

otal - 3 Operators

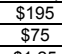

3 Total -3 Operators

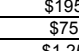

\section{5}

(2)

8) General Industry Overhead, Fees and Profit:

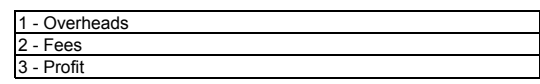

\begin{tabular}{|c|c|c|}
\hline $8 \%$ & $8 \%$ \\
\hline Empty 2\%, loaded 0\% & Empty 2\%, loaded 0\% \\
\hline $120 \mathrm{ft}$ & $120 \mathrm{ft}$ \\
\hline gravel & $40 \mathrm{ft}$ \\
\hline & gravel \\
\hline & $100 \%$ compaction & $100 \%$ compaction \\
\hline
\end{tabular}

$100 \%$ compaction

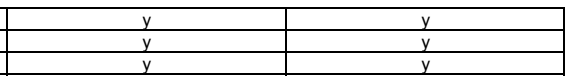

2 - Fees

9) Overall Project Crane Costs Per Facility:

9 a - Combine crew, equipment, material, expendables, mob/demob

overhead, profit, etc. into total costs per facility and per turbine

1 - Total cost per facility (50 turbines farm)

2 - Total cost per turbine

$9 \mathrm{~b}$ - Provide total schedule per facility
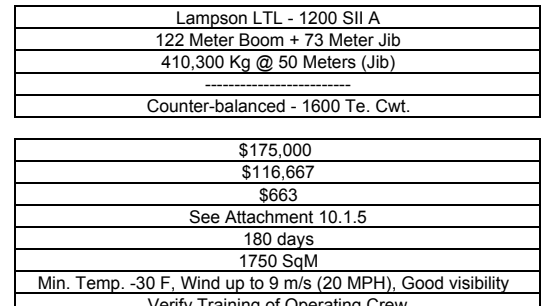

Verify Training of Operating Crew
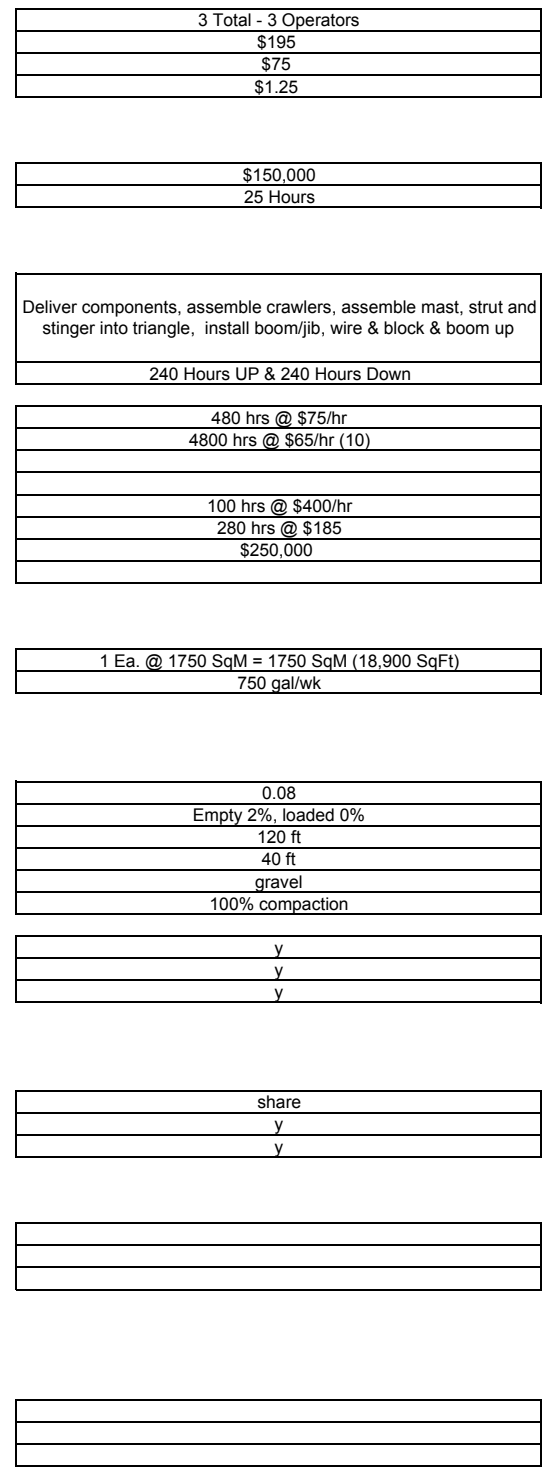
Appendix S

Crane Purchase Evaluation 


\section{Desired Analysis:}

1) Evaluate the costs of crane purchase in comparison to their rental costs.

2) Amortize the costs over the total wind farm and others in the region for erection and maintenance.

\section{Approach:}

1) Identify crane types, rental costs, purchase costs, and annual service/maintenance costs.

2) Evaluate differences between installation/erection activity and $O \& M$ activity. Differences might result in the use of different cranes.

3) Utilizing the numerator portion of the COE equation, determine the required annual crane usage that results in no impact to the COE.

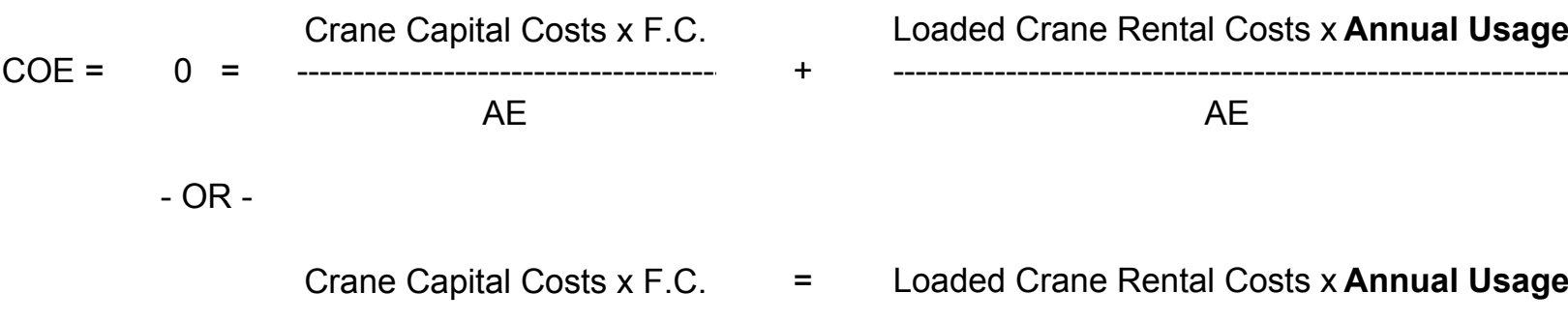

4) Solve for Annual Usage and evaluate the result to determine if such usage rates could be reasonably expected at wind energy facilities.

5) Assumed Fixed Charge Rate of $10 \%$. 
NOTE: The crane models (types) represent 'service' cranes capable of blade removal, gearbox removal, generator removal (activities typically conducted during $\mathrm{O} \& \mathrm{M}$ ). Intact nacelle removal would require the original installation crane. Costs for the original installation crane have not been evaluated but would be greater than those shown for each turbine size.

\begin{tabular}{|c|c|c|c|c|c|c|}
\hline Turbine & $\overline{\mathrm{kWW}}$ & $\overline{750}$ & $\overline{1500}$ & $\overline{2500}$ & $\overline{3500}$ & $\overline{5000}$ \\
\hline Rotor Dia & $\mathrm{m}$ & 50 & 66 & 85 & 100 & 120 \\
\hline O \& M Crane Type & & $4100-S 1$ & $4600-54$ & LTL-600 & LTL-600 & LTL-1000 \\
\hline Crane Purchase Costs & & $\$ 1,150,000$ & $\$ 2,250,000$ & $\$ 3,500,000$ & $\$ 3,500,000$ & $\$ 6,500,000$ \\
\hline Fixed Charge Rate & & 0.1 & 0.1 & 0.1 & 0.1 & 0.1 \\
\hline Capital Costs x F.C. & & $\$ 115,000$ & $\$ 225,000$ & $\$ 350,000$ & $\$ 350,000$ & $\$ 650,000$ \\
\hline Loaded Hourly Operation Rate & & $\$ 375$ & $\$ 490$ & $\$ 920$ & $\$ 920$ & $\$ 1,030$ \\
\hline Annual Usage & hrs & 307 & 459 & 380 & 380 & 631 \\
\hline Annual Usage & Months & 1.7 & 2.6 & 2.2 & 2.2 & 3.6 \\
\hline Total Usage for 20 year project & hrs & 6133 & 9184 & 7609 & 7609 & 12621 \\
\hline Usage every 3 years: & Months & 5 & 8 & 6 & 6 & 11 \\
\hline Usage every 5 years & Months & 9 & 13 & 11 & 11 & 18 \\
\hline
\end{tabular}

${ }^{1}$ Hourly operation rates based on detailed crane cost estimates presented in Scenario 1 (typical costs) and include all costs from rental yard to site work and back to rental yard.

\section{Conclusions:}

Using the $750 \mathrm{~kW}$ turbine as an example:

1) In order for crane purchase to be cheaper than rental, the annual crane usage would need to be greater than 307 hrs - for each year of the 20 year project life. Or almost 2 months of crane work would be needed each year. This usage rate is most likely not achievable at one project with 50 turbines.

2) Annual crane service/maintenance costs are not included (nor readily available from Lampson). If the present value of these annual costs were added to the capital costs, the necessary annual usage to justify crane purchase would increase further (which is undesirable).

3) 'Reasonable' crane usage could be 1 month a year or less for a given project.

4) Incorporating crane usage at other projects in the region is highly dependant upon the assumptions. However, given the value of cranes to the wind industry, it is conceivable that the annual usage rates could be met for 20 years if one crane was available for 3 to 4 projects with 50 turbines. This assumption applies to projects with turbines of similar sizes or a very large crane for MW scale turbines could handle smaller turbines.

5) If a crane sized for one project of multi-megawatt scale turbines (2.5 - $5 \mathrm{MWs}$ ) is used at neighboring projects consisting of smaller turbines (750 kW), the time for crane mob, set-up, relocation, tear-down, and demob could be prohibitively long in comparison to rental of a crane sized for $750 \mathrm{~kW}$ turbines.

6) Generally most crane work will take place during a relatively small window of low wind months. It's likely that scheduling a crane work for 3 or 4 projects may not be possible, resulting in 1 or more projects needing to rent their own. 
Public reporting burden for this collection of information is estimated to average 1 hour per response, including the time for reviewing instructions, searching existing data sources,

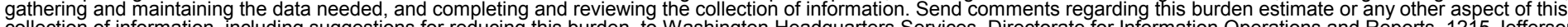
Davis Highway, Suite 1204, Arlington, VA 22202-4302, and to the Office of Management and Budget, Paperwork Reduction Project (0704-0188), Washington, DC 20503.

\begin{tabular}{|l|l|l} 
1. AGENCY USE ONLY (Leave blank) & $\begin{array}{l}\text { 2. REPORT DATE } \\
\text { June 2001 }\end{array}$ & $\begin{array}{l}\text { 3. REPORT TYPE AND DATES COVERED } \\
\text { Subcontract report }\end{array}$ \\
\hline
\end{tabular}

4. TITLE AND SUBTITLE

WindPACT Turbine Design Scaling Studies Technical Area 2: Turbine, Rotor, and Blade Logistics
5. FUNDING NUMBERS

YAM-0-30203-01

6. $\mathrm{AUTHOR}(\mathrm{S})$

Kevin Smith

7. PERFORMING ORGANIZATION NAME(S) AND ADDRESS(ES)

Global Energy Concepts LLC

5729 Lakeview Drive NE, \#100

Kirkland, WA 98033

9. SPONSORING/MONITORING AGENCY NAME(S) AND ADDRESS(ES)

National Renewable Energy Laboratory

1617 Cole Blvd.

Golden, CO 80401-3393
8. PERFORMING ORGANIZATION REPORT NUMBER

\section{SUPPLEMENTARY NOTES}

NREL Technical Monitor: Alan Laxson

12a. DISTRIBUTION/AVAILABILITY STATEMENT National Technical Information Service

U.S. Department of Commerce

5285 Port Royal Road

Springfield, VA 22161

13. ABSTRACT (Maximum 200 words) Through the National Renewable Energy Laboratory (NREL), the United States Department of Energy (DOE) implemented the Wind Partnership for Advanced Component Technologies (WindPACT) program. This program will explore advanced technologies that may reduce the cost of energy (COE) from wind turbines. The initial step in the WindPACT program is a series of preliminary scaling studies intended to determine the optimum sizes for future turbines, help define sizing limits for certain critical technologies, and explore the potential for advanced technologies to contribute to reduced COE as turbine scales increase. This report documents the results of Technical Area 2-Turbine Rotor and Blade Logistics. For this report, we investigated the transportation, assembly, and crane logistics and costs associated with installation of a range of multi-megawatt-scale wind turbines. We focused on using currently available equipment, assembly techniques, and transportation system capabilities and limitations to hypothetically transport and install 50 wind turbines at a facility in south-central South Dakota.

14. SUBJECT TERMS

15. NUMBER OF PAGES

Wind energy; turbine; transportation; research; WindPACT,

16. PRICE CODE

17. SECURITY CLASSIFICATION OF REPORT

Unclassified
18. SECURITY CLASSIFICATION OF THIS PAGE Unclassified
19. SECURITY CLASSIFICATION OF ABSTRACT Unclassified
20. LIMITATION OF ABSTRACT

UL 